\title{
Investigating the antinociceptive effects of $N$-docosahexaenoyl ethanolamine and novel kappa opioid receptor agonists
}

\section{Kelly Frances Paton}

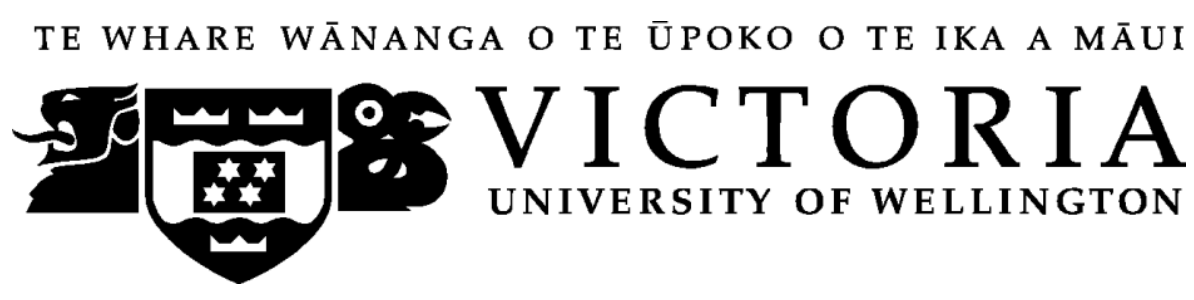

School of Biological Sciences

Te kura Matauranga Koiora

A thesis submitted to the Victoria University of Wellington in fulfilment of the requirements for the degree of Doctor of Philosophy 



\section{Abstract}

Chronic pain causes patients to endure prolonged suffering and discomfort, often having profound effects on quality of life. In New Zealand, one in five people currently suffer from chronic pain. To treat chronic pain, patients are typically prescribed drugs that activate the mu opioid receptor (MOPr), such as morphine, codeine and oxycodone. In recent years in the United States of America, there has been a rapid increase in the use of prescription and non-prescription opioid drugs, with opioid overdoses now the leading cause of accidental death. In New Zealand, daily doses of prescription opioids quadrupled in the ten year period from 2001-2011. Clearly, there is a need for the development of more effective and safe medications. This thesis evaluated two classes of non-addictive compounds: bioactive lipids and kappa opioid receptor (KOPr) agonists.

$\mathrm{N}$-docosahexaenoyl ethanolamine (DHEA) is an $\mathrm{N}$-acyl ethanolamine class lipid that is structurally similar to the endocannabinoid anandamide. DHEA has previously been shown to have immunemodulatory effects in vitro, however, the in vivo effects have not previously been tested. Using the intraplantar $2 \%$ formaldehyde model in mice, DHEA reduced inflammatory and nociceptive pain via both intraperitoneal (i.p.) and local intraplantar (i.pl.) administration. DHEA significantly reduced formaldehyde-induced footpad oedema and reduced the infiltration of neutrophils into the inflamed tissue. The antinociceptive and anti-oedematous effects were not modulated by pre-treatment with either cannabinoid 1- or 2-type receptor antagonists. DHEA did not have any effect in a thermal nociceptive pain model and did not show any motor coordination impairment or changes in thermoregulation.

In the search for non-addictive analgesics, KOPr agonists are a promising alternative. In contrast to MOPr agonists, KOPr agonists play a critical role in regulating the reward system. Salvinorin $A(S a I A)$ is a selective KOPr agonist that has antinociceptive and anti-inflammatory effects in vivo, with limited abuse potential. However, the short duration of action and aversive side effects limit the clinical usefulness. The present study aimed to investigate the antinociceptive effects of acute administration of novel analogues of SalA. In the dose-response tail withdrawal assay, SalA and the novel analogues 16-EthynyI SalA and 16-Bromo SalA were more potent than the traditional KOPr agonist U50,488, and 16-Ethynyl SalA was more efficacious. 16-Ethynyl SalA and 16-Bromo SalA both had a longer duration of action in the warm water tail withdrawal assay and the hot plate test compared to SalA. In the intraplantar 2\% formaldehyde test, SalA, 16-Ethynyl SalA and 16-Bromo SalA significantly reduced nociceptive pain and inflammatory pain, effects which were reversed by the KOPr antagonist norbinaltorphimine. SalA, 16-Ethynyl SalA and 16-Bromo SalA reduced paw oedema and reduced the 
infiltration of neutrophils into the inflamed tissue. However, SalA, 16-Ethynyl SalA and 16-Bromo SalA produced motor incoordination effects. However, 16-Ethynyl SalA did not alter thermoregulation.

The KOPr agonists were further assessed in a model of paclitaxel-induced neuropathic pain. In the acute dose-response experiment, 16-Ethynyl SalA was significantly more potent at reducing mechanical allodynia compared to morphine in both male and female mice. SalA and 16-Ethynyl SalA were more potent at reducing cold allodynia than morphine. In a chronic administration model over 22 days, for the treatment of cold and mechanical allodynia, all of the opioid treatments reduced pain, however, the traditional KOPr agonist $\mathrm{U} 50,488$, was the most potent, by reducing the male mechanical allodynia and cold allodynia in both sexes back to baseline levels. The ultrastructure of the sciatic nerves were studied, however, it was found that $\mathrm{U} 50,488$ did not reverse the effects of paclitaxel on myelin degeneration and mitochondrial damage.

Overall, this study has identified DHEA as a modest treatment for inflammatory pain, with reduced side effects and a mechanism of action in contrast to other compounds with a similar structure. The novel KOPr agonists had significant effects in acute pain models with longer duration of action than the parent compound SalA. This is the first known study to investigate the effects of KOPr agonists in a paclitaxel-induced neuropathic pain model, showing that KOPr agonists are a potential therapeutic avenue for this debilitating condition. 


\section{Acknowledgments}

This PhD study has been a long, crazy, at times stressful but thoroughly enjoyable journey and it would not have been possible without the help and support of many people to whom I am very grateful.

Firstly, to my supervisor, Dr Bronwyn Kivell, thank you for your advice, enthusiasm and support. Thank you for believing in me and giving me the opportunity to be involved in these exciting projects. You have inspired me to push myself harder than I have ever worked. To my secondary supervisor, Professor Anne La Flamme, I know I didn't call on you often but your support, knowledge and editing skills have been invaluable. Thank you to the Victoria University of Wellington Research Fund, Wellington Medical Research Foundation and the Cancer Society of New Zealand for providing funding for these projects.

I would like to acknowledge Dr Jacqui Harper, Dr Odette Shaw and Dr Kristel Kodar for their help with designing and carrying out the flow cytometry experiments. I would also like to acknowledge $\mathrm{Dr}$ St John Wakefield for helping with the electron microscopy. Thank you to Dr Janet Pitman, Adrian Bibby and Sarah Sczelecki for managing and running the Small Animal Facility. Thank you to Dr Lisa Woods for statistical advice on analysing my three-way interactions.

Projects such as these would not be possible without talented chemists to design and synthesise the compounds. I would like to acknowledge Dr Mikhail Vyssotski and Dr Rahau Shirazi from Callaghan Innovation for providing DHEA; and Professor Thomas Prisinzano at the University of Kansas for his endless knowledge on the chemistry of KOPr agonists and for providing the Salvinorin A compounds.

Over my years in the Kivell lab group, I have been lucky enough to work with a lot of amazing people, many of whom have become good friends. Thank you to Nitin, for getting me started on the pain project; to Amy \#1, for knowing everything and teaching me how to be a scientist; to Amy \#2 for being the original Honours lab friend; to Nikki, for teaching me essential skills and helping out on some of the more intense days; to Aimee \#3, for being the original lunch buddy, knitting supervisor and a great friend; to David, for somehow being endlessly positive and generally just being 'Dave the rave'; to Susan, for your ridiculously delicious baking, for always keeping us amused and crazy times in Queenstown; to Stephen, for being the lab cheerleader by always sending cute animal pictures to keep us going; to Afnan, for your wicked sense of humour and helping me out on stressful days in the lab; to Andy, for your mad marine biology skills and being my go-to statistics expert; to Diana, for sharing this crazy PhD journey with me; to Amy \#4, for being my 'lab protégé' (even if you had no choice in the matter), daily lunch buddy and fellow cat enthusiast; to the newest member of our lab group, Janine, for the daily hilarity, I already can't remember what it was like without you here; and to an 
honorary member of the lab, Varun, for being my unofficial PhD mentor, part-time life coach and provider of many delicious meals. Thank you to all of the other lab group members who have shared this journey with me: Miguel, Sam, Jasleen, Phyllida, Adam, Fraser, Mohan, Caroline, Rickiii, Harvir, Lisa and Georgia, well as the other students throughout SBS for your friendship over my time here.

Much of what we do would not be possible without the fantastic group of technicians within the SBS. A special thank you to Shaun Graham who built and fixed many pieces of equipment over the years, and to Sushila Pillai and Dr Derek Heath, who lent me solutions, microscopes and shared their knowledge on all things histology. Also thank you also to Stephen, Neville, Craig, Chris, Ange, Lesley, Pisana, Adrian and Paul for all that you do for us. Thank you to all of the SBS administration staff: Mary, Charlotte, Sandra, Lesley, Mark, Paul and Patricia for always being there when we need a helping hand, for your encouragement and for keeping this place running smoothly.

I have been very fortunate to have some great flatmates at the fire station flat over the years. A special mention must go to Britt and Sarah, who have both supported me through the ups and downs of this journey, typically with some combination of food, alcohol and trashy television. Also, a big thanks to Steph and Jeff, as well as Katrina and Boris, for letting me stay in their homes for the last couple of months. I am also very thankful for my friends outside of university who I have probably neglected over the last little while. I couldn't have made it through without all of you.

To my family who have supported me (financially and emotionally) over all these years at university. I would not be here without your love, encouragement and belief in me. Thank you for always taking an interest in whatever I was working on at the time, and sharing in my highs and lows. And thank you to my sister, Nikki, you continue to inspire me by everything you do.

Finally, thank you to all of the mice who gave their lives for this research. 


\section{Table of Contents}

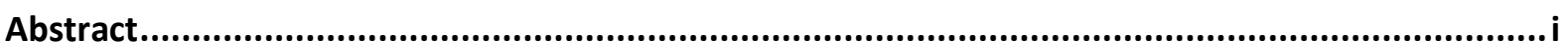

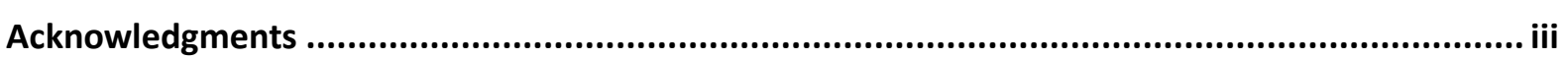

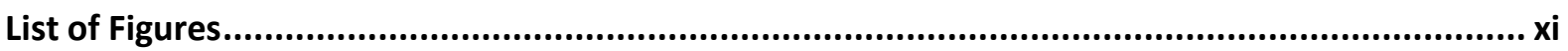

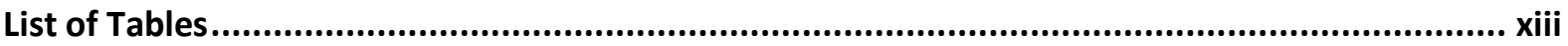

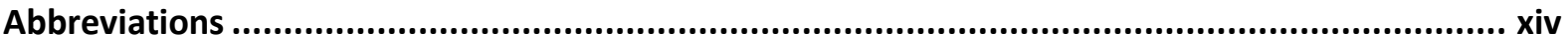

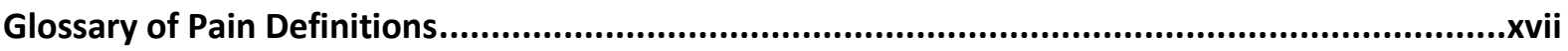

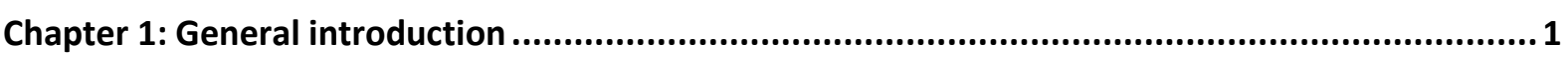

1.1 Prevalence and socioeconomic consequences of chronic pain ................................................. 1

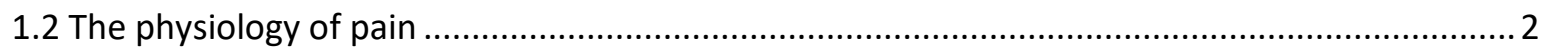

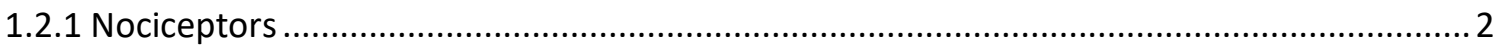

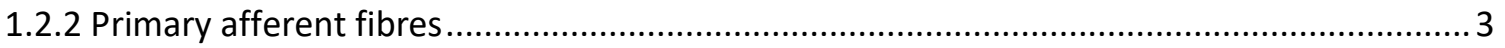

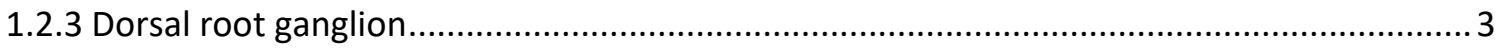

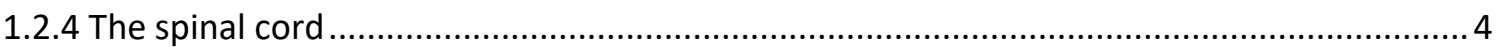

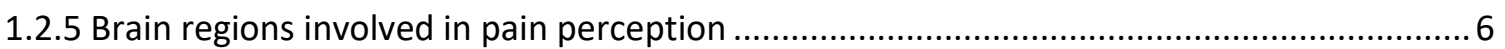

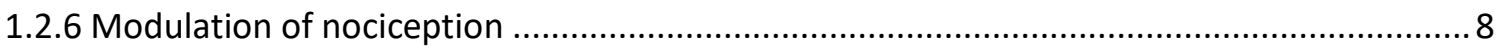

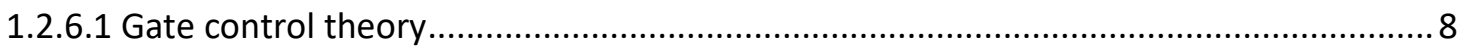

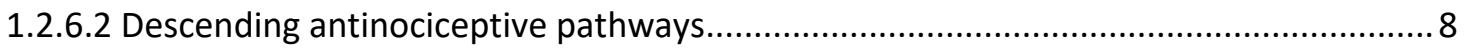

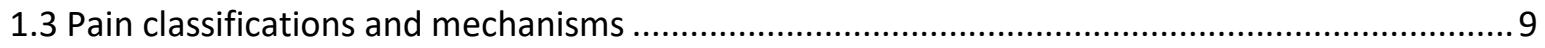

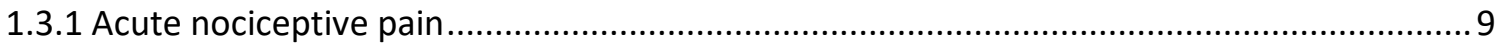

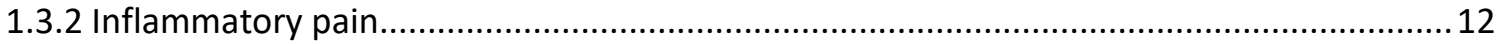

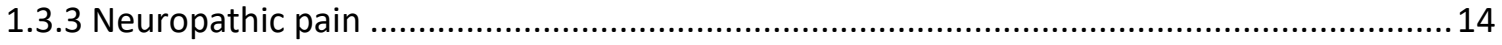

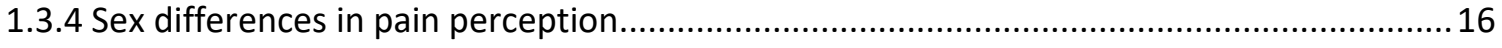

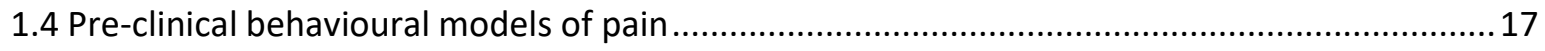

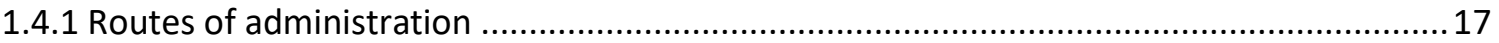

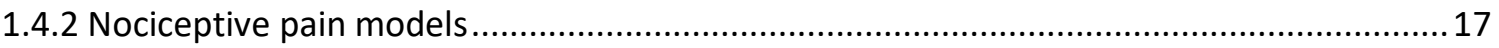

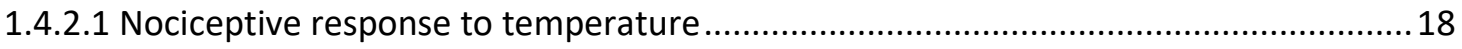

1.4.2.2 Nociceptive response to mechanical stimuli ............................................................. 19

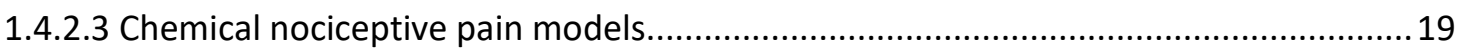

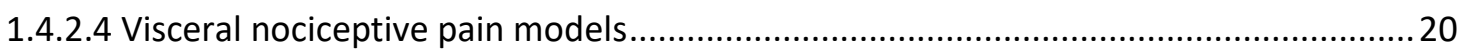

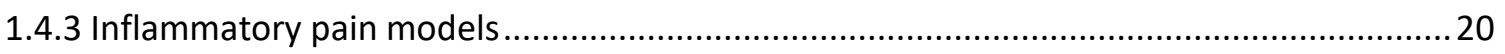

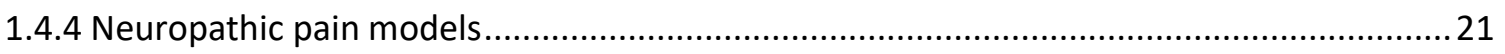

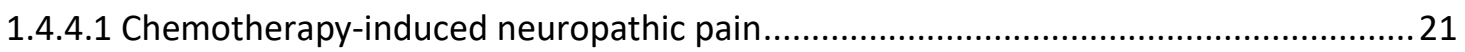

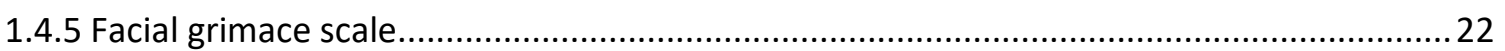

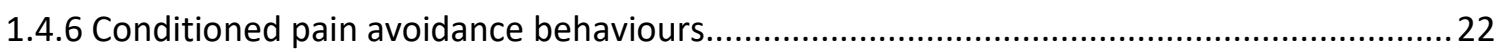




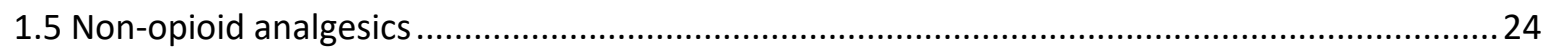

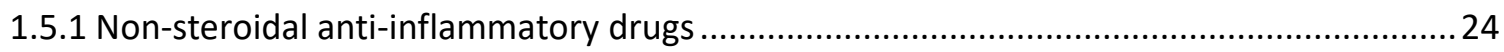

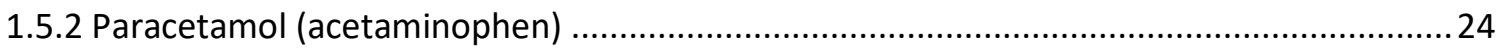

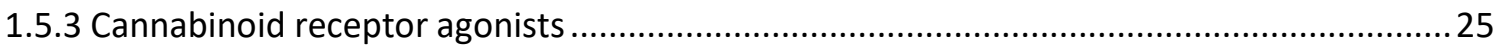

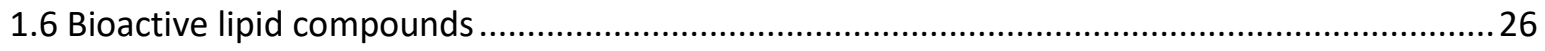

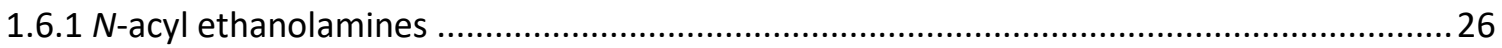

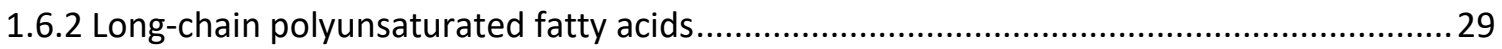

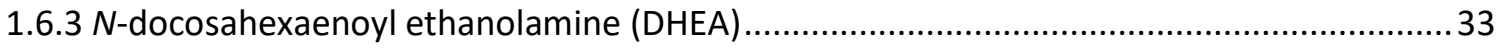

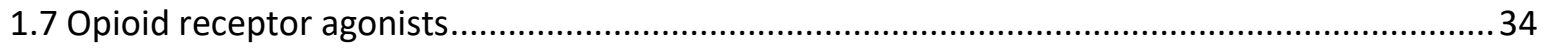

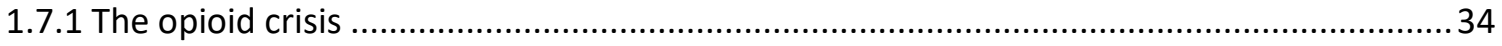

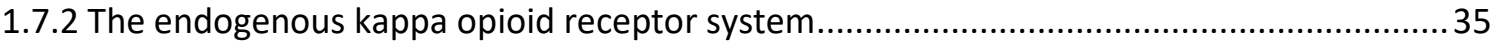

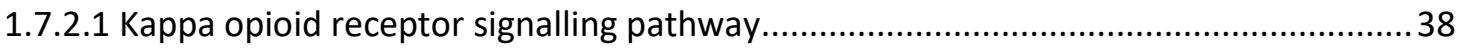

1.7.2.2 Role of the endogenous kappa opioid system in pain .................................................. 41

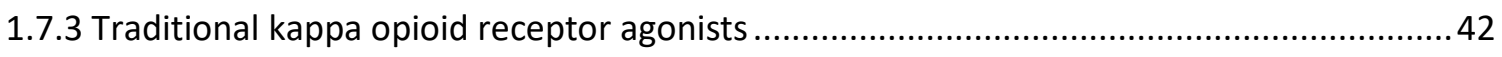

1.7.3.1 Traditional kappa opioid receptor agonists role in reward ......................................... 42

1.7.3.2 Traditional kappa opioid receptor agonists for the treatment of pain ......................... 44

1.7.3.3 Side effects of traditional kappa opioid receptor agonists.......................................... 45

1.7.4 Novel kappa opioid receptor agonists for the treatment of pain...................................... 46

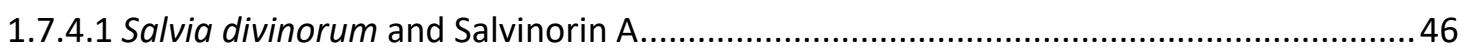

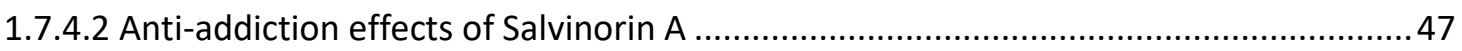

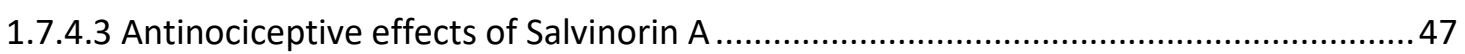

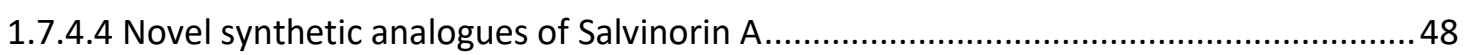

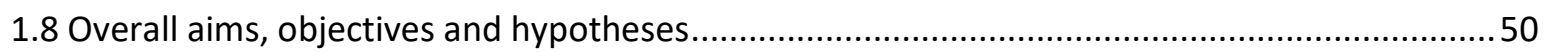

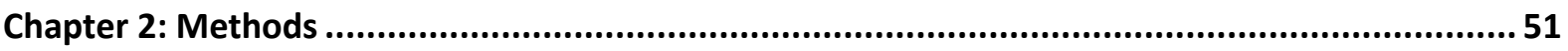

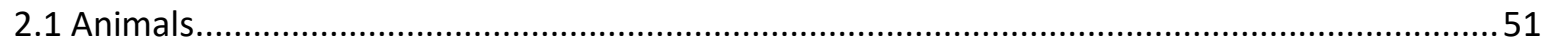

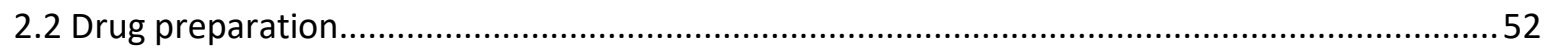

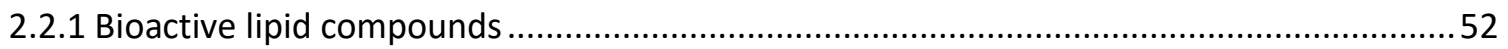

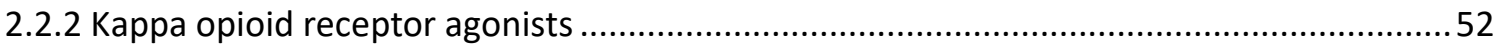

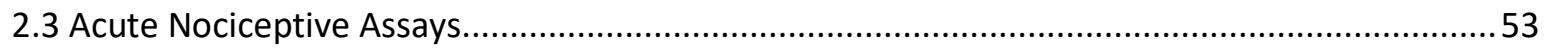

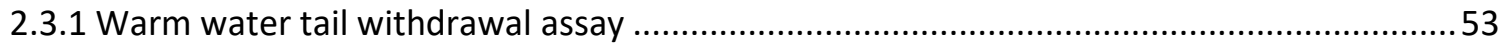

2.3.1.1 Time course warm water tail withdrawal assay .......................................................... 53

2.3.1.2 Cumulative dose-response tail withdrawal assay ....................................................... 53

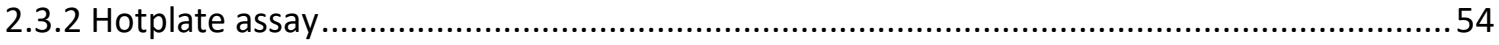

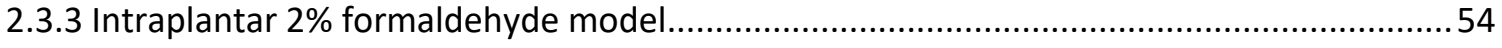

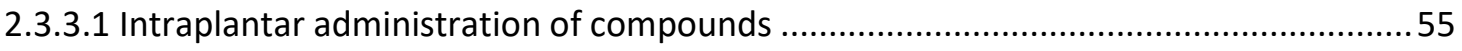

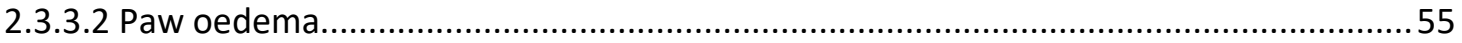




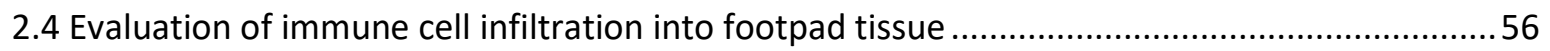

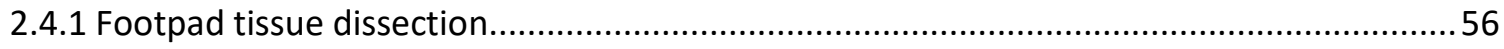

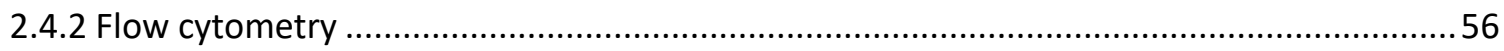

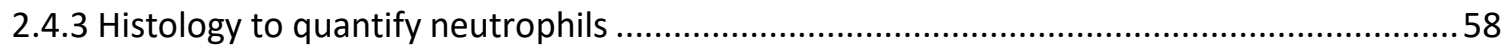

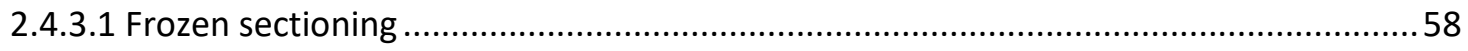

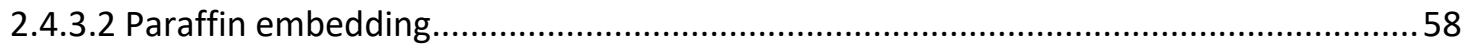

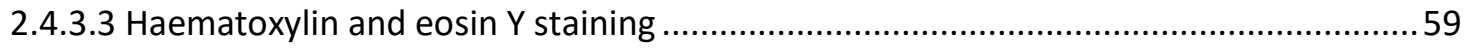

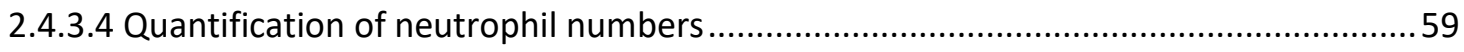

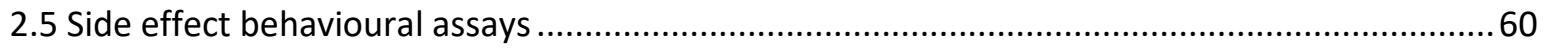

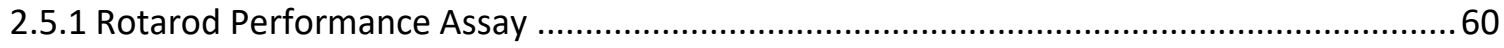

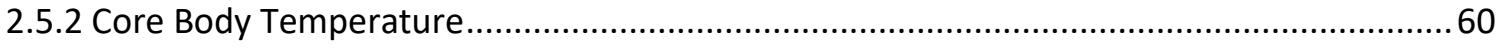

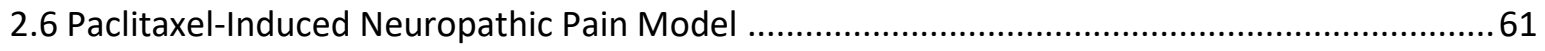

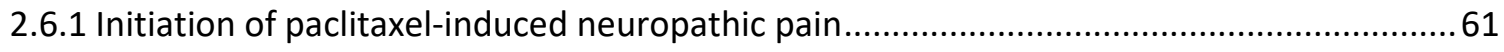

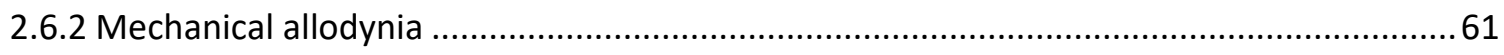

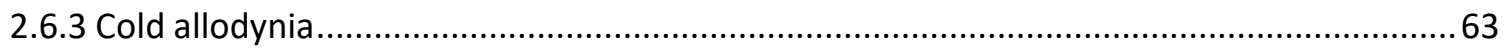

2.6.4 Acute dose-response procedure in paclitaxel-treatment mice .........................................63

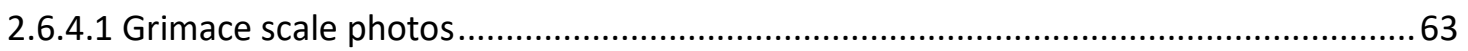

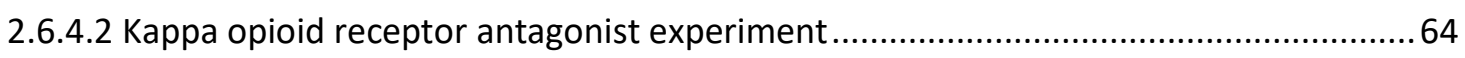

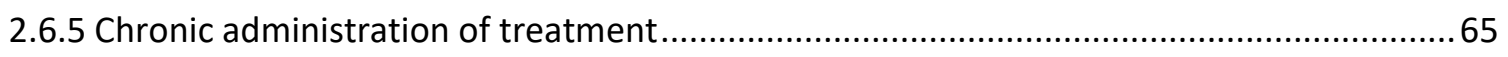

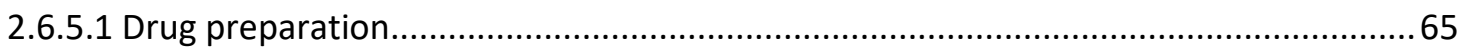

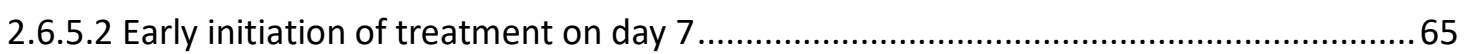

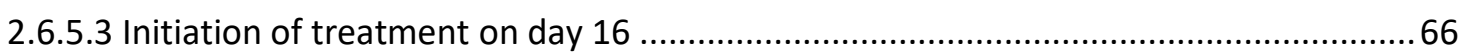

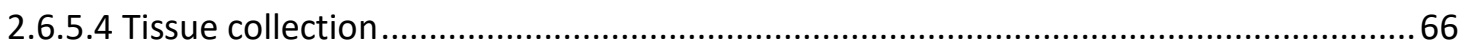

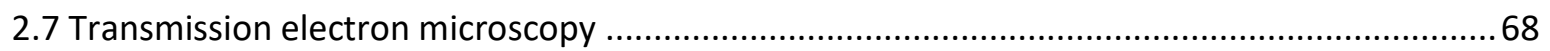

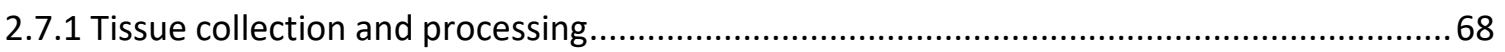

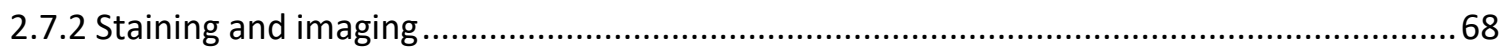

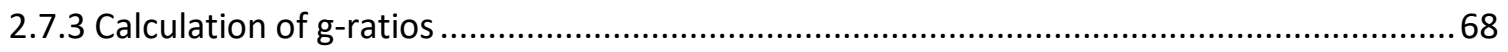

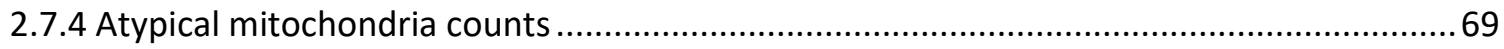

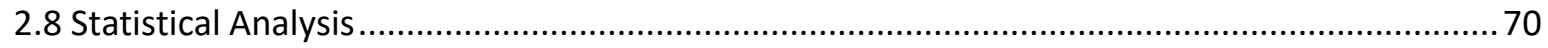

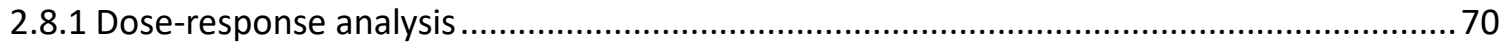

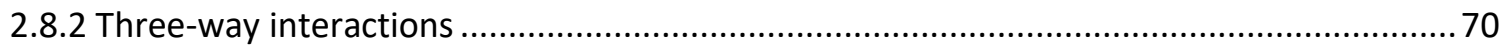

Chapter 3: Investigating the antinociceptive and anti-inflammatory effects of DHEA ................... 73

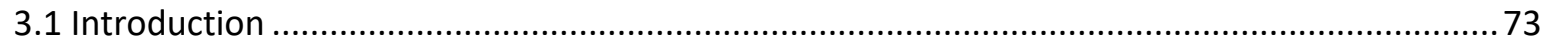

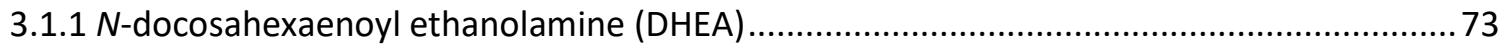

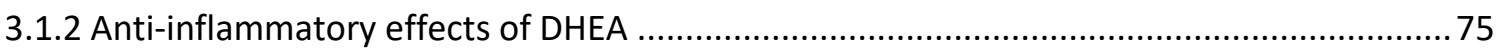

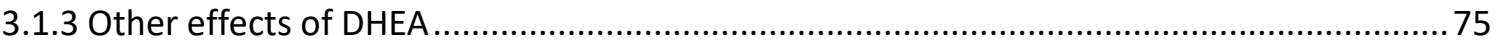


3.2 Aims and hypotheses

3.3 Results

3.3.1 The effect of intraperitoneal injection of DHEA in the intraplantar $2 \%$ formaldehyde test 77

3.3.2 The effect of DHEA on paw oedema and infiltration of neutrophils into the footpad tissue following intraplantar $2 \%$ formaldehyde administration ................................................... 79

3.3.3 The effect of local administration of DHEA in the intraplantar $2 \%$ formaldehyde test.......81

3.3.4 The effect of local injection of DHEA on the inflammatory phase of pain ......................83

3.3.5 The effect of DHEA in the warm water tail withdrawal assay ..................................... 85

3.3.6 The effect of DHEA on motor coordination and core body temperature regulation ..........85

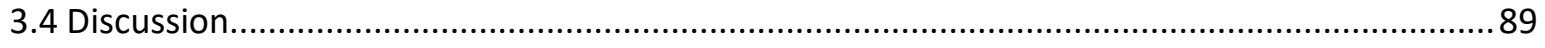

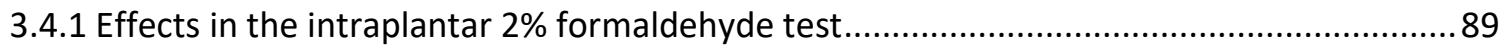

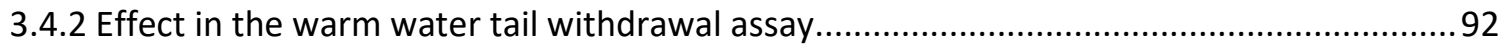

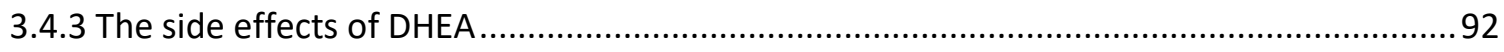

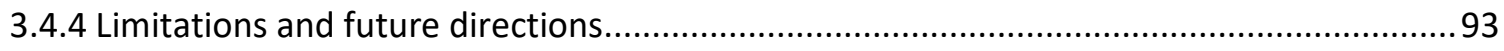

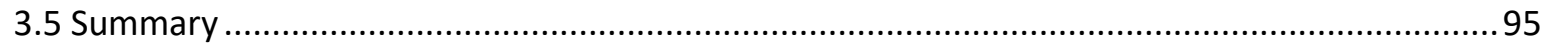

Chapter 4: Investigating the antinociceptive and anti-inflammatory effects of novel kappa opioid

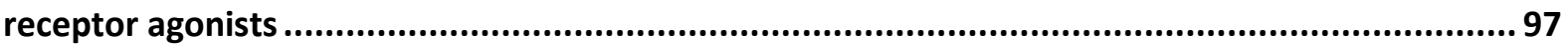

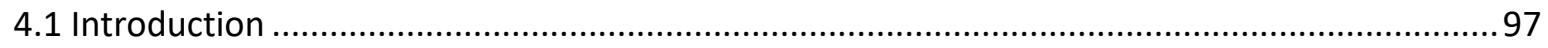

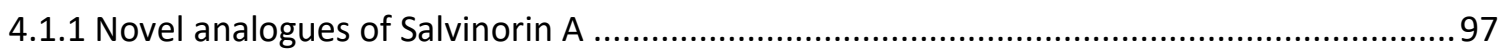

4.1.2 Biased agonism at the kappa opioid receptor .......................................................... 102

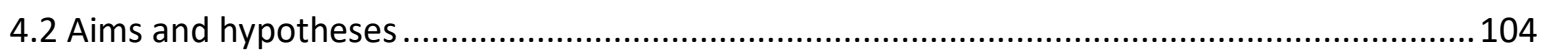

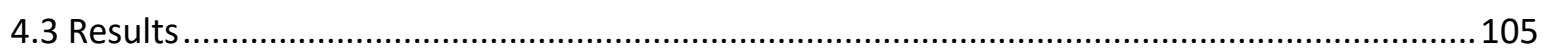

4.3.1 Cumulative dose-response effects using the warm water tail withdrawal assay.............105

4.3.2 Duration of action SalA, 16-Ethynyl SalA and 16-Bromo SalA in the warm water tail

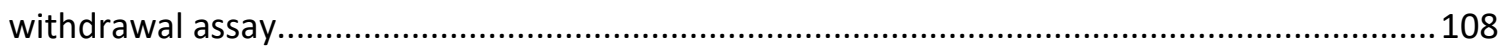

4.3.3 Duration of action in the hotplate assay......................................................... 110

4.3.4 The effect of SalA in the intraplantar 2\% formaldehyde model ................................... 112

4.3.5 The effect of 16-Ethynyl SalA in the intraplantar 2\% formaldehyde assay .....................116

4.3.6 The effect of 16-Bromo SalA in the intraplantar $2 \%$ formaldehyde assay ......................120

4.3.7 Neutrophil infiltration into inflamed footpad tissue ................................................. 124

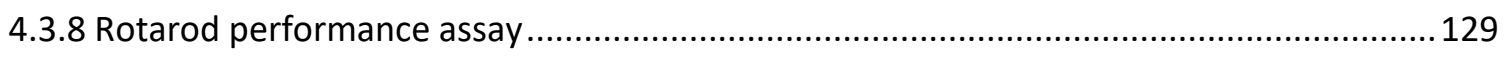

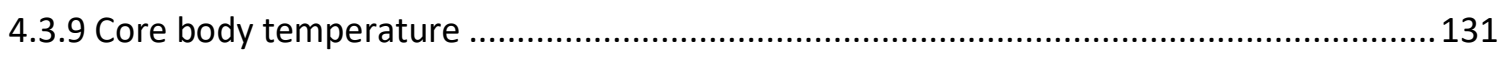

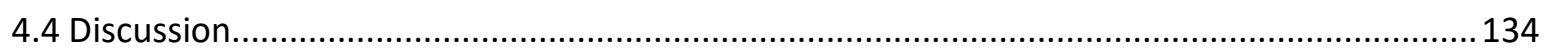

4.4.1 Antinociceptive effects in acute thermal models of pain ......................................... 134

4.4.2 Antinociceptive effects in the intraplantar $2 \%$ formaldehyde test .............................136

4.4.3 Anti-inflammatory effects of the kappa opioid receptor agonists ................................ 138 
4.4.4 Side effect profiles of the kappa opioid receptor agonists. 139

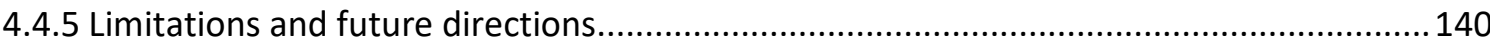

4.5 Summary 143

Chapter 5: Investigating the antinociceptive effects of kappa opioid receptor agonists on paclitaxelinduced neuropathic pain.

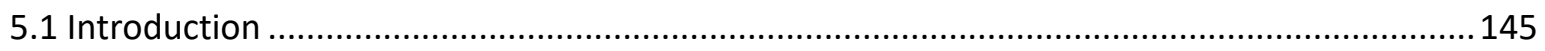

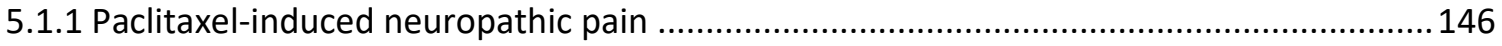

5.1.2 Sex differences in paclitaxel-induced neuropathic pain ................................................... 150

5.1.3 Potential therapies for paclitaxel-induced neuropathic pain ..........................................150

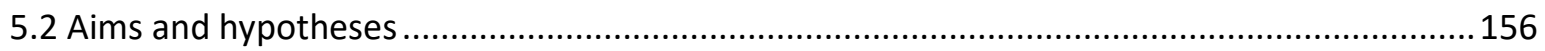

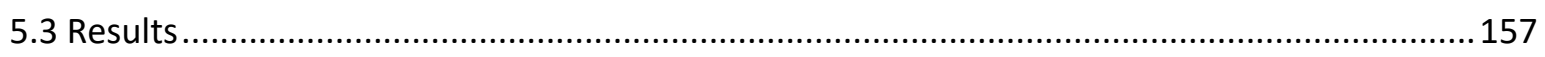

5.3.1 Establishing a model of paclitaxel-induced neuropathic pain in mice...............................157

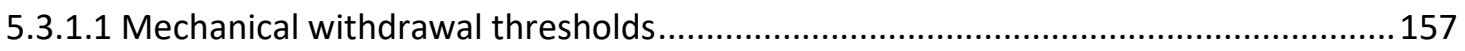

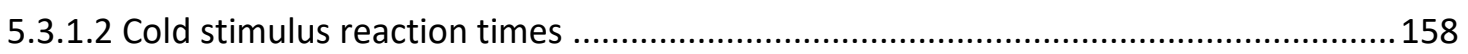

5.3.1.3 Outcome of establishing the paclitaxel-induced neuropathic pain model.................158

5.3.2 Cumulative dose-response effects of kappa opioid receptor agonists .............................163

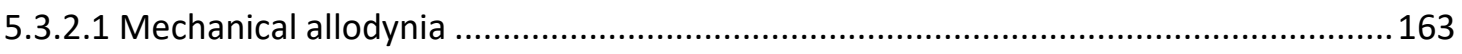

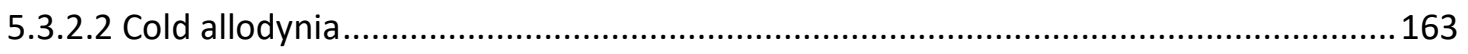

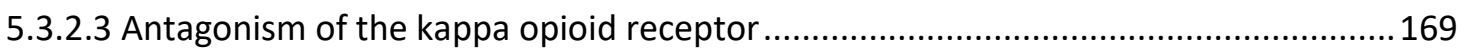

5.3.2.4 Outcome of cumulative dose-response treatment of paclitaxel-induced neuropathic

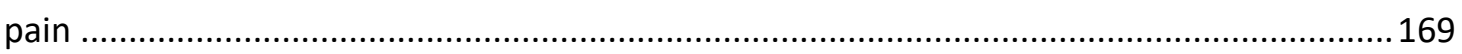

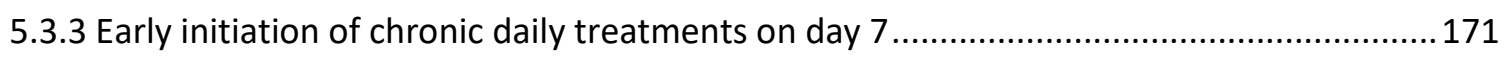

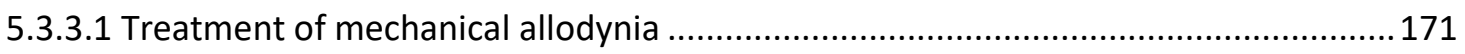

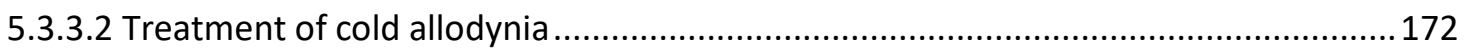

5.3.3.3 Progression of neuropathic pain in the early initiation of treatment model ..............174

5.3.3.4 Outcome of early initiation of the chronic treatment model .................................... 174

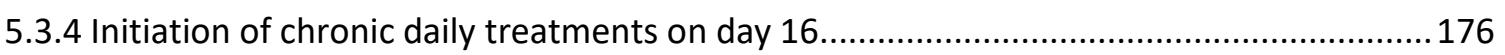

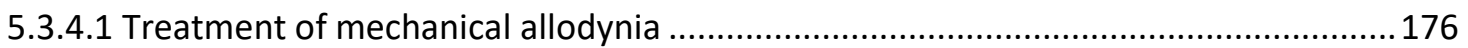

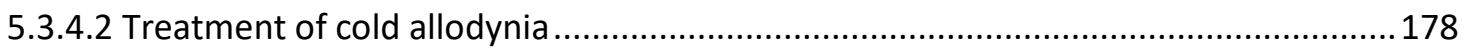

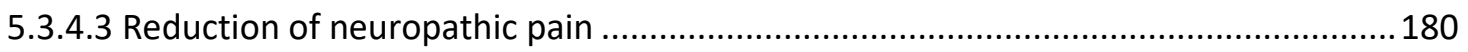

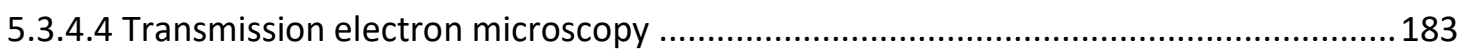

5.3.4.5 Outcome of the initiation of chronic treatment on day 16 model .............................185

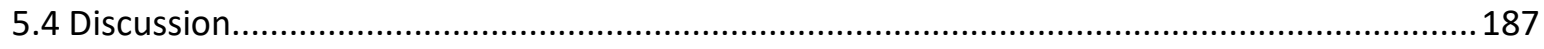

5.4.1 Establishment of the paclitaxel-induced neuropathic pain model in mice .......................187

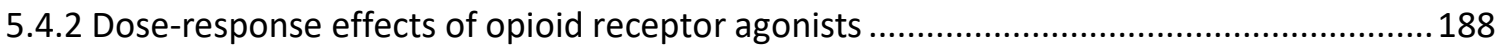

5.4.3 Chronic administration of the opioid receptor agonists .................................................190 
5.4.4 Limitations and future directions 195

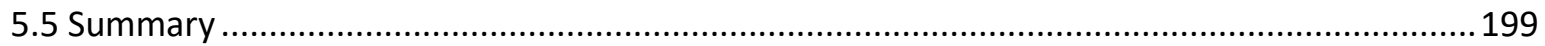

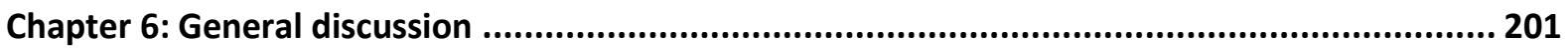

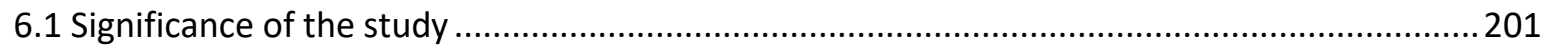

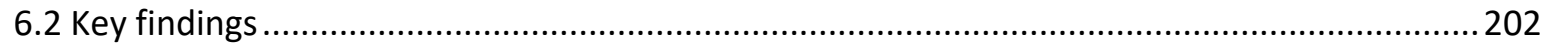

6.2.1 N-docosahexaenoyl ethanolamine reduces inflammatory pain independently of the

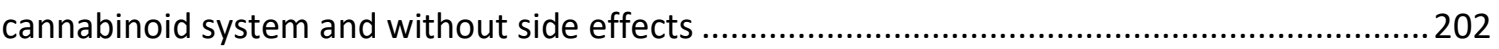

6.2.2 The novel analogues have a longer duration of action compared to Salvinorin A............202

6.2.3 16-Ethynyl SalA has potent antinociceptive effects in the intraplantar formaldehyde model

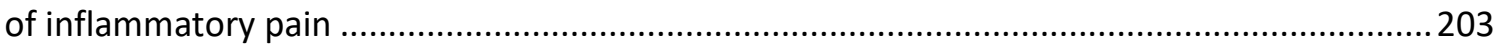

6.2.4 16-Ethynyl SalA has reduced side effects compared to parent compound Salvinorin A... 203

6.2.5 KOPr agonists have potential as a novel therapeutic for the treatment of paclitaxel-induced neuropathic pain. 204

6.2.6 U50,488 and 16-Ethynyl SalA are more effective in male mice with established paclitaxel-

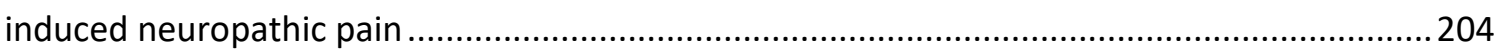

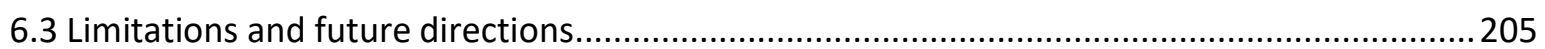

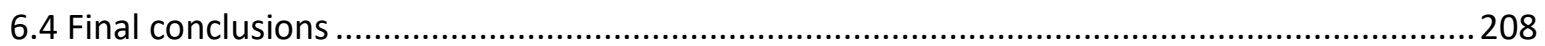

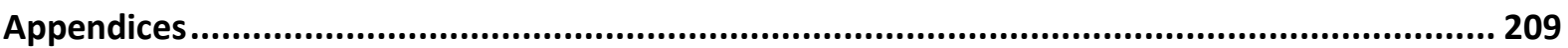

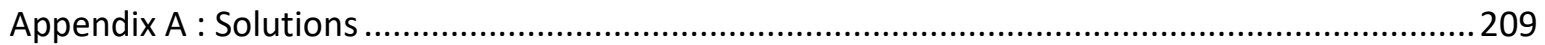

Appendix B : Analysis of routes of administration of vehicle control in the intraplantar formaldehyde

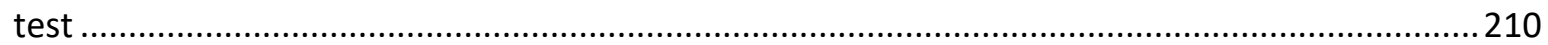

Appendix C : Warm water tail withdrawal dose-response experiment .....................................211

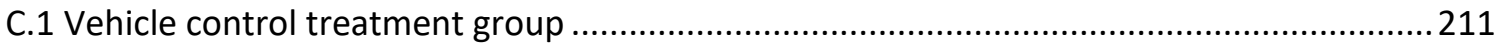

C.2 Comparison of morphine and U50,488 in the warm water tail withdrawal dose-response

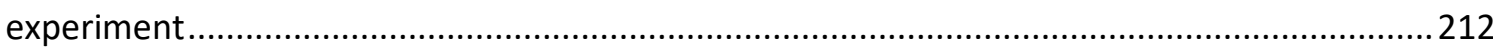

Appendix D : Acute dose-response controls in the paclitaxel-induced neuropathic pain model ..213

D.1 Baseline withdrawal thresholds

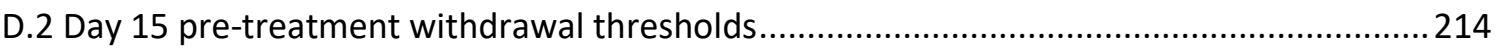

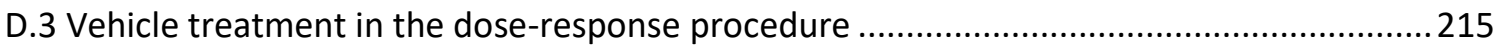

D.4 Day 15 pre-treatment withdrawal thresholds for antagonist study ..................................216

Appendix E : Controls for the chronic administration procedure in the paclitaxel-induced

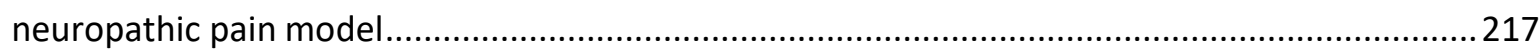

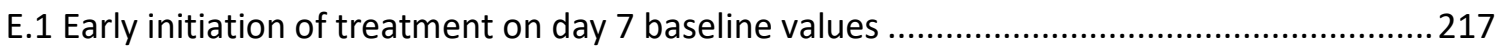

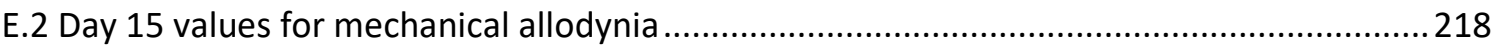

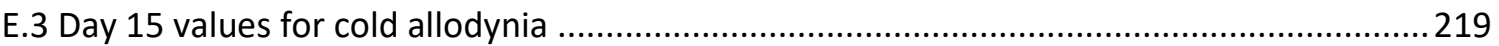

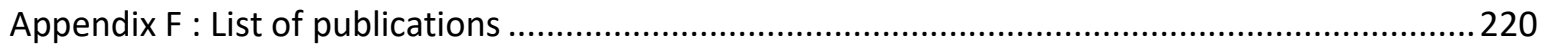

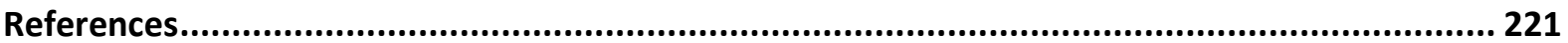




\section{List of Figures}

Figure 1.1: Pain signalling pathway from the nociceptors of the skin to the spinal cord.....................5

Figure 1.2: Ascending and descending pain pathways ................................................................. 7

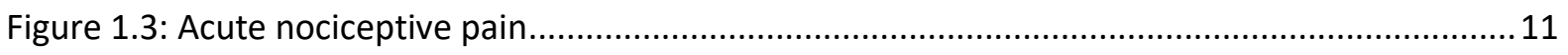

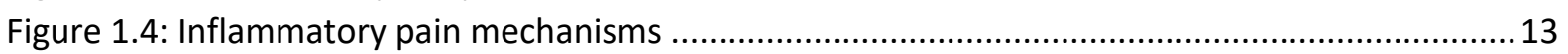

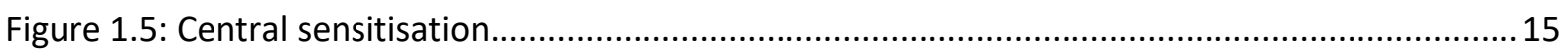

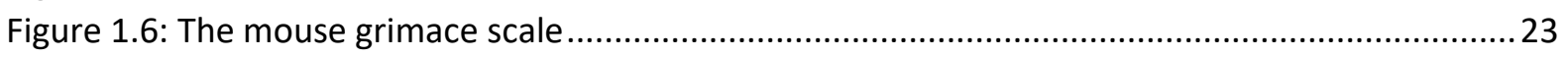

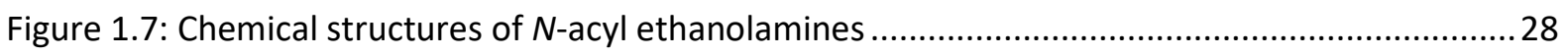

Figure 1.8: Chemical structures of $\mathrm{n}-3$ long-chain polyunsaturated fatty acids ................................... 30

Figure 1.9: Synthesis of polyunsaturated fatty acids and the ethanolamine metabolites ....................31

Figure 1.10: Brain and spinal cord regions involved in pain processing compared to kappa opioid

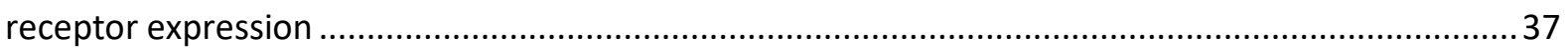

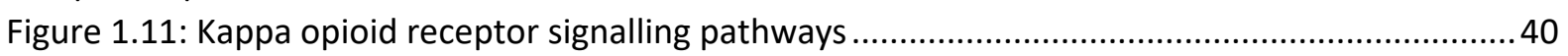

Figure 2.1: Examples of the simplified up-down von Frey filament testing scheme............................62

Figure 2.2: Experimental design for the acute dose-response procedure in paclitaxel-treated mice .64

Figure 2.3: Experimental design for the chronic treatment of the $\mathrm{KOPr}$ agonists in mice with

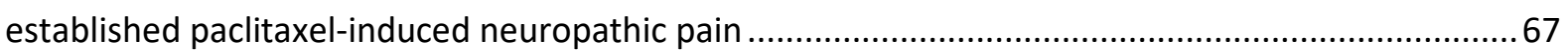

Figure 2.4: Images showing how the g-ratio and atypical mitochondria were measured ....................69

Figure 3.1: DHEA has antinociceptive and anti-inflammatory effects via intraperitoneal injection in the

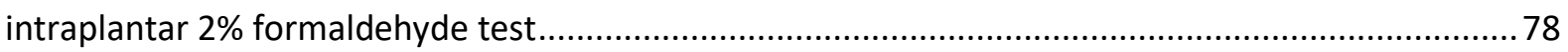

Figure 3.2: DHEA reduces paw oedema and the number of neutrophils in the footpad tissue............ 80

Figure 3.3: DHEA has antinociceptive and anti-inflammatory effects via local intraplantar injection in

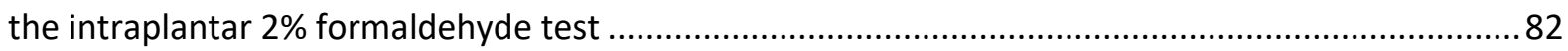

Figure 3.4: DHEA has antinociceptive and anti-inflammatory effects via local intraplantar injection in

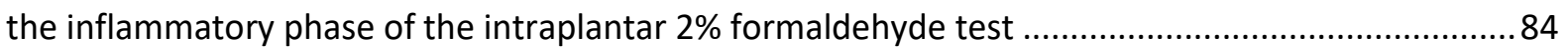

Figure 3.5: DHEA has no effect in the warm water $\left(50^{\circ} \mathrm{C}\right)$ tail withdrawal assay..................................86

Figure 3.6: DHEA had no effect on motor coordination or core body temperature ............................ 87

Figure 4.1: Dose-response effects of KOPr agonists in the warm water tail withdrawal assay .........106

Figure 4.2: Duration of action of KOPr agonists in the warm water $\left(50^{\circ} \mathrm{C}\right)$ tail withdrawal assay .....109

Figure 4.3: Duration of action of the KOPr agonists in the hotplate $\left(50^{\circ} \mathrm{C}\right)$ assay...........................111

Figure 4.4: Antinociceptive effect of SalA in the intraplantar 2\% formaldehyde assay .....................115

Figure 4.5: Antinociceptive effect of 16-Ethynyl SalA in the intraplantar 2\% formaldehyde test......119

Figure 4.6: Antinociceptive effect of 16-Bromo SalA in the intraplantar $2 \%$ formaldehyde test....... 123

Figure 4.7: KOPr agonists only reduce neutrophil cell populations in inflamed tissue ......................125

Figure 4.8: KOPr agonists reduce neutrophil counts in H\&E stained footpad tissue sections ...........126

Figure 4.9: Novel SalA analogues reduce neutrophil counts in paraffin-embedded footpad tissue

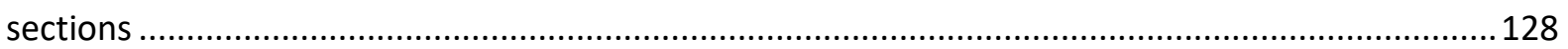

Figure 4.10: KOPr agonists cause motor incoordination in the rotarod performance assay .............130

Figure 4.11: 16-Bromo SalA reduced core body temperature in mice ..........................................132

Figure 5.1: Mechanisms of paclitaxel-induced neuropathic pain ..................................................149

Figure 5.2: Paclitaxel treatment did not cause a weight change in mice .........................................159

Figure 5.3: Representative images of the observed changes in the mouse grimace scale following

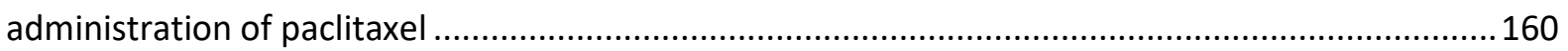

Figure 5.4: Female mice have longer baseline reaction times to a cold acetone stimulus .................161

Figure 5.5: Paclitaxel treatment induces mechanical and cold allodynia in male and female mice ..162 
Figure 5.6: Antinociceptive dose-response effects of novel KOPr agonists in mice separated by sex with paclitaxel-induced neuropathic pain

Figure 5.7: Antinociceptive dose-response effects of novel KOPr agonists in combined male and female mice with established paclitaxel-induced neuropathic pain

Figure 5.8: KOPr antagonism reduces the antinociceptive effect of the novel KOPr agonists 170

Figure 5.9: Antinociceptive effects of early initiation of chronic daily treatments in mice with paclitaxelinduced neuropathic pain.

Figure 5.10: Progression of paclitaxel-induced neuropathic pain in mice following the early initiation of daily treatments. 175

Figure 5.11: Antinociceptive effects of opioid receptor agonists on paclitaxel-induced mechanical allodynia following initiation of chronic daily treatment on day 16. 177

Figure 5.12: Antinociceptive effects of opioid receptor agonists on paclitaxel-induced cold allodynia

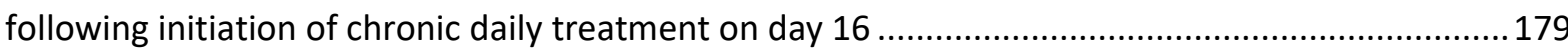

Figure 5.13: Mechanical allodynia with measurement before treatment ......................................181

Figure 5.14: Cold allodynia with measurement before daily treatment .........................................182

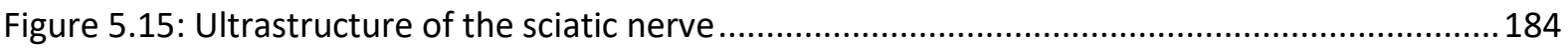

Figure 5.16: Proposed mechanism for the effects of dynorphin and the KOPr and NMDA receptor in a

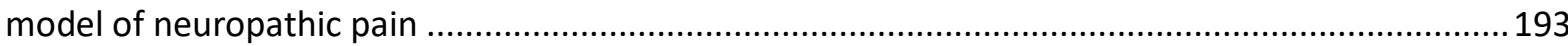

Figure B.1: The vehicle control administered via intraplantar injection produces an antinociceptive

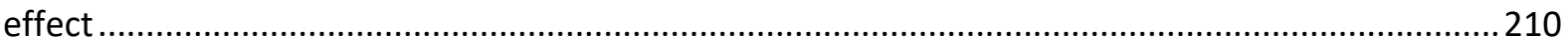

Figure C.1: Vehicle control group in the warm water dose-response experiment ..........................211

Figure C.2: Morphine is more efficacious than U50,488 in the warm water tail withdrawal assay...212 Figure D.1: The day 0 pre-paclitaxel mechanical and cold threshold values were not different between upcoming assigned dose-response treatment groups

Figure D.2: The day 15 post-paclitaxel mechanical and cold allodynia values were not different between assigned treatment groups.

Figure D.3: Vehicle treatment did not have an antinociceptive effect in mice with established

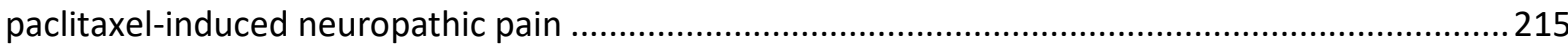

Figure D.4: KOPr antagonism with nor-BNI did not affect the day 15 pre-treatment values ...........216 Figure E.1: Day 6 values for early initiation of chronic opioid receptor agonist treatment procedure

Figure E.2: Mechanical withdrawal thresholds on day 15 prior to assignment of treatment groups 218 Figure E.3: Cold reaction times on day 15 prior to assignment of treatment groups 219 


\section{List of Tables}

Table 1.1: Comparison of dynorphin, traditional agonists and Salvinorin A in binding affinity and

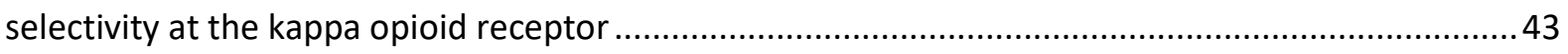

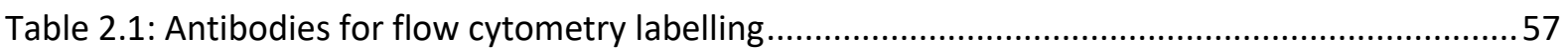

Table 2.2: Leica TP1020 processor settings for paraffin embedding footpad tissue samples...............59

Table 2.3: Target force of the von Frey filaments used to measure mechanical allodynia ...................62

Table 3.1: Comparison of the ethanolamines in binding affinity and selectivity for the cannabinoid

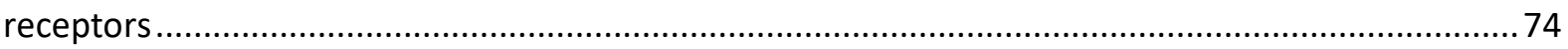

Table 3.2: Summary of the antinociceptive and side effects of $N$-docosahexaenoyl ethanolamine ... 88 Table 4.1: Comparison of Salvinorin A and analogues for binding affinity and potency for the mu and

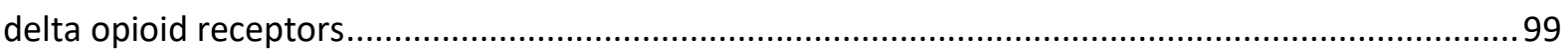

Table 4.2: Comparison of Salvinorin A and analogues in binding affinity and selectivity at the KOPr 101

Table 4.3: Statistical analysis of the dose-response effects of the KOPr agonists in the warm water tail

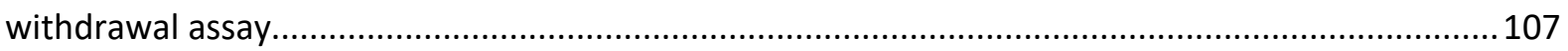

Table 4.4: Summary of the antinociceptive effects of the kappa opioid receptor agonists................133

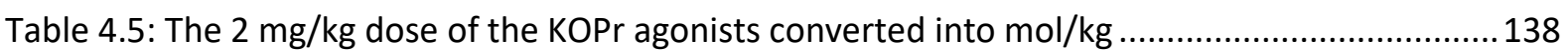

Table 5.1: Common chemotherapeutic agents that cause chemotherapy-induced neuropathic pain 145

Table 5.2: Randomised placebo-controlled clinical trial data for the treatment of established chemotherapy-induced neuropathic pain 151

Table 5.3: Preclinical results for the prevention of the development of paclitaxel-induced neuropathic

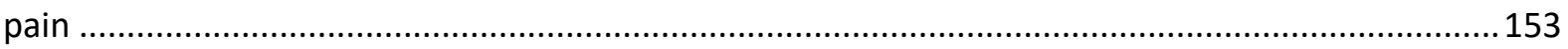

Table 5.4: Preclinical results for the treatment of paclitaxel-induced neuropathic pain ...................155 Table 5.5: Dose-response effects of the KOPr agonists in female and male mice with established paclitaxel-induced mechanical allodynia 166 Table 5.6: Dose-response effects of the KOPr agonists in female and male mice with established paclitaxel-induced cold allodynia. 167

Table 5.7: Antinociceptive dose-response effects of KOPr agonists in combined male and female mice with established paclitaxel-induced cold allodynia 168 Table 5.8: Summary of the sex differences in the paclitaxel-induced neuropathic pain experimental results. 


\section{Abbreviations}

16-Bromo SalA

16-Ethynyl SalA

AA

ACC

AEA

ALA

ANOVA

ATP

AUC

CAMP

CB1

$\mathrm{CB} 2$

CGRP

$\mathrm{CHO}$

CIMDM

CINP

CNS

COX

CPA

CPP

CREB

DAMGO

DAT

DHA

DHEA

DMSO

DNase

DOPr

DPA

DPDPE

DRG

$\mathrm{EC}_{50}$

$E D_{50}$

ELISA

$E_{\text {max }}$

EOM SalB

EPA

EPEA

ERK1/2

FAAH

FACS

FBS
16-Bromo Salvinorin A

16-Ethynyl Salvinorin A

arachidonic acid

anterior cingulate cortex

anandamide

$\alpha$-linolenic acid

analysis of variance

adenosine triphosphate

area under the curve

cyclic adenosine monophosphate

cannabinoid type 1

cannabinoid type 2

calcitonin gene-related peptide

Chinese hamster ovary

complete Iscove's modified Dulbecco's medium

chemotherapy-induced neuropathic pain

central nervous system

cyclooxygenase

conditioned place aversion

conditioned place preference

cAMP response element binding protein

[D-Ala ${ }^{2}, \mathrm{~N}$-Me-Phe ${ }^{4}, \mathrm{Gly}^{5}$-ol]-enkephalin

dopamine transporter

docosahexaenoic acid

$\mathrm{N}$-docosahexaenoyl ethanolamine

dimethyl sulfoxide

deoxyribonuclease

delta opioid receptor

docosapentaenoic acid

[D-Pen ${ }^{2}$,D-Pen ${ }^{5}$-enkephalin

dorsal root ganglion

median effective concentration (potency)

median effective dose (potency)

enzyme-linked immunosorbent assay

maximum response (efficacy)

ethoxymethyl ether Salvinorin B

eicosapentaenoic acid

$N$-eicosapentaenoyl ethanolamine

extracellular signal-regulated kinase 1 and 2

fatty acid amide hydrolase

fluorescence-activated cell sorter

foetal bovine serum 


\begin{tabular}{|c|c|}
\hline GABA & Y-aminobutyric acid \\
\hline GPCR & G-protein coupled receptor \\
\hline GRK & G-protein coupled receptor kinase \\
\hline$H \& E$ & haematoxylin and eosin $Y$ \\
\hline HEK-293 & human embryonic kidney-293 \\
\hline HPLC & high-performance liquid chromatography \\
\hline i.c.v. & intracerebroventricular \\
\hline i.p. & intraperitoneal \\
\hline i.pl. & intraplantar \\
\hline i.v. & intravenous \\
\hline IASP & International Association for the Study of Pain \\
\hline IBD & inflammatory bowel disease \\
\hline IBS & irritable bowel syndrome \\
\hline$I_{50}$ & median inhibitory dose (potency) \\
\hline IL & interleukin \\
\hline iNOS & inducible nitric oxide synthase \\
\hline JNK & c-Jun N-terminal kinase \\
\hline $\mathrm{KOPr}$ & kappa opioid receptor \\
\hline LNA & linoleic acid \\
\hline LPS & lipopolysaccharide \\
\hline LSD & lysergic acid diethylamide \\
\hline MAPK & mitogen-activated protein kinase \\
\hline MCP-1 & monocyte chemotactic protein-1 \\
\hline Mesyl SalB & mesyl Salvinorin B \\
\hline MOM SalB & methoxymethyl ether Salvinorin B \\
\hline MOPr & mu opioid receptor \\
\hline MPE & maximum possible effect \\
\hline NAE & $N$-acyl ethanolamine \\
\hline NAPE-PLD & $N$-acyl phosphatidylethanolamine-specific phospholipase D \\
\hline NAT & $N$-acyl transferase \\
\hline NMDA & N-methyl-D-aspartate \\
\hline nor-BNI & nor-binaltorphimine \\
\hline NSAID & non-steroidal anti-inflammatory drug \\
\hline OEA & $N$-oleoyl ethanolamine \\
\hline PBS & phosphate buffered saline \\
\hline PEA & $N$-palmitoyl ethanolamine \\
\hline PG & prostaglandin \\
\hline PI3K & phosphatidylinositol 3-kinase \\
\hline PIPES & piperazine-1,4-bis(2-ethanesulfonic acid) \\
\hline PKC & protein kinase $\mathrm{C}$ \\
\hline PNS & peripheral nervous system \\
\hline PPAR & peroxisome proliferator-activated receptor \\
\hline PUFA & polyunsaturated fatty acid \\
\hline S.c. & subcutaneous \\
\hline SalA & Salvinorin A \\
\hline
\end{tabular}


SD

SEM

Spiro SalB

TEM

THC

TNF $\alpha$

TRP

TRPA

TRPM

TRPV

$\beta$-FNA

$\beta$-THP SalB standard deviation

standard error of the mean

spirobutyrolactone Salvinorin B

transmission electron microscopy

tetrahydrocannabinol

tumour necrosis factor- $\alpha$

transient receptor potential

transient receptor potential subfamily ankyrin

transient receptor potential subfamily melastatin

transient receptor potential subfamily vanilloid

$\beta$-funaltrexamine

$\beta$-tetrahydropyran Salvinorin B 


\section{Glossary of Pain Definitions}

Unless otherwise stated, all definitions are reproduced from the International Association for the Study of Pain with permission (Copyright of IASP Publications, 2017)

allodynia

analgesia

central sensitisation

hyperalgesia

itch/pruritus

neuropathic pain

neuropathy

nociception

nociceptive neuron

nociceptive pain

nociceptive stimulus

nociceptor

noxious stimulus

pain

pain threshold

peripheral sensitisation

sensitisation pain due to a stimulus that does not normally provoke pain

absence of pain in response to stimulation which would normally be painful

increased responsiveness of nociceptive neurons in the central nervous system to their normal or subthreshold afferent input

increased pain from a stimulus that normally provokes pain

an unpleasant cutaneous sensation which provokes the desire to scratch (Rothman, 1941)

pain caused by a lesion or disease of the somatosensory nervous system a disturbance of function or pathological change in a nerve: in one nerve, mononeuropathy; in several nerves, mononeuropathy multiplex; if diffuse and bilateral, polyneuropathy the neural process of encoding noxious stimuli a central or peripheral neuron of the somatosensory nervous system that is capable of encoding noxious stimuli

pain that arises from actual or threatened damage to non-neural tissue and is due to activation of nociceptors

an actually or potentially tissue-damaging event transduced and encoded by nociceptors

a high-threshold sensory receptor of the peripheral somatosensory nervous system that is capable of transducing and encoding noxious stimuli

a stimulus that is damaging or threatens damage to normal tissues

an unpleasant sensory and emotional experience associated with actual or potential tissue damage, or described in terms of such damage the minimum intensity of a stimulus that is perceived as painful increased responsiveness and reduced threshold of nociceptive neurons in the periphery to the stimulation of their receptive fields increased responsiveness of nociceptive neurons to their normal input, and/or recruitment of a response to normally subthreshold inputs 


\section{Chapter 1: General introduction}

\subsection{Prevalence and socioeconomic consequences of chronic pain}

Pain is an important process for survival, acting as a warning system to detect noxious stimuli and protect against actual or potential tissue damage. While acute pain can be beneficial, chronic or dysfunctional pain causes patients to endure prolonged suffering and discomfort, often having profound effects on quality of life (Vartiainen et al., 2016). The Ministry of Health 2015/2016 selfreported survey found $21 \%$ of New Zealand adults live with chronic pain, which is expected to last or has lasted longer than 6 months, an increase from 16\% in the 2011/2012 survey (Ministry of Health, 2016). The global estimates of chronic pain vary widely. A meta-analysis of data collected in the United Kingdom shows chronic pain prevalence ranging from 35-51\% (Fayaz et al., 2016), recent studies in the United States of America (USA) range between 11-19\% (Kennedy et al., 2014; Nahin, 2015) and Australian studies have identified between $16-19 \%$ of adults suffer from chronic pain (Henderson et al., 2013; Campbell et al., 2015).

This significant portion of the population suffering from pain creates a large social and economic burden. A study in the USA estimated that healthcare and loss of productivity due to pain cost the nation up to $\$ 635$ billion annually, more than the cost of heart disease and cancer combined (Gaskin \& Richard, 2012). No reliable data could be found on the cost of chronic pain in New Zealand; however, a 2010 report on arthritis estimated $\$ 3$ billion is spent on health care and indirect costs, including $\$ 1.48$ billion in loss of productivity (Access Economics, 2010). People suffering from chronic pain often do not sleep well (Finan et al., 2013), feel stigmatised (De Ruddere \& Craig, 2016), struggle to maintain relationships (Swain \& Johnson, 2014) and have increased rates of depression (Outcalt et al., 2015), anxiety (Kroenke et al., 2013) and suicide (Campbell et al., 2015). They are also less likely to be in fulltime employment and a recent survey found that $12 \%$ of chronic pain suffers left or lost their job because of pain (Duenas et al., 2015). Pain is the most common reason for patients to seek medical attention (St Sauver et al., 2013); however, $40 \%$ of pan-European chronic pain sufferers report insufficient treatment (Breivik et al., 2006). Evidently, there are a lot of patients currently suffering from chronic pain without effective management, with high social and economic consequences, leading to a reduced quality of life. 


\subsection{The physiology of pain}

The physiologic component of pain is termed nociception, which consists of the transduction, transmission and modulation of signals produced in response to a noxious stimulus. In basic terms, the nociceptive pathway can be considered as a three-neuron chain, with the first-order neuron originating in the periphery and projecting to the spinal cord, the second-order neuron ascending the spinal cord and the third-order neuron projecting from the thalamus to the cerebral cortex. In reality, the pathway is a complex network with other sensory neurons and descending inhibitory neurons that modulate the transmission of painful stimuli (reviewed in Lamont et al., 2000; Marchand, 2008; Bourne et al., 2014).

\subsubsection{Nociceptors}

Specialised peripheral sensory neurons, named nociceptors (Sherrington, 1906), identify potentially damaging stimuli in the skin and most tissues in the body, including the muscles, joints and viscera. The skin contains many nociceptors, located in the epidermis and the dermis, which are concentrated in areas that are more likely to be exposed to injuries, such as the fingers and toes (reviewed in Chouchkov, 2012). The muscles contain fewer nociceptors than the skin, and nociceptors in the muscle are widely spread making the pain diffuse rather than localised (reviewed in Mense, 2008). In the joints, nociceptors can be activated by mechanical stimuli, such as a tear, or by inflammation, such as in rheumatoid arthritis (reviewed in Schaible et al., 2009). The internal organs are protected by the skin, muscles and bones and, therefore, have even fewer nociceptors, that are widely distributed, leading to a diffuse pain. Of the internal organs, the more highly innervated are the hollow organs, such as the intestines, bladder and uterus, which are more likely to be exposed to external hazardous agents. The solid organs, such as the liver and spleen, have fewer nociceptors, and therefore, the deterioration of these organs can go unnoticed by patients (reviewed in Cervero, 2009; Reed \& Vanner, 2017).

Nociceptors are unencapsulated, unmyelinated free nerve endings that branch off the main axon at the terminal end of primary afferent sensory neurons (Figure 1.). Ion channels on the nociceptors are activated by the inflammatory and chemical mediators released from lysed cells during tissue damage (reviewed in Dubin \& Patapoutian, 2010). Typically, nociceptors are characterised into three classes by the type of stimulus they are able to detect: high-threshold mechanical, thermal and polymodal nociceptors. The expression of different ion channels on the nociceptor determines the sensitivity to different stimuli. The largest class of ion channels involved in pain transmission are the transient receptor potential (TRP) cation channel family, which are capable of sensing noxious temperatures, 
chemicals and mechanical deformation, causing the TRP channel to open and allow $\mathrm{Na}^{+}, \mathrm{Ca}^{2+}$ and $\mathrm{Mg}^{2+}$ ions to flow into the neuron (reviewed in Zheng, 2013). The influx of cations leads to depolarisation of the membrane, and if the threshold is met, initiates voltage-gated $\mathrm{Na}^{+}$ion channel-dependent action potentials to be transmitted along the primary afferent neuron towards the spinal cord. The signal can only be carried in one direction due to the refractory period of the voltage-gated $\mathrm{Na}^{+}$ion channels and hyperpolarisation of the membrane potential due to the efflux of $\mathrm{K}^{+}$ions. Each action potential has the same strength, but the frequency of a set of action potentials encodes information on the intensity and duration of the pain (Momin \& McNaughton, 2009).

\subsubsection{Primary afferent fibres}

The pain signals generated by nociceptor activation are transmitted to the central nervous system (CNS) by the primary afferent neuron. There are two classes of nerve fibres which transmit the pain signal generated by the nociceptors: type $A$, which are medium to large diameter myelinated neurons, and type C, small diameter unmyelinated neurons (Gasser, 1941; reviewed in Manzano et al., 2008). Neurons with a larger diameter fibre conduct impulses at a higher velocity (Gasser \& Erlanger, 1927).

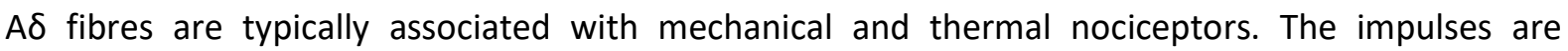
conducted rapidly $(2-30 \mathrm{~m} / \mathrm{s})$ in the large-diameter, thinly myelinated axons, giving the first phase of pain, a sharp well-localised sensation (Gasser, 1941; Adriaensen et al., 1983). In contrast, the slow transmission $(0.6-2 \mathrm{~m} / \mathrm{s})$ of polymodal nociceptor impulses is facilitated by the smaller unmyelinated C fibres (Gasser, 1941; Lukoshkova, 1976). This process requires a higher threshold of mechanical, thermal or chemical stimuli and is particularly associated with damaged tissue or inflammation. The sensation is described as secondary pain, a slower burning pain which extends beyond the removal of the noxious stimulus. Non-nociceptive information about touch, pressure and vibration are transmitted at high speed (30-70 m/s) by large, myelinated A $\beta$ fibres (Gasser, 1941). In the peripheral nervous system (PNS), single myelinating Schwann cells wrap around the axon of the neuron forming the myelin sheath. The gaps between these glial cells are termed the nodes of Ranvier, with the axon in this region expressing many ion channels that are required to propagate the action potential (reviewed in Nave \& Werner, 2014). The unmyelinated fibres are surrounded by non-myelin forming Schwann cells, with multiple axons enveloped in a single Schwann cell (reviewed in Armati \& Mathey, 2013).

\subsubsection{Dorsal root ganglion}

The cell bodies (or soma) of the primary afferent neurons are located in the dorsal root ganglion (DRG) or the trigeminal ganglion for innervations from the body and the face, respectively. The DRG are found on the dorsal root of the spinal nerve sitting between adjacent vertebrae, with one on each side 
of the spinal cord to deliver information from each side of the body (Devor, 1999; Figure 1.1). The neurons are pseudo-unipolar, meaning the branch innervating from the periphery and the branch extending into the dorsal horn of the spinal cord (or the trigeminal subnucleus caudalis for facial signals), have a single axon and form a T-junction with a short axon connecting the cell body (Lieberman \& Landon, 1976). Therefore, the electrical signals between the periphery and spinal cord follow a direct pathway, reducing the risk of conduction failure of the sensory information (Amir \& Devor, 2003). When an electrical impulse is being carried along the axon, the DRG can either further propagate the signal, block the signal or act as a low-pass filter and reduce the signal (Gemes et al., 2013). Furthermore, the cell bodies in the DRG are surrounded by satellite glial cells, which can release inflammatory mediators to sensitise the DRG neurons, further propagating the nociceptive signal (Old et al., 2015; Echeverry et al., 2017). The cell bodies in the DRG synthesise the receptors, ion channels and molecules needed within the sensory neuron. These substances are transported down the axons via microtubule-associated motor proteins. Therefore, the DRG is critical for the correct functioning of the neuron (reviewed in Sapunar et al., 2012; Bourne et al., 2014).

\subsubsection{The spinal cord}

The primary afferent neurons extend from the DRG through to the dorsal root entry zone, the boundary between the PNS and CNS. Once the neuron has entered the CNS, the glial-subtype responsible for myelination are the oligodendrocytes, which differ from Schwann cells in that the processes from one oligodendrocyte cell can wrap myelin sheaths around multiple axons (reviewed in Michalski \& Kothary, 2015). The neurons extend vertically for several segments, forming part of the dorsolateral Lissauer tract, before entering into the grey matter of the dorsal horn and synapsing with second-order neurons. Some of the primary neurons also synapse with interneurons in the dorsal horn that are associated with reflexive motor activity. The grey matter of the spinal cord is divided up into ten layers termed the Rexed's laminae, with the first six layers constituting the dorsal horn. A $\delta$ fibres typically ascend 3-4 segments of the Lissauer tract and terminate in the Rexed's lamina I (also called the marginal zone), II (substantia gelatinosa) or V (nucleus proprius). C fibres typically ascend one segment and terminate in the Rexed's lamina II. The cell body of the second-order neurons are found in the dorsal horn and the axons typically cross over (decussate) through the central canal of the spinal cord to the contralateral side and ascend to higher centres. The spinothalamic tract is the major ascending pathway carrying information on pain, temperature and crude touch, however, information is also carried by the spinoreticular, spinomesencephalic, spinotectal and spinohypothalamic tracts, which all make up the anterolateral system located within the white matter of the spinal cord (reviewed in Lamont et al., 2000; Marchand, 2008; Figure 1.1; Bourne et al., 2014). 


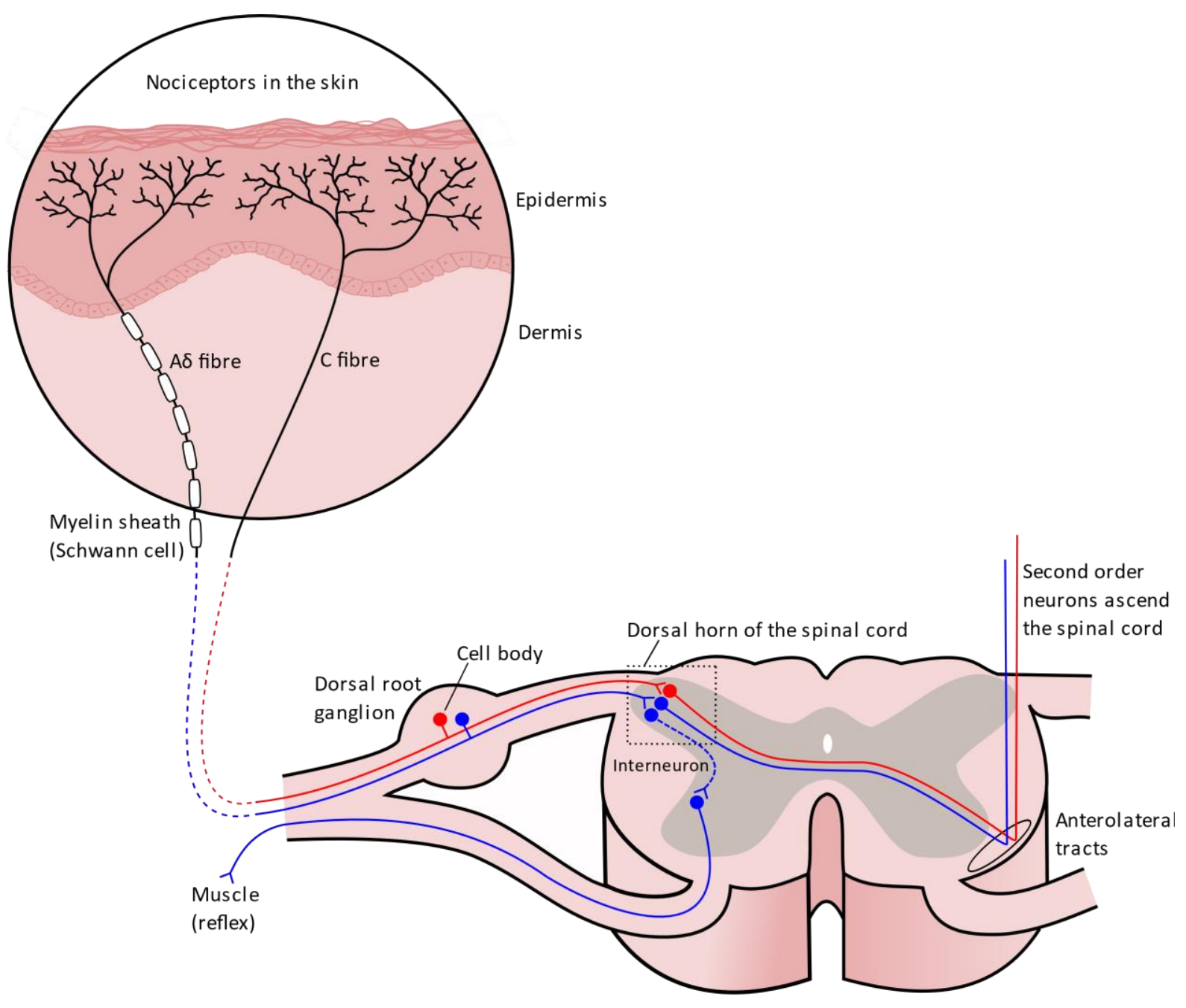

Figure 1.1: Pain signalling pathway from the nociceptors of the skin to the spinal cord

Nociceptors are unencapsulated, unmyelinated free nerve endings found in the skin and other organs that sense pain. The nociceptive signal is carried through either large-diameter, myelinated $A \delta$ fibres or smaller, unmyelinated $C$ fibres. The primary afferent neuron is pseudounipolar with the cell body in the dorsal root ganglion. The primary afferent fibres synapse with the second-order neuron in the dorsal horn of the spinal cord. The axon of the second-order neuron crosses through the central canal of the spinal cord to the contralateral side and ascends to higher centres, primarily via the spinothalamic tract. Some of the primary neurons also synapse with interneurons in the dorsal horn that are associated with reflexive motor activity. 


\subsubsection{Brain regions involved in pain perception}

Due to the complex nature of pain processing, there are some variations on the pain tracts in the literature, here I have described the simplified versions of the major pathways, however, the nomenclature may slightly vary from other sources. In the spinothalamic tract, the second-order neuron ascends the spinal cord, through the medulla and pons, and terminates in the thalamus, synapsing with third-order neurons. In this way, the thalamus acts as the relay site for signals containing sensory information. The third-order neurons deliver information involved in the localisation and discrimination of pain to the somatosensory cortex. The spinoreticular tract (also known as the spinoreticulothalamic tract) relays the message to the thalamus indirectly, as the information ascends the spinal cord and synapses in the brainstem reticular formation before projecting into the thalamus, hypothalamus and limbic system (anterior cingulate cortex and insula). Some evidence suggests that the spinoreticular tract does not cross the spinal cord, instead, the secondary neuron ascends ipsilaterally to the stimulus (Huma et al., 2015). This pathway is involved in the autonomic, reflexive, emotional and motivational-affective aspects of pain. The spinomesencephalic tract terminates in the periaqueductal grey matter and the midbrain raphe nuclei, which are involved in the modulation of the pain pathways. The spinotectal tract, which is often included as part of the spinomesencephalic tract, terminates in the superior colliculus and is involved in the reflexive movement of the head and eyes towards the painful stimulus. The spinohypothalamic tract terminates in the hypothalamus and is involved in the endocrine responses to pain (reviewed in Lamont et al., 2000; Marchand, 2008; Bourne et al., 2014) (Figure 1.2).

Many areas of the brain are involved in aspects of pain processing, perception, anticipation and relief. Due to the complex and subjective nature of pain, many brain regions form a 'pain network' which together give the pain experience. A meta-analysis found that the major regions involved in pain included the primary and secondary somatosensory cortex, involved in the perception and registering the body part involved and intensity of pain; anterior cingulate cortex and insula, involved in the processing and unpleasant feeling of pain; and the prefrontal cortex, involved in pain processing and making a plan of action (Apkarian et al., 2005; Koyama et al., 2005). A further study looked at the perception of pain through different regions and found that information is initially but simultaneously processed in the sensory, motor and limbic areas, before the conscious perception of pain has occurred; and then progresses to the prefrontal, parietal and anterior insula networks; and finally to the cingulate cortex and hippocampus where activation persists beyond the conscious perception of pain, and could be linked to memory encoding and self-awareness of pain (Bastuji et al., 2016). 


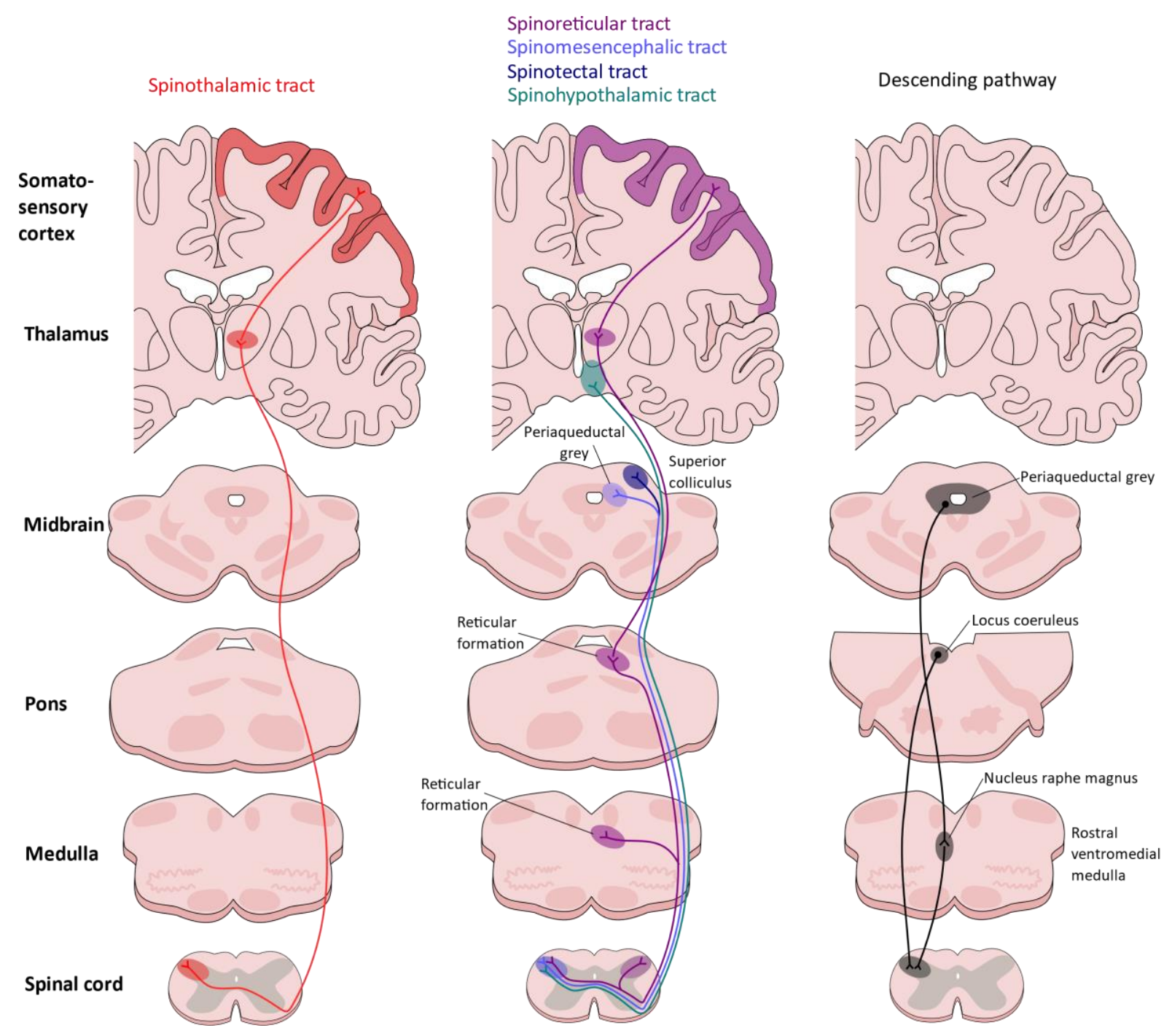

Figure 1.2: Ascending and descending pain pathways

The major pathway for the transmission of pain is the spinothalamic tract which relays information from the dorsal horn of the spinal cord to the thalamus and the somatosensory cortex. The other ascending pathways involved in pain processing are the spinoreticular tract, the spinomesencephalic tract, the spinotectal tract and the spinohypothalamic tract. The modulation of pain occurs via the descending pathway which begins in the periaqueductal grey in the midbrain and extends to the nucleus raphe magnus in the rostral ventromedial medulla and finally to the dorsal horn of the spinal cord. Figure adapted from Kandel et al. (2000). 


\subsubsection{Modulation of nociception}

\subsubsection{Gate control theory}

Gate control theory explains the observation that rubbing an injury (or an animal licking an injury) will lessen the level of pain. This theory was first published in 1965 (Melzack \& Wall, 1965), proposing that interneurons in the Rexed's lamina II (substantia gelatinosa) can have an inhibitory effect on secondorder nociceptive neurons. In this way, the substantia gelatinosa acts as a gate, either allowing the nociceptive signal to pass through or modulating it before it reaches the brain. $A \delta$ and $C$ fibres that terminate in the substantia gelatinosa inhibit the interneurons and "open the gate". A $\beta$ fibres which carry information on touch, pressure and vibration, activate the inhibitory interneurons, and therefore "close the gate". In this way, rubbing (or licking) an injury to the skin activates the $A \beta$ fibres which reduce the transmission of the pain signal. The theory is a simplistic view of the neurons in the spinal cord; however, it was revolutionary in understanding the way that nociceptive signals can be modulated at the spinal cord level (reviewed in Mendell, 2014; Katz \& Rosenbloom, 2015). It is now known that pain can also be modulated by the brain via descending antinociceptive pathways.

\subsubsection{Descending antinociceptive pathways}

Descending pathways from the brain regulate nociceptive signalling at the dorsal horn of the spinal cord (Figure 1.2). The periaqueductal grey receives information from higher brain areas, including the hypothalamus, the amygdala and the anterior cingulate cortex, and is the region primarily involved in antinociception, including opioid-mediated inhibition of pain signals. Early experiments in rabbits and rats found that microinjection of opioids or electrical stimulation of the periaqueductal grey leads to a significant analgesic effect (Tsou \& Jang, 1964; Reynolds, 1969). It is now known that the periaqueductal grey is a region rich in endogenous opioids that inhibit pain signals. Neurons from the periaqueductal grey descend into the rostral ventromedial medulla, which acts as a relay centre for pain signals, in that serotonergic neurons projecting from this region can either facilitate or inhibit pain signals at the dorsal horn of the spinal cord. There are three classes of neurons projecting from the rostral ventromedial medulla: on-cells, off-cells and neutral-cells. On-cells facilitate pain and are inhibited by opioids (Heinricher et al., 1989; Fields et al., 1995). Off-cells inhibit the nociceptive signal and are excited by opioids (Heinricher et al., 1989; Fields et al., 1995). From the rostral ventromedial medulla, these serotonergic neurons, as well as noradrenergic neurons from the locus coeruleus, project to the spinal cord and synapse with the primary afferent neurons, spinothalamic neurons or interneurons in the dorsal horn, leading to regulation of the pain signal (reviewed in Bourne et al., 2014; Ossipov et al., 2014). 


\subsection{Pain classifications and mechanisms}

Pain is a complex and subjective sensation, described by the International Association for the Study of Pain as "an unpleasant sensory and emotional experience associated with actual or potential damage, or described in terms of such damage" (IASP Task Force on Taxonomy, 1994). Despite what is known about the physiology of pain, there are still many factors which may affect an individual's experience of pain, including cognitive factors (Coghill et al., 2003; Bushnell et al., 2013), environment (Bushnell et al., 2015), past traumatic events (Nicol et al., 2016), expectations (Koyama et al., 2005; Taylor et al., 2017), gender and sex (Fillingim et al., 2009; Ramirez-Maestre \& Esteve, 2014). The clinical classification of pain can be difficult, as many factors can be considered, including initiating conditions, duration, area of the body affected or the underlying mechanism. In the current thesis, pain has been classified into three broad categories based on the underlying mechanisms: nociceptive, inflammatory and neuropathic pain. However, some pain conditions may have characteristics falling within multiple classifications. Further definitions include: allodynia, defined as pain produced by a stimulus that is not normally noxious; and hyperalgesia, defined as exaggerated pain in response to noxious stimuli.

\subsubsection{Acute nociceptive pain}

Acute nociceptive pain has a protective function and is essential to survival, as demonstrated in families with a congenital inability to feel pain (Cox et al., 2006; Bennett \& Woods, 2014; Chen et al., 2015). The fast, sharp pain acts as a warning system to detect a noxious stimulus and prevent impending or actual damage. The nociceptors have high thresholds and are only activated by intense stimuli. Therefore, only stimuli which require an immediate response produce the painful reaction and the withdrawal reflex. Due to the unpleasant sensation elicited, often the noxious stimuli will be avoided in the future. This pain may be in response to injury to the skin, muscles, visceral organs, joints, tendons or bones. For example, the pain from a cut or burn to the skin will elicit nociceptive pain (reviewed in Woolf, 2010; Baliki \& Apkarian, 2015).

Pain is sensed at the tissue level by transducer proteins and ion channels on the nociceptor (Figure 1.3). The largest group involved in sensing pain is the TRP family, which is divided into six subfamilies in mammals (reviewed in Jardin et al., 2017). The polymodal TRP subfamily vanilloid member 1 (TRPV1) was found to be responsible for detecting noxious temperatures over $42^{\circ} \mathrm{C}$ (Caterina et al., 1999); however, it has since been shown that the warm-hot temperature range can be sensed by many vanilloid TRP channels, including TRPV2, TRPV3, TRPV4 (Brauchi et al., 2006; Dhaka et al., 2006) and temperature-sensitive potassium channels TREK-1 (Alloui et al., 2006) and TREK-2 (Pereira et al., 2014). The TRP subfamily melastatin member 8 (TRPM8) cation channel has been shown to sense 
innocuous cool temperatures $\left(<28^{\circ} \mathrm{C}\right)$ (Dhaka et al., 2006; Bautista et al., 2007; Colburn et al., 2007). However, intense noxious cold temperatures may involve several channels (Lolignier et al., 2016), with evidence for the involvement of TRPM8 (Knowlton et al., 2011; Knowlton et al., 2013), TRP subfamily ankyrin member 1 (TRPA1) (Kwan et al., 2006; Gentry et al., 2010; Memon et al., 2017), voltage-gated Nav1.9 sodium channel (Leipold et al., 2015; Lolignier et al., 2015) and potassium channels TREK-1, TREK-2 and TRAAK (Alloui et al., 2006; Noel et al., 2009; Pereira et al., 2014).

The high-threshold mechanical nociceptors respond to tactile or mechanical deformation such as pinching, cutting or excessive pressure. The ion channels responsible for sensing mechanical stimuli are not as well characterised as the ion channels involved in thermal pain transduction. Although advancements have been made in understanding the channels in Caenorhabditis elegans and Drosophila melanogaster, this has not always translated in the mouse studies (Geffeney \& Goodman, 2012). There is evidence of the involvement of the TRPV1 (Walker et al., 2003; Nozadze et al., 2016), TRPV4 (Suzuki et al., 2003; Alessandri-Haber et al., 2005; Tsuno et al., 2017), TRPA1 (Brierley et al., 2009; McGaraughty et al., 2010; Lennertz et al., 2012) and acid-sensitive ion channels (Price et al., 2001; Wemmie et al., 2013), TREK-1 and TREK-2 potassium channels (Bang et al., 2000; Alloui et al., 2006), as well as stomatin-like protein 3 (Wetzel et al., 2007) and Piezo proteins (Coste et al., 2010). The TRP channels are also able to act as chemical sensors. The TRPV1 channel was first discovered to be sensitive to capsaicin from hot chilli peppers (Caterina et al., 1997), but since then many more chemical stimuli have been found the activate TRP channels including spider toxins (Siemens et al., 2006), allicin from garlic (Macpherson et al., 2005), camphor (Xu et al., 2005), oregano, thyme and clove (Xu et al., 2006), formalin (McNamara et al., 2007), mustard oil (Jordt et al., 2004), nicotine (Talavera et al., 2009), low pH (Tominaga et al., 1998), and a range of other exogenous and endogenous stimuli (reviewed in Dai, 2016). 


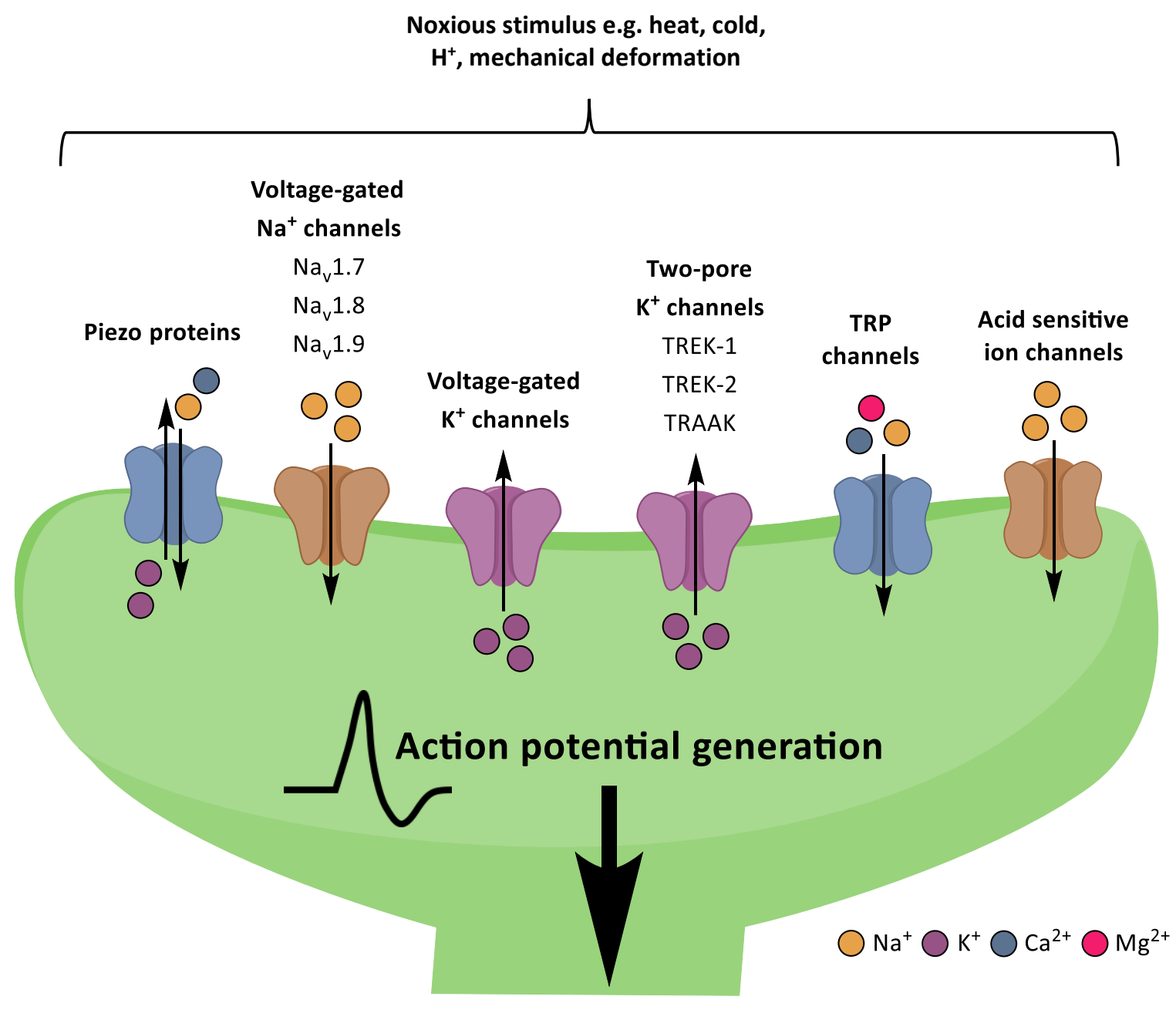

Figure 1.3: Acute nociceptive pain

Transducer proteins and ion channels on the nociceptor sense when tissue is damaged by heat, cold, mechanical deformation or a chemical stimulus. An action potential is generated in the nociceptive peripheral sensory neuron following the efflux of $\mathrm{K}^{+}$and the influx of $\mathrm{Na}^{+}, \mathrm{Ca}^{2+}$ and $\mathrm{Mg}^{2+}$. Figure adapted from Marchand et al. (2005) and Lumpkin et al. (2010). 


\subsubsection{Inflammatory pain}

Pain is one of the five cardinal signs of inflammation. Injury to tissue initiates an inflammatory response that is intended to contain pathogens, clear damaged tissue, and promote repair and tissue regeneration. Inflammatory pain has a lower threshold than nociceptive pain. By heightening sensory sensitivity after tissue damage, inflammatory pain assists in the healing of the injured body part by discouraging physical contact and movement, and promoting rest and repair. Acute inflammatory pain can occur in a range of different conditions including wound healing and infection. Chronic inflammatory pain is associated with conditions such as rheumatoid arthritis and inflammatory bowel disease (IBD) (reviewed in Linley et al., 2010; Woolf, 2010).

Inflammatory pain leads to the peripheral sensitisation of nociceptors by decreasing the activation threshold. Following tissue destruction, inflammation leads to an innate immune cascade yielding the release of active factors from blood, local and migrating inflammatory cells and injured cells (Rittner \& Stein, 2005). Activation of TRP channels can occur via the release of products from the site of injury such arachidonic acid (Watanabe et al., 2003) and the products of oxidative stress (Andersson et al., 2008). During inflammatory pain, the nociceptive terminals release inflammatory mediators such as calcitonin gene-related peptide (CGRP) and substance $P$ which increase vascular permeability, leading to the formation of oedema and the further infiltration of immune cells (Ji et al., 2014) (Figure 1.4).

Neutrophils are the most abundant leukocytes found in the circulating bloodstream. Within the first hour of the onset of inflammation, neutrophils undergo extravasation and migrate along chemical gradients to accumulate at the site of injury (Kolaczkowska \& Kubes, 2013). Neutrophil migration is associated with increased inflammatory pain and oedema (Guerrero et al., 2008). Macrophages, neutrophils and mast cells release further inflammatory mediators including chemokines (Zhang et al., 2005a), bradykinin (Sugiura et al., 2002), serotonin (Ohta et al., 2006), histamine (Kajihara et al., 2010), glutamate (Hu et al., 2002; Sikand \& Premkumar, 2007), prostaglandins (Zhang et al., 2008), adenosine triphosphate (ATP) (Tominaga et al., 2001) and nerve growth factor (Stein et al., 2006) which lead to peripheral sensitisation of the nociceptive sensory neurons. Peripheral sensitisation occurs by a reduction in the threshold or an increase in the responsiveness of the nociceptor and contributes to the hyperexcitability of the peripheral sensory neurons (Figure 1.4). This occurs when the inflammatory mediators bind to receptors on the peripheral nerve, activating downstream protein kinases that directly activate the transducer proteins and ion channels. Furthermore, binding of the inflammatory mediators leads to posttranslational changes to increase expression of the transducer proteins and ion channels, as well as increased trafficking of these proteins and channels to the cell surface (reviewed in Pinho-Ribeiro et al., 2017). 


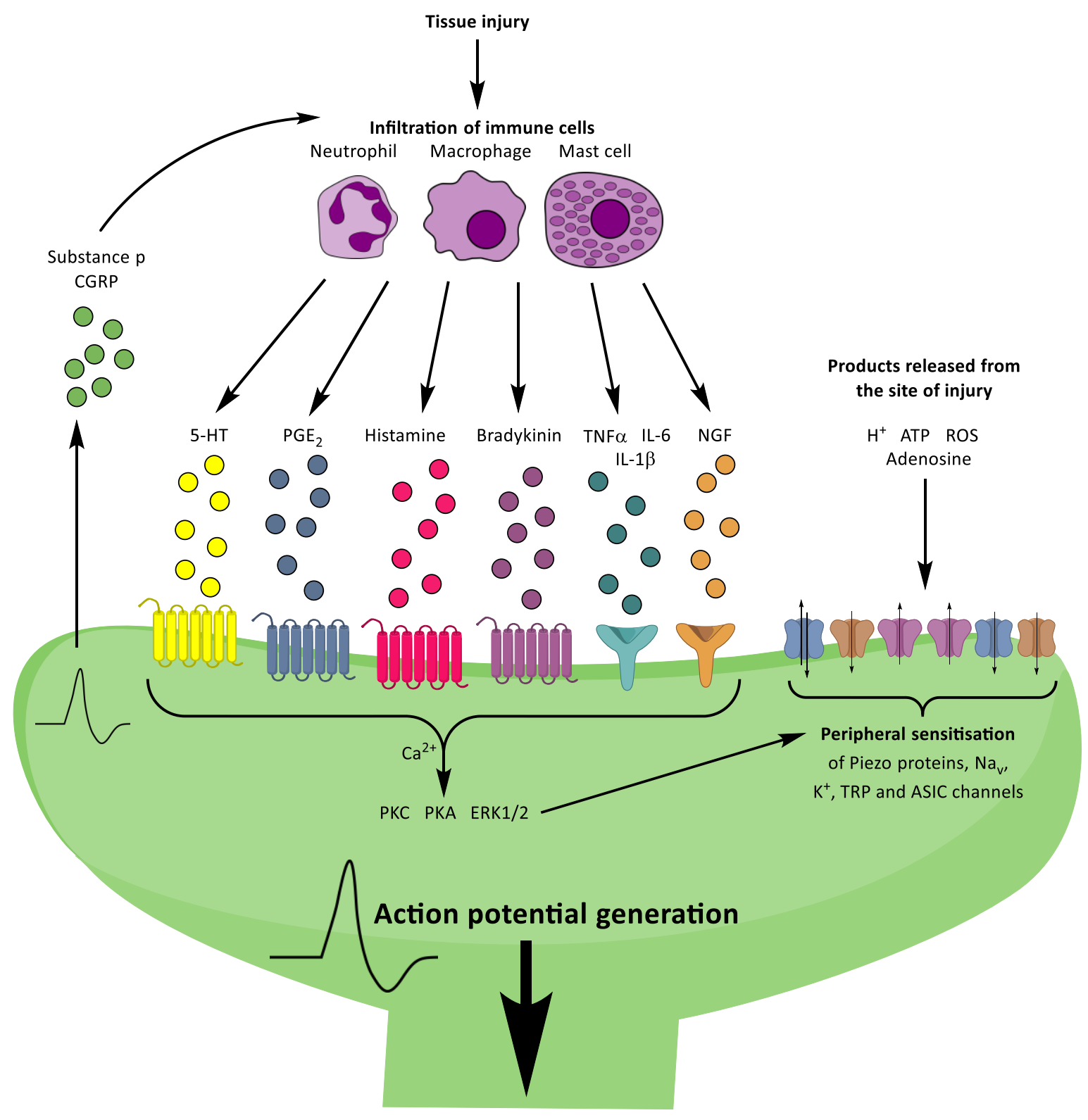

Figure 1.4: Inflammatory pain mechanisms

Following tissue injury, immune cells such as macrophages, neutrophils and mast cells infiltrate into the damaged tissue. The immune cells release inflammatory mediators such as serotonin (5-HT), prostaglandins $\left(\mathrm{PGE}_{2}\right.$ ), histamine, bradykinin, cytokines (TNF $\left.\alpha, \mathrm{IL}-1 \beta, \mathrm{IL}-6\right)$ and nerve growth factors (NGF). These factors act on the respective receptors on the nociceptor of the peripheral sensory nerve. The activation of these receptors ultimately leads to the activation of several kinases (PKA, PKC, ERK1/2) which leads to the hypersensitivity and hyperexcitability of the neuron via peripheral sensitisation of the transducer proteins and ion channels. Other products released from the site of injury also activate the transducer proteins and ion channels to generate nociceptive action potentials. The activation of the nociceptor further leads to the release of substance $P$ and calcitonin gene-related peptide (CGRP), contributing to oedema and further infiltration of immune cells. Figure adapted from Marchand et al. (2005) and Ji et al. (2014).

$A T P$, adenosine triphosphate; $C A M P$, cyclic adenosine monophosphate; ERK1/2, extracellular signalregulated kinase 1/2; IL, interleukin; $P G E_{2}$, prostaglandin $E_{2} ; P K A$, protein kinase $A ; P K C$, protein kinase $C$; ROS, reactive oxygen species; $T N F \alpha$, tumour necrosis factor- $\alpha$. 


\subsubsection{Neuropathic pain}

Neuropathic pain is not protective but maladaptive, resulting from abnormal functioning of the nervous system. Neuropathic pain occurs as a result of damage to the nervous system which is characterised by tingling, numbness and on-going spontaneous burning pain. Neuropathic pain is largely the consequence of amplified sensory signals and changes in neural processing in the CNS, termed central sensitisation, as well as peripheral sensitisation. Examples of conditions associated with neuropathic pain include spinal cord injuries, diabetes, multiple sclerosis, stroke, human immunodeficiency virus (HIV) infection and chemotherapy treatments. In contrast, dysfunctional neuropathic pain is caused by conditions in which there is no damage or apparent neuroinflammation, including fibromyalgia and tension-type headache, where substantial pain is evoked but there is no noxious stimulus accountable (reviewed in Kuner, 2010; Cohen \& Mao, 2014).

Central sensitisation is defined by the IASP as the increased responsiveness of the nociceptive sensory neurons in the CNS to their normal of subthreshold afferent input (IASP Task Force on Taxonomy, 1994). A number of different mechanisms are responsible for this phenomenon, with short-lasting effects due to homosynaptic and heterosynaptic potentiation, whilst longer-lasting maintenance effects are due to activation of glial cells, membrane excitability and transcriptional changes. Homosynaptic potentiation occurs when the synaptic activity of the nociceptive neurons of the dorsal horn is amplified, increasing the level of pain for a localised low threshold stimulus, leading to primary hyperalgesia. Heterosynaptic potentiation occurs when subthreshold nociceptive or non-nociceptive signals converge on a common dorsal horn neuron, together meeting the action potential threshold and increasing the activity of other non-activated synapses on the entire dorsal horn neuron. Heterosynaptic potentiation lasts beyond the application of the stimulus and is responsible for allodynia and secondary hyperalgesia (reviewed in Woolf, 2011; Vardeh \& Naranjo, 2017) (Figure 1.5).

Following a peripheral nerve injury, neuropathic pain is generated by spontaneous activity occurring in both the damaged and surrounding undamaged neurons. An upregulation of voltage-gated $\mathrm{Na}^{+}$ channels (Hoeijmakers et al., 2015) and downregulation of $\mathrm{K}^{+}$channels (Busserolles et al., 2016) contributes to the hyperexcitability of the peripheral neurons. In addition, expression of TRPV1 is downregulated on damaged neurons but upregulated on surrounding undamaged neurons, leading to a sensitisation to heat (Ma et al., 2005). Similarly, changes in the expression of TRPM8 leads to sensitisation to cold stimuli (Proudfoot et al., 2006). Peripheral nerve damage induces migration and activation of macrophages, which adds to the hypersensitivity of neuropathic pain by releasing inflammatory cytokines (Zhang et al., 2016). Peripheral or central nerve damage causes activation of CNS glial cells, including microglia and astrocytes, that release cytokines, inflammatory mediators and 
increase glutamate concentrations, contributing to the pathogenesis and the long-term maintenance of neuropathic pain (Old et al., 2015; Echeverry et al., 2017).

In this thesis, the model of neuropathic pain utilised is paclitaxel-induced neuropathic pain, a model that uses a chemotherapeutic to induce changes in the peripheral sensory nerve, the DRG and the dorsal horn of the spinal cord, leading to hypersensitivity to mechanical and cold stimulus. The specific mechanisms of paclitaxel-induced neuropathic pain are presented in Chapter 5.

\section{Normal}

A. Normal pain signalling

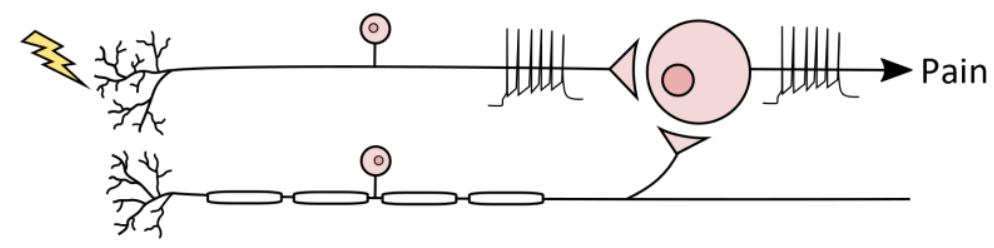

B. Normal touch signalling

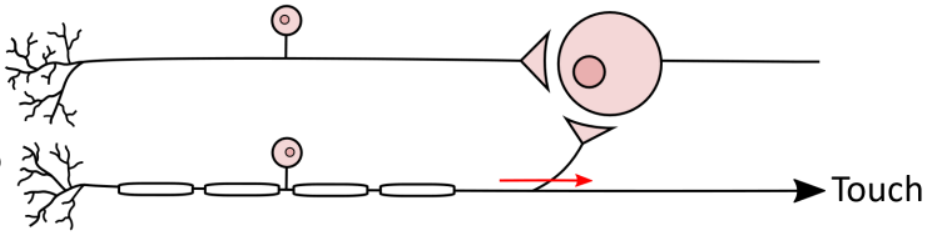

\section{Central sensitisation}

C. Homosynaptic potentiation

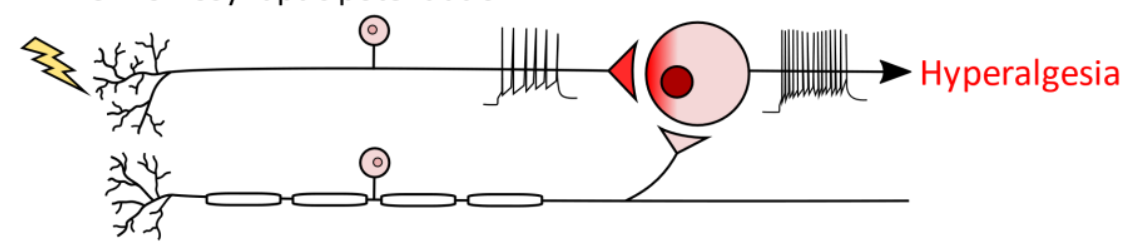

D. Heterosynaptic potentiation

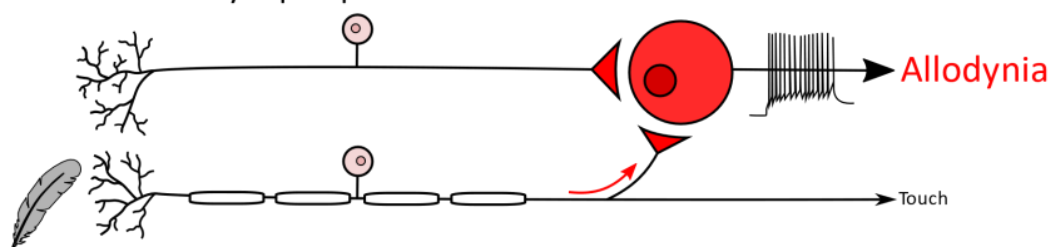

\section{Figure 1.5: Central sensitisation}

Central sensitisation occurs due to homosynaptic and heterosynaptic potentiation leading to the amplification of nociceptive signals (hyperalgesia) or activation of nociceptive neurons in the dorsal horn from a non-noxious stimulus (allodynia). Figure adapted from Latremoliere and Woolf (2009) and Woolf (2011). 


\subsubsection{Sex differences in pain perception}

Men and women perceive pain differently, with increased pain sensitivity and higher prevalence of clinical pain in women (Mogil, 2012; Bartley \& Fillingim, 2013). Specifically, there is a greater frequency of fibromyalgia (Branco et al., 2010), migraine (Victor et al., 2010; Baykan et al., 2015), chronic tensiontype headache (Karli et al., 2006), inflammatory bowel syndrome (IBS) (Pan et al., 2016) and lower back pain (Manchikanti et al., 2014) in women, as well as chronic pain syndromes which can only occur in women, such as endometriosis. There are male dominant conditions such as gout (Kuo et al., 2015) and cluster headaches (Giffin, 2016), however, there is still a greater prevalence of pain in women overall (Nahin, 2015). Pain sensitivity in humans has been tested in the laboratory and the results are complex but generally show women are more sensitive, have a lower tolerance and are better at discriminating between varying levels of pain (Fillingim et al., 2009). One meta-analysis found that the mechanical pressure pain thresholds for women were lower and that women tolerate less thermal (both heat and cold) and pressure pain than men (Racine et al., 2012).

The sex hormones have a significant effect on the sex differences in pain perception. Testosterone has been shown to have an antinociceptive effect (Gaumond et al., 2002; Ceccarelli et al., 2003; Nag \& Mokha, 2009), whereas the effect of oestradiol and progesterone are more complicated with both pro-nociceptive and antinociceptive effects (Smith et al., 2006; Craft, 2007; Kim et al., 2012). Pain sensitivity changes throughout the menstrual cycle, with increased sensitivity in the late luteal and early follicular phases (Martin, 2009). In women with high oestradiol levels, endogenous opioid activity is increased compared to low oestradiol conditions (Smith et al., 2006), suggesting that oestradiol may have an interaction with the endogenous opioid system. There are also psycho-social reasons for the differences in pain perception between the sexes, including differences in coping mechanisms (Keogh \& Eccleston, 2006), as well as sociocultural beliefs, including views around masculinity and pain (Berke et al., 2017). 


\subsection{Pre-clinical behavioural models of pain}

To test analgesic compounds, many animal behavioural models have been developed to mimic human pain conditions or to screen against specific stimuli. These pre-clinical models are used to study characteristic behaviours and in vivo mechanisms associated with pain by using mechanical, thermal, electrical, inflammatory and chemical stimuli. However, pain is the result of a complex process and one behavioural test may not be representative of the overall pain sensation, therefore, several pain models are often used to validate observations (reviewed in Mogil, 2009). In addition, it has been shown that stress on the animals can lead to an antinociceptive effect, so care must be taken to select models and techniques to reduce this effect (Parikh et al., 2011; Sorge et al., 2014). In the past, it has been standard practice to carry out experiments in only male animals to decrease variability caused by the different sexes. However, the USA National Institutes of Health $(\mathrm{NIH})$ are now requiring more extensive studies using both sexes (Clayton \& Collins, 2014), as the importance of gender differences are becoming more apparent. In this section, animal models of pain have been reviewed, with a particular focus on the models used in this thesis.

\subsubsection{Routes of administration}

Compounds of interest can be administered in different ways to mimic different clinical routes of administration. Intraperitoneal (i.p.) injection is commonly used for administration to laboratory rodents in which intravenous (i.v.) injection is difficult. The i.p. administration is considered similar to oral administration in humans as the liquid is primarily absorbed into the mesenteric vessels and the portal vein, undergoing hepatic metabolism before reaching the systemic circulation (Lukas et al., 1971). However, comparing the two routes of administration in mice, i.p. injection leads to a faster absorption into the bloodstream and higher bioavailability as drugs administered orally have to undergo luminal metabolism and efflux in the gastrointestinal system (Gavhane \& Yadav, 2012). In comparison, i.v. injection into the tail vein of rodents delivers the solution into the blood circulation, bypassing the luminal and hepatic metabolism, although smaller volumes and non-irritant solutions are required to avoid pulmonary oedema and haemolysis. Another common route of administration in rodents is via injection into the subcutaneous (s.c.) space in the skin. This method leads to a slower rate of absorption of the injected liquid into the capillaries in the skin, however, this route also avoids the initial hepatic metabolism that i.p. injected solutions undergo (reviewed in Turner et al., 2011).

\subsubsection{Nociceptive pain models}

Nociceptive models of pain are frequently used to measure the analgesic properties of compounds. One of the advantages of using these models is the nociceptive stimulus only needs to be applied for 
a short duration, therefore reducing the suffering of the animals. Often the stimulus can be retested on the same animal within a relatively short period of time, giving information on the duration of action of the analgesic compound using a repeated measures design.

\subsubsection{Nociceptive response to temperature}

Nociceptive models of pain often use a reflexive pain behaviour, frequently involving a thermal stimulus. A common model is the tail withdrawal assay, which involves immersion of the tail in warm water, typically at a constant temperature between $50-55^{\circ} \mathrm{C}$, generating a characteristic tail flick response (D'amour \& Smith, 1941). More recently, models have been developed which use an infrared beam focussed on the tail, allowing for the test to become automated. The time for the tail to flick is measured as a latency, with a longer reaction time indicating an analgesic effect. The tail withdrawal behaviour is a spinal reflex which does not require higher brain processes (Irwin et al., 1951); however, the analgesic increase in latency involves cortical, brain stem and spinal cord regions and occurs via activation of the descending pain pathways (Levy \& Proudfit, 1979; Fields et al., 1983; Roberts \& Rees, 1986).

Another common thermal nociceptive pain model is the hotplate test, where the animal is placed on a heat-conducting surface and the time for the animal to lick or withdraw a paw is measured (Woolfe \& Macdonald, 1944; Eddy \& Leimbach, 1953). The classical model uses a hotplate at a constant temperature, however, a dynamic hotplate is capable of slowly increasing the temperature, therefore measuring the threshold at which the temperature becomes noxious (Hunskaar et al., 1986). The hotplate test is a supraspinal reflex, as the act of selecting a paw to withdraw and jumping to escape the thermal stimulus involves higher processing (Chapman et al., 1985). A further test using a thermal heat stimulus on the hind paw is the Hargreaves' test, which involves placing the animal in a glass bottom enclosure with a concentrated beam of light directed at an individual paw, therefore isolating the noxious stimuli, leaving the other paw as the control (Hargreaves et al., 1988). More recently, a thermal probe test which involves applying the probe to the hind paw and rotating to initiate the warming process, with the withdrawal latency automatically recorded (Deuis \& Vetter, 2016). The advantage of this technique is that it can be carried out in the same chamber that mechanical stimuli are applied, as opposed to other techniques which require the animal to be placed in a specialised enclosure or apparatus, therefore allowing for both measurements to be tested with less acclimatisation and stress on the animal.

Nociceptive pain can also occur to a cold stimulus, using both a cold plate and a dynamic cold plate (Bennett \& Xie, 1988), however, these are less frequently used than the hotplate. Another cold thermal test uses acetone applied to the hind paw (Choi et al., 1994). The acetone evaporates on the 
skin and produces a cooling effect. The amount of time the animal spends licking, flinching or biting the affected paw is measured over time. This technique is often used as a measure of allodynia in neuropathic pain models, as naïve mice typically spend a short time responding to this stimulus, whereas animals affected by neuropathic pain will respond for a longer period of time (Deng et al., 2012; Deng et al., 2015; Mangaiarkkarasi et al., 2015; Paton et al., 2017).

\subsubsection{Nociceptive response to mechanical stimuli}

Mechanical stimuli are typically used to measure allodynia or hyperalgesia. Mechanical sensitivity is often assessed using Von Frey filaments (Von Frey, 1896), which either can be used as a set of manual nylon filaments of varying diameter, each exerting a known force; or the more recently developed electronic von Frey filament (also known as a dynamic plantar aesthesiometer). The animal is placed on an elevated mesh and the filament is held against the plantar surface of the hind paw. The manual filaments use an up-down testing paradigm where the presence or absence of the withdrawal reflex is recorded for each filament tested (Chaplan et al., 1994; Bonin et al., 2014). The electronic device records the specific force exerted that caused the withdrawal reflex (Campana \& Rimondini, 2015). This technique can be used in a range of different pain models, including the paclitaxel-induced neuropathic pain model (Deng et al., 2012; 2015; Mangaiarkkarasi et al., 2015; Paton et al., 2017), with a decrease in the withdrawal threshold force indicating allodynia and an increase in the withdrawal threshold force indicating analgesia.

The Randall-Selitto test uses pressure on the paw to measure mechanical thresholds (Randall \& Selitto, 1957). The hind paw is held in a vice-type apparatus and with an increasing pressure placed on the paw until the animal shows withdrawal behaviour, struggling or vocalisation. An improvement to this procedure was developed by using a strain gauge attached to forceps (Luis-Delgado et al., 2006). The forceps slowly increase the pressure until withdrawal of the hind paw occurs. This technique allows for the animals to be loosely restrained, rather than with the Randall-Selitto test where the animals need to be held inside an apparatus, therefore reducing stress in the animal.

\subsubsection{Chemical nociceptive pain models}

Non-reflexive nociceptive pain behaviours often involve the injection of a chemical stimulus into the hind paw and may induce both nociceptive and inflammatory pain phases. One example of this is the intraplantar formaldehyde assay, whereby formaldehyde is injected into the plantar surface of the hind paw. Formaldehyde induces a biphasic effect, with an initial nociceptive phase of pain lasting approximately 10-15 min, followed by a second inflammatory phase of pain between 20-60 min. The level of pain can be measured by behavioural observations such as weight-bearing behaviours, paw withdrawal, flinching and licking (Dubuisson \& Dennis, 1977). The effect is concentration-dependent, 
with low formaldehyde concentrations $(0.02-0.2 \%)$ showing the nociceptive phase pain only, compared to high formaldehyde concentrations ( $\geq 1 \%$ ) which result in the biphasic effects (Rosland et al., 1990). The first phase of nociceptive pain is due to the formaldehyde activating the TRP channels, directly acting on TRPA1 on the sensory neuron (McNamara et al., 2007), whilst the secondary phase is due to the inflammatory processes in the injured tissue.

\subsubsection{Visceral nociceptive pain models}

Visceral pain results from the activation of nociceptors of the thoracic, pelvic or abdominal viscera. Visceral structures are more sensitive to distention, ischaemia and inflammation rather than the more typical cutting or burning stimuli of the skin. Most visceral pain can be categorised into either nociceptive pain, including distention pain, for example by urinary stones, and ischemic pain, for example, caused by myocardial infarction; or inflammatory pain, including appendicitis and gastrointestinal disorders, such as IBD (reviewed in Cervero, 2009; Sikandar \& Dickenson, 2012). A common visceral pain model is the acetic acid writhing assay, which involves i.p. administration of acetic acid. This causes irritation to the serous membranes and leads to the characteristic writhing and abdominal contraction movements, the frequency of which is counted over time (Koster et al., 1959). Further models of visceral pain involve the injection of chemical agents into the hollow organs such as the colon or the bladder. A common model of colon distention involves the insertion of a balloon into the colon, which is subsequently inflated and the visceromotor activity of the abdominal muscles measured (Ness \& Gebhart, 1988).

\subsubsection{Inflammatory pain models}

Models of inflammatory pain often involve the injection of a chemical stimulus or inflammatory agent into the hind paw of mice or rats. The intraplantar formaldehyde model of inflammatory hyperalgesia produces a severe and immediate peripheral inflammation (Hunskaar et al., 1985; Hunskaar \& Hole, 1987; Shibata et al., 1989; Rosland et al., 1990; Saddi \& Abbott, 2000). As previously stated, the intraplantar formaldehyde model induces a nociceptive first phase of pain and the second phase of inflammatory pain. Studies have shown that analgesic drugs such as morphine and codeine suppress pain behaviours in both phases, whereas, non-steroidal anti-inflammatory drugs are only effective for treatment of the inflammatory phase (Hunskaar \& Hole, 1987).

Formaldehyde has a short duration of action, lasting about an hour (Tjolsen et al., 1992), whilst other compounds have been used to induce a longer lasting inflammatory pain. Carrageenan, phorbol ester, capsaicin and complete Freud's adjuvant are all used as inflammatory stimuli in animal models of pain (reviewed in Mogil, 2009). Carrageenan can be injected into the paw, muscle or joint, producing an acute inflammatory pain which becomes chronic at around 2 weeks (Hargreaves et al., 1988; 
Radhakrishnan et al., 2003). Complete Freud's adjuvant can also be injected into the paw, muscle or joint, producing a more persistent chronic inflammatory pain (Fehrenbacher et al., 2001). This model can be compared to human conditions such as rheumatoid arthritis and tendonitis. Injection of capsaicin produces local inflammation by activating TRPV1-positive nociceptors (Szolcsanyi et al., 1985; Tominaga et al., 1998), and induces both mechanical and thermal allodynia at the site of injection (Simone et al., 1989). Inflammatory pain can also be induced using a bacterial agent, such as Mycobacterium butyricum, that can be injected into the joints and used as a model of arthritic pain (Petty et al., 1994).

\subsubsection{Neuropathic pain models}

Neuropathic pain occurs due to damage or disease of the nerves in the PNS or CNS. To induce nerve damage, a chronic nerve injury can be created through constriction, ligation or transection of either the peripheral sciatic nerve (Bennett \& Xie, 1988) or spinal nerve at the lumbar region (Kim \& Chung, 1992). These models result in the development of thermal and mechanical allodynia and guarding behaviour of the affected limbs (Takaishi et al., 1996). Central damage can also be induced using spinal hemi-section (Christensen et al., 1996) and excitotoxin-induced spinal cord injury (Yezierski \& Park, 1993) to model traumatic spinal cord injuries. Neuropathic pain can also be induced using chemotherapeutic agents, and neuropathic pain is often measured in rodent models of diabetes, HIV infection and chronic ethanol consumption (reviewed in Colleoni \& Sacerdote, 2010; Jaggi et al., 2011; Hoke, 2012).

\subsubsection{Chemotherapy-induced neuropathic pain}

In the clinic, chemotherapeutic agents, such as the commonly used vinca alkaloids, platinum derivatives and taxanes, can induce neuropathic pain. Therefore, animal models of chemotherapyinduced neuropathic pain have been developed using the chemotherapeutic agents vincristine, cisplatin, oxaliplatin and paclitaxel (reviewed in Jaggi et al., 2011; Sisignano et al., 2014). The chemotherapy drugs are given as multiple systemic treatments, which causes the animals to develop chronic thermal and mechanical allodynia over a period of time (about 16 days for paclitaxel-induced neuropathic pain). Using a drug-induced neuropathic pain model avoids the need for surgery and is clinically more relevant for patients affected by this difficult to treat condition (reviewed in Jaggi et al., 2011; Sisignano et al., 2014). For further details on the mechanism of paclitaxel-induced neuropathic pain see Chapter 5. 


\subsubsection{Facial grimace scale}

A facial grimace scale has been developed for the measurement of pain in rodents, characterised for both mice (Langford et al., 2010) and rats (Sotocinal et al., 2011). The scale judges the facial features of the animals for orbital tightening, nose and cheek bulge, ear position and whisker changes assigning a score of either 'not present', 'moderate' or 'severe' (Figure 1.6). To set up the experiment, the mice are held in small transparent enclosures and filmed for a set period of time. From the video footage, each frame containing an in-focus unobstructed head-shot are saved for analysis. The advantage of this technique is that it allows for the measure of spontaneous pain rather than purely evoked pain behaviours. In humans, facial expressions are often used to assess levels of pain in infants and sometimes in non-verbal patients (Arif-Rahu \& Grap, 2010; Chang et al., 2015).

\subsubsection{Conditioned pain avoidance behaviours}

Pain can be measured using conditioned behaviours, giving an indication of the aversive component of pain, rather than nociceptive processing (reviewed in $\mathrm{Li}, 2013$ ). Using a two-chamber apparatus with dissimilar visual, tactile and sometimes olfactory stimuli to differentiate the chambers, both conditioned place aversion (CPA) (Johansen et al., 2001) and conditioned place preference (CPP) (Sufka, 1994) paradigms can be used to measure pain avoidance behaviour. CPA involves pairing a chamber with a noxious stimulus, and measuring whether the analgesic treatment can reverse the aversion to the paired chamber. CPP requires the use of a chronic pain model where the animal is in constant pain. The analgesic compound is given in one chamber, and the experiment tests whether the animal shows a preference to that paired chamber. Therefore, CPA paradigm tests whether the aversive nature of pain can be reduced, whereas CPP paradigm measures whether the pain relief from the analgesic was preferable to no treatment. However, one problem with using conditioned experiments is that there is a significant learning component, which the sensation of pain may disrupt.

Other examples of pain avoidance models are the thermal escape test (Mauderli et al., 2000) and place escape/avoidance paradigm (LaBuda \& Fuchs, 2000). These tests use two chambers, which the animal can move freely between. Only one chamber is paired with a noxious stimulus. For example, a chamber with a hot floor connected to a chamber with a normal temperature floor; or one chamber will have an injured paw repeatedly mechanically stimulated, whilst the other will stimulate the uninjured paw. At the beginning of the session, animals will explore both chambers, however, animals will differentiate between the chambers and escape from the noxious one, even when the nonnoxious stimulus is paired with an anxiogenic brightly-light chamber (LaBuda \& Fuchs, 2000; Pedersen \& Blackburn-Munro, 2006). When an analgesic is administered, including morphine or gabapentin, the preference for the non-noxious chamber is attenuated (Pedersen \& Blackburn-Munro, 2006). 

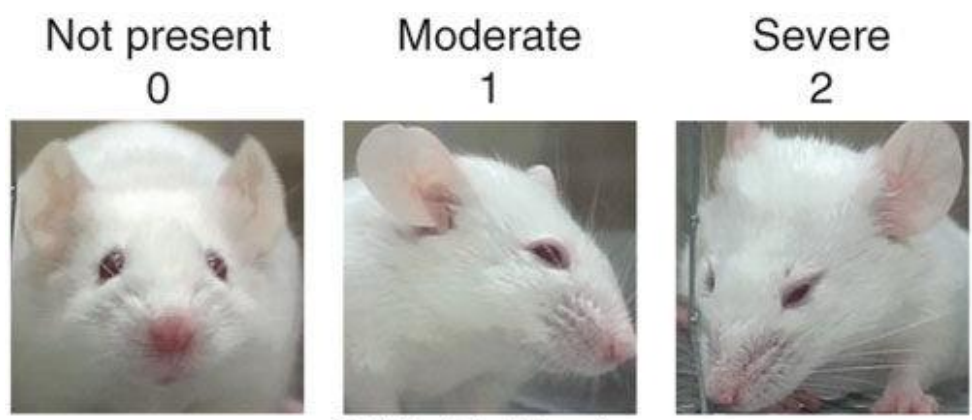

Orbital tightening
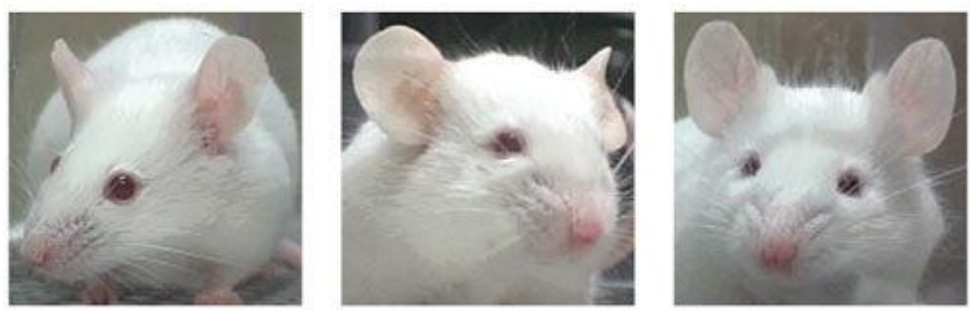

Nose bulge
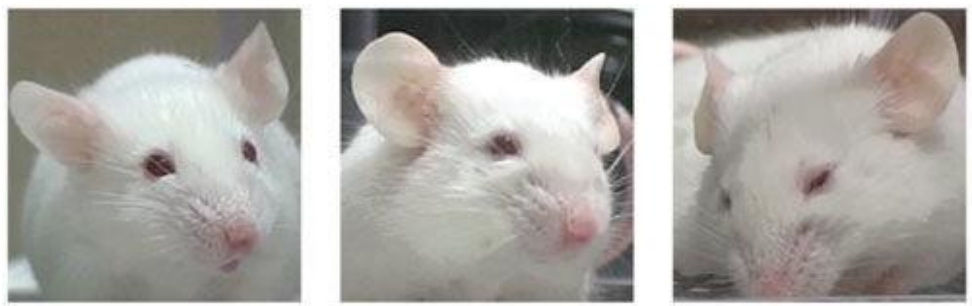

Cheek bulge
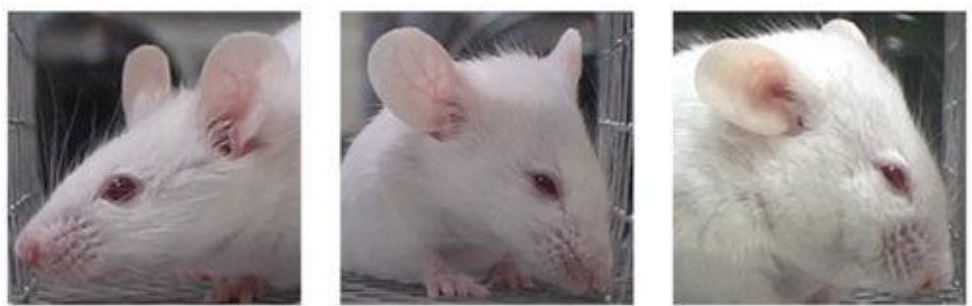

Ear position
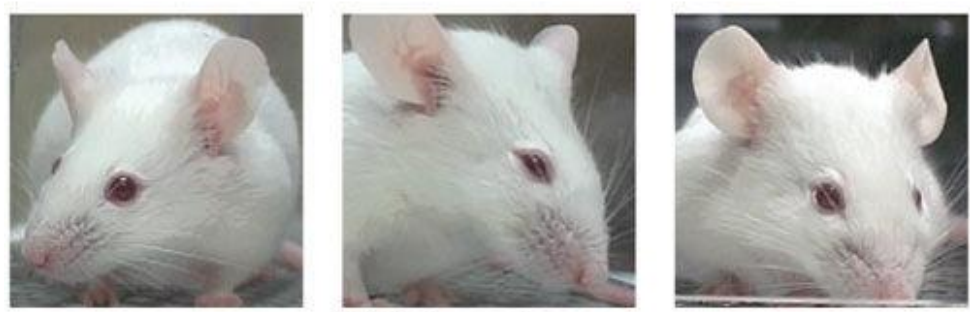

Whisker change

\section{Figure 1.6: The mouse grimace scale}

The mouse grimace scale characterises the facial features of the animals for orbital tightening, nose and cheek bulge, ear position and whisker changes assigned a score of either 'not present', 'moderate' or 'severe'. Reprinted by permission from Springer Customer Service Centre GmbH: Springer Nature, Nature Methods, Coding of facial expressions of pain in the laboratory mice, Langford et al., copyright 2010. 


\subsection{Non-opioid analgesics}

A wide range of treatments are available to relieve pain. Since the pain processes can be different, treatments are available to target distinct mechanisms for the relief of specific pain. Traditionally, analgesics have been placed into two general categories; those that act through the opioid system such as morphine and codeine and non-opioid analgesics. Non-opioid analgesics include non-steroidal anti-inflammatory drugs and paracetamol, which are widely used to treat non-clinical pain. Corticosteroids are often used as adjuvant analgesics, to be used in combination with other pain medications. Both opioid and non-opioid analgesics are efficacious and widely used, with opioids the most commonly prescribed analgesic for chronic pain (Toblin et al., 2011).

\subsubsection{Non-steroidal anti-inflammatory drugs}

One category of non-opioid analgesics are non-steroidal anti-inflammatory drugs (NSAIDs) that are used to treat inflammatory pain and include aspirin (acetylsalicylic acid), ibuprofen and naproxen. The common mechanism of action is via non-selective and reversible inhibition of cyclooxygenase (COX), causing inhibition of both isoforms COX-1 and COX-2 (Appleby et al., 1994; Ferreira et al., 1997), the enzymes responsible for producing prostaglandins and thromboxane from arachidonic acid (Smith et al., 1997; Vane \& Botting, 1998). Prostaglandins contribute to the peripheral sensitisation of nociceptors during inflammatory pain. COX-2 is upregulated during inflammation, causing vasodilation and further inflammation and pain by producing prostaglandins, and therefore, inhibition of the COX2 isoform leads to the analgesic, anti-inflammatory and antipyretic effects of NSAIDs. Non-selective COX inhibitors such as aspirin, ibuprofen and naproxen, cause negative side effects by inhibiting COX1 in the stomach lining (Ferreira, 1972; Ferreira et al., 1997), where prostaglandins have a protective role in preventing the stomach mucosa from acid erosion (Peskar, 2001). Lowered prostaglandin levels can lead to ulcers of the stomach or duodenum and internal bleeding can result (Collier \& Pain, 1985). COX-1 inhibition can have positive effects, as aspirin irreversibly inactivates COX-1 in platelets, inhibiting thromboxane $A_{2}$-dependent platelet aggregation and reducing the incidence of thrombosis (Patrono, 1994). Even though there is a range of NSAIDs available for the treatment of inflammatory pain, there are often gastrointestinal side effects or contraindications associated with taking these regimes and these drugs are frequently misused (Dhar et al., 2014; Cryer et al., 2016).

\subsubsection{Paracetamol (acetaminophen)}

While the mechanisms of action of NSAIDs are well established, the action of paracetamol (also known as acetaminophen) is not as well characterised. Paracetamol has been shown to be ineffective as an anti-inflammatory in the periphery, however, paracetamol crosses the blood-brain barrier and has 
analgesic and antipyretic effects in the CNS (Flower \& Vane, 1972; Courad et al., 2001; Engstrom Ruud et al., 2013). In the brain, paracetamol has been shown to inhibit the COX pathway in low peroxidase environments by reducing the active form of the enzyme rather than by direct binding (Boutaud et al., 2002). Potential mechanisms for the analgesic effects are via the endogenous cannabinoid system, activation of the TRPV1 and TRPA1 channels and the descending serotonergic system (Tjolsen et al., 1991).

\subsubsection{Cannabinoid receptor agonists}

The cannabinoid receptors are a class of guanine protein-coupled receptors (GPCRs) that are activated by phytocannabinoids, compounds derived from plants, such as $\Delta 9$-tetrahydrocannabinol (THC); endocannabinoids, such as anandamide; and synthetic cannabinoids. The cannabinoid type 1 (CB1) receptor is one of the most abundant neuronal receptors found in a reasonably heterogeneous fashion throughout the brain and spinal cord, with the highest binding activity in the basal ganglia, cerebellum and cerebral cortex (Glass et al., 1997; Ameri, 1999). The cannabinoid type 2 (CB2) receptor is mainly expressed on immune cells and tissue related to the immune system, such as the thymus and tonsils (Cabral \& Griffin-Thomas, 2009). Traditionally, many cultures have used cannabinoid compounds to treat pain and nausea, and subsequently, many trials have been carried out to study the analgesic effects of these compounds. Cannabinoid receptor agonists have been shown to be effective at treating neuropathic (Boychuk et al., 2015; Meng et al., 2017) and multiple sclerosis-related pain (Alvarez, 2015), but are not effective at treating acute pain (Stevens \& Higgins, 2017). However, cannabinoid receptor agonists have been known to decrease locomotor activity, and induce hypothermia and catalepsy (Compton et al., 1992), cause dizziness, drowsiness (Fitzcharles et al., 2016), and cognitive impairment (Vinals et al., 2015), and together with the psychoactive effects of THC (Gaoni \& Mechoulam, 1964) reduce their clinical potential. However, there remains interest in developing selective CB2 receptor or peripherally-restricted agonists, that would avoid the centrallymediated side effects (Pertwee, 2012; Kalliomaki et al., 2013; Seltzman et al., 2016; Adams et al., 2018). 


\subsection{Bioactive lipid compounds}

With an aim to develop more effective analgesic pharmacotherapies with fewer side effects, bioactive lipids have been identified as a potential new class of safer analgesics. Fatty acid amides are a diverse group of lipid signalling molecules with important biological effects (Di Marzo et al., 2007), including some with anti-inflammatory and immune modulatory properties (Hoareau et al., 2009; Skaper et al., 2013; Keppel Hesselink et al., 2014). The $N$-acyl ethanolamines (NAE) subclass are of particular interest, with one of the most studied compounds, $N$-palmitoyl ethanolamine (PEA) already used therapeutically to treat allergic inflammatory skin conditions in veterinary clinics (Cerrato et al., 2012; Abramo et al., 2014; Noli et al., 2015) and is now available in many countries in capsule-form as a nutritional supplement (for example, the commercial product PeaPure, JP Russel Science Ltd). Furthermore, a recent randomised, double-blind clinical trial found PEA supplementation produced a significant reduction in abdominal pain severity in IBS patients compared to placebo (Cremon et al., 2017).

\subsection{1 $\mathrm{N}$-acyl ethanolamines}

PEA was originally isolated in 1957 as a component of egg yolk, soybean and peanuts (Kuehl et al., 1957) (Figure 1.7), which explained previous findings that egg yolk contributed to a reduction in rheumatic fever (Coburn \& Moore, 1943) and peanut oil depressed the sensitivity to tuberculin in Bacillus Calmette-Guerin-infected guinea pigs (Long \& Martin, 1956). However, there was not much interest in PEA until the discovery of the closely-related NAE compound anandamide ( $N$-arachidonoyl ethanolamine, AEA, Figure 1.7). Anandamide was the first endocannabinoid compound identified (Devane et al., 1992) after the CB1 receptor was cloned in 1990 (Matsuda et al., 1990) and has since been shown to also bind to the CB2 receptor (Felder et al., 1996) and TRPV1 (Ross, 2003), producing anti-inflammatory and analgesic effects. Anandamide has antinociceptive effects in the intraplantar formaldehyde assay in mice, mediated predominately through the CB1 receptor (Calignano et al., 1998). However, other NAE compounds including PEA and N-oleoyl ethanolamine (OEA, Figure 1.7) have been shown to have less or no affinity to the cannabinoid receptors, but may have an 'entourage effect' due to the fact that the same enzyme, fatty acid amide hydrolase (FAAH), breaks down PEA, OEA and anandamide, and the competition ultimately results in higher endogenous anandamide concentrations (Ho et al., 2008). PEA does bind to a range of other receptors including the nuclear receptor peroxisome proliferator-activated receptor- $\alpha$ (PPAR- $\alpha$ ) (Lo Verme et al., 2005), TRPV1 (Ahern, 2003), the orphan GPCRs GPR55 and GPR119 (Pertwee, 2007; Borrelli \& Izzo, 2009) and potassium ion channels (Romero \& Duarte, 2012). 
Endogenous PEA is formed from palmitic acid, the most common fatty acid found in animals. Tissue concentrations of PEA are reported to be similar to or higher than anandamide in the mammalian brain (Lin et al., 2012). It has been proposed that PEA is an endogenous regulator of inflammation to maintain cellular homeostasis (PEA was originally named ALIAmide due to the compound acting as an 'autacoid local inflammation antagonist' that reduced mast cell activation) (Aloe et al., 1993; Skaper, 2017). Many studies have shown that PEA has anti-inflammatory and antinociceptive properties acting through multiple targets. In the intraplantar formaldehyde test and the acetic acid-, kaolin- and magnesium sulphate-evoked writhing tests, PEA significantly reduced pain behaviours but did not have any effect on capsaicin-induced hind paw licking or the hot-plate test in mice (Calignano et al., 2001). In a neuropathic pain model using chronic constriction of the sciatic nerve in mice, PEA reduced both mechanical and thermal allodynia mediated via CB1, PPAR- $\alpha$ and TRVP1 receptors (Costa et al., 2008), although the authors hypothesise that PEA only directly affects PPAR- $\alpha$, with the other receptors involved due to the 'entourage effect' with anandamide. Furthermore, PEA reduced carrageenan-induced mechanical hyperalgesia in mice, with the effect abolished in animals lacking PPAR- $\alpha$ (D'Agostino et al., 2009). 
PEA

OEA<smiles>CCCCC/C=C\C/C=C\C/C=C\C(C(F)(F)F)C(F)(F)F</smiles><smiles>CC/C=C\C/C=C\C/C=C\C/C=C\C/C=C\CCC(=O)NCCO</smiles><smiles>CC/C=C\C/C=C\C/C=C\C/C=C\CCC(=O)NCCO</smiles>

\section{Figure 1.7: Chemical structures of $\boldsymbol{N}$-acyl ethanolamines}

Chemical structures of $N$-palmitoyl ethanolamine (PEA), $N$-oleoyl ethanolamine (OEA), anandamide $(\mathrm{N}$-arachidonoyl ethanolamine, AEA), $\mathrm{N}$-eicosapentaenoyl ethanolamine (EPEA) and $\mathrm{N}$ docosahexaenoyl ethanolamine (DHEA). 


\subsubsection{Long-chain polyunsaturated fatty acids}

Another class of lipids with biological effects are the polyunsaturated fatty acids (PUFAs), which are fatty acids that have more than one double bond in the carbon chain. Typically, the long-chain (18 carbon chain or longer) PUFAs are separated into two groups based on the position of the first double bond from the methyl group (omega, $\omega$ ) at the end of the carbon chain: the $n-6(\omega-6)$ and the $n-3(\omega-$ 3) fatty acids. The fatty acids can also be identified with lipid numbers in the form C:Dn-3 or C:Dn-6, where $C$ is the number of carbons in the aliphatic chain, and $D$ is the number of double bonds, which are often separated by a single methylene bridge.

Mammals do not have the desaturase enzymes required to add a double bond into the $n-3$ or $n-6$ position and must obtain the fatty acids containing these bonds from dietary sources (Burr \& Burr, 1930). However, the longer chain PUFAs can be created from dietary intake of the 18-carbon fatty acid equivalents. The n-6 PUFA linoleic acid $(18: 2 n-6)$ is predominately found in corn, safflower, soybean, grapeseed and sunflower oils as well as many margarine products (Blasbalg et al., 2011), and can be converted to longer chain n-6 PUFAs including arachidonic acid (20:4n-6, Figure 1.9). Deficiency in linoleic acid has been reported to cause poor growth, scaly skin lesions and transepidermal water loss (Hansen, 1986; Phinney et al., 1993; Hansen \& Artmann, 2008).

The $n-3$ PUFA $\alpha$-linolenic acid (ALA, 18:3n-3, Figure 1.8) is found in a range of seeds, nuts and vegetable oils, such as flaxseed oil (Calder, 2011). In humans, ALA can be metabolised into eicosapentaenoic acid (EPA, 20:5n-3) and then further converted into docosapentaenoic acid (DPA, 22:5n-3) and finally to docosahexaenoic acid (DHA, 22:6n-3) (Sinclair, 1975; Emken et al., 1990) (for chemical structures see Figure 1.8, for synthesis of PUFAs see Figure 1.9). Deficiency in ALA can lead to visual impairment and polydipsia (Neuringer et al., 1984), which occurs as DHA is a major component of the phospholipid membranes in the retina and brain and is necessary for eye and nerve functions (Neuringer et al., 1986). In humans, particularly in adults, the conversion to DHA is not very efficient, with an estimated 5\% of dietary ALA producing DHA (Brenna, 2002; Burdge, 2004; Plourde \& Cunnane, 2007). The consumption of oily-type fish (for example, sardine, salmon or mackerel) and fish oil capsules deliver a higher quantity of DHA and EPA than supplementation with ALA (Brenna et al., 2009; Kris-Etherton et al., 2009). 
$\mathrm{ALA} \times(+2$<smiles>CC/C=C/C/C=C/C/C=C/C/C=C/C/C=C/CCCC(=O)O</smiles><smiles>CC/C=C\C/C=C/C/C=C/C/C=C/C/C=C/CCCCCCO</smiles>

DHA<smiles>CCC=CCC=CCC=CCC=CCC=CCC=CCCC(=O)O</smiles>

Figure 1.8: Chemical structures of n-3 long-chain polyunsaturated fatty acids

Chemical structures of $\alpha$-linolenic acid (ALA, 18:3n-3), eicosapentaenoic acid (EPA, 20:5n-3), docosapentaenoic acid (DPA, 22:5n-3) and docosahexaenoic acid (DHA, 22:6n-3). 


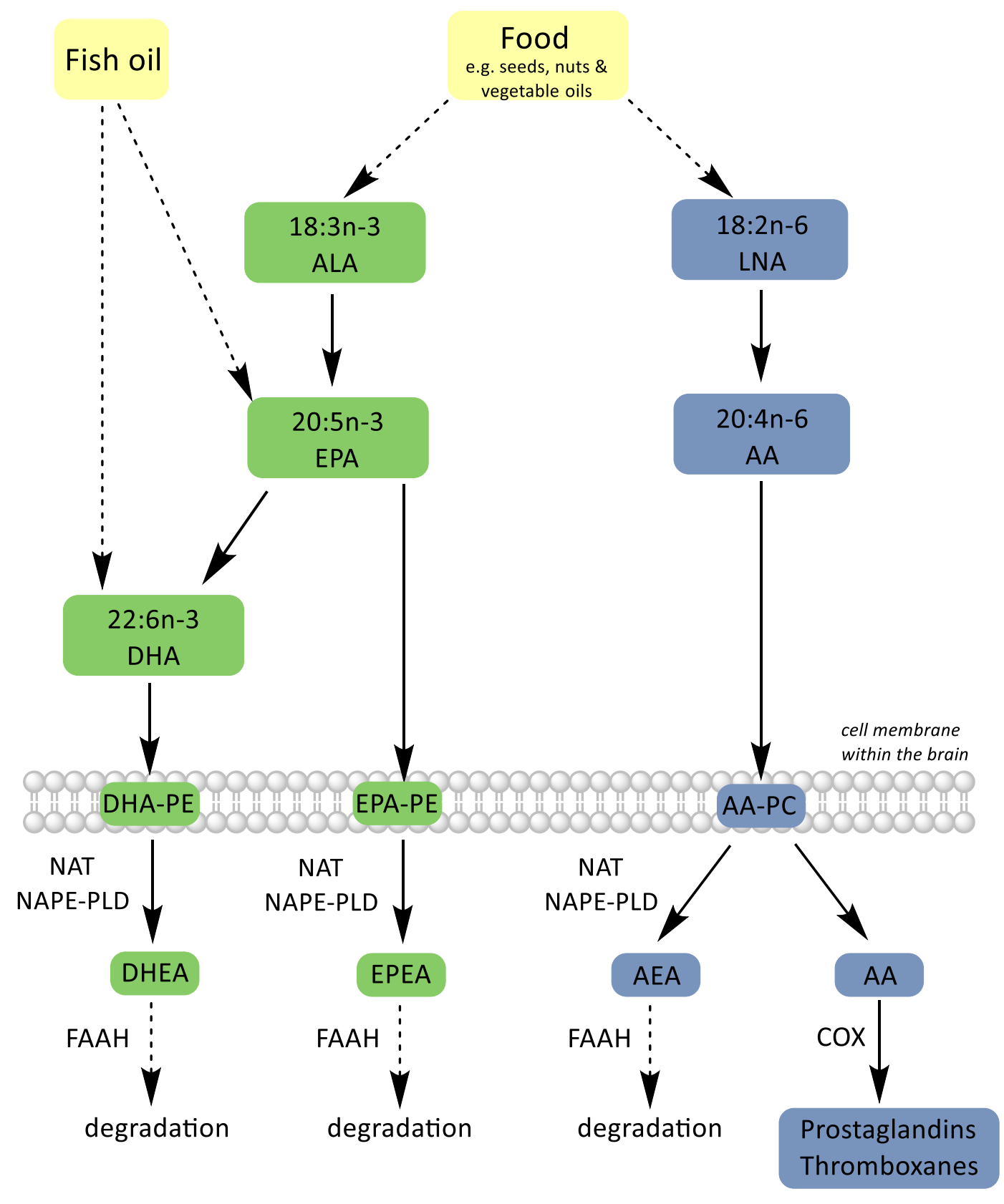

Figure 1.9: Synthesis of polyunsaturated fatty acids and the ethanolamine metabolites

Polyunsaturated fatty acids (PUFAs) must be obtained from dietary sources. The $n-3$ PUFAs (green) pathway begins with the consumption of ALA and the n-6 PUFAs pathway (blue) from LNA. EPA and DHA can be converted from ALA or gained from fish oil supplementation. The long-chain PUFAs cross the blood-brain barrier and are esterified in phospholipids of the cell membrane. The release of the esterified PUFAs from the membrane allows for the production of the $N$-acyl ethanolamines DHEA, EPEA and AEA which are all competitively synthesised and degraded into the corresponding PUFAs by the same enzymes. Arachidonic acid produces prostaglandins and thromboxane by COX enzymes. Adapted from Bosch-Bouju and Layé (2016).

$A A$, arachidonic acid; AEA, anandamide; ALA, a-linolenic acid; COX, cyclooxygenase; DHA, docosahexaenoic acid; DHEA, $N$-docosahexaenoyl ethanolamine; $E P A$, eicosapentaenoic acid; $E P E A, N$ eicosapentaenoyl ethanolamine; FAAH, fatty acid amide hydrolase; LNA, linoleic acid; NAPE-PLD; Nacyl phosphatidylethanolamine-specific phospholipase-D; NAT, N-acyl transferase; $P E$, phosphatidylethanolamine; PC, phosphatidylcholine. 
DHA and EPA are associated with many health claims. Many of these disorders that are claimed to be treated by DHA and EPA involve inflammation as either the primary driver of disease, or in an underlying secondary role, and the mechanism of action of the n-3 PUFAs is likely due to a dampening of this inflammatory response. Fish oil supplementation has been associated with the treatment of inflammatory diseases, with protection from ulcerative colitis (John et al., 2010) and improvement in symptoms in IBD patients (Belluzzi et al., 1996; Barbosa et al., 2003; Calder, 2008; Chan et al., 2014). However, other studies have found no benefit (Dichi et al., 2000; Lev-Tzion et al., 2014). There is also evidence of the therapeutic effect of fish oil in rheumatoid arthritis sufferers (Fortin et al., 1995; Lourdudoss et al., 2017). However, more randomised controlled trial studies are needed to properly understand the effect of n-3 PUFAs on disease states, as many of the current trials lack consistency in the formulation, dosage and timing of the intervention.

Many studies also focus on the positive effects on cardiac health. In 2002, the American Heart Association recommended the use of DHA and EPA for cardiovascular disease risk reduction (KrisEtherton et al., 2002). Since this statement was released, multiple randomised controlled trials have shown that DHA and EPA reduce mortality in coronary heart disease patients by $10 \%$ and in patients with heart failure, they reduce deaths and hospitalisations by $9 \%$ (Siscovick et al., 2017). Evidence also suggests n-3 PUFAs may be beneficial in decreasing cognitive decline (Dullemeijer et al., 2007; van Gelder et al., 2007; Sinn et al., 2012), reducing the risk of developing Alzheimer's disease (Shinto et al., 2014; Wu et al., 2015) and dementia (Barberger-Gateau et al., 2007; Samieri et al., 2008), as well as decreasing psychosis (Amminger et al., 2010) and depressive symptoms (Grosso et al., 2014). However, further well-designed studies may be required to fully understand the effect of these interventions on mental health and neurodegenerative diseases (Cederholm, 2017). Similarly, evidence for the positive effects of n-3 PUFAs for diabetes appears to be mixed, with studies either finding some benefit (Virtanen et al., 2014; Samimi et al., 2015) or no benefit (Wu et al., 2012b; Dasarathy et al., 2015).

The anti-inflammatory and antinociceptive effects of $n-3$ PUFAs have been shown in multiple studies in vivo. Oral administration of a supplement containing DHA and EPA has been shown to decrease visceral pain in the acetic acid-induced writhing test, decrease pain the intraplantar formaldehyde test and it had a significant effect in the thermal nociceptive hotplate test in mice (Veigas et al., 2011; Nobre et al., 2013). Furthermore, supplementation reduced carrageenan-induced paw swelling and immunohistochemistry of the inflamed paw showed a reduction in tumour necrosis factor- $\alpha$ (TNF $\alpha$ ) and the number of neutrophils present (Nobre et al., 2013). In vitro studies found that n-3 PUFA supplementation significantly decreased myeloperoxidase release from stimulated human neutrophils and did not present any cytotoxic effects on the cells as assessed using the MTT cell 
viability assay (Nobre et al., 2013). Oral administration of DHA (without EPA) also had a significant effect in the acetic acid writhing test, tail withdrawal assay and in both phases of the intraplantar formaldehyde model in mice (Nakamoto et al., 2010).

One explanation for the anti-inflammatory properties is that an increase in dietary n-3 PUFAs is reflected in an increase in the EPA and DHA in phospholipid membranes, which in turn decreases the amount of arachidonic acid available in the membranes (Lokesh et al., 1986; Chapkin et al., 1991; Peterson et al., 1998). Arachidonic acid from the phospholipid membranes is used to synthesise eicosanoids that include prostaglandins, thromboxanes and leukotrienes, which are inflammatory mediators and regulators (Dennis \& Norris, 2015) (Figure 1.9). Evidence shows that DHA and EPA have a role in the downregulated expression of the COX-2 gene and inflammatory cytokine genes, and an effect on leukocyte chemotaxis and adhesion, as well as a direct effect on GPR120 and nuclear receptor PPAR- $\gamma$ (reviewed in Calder, 2015). Furthermore, primarily EPA but also DHA, produce antiinflammatory lipid mediators called resolvins, protectins and maresins, which have a role in resolving inflammation (Serhan et al., 2000; 2002; 2008) and have been shown to treat and protect against inflammation in animal models of arthritis (Lima-Garcia et al., 2011) and IBD (Arita et al., 2005). However, DHA and EPA can both be converted into the NAE metabolites, $\mathrm{N}$-docosahexaenoyl ethanolamine (DHEA, NAE-22:6n-3, Figure 1.7) and N-eicosapentaenoyl ethanolamine (EPEA, NAE20:5n-3, Figure 1.7), and the effects of these compounds have not been tested in vivo, making it possible that these metabolites are also contributing to the antinociceptive and anti-inflammatory effect of the n-3 PUFAs.

\subsection{3 $\mathrm{N}$-docosahexaenoyl ethanolamine (DHEA)}

DHEA is the NAE metabolite of DHA which has been found in abundance in the rat brain (Sugiura et al., 1996) and bovine retina (Bisogno et al., 1999) as well as in human plasma (Balvers et al., 2013). Eating a diet high in $n-3$ PUFAs has been linked to an increased concentration of DHEA in brain tissue (Berger et al., 2001), as DHEA can be directly converted from DHA via a phospholipase D enzymatic reaction (reviewed in Kuda, 2017). DHEA is structurally similar to the endocannabinoid anandamide, and DHEA binds to both CB1 and CB2 receptors (Felder et al., 1993; Sheskin et al., 1997; Brown et al., 2010). The immune-modulatory effects of DHEA have been shown in vitro (Jaggar et al., 1998; Meijerink et al., 2011) (see Chapter 3), however, this thesis aimed to study the antinociceptive effects of DHEA in vivo, primarily using the intraplantar formaldehyde model of inflammatory pain. 


\subsection{Opioid receptor agonists}

\subsubsection{The opioid crisis}

To treat chronic pain, patients are typically prescribed drugs that activate the mu opioid receptor (MOPr), such as morphine, codeine and fentanyl (Toblin et al., 2011). The rapid increase in the use of prescription and non-prescription opioid drugs in recent years in the USA has been termed the 'opioid crisis'. Unfortunately, chronic treatment with MOPr agonists can actually potentiate pain (Celerier et al., 2000; Roeckel et al., 2016), and can lead to dependence and addiction (Compton \& Volkow, 2006). Drug addiction is a chronic, relapsing brain disorder whereby an individual prioritises drug consumption despite negative consequences. Between $21-29 \%$ of patients prescribed opioids for chronic pain misuse them and $8-12 \%$ of those that are prescribed opioids develop opioid use disorder (Vowles et al., 2015). Prescription opioids may also act as a gateway to the cheaper and sometimes more accessible drug heroin, with $79.5 \%$ of surveyed new heroin users starting out by misusing prescription opioids (Muhuri et al., 2013; Cicero et al., 2014).

Long-term use of prescription MOPr agonists results in tolerance (Chu et al., 2006), requiring escalating doses to be administered to maintain pain relief in the absence of disease progression. A significant side effect of MOPr agonists is respiratory depression (Shook et al., 1990; Dahan et al., 2001), characterised by slow or inefficient breathing. As users escalate doses due to analgesic tolerance, these high doses can lead to respiratory failure or death. Opioid overdoses are the leading cause of accidental death in the USA, killing more people than road accidents (Okie, 2010; Rudd et al., 2016). Opioid-related overdose, which combines prescription and synthetic opioids as well as heroin, in the USA for the year 2016 lead to 42,249 deaths (Jones et al., 2018). Synthetic opioids, including fentanyl, were involved in $14 \%$ of opioid-related deaths in 2010 , which rose dramatically to $46 \%$ in 2016 (Jones et al., 2018). In fact, life expectancy dropped in 2015 for Caucasian people in the USA, with an estimated 0.21-year loss in life expectancy due to opioid poisoning (Dowell et al., 2017). However, global rates of unintentional opioid overdoses have also been steadily rising (Kuehn, 2007), and opioid use disorders caused 122,100 global deaths in 2015 (GBD Mortality and Causes of Death Collaborators, 2016). In New Zealand, daily doses of prescription opioids quadrupled in the ten-year period from 2001-2011 (Berterame et al., 2016) and dispensing rates of oxycodone increased by $249 \%$ between 2007 and 2011 (Ministry of Health, 2014). Using combined data from Oceania, it is estimated that $3 \%$ of the population are addicted to opioid drugs (United Nations Office of Drug and Crime, 2017). The New Zealand Drug Harm Index for 2016 estimated 10 deaths per year related to opioid or sedative use (Ministry of Health, 2016). 
Clinically used opioids, act primarily through the MOPr (Vallejo et al., 2011). Activation of the MOPr stimulates the reward pathway by increasing dopamine in the synapse (Di Chiara \& Imperato, 1988; Titeler et al., 1988), increasing the positive reinforcement of the drug (Maldonado et al., 1997), a central process in the development of drug dependence. In the search for non-addictive analgesics, kappa opioid receptor (KOPr) agonists are a promising alternative. In contrast to MOPr agonists, $\mathrm{KOPr}$ agonists play a critical role in regulating the reward system by contributing to the negative-feedback of dopamine (Di Chiara \& Imperato, 1988). Studies have shown that acute administration of KOPr agonists show anti-addictive potential and have antinociceptive (Gallantine \& Meert, 2008), antiinflammatory (Binder et al., 2001; Bileviciute-Ljungar et al., 2006), and antipruritic effects (Kumagai et al., 2010; Akiyama et al., 2015). In contrast to MOPr agonists, $\mathrm{KOPr}$ agonists do not inhibit gastrointestinal transit (Porreca et al., 1984) and do not cause respiratory depression (Freye et al., 1983).

Development of KOPr agonists with fewer side effects (Law et al., 2013; Zhang et al., 2015) and reduced abuse potential (Morani et al., 2009) have received increased attention recently (Varadi et al., 2015; White et al., 2015; Sherwood et al., 2017b; Soeberdt et al., 2017; Spetea et al., 2017). The clinical success of nalfurafine is a promising example of the benefits of developing KOPr agonists. The potent KOPr agonist (Seki et al., 1999; also called TRK-820 and the trade-name Remitch) exhibits strong analgesic effects (Endoh et al., 1999; Endoh et al., 2000) and is the first KOPr agonist to be made clinically available, used in Japan for the treatment of medication-resistant pruritus in haemodialysis patients (Kumagai et al., 2010). Furthermore, there is no evidence of abuse liability (Ueno et al., 2013) or reinforcement of self-administration (Nakao et al., 2016), demonstrating that KOPr agonists can have therapeutic utility without abuse potential.

\subsubsection{The endogenous kappa opioid receptor system}

The KOPr is a class A (rhodopsin-like) $\gamma$ subfamily of seven-transmembrane GPCRs coupled to $G_{i / o}$ proteins, found in both the CNS and periphery. The KOPr is encoded by the OPKR1 gene, which has been cloned in the mouse (Yasuda et al., 1993; Nishi et al., 1994), rat (Chen et al., 1993; Li et al., 1993; Meng et al., 1993; Minami et al., 1993; Nishi et al., 1993) and human (Mansson et al., 1994; Simonin et al., 1995; Zhu et al., 1995). Recently, the crystal structure was solved for the human KOPr in complex with the antagonist JDTic (Wu et al., 2012a) and the agonist MP1104 (Che et al., 2018). The three classical opioid receptors (KOPr, MOPr and delta opioid receptor (DOPr)) share approximately 75\% sequence identity in the transmembrane $\alpha$-helices and $65 \%$ in their intracellular loops, however there are larger differences in the extracellular loops and $\mathrm{N}$ - and C-termini with only $35-40 \%$ sequence identity (reviewed in Pogozheva et al., 2005). Activation of the KOPr is particularly associated with 
regulation of the reward pathway, antinociception, and anxiogenic and stress-related behaviours (reviewed in Wang et al., 2010; Lalanne et al., 2014), as well as playing a role in temperature regulation (Chen et al., 2005), cardioprotection (Peart et al., 2008; Tong et al., 2011), neuroprotection (Zeynalov et al., 2006) regulation of angiogenesis (Yamamizu et al., 2015) and oligodendrocyte-mediated remyelination (Du et al., 2016; Mei et al., 2016).

The KOPr is widely distributed in the human brain and spinal cord, with high concentrations in the frontal lobe, temporal lobe, parietal lobe, occipital lobe, amygdala, olfactory bulb, nucleus accumbens, nucleus caudate, putamen, thalamus, hypothalamus, ventral tegmental area and the cerebellar cortex, as well as moderate expression in the lamina II region of the cervical, dorsal and lumbar spinal cord and the DRG (Simonin et al., 1995; Peckys \& Landwehrmeyer, 1999; Peng et al., 2012) (for KOPr expression in the pain pathways see Figure 1.10). Similar distribution patterns of the KOPr have been found in the brains of rodents (DePaoli et al., 1994; Xie et al., 1994), although there are a few differences, including the absence of KOPr found in the rat cerebellar cortex compared to high expression in humans (Peckys \& Landwehrmeyer, 1999), and variation in the relative abundance of KOPr in the hippocampus and substantia nigra (Simonin et al., 1995; Peckys \& Landwehrmeyer, 1999). The KOPr is found on both pre- and post-synaptic dopaminergic, glutamatergic, serotonergic and $\gamma$ aminobutyric acid (GABAergic) neurons in the brain (Svingos et al., 2001; Margolis et al., 2006; Li et al., 2012). In the periphery, KOPr expression is not as well characterised, with rat studies finding KOPrs in the stomach, small intestine, large intestine, adrenal gland, kidney, lung, testis, ovary and uterus (Wittert et al., 1996; Bagnol et al., 1997). The liver had no detectable transcript, whilst the spleen and heart had weak signal, although a more recent study in humans found low levels of KOPr in liver, spleen, heart tissue, and additionally (from the list in the rat above) in skeletal muscle, thymus and pancreas (Peng et al., 2012; Sobanski et al., 2014). 

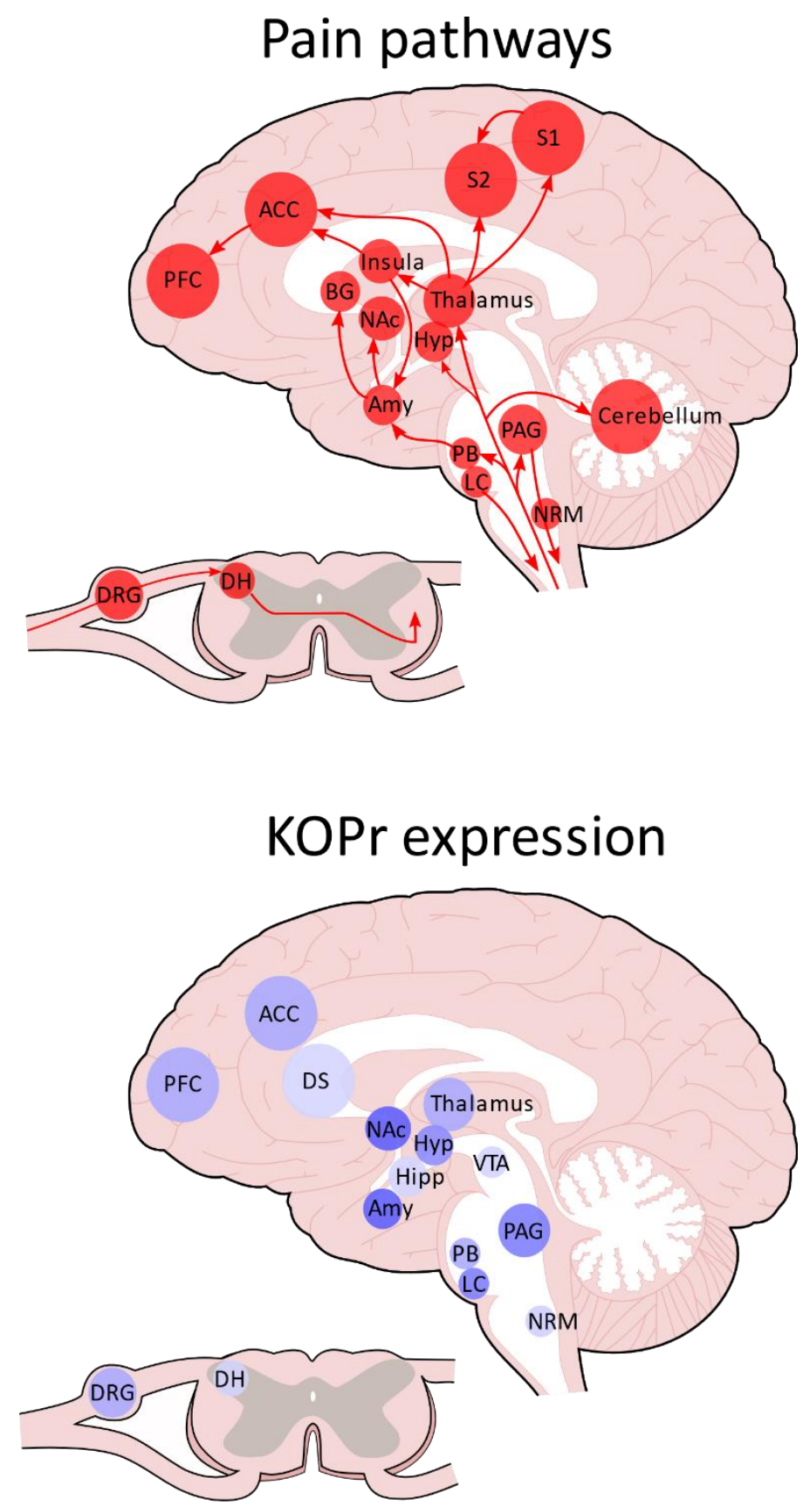

Figure 1.10: Brain and spinal cord regions involved in pain processing compared to kappa opioid receptor expression

The ascending pain pathways project to the brain regions involved in pain processing and perception, including the thalamus, somatosensory cortex and periaqueductal grey. Kappa opioid receptor (KOPr) expression is shown in the relevant areas for pain, addiction and aversion. Blue colour indicates relative abundance (darker colour $=$ more abundant). Figure adapted from Cahill et al. (2014), and KOPr expression based on radioligand binding and quantitative real-time reverse transcriptase polymerase chain reaction data from Le Merrer et al. (2009) and Peng et al. (2012) respectively. $A C C$, anterior cingulate cortex; Amy, amygdala; $D H$, dorsal horn of the spinal cord; DRG, dorsal root ganglion; DS, dorsal striatum; Hipp, hippocampus; Hyp, hypothalamus; NAc, nucleus accumbens; PAG, periaqueductal grey; $P B$, parabrachial nucleus; PFC, prefrontal cortex; S1, primary somatosensory cortex; $S 2$, secondary somatosensory cortex; VTA, ventral tegmental area. 


\subsubsection{Kappa opioid receptor signalling pathway}

When an agonist binds to the KOPr, several signalling cascades are activated (Figure 1.11). The KOPr is associated with the G-protein complex, with activation of the KOPr leading to conformational changes and dissociation of the pertussis toxin-sensitive G-protein subunits. The released $\beta \gamma$ subunits activate G-protein-gated inwardly rectifying potassium channels, enhancing $\mathrm{K}^{+}$efflux (Grudt \& Williams, 1993); and inhibit voltage-gated calcium ion channels, inhibiting $\mathrm{Ca}^{2+}$ influx (Rusin et al., 1997); which leads to the hyperpolarisation of the neuron, decreasing neuronal excitability and inhibiting action potential generation. Neurotransmitter release is inhibited due to the decrease in $\mathrm{Ca}^{2+}$ levels and subsequent inhibition of $\mathrm{Ca}^{2+}$-dependent vesicular migration. The $\mathrm{G}_{\mathrm{i}}$ subunit inhibits adenylyl cyclase activity, leading to a decrease in cyclic adenosine monophosphate (cAMP) (Taussig et al., 1993). The G-protein signalling pathway also leads the phosphorylation of c-Jun N-terminal kinase (JNK) and to the early phase phosphorylation of extracellular signal-regulated kinase 1 and 2 (ERK1/2), which is dependent on phosphatidylinositol 3-kinase (PI3K), protein kinase $\mathrm{C} \zeta$ (PKC)) and $\mathrm{Ca}^{2+}$ mobilisation (Belcheva et al., 2005). In the dorsal horn of the spinal cord, the overall effect is to decrease the excitability of the neuron, therefore inhibiting the pain signal from reaching higher processing centres to produce the analgesic effects (Porreca et al., 1984; Ruda et al., 1988). The supraspinal action involves decreasing the excitability of the inhibitory GABAergic neurons in the periaqueductal grey (Kash \& Li, 2018), which in turn reduces the inhibition of the tonically-active descending anti-nociceptive pathway system, therefore leading to an analgesic effect. In the reward centres, KOPr activation regulates dopaminergic neurons to decrease dopamine release, as well as increasing the uptake by the dopamine transporter (DAT), producing the anti-addiction effects (Di Chiara \& Imperato, 1988; Kivell et al., 2014).

KOPr agonist binding also produces a $\beta$-arrestin-dependent signalling cascade (Figure 1.11). When an agonist binds to the KOPr, the C-terminal intracellular domain of the KOPr is phosphorylated by Gprotein receptor kinase 3 (GRK3) and the scaffolding protein $\beta$-arrestin is recruited, leading to the phosphorylation of p38 mitogen-activated protein (MAP) kinase (McLaughlin et al., 2003; Bruchas et al., 2006). Pro-depressive and aversive effects of KOPr agonists have been attributed to the phosphorylation of p38 MAPK (Bruchas et al., 2007; 2011; Ehrich et al., 2015). The $\beta$-arrestindependent pathway also results in the late phase phosphorylation of ERK1/2, leading to the activation of the transcription factor CAMP response element binding protein (CREB). Multiple genes are regulated by CREB, including gene expression of dynorphin (Cole et al., 1995) and corticotrophinreleasing hormone (Itoi et al., 1996). Increased CREB activity results in KOPr-mediated pro-depressive effects (Pliakas et al., 2001; Mague et al., 2003) and is an important factor in stress-induced behavioural responses to drugs of abuse (Kreibich \& Blendy, 2004). 
The phosphorylation of the KOPr, as well as the $\beta$-arrestin-mediated uncoupling of the G-proteins, leads to desensitisation of the KOPr as the G-protein pathway can no longer be activated (reviewed in Liu-Chen, 2004). Phosphorylation of KOPr leads to the internalisation of the receptor by the formation of endocytic vesicles (Schulz et al., 2002), causing down-regulation of the KOPr on the cell surface. Recently, it was shown that PKC can also lead to the phosphorylation of the KOPr both agonistdependently and -independently, however, only GRK-mediated phosphorylation leads to the internalisation of the KOPr and contributes to KOPr agonist tolerance (McLaughlin et al., 2004; Chiu et al., 2017). The internalised KOPrs are dephosphorylated and are sorted into endosomes to be degraded or recycled back to the plasma membrane (reviewed in Liu-Chen, 2004).

Evidently, it appears that the majority of the negative side effects, as well as the development of opioid tolerance, are mediated through the $\beta$-arrestin-dependent pathway, as opposed to the analgesic and anti-addiction effects mediated through the activation of the G-protein pathway (Bruchas \& Chavkin, 2010). The idea that an agonist could be designed to preferentially activate the G-protein pathway without activating the $\beta$-arrestin-dependent pathway is termed biased agonism or functional selectivity. Recently this area of drug development has received a lot of attention (White et al., 2015; Rankovic et al., 2016; Viscusi et al., 2016; Soeberdt et al., 2017; Spetea et al., 2017), however, the application of the theoretical idea of biased agonism has proven more difficult than anticipated. Technical differences in measuring and calculating bias, as well as an increasingly complicated understanding of the signalling pathways have slowed progress in identifying potential biased analgesics (Michel \& Charlton, 2018). However, the development of biased agonists at the KOPr is discussed in further detail in Chapter 4. 


\section{G-protein signalling pathway}

\section{$\beta$-arrestin signalling pathway}

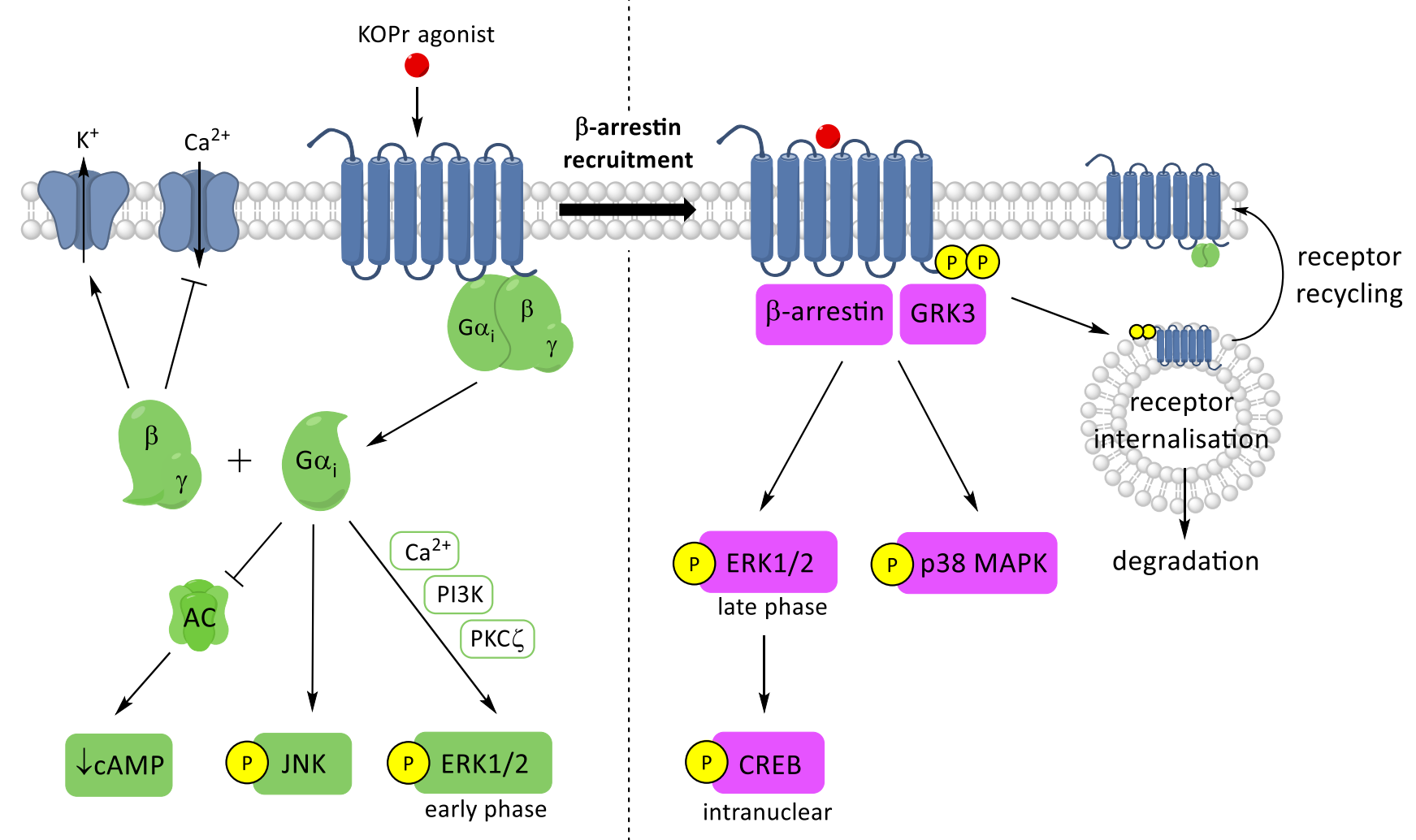

\section{Analgesic effects \\ Anti-addiction}

\section{Aversive effects \\ Depressive effects}

Figure 1.11: Kappa opioid receptor signalling pathways

Binding of an agonist to the KOPr results in the activation of both G-protein and $\beta$-arrestin signalling pathways. Activation of G-protein signalling leads to the dissociation of the G-protein complex. The $\beta \gamma$ subunits activate $\mathrm{G}$-protein-gated inwardly rectifying potassium $\left(\mathrm{K}^{+}\right)$channels and inhibit voltagegated calcium ion $\left(\mathrm{Ca}^{2+}\right)$ channels, which leads to the hyperpolarisation of the neuron. The $\mathrm{Ga}_{\mathrm{i}}$ subunit inhibits adenylyl cyclase activity, leading to a decrease in CAMP, and further signalling cascades lead to the phosphorylation of ERK1/2 and JNK. The $\beta$-arrestin-dependent pathway includes the phosphorylation of the intracellular domain of the KOPr by GRK3 and $\beta$-arrestin recruitment to the receptor. This pathway leads to a late phase phosphorylation of ERK1/2 as well as p38 MAPK and CREB. Phosphorylation of the KOPr leads to clathrin-mediated receptor internalisation within endosomes, followed by either degradation via lysosomes or recycling back to the plasma membrane. Figure adapted from Bruchas and Chavkin (2010) and Dogra and Yadav (2015).

$A C$, adenylyl cyclase; $C A M P$, cyclic adenosine monophosphate; CREB, CAMP response element binding protein; ERK1/2, extracellular signal-regulated kinase 1 and 2; $G \alpha_{i}$, G-protein $\alpha_{i}$ subunit; $G 6 \gamma$, G-protein By subunit; JNK, c-Jun N-terminal kinase; GRK3, G-protein coupled receptor kinase 3; p38 MAPK, p38 mitogen-activated protein kinase; PI3K, phosphoinositide 3-kinase; $P K C \zeta$, protein kinase $C \zeta$. 


\subsubsection{Role of the endogenous kappa opioid system in pain}

KOPr knockout $\left(\mathrm{KOPr}^{-1}\right)$ mice were generated by Simonin et al. (1998). Deletion of the KOPr had no effect on normal embryonic development and did not cause any gross abnormalities (available at Jackson Laboratory as B6.129S2-Oprk1 $\left.1^{\mathrm{tm} 1 \mathrm{Kff}} / \mathrm{J}\right)$. These animals have been tested in a range of behavioural models of pain, and show increased sensitivity in the acetic acid writhing test, indicating the KOPr system is involved in the perception of visceral pain. No differences were recorded for the warm water tail withdrawal, hot plate, tail pressure or intraplantar formaldehyde test for the $\mathrm{KOPr}^{-1-}$ mice compared to control animals (Simonin et al., 1998). However, a further study found a difference in the warm water tail withdrawal assay in female but not male KOPr ${ }^{-1-}$ mice (Martin et al., 2003). In $\mathrm{MOPr}^{-/}$mice, no difference was seen in the warm water tail withdrawal test, but there was an increased sensitivity to the hotplate, tail pressure and the intraplantar formaldehyde tests (Martin et al., 2003). This likely reflects the higher abundance of MOPrs on the descending antinociceptive tracts of the CNS and the larger role that endogenous MOPr peptides, the endorphins, play in pain relief (reviewed in Sprouse-Blum et al., 2010).

The endogenous agonists for the KOPr are the dynorphin class of opioid peptides (Goldstein et al., 1979; Chavkin et al., 1982) which are posttranslational products formed by cleavage of prodynorphin (Day et al., 1998). The most common product is dynorphin $A_{1-17}$, the natural peptide has 17 amino acids, but the shortened 13 amino acid fragment (dynorphin $A_{1-13}$ ) is often used in biological studies (Chou et al., 1996) (for amino acid sequences see Table 1.1). Intrathecal injection of dynorphin $A_{1-13}$ in the rat spinal cord has an antinociceptive effect 6-10 times more potent than morphine on a molar basis (Han \& Xie, 1982), and morphine in combination with dynorphin $A_{1-13}$ produced a synergistic antinociceptive effect in the tail withdrawal assay (Ren et al., 1985).

Dynorphin may also have a role in pain processing. Intraplantar administration into the hind paw with the inflammatory agents carrageenan, phorbol ester, yeast or complete Freund's adjuvant lead to an upregulation of dynorphin $A_{1-8}$ in rat spinal cord (ladarola et al., 1988), as well as an upregulation following spinal cord injury (Malan et al., 2000). In fact, intrathecal dynorphin $A_{1-17}$ produced mechanical allodynia in rats (Vanderah et al., 1996), which was blocked by an N-methyl-D-aspartate (NMDA) antagonist but not by the opioid antagonist naloxone (Laughlin et al., 1997), indicating a more complicated mechanism of action. Furthermore, after spinal nerve ligation, both prodynorphin knockout and wild-type mice have equal allodynia, but this neuropathy was resolved in the knockout mice by day 10 compared to no change in the wild-type, indicating that dynorphin may be required for the maintenance of neuropathic pain but not the initiation (Wang et al., 2001). Further studies found this effect could be blocked using bradykinin receptor antagonists (Lai et al., 2006; Luo et al., 
2008), suggesting the dynorphin effects may not be opioid receptor-dependent. However, it is clear that the endogenous KOPr system is involved in pain processing and can be used as a target for the development of analgesic compounds.

\subsubsection{Traditional kappa opioid receptor agonists}

There are a range of KOPr agonists that can be classified into four major categories: 1) arylacetamides, such as U50,488, U69,593, enadoline, spiradoline and ICI 204,448; 2) benzomorphans such as bremazocine, ethylketocyclazocine and cyclazocine; 3) morphinans such as 6'-guanidinonaltrindole (6'GNTI), nalmefene, nalfurafine and cyclorphan; and 4) terpenoids such as RB-64 (Yan et al., 2009), collybolide (Gupta et al., 2016) and Salvinorin A and synthetic analogues (see Section 1.7.4.1 Salvia divinorum and Salvinorin A). The two most extensively studied selective synthetic KOPr agonists are U50,488 (Szmuszkovicz \& Von Voigtlander, 1982; Von Voigtlander \& Lewis, 1982; Vonvoigtlander et al., 1983) and U69,593 (Lahti et al., 1985), which are known as traditional KOPr agonists. Both compounds are potent, full KOPr agonists, with binding affinity $K_{i}$ values of $0.70 \pm 0.09$ and $0.89 \mathrm{nM}$ for $\mathrm{U} 50,488$ and $\mathrm{U} 69,593$, respectively compared to $0.44 \pm 0.06 \mathrm{nM}$ for dynorphin $A_{1-13}$ in rhesus macaque brain membranes (Emmerson et al., 1994) (Table 1.1).

\subsubsection{Traditional kappa opioid receptor agonists role in reward}

One of the reasons why KOPr agonists are promising as analgesic medications is the low abuse potential (Vonvoigtlander et al., 1983). In the mesolimbic and nigrostriatal dopaminergic neurons, $\mathrm{U} 50,488$ and bremazocine reduced the release of dopamine, compared to the MOPr agonists morphine, methadone and fentanyl which all stimulated dopamine release (Di Chiara \& Imperato, 1988), a central process in the development of drug dependence. In fact, due to the regulation of dopamine in the reward centres of the brain by the KOPr system, traditional KOPr agonists have been studied as potential anti-addiction pharmacotherapies. Administration of the traditional $\mathrm{KOPr}$ agonists U50,488 and U69,593 reduced cocaine self-administration in rats (Glick et al., 1995; Kuzmin et al., 1997; Schenk et al., 1999; 2001) and rhesus macaques (Negus et al., 1997; Mello \& Negus, 2000) and morphine self-administration in rats (Glick et al., 1995) and mice (Kuzmin et al., 1997). Furthermore, U50,488 attenuated voluntary ethanol intake (Lindholm et al., 2001) and nicotine selfadministration in rats (Ismayilova \& Shoaib, 2010). These studies provide convincing evidence of the regulatory effect of the traditional KOPr agonists on the rewarding effects of drugs of abuse. 


\begin{tabular}{|c|c|c|c|c|}
\hline & Dynorphin peptide & U50,488 & U69,593 & Salvinorin A \\
\hline Structure & $\begin{array}{c}\text { Dynorphin A1-17 } \\
\text { H-Tyr-Gly-Gly-Phe-Leu-Arg-Arg-lle- } \\
\text { Arg-Pro-Lys-Leu-Lys-Trp-Asp-Asn- } \\
\text { Gln-OH } \\
\\
\text { Dynorphin A } \text { A }_{1-13} \text { fragment } \\
\text { H-Tyr-Gly-Gly-Phe-Leu-Arg-Arg-lle- } \\
\text { Arg-Pro-Lys-Leu-Lys-OH }\end{array}$ & & & \\
\hline $\begin{array}{l}\text { Binding affinity in } \\
\text { rhesus macaque (1) }\end{array}$ & $K_{i}=0.44 \pm 0.06 *$ & $K_{i}=0.70 \pm 0.09$ & $\mathrm{~K}_{\mathrm{i}}=0.89$ & - \\
\hline $\begin{array}{l}\text { Binding affinity at } \\
\text { the rat KOPr (2) }\end{array}$ & - & $K_{i}=0.42 \pm 0.22$ & $K_{i}=2.5 \pm 0.3$ & $\mathrm{~K}_{\mathrm{i}}=0.28 \pm 0.22$ \\
\hline $\begin{array}{l}\text { Potency and binding } \\
\text { efficiency at the } \\
\text { human KOPr (3) }\end{array}$ & $\begin{array}{c}\mathrm{EC}_{50}=3.4 \\
\mathrm{E}_{\max }=73.0 \pm 2.0\end{array}$ & $\begin{array}{c}\mathrm{EC}_{50}=30 \\
\mathrm{E}_{\max }=101 \pm 4.2\end{array}$ & $\begin{array}{c}\mathrm{EC}_{50}=94 \\
\mathrm{E}_{\max }=108 \pm 6.6\end{array}$ & $\begin{array}{c}\mathrm{EC}_{50}=23 \\
\mathrm{E}_{\max }=103 \pm 2.9\end{array}$ \\
\hline $\begin{array}{l}\text { Potency and binding } \\
\text { efficiency at the } \\
\text { human KOPr (4) }\end{array}$ & $\begin{array}{c}\mathrm{EC}_{50}=83 \\
\mathrm{E}_{\max }=107 \pm 8 * *\end{array}$ & $\begin{array}{c}E C_{50}=24 \\
E_{\max }=102 \pm 4 * *\end{array}$ & $\begin{array}{l}E C_{50}=13 \\
E_{\max }=100\end{array}$ & $\begin{array}{c}\mathrm{EC}_{50}=7 \\
\mathrm{E}_{\max }=104 \pm 7^{* *}\end{array}$ \\
\hline
\end{tabular}

\section{Table 1.1: Comparison of dynorphin, traditional agonists and Salvinorin A in binding affinity and selectivity at the kappa opioid receptor}

(1) Binding affinity $\left(\mathrm{K}_{\mathrm{i}}\right)$ in rhesus macaque brain cortex membranes with $\left[{ }^{3} \mathrm{H}\right] \mathrm{U} 69,593$ as the radioligand, measured in the presence of $\mathrm{NaCl}(150 \mathrm{mM})$ (Emmerson et al., 1994) *dynorphin $A_{1-13}$ fragment tested

(2) Binding affinity $\left(\mathrm{K}_{\mathrm{i}}\right)$ in human embryonic kidney (HEK)-293 cells stably expressing rat KOPr with [ $\left.{ }^{3} \mathrm{H}\right] \mathrm{U69,593}$ as the radioligand (Beguin et al., 2008)

(3) Potency and binding efficiency in Neuro-2A cells stably expressing human KOPr, EC 50 measured using $\left.{ }^{35} \mathrm{~S}\right] \mathrm{GTP}-\mathrm{\gamma}-\mathrm{S}$ functional assay, $\mathrm{E}_{\max }$ relative to U50,488 $(10 \mu \mathrm{M})$ (DiMattio et al., 2015)

(4) Potency and binding efficiency HEK-293 cells stably expressing human KOPr, EC 50 measured using intracellular Ca2+ mobilisation assay, $\mathrm{E}_{\max }$ relative to U69,593 (Chavkin et al., 2004)

Values expressed as mean $\pm \mathrm{SEM}\left({ }^{* *}\right.$ mean $\left.\pm \mathrm{SD}\right) . \mathrm{K}_{\mathrm{i}}$ and $\mathrm{EC}_{50}$ measured in $\mathrm{nM}, \mathrm{E}_{\max }$ measured in\%. 


\subsubsection{Traditional kappa opioid receptor agonists for the treatment of pain}

The traditional KOPr agonists have proven antinociceptive effects. In nociceptive models of pain, U50,488 has been shown to have a significant effect in thermal, mechanical and visceral models (Von Voigtlander \& Lewis, 1982; Vonvoigtlander et al., 1983; Gallantine \& Meert, 2008). In mice, U50,488 was approximately half as potent as morphine in the tail withdrawal, tail pinch and hydrochloric acid writhing test (Vonvoigtlander et al., 1983). In rats, the potency of U50,488 relative to morphine varied between the different pain tests, with U50,488 showing approximately half the potency in the air writhing assay $\left(\mathrm{ED}_{50} 1.4 \mathrm{mg} / \mathrm{kg}\right.$ vs $0.6 \mathrm{mg} / \mathrm{kg}$ ), however, $\mathrm{U} 50,488$ had a significantly lower potency in the tail withdrawal compared to morphine ( $E_{50} 32 \mathrm{mg} / \mathrm{kg}$ vs $0.6 \mathrm{mg} / \mathrm{kg}$ ) (Vonvoigtlander et al., 1983). Morphine was also more potent than $\mathrm{U} 50,488$ in the warm plate $\left(49.5^{\circ} \mathrm{C} ; \mathrm{ED}_{50} 1.0 \mathrm{mg} / \mathrm{kg}\right.$ vs $\left.4.4 \mathrm{mg} / \mathrm{kg}\right)$ and hot plate $\left(54.5^{\circ} \mathrm{C}\right.$ ) assay $\left(\mathrm{ED}_{50} 1.2 \mathrm{mg} / \mathrm{kg}\right.$ vs $7.0 \mathrm{mg} / \mathrm{kg}$ ) (Vonvoigtlander et al., 1983). In rhesus macaques, a dose of $3.2 \mathrm{mg} / \mathrm{kg}$ of U50,488 was required to reach maximum effect in the $55^{\circ} \mathrm{C}$ tail withdrawal assay (France et al., 1994).

In a more recent paper, $\mathrm{U} 50,488$ was further compared to morphine using the tail withdrawal assay in Sprague Dawley rats (Gallantine \& Meert, 2008). The experiment showed a similar trend to Vonvoigtlander et al. (1983), with the $\mathrm{ED}_{50}$ of morphine at $1.26 \mathrm{mg} / \mathrm{kg}$ whereas $\mathrm{U} 50,488$ didn't produce an antinociceptive effect even at the highest $(40 \mathrm{mg} / \mathrm{kg}$ ) dose (Gallantine \& Meert, 2008). However, in the acetic acid writhing test, the potency of morphine $\left(E_{50} 0.79 \mathrm{mg} / \mathrm{kg}\right)$ and $U 50,488\left(E_{50} 1.00\right.$ $\mathrm{mg} / \mathrm{kg}$ ) were more comparable (Gallantine \& Meert, 2008). Interestingly, co-administration of U50,488 was able to block the development of morphine tolerance in male Wistar rats and the antinociceptive effect of morphine was restored to morphine-tolerant animals following administration of $\mathrm{U} 50,488$, as measured using the hotplate $\left(55^{\circ} \mathrm{C}\right)$ test (Yamamoto et al., 1988).

In inflammatory models of pain, $\mathrm{U} 50,488$ produced dose-dependent antinociception in the intraplantar formaldehyde assay via intrathecal injection (Pelissier et al., 1990) and via intracerebroventricular (i.c.v.) injection, although the MOPr agonist DAMGO ([D-Ala ${ }^{2}, \mathrm{~N}-\mathrm{Me}-\mathrm{Phe}^{4}, \mathrm{Gly}^{5}-$ ol]-enkephalin) and the DOPr agonist DPDPE ([D-Pen ${ }^{2}$, D-Pen ${ }^{5}$-enkephalin) had higher efficacy in the assay (Calcagnetti et al., 1988). The intraplantar formaldehyde assay was also tested in the Gallantine and Meert (2008) study, which showed an $E D_{50}$ of $5.2 \mathrm{mg} / \mathrm{kg}$ for morphine in both phases of pain, whereas U50,488 had an ED 50 of $3.81 \mathrm{mg} / \mathrm{kg}$ in phase I and $1.66 \mathrm{mg} / \mathrm{kg}$ in phase II. U50,488 has also been found to dose-dependently reduce arthritis disease score (Wilson et al., 1996). In an arthritis pain model, Mycobacterium butyricum was injected in the right hind paw in rats, contralateral administration of $U 50,488$ reduced paw oedema, the spread of inflammation and mechanical allodynia (Bileviciute-Ljungar et al., 2006). U50,488 also relieved mechanical hyperalgesia induced by the injection of complete Freund's adjuvant in the rat hind paw (Auh \& Ro, 2012). 
Following chronic constriction of the sciatic nerve in rats, administration of U50,488 in the contralateral hind paw reduced mechanical but not thermal allodynia, which was reversed by the peripherally restricted antagonist naloxone methiodide (Bileviciute-Ljungar \& Spetea, 2004). Further experiments with sciatic nerve ligation, measured the autotomy score for six weeks and found that U50,488 reduced this behaviour (Bileviciute-Ljungar \& Spetea, 2004).

The agonist U69,593 has been less extensively studied than U50,488. However, for U69,593 i.p. administration has shown antinociceptive effects in the hot plate $\left(55^{\circ} \mathrm{C}\right)$ in rats (La Regina et al., 1988) and the tail withdrawal assay in rhesus macaques (France et al., 1994; Negus et al., 2008). It has also been found that U69,593 treatment in female rats shows a faster peak effect at 5-15 min, compared to $30 \mathrm{~min}$ in males using the tail withdrawal assay, whilst there were no sex differences in the hot plate test (Bartok \& Craft, 1997). In the complete Freund's adjuvant model of arthritis pain, U69,593 significantly reduced paw volume and histological score, as well as reducing the mechanical allodynia in a paw pressure threshold test for up to 120 min (Binder et al., 2001).

\subsubsection{Side effects of traditional kappa opioid receptor agonists}

Unfortunately, traditional KOPr agonists have side effects that have limited the clinical development of these compounds. Administration of U50,488 has been shown to have pro-depressive effects in the forced-swim test in mice with an $\mathrm{ED}_{50}$ of $2.35 \mathrm{mg} / \mathrm{kg}$ (Zhang et al., 2015). Similarly, pro-depressive effects have also been reported with the administration of U69,593 in rats (Mague et al., 2003). The traditional KOPr agonists show aversion in the CPA test (Mucha \& Herz, 1985; Suzuki et al., 1992; BalsKubik et al., 1993; Skoubis et al., 2001; Ehrich et al., 2015) and in the conditioned taste aversion test (Mucha \& Herz, 1985). The compounds also cause anxiety measured using the elevated plus maze, light/dark test and open field test (Privette \& Terrian, 1995; Kudryavtseva et al., 2004; Vunck et al., 2011; Wang et al., 2016), muscle weakness and sedation (Dykstra et al., 1987; Zhang et al., 2015) and diuresis (Tang \& Collins, 1985; Dykstra et al., 1987). Due to these side effects, the traditional KOPr agonists are not able to be developed any further clinically; however, an alternative strategy is to evaluate the potential of structurally-unique KOPr agonists such as Salvinorin A for the development of non-addictive pain medications with reduced side effects. 


\subsubsection{Novel kappa opioid receptor agonists for the treatment of pain}

\subsubsection{Salvia divinorum and Salvinorin A}

The neo-clerodane diterpene, Salvinorin A (SalA) was discovered as the first naturally-occurring, nonnitrogenous KOPr agonist (Chavkin et al., 2004; Roth et al., 2002). SalA is the active ingredient of Salvia divinorum, a plant from the Sage family native to Mexico (Valdes, 1994). The plant has traditionally been used in the spiritual practices of the Mazatec Indians to produce hallucinogenic experiences as well as treating a variety of ailments including headache, diarrhoea, rheumatism and given as palliative care (Valdes et al., 1983). Leaves of S. divinorum can either be chewed or smoked, with ingestion of the leaves leading to a weak absorption of SalA (Siebert, 1994), whilst inhalation of the smoke gives a short-acting, potent hallucinogenic effect which is unique to that of traditional hallucinogens (Hutton et al., 2016).

Today, S. divinorum is used as a recreational hallucinogenic drug (known as Salvia), especially among young adults. In several countries, Salvia is legal and is easily accessible over the internet (Hoover et al., 2008; Moreira et al., 2014). In New Zealand, Salvia is currently illegal under the 2014 Psychoactive Substances Amendment Act. Salvia has been reported to be non-toxic, with no serious adverse effects (death or hospitalisation) (Baggott et al., 2010). In a double-blind, placebo-controlled, randomised study, 30 males were given Salvia to self-administer over two weeks, with no adverse effects observed or reported at an 8-week follow-up (Addy, 2012). Despite the rapid-onset hallucinogenic effects, Salvia has a low potential for abuse, with $87 \%$ of individuals from a self-administered Salvia study saying they would not like to use the drug again (Addy et al., 2015).

SalA is unique among hallucinogens, as it does not bind to the serotonin $2 \mathrm{~A}$ receptor $\left(5-\mathrm{HT}_{2 \mathrm{~A}}\right)$, the receptor responsible for the actions of classical hallucinogens such as lysergic acid diethylamide (LSD). SalA did not substitute for the discriminative effects of the serotonergic hallucinogen LSD or the glutamatergic hallucinogen ketamine in rats, indicating that the effects of SalA are distinct from these traditional hallucinogenic compounds (Killinger et al., 2010). SalA instead binds with high affinity and selectivity at the KOPr (Roth et al., 2002). SalA is a full KOPr agonist with similar binding affinity and efficacy to the traditional agonists U50,488 and U69,593 (Chavkin et al., 2004; Beguin et al., 2008; DiMattio et al., 2015) (Table 1.1). Interestingly, the structure of SalA has no resemblance to the traditional KOPr agonists and was the first KOPr agonist discovered which did not contain a polar nitrogen atom, which was originally considered essential for opioid receptor binding (Roth et al., 2002). 


\subsubsection{Anti-addiction effects of Salvinorin A}

SalA also has potential as a pharmacotherapy for drug addiction. SalA has been shown to attenuate dopamine release in the dorsal striatum (Zhang et al., 2005b; Gehrke et al., 2008) and nucleus accumbens (Ebner et al., 2010), as well as regulate dopamine transporter activity (Kivell et al., 2014). SalA has been studied in several models of reward, particularly for cocaine use, for which there is currently no clinically approved therapeutics. SalA has been shown to decrease cocaine-induced behavioural sensitisation (Morani et al., 2012) and acute administration of SalA attenuated cocaineinduced drug-seeking behaviour in a self-administration model in Sprague Dawley rats, without causing sedation in spontaneous locomotion tests (Morani et al., 2009) or causing taste aversion to a saccharin solution (Morani et al., 2012). In a study by Freeman et al. (2014), SalA acted as a punisher of drug-seeking behaviour for the self-administration of cocaine and the MOPr agonist remifentanil in rhesus macaques.

\subsubsection{Antinociceptive effects of Salvinorin A}

SalA has antinociceptive effects in animal behavioural models of thermal and visceral nociceptive pain. SalA produced antinociceptive effects to a thermal stimulus in male Swiss mice, in both the tail withdrawal and hot plate $\left(52^{\circ} \mathrm{C}\right)$ tests, showing a short 20 min duration of action in the tail withdrawal assay (2 $\mathrm{mg} / \mathrm{kg}$ i.p.), which was attenuated by pre-treatment with the $\mathrm{KOPr}$ antagonist norbinaltorphimine (nor-BNI) (McCurdy et al., 2006). The antinociceptive effects of SalA were also measured in the tail withdrawal assay using intrathecal administration (injected between the lumbar regions L5 and L6 of the spinal cord) in male CD-1 mice. SalA produced a dose-dependent antinociceptive effect up to $10 \mathrm{~min}$ (at the $23.1 \mathrm{nmol}$ dose), an effect that was reversed by pretreatment with nor-BNI, but not with the MOPr antagonist $\beta$-funaltrexamine ( $\beta-F N A$ ) or the DOPr antagonist naltrindole, indicating the antinociceptive effect is mediated selectively through the KOPr (John et al., 2006). In the acetic acid writhing assay of visceral nociceptive pain, SalA significantly reduced the number of responses for up to $15 \mathrm{~min}(0.5 \mathrm{mg} / \mathrm{kg}$ i.p.) in male Swiss mice (McCurdy et al., 2006).

SalA also has antinociceptive effects in inflammatory pain models. In male ICR mice, SalA significantly reduced lipopolysaccharide (LPS)- and carrageenan-induced paw oedema, which was further studied in vitro, showing SalA reduced LPS-stimulated nitrite, TNF $\alpha$ and interleukin-10 (IL-10) levels, and inducible nitric oxide synthase (iNOS) expression in mouse peritoneal macrophages (Aviello et al., 2011). In an inflammatory pain model of colitis, male CD-1 mice administered SalA show significant decreases in disease score via both i.p. administration $(3 \mathrm{mg} / \mathrm{kg})$ and oral administration $(10 \mathrm{mg} / \mathrm{kg})$ when given twice daily for 3 days (Fichna et al., 2012). SalA (3 mg/kg i.p. or $10 \mathrm{mg} / \mathrm{kg}$ intracolonic) produced an antinociceptive effect measured by a decrease in pain responses following intracolonic 
instillation of mustard oil, which was reversed by nor-BNI and the CB1 receptor antagonist AM251 (Fichna et al., 2012).

In the intraplantar formaldehyde assay, SalA reduced the nociceptive and inflammatory phases of pain in a dose-dependent manner (0.5-2 mg/kg i.p.), which was reversed by pre-treatment of nor-BNI and the CB1 receptor antagonist rimonabant (Aviello et al., 2011). A further study extended the intraplantar formaldehyde test to measure the persistent formaldehyde-induced pain effects of over 7 days. Daily treatment of SalA (2 $\mathrm{mg} / \mathrm{kg}$ i.p.) significantly reduced mechanical allodynia up to day 7 (Guida et al., 2012). Both nor-BNI and the CB1 receptor antagonist AM251, but not the CB2 receptor antagonist AM630 reduced the effect of SalA (Guida et al., 2012). Electrophysiology experiments were carried out to measure the changes in nociceptive specific neuron activity in the spinal cord, with SalA transiently normalising the formaldehyde-induced nociceptive activity (Guida et al., 2012). Furthermore, SalA reduced the formaldehyde-evoked glial and microglial activation and normalised the levels of IL-10 and iNOS expression in the spinal cord (Guida et al., 2012).

In the sciatic nerve ligation model of neuropathic pain, microinjection of SalA into the insular cortex of male Wistar rats produced a significant antinociceptive effect measured with the thermal Hargreaves' test and electronic von Frey aesthesiometer (Coffeen et al., 2018). The effect was blocked by the administration of the KOPr antagonist nor-BNI and the CB1 receptor antagonist AM251 (Coffeen et al., 2018). Interestingly, in several of the models, the antinociceptive effect was reduced or attenuated by pre-treatment with CB1 receptor antagonists (Aviello et al., 2011; Fichna et al., 2012; Guida et al., 2012; Coffeen et al., 2018), however, binding studies show that SalA is a selective KOPr agonist (Roth et al., 2002) and SalA does not substitute in mice trained to discriminate THC (Walentiny et al., 2010). Cannabinoid receptor agonists have been shown to have a synergistic action with opioid receptor agonists (Grenald et al., 2017) and may form heterodimers with the opioid receptors (Sierra et al., 2017). In addition, the CB1 antagonist rimonabant has been shown to attenuate U69,593 binding to the KOPr, indicating that rimonabant may have antagonistic effects on the KOPr (Walentiny et al., 2010; Zador et al., 2015).

\subsubsection{Novel synthetic analogues of Salvinorin A}

SalA has reduced side effects compared to the traditional KOPr agonists. However, SalA has been shown to cause aversion in CPA (Zhang et al., 2005b), anxiety in the elevated plus maze (Braida et al., 2009), pro-depressive effects in the forced swim test and intracranial self-stimulation model (Carlezon et al., 2006), learning and memory impairments in the eight-arm radial maze and object recognition tasks (Braida et al., 2011), and motor incoordination in the inverted screen task (Fantegrossi et al., 2005) in rodent models. Using male and female rhesus macaques as a non-human primate model, 
SalA had a sedative effect (0.01-0.1 mg/ $/ \mathrm{kg}$ i.v.), as measured by unresponsiveness to environmental stimuli and postural relaxation (Butelman et al., 2009). Furthermore, SalA has a short duration of action in vivo (Butelman et al., 2007; Ranganathan et al., 2012) as it is quickly metabolised to the inactive product Salvinorin B (Schmidt et al., 2005b; Kutrzeba et al., 2009), all of which has limited the clinical usefulness of SalA.

However, longer acting SalA analogues have the potential for development of non-addictive analgesics if centrally mediated side effects can be reduced. To improve the therapeutic potential for SalA, two strategies for designing analogues have been considered. Firstly, creating a peripherally restricted analogue of SalA would potentially remove the negative centrally-mediated side effects (Kivell \& Prisinzano, 2010). This could be particularly useful at treating inflammatory pain, as inflammation leads to the increase in synthesis of opioid receptors in the DRG which are then distributed at higher density in the PNS and CNS (Zollner et al., 2003; Puehler et al., 2006). However, compounds tested so far have only produced moderate antinociceptive effects (Vadivelu et al., 2011), although, the peripherally-restricted KOPr agonist CR665 developed by Cara Pharmaceuticals was advanced to Phase II clinical trials (Arendt-Nielsen et al., 2009; Olesen et al., 2013). The second strategy is to take advantage of the concept of biased agonism where specific ligands activate distinct downstream pathways (Bruchas \& Chavkin, 2010). Analogues which can activate the analgesic G-protein pathways without the $\beta$-arrestin pathway could have reduced side effects (Bruchas \& Chavkin, 2010). Recently, it has been shown that the clinically-available agonist nalfurafine is 'extremely' G-protein biased at the human KOPr (Schattauer et al., 2017).

Our collaborator, Prof. Thomas Prisinzano at the University of Kansas, has created a library containing structural analogues of SalA. Many of the modifications have focussed on the functional alterations at the carbon-2 and carbon-16 positions. Modifications at the carbon-2 have led to increased potency and binding affinity by adding metabolism-protective groups and removing the hydrolysable ester (Munro et al., 2008). Modifications at the carbon-16 position may hinder the action of cytochrome P450 enzymes metabolising the furan ring and therefore improve the metabolic stability (Wilson et al., 1990). In vivo testing of these carbon-2 and carbon-16 SalA analogues have been shown to attenuate drug-seeking behaviours with reduced side effect profiles (Riley et al., 2014; Simonson et al., 2015; Ewald et al., 2017). This thesis aimed to screen the antinociceptive and anti-inflammatory effects of novel analogues of SalA, and further study the promising compounds as potential treatments for chemotherapy-induced neuropathic pain. 


\subsection{Overall aims, objectives and hypotheses}

Currently, there is an urgent global need for the development of non-addictive analgesic medications. This research aimed to screen and evaluate the antinociceptive and anti-inflammatory effects of several novel compounds including bioactive lipids and KOPr agonists, all of which have reduced abuse potential. The effect of acute administration of the compounds were evaluated in animal models of nociceptive and inflammatory pain in mice. Based on previous studies with SalA, it was hypothesised that the novel potent compounds would have antinociceptive and anti-inflammatory effects in vivo. Further studies into the sedative and thermoregulatory side effects were also evaluated, with the hypothesis that DHEA and novel KOPr agonists would show a reduced side effect profiles.

Additionally, chemotherapy-induced neuropathic pain is a serious problem and can be the reason for limiting cancer treatments. This thesis aimed to investigate the efficacy of treating paclitaxel-induced neuropathic pain with KOPr agonists and further assess the mechanism of action by quantifying demyelination in the sciatic nerve.

The specific aims of the study were:

1. To evaluate the acute antinociceptive effects and side effects of the bioactive lipid compound DHEA in preclinical models of pain in mice.

2. To evaluate the acute antinociceptive effects and side effects of novel KOPr agonists in preclinical models of pain in mice.

3. Assess the efficacy of the novel KOPr agonists on paclitaxel-induced neuropathic pain using an acute dose-response model for mechanical and cold allodynia.

4. To further test the KOPr agonists in a chronic model of paclitaxel-induced neuropathic pain and evaluate the effects on demyelination in the sciatic nerve. 


\section{Chapter 2: Methods}

\subsection{Animals}

Adult female and male C57BL/6J mice (18-30 g, 8+ weeks old) were used in the paclitaxel-induced neuropathic pain experiments. Adult male B6-SJL mice (22-30 g, 8+ weeks old) were used for all other experimental procedures. Animals were bred and housed at the Victoria University of Wellington (VUW) Animal Facility, New Zealand, however initial breeding stock were supplied from the Biomedical Research Unit at the Malaghan Institute of Medical Research, Wellington, New Zealand. Animals were originally sourced from the Jackson Laboratories (Bar Harbour, ME, USA).

All animals were group-housed (maximum 5 mice/cage) in a temperature $\left(20-22^{\circ} \mathrm{C}\right.$ ) and humidity (55\%) controlled environment. The animals were maintained on a 12-hour light/dark cycle with lights on at $7 \mathrm{am}$. All procedures were undertaken during the light cycle, with procedures never beginning in the first hour the lights turned on. Procedures were carried out in the presence of white noise to mask the effects of background noise. Access to food pellets (Diet 86, Sharpes Stock Feed, Carterton, NZ) and tap water was provided ad libitum except during experimental sessions. For all paclitaxelinduced neuropathic pain experiments, soft paper/pulp-based Carefresh Natural bedding (Masterpet, Lower Hutt, NZ) was used in the home cage to avoid any mechanical stimulation to the paw. In other experimental procedures, Kay pine bedding (Masterpet) was used. Each cage had shredded nesting material and cardboard rolls as environmental enrichment.

Animals were handled for at least two days before testing in order to acclimatise to handling and prevent stress during experimental procedures. Animals were habituated to the experimental room for $30 \mathrm{~min}$ each day. If a male observer was present, the animals were habituated to the observer for $60 \mathrm{~min}$ daily before commencing the experiment to reduce stress-induced analgesia (Sorge et al., 2014). All procedures were carried out with the approval of the VUW Animal Ethics Committee (bioactive lipids project, approval number 2013R31; opioid agonist project, approval number 21480). All procedures were carried out in agreement with the New Zealand Animal Welfare Act, 1999. In accordance with the 3 Rs principles for the ethical use of animals in research (Russell \& Burch, 1959), efforts were made to reduce total animal numbers by using mice in multiple behavioural experiments. Mice which were reused for multiple procedures had a minimum rest period of seven days between tests. Animals were reused for the time course tail withdrawal assay, rotarod, temperature change and intraplantar formaldehyde assay. All animals which underwent the intraplantar formaldehyde assay were immediately culled afterwards. Animals used in repeated procedures did not receive the same treatment twice to reduce the effects of opioid tolerance. Treatment-naive animals were used 
for the warm water tail withdrawal dose-response experiments and all paclitaxel-induced neuropathic pain experiments.

\subsection{Drug preparation}

\subsubsection{Bioactive lipid compounds}

DHEA was provided by Dr Mikhail Vyssotski at Callaghan Innovation (Lower Hutt, NZ). DHEA was stored under argon in the $-80^{\circ} \mathrm{C}$ freezer and freshly prepared on each day of use. DHEA was dissolved in dimethyl sulfoxide (DMSO; Sigma-Aldrich, St Louis, MO, USA) and propylene glycol (Sigma-Aldrich) at a ratio of 1:4 to make a $2 \mathrm{mg} / \mathrm{mL}$ stock solution. This stock was further diluted with phosphate buffered saline (PBS, $140 \mathrm{mM} \mathrm{NaCl}, 2.68 \mathrm{mM} \mathrm{KCl}, 8.1 \mathrm{mM} \mathrm{Na}_{2} \mathrm{HPO}_{4}, 1.47 \mathrm{mM} \mathrm{KH} 2 \mathrm{PO}_{4}$; Appendix A) to make a $0.2 \mathrm{mg} / \mathrm{mL}$ solution via i.p. injection to give a $2 \mathrm{mg} / \mathrm{kg}$ dose (delivered at $10 \mu \mathrm{L} / \mathrm{g}$ ). The cannabinoid receptor antagonists AM251 and AM630 were purchased from Tocris Bioscience (Bristol, UK). AM630 and AM251 (1 mg/kg i.p.) were administered with a vehicle containing 1:9 ratio of DMSO and physiological $(0.9 \% \mathrm{NaCl})$ saline prior to DHEA administration, with a pre-treatment time of $30 \mathrm{~min}$ and 40 min respectively.

\subsubsection{Kappa opioid receptor agonists}

SalA and the analogues were provided by Prof. Thomas Prisinzano (University of Kansas, Lawrence, KS, USA). SalA was isolated and purified from S. divinorum leaves and assessed for purity (>98\%) using high-performance liquid chromatography (HPLC) (Munro \& Rizzacasa, 2003; Tidgewell et al., 2004). The SalA analogues were synthesised as previously published: 16-Ethynyl SalA and 16-Bromo SalA described in Riley et al. (2014); $\beta$-THP SalB and EOM SalB described in Prevatt-Smith et al. (2011). All the novel SalA analogues were tested for purity (>95\%) with HPLC. The traditional KOPr agonist U50,488 was purchased from Sigma-Aldrich. Morphine sulphate (Hospira NZ Ltd., $10 \mathrm{mg} / \mathrm{mL}$ in saline) was purchased from the Capital and Coast District Health Board Pharmacy (Wellington, NZ). All of the KOPr agonists and morphine were light-protected and stored at $4^{\circ} \mathrm{C}$.

All of the KOPr agonists were dissolved in a vehicle containing DMSO, Tween-80 (Sigma-Aldrich) and physiological saline at a ratio of 2:1:7 respectively. Morphine came pre-prepared in saline but was diluted by adding DMSO, Tween-80 and further saline, to achieve an identical vehicle. The KOPr agonists were delivered at a volume of $10 \mu \mathrm{L} / \mathrm{g}$ of weight via i.p. injection and delivered at $5 \mu \mathrm{L} / \mathrm{g}$ via s.c. injection in the warm water tail withdrawal dose-response experiments. The KOPr antagonist norBNI (Sigma-Aldrich) was dissolved in physiological saline and injected s.c. $24 \mathrm{~h}$ prior to testing to selectively antagonise the KOPr, as earlier pre-treatment intervals have been shown to also antagonise the MOPr (Endoh et al., 1992; Kishioka et al., 2013). 


\subsection{Acute Nociceptive Assays}

\subsubsection{Warm water tail withdrawal assay}

On the two days prior to the test day, mice were restrained in transparent plastic tube restrainers (internal diameter $24 \mathrm{~mm}$ ) for $5 \mathrm{~min}$ daily, to acclimatise the animals to the procedure and reduce restraint stress. Tail withdrawal latencies were measured by immersing one-third of the distal portion of the tail in a warm water bath $\left(50 \pm 0.5^{\circ} \mathrm{C}\right)$ and the time to show the withdrawal response recorded with a stopwatch. A maximum tail immersion cut-off of $10 \mathrm{~s}$ was used to avoid tissue damage and allow for repeated exposure to the thermal stimulus. On the day of the experiment, the baseline latency for each animal was measured by averaging three drug-naive tail withdrawal latencies, with successive measurements taken at least 5 min apart. Following the experimental procedure, the percentage maximum possible effect (\%MPE) was calculated for each animal at each time point by using the following formula:

$$
\% M P E=\left(\frac{\text { test latency }- \text { baseline latency }}{10-\text { baseline latency }}\right) \times 100
$$

\subsubsection{Time course warm water tail withdrawal assay}

To measure the duration of action, the tail withdrawal assay was measured over a time course (Simonson et al., 2015; Paton et al., 2017). Following the baseline latency measurements, mice were given an i.p. injection of either DHEA, KOPr agonist or vehicle and the latency to tail withdrawal behaviour was determined at 5, 10, 15, 30, 45, 60, 90 and 120 min post-injection for each animal in a repeated measures design. One latency measurement was taken at each time point.

\subsubsection{Cumulative dose-response tail withdrawal assay}

The cumulative dose-response effects were evaluated using a within animal design (Bohn et al., 2000; Paton et al., 2017; Sherwood et al., 2017a). The mice were given s.c. injections at increasing concentrations to create cumulative doses. At 30 min post-injection, the tail withdrawal latency was measured and the next cumulative dose administered immediately after ( 30 min time interval based on the previous duration of action experiments in the Kivell laboratory). The doses were delivered at a volume of $5 \mu \mathrm{L} / \mathrm{g}$ of weight, and the s.c. injections delivered into the scruff of the neck, the left flank and the right flank in sequence to avoid continuous injections into the same area. SalA and the analogue $\beta$-THP SalB, were measured at the following doses: 0.3, 0.6, 1.0, 2.5, 5.0, 7.5, $10.0 \mathrm{mg} / \mathrm{kg}$. 16-Ethynyl SalA and 16-Bromo SalA were measured at the following doses: 0.3, 0.6, 1.0, 2.5, 5.0, 7.5, $10.0,12.5 \mathrm{mg} / \mathrm{kg}$. EOM SalB was measured at the following doses: 0.1, 0.3, 0.6, 1.0, 2.5, 5.0, 7.5 and $10.0 \mathrm{mg} / \mathrm{kg}$. U50,488 was measured at the following doses: 1.0, 2.5, 5.0, 7.5, 10.0, 20.0 and 30.0 $\mathrm{mg} / \mathrm{kg}$. Typically, higher doses were prepared for the initial experiment, as the effectiveness of the 
novel agonists in vivo were unknown. However, a judgement was made to terminate the experiment when two consecutive results had no substantial increase in latency or the animals were too sedated to enter the restrainers. The mice were culled at the end of the procedure. Non-linear regression analysis was used to determine potency (median effective dose $\left(E D_{50}\right)$ ) and efficacy calculated by normalising data to the traditional KOPr agonist $\mathrm{U} 50,488$ (maximum dose achieved $\left(\mathrm{E}_{\max }\right)$ ).

\subsubsection{Hotplate assay}

Mice were tested using a hotplate (IITC Life Science, Woodland Hills, CA, USA) set at a constant temperature of $50^{\circ} \mathrm{C}$. The mice were placed in a clear plastic enclosure with a removable lid sitting on top of the hotplate surface. The timing began when the mouse was placed on the hotplate and all four paws were touching the surface. The time for the mouse to either shake or lick one of the hind paws or to exhibit escape behaviour (trying to jump out of the enclosure) was measured with a cut-off of $30 \mathrm{~s}$ to avoid tissue damage. The mice were acclimatised to the sound of the hotplate machine for 30 min per day and baseline measurements were taken once per day for three days prior to testing. The baseline latency was taken as the average of the three measurements. On the test day, an i.p. injection of the KOPr agonist or vehicle was administered and measurements were taken at 15, 30 and 60 min post-injection. The percentage change at each time point was calculated using the following formula:

$$
\% \text { change }=\left(\frac{\text { test latency }- \text { baseline latency }}{\text { baseline latency }}\right) \times 100
$$

\subsubsection{Intraplantar $2 \%$ formaldehyde model}

The apparatus for the intraplantar $2 \%$ formaldehyde model used a wooden box $(27.5 \times 18.5 \mathrm{~cm})$ with an open bottom and top (height $30 \mathrm{~cm}$ ). This box was placed on top of a glass surface with a $45^{\circ}$ angle mirror placed underneath to facilitate observations of the hind paws via a digital video camera. The mice were allowed to habituate to the test enclosure for $15 \mathrm{~min}$ before testing. The mice were given KOPr agonist, DHEA or vehicle treatment via i.p. injection 5 min prior to the administration of $20 \mu \mathrm{L}$ of formaldehyde (2\% in PBS) or PBS control via i.pl. injection. $2 \%$ formaldehyde concentration was selected based on previous concentrations tested in the Kivell laboratory, which showed $2 \%$ was sufficient to generate a robust biphasic nociceptive effect. For the i.pl. injection, the mice placed in a restrainer and drug or vehicle administered with a 29-gauge needle orientated bevel upwards. The needle was inserted under the plantar surface in the centre of the right hind paw, with care to avoid blood vessels. In a small number of cases, the injected liquid came back out of the injection site or a blood vessel was hit causing bleeding, these animals were excluded from the experimental results. 
The pain behaviours were recorded for $60 \mathrm{~min}$. The methods of Dubuisson and Dennis (1977) were used to assess the pain behaviour of the injected paw using a weight-bearing score. The pain was scored as 0 if the mouse showed normal behaviour; 1 for partial weight-bearing with only the digits touching the floor; 2 with no weight-bearing with the paw raised; and 3 where the paw was bitten, licked or shaken. The videos were blinded and the pain behaviour scores were assigned at $5 \mathrm{~s}$ intervals for the total $60 \mathrm{~min}$. An average pain score was calculated for each $5 \mathrm{~min}$ time period. For the area under the curve analysis, the two phases of pain were separated out as follows: 0-15 min phase I nociceptive pain; and 20-60 min phase II inflammatory pain. Mice were immediately culled after the experimental procedure was completed.

\subsubsection{Intraplantar administration of compounds}

To test the local effects of the compounds, treatments were given as an i.pl. injection 5 min prior to and in the same hind paw as the $2 \%$ formaldehyde i.pl. injection. The initial test compound injection was delivered slightly to the left of the centre point of the hind paw, and the second injection of formaldehyde was injected slightly to the right. The KOPr agonist, DHEA or vehicle were delivered at $1 \mu \mathrm{L} / \mathrm{g}$ with a maximum of $30 \mu \mathrm{L}$. The KOPr agonists were in a vehicle containing DMSO only, and DHEA was in a vehicle containing 1:4 ratio of DMSO and propylene glycol.

To further investigate the effects of DHEA on inflammatory pain, the DHEA i.pl. injection was given 15 min following the $2 \%$ formaldehyde injection. In the time between the injections, the mice were placed into the test enclosure but were not recorded.

\subsubsection{Paw oedema}

The mice were restrained and the size of the right hind paw was measured with digital callipers prior to the beginning of the experimental procedure. The depth, width and height of the paw were measured, however, the decision was made to only use the depth in the oedema calculations as these were the only measurements that changed following the administration of formaldehyde. Following the 60 min test period, the hind paw was measured again to calculate the percentage change in paw depth, using the following formula:

$$
\% \text { change }=\left(\frac{d_{2}-d_{1}}{d_{1}}\right) \times 100
$$

Where $d_{1}$ is the original depth of the paw and $d_{2}$ is the depth of the paw 60 min after $2 \%$ formaldehyde or PBS vehicle i.pl. injection. 


\subsection{Evaluation of immune cell infiltration into footpad tissue}

\subsubsection{Footpad tissue dissection}

The footpad tissue from the intraplantar formaldehyde model was assessed for immune cell infiltration. Animals were euthanised by $\mathrm{CO}_{2}$ asphyxiation followed by cervical dislocation. The right hind paw was removed and the footpad tissue was dissected using a razor blade to slice off the tissue from above the bone. The tissue was further processed for either flow cytometry or histology.

\subsubsection{Flow cytometry}

Cell isolation from footpad tissue was carried out using methods modified from Leong et al. (2012) and as described in Paton et al. (2017). Immediately following the formaldehyde test (60 min following formaldehyde injection), the footpad tissue was dissected and digested for $2 \mathrm{~h}$ in complete Iscove's modified Dulbecco's medium (cIMDM with 10\% heat-inactivated foetal bovine serum (FBS), $1 \%$ penicillin-streptomycin) containing $125 \mu \mathrm{g} / \mathrm{mL}$ Deoxyribonuclease I (DNase; from bovine pancreas, Type II-S, Sigma-Aldrich) and $5 \mathrm{mg} / \mathrm{mL}$ collagenase I (from Clostridium histolyticum, Sigma-Aldrich) at $37^{\circ} \mathrm{C}$. The digest was pressed through a $40 \mu \mathrm{m}$ filter (BD Bioscience, San Jose, CA, USA) and the cells were washed and resuspended in cIMDM and stored on ice until all samples were ready to be further processed. The cells were washed in fluorescence-activated cell sorter (FACS) buffer, resuspended in an antibody solution in FACS buffer and incubated in the dark for $15 \mathrm{~min}$ at $4^{\circ} \mathrm{C}$. The antibody solution contained antibodies for the cell surface markers stated in Table 2.1. 


\begin{tabular}{|c|c|c|c|c|c|}
\hline Cell surface marker & Description of cell marker & Fluorophore & Species & Manufacturer & $\begin{array}{l}\text { Optimal } \\
\text { dilution }\end{array}$ \\
\hline CD45 & lymphocyte common antigen & PerCP & Rat anti-mouse & Biolegend & $1: 800$ \\
\hline CD11c & common dendritic cell marker & APC & Hamster anti-mouse & BD Bioscience & $1: 200$ \\
\hline $\mathrm{CD} 11 \mathrm{~b}$ & macrophage, neutrophil and monocyte marker & FITC & Rat anti-mouse & BD Bioscience & $1: 400$ \\
\hline $\mathrm{F} 4 / 80$ & macrophage marker & $\mathrm{PE}$ & Rat anti-mouse & Biolegend & $1: 200$ \\
\hline Gr-1 & myeloid differentiation marker & Pacific Blue & Rat anti-mouse & Biolegend & $1: 400$ \\
\hline Ly6C & monocyte marker & AF 700 & Rat anti-mouse & BD Bioscience & $1: 200$ \\
\hline CD3 & T cell marker & $\mathrm{PE}-\mathrm{Cy} 7$ & Rat anti-mouse & Biolegend & $1: 600$ \\
\hline Live/dead stain & live/dead fixable blue for UV excitation & $\begin{array}{c}\text { Ext/Em } \\
\sim 350 / \sim 450 \mathrm{~nm}\end{array}$ & - & Life Technologies & $1: 500$ \\
\hline
\end{tabular}

Table 2.1: Antibodies for flow cytometry labelling

AF, Alexa Fluor; APC, allophycocyanin; Ext/Em, excitation/emission; FITC, fluorescein isothiocyanate; PerCP, peridinin-chlorophyll-protein; PE, phycoerythrin. 
Cells were resuspended and fixed in $10 \%$ formalin at room temperature for $10 \mathrm{~min}$ and resuspended in FACS buffer, to be analysed by flow cytometry the next day. Flow cytometry was analysed on a LSRII Special Order Research Product (BD Bioscience) at the Malaghan Institute of Medical Research with the help of Odette Shaw and Kristel Kodar. Single-stained samples of negative control cells were included for each fluorophore. Leukocytes were distinguished from non-haematological cells by their CD45+ expression. Neutrophils were identified as CD45+/CD11b+/Ly6C-/CD11C-/Gr-1 ${ }^{\text {high }}$. Monocytes were identified as CD45+/CD11b+/Ly6C+/CD11C-/F4-80-/Gr-1 ${ }^{\text {low }}$. Macrophages were identified as CD45+/CD11b+/Ly6C-/CD11C-/F4-80+/Gr-1 1ow. Dendritic cells were identified as CD45+/CD11b/CD11c+. T cells were sorted out with CD3 expression. All gating strategies removed the dead cells using Live/Dead Fixable blue dead cell stain kit. The data were analysed using FlowJo version 10.0 (FlowJo LLC, Ashland, OR).

\subsubsection{Histology to quantify neutrophils}

\subsubsection{Frozen sectioning}

The footpad tissue samples from bioactive lipid experiments were fixed in $4 \%$ formaldehyde overnight and cryoprotected in $30 \%$ sucrose in PBS for $24-48$ hours until the tissue sunk to the bottom of the container. The sections were embedded in Jung tissue freezing medium (Leica Biosystems) and frozen at $-80^{\circ} \mathrm{C}$ for long-term storage. Sagittal $10 \mu \mathrm{m}$ sections were cut using a Leica CM3050 S cryostat microtome (Leica Biosystems) and mounted on Superfrost plus slides (Thermo Fisher Scientific). To enhance fixation and adherence to the slide, samples were immersed in ice-cold acetone for $10 \mathrm{~min}$ prior to staining and air-dried overnight.

\subsubsection{Paraffin embedding}

The footpad tissue samples from the KOPr agonist experiments were fixed in $4 \%$ formaldehyde overnight and then stored in $70 \%$ ethanol at $4^{\circ} \mathrm{C}$. The tissue was placed into a tissue processing cassette (Thermo Fisher Scientific) and processed on a Leica TP1020 processor (Leica Biosystems, Wetzlar, Germany) using the settings in Table 2.2. The tissue was removed from cassettes and embedded in paraffin (Histoplast PE, Thermo Fisher Scientific) using a Leica EG1160 tissue embedding system (Leica Biosystems). Sagittal $5 \mu \mathrm{m}$ sections were cut using a Leica RM 2235 microtome (Leica Biosystems) and mounted on Superfrost plus slides. Sections were deparaffinised with xylene (histological grade; $2 \times 5 \mathrm{~min}$ ) and then rehydrated through a sequence of ethanol concentrations starting at $100 \%$ ( $2 \times 5 \mathrm{~min})$ through to $70 \%$ ethanol $(1 \times 5 \mathrm{~min})$. 


\begin{tabular}{cc}
\hline Solution & Time \\
\hline $70 \%$ Ethanol & 2 hours \\
\hline $80 \%$ Ethanol & 1.5 hours \\
\hline $95 \%$ Ethanol & 1 hour \\
\hline $95 \%$ Ethanol & 1 hour \\
\hline $100 \%$ Ethanol & 1.5 hours \\
\hline $100 \%$ Ethanol & 1.25 hours \\
\hline $100 \%$ Ethanol & 1.25 hours \\
\hline $100 \%$ Ethanol/Xylene & 1.5 hours \\
\hline Xylene & 1 hour \\
\hline Xylene & 1 hour \\
\hline Paraffin wax & 1.5 hours \\
\hline Paraffin wax (with vacuum) & 2 hours \\
\hline
\end{tabular}

Table 2.2: Leica TP1020 processor settings for paraffin embedding footpad tissue samples

\subsubsection{Haematoxylin and eosin Y staining}

All of the sections were stained with haematoxylin and eosin $Y$ (H\&E) to identify neutrophils. After the processing mentioned above, slides were washed with tap water for 5 min and then immersed in haematoxylin (Gill's No. 3, Sigma-Aldrich) for $5 \mathrm{~min}$, before being dipped in tap water until the slides were clear of excess stain. The slides were then dipped in acid alcohol $(1 \% \mathrm{HCl}$ in $70 \%$ ethanol) approximately 10 times and placed back into tap water for $10 \mathrm{~min}$. Slides were then placed in $1 \%$ eosin Y (Acros Organics, Thermo Fisher Scientific) in an acid alcohol solution (0.5\% glacial acetic acid in 70\% ethanol, Appendix A) for 2 min. Slides were washed in water and dehydrated using 70\% (quick pass) and then $100 \%$ ethanol for $5 \mathrm{~min}$, before being placed in two successive washes with xylene for $5 \mathrm{~min}$ each. Coverslips were mounted using DPX mounting medium (Thermo Fisher Scientific). In more recent experiments, CC aqueous tissue mounting medium (Sigma-Aldrich) was used to replace the ethanol and xylene steps at the end. After the eosin $Y$ solution, slides were dipped into tap water until clear and then the coverslip was mounted with the CC mountant.

\subsubsection{Quantification of neutrophil numbers}

The stained sections were examined using an Olympus IX53 microscope (Olympus New Zealand Ltd.) with an Olympus TH4-200 white light and images captured with an Olympus DP73 camera using CellSens software. The sections were assessed at $4 x$ magnification and a grid with rectangles measuring $140 \times 100 \mu \mathrm{m}$ was placed randomly upon the section. The grid squares that completely contained dermis layer were numbered. Using a random number generator, 5 fields of view at $100 \mathrm{x}$ magnification were assessed for neutrophil numbers in 6 sections per animal (30 fields of view). 
Neutrophils were identified by the unique multilobular nucleus. Neutrophils were only counted when the complete cell was visualised within the field of view, however, neutrophils within blood vessels were not counted. The total number of neutrophils per animal was divided by the area of the sections counted. The cell counts were performed blind to the treatment.

\subsection{Side effect behavioural assays}

\subsubsection{Rotarod Performance Assay}

Mice were assessed for motor coordination on a rotarod apparatus (32 mm diameter barrel; Harvard Apparatus, MA, USA) as previously described in Crowley et al. (2016). The mice were initially trained on the apparatus in a series of 4 sessions/day over 4 days. The rotarod procedure involved placing the mice on the apparatus which was spinning at $4 \mathrm{rpm}$. Following a few seconds on the apparatus and once the mice were positioned correctly so that they were running against the spin of the barrel, the acceleration began with the increase from 4-40 rpm over $300 \mathrm{~s}$. The latency to fall from the apparatus was measured, with the mice removed from the apparatus if they still remained on at $300 \mathrm{~s}$. On the test day, triplicate baseline measurements were taken and only mice able to remain on the rotarod for longer than $240 \mathrm{~s}$ in every trial were included in the experiment. This was done to exclude any animals with pre-existing motor incoordination. The mice were administered an i.p. injection of either DHEA, KOPr agonist or vehicle and the latency to fall was measured repeatedly at $15,30,45,60,90$, 120,180 and $240 \mathrm{~min}$. The results were expressed as a percentage of the individual animal's baseline, with no value larger than $100 \%$.

\subsubsection{Core Body Temperature}

The core body temperature of the mice was measured via a rectal probe (Acorn series, Singapore). The temperature probe was calibrated to $37^{\circ} \mathrm{C}$ each day prior to use. The mice were placed in a transparent plastic tube restrainer and the probe was inserted $1 \mathrm{~cm}$ into the anal canal and held in place until the temperature stabilised (for no more than $5 \mathrm{~s}$ ). A baseline measurement was taken 30 min before the experiment began. Following i.p administration of DHEA, KOPr agonist or vehicle, with a single measurement taken at 30,60,90 and 120 min post-injection. The change in core body temperature was calculated based on the baseline measurement. 


\subsection{Paclitaxel-Induced Neuropathic Pain Model}

\subsubsection{Initiation of paclitaxel-induced neuropathic pain}

Paclitaxel was purchased from Tocris Bioscience (Taxol \#RDS109750) and stored light-protected at $4^{\circ} \mathrm{C}$. Paclitaxel was made up fresh every day by dissolving in absolute ethanol, cremophor EL (SigmaAldrich) and physiological saline at a ratio of 1:1:18 respectively. The powder had to be completely dissolved in the solvent (ethanol) before adding the emulsifier (cremophor) and finally, the saline was added immediately prior to use in order to avoid precipitation of the drug. Experimental procedures were modified from methods found in Deng et al. (2015) and as described in Paton et al. (2017). Male and female C57BL/6J mice were administered paclitaxel $4 \mathrm{mg} / \mathrm{kg}$ i.p. injections on four alternate days to give a cumulative dose of $16 \mathrm{mg} / \mathrm{kg}$ (see Figure 2.2). The paclitaxel was made as a $0.4 \mathrm{mg} / \mathrm{ml}$ solution and delivered at $10 \mu \mathrm{l} / \mathrm{g}$. Control mice were given equivalent doses of the cremophor vehicle and were housed in separate cages to the paclitaxel-treated animals. Mechanical and cold allodynia were assessed every second day to measure the progression of paclitaxel-induced effects. Mice were placed in transparent plastic chambers upon a metal mesh stand. After a 20 min habituation to the apparatus, each hind paw was measured in duplicate for each type of stimulation, always beginning with mechanical. On days with behavioural measurements and a paclitaxel dose, measurements were always taken prior to the administration of paclitaxel.

\subsubsection{Mechanical allodynia}

Mechanical allodynia was measured using a 20-piece set of Semmes Weinstein von Frey filaments (Touch Test Sensory Evaluator Kit for Animal Research, \#58011, Stoelting, IL, USA). Filaments numbered from 2 to 9 were used, with testing always beginning with filament number 5 . The filament was applied at a right angle to the plantar surface of the hind paw with enough force to produce a slight bend in the nylon filament. The force that each filament bends at is proportional to the diameter and inversely proportional to the length (see Table 2.3 for target forces of each monofilament). The filaments were held for $3 \mathrm{~s}$ or until a positive withdrawal response was observed. A positive withdrawal response was defined as a sharp withdrawal of the paw upon application of the filament or flinching immediately on removal of the filament. Mechanical allodynia was measured using a simplified up-down method (Bonin et al., 2014), where a withdrawal response resulted in the use of next lower filament in the subsequent test; if no withdrawal response was observed, the next higher filament was used (for examples see Figure 2.1). This continued until 5 filaments had been administered. The paw withdrawal threshold was calculated by using the outcome of the fifth filament used in each testing sequence and adding or subtracting a value of 0.5 filament intervals if the response to the fifth filament was negative or positive, respectively (Bonin et al., 2014). Mechanical 
allodynia for each animal was calculated by averaging the paw withdrawal thresholds from duplicate values for each hind paw.

\begin{tabular}{cccc}
\hline Filament no. & Evaluator size & Target force* $^{*}(\mathbf{g})$ & Target force* $^{(\mathbf{m N})}$ \\
\hline 2 & 2.36 & 0.02 & 0.196 \\
\hline 3 & 2.44 & 0.04 & 0.392 \\
\hline 4 & 2.83 & 0.07 & 0.686 \\
\hline 5 & 3.22 & 0.16 & 1.569 \\
\hline 6 & 3.61 & 0.40 & 3.922 \\
\hline 7 & 3.84 & 0.60 & 5.882 \\
\hline 8 & 4.08 & 1.00 & 9.804 \\
\hline 9 & 4.17 & 1.40 & 13.725 \\
\hline
\end{tabular}

Table 2.3: Target force of the von Frey filaments used to measure mechanical allodynia

Manufacturers (Stoelting Co.) details on the individual von Frey filaments used to measure mechanical allodynia. The evaluator size refers to the value of $\log _{10}(10 \mathrm{x}$ force in milligrams) and is used as a handle marking to identify the filaments. *The target forces are derived from tests performed by the manufacturer and individually calibrated within a $5 \%$ standard deviation.

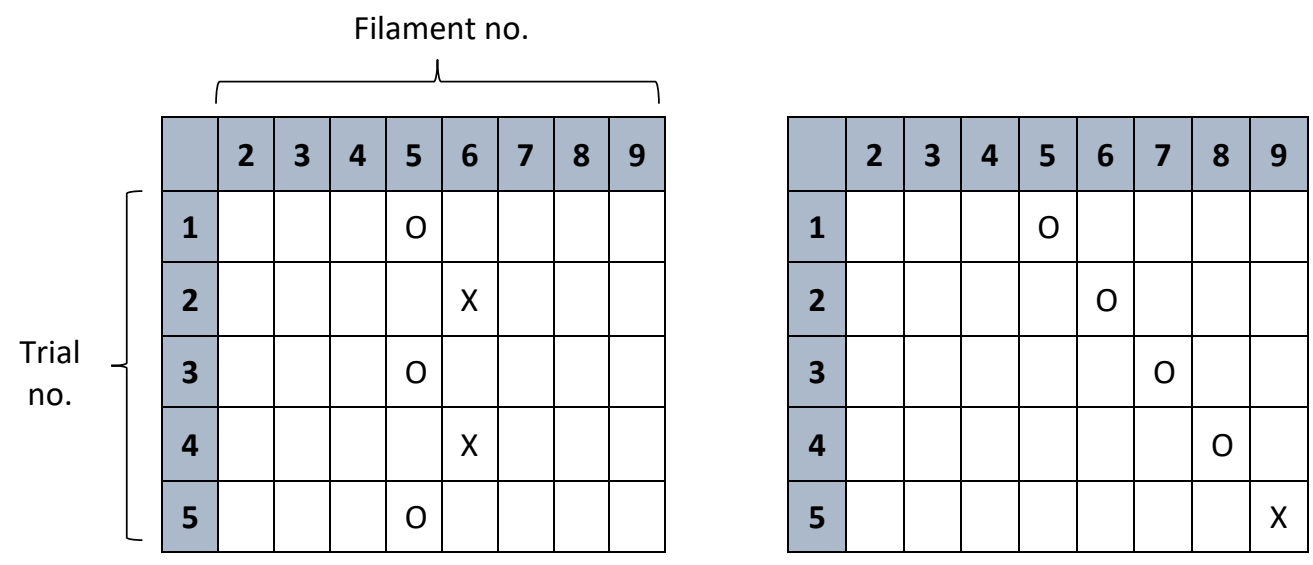

Figure 2.1: Examples of the simplified up-down von Frey filament testing scheme

Two examples of the simplified up-down method using von Frey filaments to test for mechanical allodynia. The testing always begins with filament number 5 . No withdrawal response $(0)$ means the next highest fibre is subsequently used. A positive withdrawal response $(X)$ means that the next lowest fibre is used next. The withdrawal scores are 5.5 and 8.5 for these two examples. 


\subsubsection{Cold allodynia}

Using a $1 \mathrm{~mL}$ syringe, a bubble of acetone was administered to the plantar surface of the hind paw with care not to cause any mechanical stimulation with the end of the syringe. The amount of time the animal reacted to the stimulus was recorded within $60 \mathrm{~s}$ of the application. A positive reaction was defined as elevating, licking, biting or shaking of the paw. Two measurements were taken for each hind paw alternately with 5 min between consecutive applications. Cold allodynia for each animal was calculated by averaging the duration of time (s) spent responding to the acetone across the 4 applications.

\subsubsection{Acute dose-response procedure in paclitaxel-treatment mice}

On day 15 following the initial paclitaxel injection, the cumulative dose-response effects of the $\mathrm{KOPr}$ agonists and morphine were assessed in the paclitaxel-treated male and female mice using a withinanimals design (Figure 2.2). The KOPr agonists, morphine, or equivalent volumes of the vehicle were administered via s.c. injection every $30 \mathrm{~min}$ at increasing concentrations to create cumulative doses, with the mechanical and cold allodynia measured $30 \mathrm{~min}$ following each dose. The effects were measured in each hind paw once for each dose. The treatments were delivered at a volume of $5 \mu \mathrm{l} / \mathrm{g}$ of weight, and the s.c. injections delivered into the scruff of the neck, the left flank and the right flank in sequence to avoid continuous injections into the same area. SalA and the analogues $\beta$-THP SalB, 16Ethynyl SalA and 16-Bromo SalA were measured the following doses: 0.3, 0.6, 1.0, 2.0, 5.0 and 10 $\mathrm{mg} / \mathrm{kg}$. The traditional KOPr agonist U50,488 and morphine were measured at the following doses: $0.5,1.0,2.0,5.0,10$ and $20 \mathrm{mg} / \mathrm{kg}$. Non-linear regression analysis was used to determine potency $\left(E D_{50}\right)$ with the data compared to morphine.

\subsubsection{Grimace scale photos}

During the first experimental replicate of the acute dose-response procedure, the mice treated with paclitaxel were closely monitored as this was the first instance of using this procedure in the Kivell laboratory. On days $0,4,7,11$ and 15 , the mice that received paclitaxel were placed in the behavioural observation platform and observed for $5 \mathrm{~min}$ each, with behavioural observations related to spontaneous pain were recorded (Langford et al., 2010). The scale judges the facial features of the animals for orbital tightening, nose and cheek bulge, ear position and whisker changes assigning a score of either 'not present', 'moderate' or 'severe' (Figure 1.6). Images were taken to represent the behaviours observed. The grimace scale was not measured in any further experimental replicates as spontaneous pain in the mice was deemed to either be not present or at very low levels. 


\subsubsection{Kappa opioid receptor antagonist experiment}

To understand the involvement of the KOPr in the anti-allodynic effects of the novel agonists, the KOPr antagonist nor-BNI was used. Nor-BNI is a long-acting KOPr antagonist (up to 28 days) which has transient MOPr antagonism for up to 4 hours after administration (Munro et al., 2012). To selectively antagonise the KOPr, nor-BNI was administered s.c. 24 hours before the dose-response procedure with 16-Ethynyl SalA and 16-Bromo SalA. For this procedure, 3 male and 3 female mice were used per treatment group.

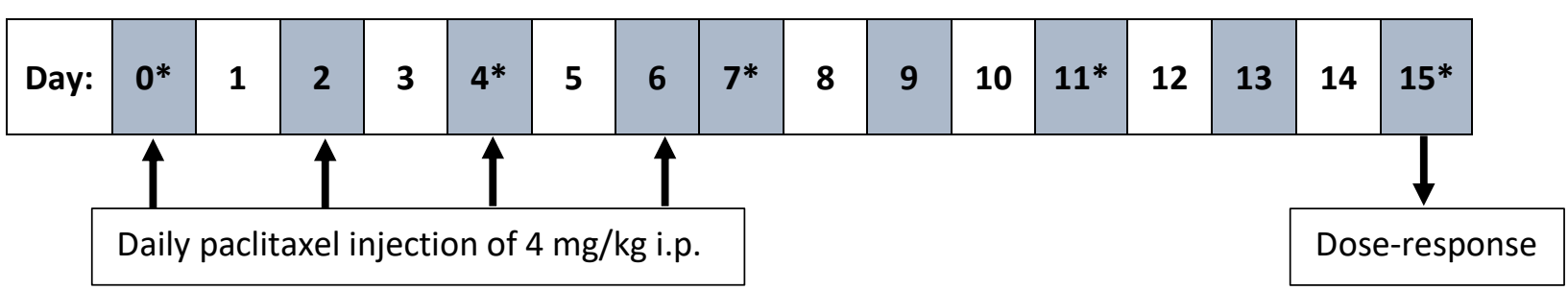

Figure 2.2: Experimental design for the acute dose-response procedure in paclitaxel-treated mice

Schematic showing the experimental design for the acute dose-response experiment. Animals received $4 \mathrm{mg} / \mathrm{kg}$ i.p. of paclitaxel on days $0,2,4$ and 6 . The shaded days indicate when animals were assessed for mechanical and cold allodynia. On days where allodynia measurements and paclitaxel administration both occurred, measurements were always taken prior to injections. The doseresponse experiments were carried out on day 15, after the baseline mechanical and cold allodynia measurements. * indicates days during the first experimental replicate that images were taken of the paclitaxel-treated mice to analyse on the grimace scale. 


\subsubsection{Chronic administration of treatment}

The efficacy and tolerance effects of chronic administration of the KOPr agonists were measured in C57BL/6J male and female mice with established paclitaxel-induced neuropathic pain. Two different treatment regimens were tested, with the initial dose beginning on either day 7 or day 16 following the first treatment of paclitaxel (for comparison of experimental design see Figure 2.3).

\subsubsection{Drug preparation}

The drugs for each of the chronic administration experiments were made up in stock solutions and used for a maximum of 7 days before a fresh solution was prepared. The stock solution contained the DMSO and Tween-80 portion of the vehicle solution. Every day an aliquot of the stock solution was taken and physiological saline added to create the final drug solution in a 2:1:7 vehicle containing DMSO:Tween-80:saline. The stock solutions made up in the same volume and blinded by another student in the laboratory. The drug treatments were not decoded until after the conclusion of the experiment.

In the early initiation of treatment on day 7 procedure, the doses were based on the $\mathrm{ED}_{60}$ values from the dose-response experiment. This dose was selected to better reflect a clinical setting where drugs are administered at lower doses and are titrated higher when required. However, following the results of the early initiation experiment, the higher $\mathrm{ED}_{80}$ dose was administered in the initiation of treatment on day 16 experiments to improve the potency of the antinociceptive effects and to produce tolerance effects in the time frame measured.

\subsubsection{Early initiation of treatment on day 7}

To test the effect of chronic treatment on early stages of paclitaxel-induced neuropathic pain, initial treatments began on day 7 (Figure 2.3A). The doses used were the $\mathrm{ED}_{60}$ value of the mechanical allodynia dose-response results for the combined male and female mice. The treatments were as follows: 16-Ethynyl SalA, $1.3 \mathrm{mg} / \mathrm{kg}$; 16-Bromo SalA, $1.9 \mathrm{mg} / \mathrm{kg}$; U50,488, $3.2 \mathrm{mg} / \mathrm{kg}$; morphine, 4.3 $\mathrm{mg} / \mathrm{kg}$; and vehicle (2:1:7 ratio containing DMSO:Tween-80:saline). Animals were given a daily i.p. injection starting on day 7. On all the odd-numbered days the treatment was given 30 min prior to mechanical and cold allodynia testing. On the even-numbered days, the mechanical and cold allodynia testing occurred prior to the treatment to understand the development of the paclitaxel-induced pain symptoms without treatment. The experimenter was blinded to the treatments each animal received. The experiment continued up to day 24 when the animals were euthanised. 


\subsubsection{Initiation of treatment on day 16}

To test the effect of the treatments on the stable paclitaxel-induced neuropathic pain, initial treatments began on day 16 (Figure 2.3B). Following the measurements on day 15, animals were assigned to treatment groups to ensure an equivalent average mechanical allodynia score across all groups, with care taken to reduce the number of animals from the same cage (and therefore the same litter) receiving the same treatment. The experimenter was blinded to the treatments each animal received. The doses used were based on the $\mathrm{ED}_{80}$ value obtained from the mechanical allodynia doseresponse results. Animals were given daily i.p. injections starting on day 16 . The treatments were as follows: 16-Ethynyl SalA, 3 mg/kg; 16-Bromo SalA, 4 mg/kg; U50,488, 10 mg/kg; morphine, 10 mg/kg; and vehicle (2:1:7 ratio containing DMSO:Tween-80:saline). On all the even numbered days the treatment was given $30 \mathrm{~min}$ prior to mechanical and cold allodynia testing. On the odd numbered days, the mechanical and cold allodynia testing occurred prior to the treatment. Treatments continued up to day 38 , with the animals euthanised on day 39 and the sciatic nerve, spinal cord and DRG tissue collected.

\subsubsection{Tissue collection}

Animals were euthanised by $\mathrm{CO}_{2}$ asphyxiation and sprayed with $70 \%$ ethanol to reduce the fur from contaminating the dissection samples. An incision was made into the peritoneal cavity and the portal vein under the liver was cut. The diaphragm was removed to expose the heart and the animals were perfused with $20 \mathrm{~mL}$ of $1 \times$ PBS using a 23 gauge needle. The animals were further perfused with 10 $\mathrm{mL} 4 \%$ formaldehyde solution (in PBS, Appendix A). The sciatic nerve was isolated by making an incision along the mid-thigh and the skin retracted. The posterior thigh muscles were split to expose the sciatic nerve. The nerve was lifted using forceps and each end of the nerve cut. The right sciatic nerve was used for immunohistochemistry and the left sciatic nerve was used for electron microscopy. The mice were decapitated and the spinal column cut at the sacral region. The spinal cord was removed using hydraulic extrusion, where a 19 gauge needle was inserted into the spinal column at the distal end and flushed out into a petri dish using $10 \mathrm{~mL}$ of $1 \times$ PBS. The DRG was then dissected by isolating the lumbar spinal column and identifying the T13 vertebra from the attachment of the most distal costae. The L1 vertebra and DRG were identified as the next proximal after T13. The lumbar spinal column was split in half longitudinally on the midline and placed under a dissecting microscope medial side facing up. The meninges were peeled back and removed with forceps and individual lumbar DRG removed with care taken to remove the axons. 
A

\begin{tabular}{|l|l|l|l|l|l|l|l|l|l|}
\hline Day: & 0 & 1 & 2 & 3 & 4 & 5 & 6 & $\begin{array}{c}\text { Days 7-23: daily administration of KOPr or MOPr agonist or vehicle } \\
\text { Odd numbered days: treatment } 30 \text { min before measurements } \\
\text { Even numbered days: measurements before treatment }\end{array}$ & $\begin{array}{l}24 \\
\text { Cull day }\end{array}$ \\
\hline
\end{tabular}

B

\begin{tabular}{|c|c|c|c|c|c|c|c|c|c|c|c|c|c|c|c|c|c|c|}
\hline Day: & 0 & 1 & 2 & 3 & 4 & 5 & 6 & 7 & 8 & 9 & 10 & 11 & 12 & 13 & 14 & 15 & $\begin{array}{l}\text { Days 16-38: daily administration of KOPr, MOPr agonist or vehicle } \\
\text { Even numbered days: treatment } 30 \text { min before measurements } \\
\text { Odd numbered days: measurements before treatment }\end{array}$ & 39 \\
\hline Dail & & & & & & & & & & & & & & & & & & Cull da \\
\hline
\end{tabular}

Figure 2.3: Experimental design for the chronic treatment of the KOPr agonists in mice with established paclitaxel-induced neuropathic pain

Schematic showing the experimental plan for the chronic treatment with either KOPr agonist, morphine or vehicle in mice with paclitaxel-induced neuropathic pain. The shaded days indicate when animals were assessed for mechanical and cold allodynia. On days where allodynia measurements and paclitaxel administration both occurred, measurements were always taken prior to injections. (A) Early initiation of treatment had the administration of the opioid agonists or vehicle beginning on day 7 and finishing on day 24. (B) The second treatment regime had the Initiation of treatment on day 16 and the cull day on day 39 where sciatic nerve, lumbar spinal cord and dorsal root ganglion tissue were collected. 


\subsection{Transmission electron microscopy}

\subsubsection{Tissue collection and processing}

Sciatic nerve tissue samples were collected as previously described (Section 2.6.5.4) for analysis with transmission electron microscopy (TEM). The samples used were from the second replicate of the chronic administration of the opioid treatments which begun on day 16 . The male mice that received the vehicle/vehicle, vehicle/paclitaxel or U50,488/paclitaxel treatment were used ( $n=3 /$ treatment group). The samples were fixed for up to 2 hours in piperazine-1,4-bis(2-ethanesulfonic acid) (PIPES) buffer (0.1 M PIPES, $1 \mathrm{~N} \mathrm{NaOH}, \mathrm{pH}$ 7.4, see Appendix A) containing 2.5\% glutaraldehyde (EM grade, distillation purified, ProSciTech, Kirwan, Australia). The glutaraldehyde was added to the PIPES buffer immediately prior to use to reduce polymerisation.

\subsubsection{Staining and imaging}

The staining and imaging were carried out by Dr St John Wakefield at the Department of Pathology and Molecular Medicine at the University of Otago, Wellington campus. Samples were processed in $1 \%$ osmium tetroxide in PIPES buffer for 1 hour, then dehydrated through an ethanol series $(50-100 \%)$ and embedded in Procure 812 epoxy resin (Proscitech, Australia). Thin sections were cut on an Ultracut T ultramicrotome (Leica Microsystems) and stained with a saturated alcoholic solution of uranyl acetate for $15 \mathrm{~min}$, followed by Reynolds lead citrate for $5 \mathrm{~min}$. Images were captured on a Philips CM-100 transmission electron microscope operated at $80 \mathrm{kV}$. Images were taken of 20 fields of view at $2500 x$ magnification. The images received from $\mathrm{Dr}$ Wakefield were coded to allow for blind analysis of the g-ratio and atypical mitochondria counts.

\subsubsection{Calculation of g-ratios}

The g-ratio gives a measure of the myelin relative to the size of the axon. G-ratios were calculated for individual myelinated axons in the sciatic nerve samples using a plugin (available online at http://gatio.efil.de/) for the ImageJ software (version 1.52b, USA National Institutes of Health, Bethesda, MD, USA). The semi-automated plugin was used to analyse transverse nerve sections by randomly selecting a target fibre, which the user manually draws around the outer border of the myelin sheath and subsequently draws the outside of the axon (Figure 2.4). The plugin automatically calculates the g-ratio (axon diameter/outer myelin border diameter). The 20 images for each animal were used to count 100 random selected myelinated axons. Only axons which were in full view within the image were included. The average g-ratio was calculated for each animal. 


\subsubsection{Atypical mitochondria counts}

The percentage of atypical mitochondria were also counted in the images produced by electron microscopy. All of the mitochondria present in 100 myelinated axons (between 301-362 individual mitochondria/mouse) were counted and either categorised as typical or atypical. Swollen, enlarged or vacuolated mitochondria were determined to be atypical (Figure 2.4, based on methodology in Chen et al., 2017a).
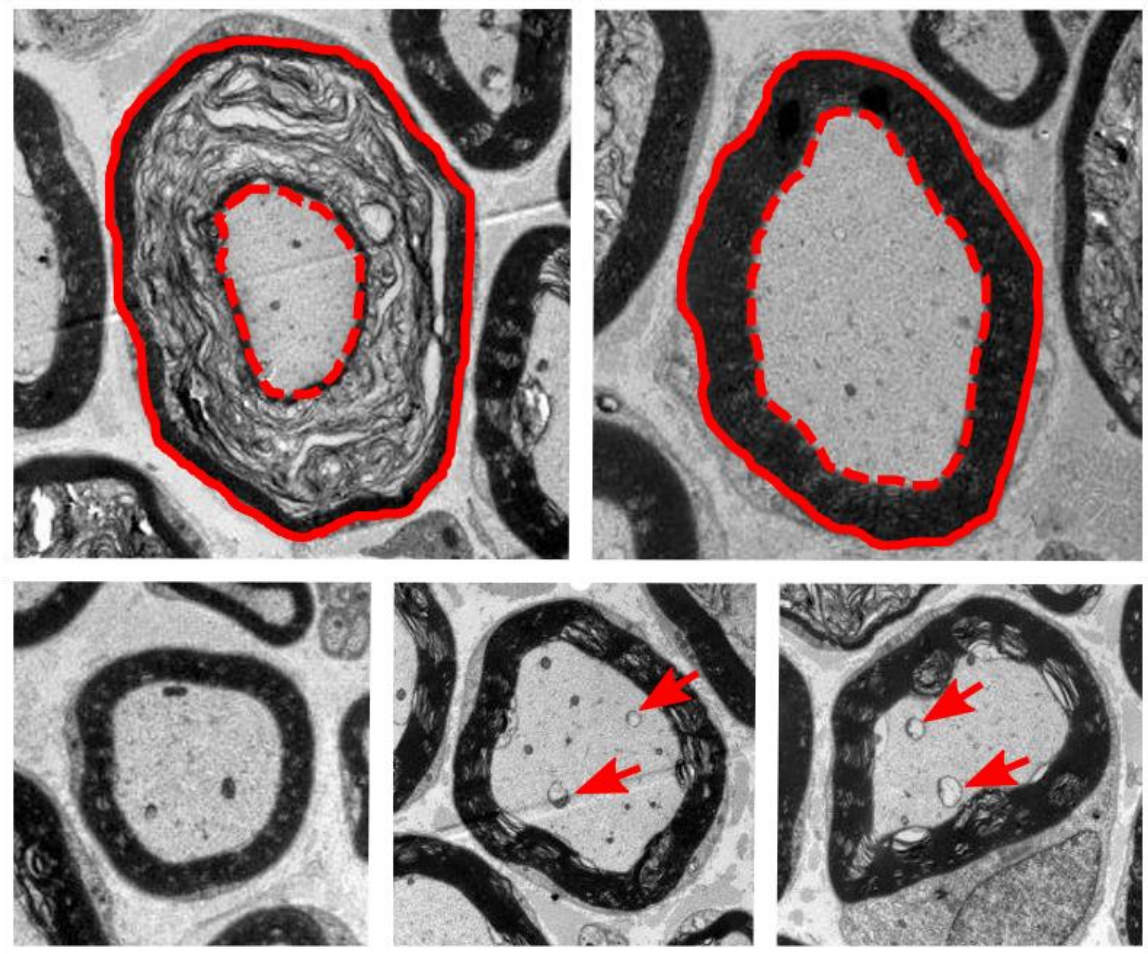

Figure 2.4: Images showing how the g-ratio and atypical mitochondria were measured

Transmission electron microscopy images of the sciatic nerve. The g-ratio was calculated using a plugin for ImageJ that calculates the ratio of the axon diameter (red dashed line) to the myelin diameter (red solid line). Swollen and vacuolated atypical mitochondria are shown with red arrows. Examples of typical mitochondria are seen in the first panel. 


\subsection{Statistical Analysis}

GraphPad Prism (version 7.03, GraphPad Software, La Jolla, CA, USA) and SPSS Statistics (version 25, IBM, Armonk, NY, USA) were used to determine statistical significance. All graphs were prepared using GraphPad Prism. Values represented as the mean \pm standard error of the mean (SEM) and were considered significant when $p<0.05$. The data sets were tested for normality using the D'Agostino and Pearson omnibus normality test unless otherwise stated. Data sets with $p<0.05$ in the normality test were analysed with a non-parametric test, the Mann-Whitney test instead as Gaussian distribution could not be assumed. Unpaired t-tests were used to compare between two groups. Comparison of multiple treatment data was analysed using one-way analysis of variance (ANOVA) followed by Bonferroni post-tests. Comparisons of multiple effects were analysed using two-way ANOVA followed by Bonferroni post-tests. Two-way repeated measures ANOVA was used when one variable was measured over time. Post-hoc testing was only performed if the interaction result from the ANOVA was significant. The $p$ values reported for the post-tests were adjusted for multiple families of comparisons.

\subsubsection{Dose-response analysis}

To understand the relationship between increasing the dose and the effect on tail withdrawal or mechanical and cold allodynia were analysed by creating a dose-response curve in GraphPad Prism. The data underwent a logarithmic (base 10) transformation, before a non-linear regression was carried out. The four parameter variable slope with least squares ordinary fit was used to fit the curve to the data sets. For the tail withdrawal experiment, on constraints were set. For mechanical allodynia, the top constraint was set no more than 9.5. For cold allodynia, the bottom constraint was set at no less than 0 . The extra sum-of-squares $F$ test with the bottom, top, $\log E D_{50}$ and hillslope parameters was used to compare the treatment curves, with the null hypothesis that one curve fits all data sets. If the results showed a different curve fit each data set, then the $E D_{50}$ and $E_{\max }$ values were compared with one-way ANOVA analysis. The $\log E D_{50} \pm$ SEM were used for comparison, as the values were calculated on a logarithmic scale and the $\mathrm{ED}_{50}$ values would, therefore, have an asymmetrical SEM. The $E_{\max }$ values were calculated as a percentage of the control treatment.

\subsubsection{Three-way interactions}

In the paclitaxel-induced neuropathic pain model, the effects of treatment, sex and time on either the mechanical withdrawal thresholds or the cold reaction times were analysed using SPSS Statistics. This analysis was carried out with a three-way repeated measures mixed ANOVA, with treatment and sex set as between-subjects variables and time as the within-subjects variable. The normality of the data 
was assessed with the Shapiro-Wilk test using the standardised residuals. The homogeneity of variances was measured using the Levene's test of equality of error variances. If the data was nonnormal and had unequal variances at some time points, then the data was transformed. The box plots were inspected to ascertain whether the data was negatively or positively skewed. Slightly negativelyskewed data was corrected with square root transformation. Moderately negatively-skewed data was corrected with logarithmic transformation. Slightly positively-skewed data was corrected with a reflect and square root transformation. The sphericity of the data was tested using Mauchly's test. If the $p<0.05$, the assumption of sphericity was violated and the Greenhouse-Geisser correction was applied. If there was a significant three-way interaction of treatment, sex and time, then the two-way interactions of treatment and sex were assessed at each time point. If there was no significant threeway interaction, the simple main effects of any significant two-way interactions were assessed. The Bonferroni correction applied for multiple families of comparisons and the adjusted $\alpha$ level reported. 


\section{Chapter 3: Investigating the antinociceptive and anti- inflammatory effects of DHEA}

\subsection{Introduction}

\subsection{1 $\mathrm{N}$-docosahexaenoyl ethanolamine (DHEA)}

DHEA (also known as synaptamide) was originally discovered in rat brain tissue (Sugiura et al., 1996) and bovine retina (Bisogno et al., 1999). Since being discovered, DHEA has also been found in human plasma in both free and esterified states, at similar levels as anandamide (Balvers et al., 2013). DHEA can be synthesised from DHA-containing phosphatidylethanolamine within cell membranes of the brain and is released on demand (reviewed in Kuda, 2017). In piglets, consuming a diet high in n-3 PUFAs led to a large increase in the amount of DHEA (9-10 fold) and EPEA ( 5 fold) in the brain tissue (Berger et al., 2001). Since this finding, a further study confirmed that mice fed a DHA-rich fish oil diet had increased DHEA concentrations in both brain tissue and plasma, however, concentrations of PEA, OEA and EPEA did not change, and there was a decrease in anandamide concentration in the brain tissue (Wood et al., 2010). Similar results were found in human plasma, with fish oil dietary supplementation leading to an increase in DHEA, with a decrease seen in OEA, PEA and anandamide concentrations (Balvers et al., 2013).

DHEA is similar in structure to the most studied NAE compound, anandamide, which has antiinflammatory effects mediated through the cannabinoid receptors (Jaggar et al., 1998). DHEA also binds relatively weakly to both cannabinoid receptors, with greater affinity to the CB1 receptor (Brown et al., 2010). However, the affinity of DHEA at the CB1 receptor is approximately 8-times less than anandamide (Sheskin et al., 1997) (Table 3.1). DHEA is degraded by FAAH, the same enzyme responsible for the breakdown of anandamide, however, evidence suggests DHEA is a worse substrate than anandamide (Balvers et al., 2010; Sonti et al., 2017). The binding affinity of DHEA was measured in the presence of phenylmethylsulfonyl fluoride, a non-selective protease inhibitor, which inhibits the action of $F A A H$, and these conditions lead to a 5-fold increase in the binding affinity of DHEA for the CB1 receptor (Brown et al., 2010) (Table 3.1). DHEA has also been shown to have effects mediated through the ligand-dependent transcription factors PPAR- $\alpha$ (Meijerink et al., 2015) and PPAR- $\gamma$ (Meijerink et al., 2015), which have well-documented effects in lipid metabolism, as well as essential roles in the expression of inflammatory cytokines and differentiation of immune cells (Meijerink et al., 2011). 


\begin{tabular}{|c|c|c|c|}
\hline & Anandamide & DHEA & EPEA \\
\hline \multicolumn{4}{|l|}{ Structure } \\
\hline Binding affinity (1) & $\mathrm{K}_{\mathrm{i}}=39.2 \pm 5.7 \mathrm{nM}$ & $K_{i}=324.1 \pm 9.2 n M$ & $\mathrm{~K}_{\mathrm{i}}=162.3 \pm 13.6 \mathrm{nM}$ \\
\hline \multirow{2}{*}{ Binding affinity (2) } & CB1 & $\mathrm{K}_{\mathrm{i}}=633(292$ to 1372$) \mathrm{nM}$ & $\mathrm{K}_{\mathrm{i}}=2000$ (989 to 4045$) \mathrm{nM}$ \\
\hline & $\mathrm{CB} 2$ & $\mathrm{~K}_{\mathrm{i}}=3843(2995$ to 4932$) \mathrm{nM}$ & $\mathrm{K}_{\mathrm{i}}=9027$ (6451 to 12630$) \mathrm{nM}$ \\
\hline \multirow{2}{*}{$\begin{array}{l}\text { Binding affinity with } \\
\text { PMSF protease } \\
\text { inhibitor (2) }\end{array}$} & CB1 & $\mathrm{K}_{\mathrm{i}}=124$ (94.8 to 162$) \mathrm{nM}$ & $\mathrm{K}_{\mathrm{i}}=55$ (37.5 to 80.9) nM \\
\hline & CB2 & $\mathrm{K}_{\mathrm{i}}=2441$ (1320 to 3473 ) nM & $\mathrm{K}_{\mathrm{i}}=3440$ (2324 to 5092$) \mathrm{nM}$ \\
\hline \multirow{2}{*}{$\begin{array}{l}\text { Potency and binding } \\
\text { efficiency (2) }\end{array}$} & CB1 & $\begin{array}{c}\mathrm{EC}_{50}=50(2.74 \text { to } 911) \mathrm{nM} \\
\mathrm{E}_{\max }=26.5(16.3 \text { and } 36.8) \%\end{array}$ & $\begin{array}{c}\left.\mathrm{EC}_{50}=1361 \text { (223 to } 8288\right) \mathrm{nM} \\
\mathrm{E}_{\max }=40.6(24.7 \text { to } 56.6) \%\end{array}$ \\
\hline & $\mathrm{CB} 2$ & $\begin{array}{c}E C_{50}=42(2.6 \text { to } 676) n M \\
E_{\max }=23.1(15.7 \text { to } 30.5) \%\end{array}$ & $\begin{array}{c}\mathrm{EC}_{50}=397.1(22.2 \text { to } 7085) \mathrm{nM} \\
\mathrm{E}_{\max }=22.9(13.4 \text { to } 32.5) \%\end{array}$ \\
\hline
\end{tabular}

\section{Table 3.1: Comparison of the ethanolamines in binding affinity and selectivity for the cannabinoid receptors}

(1) Binding affinity $\left(\mathrm{K}_{\mathrm{i}}\right)$ in synaptosomal brain preparation from whole rat brain with $\left[{ }^{3} \mathrm{H}\right] \mathrm{HU}-243$ as the radioligand. Data expressed as mean \pm SEM (Sheskin et al., 1997)

(2) CB1 receptor measured in MF1 mouse whole brain membranes and the CB2 receptor in CHO-hCB2 cell membranes. Binding affinity ( $\mathrm{K}_{\mathrm{i}}$ ) calculated for displacement of $\left[{ }^{3} \mathrm{H}\right] \mathrm{CP} 55940$ in the presence and absence of the non-selective protease inhibitor, phenylmethylsulfonyl fluoride (PMSF, $\left.100 \mu \mathrm{MM}\right)$. Potency $\left(E C_{50}\right)$ and binding efficiency $\left(E_{\text {max }}\right)$ calculated for an increase in stimulation of $\left[{ }^{35} \mathrm{~S}\right] \mathrm{GTP} \gamma \mathrm{S}$ binding from basal levels. Data expressed as mean $(95 \%$ confidence limits) (Brown et al., 2010) 


\subsubsection{Anti-inflammatory effects of DHEA}

DHEA has potent immune-modulatory effects in vitro, however, the mechanism of action is unclear, with different studies showing the effects to be both cannabinoid receptor-dependent and independent. In cultured mouse 3T3-L1 adipocytes, administration of DHEA (10 nM) decreased the levels of the LPS-stimulated inflammatory cytokines, IL-6 and monocyte chemotactic protein-1 (MCP1) (Balvers et al., 2010). The IL-6 release was attenuated by a combination of both CB2 receptor and PPAR- $\gamma$ antagonists, however, the antagonists had no effect on the MCP-1 levels. The parent compound DHA did not have any effect on IL-6 or MCP-1 levels, showing that the effects of DHEA were not due to the conversion into DHA. In the murine RAW264.7 macrophage cell line and in murine peritoneal macrophages, DHEA dose-dependently (0.1-10 $\mu \mathrm{M})$ reduced LPS-stimulated inflammatory nitric oxide release and suppressed the production of MCP-1, with more potent effects than anandamide, OEA and DHA (Meijerink et al., 2011). A further study found that the effect of DHEA did not involve the CB1 and CB2 receptors or PPAR- $y$, however, DHEA did decrease the levels of prostaglandins and thromboxanes in the RAW264.7 macrophages, which are COX-2 mediated eicosanoids, indicating that the mode of action of DHEA may be related to the COX-2 enzyme (Meijerink et al., 2015).

\subsubsection{Other effects of DHEA}

Evidence from in vitro studies show that DHEA has antiproliferative and cell growth inhibitory effects in prostate cancer cell lines, which were not blocked by CB1 or CB2 receptor antagonists (Brown et al., 2010). In contrast, the anti-proliferative effects of EPEA in one cell line was reduced by selective CB1 and CB2 receptor antagonists (Brown et al., 2010). However, the concentrations required for the antiproliferative effects are likely physiologically-impossible, so the clinical effects remain unclear. Further studies have shown that DHEA stimulates neurite growth and synaptogenesis in developing hippocampal neurons independently of the cannabinoid receptors (Kim et al., 2011a; Kim et al., 2011b) and promotes axon growth in cultured cortical neurons (Kharebava et al., 2015). 


\subsection{Aims and hypotheses}

DHEA has previously been shown to have immune-modulatory effects in vitro, however, the in vivo effects have not been tested. The overall aim of this study was to investigate the in vivo effects of DHEA in mice, primarily using the intraplantar formaldehyde model, which gives a biphasic effect of both nociceptive pain and inflammatory pain. It was hypothesised that DHEA would have a significant antinociceptive effect in the inflammatory phase of pain, similar to the parent compound DHA and other NAE class lipids.

The specific aims of the study were to:

1. Determine whether DHEA had an antinociceptive effect in nociceptive and inflammatory pain using the intraplantar $2 \%$ formaldehyde assay. To further understand the involvement of the cannabinoid receptors, the effects of DHEA were tested following pre-treatment with the CB1 receptor antagonist AM251 and the CB2 receptor antagonist AM630.

2. Investigate the effects of DHEA on formaldehyde-induced paw oedema and local inflammation using H\&E staining to identify neutrophils in the swollen footpad tissue.

3. Assess the duration of action of the antinociceptive effect of DHEA using the warm water tail withdrawal assay.

4. Evaluate the side effect profile of DHEA using the accelerating rotarod performance assay and the change in core body temperature. 


\subsection{Results}

\subsubsection{The effect of intraperitoneal injection of DHEA in the intraplantar $2 \%$ formaldehyde test}

The intraplantar formaldehyde test produced two phases of pain, the initial nociceptive pain lasting up to $15 \mathrm{~min}$, and the secondary inflammatory phase of pain lasting from 20 to $60 \mathrm{~min}$. The administration of DHEA ( $2 \mathrm{mg} / \mathrm{kg}$ i.p.) in the intraplantar formaldehyde model showed a significant interaction of treatment $x$ time $\left[F_{(60,408)}=3.918, p<0.0001\right]$ (Two-way repeated measures ANOVA; Figure 3.1A). Bonferroni post-tests showed that DHEA ( $2 \mathrm{mg} / \mathrm{kg}$ ) significantly reduced the pain score in the inflammatory phase of pain at $25(p=0.0083), 30(p<0.0001)$ and $35 \mathrm{~min}(p<0.0001)$ when compared to the vehicle/formaldehyde-treated positive control (Figure 3.1A). Further animals were treated with DHEA following a pre-treatment with either the CB1 receptor antagonist AM251, the CB2 receptor antagonist AM630 or a combination of both antagonists, however, no antagonist groups were significantly different to the DHEA treatment group at any time points $(p>0.05)$.

The area under the curve (AUC) was calculated for both phase I nociceptive pain (0-15 $\mathrm{min}$ ) and phase II inflammatory pain (20-60 min). One-way ANOVA showed a significant effect of treatment for both phase I $\left[\mathrm{F}_{(5,34)}=50.10, p<0.0001\right]$ (Figure 3.1B) and phase II pain $\left[F_{(5,34)}=78.78, p<0.0001\right]$ (Figure 3.1C). Bonferroni post-tests showed that DHEA $(2 \mathrm{mg} / \mathrm{kg}$ ) significantly reduced the phase I pain ( $p=$ 0.0085; Figure 3.1B) and phase II inflammatory pain ( $p<0.0001$; Figure $3.1 \mathrm{C})$. When compared to the DHEA treated group, the cannabinoid antagonists AM251 or AM630 or AM251 and AM630 combined were not significantly different in either phase I $(p=0.6935, p>0.9999$ and $p>0.9999$ respectively; Figure 3.1B) or in phase II ( $p=0.4328, p>0.9999$ and $p>0.9999$ respectively; Figure 3.1C). Therefore, the results show that i.p. administration of DHEA leads to a reduction in the overall nociceptive pain and inflammatory pain, which was not mediated through the cannabinoid receptor system. 

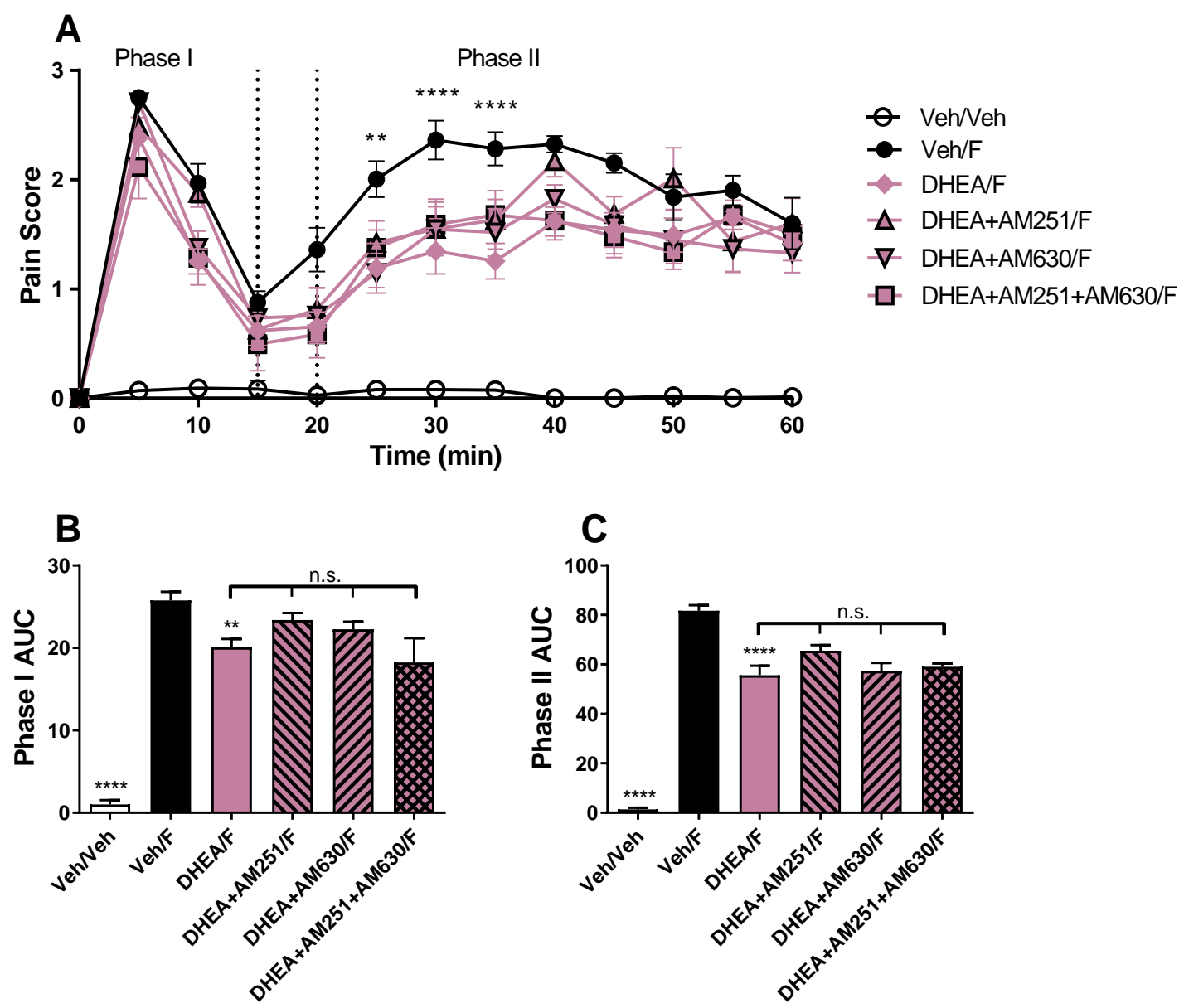

Figure 3.1: DHEA has antinociceptive and anti-inflammatory effects via intraperitoneal injection in the intraplantar $2 \%$ formaldehyde test

(A) Time course of pain behaviour following intraplantar $2 \%$ formaldehyde $(F)$ injection into the right hind paw. DHEA $(2 \mathrm{mg} / \mathrm{kg}$ ) treatment showed a significant reduction in pain compared to the vehicle/formaldehyde-treated positive control. Antagonist treatments did not produce a significant difference at any time point compared to the DHEA treatment group. Two-way repeated measures ANOVA with Bonferroni post-tests. (B-C) Area under the curve (AUC) was calculated for phase I nociceptive pain (0-15 min, B) and phase II inflammatory pain (20-60 min, C). DHEA showed a significant difference in phase I and II pain compared to the vehicle/formaldehyde-treated positive control. The antagonist treatments did not show a significant difference compared to the DHEA treatment group. One-way ANOVA with Bonferroni post-tests. ${ }^{* *} p<0.01,{ }^{* * *} p<0.001,{ }^{* * * *} p<$ 0.0001 compared to the vehicle/formaldehyde treated control. n.s. = not significant. Values presented as mean $\pm S E M, n=7-11$ per group and $n=5$ for all antagonist treatment groups. 


\subsubsection{The effect of DHEA on paw oedema and infiltration of neutrophils into the footpad tissue following intraplantar $2 \%$ formaldehyde administration}

Following the injection of formaldehyde in the footpad, an inflammatory reaction occurs leading to an acute oedema of the footpad tissue. The percentage change in the depth of the footpad injected with $2 \%$ formaldehyde was measured to give an indication of the inflammatory reaction that had occurred, and whether DHEA dampened this effect. One-way ANOVA showed a significant effect of treatment $\left[F_{(5,34)}=19.82, p<0.0001\right]$ (Figure 3.2A). Bonferroni post-tests revealed that DHEA $(2 \mathrm{mg} / \mathrm{kg}$ ) significantly reduced $(p<0.0001)$ the level of paw oedema compared to mice only exposed to the pain stimulus with vehicle treatment. There was no significant effect in the groups treated with DHEA and cannabinoid antagonists $\operatorname{AM} 251(p=0.1843), \operatorname{AM} 630(p=0.8023)$ or with a combination of both antagonists administered together $(p=0.1221)$.

Within the first hour of the onset of inflammation, neutrophils undergo extravasation and migrate along chemical gradients to accumulate at the site of injury (Kolaczkowska \& Kubes, 2013). Neutrophil migration is associated with increased inflammatory pain and oedema (Guerrero et al., 2008). Therefore, as an indication of the inflammatory response, the migration of neutrophils into the area was measured histologically. The footpad tissue was collected at the 60 min endpoint, and the H\&E stain was used to identify and count the number of neutrophils present. One-way ANOVA showed a significant effect of treatment $\left[F_{(3,15)}=36.87, p<0.0001\right]$ (Figure 3.2B). Bonferroni post-test analysis showed a significant effect between the vehicle/formaldehyde control group and the DHEA treatment group $(p<0.0001)$. There was no significant effect when the cannabinoid receptor antagonists were administered $(p>0.9999)$. Therefore, DHEA reduced the local level of swelling and infiltration of neutrophils in the formaldehyde-mediated inflamed tissue, however, the effects of DHEA were not mediated via the cannabinoid receptors. 
A
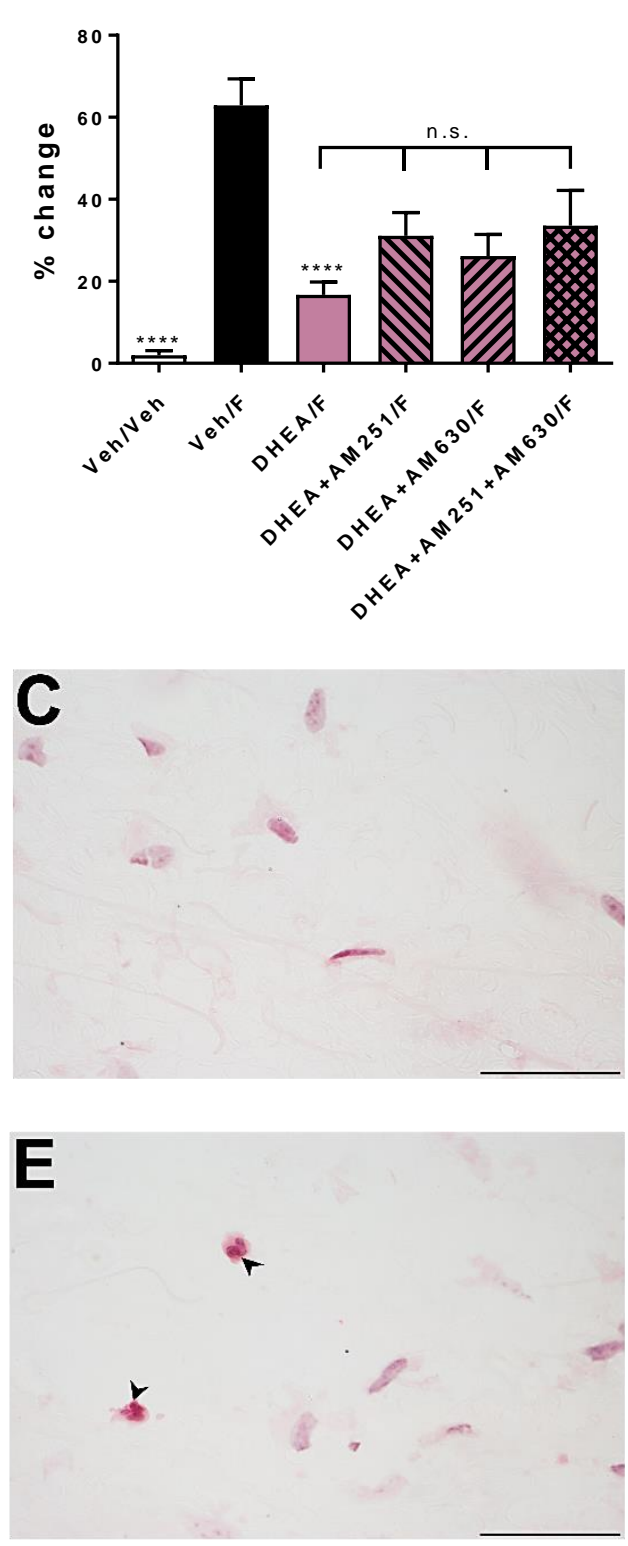

B
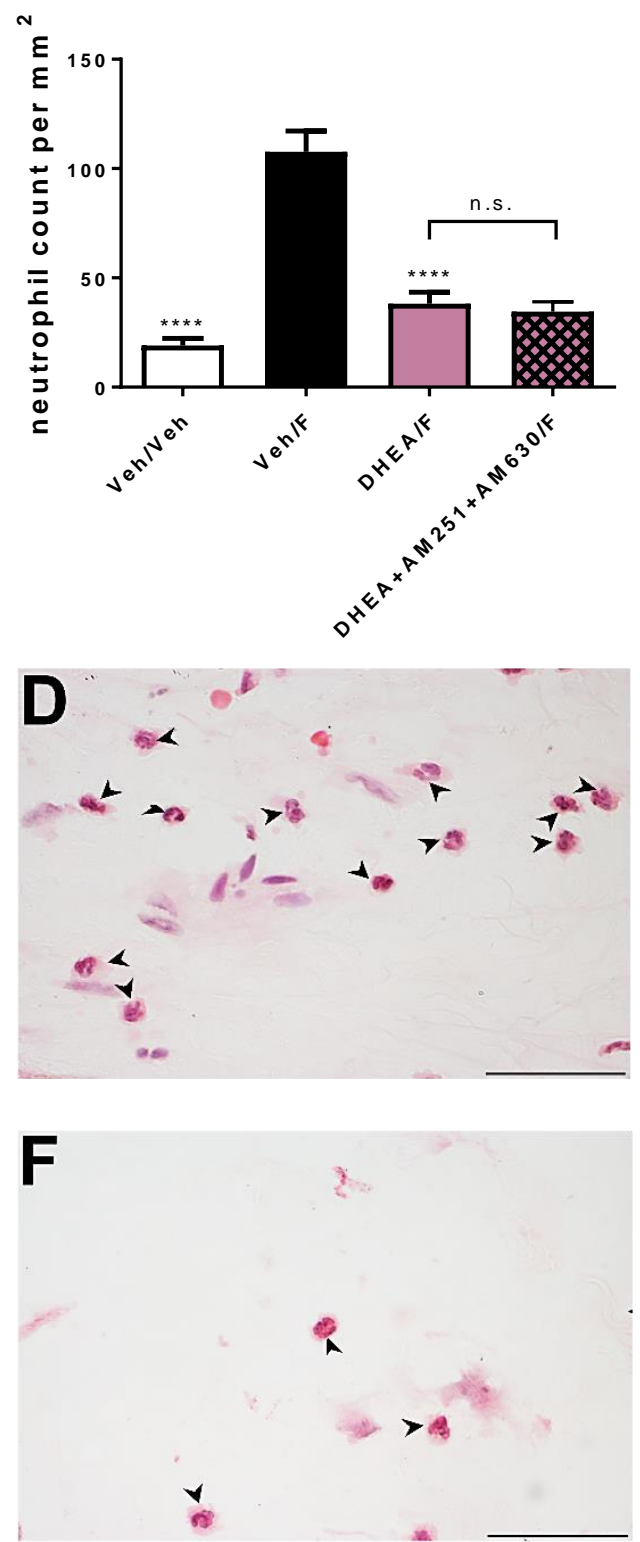

Figure 3.2: DHEA reduces paw oedema and the number of neutrophils in the footpad tissue

(A) The depth of the right hind paw was measured before the intraplantar $2 \%$ formaldehyde injection and measured again at the end of the testing period $(60 \mathrm{~min})$ and the percentage change calculated. DHEA $(2 \mathrm{mg} / \mathrm{kg})$ showed a significant reduction in paw oedema compared to the vehicle/formaldehyde-treated positive control. (B) Neutrophil numbers were counted in H\&E stained footpad tissue following the intraplantar formaldehyde test. DHEA $(2 \mathrm{mg} / \mathrm{kg})$ reduced the number of neutrophils in the inflamed tissue, which was not mediated by the cannabinoid receptors. $n=4-6$ per group. (C-F) Representative images showing neutrophil infiltration into the inflamed footpad tissue following (C) vehicle/vehicle, (D) vehicle/formaldehyde, (E) DHEA/formaldehyde and (F) DHEA/formaldehyde with AM251 and AM630 pre-treatment. Markers indicate neutrophils. One-way ANOVA with Bonferroni post-tests. $* * * * p<0.0001$ compared to the vehicle/formaldehyde treated control. $n . s .=$ not significant. $n=7-11$ per group and $n=5$ for all antagonist treatment groups. Values presented as mean \pm SEM. Scale bar $=20 \mu \mathrm{m}$. 


\subsubsection{The effect of local administration of DHEA in the intraplantar $2 \%$ formaldehyde test}

To further evaluate whether the effects of DHEA were locally mediated, the treatment was given as an intraplantar (i.pl.) injection in the same hind paw as the $2 \%$ formaldehyde injection (based on experimental procedures in Lamb et al., 2012). Two-way repeated measures ANOVA showed a significant interaction of treatment $x$ time $\left[F_{(36,300)}=2.41, p<0.0001\right]$ (Figure 3.3A). Bonferroni posttests showed DHEA ( $2 \mathrm{mg} / \mathrm{kg}$ ) significantly reduced the formaldehyde-mediated pain at $30 \mathrm{~min}(p=$ 0.0003). Using the i.pl. administration of the 2:1:7 vehicle lead to a significant level of pain compared to the i.p. administration in the previous experiment (for analysis see Appendix B). Since the vehicle/vehicle control showed a pain response, a DHEA/vehicle treatment was also administered to a group of animals. In the time course, there was a significant difference between the DHEA/vehicle treatment group and the vehicle/vehicle treatment group at $5 \mathrm{~min}(p=0.0186)$. However, no other time points showed a statistically significant effect $(p>0.05)$.

The AUC analysis revealed a significant effect of treatment for phase I $\left[F_{(3,25)}=43.66, p<0.0001\right]$ (Figure 3.3B) and phase II pain responses $\left[F_{(3,25)}=12.59, p<0.0001\right]$ (Figure 3.3C). DHEA $(2 \mathrm{mg} / \mathrm{kg}$ ) significantly reduced the level of nociceptive pain in phase I pain ( $p=0.0004$; Figure 3.3B) and inflammatory pain in phase II ( $p=0.0429$; Figure $3.3 C$ ). Since the vehicle/vehicle control showed pain in both phases, it was compared to the DHEA/vehicle group. DHEA reversed the effects of the vehicle injection in phase I pain ( $p=0.0008$; Figure 3.3B) but not phase II pain ( $p>0.9999$; Figure $3.3 \mathrm{C})$. Therefore, DHEA significantly reduced pain locally, however, DHEA did not reduce the pain level below that of the vehicle/vehicle control.

The paw oedema was measured to assess the level of inflammation in the hind paw. One-way ANOVA showed a significant effect of treatment $\left[F_{(3,24)}=3.047, p=0.0481\right]$ (Figure 3.3D). There was a significant difference between the DHEA/formaldehyde group and the vehicle/formaldehyde group ( $p$ $=0.0234)$. This indicates that DHEA is having an effect on the level of inflammation at the site of the local injection. However, there was no significant difference between DHEA/vehicle and vehicle/vehicle $(p=0.7594)$, showing that DHEA treatment could not reduce the swelling caused by the vehicle/vehicle control. 

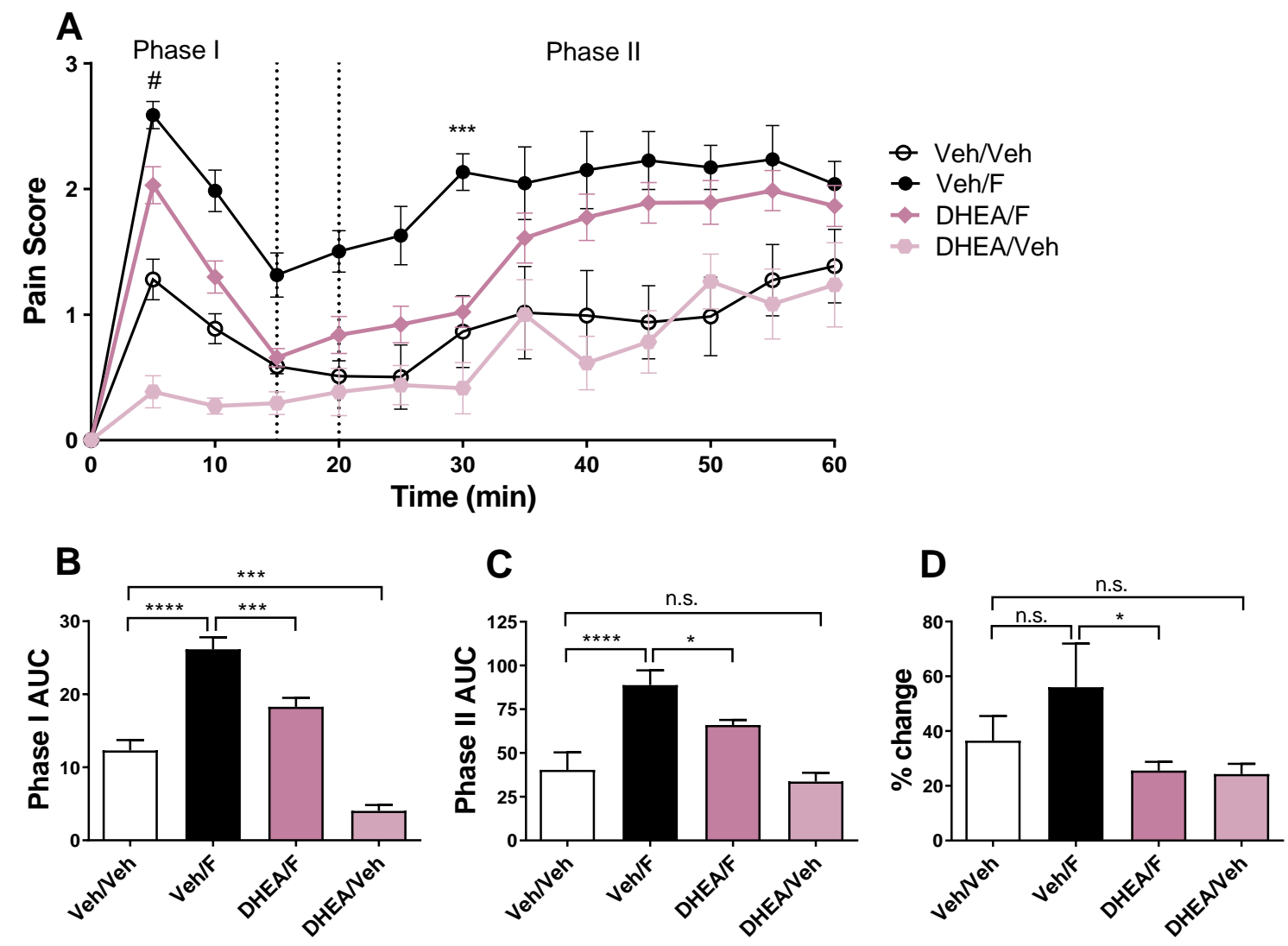

Figure 3.3: DHEA has antinociceptive and anti-inflammatory effects via local intraplantar injection in the intraplantar $2 \%$ formaldehyde test

(A) Time course of pain behaviour following intraplantar $2 \%$ formaldehyde injection into the right hind paw. DHEA $(2 \mathrm{mg} / \mathrm{kg}$ ) treatment showed a significant reduction in pain compared to the vehicle/formaldehyde-treated positive control. Antagonist treatments did not produce a significant difference at any time point compared to the DHEA treatment group. Two-way repeated measures ANOVA with Bonferroni post-tests. ${ }^{* * *} p<0.001$ for DHEA/formaldehyde group compared to the vehicle/formaldehyde treated control. ${ }^{\#} p<0.05$ for the DHEA/vehicle group compared to vehicle/vehicle treatment group. (B-C) Area under the curve (AUC) was calculated for phase I nociceptive pain (0-15 $\mathrm{min}, \mathrm{B}$ ) and phase II inflammatory pain (20-60 $\mathrm{min}, \mathrm{C})$. DHEA significantly decreased the effect of formaldehyde in phase I and II pain compared to the vehicle/formaldehydetreated positive control and decreased the effect of the vehicle in phase I pain. (D) The percentage change in the paw oedema depth revealed a significant difference between the DHEA/formaldehyde treated animals and the vehicle/formaldehyde treated animals. One-way ANOVA with Bonferroni post-tests. ${ }^{*} p<0.05,{ }^{* *} p<0.01,{ }^{* * *} p<0.001$, n.s. $=$ not significant. Values presented as mean $\pm \mathrm{SEM}$, $\mathrm{n}=6-9$ per treatment group. 


\subsubsection{The effect of local injection of DHEA on the inflammatory phase of pain}

To understand the effect of DHEA on the inflammatory phase II of pain, DHEA was administered locally following the phase I pain. The animals were given an i.pl. injection with $2 \%$ formaldehyde and after $15 \mathrm{~min}$, DHEA (2 mg/kg) was also administered via i.pl. injection. Two-way repeated measures ANOVA showed a significant interaction of treatment $x$ time $\left[F_{(16,120)}=2.152, p=0.0101\right]$ (Figure 3.4A). Control (vehicle/vehicle) mice showed a significant difference compared to the vehicle/formaldehyde control at $50 \mathrm{~min}(p=0.0050)$ and $55 \mathrm{~min}(p=0.0015)$. The DHEA/formaldehyde treatment group did not show a significant difference at any time point compared to the vehicle/formaldehyde treatment group $(p>0.05)$. AUC analysis showed a significant effect of treatment $\left[F_{(2,15)}=6.413, p=0.0097\right]$ (Figure 3.4B). Bonferroni post-tests revealed a significant difference between the negative control group (vehicle/vehicle) and the positive control group (vehicle/formaldehyde) treatments ( $p=$ 0.0083). However, there was no difference between the DHEA/formaldehyde and vehicle/formaldehyde groups $(p>0.9999)$. Paw oedema measurements were taken at the start of the experiment and at the $60 \mathrm{~min}$ time point, and the percentage change calculated. One-way ANOVA showed no significant effect of treatment in the paw oedema $\left[F_{(2,15)}=2.048, p=0.1635\right]$ (Figure 3.4C), indicating that there was the same level of swelling in both the negative and positive controls, indicating that the test is not sensitive enough. In conclusion, the positive and negative controls showed a significant difference overall in the AUC analysis, however, DHEA was not able to significantly reduce the level of pain in this experiment. 


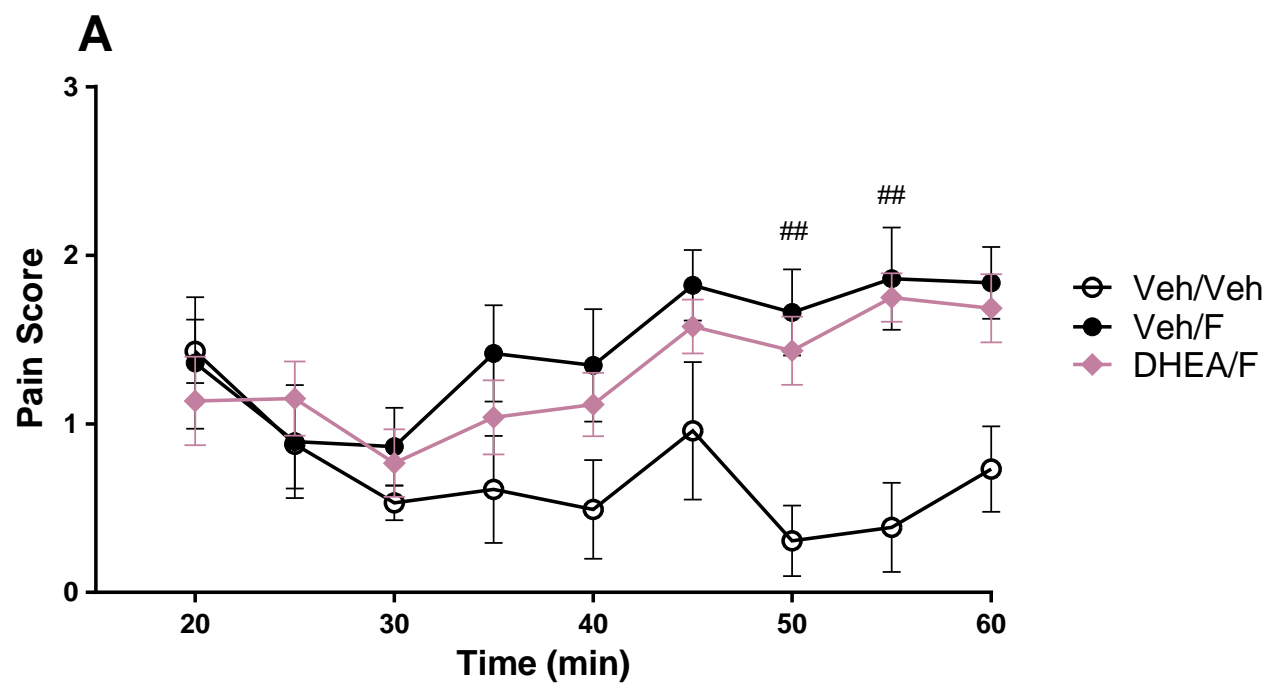

B

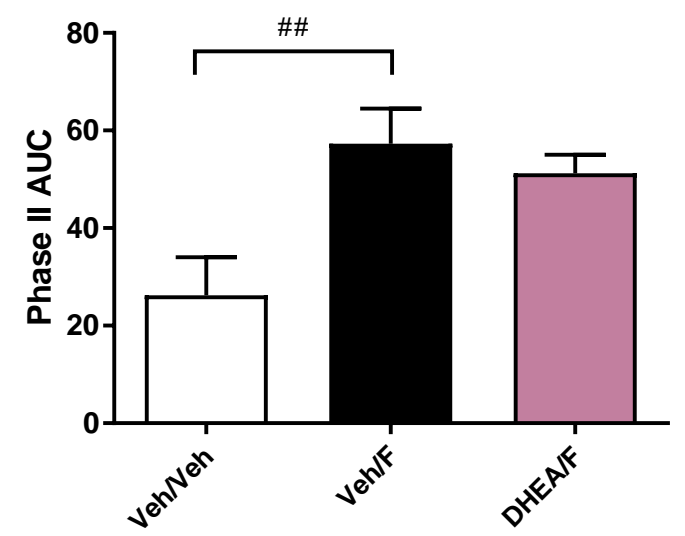

C

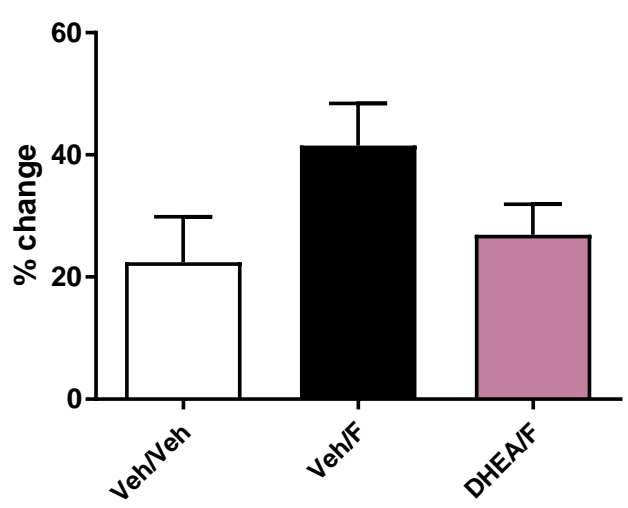

Figure 3.4: DHEA has antinociceptive and anti-inflammatory effects via local intraplantar injection in the inflammatory phase of the intraplantar $2 \%$ formaldehyde test

(A) Time course of pain behaviour following intraplantar $2 \%$ formaldehyde injection into the right hind paw. DHEA ( $2 \mathrm{mg} / \mathrm{kg}$ ) was administered at 15 min post-formaldehyde and did not show a significant difference compared to the vehicle/formaldehyde control. Two-way repeated measures ANOVA with Bonferroni post-tests. (B) Area under the curve (AUC) analysis of the pain behaviour revealed a difference between vehicle/vehicle and vehicle/formaldehyde groups but DHEA did not reverse the effects of the formaldehyde injection. One-way ANOVA with Bonferroni post-tests. (C) The percentage change in paw swelling showed no effect of treatments. One-way ANOVA with Bonferroni post-tests. ${ }^{\#} p<0.01$ for vehicle/vehicle group vs vehicle/formaldehyde group. Values presented as mean $\pm \mathrm{SEM}$, $\mathrm{n}=6$ per treatment group. 


\subsubsection{The effect of DHEA in the warm water tail withdrawal assay}

The warm water tail withdrawal assay measures the spinal reflex in reaction to a thermal stimulus (Irwin et al., 1951). Typically, this assay is used to measure the antinociceptive effects and the duration of action of the compound of interest compared to the vehicle control. The assay was used to measure the antinociceptive effect of DHEA ( $2 \mathrm{mg} / \mathrm{kg}$ ) via i.p. administration. Two-way repeated measures ANOVA showed no significant interaction of treatment and time $\left[\mathrm{F}_{(8,96)}=0.703, p=0.6881\right]$, and no main effect of treatment $\left[F_{(1,12)}=0.2303, p=0.6399\right]$ or time $\left[F_{(8,96)}=1.168, p=0.3264\right]$ (Figure 3.5), indicating that DHEA has no effect in this assay at the dose tested compared to the vehicle-treated control group.

\subsubsection{The effect of DHEA on motor coordination and core body temperature regulation}

Since DHEA has been previously shown to have activity at the cannabinoid receptor, side effect behavioural models were chosen which have relevance to activation of the cannabinoid receptors. The cannabinoid receptor system has been shown to induce motor incoordination via the CB1 receptor, with THC administration (15-30 $\mu \mathrm{g}$ intracerebellar microinfusion) leading to a reduced latency in the rotarod performance test (Dar, 2000). Anandamide has been shown to reduce locomotor activity in rats in the open field test (Bruijnzeel et al., 2016), however, the effect of anandamide on the rotarod performance test is unclear. Therefore, the rotarod performance assay was carried out to assess the level of motor coordination or sedation in the animals treated with DHEA $(2 \mathrm{mg} / \mathrm{kg}$ ) compared to the vehicle treatment over $120 \mathrm{~min}$. Two-way repeated measures ANOVA showed no significant interaction of treatment $x$ time $\left[F_{(6,60)}=16.74, p=0.5842\right]$ and no main effect of treatment $\left[F_{(1,10)}=0.0024, p=0.9621\right]$ (Figure 3.6A), indicating that DHEA does not have an effect on motor coordination at the dose tested.

Cannabinoid receptor agonists are known to cause hypothermia. Anandamide (Crawley et al., 1993; Fride \& Mechoulam, 1993; Smith et al., 1994) and THC (Varvel et al., 2005) are known to produce hypothermic effects in mice. The core body temperature of DHEA treated mice were measured to ascertain whether there were any cannabinoid receptor-dependent effects on thermoregulation. The change in core body temperature was measured using a rectal probe over a 120 min time-course. Two-way repeated measures ANOVA showed no significant interaction of treatment and time $\left[F_{(4,48)}=\right.$ $1.298, p=0.2842]$ or main effect of treatment $\left[F_{(1,12)}=1.054, p=0.3249\right]$ (Figure 3.6B), indicating that DHEA ( $2 \mathrm{mg} / \mathrm{kg}$ ) does not affect the level of core body temperature. 


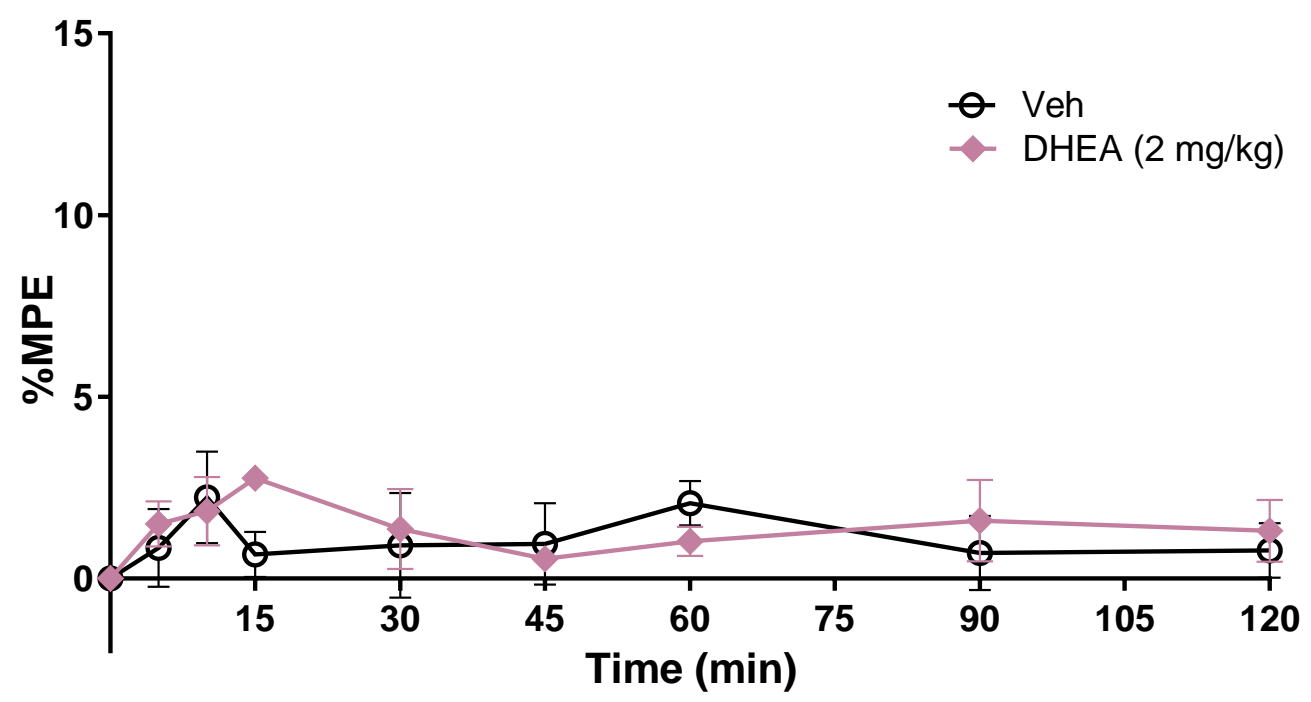

Figure 3.5: DHEA has no effect in the warm water $\left(50^{\circ} \mathrm{C}\right)$ tail withdrawal assay

The maximal possible effect (\%MPE) at each time point was calculated as a percentage based on pretreatment baseline latencies. Mice were treated with either vehicle or DHEA ( $2 \mathrm{mg} / \mathrm{kg}$ i.p.) and the tail withdrawal latencies measured up to $120 \mathrm{~min}$. DHEA showed no significant effect of treatment compared to vehicle control. Two-way repeated measures ANOVA. Values presented as mean $\pm S E M$, $\mathrm{n}=7$ per group. 

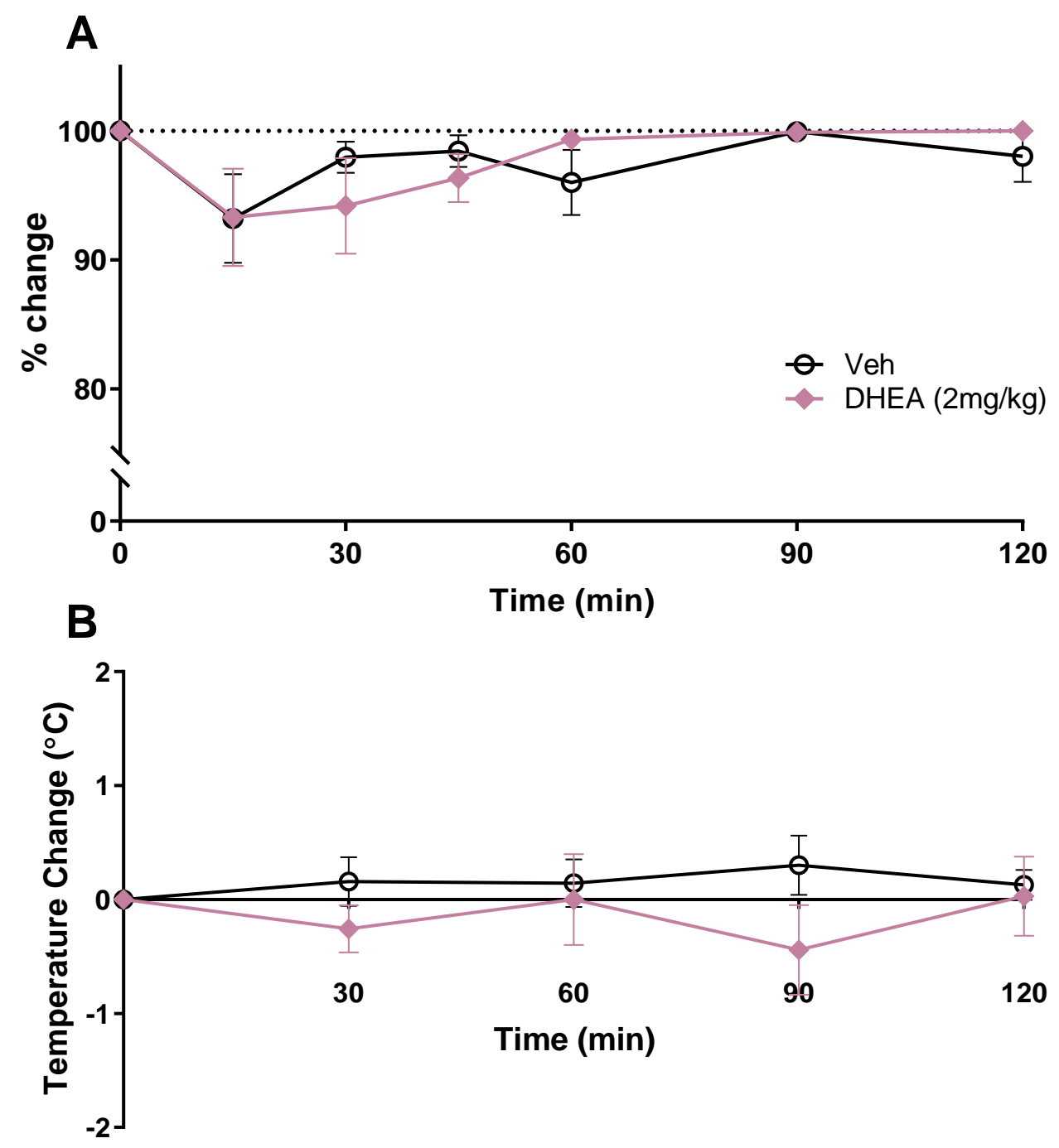

Figure 3.6: DHEA had no effect on motor coordination or core body temperature

(A) Mice were treated with either vehicle or DHEA (2 mg/kg i.p.) and the rotarod performance measured up to $120 \mathrm{~min}$. DHEA showed no significant effect of treatment compared to vehicle control. $\mathrm{n}=6$ per group. (B) Mice were treated with either vehicle or DHEA $(2 \mathrm{mg} / \mathrm{kg}$ i.p.) and the core body temperature measured up to $120 \mathrm{~min}$. DHEA showed no significant effect of treatment compared to vehicle control. $n=7$ per group. Two-way repeated measures ANOVA. Values presented as mean \pm SEM. 
The main effects of the DHEA study are summarised in Table 3.2 below.

\begin{tabular}{|c|c|c|c|c|c|}
\hline & DHEA (i.p.) & $\begin{array}{c}\text { DHEA (i.p.) + } \\
\text { AM251 }\end{array}$ & $\begin{array}{c}\text { DHEA (i.p.) + } \\
\text { AM630 }\end{array}$ & $\begin{array}{c}\text { DHEA (i.p.) + } \\
\text { AM251 + } \\
\text { AM630 }\end{array}$ & DHEA (i.pl.) \\
\hline \multicolumn{6}{|c|}{ Formaldehyde model } \\
\hline - Time course & $25-35 \mathrm{~min}$ & n.s. & n.s. & n.s. & $5,30 \mathrm{~min}$ \\
\hline - Phase I pain & $\downarrow$ & n.s. & n.s. & n.s. & $\downarrow$ \\
\hline - Phase II pain & $\downarrow$ & n.s. & n.s. & n.s. & $\downarrow$ \\
\hline - Paw oedema & $\downarrow$ & n.s. & n.s. & n.s. & $\downarrow$ \\
\hline - Neutrophil count & $\downarrow$ & - & - & n.s. & - \\
\hline \multicolumn{6}{|l|}{ Tail withdrawal } \\
\hline - Time course & n.e. & - & - & - & - \\
\hline \multicolumn{6}{|l|}{ Rotarod } \\
\hline - Time course & n.e. & - & - & - & - \\
\hline \multicolumn{6}{|l|}{ Core temperature } \\
\hline - Time course & n.e. & - & - & - & - \\
\hline
\end{tabular}

Table 3.2: Summary of the antinociceptive and side effects of $\boldsymbol{N}$-docosahexaenoyl ethanolamine

$\downarrow$ indicates a reduction compared to the vehicle/formaldehyde treatment. n.s. $=$ antagonist treatment does not significantly alter effects of DHEA. n.e. = no effect of DHEA compared to vehicle control. 


\subsection{Discussion}

The importance of $n-3$ PUFAs in the diet is well known and fish oil tablets containing DHA and EPA are one of the most popular dietary supplements in the developed world (Clarke et al., 2015). These lipid compounds have beneficial effects on a range of disease states, particularly in conditions with an inflammatory aspect (Calder, 2015; Lorente-Cebrian et al., 2015; Cederholm, 2017; Siscovick et al., 2017). The anti-inflammatory and antinociceptive effects of $n-3$ PUFAs have been shown in multiple studies in vivo and in vitro (Nakamoto et al., 2010; Veigas et al., 2011; Nobre et al., 2013). However, there are many different potential mechanisms of action for these compounds. One downstream mechanism which has not been well explored is the effect of the NAE metabolites, a class of lipid compounds which have proven anti-inflammatory properties. Specifically, DHEA is the NAE metabolite of DHA which has been found in abundance in the brain and retina, and increases in DHEA are seen following an n-3 PUFA enriched diet (Sugiura et al., 1996; Bisogno et al., 1999; Berger et al., 2001). DHEA has been previously shown to bind to both the CB1 and CB2 receptors (Felder et al., 1993; Sheskin et al., 1997; Brown et al., 2010) and has anti-inflammatory properties in vitro (Meijerink et al., 2015). Therefore, this study was designed to understand the in vivo effects of DHEA.

\subsubsection{Effects in the intraplantar $2 \%$ formaldehyde test}

To the best of our knowledge, this is the first study to investigate the in vivo effects of DHEA using behavioural models of pain. The intraplantar formaldehyde test was selected for the acute inflammatory pain reaction produced, as well as the nociceptive pain phase. DHA, the parent structure of DHEA, was effective in both phases of the intraplantar formaldehyde test in mice when administered orally (Nakamoto et al., 2010; Nobre et al., 2013). The endocannabinoid NAE compound anandamide was effective via i.p. administration in the second phase of the intraplantar formaldehyde test in Wistar rats (Jaggar et al., 1998). Conversely, a study in Swiss male mice found that anandamide was not effective in either phase of pain when administered via i.p. injection, although this study only assessed the effect of formaldehyde over a $30 \mathrm{~min}$ time period, as opposed to the typical $60 \mathrm{~min}$ (Calignano et al., 1998). In the present study, the i.p. administration of DHEA produced a significant decrease in both the nociceptive and inflammatory phases of pain (Figure 3.1). Analysis of the time course showed a significant effect of DHEA from 25-35 min, but the area under the curve analysis indicated a significant effect on the nociceptive phase of pain, indicating a relatively short onset of action of the compound. This experiment indicates that i.p. administration is an appropriate method for administration of this compound to produce antinociceptive effects. 
Recently, a radiolabelling experiment with DHEA has shown that the compound is present in the brain $15 \mathrm{~s}$ following i.v. administration in the tail vein of male Swiss-Webster mice (Sonti et al., 2017). The brain concentration remained between 1.7-2.0\% $\mathrm{I} / \mathrm{g}$ (percent injected activity per gram) from the 15 $\mathrm{s}$ time point up to $60 \mathrm{~min}$, with the highest concentrations in the midbrain, brain stem and hypothalamus. The urine concentration increased significantly from $15 \mathrm{~min}$. Similarly, in an experiment carried out for this thesis, $\left[{ }^{13} \mathrm{C}\right]$-DHEA was administered via i.p. injection and the concentration in whole brain samples assessed by mass spectroscopy (analysed at Callaghan Innovation). The results indicated that at the two time points measured, 10 and $30 \mathrm{~min}$, DHEA had reached the brain at an approximate concentration of $18 \mathrm{ng} / \mathrm{mL}$ (same average for both time points). However, due to a small sample size per treatment group and the low concentration being at the limit of the sensitivity of the mass spectroscopy instrument, the results must be interpreted with caution. However, the evidence does suggest that DHEA is able to cross the blood-brain barrier and enter the brain tissue rapidly, although, this does not necessarily mean the mode of action of the antinociceptive effects occurs in the CNS.

In the Calignano et al. (1998) study, anandamide was ineffective when administered via i.p. injection, however, it was effective in the first phase of the intraplantar formaldehyde test when administered via local i.pl. injection and systemic i.v. injection. However, the dose-response of the effects showed local administration to be 100 times more effective than the systemic administration (ID 50 values of $0.1 \mathrm{mg} / \mathrm{kg} /$ i.pl. vs. $10 \mathrm{mg} / \mathrm{kg} /$ i.v.). Another NAE compound, PEA, showed a significant effect in both phases following i.pl. administration. A further study found that local injection of anandamide reduced pain in both phases in male Wistar rats, whereas injection into the contralateral hind paw did not show any reduction in pain scores (Guindon et al., 2006). Therefore, it is likely that these NAE compounds are producing antinociceptive effects by acting in the periphery rather than CNS. In the present study, the local administration of DHEA had a significant effect in both the nociceptive and inflammatory phases of pain when compared to the vehicle control (Figure 3.3), indicating a potential peripheral mechanism of action. Nevertheless, it is possible that the compound crossed into the bloodstream via capillaries in the skin and into the CNS to produce the antinociceptive effects. Unfortunately, the local administration of the vehicle solution led to a significant level of pain in both phases compared to the vehicle control for the i.p. administration (for analysis see Appendix B). To further test the effects of DHEA, the vehicle control was compared to DHEA without formaldehydeinduced pain. In this case, DHEA reduced the first phase of pain only (Figure 3.3), indicating that the level of inflammation and therefore inflammatory pain, induced by the vehicle or the double injection procedure could not be reduced by DHEA. 
For the final experiment using the intraplantar formaldehyde test, DHEA was administered i.pl. at the beginning of the inflammatory phase of pain, to test whether the onset of action would change the effectiveness of the compound. In this experiment, there was a significant difference between the vehicle control and the formaldehyde control, confirming that the procedure had worked, although, over the time course, there was only significant difference at the 50-55 min time points (Figure 3.4). Since the paw would have already been inflamed by the i.pl. injection of both the formaldehyde and the inflammatory vehicle, in the time before the second injection (at the $15 \mathrm{~min}$ time point) nociceptors would have undergone peripheral sensitisation, and therefore the second i.pl. injection induced the same amount of pain irrespective of what was administered in the previous injection. DHEA did not show a reduction in the level of pain. This could be due to DHEA requiring a longer time to produce antinociceptive effects and DHEA could have a mechanism of action which prevents the formation of inflammation rather than reducing established inflammation. However, it is likely that the experiment was not sensitive enough to measure any effects of the DHEA treatment, as there was not a large change in the inflammatory pain level between the positive and negative control and there was no significant difference between any of the treatments for the paw oedema measurements.

Injection of formaldehyde into the footpad tissue causes an acute local inflammatory reaction. Measuring the size of oedema induced by the formaldehyde gives an indication of the level of inflammation that has occurred. Previous studies show that anandamide did not reduce formaldehyde-induced (Guindon et al., 2006) or carrageenan-induced paw oedema, although PEA did show anti-oedema effects in the carrageenan model (Wise et al., 2008). In the results presented here, both the i.p. administration and the local i.pl. administration of DHEA reduced the formaldehydeinduced paw oedema over a $60 \mathrm{~min}$ time period (Figure 3.2; Figure 3.3). DHEA also reduced the level of neutrophil infiltration into the inflamed tissue at the dose tested (Figure 3.2). Both of these processes were independent of the cannabinoid receptors.

DHEA is structurally similar to the endocannabinoid NAE compound anandamide and has proven binding affinity to the CB1 and CB2 receptors (Brown et al., 2010). The anti-inflammatory effects of DHEA in vitro in 3T3-L1 adipocyte cells were mediated through activation of the CB2 receptor in a previous study (Balvers et al., 2010). Therefore, in assessing the effects of DHEA, it was decided to antagonise the cannabinoid receptor system to evaluate the in vivo mechanism of action. The results show that the antinociceptive effects in the intraplantar formaldehyde test were independent of both CB1 and CB2 receptors (Figure 3.1). In addition, antagonism of the $C B 1$ and $C B 2$ receptors did not change the ability of DHEA to reduce paw oedema or decrease neutrophil infiltration into inflamed tissue (Figure 3.2). It also shows that the effect of DHEA is not due to the 'entourage effect' whereby increasing the levels of NAEs leads to competition at the degrading FAAH enzyme, leading to an 
increase in anandamide levels. Since anandamide acts through the cannabinoid receptors, if the entourage effect was responsible for the effects of DHEA, it would be expected that the antinociceptive effects would be blocked by AM251 and AM630 administration.

After the study in this chapter had been carried out, a paper was published showing DHEA had COX-2 inhibitory properties in vitro (Meijerink et al., 2015), although there had been earlier speculation to this effect, with discussion of unpublished data in a review article by the same group (Meijerink et al., 2013). The paper found that in the RAW264.7 macrophage cell line, the CB1 and CB2 receptors and PPARs were not involved in the anti-inflammatory effects, but instead, DHEA reduced levels of eicosanoids produced by COX-2 (Meijerink et al., 2015). With the addition of this information, it is possible that the effects presented here for DHEA could be due to COX-2 inhibition, which is the analgesic mechanism of action of NSAIDs. This could explain why DHEA shows different results in the intraplantar formaldehyde test compared to anandamide and PEA which are predominately mediated by the CB1 and CB2 receptor respectively (Calignano et al., 1998).

\subsubsection{Effect in the warm water tail withdrawal assay}

The warm water tail withdrawal assay can be used to assess antinociceptive effects on thermal nociceptive pain, and give an indication of the onset and duration of action of test compounds. Unfortunately, DHEA did not produce a significant effect in this assay (Figure 3.5). Since the warm water tail withdrawal assay assesses the nociceptive processes at the spinal cord, the results indicate that DHEA may act in the periphery rather than the CNS. Although it is possible that DHEA does act in the CNS but is inactive in this model of pain. On the contrary, oral administration of $n-3$ PUFAs or DHAenriched supplement does produce a significant effect in the tail withdrawal assay (Nakamoto et al., 2010; Nobre et al., 2013). Therefore, DHA, the precursor to DHEA, may have a different mechanism of action or the metabolism of these lipid compounds following oral vs. i.p. administration may affect the bioavailability and antinociceptive effects. The lipid mediators resolvins are hydroxylated derivatives of EPA (E-resolvins) and DHA (D-resolvins) and have a role in resolving or dampening inflammation (Serhan et al., 2000; Serhan et al., 2002; Serhan et al., 2008), therefore it is possible that this is the mechanism of action of the n-3 PUFAs.

\subsubsection{The side effects of DHEA}

Since the structure of DHEA is similar to the endocannabinoid anandamide, and DHEA has previously been shown to have CB1 and CB2 activation (Brown et al., 2010), two side effects associated with cannabinoid receptor activation were selected to be tested. The motor coordination of the animals was tested using the accelerating rotarod performance test, although DHEA treatment did not have 
any effect on motor coordination (Figure 3.6A). The effect of DHEA on thermoregulation was tested using a rectal probe to assess the core body temperature changes over time, and similarly, the administration of DHEA did not have any effect on the core body temperature (Figure 3.6B). Conversely, anandamide has been shown to cause hypothermia in mice (Crawley et al., 1993; Fride \& Mechoulam, 1993; Smith et al., 1994) and reduce locomotor activity in rats in the open field test (Bruijnzeel et al., 2016), however, the effect of anandamide on the rotarod performance test is unclear. Other cannabinoid receptor agonists have been shown to cause motor incoordination in the rotarod performance test in mice, such as THC (Dar, 2000), and synthetic agonists HU-210 and CP55,940 (DeSanty \& Dar, 2001). Together this indicates that, unlike anandamide, DHEA does not have side effects which are related to cannabinoid receptor activation.

\subsubsection{Limitations and future directions}

The in vivo antinociceptive effects of DHEA had not previously been assessed, and therefore information about the onset and duration of action were unknown. When screening a drug for antinociceptive effects, a repeated-measures test can be used to understand these dynamics. Typically, we would use the tail withdrawal assay, due to the fact that the warm water gives a nociceptive response but doesn't cause tissue damage, so that it can be repeated over a set amount of time. However, DHEA did not have any effect in the warm water tail withdrawal. Therefore, in the intraplantar formaldehyde test, DHEA was given a short 5 min pre-treatment time to avoid losing any early effects of the compound. As an indication, anandamide, PEA and other NAE compounds had a 30 min pre-treatment with i.p. administration in the carrageenan-induced oedema test (Wise et al., 2008) and 15 min pre-treatment when administered i.pl. in the intraplantar formaldehyde test (Guindon et al., 2006). DHA has been given 30 min before carrying out the intraplantar formaldehyde test, however, this was via oral administration (Nakamoto et al., 2010; Nobre et al., 2013). The intraplantar formaldehyde test could potentially be repeated with a longer pre-treatment time and a higher dose to test for a dose-dependent effect. Anandamide, PEA and other NAE compounds have been tested up to $50 \mathrm{mg} / \mathrm{kg}$ via i.p. administration (Wise et al., 2008). Finally, to assess the site of action, the DHEA compound could be administered via intrathecal or i.c.v. injection, and if no antinociceptive effects were produced this would show that the effects were occurring in the peripheral tissue.

Since DHEA is structurally similar to anandamide, and DHEA has binding affinity to both the CB1 and CB2 receptors, the cannabinoid system was antagonised in the present study. However, the results show the antinociceptive effects of DHEA were not mediated through the CB1 or CB2 receptors. Evidence from a previous in vitro study indicates that DHEA may be a COX-2 inhibitor (Meijerink et al., 
2015). To measure whether DHEA is inhibiting COX-2, plasma and tissue homogenate samples could be taken from mice and the concentration of COX-mediated eicosanoids in the samples measured using enzyme-linked immunosorbent assay (ELISA) or liquid-chromatography mass spectroscopy analysis as the Meijerink et al. (2015) paper carried out (LC-MS/MS). The eicosanoids produced by the COX pathways that could be analysed include prostaglandin $(\mathrm{PG}) \mathrm{E}_{2}, \mathrm{PGD}_{2}, \mathrm{PGF}_{2 \alpha}$ and thromboxane $\mathrm{B}_{2}$ and could be compared to metabolites of other enzymatic pathways, including lipoxygenase and cytochrome P450. The results could be compared to a known selective COX-2 inhibitor, such as Celecoxib or SC-58125; and a known selective COX-1 inhibitor such as SC-560 or FR-122047.

However, it is possible that the effects seen for DHEA could be due to the FAAH enzyme hydrolysing DHEA into DHA and ethanolamine, and the DHA having antinociceptive effects. This could be ruled out by administering a FAAH inhibitor, which should not alter or increase the effects of DHEA, as more DHEA would be available since the method of degradation would be removed. If a FAAH inhibitor decreased the action of DHEA, it could be concluded that some of the effects were due to conversion to DHA. However, since FAAH is not specific for this pathway, inhibiting this enzyme would also affect the levels of anandamide and arachidonic acid. FAAH inhibition would increase anandamide levels, which would produce a cannabinoid receptor-mediated antinociceptive effect.

Further experiments could be carried out with other inflammatory pain models, to build a greater body of evidence for the antinociceptive effect of DHEA on inflammatory pain. Examples of potential models include carrageenan- or capsaicin-induced inflammatory pain, as well as arthritis models involving the injection of complete Freund's adjuvant into joints, and models of visceral inflammation, including the acetic acid writhing test. DHA and NAE compounds have known antinociceptive effects in these models (Calignano et al., 2001; Wise et al., 2008; Clapper et al., 2010; Nakamoto et al., 2010; Nobre et al., 2013). However, the scope of this thesis was to screen potential antinociceptive drugs and then expand further study on the most promising compounds. The selection criteria were predominately based on the dose-response and duration of action effects in the warm water tail withdrawal assay, which could not be calculated for DHEA, as the compound was not active in this model at the concentration tested. The KOPr agonist compounds presented in Chapter 4 had more potential in the acute antinociceptive models, and therefore these compounds were given priority for further development. 


\subsection{Summary}

DHEA reduced inflammatory and nociceptive pain the in the intraplantar formaldehyde test in mice with both i.p. and local i.pl. administration, which did not appear to be modulated by pre-treatment with $\mathrm{CB} 1$ and $\mathrm{CB} 2$ receptor antagonists. DHEA significantly reduced formaldehyde-induced footpad oedema and reduced the infiltration of neutrophils into the inflamed footpad tissue independent of the cannabinoid receptor activation. Further studies are required to understand the mechanism of these antinociceptive effects, although one likely mechanism is via COX-2 inhibition. DHEA did not have any effect in the warm water tail withdrawal assay and did not show any motor coordination impairment or changes in thermoregulation, indicating the mechanism of action is likely to be in the periphery rather than the CNS. 


\section{Chapter 4: Investigating the antinociceptive and anti- inflammatory effects of novel kappa opioid receptor agonists}

\subsection{Introduction}

In the search for non-addictive analgesics, KOPr agonists are a promising alternative to MOPr agonists. SalA has proven antinociceptive and anti-inflammatory effects in vivo (Ansonoff et al., 2006; John et al., 2006; McCurdy et al., 2006; Aviello et al., 2011; Fichna et al., 2012; Guida et al., 2012; Rossi et al., 2016), however, a short duration of action (Prisinzano, 2005; Butelman et al., 2009; Teksin et al., 2009; Ranganathan et al., 2012), plus aversive (Zhang et al., 2005b) and anxiogenic side effects (Braida et al., 2009) have limited their clinical development (see Section 1.7.4.4 for further details on side effects of SalA). In the current chapter, the antinociceptive and anti-inflammatory effects of novel KOPr analogues of SalA have been assessed in behavioural models in mice.

\subsubsection{Novel analogues of Salvinorin A}

Many SalA analogues have been created with functional alterations at the carbon-2 position. Modifications at this position have increased the duration of action by adding metabolism-protective groups and removing the hydrolysable ester. Two novel compounds which have been created by altering the functional group at the carbon-2 position are methoxymethyl ether SalB (MOM SalB) and ethoxymethyl ether SalB (EOM SalB), which both have increased binding affinity and potency compared to parent compound SalA in Chinese hamster ovary $(\mathrm{CHO})$ cells expressing the human KOPr (Munro et al., 2008; Prevatt-Smith et al., 2011) (Table 4.2). EOM SalB also has a binding affinity to the human MOPr of $41 \pm 3 \mathrm{nM}$ (Prevatt-Smith et al., 2011) (Table 4.1), however, a previous study had found the affinity at the rat MOPr to be $>1,000 \mathrm{nM}$ using competitive inhibition of $\left[{ }^{3} \mathrm{H}\right]$ diprenorphine (Munro et al., 2008).

EOM SalB was more metabolically stable in an in vitro rat liver microsome assay compared to SalA (Ewald et al., 2017), and was more stable in baboons when measured with positron emission tomography (PET) and in rats administered via i.p. injection brain concentrations declined more slowly than SalA (Hooker et al., 2009). EOM SalB has been shown to attenuate drug-seeking behaviour in cocaine-induced reinstatement of self-administration and attenuated the locomotor effects of cocaine, without causing sedation in the spontaneous locomotor activity test, anxiety in the elevated plus maze or depressive-like effects in the forced swim test (Ewald et al., 2017). MOM SalB attenuated cocaine-induced drug seeking, however, the compound also reduced natural reward behaviours in 
sucrose self-administration studies and showed pro-depressive effects in the forced swim test in rats (Morani et al., 2013). In discrimination studies, MOM SaIB and EOM SalB both substituted for U69,593 (Baker et al., 2009) and SalA (Peet \& Baker, 2011) in rats, indicating that the animals could not recognise differences between these compounds.

Further carbon-2 modifications have been made to the SalA structural scaffold to create $\beta$ tetrahydropyran SaIB ( $\beta$-THP SalB) and Mesyl SalB, and both have similar binding affinity and potency compared to SalA when measured in CHO cells expressing the human KOPr (Harding et al., 2005; Prevatt-Smith et al., 2011; Kivell et al., 2018) (Table 4.2). Both attenuated drug-seeking behaviour in cocaine-induced reinstatement self-administration studies in rats without causing sedation in spontaneous locomotor tests (Morani et al., 2013; Simonson et al., 2015). Mesyl SalB has also been shown to reduce alcohol intake in mice subjected to chronic escalation drinking of ethanol but did not reduce sucrose self-administration (Zhou et al., 2017). We have previously shown that both compounds have a longer duration of action than SalA (45 min for $\beta$-THP SalB and 60 min for Mesyl SalB vs 30 min for SalA) in the warm water tail withdrawal assay in mice (Simonson et al., 2015; Paton et al., 2017). Recently, further modification at the carbon-2 produced the conformationally-restricted compound spirobutyrolactone SalB (Spiro SalB), with similar potency and selectivity to SalA (Sherwood et al., 2017a). We also showed that Spiro SalB had similar antinociceptive effects to SalA in the intraplantar $2 \%$ formaldehyde test and the warm water tail withdrawal assay (Sherwood et al., 2017a).

Modifications have also been made at the carbon-16 position on the furan ring of SalA (Riley et al., 2014). The addition of a bromine group at this carbon-16 position produced the compound 16-Bromo SalA and the addition of an ethynyl group produced the compound 16-Ethynyl SalA. Both compounds have similar potency at the KOPr compared to the parent compound SalA measured in CHO cells expressing the human KOPr (Beguin et al., 2009; Riley et al., 2014) (Table 4.2). In rats, 16-Bromo SalA and 16-Ethynyl SalA attenuated drug-seeking in cocaine prime-induced reinstatement without decreasing spontaneous locomotor activity (Riley et al., 2014). The compounds also reduce the rewarding value of cocaine in progressive ratio self-administration but do not attenuate the natural reward pathway in sucrose self-administration studies (Ewald, 2015; Young, 2015). 


\begin{tabular}{|c|c|c|}
\hline & MOPr & DOPr \\
\hline SalA & $>1,000 \mathrm{nM}^{(2)}$ & $>10,000 \mathrm{nM}^{(1)}$ \\
\hline MOM SalB ${ }^{(1)}$ & $390 \pm 20 \mathrm{nM}$ & $2,840 \pm 180 \mathrm{nM}$ \\
\hline EOM SalB ${ }^{(1)}$ & $41 \pm 3 \mathrm{nM}$ & $1,017 \pm 99 \mathrm{nM}$ \\
\hline$\beta$-THP SalB ${ }^{(1)}$ & $>10,000 \mathrm{nM}$ & $>10,000 \mathrm{nM}$ \\
\hline Mesyl SalB (2) & $6,820 \pm 660 \mathrm{nM}$ & $>10,000 \mathrm{nM}$ \\
\hline Spiro SalB ${ }^{(5)}$ & $E_{\max }=0 \%$ up to $10,000 \mathrm{nM}$ & $E_{\max }=0 \%$ up to $10,000 \mathrm{nM}$ \\
\hline 16-Ethynyl SalA ${ }^{(4)}$ & $\mathrm{EC}_{50} \geq 10,000 \mathrm{nM}$ & n.d. \\
\hline 16-Bromo SalA & $\mathrm{EC}_{50} \geq 10,000 \mathrm{nM}^{(4)}$ & $>1,000 \mathrm{nM}^{(3)}$ \\
\hline
\end{tabular}

Table 4.1: Comparison of Salvinorin A and analogues for binding affinity and potency for the mu and delta opioid receptors

(1) Binding affinity in Chinese hamster ovary ( $\mathrm{CHO}$ ) cells expressing hMOPr or hDOPr with [ $\left.{ }^{3} \mathrm{H}\right] \mathrm{DAMGO}$ and $\left[{ }^{3} \mathrm{H}\right] \mathrm{DADLE}$ as the radioligands respectively (Prevatt-Smith et al., 2011)

(2) Binding affinity in $\mathrm{CHO}$ cells stably expressing hMOPr and hDOPr with [ ${ }^{125}$ ] IOXY as the radioligand (Harding et al., 2005)

(3) Binding affinity in $\mathrm{CHO}$ cells stably expressing mDOPr with $\left[{ }^{3} \mathrm{H}\right]$ diprenorphine as the radioligand (Beguin et al., 2009)

(4) Potency $\left(\mathrm{EC}_{50}\right)$ for inhibition of forskolin-induced cAMP accumulation in $\mathrm{CHO}$ cells expressing hMOPr (Riley et al., 2014)

(5) Efficacy $\left(E_{\max }\right)$ for inhibition of forskolin-induced cAMP accumulation in $\mathrm{CHO}$ cells expressing hMOPr and hDOPr (Sherwood et al., 2017a)

n.d. $=$ not determined. Values presented as mean \pm SD. 


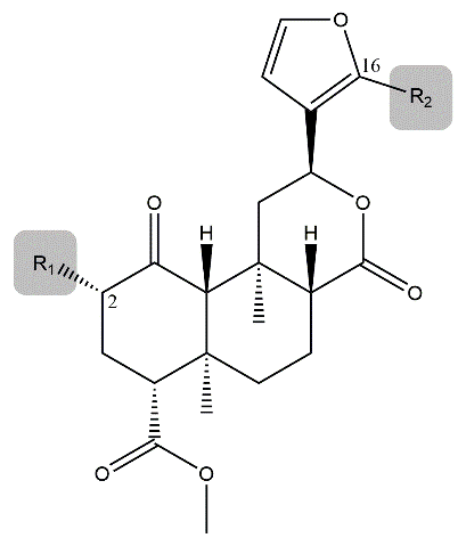

\begin{tabular}{|c|c|c|c|c|c|c|c|c|c|c|}
\hline & U50,488 & SalA & MOM SalB & EOM SalB & $\beta$-THP SalB & Mesyl SalB & Spiro SalB & $\begin{array}{c}\text { 16-Ethynyl } \\
\text { SalA }\end{array}$ & $\begin{array}{c}\text { 16-Bromo } \\
\text { SalA }\end{array}$ & Ref \\
\hline $\mathbf{R}_{\mathbf{2}}$ & - & $-H$ & $-H$ & $-H$ & $-H$ & $-H$ & $-H$ & $\xi \quad \rightleftharpoons$ & $-B r$ & \\
\hline \multirow{2}{*}{$\begin{array}{c}\text { Binding affinity, } \\
K_{i}(n M)\end{array}$} & & $1.9 \pm 0.2$ & & & & $2.3 \pm 0.1$ & & & & (2a) \\
\hline & $2.2 \pm 0.2$ & $2.5 \pm 0.6$ & & & & & & & $2.9 \pm 0.3$ & (3a) \\
\hline \multirow{2}{*}{$\begin{array}{c}\mathrm{EC}_{50}(\mathrm{nM}) \\
{\left[{ }^{35} \mathrm{~S}\right] \mathrm{GTP}-\gamma-\mathrm{S} \text { assay }}\end{array}$} & & $40 \pm 10$ & $6 \pm 1$ & $0.65 \pm 0.17$ & $60 \pm 6$ & $30 \pm 5^{(2 b)}$ & & & & (1b) \\
\hline & $2.9 \pm 0.2$ & $2.1 \pm 0.6$ & & & & & & & $2.4 \pm 0.2$ & (3b) \\
\hline $\begin{array}{c}\text { Emax (\%) } \\
{\left[{ }^{35} \text { S]GTP- }- \text {-S assay }\right.}\end{array}$ & & $120 \pm 2$ & $118 \pm 2$ & $127 \pm 5$ & $109 \pm 3$ & $112 \pm 4^{(2 b)}$ & & & & (1b) \\
\hline
\end{tabular}


Table 4.2: Comparison of Salvinorin A and analogues in binding affinity and selectivity at the KOPr

(1) (a) Binding affinity $\left(\mathrm{K}_{\mathrm{i}}\right)$ in Chinese hamster ovary $(\mathrm{CHO})$ cells expressing hKOPr with $\left[{ }^{3} \mathrm{H}\right] \mathrm{U} 69,593$ as the radioligand. (b) Potency $\left(\mathrm{EC}_{50}\right)$ and efficacy $\left(\mathrm{E}_{\max }\right)$ measured with the $\left[{ }^{35} \mathrm{~S}\right]$ GTP- $\gamma$-S functional assay in CHO cells expressing the hKOPr and $\mathrm{E}_{\max }$ compared to U50,488 (Prevatt-Smith et al., 2011)

(2) (a) Binding affinity $\left(\mathrm{K}_{\mathrm{i}}\right)$ in $\mathrm{CHO}$ cells stably expressing hKOPr with $\left[{ }^{125} \mathrm{I}\right] \mathrm{IOXY}$ as the radioligand. (b) Potency $\left(\mathrm{EC}_{50}\right)$ and efficacy $\left(\mathrm{E}_{\max }\right)$ measured with the $\left[{ }^{35}\right.$ S] GTP- $\gamma$-S functional assay in CHO cells stably expressing the hKOPr and $E_{\max }$ compared to U50,488 (Harding et al., 2005)

(3) (a) Binding affinity $\left(\mathrm{K}_{\mathrm{i}}\right)$ in $\mathrm{CHO}$ cells stably expressing hKOPr with $\left[{ }^{3} \mathrm{H}\right]$ diprenorphine as the radioligand. (b) Potency $\left(\mathrm{EC}_{50}\right)$ and efficacy $\left(\mathrm{E}_{\mathrm{max}}\right)$ measured with the $\left.{ }^{35} \mathrm{~S}\right] \mathrm{GTP}-\gamma-\mathrm{S}$ functional assay in $\mathrm{CHO}$ cells stably expressing the hKOPr and $\mathrm{E}_{\max }$ compared to U50,488 (Beguin et al., 2009)

(4) Potency $\left(\mathrm{EC}_{50}\right)$ for inhibition of forskolin-induced cAMP accumulation in $\mathrm{CHO}$ cells expressing hKOPr, with all compounds reported showed an $\mathrm{E}_{\max }$ of $100 \%$. *value for U69,593 rather than U50,488 (Riley et al., 2014)

(5) Potency $\left(\mathrm{EC}_{50}\right)$ for inhibition of forskolin-induced cAMP accumulation in $\mathrm{CHO}$ cells expressing hKOPr. All reported compounds showed an $\mathrm{E}_{\mathrm{max}}$ of $100 \%$ (Sherwood et al., 2017a)

Values presented as mean \pm SD (Ref 1-2) and mean \pm SEM (Ref 3-5) 


\subsubsection{Biased agonism at the kappa opioid receptor}

Traditional KOPr agonists, such as U50,488, are associated with many side effects including sedation, anxiety, aversion and dysphoria (Mucha \& Herz, 1985; Suzuki et al., 1992; Bals-Kubik et al., 1993; Privette \& Terrian, 1995; Skoubis et al., 2001; Mague et al., 2003; Kudryavtseva et al., 2004; Vunck et al., 2011; Ehrich et al., 2015; Zhang et al., 2015; Wang et al., 2016). However, the concept of biased agonism, where a ligand can preferentially activate desired signalling pathways, has renewed interest in designing improved KOPr agonist analgesics. It is proposed that the antinociceptive effects of the $\mathrm{KOPr}$ are associated with G-protein regulation of ion channels, resulting in reduced neuronal excitability. Whereas, the negative side effects tend to be associated with the $\beta$-arrestin-dependent signalling cascades (see Section 1.7.2.1 for further details on KOPr signalling pathways).

Side effect data collected in the Kivell laboratory group in Sprague Dawley rats suggests that some of the novel SalA analogues may be G-protein biased. 16-Ethynyl SalA and 16-Bromo SalA did not cause sedation in spontaneous locomotor tests (Riley et al., 2014) and do not cause anxiety in the elevated plus maze or light-dark test, or have depressive-like effects in the forced swim test (Ewald, 2015; Young, 2015; Culverhouse, 2015). In the CPA paradigm, 16-Ethynyl SalA did not show aversive effects (Culverhouse, 2015), however, 16-Bromo SalA was aversive (Ewald, 2015). Neither 16-Ethynyl SalA nor 16-Bromo SalA produced long-term memory impairments in the novel object recognition task (Welsh, 2017). Similarly, EOM SalB and $\beta$-THP SalB did not cause sedation in spontaneous locomotor activity, anxiety in the elevated plus maze or depressive-like effects in the forced swim test (Ewald et al., 2017). EOM SalB did not induce aversive effects in CPA, however, $\beta$-THP SalB did (Ewald et al., 2017).

Negative side effects have been associated with late phase ERK1/2 and p38 MAPK activation (Bruchas \& Chavkin, 2010). The phosphorylation of these kinases were measured using western blotting studies, which revealed that 16-Bromo SalA did not activate ERK1/2 or p38 MAPK in the rat dorsal striatum or prefrontal cortex, however, in the nucleus accumbens, there was significant phosphorylation of p38 MAPK but not ERK1/2 (Ewald, 2015). This indicates that 16-Bromo SalA may be acting preferentially through the G-protein biased pathways.

The bias can be calculated by using the potency and efficacy results from both G-protein and $\beta$-arrestin assays, using the following formula as in Crowley et al. (2016):

$\log ($ bias factor $)=\log \left(\frac{E_{\max (\text { test })} \times E C_{50(\text { control })}}{E C_{50(\text { test })} \times E_{\max (\text { control })}}\right)_{\beta \text {-arrestin }}-\log \left(\frac{E_{\max (\text { test }) \times E C_{50(\text { control })}}}{E C_{50(\text { test })} \times E_{\max (\text { control })}}\right)_{G-\text { protein }}$ 
Where the control compound is a balanced agonist with similar potency in both assays, which has been set as U50,488 (Schattauer et al., 2017), SalA (White et al., 2015) or dynorphin $A_{1-17}$ (DiMattio et al., 2015) in previous studies. Using this formula, a bias factor of 1 is a balanced agonist, less than 1 is G-protein biased and more than 1 is $\beta$-arrestin biased. As a further complication, the importance of understanding the bias factor in different species has been shown in multiple studies. DiMattio et al. (2015) calculated the bias factor for a range of KOPr agonists in N2a cells stably transfected with either the human or mouse KOPr. The results showed that SalA was significantly biased towards the $\beta$ arrestin pathway for the human receptor, but had similar activation of both pathways at the mouse KOPr. On the contrary, U50,488 was biased towards the $\beta$-arrestin pathway at the mouse receptor but was unbiased at the human KOPr. Similarly, a recent study found that nalfurafine was extremely Gprotein biased at the human KOPr but was biased to a much lesser extent at the rat KOPr (Schattauer et al., 2017).

However, investigations into the cell signalling pathways induced by the novel SalA analogues are ongoing in the Kivell laboratory and are not the focus of this thesis. The analogues tested in this thesis have reduced side effects in vivo, and therefore the aim was to assess the antinociceptive potential and the effects on motor coordination and thermoregulation. 


\subsection{Aims and hypotheses}

SalA is a KOPr agonist that has antinociceptive and anti-inflammatory effects in vivo, with limited abuse potential. However, the short duration of action and aversive side effects limit the clinical usefulness. The present study aimed to investigate the antinociceptive and anti-inflammatory effects as well as the side effects of acute administration of novel analogues of SalA in mice. It was hypothesised that altering the functional group at the carbon-16 position would increase the duration of action and potency at the KOPr and alter the bias factor towards a compound with fewer side effects.

The specific aims of the study were to:

1. Screen the potency and efficacy of the novel KOPr agonists by measuring the dose-response effect in the warm water tail withdrawal assay. From these results, select efficacious compounds to assess in further assays.

2. Assess the antinociceptive effects and duration of action to a thermal stimulus in the warm water tail withdrawal assay and hotplate assay.

3. Determine whether the novel compounds 16-Ethynyl SalA and 16-Bromo SalA are effective in the intraplantar $2 \%$ formaldehyde test and antagonise the KOPr using nor-BNI.

4. Investigate the effects of the novel KOPr agonists on formaldehyde-induced paw oedema and the level of local inflammation in the swollen paw using flow cytometry and histology to identify immune cell populations.

5. Evaluate the side effect profile by assessing motor coordination using the accelerating rotarod performance assay and thermoregulation using core body temperature measurements. 


\subsection{Results}

\subsubsection{Cumulative dose-response effects using the warm water tail withdrawal assay}

The warm water tail withdrawal assay measures the spinal reflex in response to a thermal stimulus. In this procedure, an escalating cumulative dose of the KOPr agonists were administered via s.c. injection and the tail withdrawal behaviour measured at $30 \mathrm{~min}$ intervals. Non-linear regression analysis was used to determine the potency (median effective dose, $E D_{50}$ ) and the efficacy (maximum response, $\left.E_{\max }\right)$ and the values were compared to the traditional KOPr agonist, U50,488. Analysis of the doseresponse curves revealed that a different curve fits each data set, demonstrating a significant effect of treatment $\left[\mathrm{F}_{(20,244)}=25.92, p<0.0001\right]$ (Figure 4.1). All of the data sets passed normality testing ( $D^{\prime}$ Agostino \& Pearson omnibus K2 normality test). The $E D_{50}$ values showed that SalA and all of the novel SalA analogues were significantly more potent when compared to U50,488 (for $p$ values see Table 4.3). The $E_{\max }$ values showed that only 16-Ethynyl SalA had a significantly higher maximum effect than $\mathrm{U} 50,488$ (for $p$ values see Table 4.3). The vehicle control group were administered the same number of injections as the KOPr treated animals, with no significant increase in the \%MPE over time ( $p=0.4032$, see Appendix C.1).

For this thesis, 16-Ethynyl SalA and 16-Bromo SalA were selected for further investigation in additional models of acute pain, because of the high efficacy of 16-Ethynyl SalA and the fact that both compounds had been extensively tested for side effects in rats, with both compounds showing a reduced side effect profile compared to SalA. $\beta$-THP SalB was investigated in conjunction with Master's student Nitin Kumar and published in the European Journal of Pain (Paton et al., 2017). Studies into the antinociceptive effects of EOM SalB are ongoing in the Kivell laboratory. 


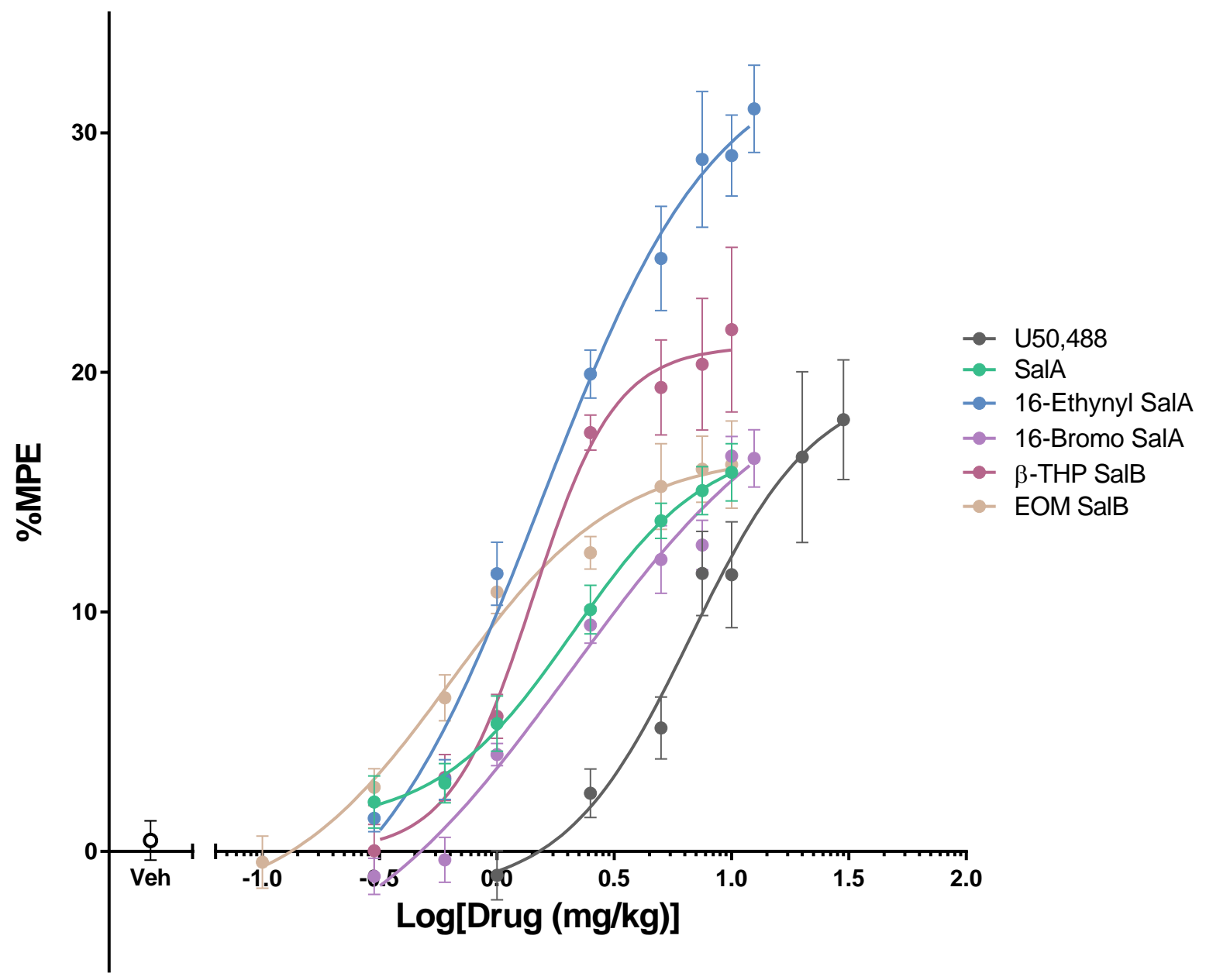

Figure 4.1: Dose-response effects of KOPr agonists in the warm water tail withdrawal assay

Cumulative dose-response effects of KOPr agonists. The maximal possible effect (\%MPE) at each dose was calculated as a percent based on the pre-treatment baseline latencies. Values presented as mean \pm SEM, $n=6$. The U50,488, SalA and $\beta$-THP SalB data sets are also presented in Paton et al. (2017). 


\begin{tabular}{|c|c|c|c|c|c|c|c|c|}
\hline & $\begin{array}{c}\text { Potency } \\
\text { ED }_{50}(\mathrm{mg} / \mathrm{kg})\end{array}$ & $\operatorname{logED} D_{50} \pm S E M$ & \multicolumn{2}{|c|}{$\begin{array}{l}p \text { value for } \\
\mathrm{ED}_{50} \text { compared to } \\
\mathrm{U} 50,488^{(1)}\end{array}$} & $\begin{array}{c}\text { Efficacy } \\
E_{\max } \pm \mathrm{SEM}^{(2)}\end{array}$ & \multicolumn{2}{|c|}{$\begin{array}{l}p \text { value for } \\
E_{\max } \text { compared to } \\
U 50,488^{(3)}\end{array}$} & $R^{2}$ value \\
\hline U50,488 & 6.28 & $0.825 \pm 0.131$ & - & - & 100 & - & - & 0.689 \\
\hline SalA & 2.11 & $0.324 \pm 0.109$ & 0.0494 & $*$ & $87.8 \pm 12.1$ & $>0.9999$ & n.s. & 0.850 \\
\hline 16-Ethynyl SalA & 1.54 & $0.187 \pm 0.122$ & 0.0036 & $* *$ & $171.0 \pm 21.5$ & 0.0343 & $*$ & 0.889 \\
\hline 16-Bromo SalA & 2.06 & $0.314 \pm 0.174$ & 0.0326 & $*$ & $107 \pm 32.7$ & 0.1012 & n.s. & 0.887 \\
\hline$\beta$-THP SalB & 1.42 & $0.152 \pm 0.0865$ & 0.0029 & $* *$ & $108 \pm 7.61$ & $>0.9999$ & n.s. & 0.787 \\
\hline EOM SalB & 0.632 & $-0.200 \pm 0.135$ & $<0.0001$ & $* * * *$ & $84.9 \pm 8.19$ & > 0.9999 & n.s. & 0.806 \\
\hline
\end{tabular}

Table 4.3: Statistical analysis of the dose-response effects of the KOPr agonists in the warm water tail withdrawal assay

(1) One-way ANOVA with Bonferroni post-tests, reported $p$ values are for comparison to the $\mathrm{ED}_{50}$ of $\mathrm{U} 50,488$

(2) $E_{\max }$ calculated as a percentage of the maximum height of the U50,488 curve

(3) One-way ANOVA with Bonferroni post-tests, reported $p$ values are for comparison to the $E_{\max }$ of $U 50,488$

n.s. = not significant, $* p<0.05, * * p<0.01, * * * p<0.001, * * * * p<0.0001$ 


\subsubsection{Duration of action SalA, 16-Ethynyl SalA and 16-Bromo SalA in the warm water tail withdrawal assay}

To measure the onset and duration of action of the KOPr agonists, the warm water tail withdrawal assay technique was used again, however, the animals were administered a single i.p. injection, and the tail withdrawal behaviour repeatedly measured over time. For the SalA cohort, two-way repeated measures ANOVA showed a significant effect of treatment $\left[F_{(2,26)}=19.18, p<0.0001\right]$, time $\left[F_{(8,208)}=\right.$ 11.74, $p<0.0001]$ and interaction of treatment $x$ time $\left[F_{(16,208)}=3.923, p<0.0001\right]$ (Figure 4.2A). Bonferroni post-tests showed that SalA had a significant antinociceptive effect for the $1 \mathrm{mg} / \mathrm{kg}$ dose at 5-30 $\mathrm{min}(p<0.05)$ and a significant effect for the $2 \mathrm{mg} / \mathrm{kg}$ dose at 5-30 $\mathrm{min}(p<0.05)$. 16-Ethynyl SalA also showed a significant effect of treatment $\left[F_{(2,18)}=15.44, p=0.0001\right]$, time $\left[F_{(8,144)}=16.25, p<\right.$ $0.0001]$ and interaction of treatment $x$ time $\left[F_{(16,144)}=3.546, p<0.0001\right]$ (Figure 4.2B). 16-Ethynyl SalA showed a significant antinociceptive effect for the $1 \mathrm{mg} / \mathrm{kg}$ dose at 10-15 $\mathrm{min}$ and a significant effect for the $2 \mathrm{mg} / \mathrm{kg}$ dose at 5-60 $\mathrm{min}(p<0.05)$. Finally, 16-Bromo SalA showed a significant effect of treatment $\left[F_{(2,18)}=12.85, p=0.0003\right]$, time $\left[F_{(8,208)}=11.74, p<0.0001\right]$ and interaction of treatment $\mathrm{x}$ time $\left[F_{(16,208)}=3.923, p<0.0001\right]$ (Figure $4.2 C$ ). 16-Bromo SalA had a significant antinociceptive effect at $1 \mathrm{mg} / \mathrm{kg}$ at $10-60 \mathrm{~min}$ and for the $2 \mathrm{mg} / \mathrm{kg}$ dose at $10-15$ and $45-60 \mathrm{~min}(p<0.05)$.

The AUC was calculated for the each of the treatments, with the vehicle controls pooled together, to understand the overall antinociceptive effect of the KOPr agonists. Comparison of the $1 \mathrm{mg} / \mathrm{kg}$ doses of the KOPr agonists showed a significant effect of treatment $\left[F_{(3,43)}=32.55, p<0.0001\right]$ (Figure 4.2D), with SalA $(p<0.0001), 16$-Ethynyl SalA $(p=0.0008)$ and 16-Bromo SalA $(p=0.0009)$ showing an increased level of antinociceptive effect compared to vehicle. SalA had a significantly larger effect than 16-Ethynyl SalA ( $p=0.0059)$ and 16 -Bromo SalA $(p=0.0053)$. There was no difference between 16 Ethynyl SalA and 16-Bromo SalA ( $p>0.9999)$. The $2 \mathrm{mg} / \mathrm{kg}$ doses also showed a significant effect of treatment $\left[F_{(3,37)}=20.3, p<0.0001\right]$ (Figure 4.2E). SalA $(p<0.0001), 16$-Ethynyl SalA $(p<0.0001)$ and 16-Bromo SalA $(p=0.0040)$ had a significant effect compared to vehicle. There was no difference between any of the KOPr agonists at the higher dose $(p>0.05)$. 
A

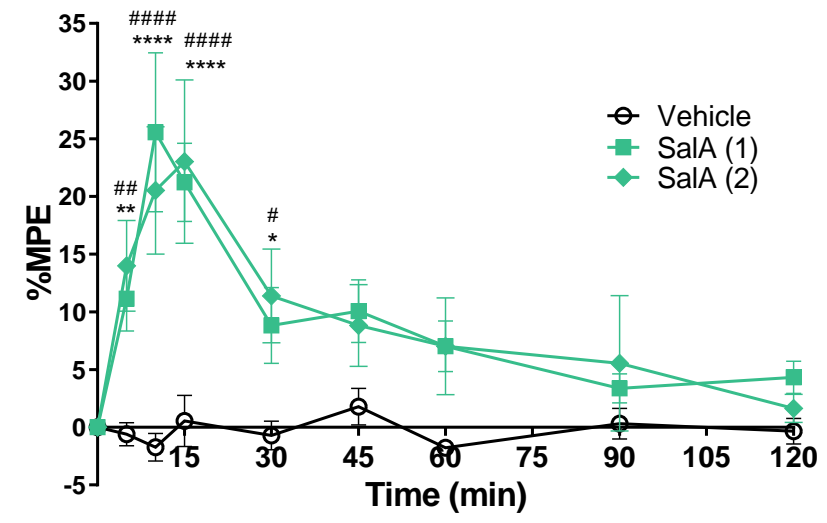

B
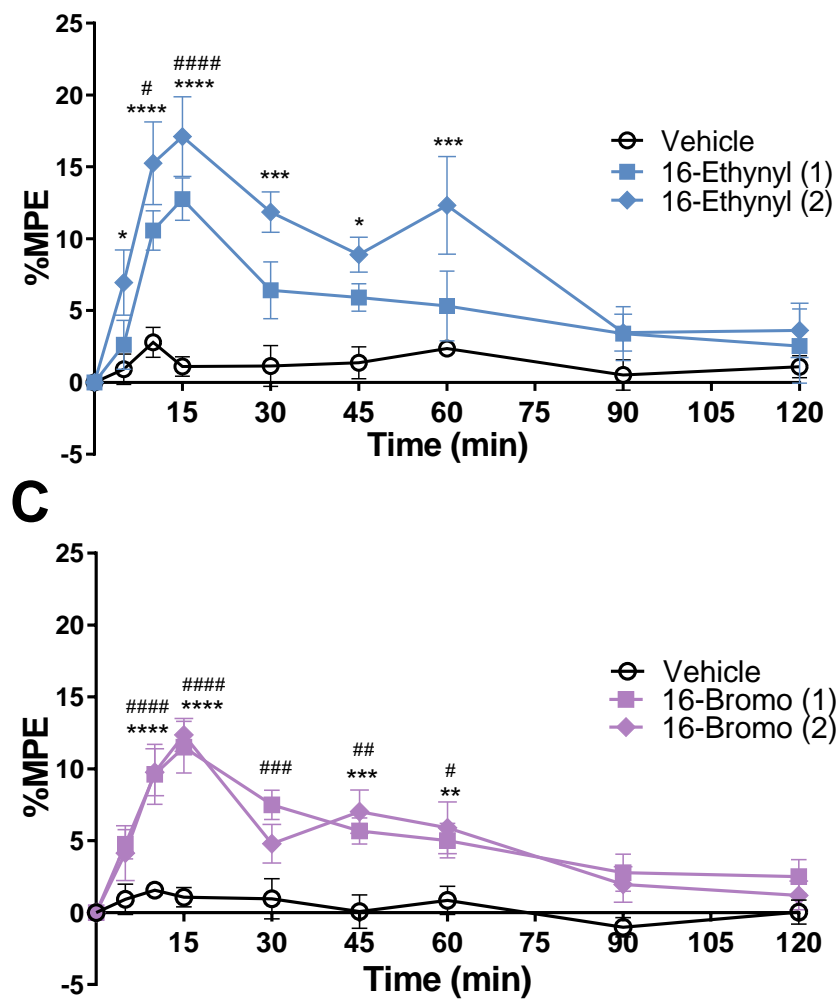

D

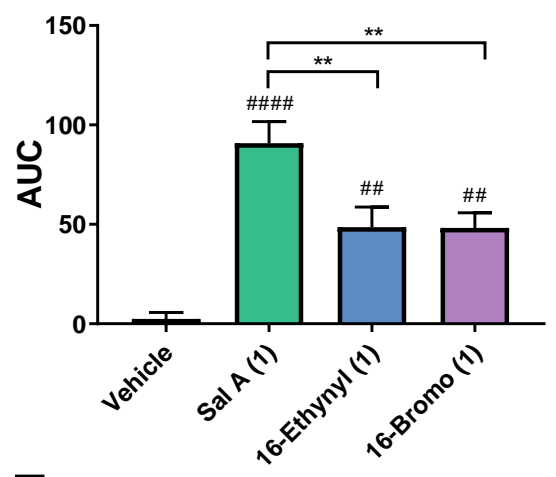

E

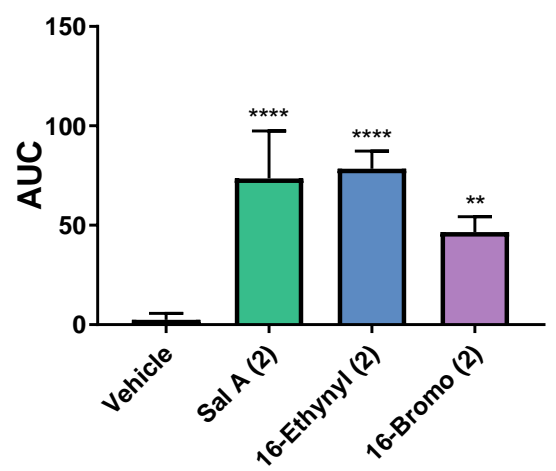

Figure 4.2: Duration of action of KOPr agonists in the warm water $\left(50^{\circ} \mathrm{C}\right)$ tail withdrawal assay

The maximum possible effect (\%MPE) at each time point was calculated as a percentage based on the pre-treatment baseline latencies. Mice were treated with either vehicle, SalA, 16-Ethynyl SalA or 16Bromo SalA and the warm water $\left(50^{\circ} \mathrm{C}\right)$ tail withdrawal latencies measured up to $120 \mathrm{~min}$. (A) SalA showed a significant duration of action up to $30 \mathrm{~min}$. (B) 16-Ethynyl SalA showed a significant effect up to $15 \mathrm{~min}$ for the $1 \mathrm{mg} / \mathrm{kg}$ dose and $60 \mathrm{~min}$ for the $2 \mathrm{mg} / \mathrm{kg}$ dose. (C) $16-B$ romo SalA showed a significant duration of action up to $60 \mathrm{~min}$ at both doses. Two-way repeated measures ANOVA followed by Bonferroni post-tests. (D-E) Area under the curve (AUC) analysis of the tail withdrawal behaviour, comparing the $1 \mathrm{mg} / \mathrm{kg}$ doses (D) and the $2 \mathrm{mg} / \mathrm{kg}$ doses (E). SalA (1 mg/kg) had an overall increased antinociceptive effect compared to both 16-Ethynyl SalA and 16-Bromo SalA. One-way ANOVA with Bonferroni post-tests. ${ }^{*} p<0.05,{ }^{* *} p<0.01,{ }^{* * *} p<0.001, * * * * p<0.0001$ for $2 \mathrm{mg} / \mathrm{kg}$

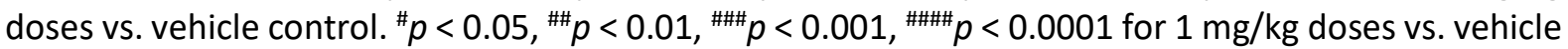
control. Values presented as mean $\pm \mathrm{SEM}, \mathrm{n}=7-12$. 


\subsubsection{Duration of action in the hotplate assay}

The hotplate assay measures the withdrawal response from a thermal stimulus to the paws, which involves supraspinal processes, as opposed to the warm water tail withdrawal assay which evaluates a spinal reflex. Similar to the previous experiment, the onset and duration of action can be measured using this technique. Two-way repeated measures ANOVA showed there was a significant effect of treatment $\left[F_{(3,20)}=4.365, p=0.0161\right]$, time $\left[F_{(3,60)}=18.95, p<0.0001\right]$ and treatment $x$ time interaction $\left[F_{(9,60)}=3.586, p=0.0013\right]$ (Figure 4.3A). Bonferroni post-tests showed that SalA $(2 \mathrm{mg} / \mathrm{kg}$ ) had a significant antinociceptive effect at $15 \mathrm{~min}(p=0.0293)$. Whereas, both the novel analogues showed a longer duration of action, with 16-Ethynyl SalA significant at $15(p=0.0024)$ and $30 \mathrm{~min}(p<0.0001)$, and 16-Bromo SalA at $15(p=0.0007)$ and $30 \mathrm{~min}(p=0.0011)$. The AUC analysis analysed the total antinociceptive effect and had a significant effect of treatment $\left[F_{(3,20)}=4.795, p=0.0112\right]$ (Figure 4.3B). 16-Ethynyl SalA ( $p=0.0142)$ and 16-Bromo SalA $(p=0.0076)$ had a significant effect compared to vehicle, whereas SalA did not $(p=0.1549)$. 

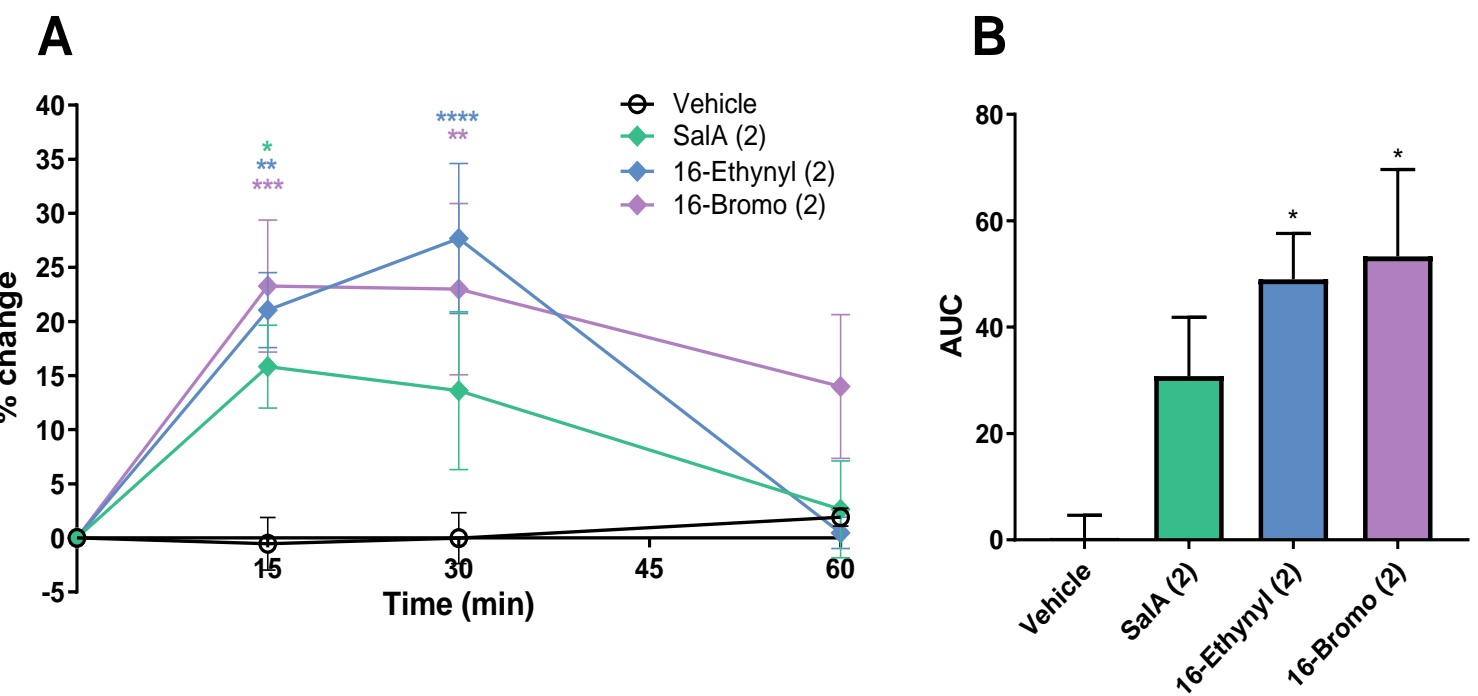

Figure 4.3: Duration of action of the KOPr agonists in the hotplate $\left(50^{\circ} \mathrm{C}\right)$ assay

The percentage change in the paw withdrawal time on the hotplate $\left(50^{\circ} \mathrm{C}\right)$ was calculated based on pre-treatment baseline latencies. (A) Mice were treated with either vehicle, SalA ( $2 \mathrm{mg} / \mathrm{kg}$ ), 16-Ethynyl SalA $(2 \mathrm{mg} / \mathrm{kg}$ ) or $16-$ Bromo SalA $(2 \mathrm{mg} / \mathrm{kg}$ ) and the withdrawal latencies measured up to $60 \mathrm{~min} .16-$ Ethynyl SalA and 16-Bromo SalA showed a significant duration of action to $30 \mathrm{~min}$, whilst SalA only had a significant effect at $15 \mathrm{~min}$. Two-way repeated measures ANOVA followed by Bonferroni posttests. (B) Area under the curve (AUC) analysis of the antinociceptive effects showed a significant effect for 16-Ethynyl SalA and 16-Bromo SalA. One-way ANOVA with Bonferroni post-tests. ${ }^{*} p<0.05,{ }^{* *} p<$ $0.01,{ }^{* * *} p<0.001,{ }^{* * * *} p<0.0001$ vs. vehicle control. Values presented as mean $\pm \mathrm{SEM}, \mathrm{n}=6$. 


\subsubsection{The effect of SalA in the intraplantar $2 \%$ formaldehyde model}

The intraplantar formaldehyde model was used to assess the ability of the KOPr agonists to reduce nociceptive and inflammatory pain. SalA was initially administered via i.p. injection, with two-way repeated measures ANOVA showed a significant effect of treatment $\left[F_{(4,39)}=24.7, p<0.0001\right]$, time $\left[F_{(12,468)}=27.78, p<0.0001\right]$ and interaction of treatment $x$ time $\left[F_{(48,468)}=5.153, p<0.0001\right]$ (Figure 4.4A). Bonferroni post-tests showed that SalA reduced the effects of the i.pl. administration of $2 \%$ formaldehyde from 5-50 $\mathrm{min}(p<0.05)$. The KOPr antagonist reversed the effects of SalA at 5 and 30$40 \mathrm{~min}(p<0.05)$. Because previous studies have shown that cannabinoid receptor antagonism reduces the antinociceptive effects of SalA (Aviello et al., 2011; Fichna et al., 2012; Guida et al., 2012; Coffeen et al., 2018), the CB1 receptor antagonist AM251 administered as a pre-treatment, however, this did not alter the effects of SalA. One-way ANOVA showed a significant effect of treatment for the AUC analyses of phase I $\left[\mathrm{F}_{(4,39)}=4.179, p<0.0001\right]$ (Figure 4.4B) and phase II pain $\left[\mathrm{F}_{(4,39)}=5.131, p<\right.$ 0.0001 ] (Figure 4.4C). SalA reduced the effects of formaldehyde in phase I $(p<0.0001)$ and phase II ( $p$ $<0.0001$ ) and in both phases, nor-BNI significantly reversed the effect of SalA (phase I, $p=0.0242$; phase II, $p=0.0185$ ), whilst AM251 treatment had no significant effect (phase I, $p=0.5302$; phase II, $p>0.9999)$. The paw oedema measurements showed a significant effect of treatment $\left[F_{(4,27)}=15.46\right.$, $p<0.0001$ ] (Figure 4.4D). SalA significantly reduced paw oedema compared to the vehicle/formaldehyde treatment $(p<0.0001)$, and there was a significant effect with the pretreatment of nor-BNI $(p=0.0146)$ but not AM251 $(p>0.9999)$.

A second experiment had SalA administered via i.pl. injection at the same paw as the $2 \%$ formaldehyde. There was a significant effect of treatment $\left[F_{(2,15)}=25.95, p<0.0001\right]$, time $\left[F_{(2,180)}=\right.$ 18.7, $p<0.0001]$ and interaction of treatment $x$ time $\left[F_{(24,180)}=3.11, p<0.0001\right]$ (Figure 4.4E). Bonferroni post-tests showed that SalA reduced the formaldehyde-induced pain from 5-10 and 20-60 $\min (p<0.05)$, and at the $10 \mathrm{~min}$ time point SalA also reduced the effect of the vehicle $(p=0.0423)$. AUC analysis showed a significant effect of treatment for phase I $\left[F_{(2,15)}=25.89, p<0.0001\right]$ (Figure $4.4 \mathrm{~F}$ ) and phase II $\left[F_{(2,15)}=20.03, p<0.0001\right]$ (Figure 4.4G). SalA significantly reduced the effects of formaldehyde in phase I $(p<0.0001)$ and phase II $(p<0.0001)$. The paw oedema measurements showed a significant effect of treatment $\left[F_{(2,14)}=6.782, p=0.0087\right]$ (Figure 4.4H), however, there was no significant effect of SalA treatment on formaldehyde-induced paw oedema $(p>0.9999)$. 

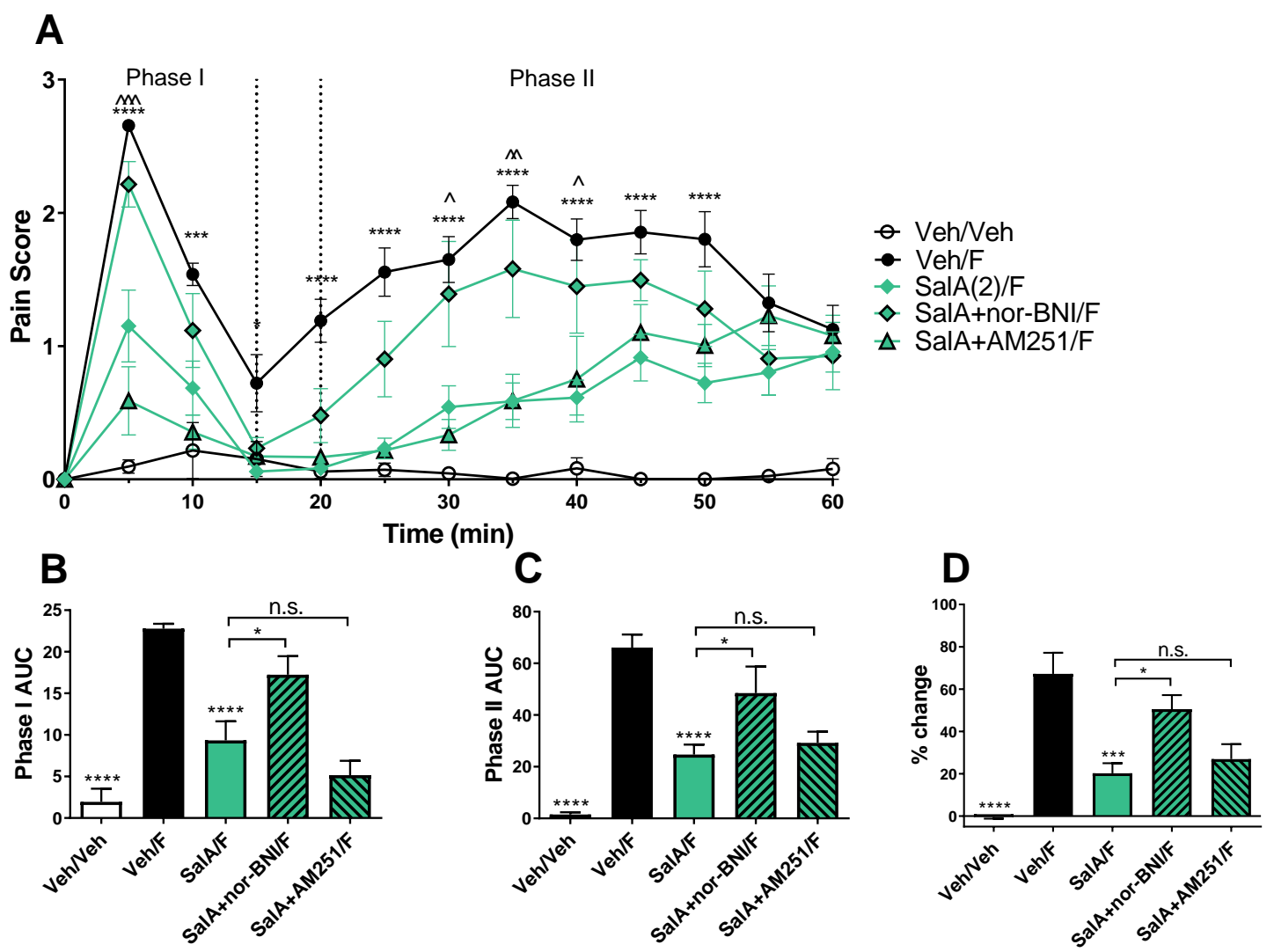

\section{E}
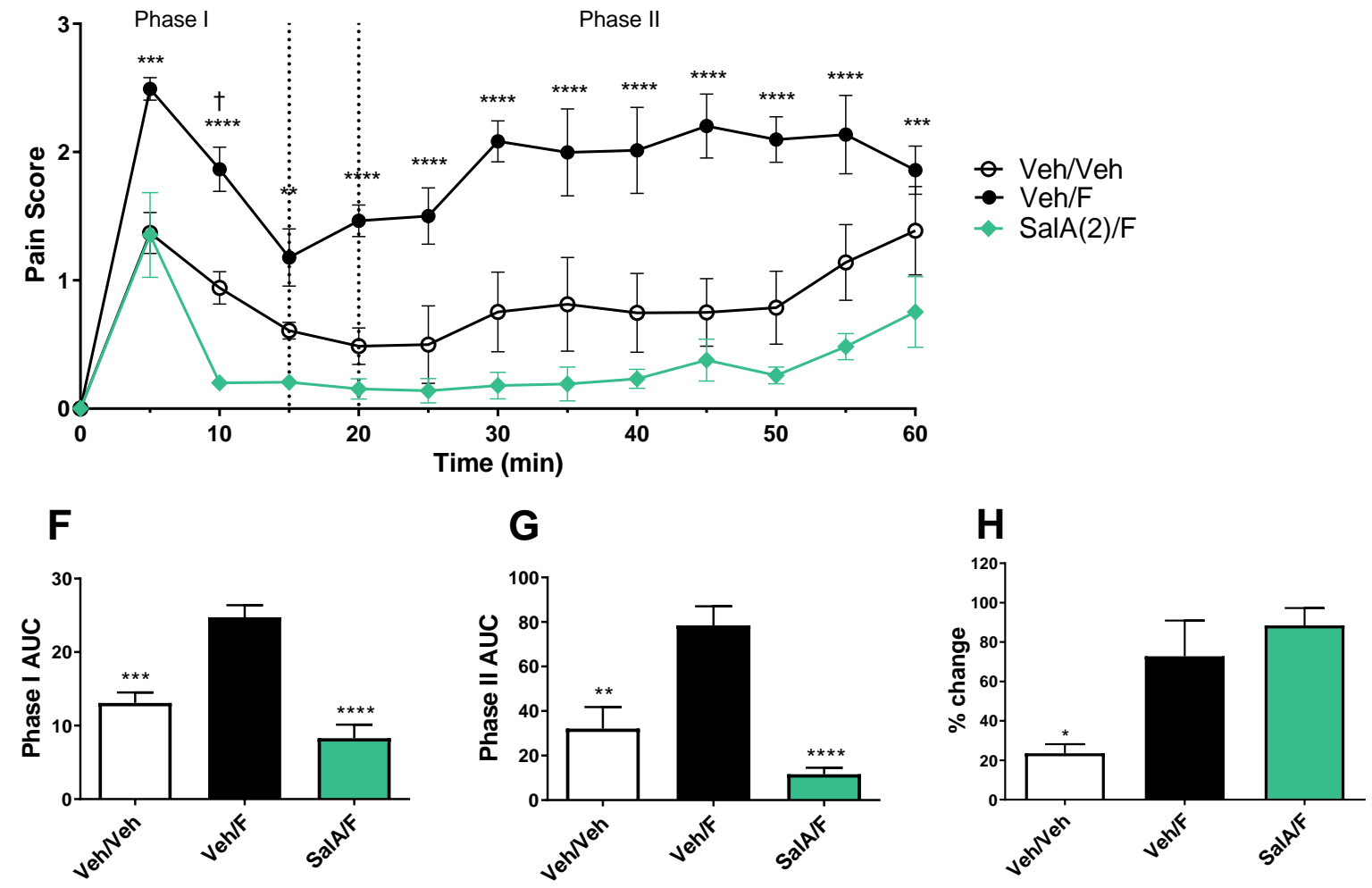


\section{Figure 4.4: Antinociceptive effect of SalA in the intraplantar $2 \%$ formaldehyde assay}

(A) Time course of pain behaviour following intraplantar $2 \%$ formaldehyde injection into the right hind paw. SalA $(2 \mathrm{mg} / \mathrm{kg}$ i.p.) treatment showed a significant reduction in pain compared to the vehicle/formaldehyde treated control.

(B-C) Area under the curve (AUC) was calculated for phase I nociceptive pain (0-15 min, B) and phase II inflammatory pain (20-60 min, C). SalA showed a significant reduction in both phases of pain compared to the vehicle/formaldehyde treatment. The antagonist nor-BNI treatment reversed the effects of SalA.

(D) The percentage change in the paw oedema depth revealed a significant difference between the vehicle/formaldehyde control and SalA. The antagonist nor-BNI reversed this effect.

(E) Time course of pain behaviour following intraplantar $2 \%$ formaldehyde injection into the right hind paw. SalA ( $2 \mathrm{mg} / \mathrm{kg}$ i.pl.) treatment showed a significant reduction in pain compared to the vehicle/formaldehyde treated control.

(F-G) AUC analysis showed SalA significantly reduced phase I (F) and phase II (G) pain.

(H) The percentage change in the paw oedema depth revealed no significant difference between the vehicle/formaldehyde control and SalA.

(A, E) Two-way repeated measures ANOVA with Bonferroni post-tests. (B-D, F-H) One-way ANOVA with Bonferroni post-tests. ${ }^{*} p<0.05,{ }^{* *} p<0.01,{ }^{* * *} p<0.001,{ }^{* * * *} p<0.0001$ for SalA vs. vehicle/formaldehyde control. $\wedge p<0.05, \wedge \wedge p<0.01, \wedge \wedge \wedge p<0.001$ for SalA vs. SalA + nor-BNI. $+p<$ 0.05 for SalA vs vehicle/vehicle. $n$.s. $=$ not significant. Values presented as mean $\pm S E M, n=13$ for vehicle/formaldehyde control group (i.p. experiment) and SalA ( $2 \mathrm{mg} / \mathrm{kg}$ i.p.), all other groups $n=6$. Number in brackets indicates dose in $\mathrm{mg} / \mathrm{kg}$ and $\mathrm{F}=$ formaldehyde i.pl. administration. 


\subsubsection{The effect of 16-Ethynyl SalA in the intraplantar $2 \%$ formaldehyde assay}

The novel KOPr agonist, 16-Ethynyl SalA, was tested via i.p. injection in the intraplantar formaldehyde assay, and two-way repeated measures ANOVA showed a significant effect of treatment $\left[F_{(4,26)}=26.06\right.$, $p<0.0001]$, time $\left[F_{(12,312)}=35.97, p<0.0001\right]$ and interaction of treatment $x$ time $\left[F_{(48,312)}=4.324, p<\right.$ 0.0001 ] (Figure 4.5A). The $2 \mathrm{mg} / \mathrm{kg}$ dose had a significant antinociceptive effect from 5-10 min, 25-45 $\min$ and $60 \mathrm{~min}(p<0.05)$, and the $1 \mathrm{mg} / \mathrm{kg}$ dose had a significant effect at $25-35 \mathrm{~min}(p<0.05)$ compared to the vehicle/formaldehyde treatment. The KOPr antagonist nor-BNI reversed the effect of the $2 \mathrm{mg} / \mathrm{kg}$ dose at 5 and 25-40 $\mathrm{min}(p<0.05)$. One-way ANOVA analysis of the AUC data showed a significant effect of treatment in phase I $\left[F_{(4,26)}=24.58, p<0.0001\right]$ (Figure 4.5B) and phase II $\left[F_{(4,26)}\right.$ $=21.1, p<0.0001$ ] (Figure 4.5C). For the nociceptive phase I pain, 16-Ethynyl SalA reduced the pain at $2 \mathrm{mg} / \mathrm{kg}(p=0.0001)$ but not $1 \mathrm{mg} / \mathrm{kg}(p=0.1019)$. Nor-BNI significantly reversed the effect of the 2 $\mathrm{mg} / \mathrm{kg}$ dose $(p=0.0054)$. In the inflammatory phase, the pain was reduced by the $2 \mathrm{mg} / \mathrm{kg}(p<0.0001)$ and $1 \mathrm{mg} / \mathrm{kg}$ dose $(p=0.0200)$, and nor-BNI reversed the effect of $2 \mathrm{mg} / \mathrm{kg}$ dose $(p=0.0012)$. The treatments also had a significant effect on the level of paw oedema $\left[F_{(4,27)}=12.79, p<0.0001\right]$ (Figure 4.5D), with the $2 \mathrm{mg} / \mathrm{kg}$ dose of 16-Ethynyl SalA showing a reduction in the level of swelling ( $p=$ 0.0096), which was reversed by pre-treatment with nor-BNI $(p=0.0009)$, however, the $1 \mathrm{mg} / \mathrm{kg}$ dose did not have an effect $(p>0.9999)$.

The antinociceptive effects of 16-Ethynyl SalA were further assessed by administering via i.pl. injection, and two-way repeated measures ANOVA showed a significant effect of treatment $\left[F_{(2,15)}=\right.$ $25.3, p<0.0001]$, time $\left[F_{(12,180)}=17.89, p<0.0001\right]$ and interaction of treatment $x$ time $\left[F_{(24,180)}=3.693\right.$, $p<0.0001$ ] (Figure 4.5E). 16-Ethynyl SalA ( $2 \mathrm{mg} / \mathrm{kg}$ ) had a significant effect from 5-60 min compared to the vehicle/formaldehyde control. For this route of administration, one-way ANOVA showed there was a significant effect of treatment in phase I $\left[F_{(2,15)}=26.47, p<0.0001\right]$ (Figure 4.5F), phase II $\left[F_{(2,15)}\right.$ $=21.12, p<0.0001$ ] (Figure 4.5G) and the paw oedema measurements $\left[F_{(2,14)}=6.501, p=0.0101\right]$ (Figure 4.5H). 16-Ethynyl SalA significantly reduced pain in phase I $(p<0.0001)$ and phase II $(p<0.0001)$ but did not reduce the level of paw oedema compared to the vehicle/formaldehyde treatment group $(p>0.9999)$. 

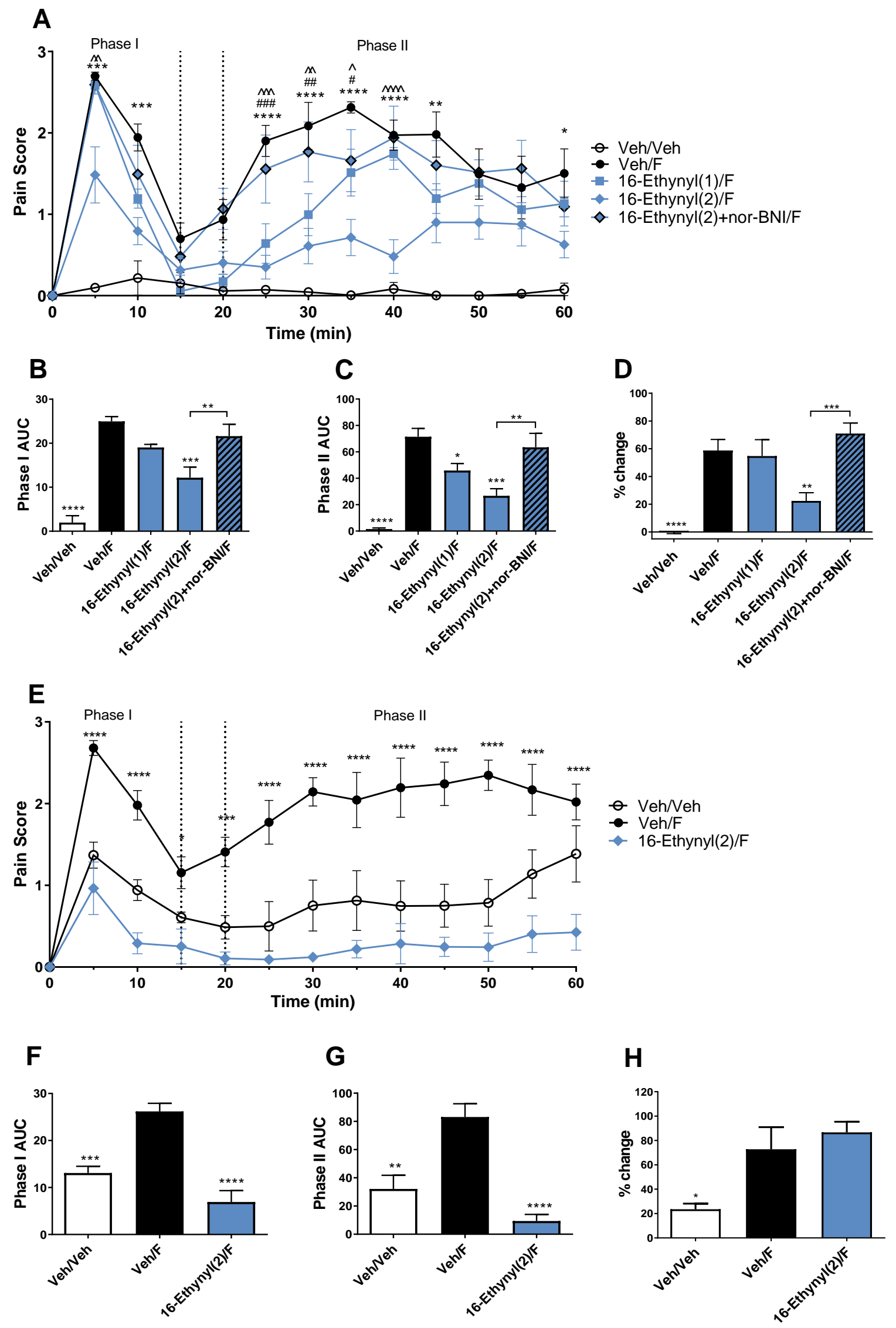


\section{Figure 4.5: Antinociceptive effect of 16-Ethynyl SalA in the intraplantar $2 \%$ formaldehyde test}

(A) Time course of pain behaviour following intraplantar $2 \%$ formaldehyde injection into the right hind paw. 16-Ethynyl SalA (1-2 mg/kg i.p.) treatment showed a significant reduction in pain compared to the vehicle/formaldehyde treated control group.

(B-C) Area under the curve (AUC) was calculated for phase I nociceptive pain (0-15 min, B) and phase II inflammatory pain (20-60 min, C). 16-Ethynyl SalA showed a significant reduction in both phases of pain compared to the vehicle/formaldehyde treatment, which was reversed using the KOPr antagonist nor-BNI.

(D) The percentage change in the paw oedema depth revealed a significant difference between the vehicle/formaldehyde treatment and the $2 \mathrm{mg} / \mathrm{kg}$ dose of 16-Ethynyl SalA. The antagonist nor-BNI reversed the effect of the $2 \mathrm{mg} / \mathrm{kg}$ dose.

(E) Time course of pain behaviour following intraplantar $2 \%$ formaldehyde injection into the right hind paw. 16-Ethynyl SalA ( $2 \mathrm{mg} / \mathrm{kg}$ i.pl.) treatment showed a significant reduction in pain compared to the vehicle/formaldehyde treated control.

(F-G) AUC analysis showed 16-Ethynyl SalA significantly reduced in phase I (F) and II (G) pain compared to the vehicle/formaldehyde-treated positive control.

(H) The percentage change in the paw oedema depth revealed no significant difference between the vehicle/formaldehyde control and 16-Ethynyl SalA.

(A, E) Two-way repeated measures ANOVA with Bonferroni post-tests. (B-D, F-H) One-way ANOVA with Bonferroni post-tests. ${ }^{*} p<0.05,{ }^{* *} p<0.01$ for $2 \mathrm{mg} / \mathrm{kg}$ doses vs. vehicle control. ${ }^{*} p<0.05,{ }^{* *} p$ $<0.01,{ }^{* * *} p<0.001,{ }^{* * * *} p<0.0001$ for $2 \mathrm{mg} / \mathrm{kg}$ dose vs. vehicle/formaldehyde control. ${ }^{\#} p<0.05$, \#\#

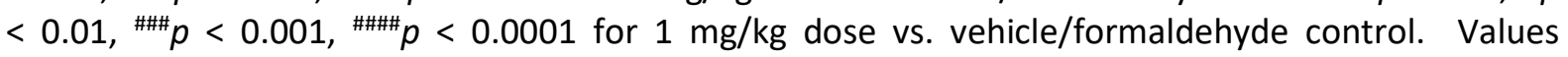
presented as mean \pm SEM, $n=6-7$ and $n=5$ for nor-BNI antagonist group. Number in brackets indicates dose in $\mathrm{mg} / \mathrm{kg}$ and $\mathrm{F}=$ formaldehyde i.pl. administration. 


\subsubsection{The effect of 16-Bromo SalA in the intraplantar $2 \%$ formaldehyde assay}

The novel KOPr agonist, 16-Bromo SalA, was tested via i.p. administration in the intraplantar formaldehyde model, and two-way repeated measures ANOVA showed a significant effect of treatment $\left[F_{(4,28)}=43.75, p<0.0001\right]$, time $\left[F_{(12,336)}=58.07, p<0.0001\right]$ and interaction of treatment $x$ time $\left[F_{(48,336)}=5.046, p<0.0001\right]$ (Figure $\left.4.6 \mathrm{~A}\right)$. Over the time course, Bonferroni post-tests showed the $2 \mathrm{mg} / \mathrm{kg}$ dose of $16-$ Bromo SalA reduced pain at $30 \mathrm{~min}(p=0.0207)$ and the $1 \mathrm{mg} / \mathrm{kg}$ dose reduced at $25(p=0.0269)$ and $30 \mathrm{~min}(p=0.0006)$. One-way ANOVA analysis showed a significant effect of treatment for the AUC analysis of phase I $\left[F_{(4,28)}=46.59, p<0.0001\right]$ (Figure $4.6 \mathrm{~B}$ ) and phase II $\left[F_{(4,28)}=\right.$ 35.8, $p<0.0001$ ] (Figure 4.6C), as well as for the paw oedema measurements $\left[F_{(4,28)}=11.06, p<\right.$ 0.0001] (Figure 4.6D). 16-Bromo SalA did not show a significant reduction in phase I or phase II pain for either dose $(p>0.05)$. However, there was a reduction in the level of paw oedema at $1 \mathrm{mg} / \mathrm{kg}(p=$ $0.0083)$ and $2 \mathrm{mg} / \mathrm{kg}(p=0.0218)$, and the pre-treatment of nor-BNI significantly reversed the effects of the $2 \mathrm{mg} / \mathrm{kg}$ dose $(p=0.0044)$.

The local effects of 16-Bromo SalA were further tested by administering the compound via i.pl. administration, which showed a significant effect of treatment $\left[F_{(2,15)}=8.141, p=0.0040\right]$, time $\left[F_{(12,180)}\right.$ $=24.12, p<0.0001]$ and interaction of treatment $x$ time $\left[F_{(24,180)}=2.542, p=0.0003\right]$ (Figure 4.6E). 16Bromo SalA showed significant effects at $10,20-35$ and $55 \mathrm{~min}(p<0.05)$. There was a significant effect of treatment for the phase I AUC data $\left[F_{(2,15)}=11.21, p=0.0011\right]$ (Figure 4.6F), phase II AUC data $\left[F_{(2,15)}\right.$ $=6.507, p=0.0092]$ (Figure 4.6G) and the paw oedema measurements $\left[F_{(2,13)}=4.785, p=0.0277\right]$ (Figure 4.6H). 16-Bromo SalA produced a significant reduction in nociceptive pain $(p=0.0108)$, but not inflammatory pain ( $p=0.1153$ ) or paw oedema $(p=0.2260)$. 

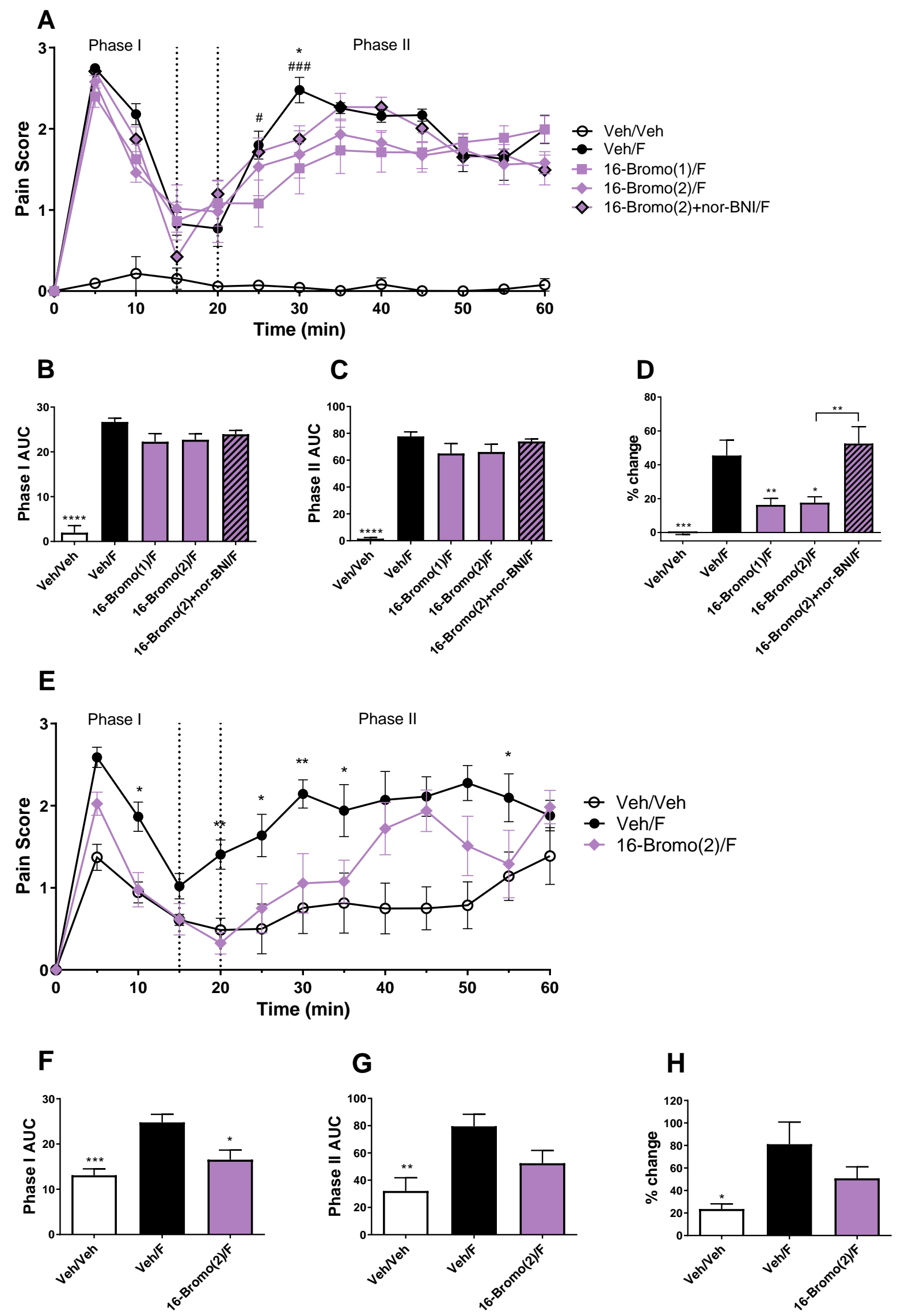


\section{Figure 4.6: Antinociceptive effect of 16 -Bromo SalA in the intraplantar $2 \%$ formaldehyde test}

(A) Time course of pain behaviour following intraplantar $2 \%$ formaldehyde injection into the right hind paw. 16-Bromo SalA (1-2 mg/kg i.p.) treatment showed a significant reduction in pain compared to the vehicle/formaldehyde treated positive control group.

(B-C) Area under the curve (AUC) was calculated for phase I nociceptive pain (0-15 min, B) and phase II inflammatory pain (20-60 min, C). 16-Bromo SalA did not show a significant reduction in both phases of pain compared to the vehicle/formaldehyde control group.

(D) The percentage change in the paw oedema depth revealed a significant difference between the positive control and both doses of 16-Bromo SalA. The antagonist nor-BNI reversed the effect of the $2 \mathrm{mg} / \mathrm{kg}$ dose.

(E) Time course of pain behaviour following intraplantar $2 \%$ formaldehyde injection into the right hind paw. 16-Bromo SalA ( $2 \mathrm{mg} / \mathrm{kg}$ i.pl.) treatment showed a significant reduction in pain compared to the vehicle/formaldehyde treated controls.

(F-G) AUC analysis of 16-Bromo SalA showed a significant reduction in phase I pain compared to the vehicle/formaldehyde treated controls.

(H) The percentage change in the paw oedema depth revealed no significant difference between the vehicle/formaldehyde group and 16-Bromo SalA treatment.

(A, E) Two-way repeated measures ANOVA with Bonferroni post-tests. (B-D, F-H) One-way ANOVA with Bonferroni post-tests. ${ }^{*} p<0.05,{ }^{* *} p<0.01$ for $2 \mathrm{mg} / \mathrm{kg}$ doses vs. vehicle/formaldehyde control. ${ }^{\#} p<0.05, " \# p<0.01, \# \#<0.001$ for $1 \mathrm{mg} / \mathrm{kg}$ doses vs. vehicle/formaldehyde control. Values presented as mean \pm SEM, $n=6-8$. Number in brackets indicates dose in $\mathrm{mg} / \mathrm{kg}$ and $F=$ formaldehyde i.pl. administration. 


\subsubsection{Neutrophil infiltration into inflamed footpad tissue}

To further assess the anti-inflammatory effects of the KOPr agonists, we used flow cytometry as a preliminary screen to understand the effect of the KOPr agonists on inflammatory cell populations. ICI 204,448 was used as a known KOPr agonist with peripherally-restricted antinociceptive effects (Shaw et al., 1989; Inan \& Cowan, 2004). One-way ANOVA analysis showed a significant effect of treatment $\left[F_{(3,55)}=5.387, p=0.0016\right]$ for the neutrophil population (Figure $\left.4.7 \mathrm{~A}\right)$. Administration $(2 \mathrm{mg} / \mathrm{kg}$ i.p.) of SalA $(p=0.0387)$ and $\mathrm{ICI} 204,448(p=0.0045)$ both lead to a significant reduction in neutrophil cell counts in the inflamed tissue. The monocyte analysis showed a significant effect of treatment $\left[F_{(3,53)}=\right.$ 3.639, $p=0.0184]$, however, only the vehicle/vehicle and vehicle/formaldehyde controls were significantly different ( $p=0.0229$; Figure 4.7B). There was no significant effect of treatment in the macrophage $\left[F_{(3,53)}=1.689, p=0.1804\right]$ (Figure $4.7 C$ ) or dendritic cell populations $\left[F_{(3,55)}=0.0910, p=\right.$ 0.9647] (Figure 4.7D).

Following on from the flow cytometry experiment, the neutrophil cell counts were further quantified in H\&E stained footpad sections. The tissue from this portion of the study was collected by Master's student Nitin Kumar, cryoprotected and stored in the $-80^{\circ} \mathrm{C}$ freezer and sectioned using a cryostat microtome. One-way ANOVA revealed a significant effect of treatment $\left[F_{(4,31)}=43.37, p<0.0001\right]$ (Figure 4.8). Administration ( $2 \mathrm{mg} / \mathrm{kg}$ i.p.) of ICl 204,448 $(p<0.0001)$, SalA $(p<0.0001)$ and $\beta$-THP SalB $(p<0.0001)$ all lead to a significant reduction in neutrophil counts compared to the vehicle/formaldehyde treatment. 
A

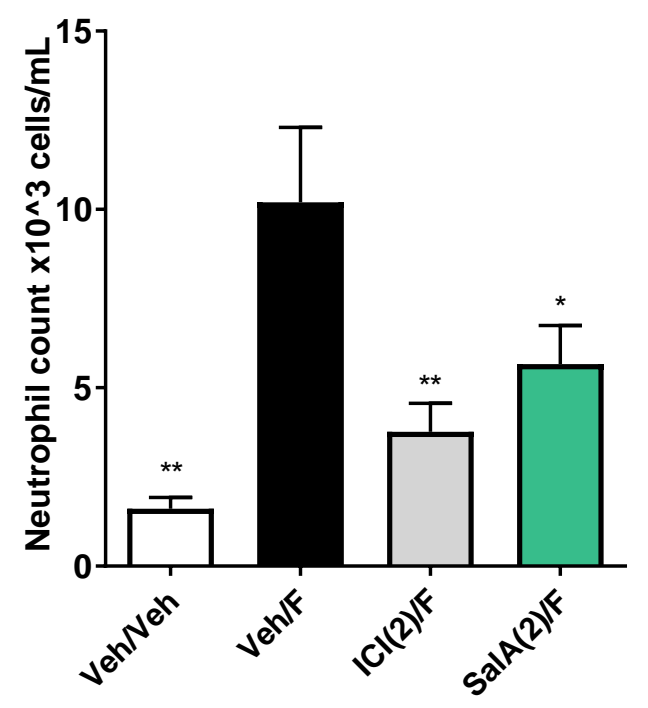

C

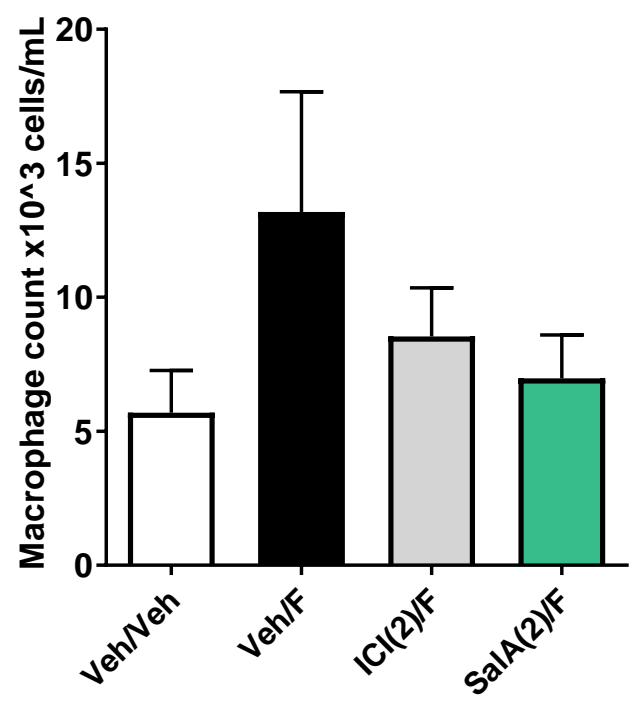

\section{B}
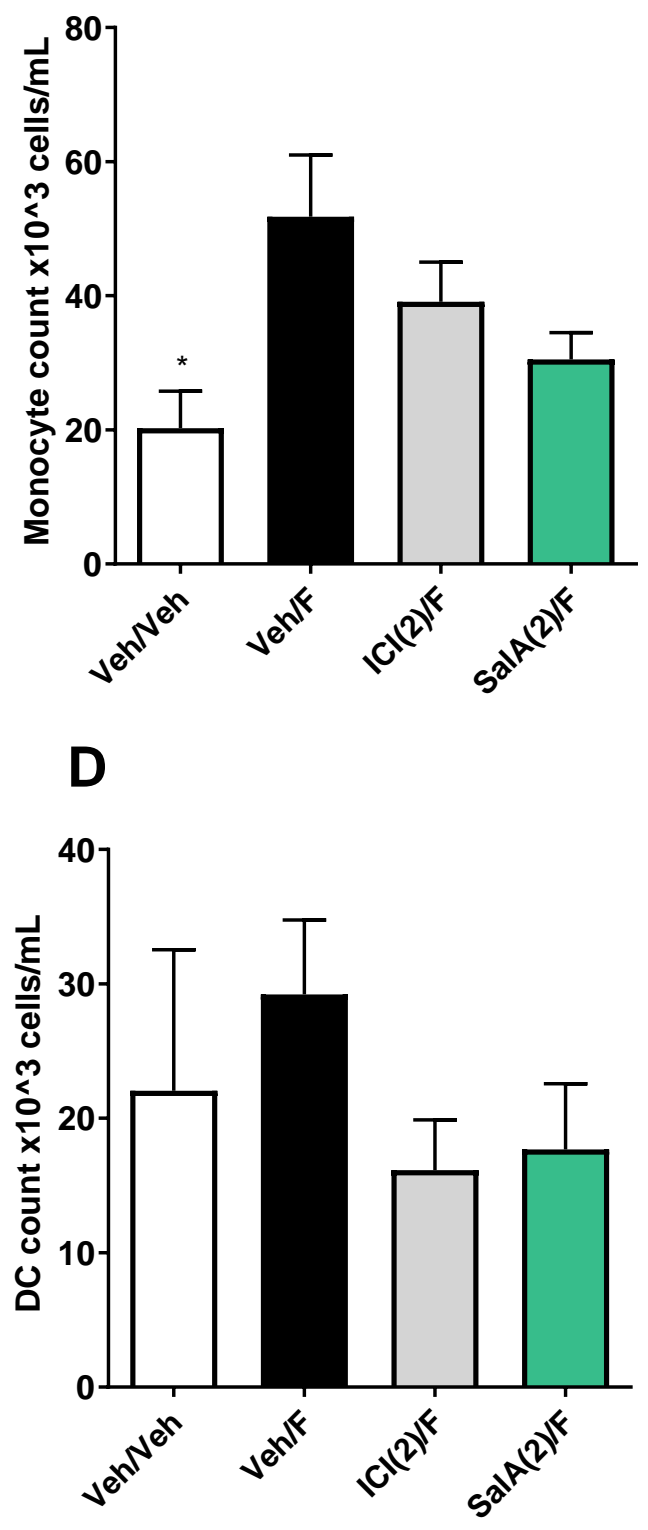

Figure 4.7: KOPr agonists only reduce neutrophil cell populations in inflamed tissue

The immune cell populations in footpad tissue were evaluated using flow cytometry following the administration of $\mathrm{ICl} 204,448$ or SalA (2 mg/kg i.p.) (A) Both KOPr agonists reduced the level of neutrophils in the tissue. (B-D) The KOPr agonists had no effect on macrophage (B), monocyte (C) or dendritic cell (D) counts. One-way ANOVA with Bonferroni post-tests. ${ }^{*} p<0.05,{ }^{* *} p<0.01$ vs. vehicle/formaldehyde control. Number in brackets indicates dose in $\mathrm{mg} / \mathrm{kg}$ and $\mathrm{F}=$ formaldehyde i.pl. administration. Values presented as mean \pm SEM, $n=13-23$ and $n=8$ for vehicle control. Data also presented in Paton et al. (2017). 

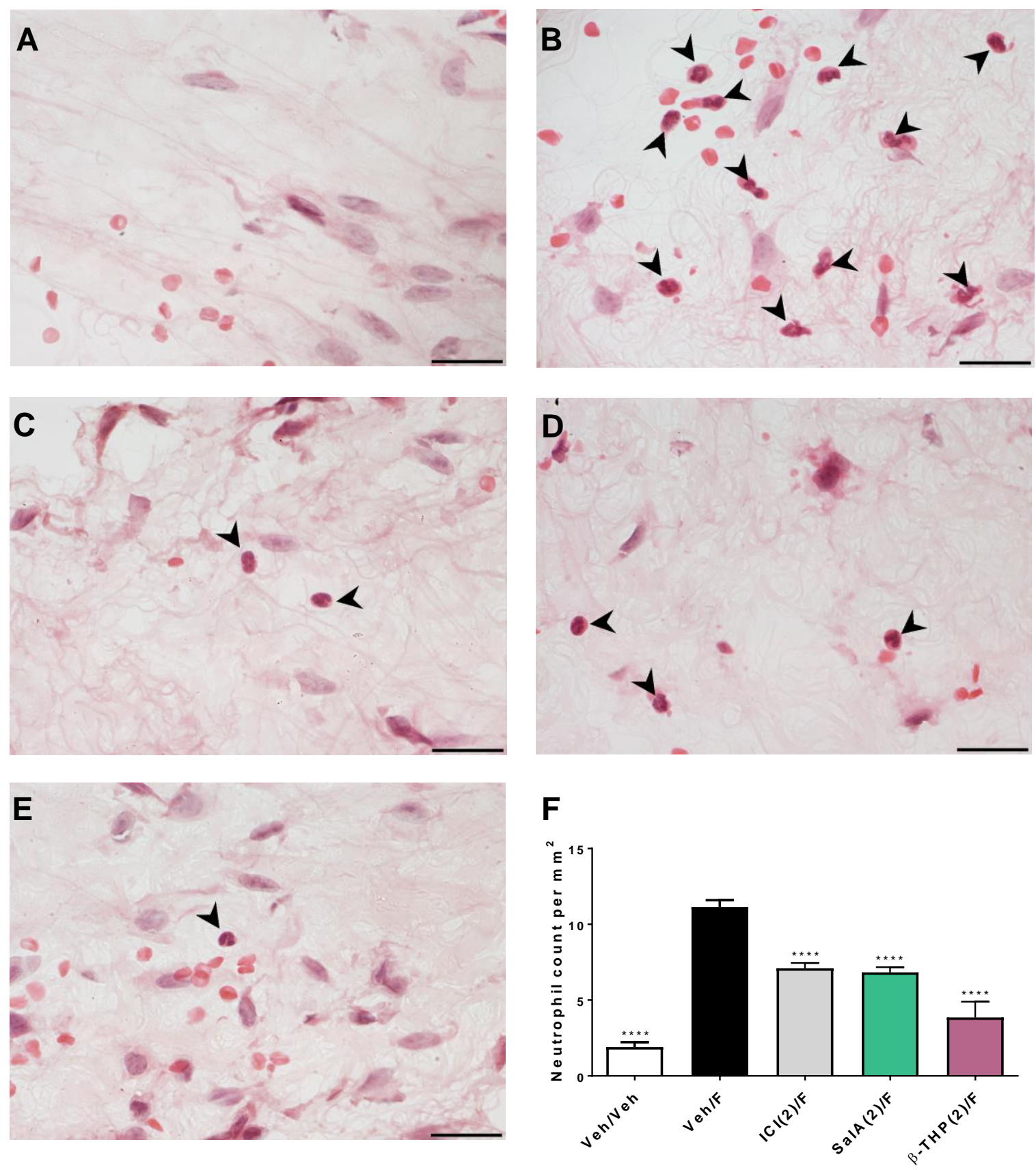

Figure 4.8: KOPr agonists reduce neutrophil counts in H\&E stained footpad tissue sections

(A-E) Representative images showing neutrophil infiltration into the footpad tissue following the intraplantar formaldehyde test with the treatments (A) vehicle/vehicle, (B) vehicle/formaldehyde, (C) ICI 204,448 (2 mg/kg)/formaldehyde, (D) SalA (2 mg/kg)/formaldehyde and (E) $\beta$-THP SalB (2 $\mathrm{mg} / \mathrm{kg}$ )/formaldehyde. Markers indicate neutrophils. Images were taken at $100 x$ magnification. Scale bar $=20 \mu \mathrm{m}$. (F) Neutrophil counts in H\&E stained footpad tissue, showing all KOPr agonists reduced the infiltration of neutrophils compared to the vehicle/formaldehyde control. One-way ANOVA with Bonferroni post-tests. ${ }^{* * * *} p<0.0001$ compared to the vehicle/formaldehyde treatment group. Values presented as mean \pm SEM. $n=6-12$. Data also presented in Paton et al. (2017). 
Since the H\&E stained sections showed a similar result to the flow cytometry experiment, I sought to analyse the tissue collected from the intraplantar $2 \%$ formaldehyde assays presented earlier in this chapter (Figure 4.4, 4.5 and 4.6). However, to improve the cell morphology of the tissue sections, paraffin-embed sections were used rather than the previous procedure which used frozen sections due to the samples being stored in the $-80^{\circ} \mathrm{C}$ freezer. The aim was to replicate the results for SalA, as well as generate results for the novel analogues 16-Ethynyl SalA and 16-Bromo SalA and include the animals that received the antagonist treatment schemes.

One-way ANOVA revealed there was a significant effect of treatment $\left[F_{(7,24)}=36.83, p<0.0001\right]$ (Figure 4.9). The findings from the previous studies were further confirmed as SalA significantly reduced the number of neutrophils in the tissue sections $(p<0.0001)$. The novel agonists, $16-$ Ethynyl SalA and $16-$ Bromo SalA, also both reduced the neutrophil counts compared to the vehicle/formaldehyde control $(p<0.0001)$. Pre-treatment with nor-BNI reversed the effects of SalA $(p<0.0001), 16$-Ethynyl SalA ( $p$ $=0.0007)$ and $16-$ Bromo SalA $(p<0.0001)$, indicating that the reduction in the neutrophils in the tissue was KOPr-mediated. 


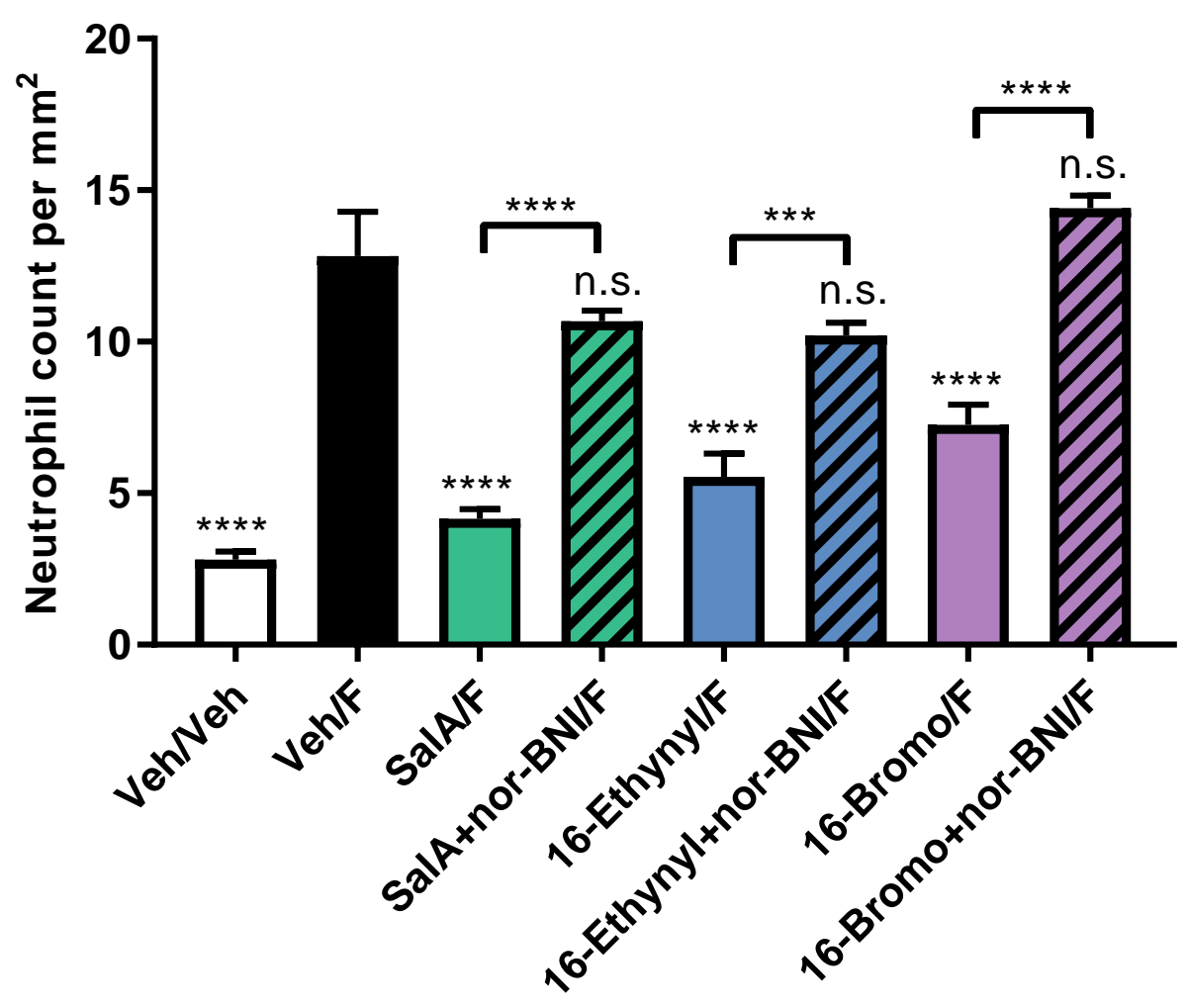

Figure 4.9: Novel SalA analogues reduce neutrophil counts in paraffin-embedded footpad tissue sections

Neutrophil counts in paraffin-embedded, H\&E stained footpad tissue. SalA, 16-Ethynyl SalA and 16Bromo SalA reduced the level of neutrophil infiltration into the footpad tissue compared to the vehicle/formaldehyde positive control. Pre-treatment with the KOPr antagonist nor-binaltorphimine (nor-BNI) reversed the effect of all the KOPr agonists. One-way ANOVA with Bonferroni post-tests. $* * * * p<0.0001$, n.s. $=$ not significant, compared to the vehicle/formaldehyde treatment group. Values presented as mean \pm SEM. $n=4$. 


\subsubsection{Rotarod performance assay}

The rotarod performance test measures motor coordination and is frequently used to assess the sedative effects of novel drugs. The KOPr agonists were compared to a standard dose of morphine (10 $\mathrm{mg} / \mathrm{kg}$ i.p.). Morphine significantly reduced the time spent on the rotarod apparatus for 15-120 min $(p<0.05)$, and this data has been previously published (Crowley et al., 2016). Each of the KOPr agonists was compared to the morphine results, to compare the relative sedative effects.

Two-way repeated measures ANOVA showed that the SalA cohort had a significant effect of treatment $\left[F_{(2,15)}=10.21, p=0.0016\right]$, time $\left[F_{(8,120)}=20.52, p<0.0001\right]$ and interaction of treatment $x$ time $\left[F_{(16,120)}\right.$ $=11.41, p<0.0001$ ] (Figure 4.10A). Bonferroni post-tests showed a reduction in the latency at 15-30 $\min (p<0.05)$. At $15 \mathrm{~min}$, SalA induced more sedation than morphine $(p<0.0001)$. However, at $30-$ 120 min morphine was more sedative $(p<0.05)$. One-way ANOVA showed a significant effect of treatment for the AUC analysis $\left[F_{(2,15)}=10.45, p=0.0014\right]$ (Figure 4.10B), with Bonferroni post-tests showing no significant effect between SalA and vehicle $(p=0.2553)$, however, there was a significant effect when morphine was compared to both vehicle $(p=0.0012)$ and SalA $(p=0.0493)$.

For the 16-Ethynyl SalA experiment, two-way repeated measures ANOVA showed a significant effect of treatment $\left[F_{(3,20)}=8.036, p=0.0010\right]$, time $\left[F_{(8,160)}=17.01, p<0.0001\right]$ and interaction of treatment $x$ time $\left[F_{(24,160)}=3.783, p<0.0001\right]$ (Figure $\left.4.10 \mathrm{C}\right)$. The $1 \mathrm{mg} / \mathrm{kg}$ dose was sedative at $30 \mathrm{~min}(p=0.0049)$ and the $2 \mathrm{mg} / \mathrm{kg}$ dose at $15(p=0.0180)$ and $30 \mathrm{~min}(p=0.0234)$. Morphine had significantly more sedation at 45-120 min compared to the $1 \mathrm{mg} / \mathrm{kg}$ dose; and 45-60 and $120 \mathrm{~min}$ compared to the 2 $\mathrm{mg} / \mathrm{kg}$ dose $(p<0.05)$. The AUC analysis showed a significant effect of treatment $\left[F_{(3,20)}=8.147, p=\right.$ 0.0010] (Figure 4.10D). There was no significant difference between vehicle and both 16-Ethynyl SalA at $1 \mathrm{mg} / \mathrm{kg}(p>0.9999)$ and $2 \mathrm{mg} / \mathrm{kg}(p=0.8507)$, however, there was significant effect for morphine compared to the $1 \mathrm{mg} / \mathrm{kg}$ dose $(p=0.0065)$ and the $2 \mathrm{mg} / \mathrm{kg}$ dose of 16 -Ethynyl SalA $(p=0.0349)$.

The 16-Bromo SalA experiment showed a significant effect of treatment $\left[F_{(3,20)}=7.519, p=0.0015\right]$, time $\left[F_{(8,160)}=12.46, p<0.0001\right]$ and interaction of treatment $x$ time $\left[F_{(24,160)}=2.524, p=0.0003\right]$ (Figure 4.10E). Only the $1 \mathrm{mg} / \mathrm{kg}$ dose of $16-$ Bromo SalA had a significant effect at $30 \mathrm{~min}(p=0.0199)$. Morphine had significantly more sedative effect compared to the $2 \mathrm{mg} / \mathrm{kg}$ dose at 30-60 min and 120 $\min (p<0.05)$; and compared to the $1 \mathrm{mg} / \mathrm{kg}$ dose at 60 and $120 \mathrm{~min}(p<0.05)$. The AUC analysis showed a significant effect of treatment $\left[F_{(3,20)}=7.593, p=0.0014\right]$ (Figure 4.10F). Bonferroni posttests showed no significant effect between vehicle and both the $1 \mathrm{mg} / \mathrm{kg}(p=0.5406)$ and $2 \mathrm{mg} / \mathrm{kg}(p$ $>0.9999$ ) dose of 16-Bromo SalA. There was a significant effect between morphine and the $2 \mathrm{mg} / \mathrm{kg}$ dose of 16-Bromo SalA ( $p=0.0138)$, but no effect between morphine and the $1 \mathrm{~m} / \mathrm{kg}$ dose $(p=0.0691)$. 
A

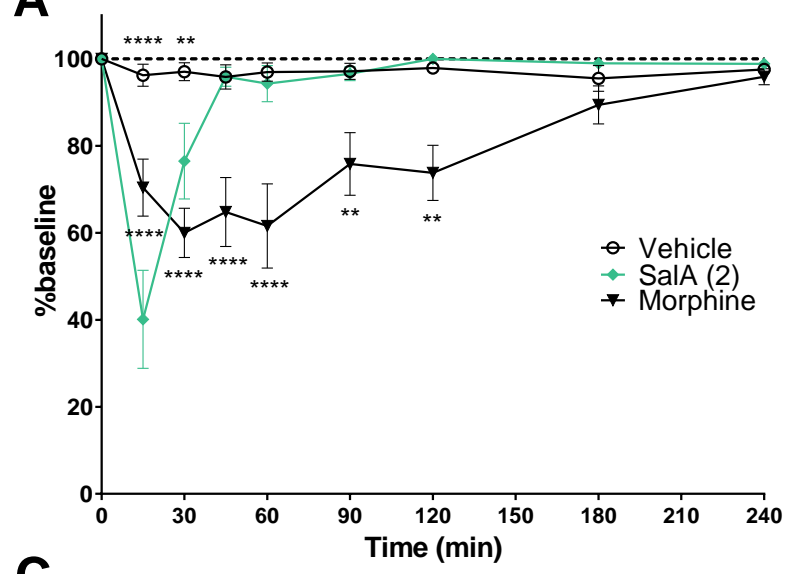

C

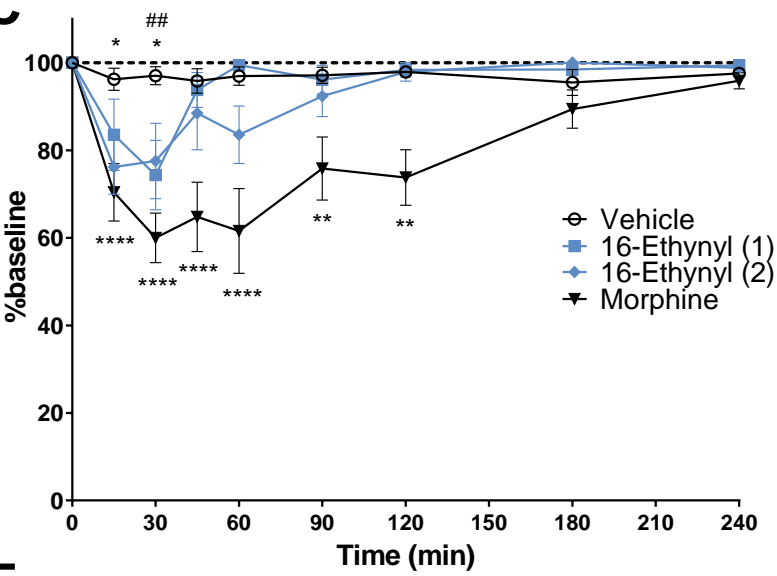

$\mathbf{E}$

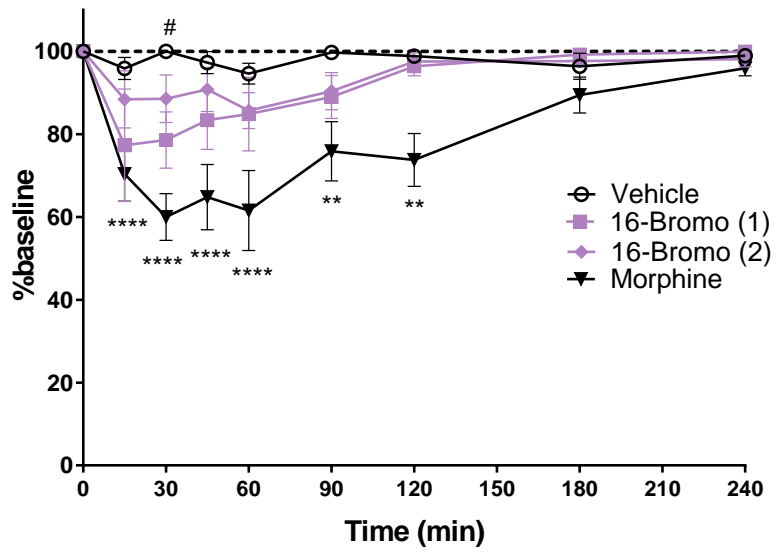

B

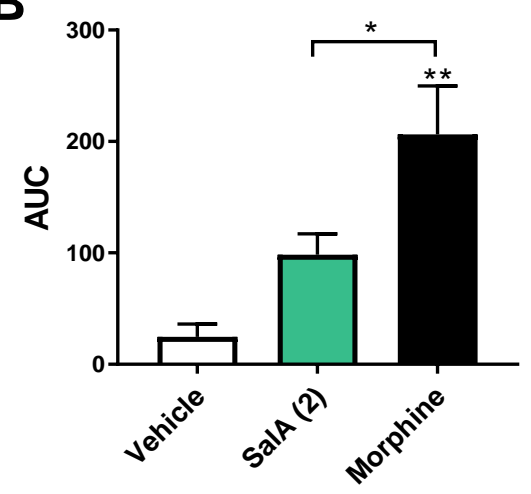

D

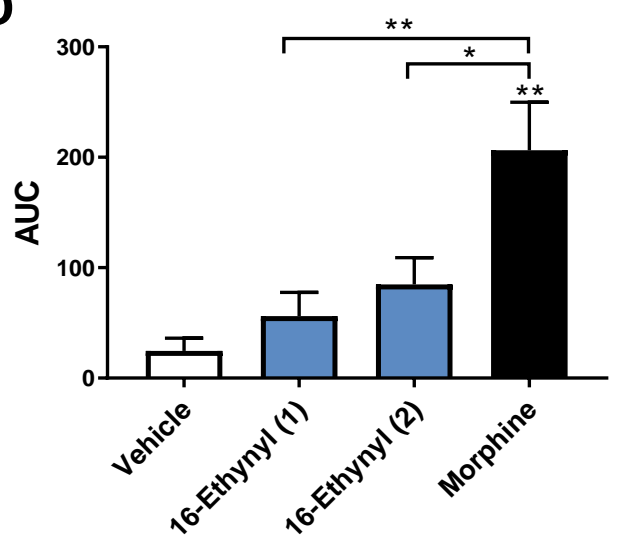

$\mathbf{F}$

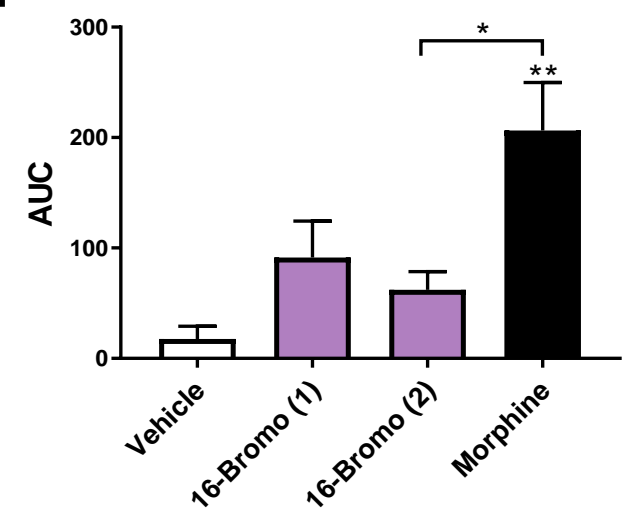

Figure 4.10: KOPr agonists cause motor incoordination in the rotarod performance assay

Using a rotarod apparatus set to accelerate from $4-40 \mathrm{rpm}$ over $300 \mathrm{~s}$, morphine $(10 \mathrm{mg} / \mathrm{kg}$ i.p.) showed a significant decrease in motor coordination. (A) SalA showed a significant effect at 15-30 min. (B) Area under the curve (AUC) analysis showed no difference between vehicle and SalA. (C) 16Ethynyl showed a significant effect at 15-30 min. (D) AUC analysis showed no effect between the vehicle and both doses of 16-Ethynyl SalA. (E) 16-Bromo SalA had a significant impairment at $30 \mathrm{~min}$ at $1 \mathrm{mg} / \mathrm{kg}$. (F) AUC analysis showed no overall effect of 16-Bromo SalA on sedation when compared to vehicle. (A, C, E) Two-way repeated measures ANOVA, (B, D, F) One-way ANOVA, all with Bonferroni post-tests. ${ }^{*} p<0.05,{ }^{* *} p<0.01,{ }^{* * *} p<0.001,{ }^{* * *} p<0.0001$ for $2 \mathrm{mg} / \mathrm{kg}$ dose (above) or morphine (below) vs. vehicle. ${ }^{\#} p<0.05,{ }^{\# \#} p<0.01,{ }^{\# \#} p<0.001$ for $1 \mathrm{mg} / \mathrm{kg}$ dose vs. vehicle. Values presented as mean $\pm S E M, n=6$. Morphine dataset also appears in Crowley et al. (2016). 


\subsubsection{Core body temperature}

The core body temperature was measured to assess the effect of the KOPr agonists on thermoregulation. Previous work from the Kivell laboratory has shown that SalA caused a decrease in core body temperature from 20-60 $\mathrm{min}$ at $2 \mathrm{mg} / \mathrm{kg}$ (Kumar, 2014). Two-way repeated measures ANOVA showed that 16-Ethynyl SalA had no effect of treatment $\left[F_{(2,15)}=1.098, p=0.3590\right]$, time $\left[F_{(4,60)}\right.$ $=1.532, p=0.2043]$ or interaction of treatment $x$ time $\left[F_{(8,60)}=1.115, p=0.3666\right]$ (Figure 4.11A). The AUC analysis also showed no significant effect of treatment $\left[F_{(2,15)}=1.061, p=0.3706\right]$ (Figure 4.11B). However, for the 16-Bromo SalA cohort, there was a significant effect of treatment $\left[F_{(2,15)}=16.67, p=\right.$ $0.0002]$, time $\left[F_{(4,60)}=9.581, p<0.0001\right]$ and interaction of treatment $x$ time $\left[F_{(8,60)}=7.816, p<0.0001\right]$ (Figure 4.11C). The $2 \mathrm{mg} / \mathrm{kg}$ dose of 16-Bromo SalA significantly reduced core body temperature at $30-90 \mathrm{~min}$ and the $1 \mathrm{mg} / \mathrm{kg}$ dose at 60-90 $\mathrm{min}(p<0.05)$. The AUC analysis showed a significant effect of treatment $\left[F_{(2,15)}=17.16, p=0.0001\right]$ (Figure 4.11D) and Bonferroni post-tests showed 16-Bromo SalA had a significant effect at $1 \mathrm{mg} / \mathrm{kg}(p=0.0264)$ and $2 \mathrm{mg} / \mathrm{kg}(p<0.0001)$. Overall, this indicates that like the parent compound SalA, 16-Bromo SalA is having a hypothermic effect on the mice, whereas 16-Ethynyl SalA did not cause any change in thermoregulation. 
A

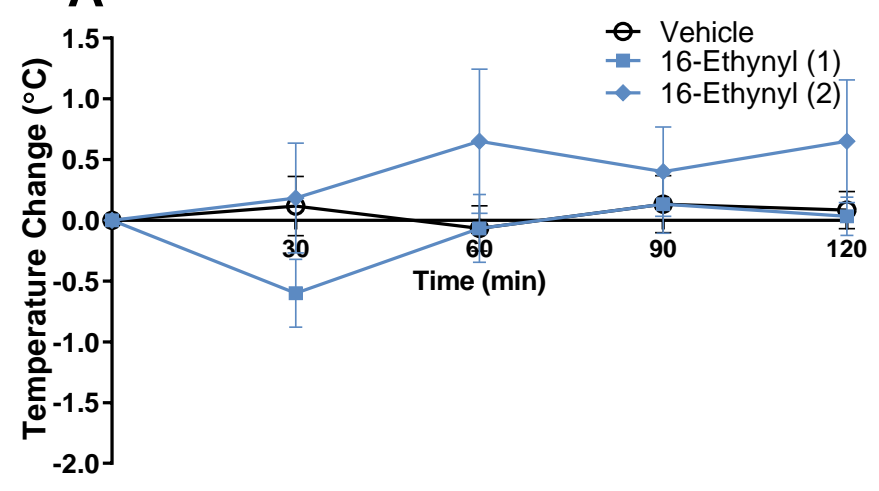

C

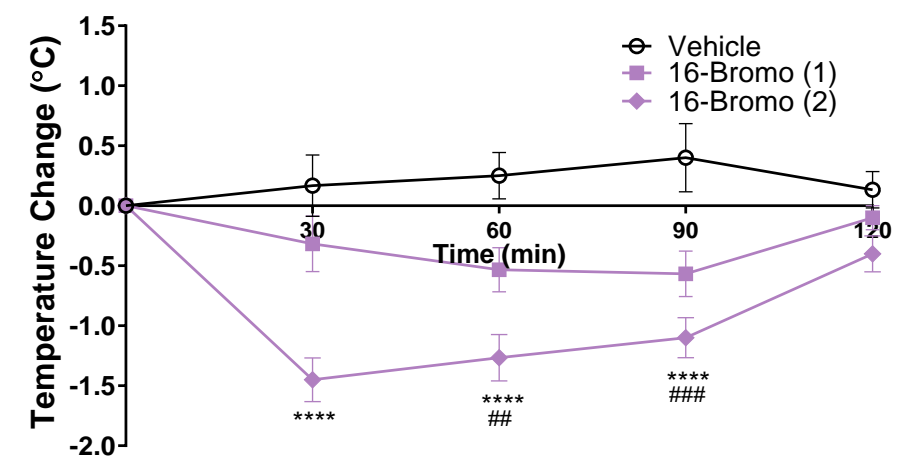

B

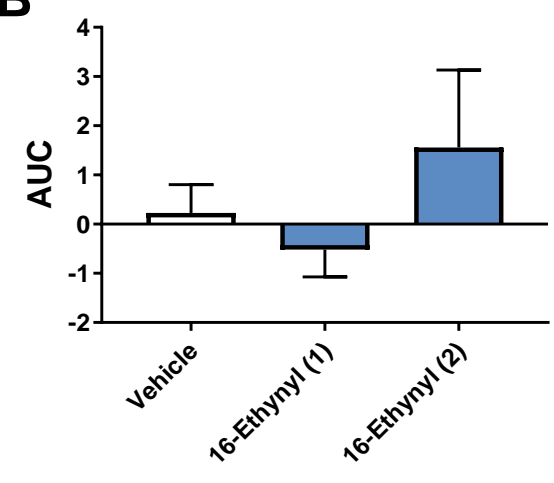

D

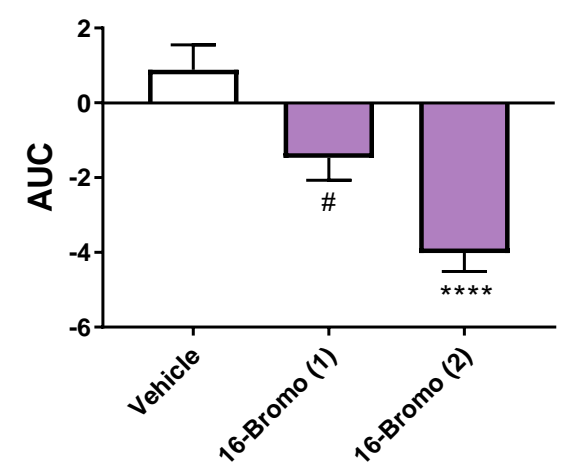

\section{Figure 4.11: 16-Bromo SalA reduced core body temperature in mice}

Mice were given an i.p. injection of either vehicle, 16-Ethynyl SalA or 16-Bromo SalA. Core body temperature change was calculated as the difference from the baseline measurements. (A) 16-Ethynyl SalA showed no effect on core body temperature. (B) Area under the curve (AUC) analysis showed no overall significant effect of 16-Ethynyl SalA. (C) 16-Bromo SalA significantly decreased the core body temperature up to 90 min. (D) AUC analysis showed a significant effect of 16-Bromo SalA for both doses. (A, C) Two-way ANOVA, (B, D) One-way ANOVA, all with Bonferroni post-tests. ${ }^{*} p<0.05,{ }^{* *} p<$ $0.01,{ }^{* * *} p<0.001,{ }^{* * * *} p<0.0001$ for $2 \mathrm{mg} / \mathrm{kg}$ doses vs. vehicle control. ${ }^{\#} p<0.05,{ }^{\# \#} p<0.01,{ }^{* \# \#} p<$ 0.001 for $1 \mathrm{mg} / \mathrm{kg}$ doses vs. vehicle control. Values presented as mean $\pm \mathrm{SEM}, \mathrm{n}=6$. 
The main effects of the acute KOPr agonist study are summarised in Table 4.4 below.

\begin{tabular}{|c|c|c|c|c|c|c|}
\hline & SalA (2) & $\begin{array}{c}\text { SalA }(2)+ \\
\text { nor-BNI }\end{array}$ & $\begin{array}{l}\text { 16-Ethynyl } \\
\text { (2) }\end{array}$ & $\begin{array}{l}\text { 16-Ethynyl (2) } \\
+ \text { nor-BNI }\end{array}$ & $\begin{array}{c}\text { 16-Bromo } \\
\text { (2) }\end{array}$ & $\begin{array}{l}\text { 16-Bromo (2) } \\
+ \text { nor-BNI }\end{array}$ \\
\hline \multicolumn{7}{|l|}{ Tail withdrawal } \\
\hline - Dose-response $\mathrm{ED}_{50}$ & $>$ U 50,488 & - & $>$ U 50,488 & - & $>$ U 50,488 & - \\
\hline - Dose-response $E_{\max }$ & $=U 50,488$ & - & $>$ U 50,488 & - & $=\mathrm{U} 50,488$ & - \\
\hline - Time course & $5-30 \mathrm{~min}$ & - & $5-60 \mathrm{~min}$ & - & $10-60 \mathrm{~min}$ & - \\
\hline \multicolumn{7}{|l|}{ Hotplate } \\
\hline - Time course & $15 \mathrm{~min}$ & - & $15-30 \mathrm{~min}$ & - & $15-30 \mathrm{~min}$ & - \\
\hline \multicolumn{7}{|l|}{$\begin{array}{l}\text { Formaldehyde model } \\
\text { (i.p. admin) }\end{array}$} \\
\hline - Phase I pain & $\downarrow$ & $\uparrow$ & $\downarrow$ & $\uparrow$ & n.e. & - \\
\hline - Phase II pain & $\downarrow$ & $\uparrow$ & $\downarrow$ & $\uparrow$ & n.e. & - \\
\hline - Paw oedema & $\downarrow$ & $\uparrow$ & $\downarrow$ & $\uparrow$ & $\downarrow$ & $\uparrow$ \\
\hline -Neutrophil infiltration & $\downarrow$ & $\uparrow$ & $\downarrow$ & $\uparrow$ & $\downarrow$ & $\uparrow$ \\
\hline \multicolumn{7}{|l|}{$\begin{array}{l}\text { Formaldehyde model } \\
\text { (i.pl. admin) }\end{array}$} \\
\hline - Phase I pain & $\downarrow$ & - & $\downarrow$ & - & $\downarrow$ & - \\
\hline - Phase II pain & $\downarrow$ & - & $\downarrow$ & - & n.e. & - \\
\hline - Paw oedema & n.e. & - & n.e. & - & n.e. & - \\
\hline
\end{tabular}

Table 4.4: Summary of the antinociceptive effects of the kappa opioid receptor agonists

Summary of the main effects of the KOPr agonists at the $2 \mathrm{mg} / \mathrm{kg}$ dose. $\downarrow$ indicates a reduction compared to the vehicle/formaldehyde treatment. $\uparrow$ indicates antagonist treatment significantly reversed the effects of the KOPr agonist. n.e. $=$ no effect of KOPr agonist compared to control. 


\subsection{Discussion}

There is a large proportion of the global population suffering from chronic pain without effective management, clearly showing there is a need for the development of more effective and safe medications. Specifically, the development of efficacious analgesics without addictive properties remains a high priority. KOPr agonists are unique, as contrary to MOPr agonists, KOPr agonists do not have abuse potential. SalA has proven antinociceptive and anti-inflammatory effects in vivo (Ansonoff et al., 2006; John et al., 2006; McCurdy et al., 2006; Aviello et al., 2011; Fichna et al., 2012; Guida et al., 2012; Rossi et al., 2016), however, a short duration of action (Prisinzano, 2005; Butelman et al., 2009; Teksin et al., 2009; Ranganathan et al., 2012), plus aversive (Zhang et al., 2005b) and anxiogenic side effects (Braida et al., 2009) have limited the clinical development. The novel analogues 16-Ethynyl SalA and 16-Bromo SalA have reduced side effects in vivo (Riley et al., 2014; Kivell laboratory unpublished data). This study was designed to understand the antinociceptive effect of these compounds in behavioural models of pain in mice.

\subsubsection{Antinociceptive effects in acute thermal models of pain}

The KOPr agonists were assessed for the antinociceptive dose-response effects using the acute warm water tail withdrawal assay. SalA and the novel analogues all had an increased potency (ED $D_{50}$ value) compared to the traditional agonist U50,488, however, there were no significant differences between any of the other KOPr agonists (Figure 4.1; Table 4.1). This shows that SalA and the novel analogues have an improved antinociceptive effect compared to the traditional agonist, although the novel analogues were no more potent than the parent compound SalA. The only compound which was more efficacious (higher $E_{\max }$ value) than U50,488 was 16-Ethynyl SalA (Figure 4.1; Table 4.3), which is an important improvement as the KOPr agonists display relatively modest effects in the warm water tail withdrawal assay in comparison to MOPr agonists. Comparison of the dose-response effects of $\mathrm{U} 50,488$ to morphine found that the $E D_{50}$ values were not significantly different, however, the efficacy of morphine was significantly higher at $100 \%$ compared to $19.7 \pm 4.6 \%$ for $U 50,488$ (for a full analysis, see Appendix C.2). This shows that the whilst the traditional KOPr agonist U50,488 has a similar potency to morphine, the overall antinociceptive benefit is considerably lower. These results are similar to findings in Simonin et al. (1998), where it was found that $\mathrm{KOPr}^{-/}$mice did not have any altered effect in the warm water $\left(50^{\circ} \mathrm{C}\right)$ tail withdrawal assay. Although a follow-up study found that female $\mathrm{KOPr}^{-/-}$mice had decreased tail withdrawal latencies (Martin et al., 2003). In Sprague Dawley rats, morphine had dose-dependent antinociceptive effects in the warm water $\left(55^{\circ} \mathrm{C}\right)$ tail withdrawal assay with an $E D_{50}$ value of $1.26 \mathrm{mg} / \mathrm{kg}$, whilst even up to a dose of $40 \mathrm{mg} / \mathrm{kg} U 50,488$ did not have any antinociceptive effect (Gallantine \& Meert, 2008). The results indicate that the traditional KOPr 
agonists are not very effective in this thermal model of pain, however, the model is a useful way of repeatedly measuring the antinociceptive effects of the compounds as a fast screening tool, whilst reducing the suffering of the animals due to the short duration of the nociceptive stimulus.

The KOPr agonists were also assessed in the warm water tail withdrawal assay using a repeated measures paradigm to measure the duration of action. One of the problems with SalA is that the structure is rapidly metabolised to the inactive Salvinorin B, and therefore the antinociceptive effects of SalA are short-lasting which is not optimal for clinical use. In this study, the effects of SalA at the higher dose (2 mg/kg i.p.) lasted from 5-30 min (Figure 4.2A). The novel agonist 16-Ethynyl SalA (2 $\mathrm{mg} / \mathrm{kg}$ i.p.) had a longer total duration, with significant effects from 5-60 min (Figure 4.2B). The duration of action was the same for 16-Bromo SalA ( $2 \mathrm{mg} / \mathrm{kg}$ i.p.), but there was a slower onset of action, with significant antinociceptive effects observed between 10-60 min (Figure 4.2C). The peak antinociceptive effects for each of the compounds was $15 \mathrm{~min}$, indicating that the cumulative doseresponse effects in the previous experiment may have been more potent if administered every $15 \mathrm{~min}$ rather than $30 \mathrm{~min}$. However, the dose-response experiment for SalA was repeated at both a $15 \mathrm{~min}$ and $30 \mathrm{~min}$ interval between subsequent injections by another member of the Kivell laboratory, showing consistent results for both time schedules with the $E_{50}$ values at $1.30 \mathrm{mg} / \mathrm{kg}$ and $1.29 \mathrm{mg} / \mathrm{kg}$ respectively (Sherwood et al., 2017a; unpublished data). In the second model of thermal nociceptive pain, the hotplate test was used at the same temperature as the warm water tail withdrawal assay. At the $2 \mathrm{mg} / \mathrm{kg}$ (i.p.) dose, SalA had a duration of action of $15 \mathrm{~min}$, whilst both the novel analogues had a duration of 15-30 min (Figure 4.3).

The results for SalA were similar to those found in the literature. McCurdy et al. (2006) found that SalA produced antinociceptive effects in the tail withdrawal assay in male Swiss mice, at 2-4 mg/kg i.p. showing a 20 min duration of action. However, the study used an automated analgesia meter with a light beam as the thermal stimulus, which may not be directly comparable to immersion of the tail into warm water as the procedure in the current thesis. The hotplate $\left(52^{\circ} \mathrm{C}\right)$ model was also carried out in the McCurdy et al. (2006) study, however, the measurements were only taken at one time point, $10 \mathrm{~min}$ following i.p. injection of SalA, which had a significant effect at the $1 \mathrm{mg} / \mathrm{kg}$ dose, but not 2 $\mathrm{mg} / \mathrm{kg}$. The longer duration of action of the novel KOPr agonists compared to SalA indicates that the novel compounds may have improved pharmacokinetic profiles. These analogues were designed to decrease the metabolism of the furan ring of the SalA scaffold (Riley et al., 2014). It has been previously shown that carbon-2 alterations of the SalA structure, such as the analogue EOM SalB, are more metabolically stable (Ewald et al., 2017), while less is known about the effects of carbon-16 alterations, it has been predicted that modifications at this position may hinder the action of cytochrome P450 enzymes (Wilson et al., 1990). Since the short duration of action of SalA is one factor 
that has limited the clinical development of the KOPr agonist, the increase in the duration for the novel analogues is a promising development.

\subsubsection{Antinociceptive effects in the intraplantar $2 \%$ formaldehyde test}

The antinociceptive effects of the KOPr agonists were further assessed using the intraplantar formaldehyde model of both nociceptive and inflammatory pain. Initially, SalA was tested via i.p. injection at a single dose to test whether the results were consistent with previous work from our laboratory and the literature. At $2 \mathrm{mg} / \mathrm{kg}$, SalA produced antinociceptive effects between 5 and 50 min, with a significant reduction in both phase I and phase II pain observed (Figure 4.4A-C). Previous results from the Kivell laboratory has found that SalA ( $2 \mathrm{mg} / \mathrm{kg}$ i.p.) reduced pain from 5-10 and 25-40 min (Paton et al., 2017; Kumar, 2014) and consistent with other studies which show effects between 5-10 and 20-60 $\mathrm{min}$ (Aviello et al., 2011). It was hypothesised that the majority of the antinociceptive effects would be mediated selectively via KOPr activation, however, previous studies have shown that the CB1 receptor may also be involved (Aviello et al., 2011; Guida et al., 2012). However, the CB1 receptor antagonist rimonabant, used in the Aviello et al. (2011) study, has been shown to attenuate U69,593 binding to the KOPr, indicating that rimonabant may have antagonistic effects on the KOPr (Walentiny et al., 2010; Zador et al., 2015) and the CB1 antagonist AM251 has been shown to have antagonism at the MOPr (Seely et al., 2012; Ostadhadi et al., 2016). Pre-treatment with the KOPr antagonist nor-BNI reversed the effects of SalA at 5 and 30-40 min and significantly increased the levels of pain in phase I and II AUC analysis. However, AM251 did not have any effect on the pain score. This is consistent with the binding studies that show SalA is a selective KOPr agonist (Roth et al., 2002). Cannabinoid and opioid receptor agonists have been shown to have a synergistic action (Grenald et al., 2017), possibly via convergence of downstream GPCR signalling pathways or via the formation of heterodimers (Hojo et al., 2008; Sierra et al., 2017), which has been used as the explanation for the previous findings that SalA was partially attenuated by cannabinoid receptor antagonism. However, since the current study did not show any significant change with the pretreatment of AM251, further studies were not carried out to investigate any other cannabinoid receptor-mediated effects.

Aviello et al. (2011) speculated that higher doses of SalA ( $2 \mathrm{mg} / \mathrm{kg}$ i.p.) may have a central target, whilst lower doses $(0.5 \mathrm{mg} / \mathrm{kg})$ appeared to have a peripheral effect greater than the central effects. In the present study, SalA was administered via i.pl. injection at the site of inflammation, to assess the local effects of the KOPr agonist. The results show that SalA ( $2 \mathrm{mg} / \mathrm{kg}$ i.pl.) reduced pain between 5$60 \mathrm{~min}$ and reduced the total pain levels in phase I and II AUC analysis (Figure 4.4E-G). Interestingly, SalA did not reduce the level of paw oedema when administered locally, whereas via i.p. injection the 
swelling was reduced in a nor-BNI-sensitive manner (Figure 4.4). It is likely that the combination of two injections into the same site, the inflammatory effect of the DMSO vehicle and the short pretreatment time may have played a role. The combination of both i.pl. injections ( $20 \mu \mathrm{L}+\leq 30 \mu \mathrm{L}$ ) is less than or equal to the maximum volume $(50 \mu \mathrm{L}$ ) recommended for this route of administration (Shimizu, 2004; Turner et al., 2011), although the combination of both injections appears to have produced an inflammatory and nociceptive response (paw oedema in the vehicle/vehicle control for i.p. administration was $-0.393 \pm 0.826 \%$ compared to the vehicle/vehicle control for the i.pl. experiment which increased paw depth by $23.6 \pm 4.6 \%$ ). Whether this response is due to the two injections causing increased injury to the area, or from irritation caused by the DMSO vehicle is unclear. The procedure was based on methods from Lamb et al. (2012), however, this study used rats, which have a much larger paw volume and area to inject. Also, the drug of interest in this study, herkinorin, was delivered in the solvent dimethylacetamide, which may have different effects to the vehicle used in the present thesis. The novel compounds 16-Ethynyl SalA (Figure 4.5D,H) and 16-Bromo SalA (Figure 4.6D,H) both did not reduce paw oedema via i.pl. administration, however, data presented for DHEA in Chapter 3, showed a reduction in paw oedema when administered locally, although DHEA was dissolved in a vehicle containing propylene glycol.

Following the SalA results in the intraplantar formaldehyde model, both of the novel analogues were tested for dose-dependent antinociceptive effects. The i.p. administration of 16-Ethynyl SalA produced antinociceptive effects in phase I at $2 \mathrm{mg} / \mathrm{kg}$ and in phase II at both doses, which was reversed by nor-BNI (Figure 4.5A-C). 16-Bromo SalA did not overall reduce either phase of pain but did have an effect on the 25-30 min time points when the data was analysed over a time course (Figure 4.6E-G). For the i.pl. administration, again 16-Ethynyl reduced both phases of pain (Figure 4.5E-G), however, 16-Bromo SalA only reduced phase I pain, although, at the 25-35 and 55 min time points within the inflammatory phase, 16-Bromo SalA induced a reduction in pain score (Figure 4.6E-G). For the results with SalA and 16-EthynyI SalA, there was a reduction in inflammatory pain (phase II) and a reduction in oedema, however, 16-Bromo SalA reduced oedema but did not significantly reduce the overall inflammatory pain. This indicates that $16-$ Bromo SalA may be having a more potent antiinflammatory effect as opposed to an antinociceptive effect, although oedema induces inflammatory pain, and similarly inflammatory pain induces oedema via substance P and CGRP release (Ji et al., 2014). However, oedema is not the only marker of inflammation, and 16-Bromo SalA did reduce inflammatory pain at two time points when the results were compared over the time course. The reduction in antinociceptive potency of 16-Bromo SalA when compared to the other two compounds may be partially due to the fact that 16-Bromo SalA has a larger molecular mass and therefore the doses of SalA, 16-Ethynyl SalA and 16-Bromo SalA are not equimolar. Conversion of the $2 \mathrm{mg} / \mathrm{kg}$ dose 
into the $\mathrm{mol} / \mathrm{kg}$ equivalent is shown in Table 4.5. To directly compare the results, the KOPr agonists could be administered as $\mathrm{mol} / \mathrm{kg}$ instead of $\mathrm{mg} / \mathrm{kg}$ or given a dosing scheme based on the results of the warm water tail withdrawal dose-response experiment.

\begin{tabular}{ccc}
\hline KOPr agonist & Molecular mass (g/mol) & Equivalent dose $(\mathrm{mol} / \mathbf{k g})$ \\
\hline SalA & 432.46 & $4.62 \times 10^{-6}$ \\
\hline 16-Ethynyl SalA & 456.49 & $4.38 \times 10^{-6}$ \\
\hline 16-Bromo SalA & 535.38 & $4.03 \times 10^{-6}$ \\
\hline
\end{tabular}

Table 4.5: The $2 \mathrm{mg} / \mathrm{kg}$ dose of the KOPr agonists converted into $\mathrm{mol} / \mathrm{kg}$

\subsubsection{Anti-inflammatory effects of the kappa opioid receptor agonists}

Previous studies have shown SalA to have anti-oedema and anti-inflammatory properties (Aviello et al., 2011; Fichna et al., 2012). Aviello et al. (2011) found that SalA (0.5 mg/kg i.p.) reduced LPS (0.05\%)and carrageenan (2\%)-induced paw volume in male ICR mice for 2-6 $\mathrm{h}$ following the inflammatory insult. In the present study, i.p. administration of SalA and both the novel analogues significantly reduced the formaldehyde-induced paw oedema. To better understand the effect of the KOPr agonists on formaldehyde-induced inflammation, two methods were utilised to quantify the infiltration of immune cells. As a preliminary screen to understand the immune cell populations in digested footpad tissue, cells were labelled with an array of antibodies to known immune cell surface markers and flow cytometry used to profile immune cell infiltration. Both SaIA and ICI 204,448 significantly reduced neutrophil infiltration into the paw (Figure 4.7). In the present study, however, we did not see a reduction in the macrophage, monocytes or dendritic cell numbers. This is likely due to the short onehour duration of the intraplantar formaldehyde model of acute inflammation. Neutrophils were further assessed via histological counts in H\&E stained footpad tissue-matched from the animals in the intraplantar formaldehyde assay. The results confirmed that SaIA and ICI 204,448 and well as $\beta$ THP SalB significantly reduced neutrophil numbers compared to the vehicle/formaldehyde control (Figure 4.8).In a further experiment, 16-Ethynyl SalA and 16-Bromo SalA were also found to reduce the level of neutrophil infiltration into the inflamed tissue, in a nor-BNI reversible manner (Figure 4.9). During inflammatory pain events in the peripheral tissue, the nociceptive terminals release CGRP and substance $P$ which increase vascular permeability, leading to the formation of oedema and the further infiltration of neutrophils into the inflamed tissue (see Figure 1.4). In addition, NGF and cytokine signalling during inflammation leads to an increase in synthesis of opioid receptors in the DRG, leading to a higher distribution of the receptors at the peripheral nociceptive terminals (Zollner et al., 2003; Puehler et al., 2006). Therefore, the conclusion could be made that SalA and the novel analogues bind 
to the KOPr on the nociceptor, leading to hyperpolarisation of the sensory neuron, and therefore reducing the release of CGRP and substance $P$, which in turn reduces the level of oedema and neutrophil infiltration into the footpad tissue.

\subsubsection{Side effect profiles of the kappa opioid receptor agonists}

Opioid drugs have known motor incoordination and sedative effects. The traditional KOPr agonist U50,488 induced motor incoordination assessed on the rotarod performance assay at the doses that produced antinociception in the acetic acid writhing test in both mice and rats (Vanderah et al., 2004; 2008). White et al. (2015) found that SalA induced motor incoordination in the rotarod performance test for $30 \mathrm{~min}$ at $3-10 \mathrm{mg} / \mathrm{kg}$ (s.c.) in female and male C57BL/6J mice, compared to the G-protein biased agonist RB-64 which did not cause any motor deficits. In male NIH Swiss mice, SalA (0.5-2 mg/kg i.p.) disrupted climbing behaviour in the inverted screen task, a model of motor coordination, with the effect lasting only $5 \mathrm{~min}$, compared to U69,593 (1 mg/kg i.p.) for which the effects lasted $10 \mathrm{~min}$ (Fantegrossi et al., 2005). In male and female Rhesus macaques as a non-human primate model, SalA had a sedative effect (0.01-0.1 mg/kg i.v.), as measured by unresponsiveness to environmental stimuli and postural relaxation (Butelman et al., 2009).

In the present study, the effects of the KOPr agonists on motor coordination were measured over 240 min and compared to morphine (10 mg/kg i.p.) as a positive control (Figure 4.10). SalA and 16-Ethynyl SalA ( $2 \mathrm{mg} / \mathrm{kg}$ i.p.) showed a decrease in latency from 15-30 min. Both novel analogues at the $1 \mathrm{mg} / \mathrm{kg}$ dose decreased the latency at the $30 \mathrm{~min}$ time point. The SalA results show a longer duration than a previous study in the Kivell laboratory (15 $\mathrm{min}$ vs $30 \mathrm{~min}$ ), however, the previous study used a stablespeed (16 rpm for $120 \mathrm{~s}$ ) rotarod procedure, whereas the current study used an accelerating procedure (4-40 rpm over $300 \mathrm{~s}$ ), which is a more sensitive measure. Morphine produced the longest duration of motor incoordination effects with significantly impaired motor performance seen up to $120 \mathrm{~min}$. However, morphine has a longer antinociceptive duration of action compared to the KOPr agonists evaluated (120 $\mathrm{min}$ for $10 \mathrm{mg} / \mathrm{kg}$ i.p. morphine in the warm water tail withdrawal assay; Shivaperumal, 2017), which could simply explain the longer duration of sedation produced. At the 15 min time point, SalA was more sedative than morphine, however, morphine had a significantly greater effect on motor coordination than the KOPr agonists for the overall AUC analysis (Figure 4.10).

The core body temperature was measured due to the effect of opioid analgesics on thermoregulation. At the primary site for the control of body temperature, the preoptic anterior hypothalamus, i.c.v. administration of MOPr agonists caused hyperthermic responses, whereas KOPr agonists caused hypothermic responses (Xin et al., 1997). Morphine has a biphasic effect in rodents, with low doses inducing a hyperthermic effect, whilst high doses produce hypothermia (Baker \& Meert, 2002; 
Safrany-Fark et al., 2015; Lu et al., 2017). Previous studies show that U50,488 produces a hypothermic effect in guinea pigs (Adler et al., 1991), Sprague Dawley rats (Spencer et al., 1988) and Swiss Webster mice ( $25 \mathrm{mg} / \mathrm{kg}$ i.p.) (Bhalla et al., 2010). In $129 S 6$ mice, SalA (50 $\mu$ g i.c.v.) but not Salvinorin B, reduced body temperature for up to $120 \mathrm{~min}$, an effect that was not seen in KOPr knock-out mice (Ansonoff et al., 2006). In a self-reported human study, participants reported experiencing changes in body temperature in previous experiences with the drug (Maqueda et al., 2015), however, two other human studies have reported no changes in body temperatures, although these studies administered SalA via smoking (Addy, 2012) or sublingually (Mendelson et al., 2011), and these routes of administration may alter the effects. Prior to the current thesis study, our laboratory showed that SalA ( $2 \mathrm{mg} / \mathrm{kg}$ i.p.) had a significant hypothermic effect between 20-40 min in mice (measurements were taken at $10 \mathrm{~min}$ intervals up to $60 \mathrm{~min}$; Kumar, 2014). In the present study, 16-Ethynyl SalA had no effect on thermoregulation, whereas 16-Bromo SalA produced a significant decrease in core body temperature from 30-90 min (Figure 4.11). The fact that 16-Ethynyl SalA did not alter body temperature indicates the compound may be a better candidate for clinical development compared to SalA or 16-Bromo SalA.

\subsubsection{Limitations and future directions}

One of the confounding features of the warm water tail withdrawal assay and hotplate test is that the initial skin temperature can alter response latencies. In rodents, the tail is important in thermoregulation as vasodilation in the extremities is used to lose heat, with longer tails found in mice exposed to high temperatures during development and shorter tails in those exposed to low temperatures (Al Hilli \& Wright, 1983; Gordon et al., 2014). The withdrawal behaviour occurs when a critical temperature is sensed by the nociceptors in the skin of the tail or hind paw. Therefore, the time it takes to reach the nociceptive threshold temperature and antinociceptive effect is dependent on the initial skin temperature (Berge et al., 1988; Hole \& Tjolsen, 1993). The ambient temperature directly correlates to the hind paw and tail skin temperatures, and both temperatures are inversely correlated to the thermal withdrawal latency (Vitkova et al., 2015). In the current study, the ambient temperature was controlled at $20-22^{\circ} \mathrm{C}$, but the procedures did not take into account the tail or hind paw skin temperature. Since SalA and 16-Bromo SalA caused significant decreases in core body temperature (Figure 4.11), this could lead to vasoconstriction in the tail, lowering the skin temperature and increasing the latency, which would be interpreted as an antinociceptive effect. In addition, restraint stress in rats leads to a decrease in skin temperature at standard laboratory conditions (Aydin et al., 2011) and an increase in core body temperature (Cristina-Silva et al., 2017). From the available literature, it is also unclear if this restraint effect is limited to rats or also extends to mice. However, 
in the present study, restraint stress was reduced by acclimatising the animals to the restrainer for two days prior to the experiment.

To improve upon the warm water tail withdrawal procedure, tail skin temperatures could be recorded prior to each withdrawal measurement and a correction applied to the latency depending on the skin temperature (Tjolsen et al., 1989). However, this notion was questioned by Lichtman et al. (1993), who found that the tail withdrawal latency was not influenced by skin or core body temperature. The Lichtman et al. (1993) study showed that both morphine and THC reduced core body temperature in male ICR mice, but this did not lead to a reduction in tail skin temperature, and raising the ambient temperature completely attenuated the hypothermic effects without influencing the antinociceptive effects measured using the warm water tail withdrawal assay. Even if the lowered core body temperature led to a decrease in tail skin temperature, it is likely that the change in tail withdrawal latency would be beyond the sensitivity of the assay. Previous studies had used a lower temperature stimulus on rats with baseline tail withdrawal latencies in the range of 5-8 $s$, which is more sensitive to subtle changes compared to the current study which had a mean baseline latency of $0.90 \pm 0.02 \mathrm{~s}$ (Berge et al., 1988; Tjolsen et al., 1989; Hole \& Tjolsen, 1993; Vitkova et al., 2015). Therefore, it can be concluded that the hypothermic effects of the KOPr agonists would likely have a negligible effect on the tail and paw withdrawal latencies.

A factor that may have more influence is the variance caused by the experimenter. In this study, the warm water tail withdrawal assay and hotplate latencies were measured using a stopwatch, which relies heavily on the reaction times of the experimenter. Since the KOPr agonists have reasonably weak effects to thermal stimuli, the difference between the baseline and test latencies is only a few seconds, which allows for greater influence by the experimenter, who has to first observe the behaviour, then make a decision as to whether the behaviour meets the criteria and finally press the button on the stopwatch the end the experiment. The accuracy of this assay relies on quick reaction times and likely influenced by how experienced and confident the experimenter is at carrying out the procedure and potentially leading to day-to-day experimental variance. Although this effect was likely lessened by carrying out vehicle controls within every cohort tested, the accuracy could be improved by using an automated apparatus. In addition, in future experiments, instead of purely measuring the depth of the footpad, a plethysmometer could be used to measure the entire paw volume for a more accurate, less subjective measure, however, this methodology is not currently available in our laboratory.

SalA and 16-Ethynyl SalA had potent effects to a chemical stimulus in the intraplantar formaldehyde assay, however, it is important to test compounds in multiple behavioural models, especially since the 
compounds have a motor incoordination effect which may have reduced the capability of the animals to lift their paws in reaction to the formaldehyde stimulus. Other models that could be used are carrageenan- or capsaicin-induced inflammatory pain models. U50,488 was effective in the $M$. butyricum arthritic pain model (Bileviciute-Ljungar et al., 2006) and reduced complete Freund's adjuvant-induced hyperalgesia in rats (Auh \& Ro, 2012), protocols which could be repeated with the novel KOPr analogues. In addition, the endogenous KOPr system appears to be important for sensing visceral pain (Simonin et al., 1998), and peripherally-restricted KOPr agonists are particularly potent in visceral pain models (Rivière, 2004; Arendt-Nielsen et al., 2009; Albert-Vartanian et al., 2016). Therefore, the novel KOPr analogues could be tested using the acetic acid writhing test in mice. In this model, SalA dose-dependently reduced the mean number of responses for $15 \mathrm{~min}$ (McCurdy et al., 2006). Since the novel SalA analogues have a longer duration of action in both of the thermal models tested in the current thesis, it would be hypothesised that 16-Ethynyl SalA and 16-Bromo SalA would have a longer effect than SalA. 


\subsection{Summary}

In the dose-response tail withdrawal assay, SalA and the novel analogues 16-Ethynyl SalA, 16-Bromo SalA, EOM SalB and $\beta$-THP SalB were more potent that U50,488 and 16-Ethynyl SalA was more efficacious. 16-Ethynyl SalA and 16-Bromo SalA both had a longer duration of action in the warm water tail withdrawal assay (60 min vs. $30 \mathrm{~min}$ for SalA) and the hot plate test ( $30 \mathrm{~min}$ vs. $15 \mathrm{~min}$ for SalA). In the intraplantar formaldehyde test, SalA and 16-Ethynyl SalA significantly reduced nociceptive pain and inflammatory pain when administered by both via i.p. and local i.pl. injection, effects which were reversed by the antagonist nor-BNI. 16-Bromo SalA reduced nociceptive pain following local i.pl. injection and inflammatory pain from 25-30 min via i.p. injection. SalA, 16-Ethynyl SalA and 16-Bromo SalA all reduced paw oedema when administered via i.p. injection, but not when administered locally. The formaldehyde-injected footpad tissue was collected and flow cytometry analysis revealed a significant reduction in neutrophil cell counts following administration of SalA. This result was confirmed in H\&E stained sections, with further analysis showing 16-Ethynyl SalA and 16-Bromo SalA reduced neutrophil numbers, an effect that was nor-BNI reversible. Unfortunately, in the rotarod performance test, SalA, 16-Ethynyl SalA and 16-Bromo SalA all produced motor incoordination effects. In addition, 16-Bromo SalA also produced hypothermic effects up to $90 \mathrm{~min}$, however, 16-Ethynyl SalA did not have any effect on core body temperature. Overall, 16-Ethynyl SalA appears to be a promising novel candidate for the treatment of acute inflammatory pain, with reduced side effects compared to the parent compound SalA. 


\section{Chapter 5: Investigating the antinociceptive effects of kappa opioid receptor agonists on paclitaxel-induced neuropathic pain}

\subsection{Introduction}

Chemotherapy-induced neuropathic pain (CINP) is a common side effect of treating cancer (Sisignano et al., 2014; Addington \& Freimer, 2016) with 68\% of chemotherapy patients reporting CINP within the first month of treatment (Seretny et al., 2014). Many cancers are treated with chemotherapy drugs that induce CINP, such as the commonly used vinca alkaloids, platinum derivatives and taxanes, and specifically vincristine, cisplatin, oxaliplatin and paclitaxel (Table 5.1; reviewed in Jaggi et al., 2011; Sisignano et al., 2014; Ewertz et al., 2015). CINP is often characterised by spontaneous tingling or burning pain, hypersensitivity to mechanical and cold stimuli, and numbness (Forman, 1990; Dougherty et al., 2004). CINP is chronic in nature and consequently can be very debilitating, significantly impacting the quality of life and independence of cancer sufferers (Beijers et al., 2014; Mols et al., 2014). Often CINP is identified as the reason for limiting either the dose or length of chemotherapy treatment. In severe cases, this can even result in the termination of treatment (Holmes et al., 1991; Rowinsky et al., 1993). Furthermore, CINP may persist for months following cessation of chemotherapy treatment, increasing the negative impact on patients (van den Bent et al., 1997). Unfortunately, the problem is also hindered by the lack of effective medications to treat chronic neuropathic pain (Hershman et al., 2014).

\begin{tabular}{cccc}
\hline Taxanes & Platinum-derived & Vinca alkaloids & Others \\
\hline Paclitaxel & Cisplatin & Vincristine & Bortezomib \\
\hline Docetaxel & Carboplatin & Vinblastine & Thalidomide \\
\hline & Oxaliplatin & Vinorelbine & \\
\hline & Vindesine & \\
\hline
\end{tabular}

Table 5.1: Common chemotherapeutic agents that cause chemotherapy-induced neuropathic pain 


\subsubsection{Paclitaxel-induced neuropathic pain}

Paclitaxel (clinically available under the brand name Taxol) was originally isolated from the bark of the Pacific yew tree (Taxus brevifolia) (Wani et al., 1971). Paclitaxel was approved as a taxane chemotherapy drug in the 1990s and is now widely used to treat solid tumours such as ovarian, breast, cervical, prostate, non-small cell lung, gastric, head and neck, Kaposi's sarcoma and pancreatic cancers (reviewed in Khanna et al., 2015). Paclitaxel has antineoplastic effects by binding to $\beta$-tubulin, promoting polymerisation and stabilisation of microtubules (Schiff et al., 1979), in contrast to other chemotherapy drugs like vinca alkaloids which prevent microtubule polymerisation (Bensch \& Malawista, 1968). Cells treated with paclitaxel arrest at metaphase, stopping progression into anaphase, inhibiting mitosis and ultimately leading to cellular death by apoptosis (reviewed in Weaver, 2014). However, arrest at the mitotic checkpoint as the sole mechanism of action may not be sufficient to explain the considerable efficacy of paclitaxel. Evidence suggests that at lower, more clinically relevant doses of paclitaxel, cells proceed through mitosis into anaphase, however, chromosomes divide on multipolar spindles, dividing the chromosomes in multiple directions, resulting in cells with the incorrect number of chromosomes and, consequently, cell death (Zasadil et al., 2014).

The pathogenesis of paclitaxel-induced neuropathic pain involves dying-back axonal damage, causing the distal sensory axons to degenerate in the PNS, and sensitisation of nociceptive afferents, leading to neuropathic pain symptoms in the hands and feet in a 'stocking and glove'-type distribution (Forman, 1990; Dougherty et al., 2004). Neuropathy also occurs in the DRG, where the cell bodies of the peripheral sensory neurons are located. The DRG lack an efficient neurovascular blood-brain barrier (Allen \& Kiernan, 1994), allowing for diffusion of neurotoxic compounds, such as paclitaxel, which has been shown to accumulate in the DRG (Cavaletti et al., 2000; Wozniak et al., 2016). However, the exact mechanism of action of paclitaxel-induced neuropathic pain is not fully defined and numerous underlying mechanisms have been published. The current chapter will focus on the most significant mechanisms of paclitaxel-induced neuropathic pain (see Figure 5.1).

One mechanism of action is by disruption of axonal transport in the sensory neurons that relay the pain signal. Neurons are particularly reliant on intracellular transport due to the elongated axon, with proteins and other essential materials transported from the cell body in the DRG, as well as clearing toxic materials from the axon back to the cell body (reviewed in Yogev et al., 2016; Prior et al., 2017). This is especially important for innervations into the epidermis, as the distal portion of the axons must withdraw and reinnervate as the epidermal cells regularly turn over (Cheng et al., 2010). Since paclitaxel leads to microtubule dysfunction, this can hinder axonal transport, leading to axonal degradation and loss of nociceptive innervations (Siau et al., 2006; LaPointe et al., 2013; Bober et al., 
2015; Gornstein \& Schwarz, 2017; Pease-Raissi et al., 2017). It has been suggested that the 'stocking and glove' distribution of paclitaxel-induced neuropathic pain could be due to the greater effect of axonal transport dysfunction in the long axons that extend to the extremities (Gornstein \& Schwarz, 2014).

Paclitaxel treatment also causes mitochondrial damage in the axons of the peripheral sensory neurons and lumbar DRG, characterised with an increased frequency of swollen and vacuolated mitochondria (Flatters \& Bennett, 2006; Barriere et al., 2012). Paclitaxel leads to the opening of the mitochondrial permeability transition pore, leading to swollen or vacuolated mitochondria which have dysfunctional respiration and ATP production due to disrupted proton gradient across the inner membrane (Zheng et al., 2011). Increased levels of the atypical mitochondria and mitochondrial reactive oxygen species have been correlated to increased paclitaxel-induced neuropathy (Flatters \& Bennett, 2006; Jin et al., 2008; Duggett et al., 2016). Interestingly, this mitotoxic effect was observed in the sensory axons of the dorsal root of the spinal cord, however not in the motor neurons of the ventral root, which is consistent with the fact that paclitaxel induces sensory but not motor deficits (Xiao et al., 2011).

Intracellular $\mathrm{Ca}^{2+}$ levels are increased in paclitaxel-induced neuropathy (Siau \& Bennett, 2006), which can alter the neuronal excitability, neurotransmitter release and synaptic plasticity of the primary sensory neurons. In a rat model of paclitaxel-induced neuropathic pain, hypersensitivity of the skin was found in the glabrous skin of the hind paw, but not the hairy skin of the hind paw or thigh, and the nociceptive neurons innervating the hind paw were associated with higher intracellular $\mathrm{Ca}^{2+}$ compared to the neurons innervating the thigh (Yilmaz \& Gold, 2015; Yilmaz et al., 2017). Mitochondria are important in maintaining intracellular $\mathrm{Ca}^{2+}$ homeostasis by uptaking $\mathrm{Ca}^{2+}$ (Rizzuto et al., 2012), however, the paclitaxel-induced myotoxicity impairs this function (Kidd et al., 2002). Paclitaxel also increases $\mathrm{Ca}^{2+}$ levels via the phosphoinositide signalling pathway and from endoplasmic reticulum stores (Boehmerle et al., 2006; Boehmerle et al., 2007). Additionally, paclitaxel induces changes in the expression of membrane ion channels (Zhang \& Dougherty, 2014), leading to the development of spontaneous action potential generation in the sensory neurons (Samineni et al., 2017). Paclitaxel treatment increased the mRNA and protein levels of the $\alpha 2 \delta-1$ subunit of voltagegated $\mathrm{Ca}^{2+}$ channels (Xiao et al., 2007; Kawakami et al., 2012), the molecular target of the neuropathic pain drug gabapentin (Gee et al., 1996). In rats, gabapentin significantly reduced paclitaxel-induced mechanical allodynia (Xiao et al., 2007; Mangaiarkkarasi et al., 2015). Voltage-gated $\mathrm{Na}^{+}$channels also appear to be involved in the development of paclitaxel-induced neuropathy, with upregulation of $\mathrm{Na}_{v} 1.7$ following paclitaxel treatment in Sprague Dawley rats (Zhang \& Dougherty, 2014) and in human DRG neurons in vitro (Chang et al., 2018; Li et al., 2018). Furthermore, in rodents, selective inhibition of the TRP channels TRPV1 (Hara et al., 2013; Kalynovska et al., 2017), TRPA1 (Materazzi et al., 2012) 
and TRPV4 (Alessandri-Haber et al., 2008; Costa et al., 2018) reduced paclitaxel-induced neuropathic pain.

Inflammation also has a significant effect on the development of paclitaxel-induced neuropathic pain. In Sprague Dawley rats, activating transcription factor 3 (ATF3), a marker of neuronal injury (Tsujino et al., 2000), had increased expression in the nuclei of myelinated sensory neurons and satellite cells in the DRG, as well as in myelinating Schwann cells in the sciatic nerve of paclitaxel-treated animals (Peters, 2007). Schwann cells form the myelin sheath in the PNS and paclitaxel treatment leads to degeneration or breakdown in myelin (Jia et al., 2017), by inducing Schwann cell dedifferentiation into an immature state (Imai et al., 2017), an effect that was found at higher levels in the distal segment of the sciatic nerve compared to the proximal section (Tasnim et al., 2016). In Sprague Dawley rats, paclitaxel-induced the activation of spinal astrocytes, measured by the presence of glial fibrillary acidic protein (GFAP), however, there was no activation of microglia, measured using OX42, Iba-1 and phosphorylated p38 (Zhang et al., 2012). Increased expression of GFAP was also found in the satellite glial cells of the DRG, however, on the contrary, this study also found that there was an increase in OX42-positive microglia in the dorsal horn of the lumbar spinal cord (Peters, 2007). Activation of the glial cells triggers the release of proinflammatory cytokines, including TNF $\alpha$, IL-1 $\beta$ and IL-6, leading to neuronal hypersensitivity, neurogenic inflammation and neuropathic pain (Old et al., 2015; Echeverry et al., 2017). IL-8 also appears to be important in the development of paclitaxel-induced neuropathic pain, as a selective inhibitor of the CXC motif chemokine receptor $1 / 2$ (CXCR1/2) suppressed the progression of mechanical and cold allodynia in Wistar rats (Brandolini et al., 2017).

In rodent models, paclitaxel treatment led to the infiltration of CD68-positive macrophages into the DRG and peripheral sciatic nerve (Peters, 2007; Tasnim et al., 2016). Paclitaxel activates Toll-like receptor 4 (TLR4) and increased expression of MCP-1 in the DRG (Zhang et al., 2013; Li et al., 2014), resulting in the infiltration of macrophages that release proinflammatory cytokines, including TNF $\alpha$, inducing persistent neuropathic pain (Zhang et al., 2016). When Sprague Dawley rats were treated with the clodronate to inhibit macrophage infiltration or the TLR4 antagonist LPS-RS, paclitaxelinduced loss of intra-epidermal nerve fibres and hind paw sensitivity were reduced (Zhang et al., 2016). The paclitaxel-induced loss of intra-epidermal nerve fibres in the hind paws of rats is also associated with increased numbers of Langerhans cells in the affected skin (Siau et al., 2006). Cutaneous Langerhans cells may cause sensitisation of the remaining epidermal nociceptive neurons by the release of nitric oxide (Qureshi et al., 1996) and TNF $\alpha$ (Deng et al., 2003). 


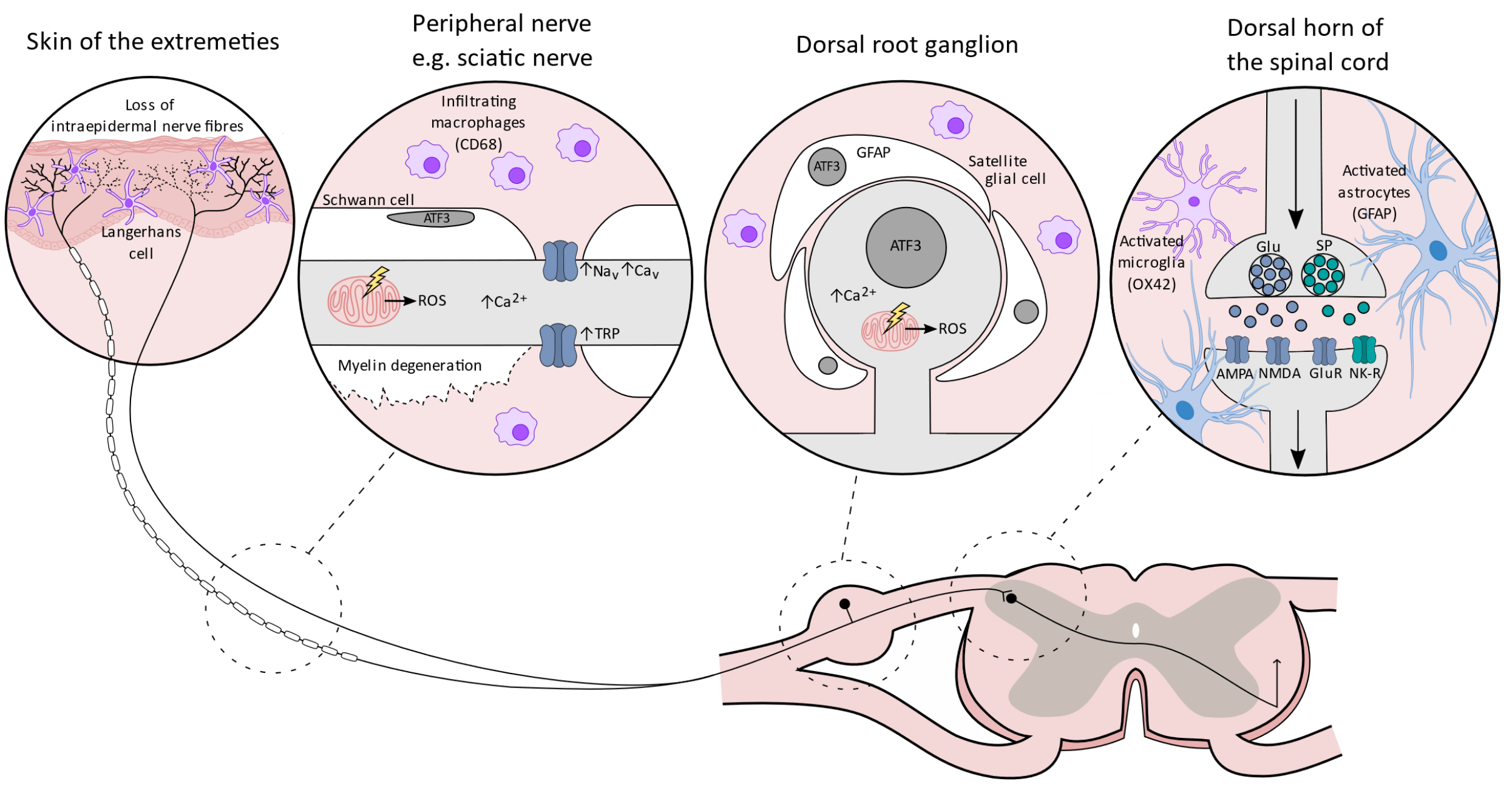

Figure 5.1: Mechanisms of paclitaxel-induced neuropathic pain

Paclitaxel induces many changes in the skin, peripheral nerve, dorsal root ganglion and dorsal horn of the spinal cord that lead to neuropathic pain. AMPA, a-amino-3-hydroxy-5-methyl-4-isoxazolepropionic acid receptor; ATF3, activating transcription factor 3; GFAP, glial fibrillary acidic protein; Glu, glutamate; GluR, metabotropic glutamate receptor; NK-R, neurokinin-1 receptor; NMDA, N-methyl-D-aspartate receptor; ROS, reactive oxygen species; SP, substance $P$; TRP, transient receptor potential ion channel. 


\subsubsection{Sex differences in paclitaxel-induced neuropathic pain}

Paclitaxel is frequently used in female patients (used as a chemotherapeutic for breast, ovarian and cervical cancers, as well as other cancers in both sexes), however, the majority of preclinical studies used male mice or rats (Table 5.3 and Table 5.4). In chronic pain studies, women typically have increased pain sensitivity and a higher prevalence of clinical pain (Mogil, 2012; Bartley \& Fillingim, 2013). The paclitaxel-induced neuropathic pain was measured in male and female mice in 10 inbred strains. Mechanical allodynia was measured using von Frey filaments, which found that males had significantly increased levels of allodynia for all the strains combined, but there was no significant effect of strain and sex interactions (Smith et al., 2004). In a further study using NMRI mice, the female animals had increased paw licking using the acetone model of cold allodynia, specifically in the onset of paclitaxel-induced neuropathic pain from days 7-11 (Naji-Esfahani et al., 2016). However, there was no significant difference between the sexes in the development of mechanical allodynia measured using von Frey filaments. In Sprague Dawley rats, no sex differences were observed during the manifestation and maintenance phase of paclitaxel-induced mechanical allodynia and in the doseresponse effects of morphine treatment (Hwang et al., 2012). However, further research is required to understand the sex difference effects of paclitaxel treatment.

\subsubsection{Potential therapies for paclitaxel-induced neuropathic pain}

The American Society of Clinical Oncology clinical practice guideline states that there are currently no therapeutics that can be recommended for the prevention of CINP as there is not sufficient or consistent evidence from any randomised placebo-controlled trials (Hershman et al., 2014). For the treatment of established CINP, the serotonin-norepinephrine reuptake inhibitor (SNRI) duloxetine is the only agent which is moderately recommended (Hershman et al., 2014; for clinical trial details see Table 5.2). However, the clinical trial which found efficacy with duloxetine included patients with a mixture of paclitaxel, oxaliplatin, docetaxel and cisplatin CINP (Smith et al., 2013). Serotonergic and noradrenergic signalling has been associated in the development of vinca alkaloid and platinum derived CINP (Thibault et al., 2008; Hansen et al., 2011; Aziz et al., 2014; Barzegar-Fallah et al., 2014) and another SNRI venlafaxine has been shown to be effective at reducing oxaliplatin neurotoxicity in a relatively small clinical trial (Durand et al., 2012). However, duloxetine is weaker at treating paclitaxel-induced neuropathic pain (Smith et al., 2013). 


\begin{tabular}{|c|c|c|c|c|c|c|}
\hline Therapeutic & Mechanism & Dose & Duration & Patients & Results & Ref \\
\hline Duloxetine & $\begin{array}{l}\text { Serotonin- } \\
\text { norepinephrine } \\
\text { reuptake inhibitor }\end{array}$ & $\begin{array}{l}\text { Oral } 30 \mathrm{mg} \text { daily for } 1 \text { week, } \\
\text { then } 60 \mathrm{mg} \text { daily for } 4 \text { weeks }\end{array}$ & 5 weeks & $\begin{array}{l}231 \text { patients with paclitaxel, } \\
\text { oxaliplatin, docetaxel or cisplatin CINP }\end{array}$ & $\begin{array}{l}\text { Reduced CINP compared to } \\
\text { placebo }\end{array}$ & (1) \\
\hline Gabapentin & $\begin{array}{l}\text { Modulates } \alpha 2 \delta-1 \\
\text { subunit of } \\
\text { voltage-gated } \\
\mathrm{Ca}^{2+} \text { channels }\end{array}$ & $\begin{array}{l}\text { Oral dose escalated up to } \\
2700 \mathrm{mg} \text { daily }\end{array}$ & 6 weeks & $\begin{array}{l}115 \text { patients with paclitaxel, docetaxel, } \\
\text { carboplatin, cisplatin, oxaliplatin, } \\
\text { vincristine or vinblastine CINP }\end{array}$ & No difference to placebo & (2) \\
\hline Lamotrigine & $\begin{array}{l}\text { Voltage-gated } \mathrm{Na}^{+} \\
\text {channel blocker }\end{array}$ & $\begin{array}{l}\text { Oral } 25 \text { mg daily, escalated } \\
\text { every } 2 \text { weeks up to } 300 \mathrm{mg} \\
\text { daily }\end{array}$ & 10 weeks & $\begin{array}{l}131 \text { with paclitaxel, docetaxel, } \\
\text { carboplatin, cisplatin, oxaliplatin, } \\
\text { vincristine or vinblastine CINP }\end{array}$ & No difference to placebo & (3) \\
\hline Nortriptyline & $\begin{array}{l}\text { Serotonin- } \\
\text { norepinephrine } \\
\text { reuptake inhibitor }\end{array}$ & $\begin{array}{l}\text { Oral } 25 \mathrm{mg} \text { daily, escalated } \\
\text { every week up to } 100 \mathrm{mg} \\
\text { daily }\end{array}$ & 9 weeks & 51 patients with cisplatin CINP & No difference to placebo & (4) \\
\hline Amitriptyline & $\begin{array}{l}\text { Serotonin- } \\
\text { norepinephrine } \\
\text { reuptake inhibitor }\end{array}$ & $\begin{array}{l}\text { Oral } 10 \mathrm{mg} \text { daily, escalated } \\
\text { every week up to } 50 \mathrm{mg} \\
\text { daily }\end{array}$ & 8 weeks & $\begin{array}{l}44 \text { patients with vinca alkaloid, } \\
\text { platinum derivative or taxane CINP }\end{array}$ & No difference to placebo & (5) \\
\hline Ketamine & $\begin{array}{l}\text { NMDA receptor } \\
\text { antagonist }\end{array}$ & $\begin{array}{l}\text { Oral } 40 \mathrm{mg} \text { daily, escalated } \\
\text { up to } 400 \mathrm{mg}\end{array}$ & $\begin{array}{l}16 \text { days of } \\
\text { stable dose }\end{array}$ & 214 patients with CINP & No difference to placebo & (6) \\
\hline Nabiximols & $\begin{array}{l}\text { Cannabinoid } \\
\text { receptor agonists }\end{array}$ & $\begin{array}{l}\text { Oral spray containing THC } \\
\text { and cannabidiol, } 1-12 \text { sprays }\end{array}$ & $\begin{array}{l}4 \text { weeks of } \\
\text { stable dose }\end{array}$ & $\begin{array}{l}16 \text { patients with paclitaxel, vincristine } \\
\text { or cisplatin CINP }\end{array}$ & No difference to placebo & (7) \\
\hline $\begin{array}{l}\text { Amitriptyline, } \\
\text { baclofen \& } \\
\text { ketamine } \\
\text { (topical) }\end{array}$ & $\begin{array}{l}\text { Baclofen: GABA } \\
\text { receptor agonist }\end{array}$ & $\begin{array}{l}1.31 \mathrm{~g} \text { gel with } 40 \mathrm{mg} \\
\text { amitriptyline, } 10 \mathrm{mg} \\
\text { baclofen \& } 20 \mathrm{mg} \text { ketamine, } \\
\text { one spoonful twice daily }\end{array}$ & 4 weeks & $\begin{array}{l}208 \text { with vinca alkaloid, platinum } \\
\text { derivative or taxane CINP }\end{array}$ & $\begin{array}{l}\text { Trend towards benefit over } \\
\text { placebo for sensory } \\
\text { symptoms ( } p=0.053)\end{array}$ & (8) \\
\hline $\begin{array}{l}\text { Amitriptyline \& } \\
\text { ketamine } \\
\text { (topical) }\end{array}$ & As above & $\begin{array}{l}\text { Cream containing } \\
2 \% \text { ketamine } \& 4 \% \\
\text { amitriptyline, twice daily }\end{array}$ & 6 weeks & $\begin{array}{l}462 \text { with vinca alkaloid, platinum } \\
\text { derivative or taxane CINP }\end{array}$ & No difference to placebo & (9) \\
\hline
\end{tabular}

\section{Table 5.2: Randomised placebo-controlled clinical trial data for the treatment of established chemotherapy-induced neuropathic pain}

References: (1) Smith et al. (2013), (2) Rao et al. (2007), (3) Rao et al. (2008), (4) Hammack et al. (2002), (5) Kautio et al. (2008), (6) Fallon et al. (2018), (7) Lynch et al. (2014), (8) Barton et al. (2011), (9) Gewandter et al. (2014). 
Despite a lack of significant and consistent evidence from clinical trials, a range of medications are used for the clinical management of CINP (reviewed in Kim et al., 2015; Flatters et al., 2017). Typically, the first-line treatment is with voltage-gated $\mathrm{Ca}^{2+}$ channel-modulating anticonvulsants (gabapentin, pregabalin), tricyclic antidepressants (amitriptyline, nortriptyline), SNRIs (duloxetine) or topical lidocaine patches (voltage-gated $\mathrm{Na}^{+}$channel inhibitor). Many of these therapies have been tested in preclinical studies with rodent models showing potential in treating and preventing paclitaxel-induced neuropathic pain (Table 5.3 and Table 5.4). Gabapentin reduced established paclitaxel-induced mechanical allodynia and thermal hyperalgesia in male ddY mice (Matsumoto et al., 2006), female BALB/c mice (Thangamani et al., 2013) and Sprague Dawley rats (Xiao et al., 2007). Amitriptyline reduced paclitaxel-induced thermal hyperalgesia in BALB/c mice (Thangamani et al., 2013). However, many of these compounds lack efficacy in the randomised placebo-controlled clinical trials (Table 5.2).

Opioid receptor analgesics, including hydrocodone, morphine, oxycodone, methadone, fentanyl patches or tramadol are considered a third-line therapy as long-term treatment with MOPr agonists is not recommended (Finnerup et al., 2015; Grace et al., 2016). However, one benefit of using opioid analgesics is the immediate relief from pain, compared to the first-line treatments that require titration up to the correct dosage (reviewed in Kim et al., 2015; Flatters et al., 2017). In male Sprague Dawley rats, Flatters and Bennett (2004) found that $4 \mathrm{mg} / \mathrm{kg}$ morphine was ineffective at treating paclitaxel-induced mechanical allodynia and $8 \mathrm{mg} / \mathrm{kg}$ only produced a $50 \%$ reversal of mechanical allodynia. However, in a further study by Rahn et al. (2008), it was found that $4 \mathrm{mg} / \mathrm{kg}$ normalised the mechanical withdrawal thresholds to pre-paclitaxel baseline levels. In paclitaxel-treated male Sprague Dawley rats, it was found that the order of potency for MOPr agonists was: morphine < oxycodone < fentanyl (Mori et al., 2014). Unfortunately, MOPr agonists are associated with many side effects and can induce hyperalgesia (Grace et al., 2016), tolerance (Chu et al., 2006) and addiction (Compton \& Volkow, 2006). In comparison, KOPr agonists have fewer side effects and could be developed as an improved analgesic for paclitaxel-induced neuropathic pain. From the available evidence, it appears that KOPr agonists have not been previously assessed in a model of paclitaxel-induced neuropathic pain or CINP. 


\begin{tabular}{|c|c|c|c|c|c|c|}
\hline Therapeutic & Mechanism & Rodent model & $\begin{array}{l}\text { Paclitaxel } \\
\text { regimen }\end{array}$ & Treatment & Outcome of study & Ref \\
\hline WIN 55,212-2 & $\mathrm{CB}$ receptor agonist & Male Wistar rats & $\begin{array}{l}1 \mathrm{mg} / \mathrm{kg} \text { i.p. } \times 4 \\
\text { alternate days }\end{array}$ & $\begin{array}{l}\text { Daily on days } \\
1-14\end{array}$ & $\begin{array}{l}1 \mathrm{mg} / \mathrm{kg} \text { i.p. prevented the development of thermal hyperalgesia } \\
\text { and mechanical allodynia }\end{array}$ & (1) \\
\hline Cannabidiol & $\begin{array}{l}\text { Weak } \mathrm{CB} \text { receptor } \\
\text { agonist }\end{array}$ & $\begin{array}{l}\text { Male and female } \\
\text { C57BL/6 mice }\end{array}$ & $\begin{array}{l}8 \mathrm{mg} / \mathrm{kg} \text { i.p. } \times 4 \\
\text { alternate days }\end{array}$ & $\begin{array}{l}\text { Daily on days } \\
1-14\end{array}$ & $\begin{array}{l}5-10 \mathrm{mg} / \mathrm{kg} \text { i.p. prevented development of cold and mechanical } \\
\text { allodynia }\end{array}$ & $(2)$ \\
\hline Cannabidiol & $\begin{array}{l}\text { Weak } \mathrm{CB} \text { receptor } \\
\text { agonist }\end{array}$ & $\begin{array}{l}\text { Female } \mathrm{C} 57 \mathrm{BL} / 6 \\
\text { mice }\end{array}$ & $\begin{array}{l}8 \mathrm{mg} / \mathrm{kg} \text { i.p. } \times 4 \\
\text { alternate days }\end{array}$ & $\begin{array}{l}\text { Daily on days } \\
1,3,5 \text { and } 7\end{array}$ & $\begin{array}{l}\text { 2.5-5 mg/kg i.p. prevented mechanical hyperalgesia, reversed by } \\
5-H T_{1 A} \text { antagonist but not CB1 or CB2 receptor antagonists }\end{array}$ & (3) \\
\hline Desipramine & $\begin{array}{l}\text { Serotonin- } \\
\text { norepinephrine } \\
\text { reuptake inhibitor }\end{array}$ & $\begin{array}{l}\text { Male Sprague } \\
\text { Dawley rats }\end{array}$ & $\begin{array}{l}2 \mathrm{mg} / \mathrm{kg} \text { i.p. } \times 4 \\
\text { alternate days }\end{array}$ & $\begin{array}{l}\text { Daily from day } \\
-6 \text { to day } 22\end{array}$ & $\begin{array}{l}10 \mathrm{mg} / \mathrm{kg} \text { daily via osmotic pump (s.c.) blocked development of } \\
\text { mechanical and cold allodynia }\end{array}$ & (4) \\
\hline Tetrodotoxin & $\begin{array}{l}\text { Inhibits voltage- } \\
\text { gated } \mathrm{Na}^{+} \text {channels }\end{array}$ & Female CD-1 mice & $\begin{array}{l}2 \mathrm{mg} / \mathrm{kg} \text { i.p. } \times 5 \\
\text { daily }\end{array}$ & $\begin{array}{l}\text { Daily on days } \\
1-5\end{array}$ & $\begin{array}{l}\text { 3-6 } \mu \mathrm{g} / \mathrm{kg} \text { s.c. prevented development of cold allodynia and } \\
\text { mechanical allodynia but not heat hyperalgesia }\end{array}$ & $(5)$ \\
\hline Minoxidil & $\mathrm{K}^{+}$channel opener & $\begin{array}{l}\text { Female } \mathrm{C} 57 \mathrm{BL} / 6 \mathrm{~J} \\
\text { mice }\end{array}$ & $\begin{array}{l}4.5 \mathrm{mg} / \mathrm{kg} \text { i.p. } \times 4 \\
\text { alternate days }\end{array}$ & $\begin{array}{l}\text { On the } 4 \\
\text { alternate days }\end{array}$ & $\begin{array}{l}25-50 \mathrm{mg} / \mathrm{kg} \text { i.p. protected from thermal insensitivity and } \\
\text { alleviated mechanical allodynia }\end{array}$ & (6) \\
\hline $\begin{array}{l}\text { FeTMPyP \& } \\
\text { MnTE-2-PyP }\end{array}$ & $\begin{array}{l}\text { Peroxynitrite } \\
\text { decomposition }\end{array}$ & $\begin{array}{l}\text { Male Sprague } \\
\text { Dawley rats }\end{array}$ & $\begin{array}{l}2 \mathrm{mg} / \mathrm{kg} \text { i.p. } \times 4 \\
\text { alternate days }\end{array}$ & $\begin{array}{l}\text { Daily up to } \\
\text { day } 15\end{array}$ & $\begin{array}{l}\text { 1-10 mg/kg s.c. prevented development of mechanical allodynia } \\
\text { and hyperalgesia }\end{array}$ & (7) \\
\hline IB-MECA & $\begin{array}{l}A_{3} \text { adenosine } \\
\text { receptor agonist }\end{array}$ & $\begin{array}{l}\text { Male Sprague } \\
\text { Dawley rats }\end{array}$ & $\begin{array}{l}2 \mathrm{mg} / \mathrm{kg} \text { i.p. } \times 4 \\
\text { alternate days }\end{array}$ & $\begin{array}{l}\text { Daily on days } \\
1-16\end{array}$ & $0.1 \mathrm{mg} / \mathrm{kg}$ i.p. prevented mechanical hyperalgesia & (8) \\
\hline Minocycline & Inhibits microglia & $\begin{array}{l}\text { Male Sprague } \\
\text { Dawley rats }\end{array}$ & $\begin{array}{l}8 \mathrm{mg} / \mathrm{kg} \text { i.p. } \times 3 \\
\text { alternate days }\end{array}$ & $\begin{array}{l}\text { Daily on days } \\
1-8, \text { measured } \\
\text { up to day } 12 \\
\end{array}$ & $\begin{array}{l}30-50 \mathrm{mg} / \mathrm{kg} \text { i.p. prevented development of mechanical } \\
\text { allodynia, reduced degeneration of intra-epidermal nerve fibre } \\
\text { loss, }\end{array}$ & (9) \\
\hline Minocycline & Inhibits microglia & Male Wistar rats & $\begin{array}{l}1 \mathrm{mg} / \mathrm{kg} \text { i.p. } \times 4 \\
\text { alternate days }\end{array}$ & $\begin{array}{l}\text { Daily on days } \\
1-14\end{array}$ & $\begin{array}{l}15 \mathrm{mg} / \mathrm{kg} \text { i.p. prevented the development of thermal } \\
\text { hyperalgesia and mechanical allodynia }\end{array}$ & (1) \\
\hline $\begin{array}{l}\text { Ibudilast } \\
\text { (AV411) }\end{array}$ & $\begin{array}{l}\text { Suppresses glial } \\
\text { activation }\end{array}$ & $\begin{array}{l}\text { Male Sprague } \\
\text { Dawley rats }\end{array}$ & $\begin{array}{l}1 \mathrm{mg} / \mathrm{kg} \text { i.p. } \times 4 \\
\text { alternate days }\end{array}$ & $\begin{array}{l}\text { Daily on days } \\
12-19\end{array}$ & $7.5 \mathrm{mg} / \mathrm{kg}$ i.p. mechanical allodynia remained stable & (10) \\
\hline $\begin{array}{l}\text { Acetyl-L- } \\
\text { carnitine }\end{array}$ & $\begin{array}{l}\text { Neuroregenerative } \\
\text { properties }\end{array}$ & $\begin{array}{l}\text { Male Sprague } \\
\text { Dawley rats }\end{array}$ & $\begin{array}{l}2 \mathrm{mg} / \mathrm{kg} \text { i.p. } \times 4 \\
\text { alternate days }\end{array}$ & $\begin{array}{l}\text { Daily on days } \\
1-20\end{array}$ & $\begin{array}{l}50-100 \mathrm{mg} / \mathrm{kg} \text { oral gavage prevented the development of } \\
\text { mechanical allodynia }\end{array}$ & $(11)$ \\
\hline Olesoxime & $\begin{array}{l}\text { Neuroregenerative } \\
\text { properties } \\
\text { (mitochondria) }\end{array}$ & $\begin{array}{l}\text { Male Sprague } \\
\text { Dawley rats }\end{array}$ & $\begin{array}{l}2 \mathrm{mg} / \mathrm{kg} \text { i.p. } \times 4 \\
\text { alternate days }\end{array}$ & $\begin{array}{l}\text { Daily on days - } \\
1 \text { until day } 15\end{array}$ & $\begin{array}{l}\text { 3-30 mg/kg oral gavage prevented mechanical allodynia and } \\
\text { hyperalgesia }\end{array}$ & $(12)$ \\
\hline
\end{tabular}

Table 5.3: Preclinical results for the prevention of the development of paclitaxel-induced neuropathic pain

References: (1) Burgos et al. (2012), (2) Ward et al. (2011), (3) Ward et al. (2014), (4) Deng et al. (2016), (5) Flatters et al. (2006), (6) Chen et al. (2017a), (7) Doyle et al. (2012), (8) Janes et al. (2014), (9) Liu et al. (2010), (10) Ledeboer et al. (2006), (11) Nieto et al. (2008), (12) Xiao et al. (2009). 


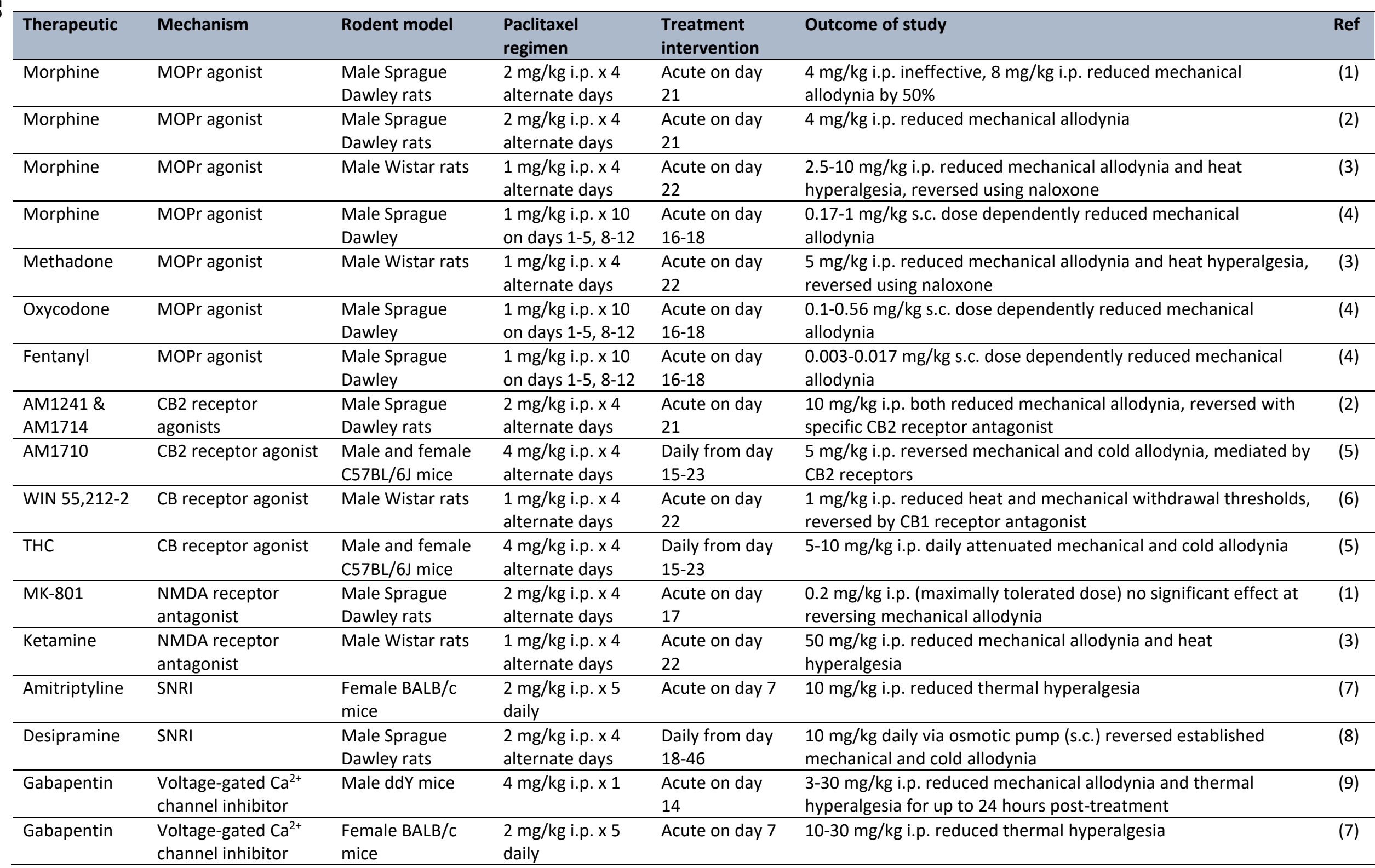




\begin{tabular}{|c|c|c|c|c|c|c|}
\hline Gabapentin & $\begin{array}{l}\text { Voltage-gated } \mathrm{Ca}^{2+} \\
\text { channel inhibitor }\end{array}$ & $\begin{array}{l}\text { Male Sprague } \\
\text { Dawley rats }\end{array}$ & $\begin{array}{l}2 \mathrm{mg} / \mathrm{kg} \text { i.p. } \times 4 \\
\text { alternate days }\end{array}$ & $\begin{array}{l}\text { From day } 26 \\
\text { daily for } 4 \text { days }\end{array}$ & $\begin{array}{l}100 \mathrm{mg} / \mathrm{kg} \text { i.p. reduced mechanical allodynia and expression of } \\
\alpha 2 \delta-1 \text { subunit normalised in the spinal cord }\end{array}$ & (10) \\
\hline Ethosuximide & $\begin{array}{l}\text { Voltage-gated } \mathrm{Ca}^{2+} \\
\text { channel blocker }\end{array}$ & $\begin{array}{l}\text { Male Sprague } \\
\text { Dawley rats }\end{array}$ & $\begin{array}{l}2 \mathrm{mg} / \mathrm{kg} \text { i.p. } \times 4 \\
\text { alternate days }\end{array}$ & $\begin{array}{l}\text { Acute on day } \\
27\end{array}$ & 450 mg/kg i.p. reduced mechanical allodynia & (1) \\
\hline Tetrodotoxin & $\begin{array}{l}\text { Inhibits voltage- } \\
\text { gated } \mathrm{Na}^{+} \text {channels }\end{array}$ & Female CD-1 mice & $\begin{array}{l}2 \mathrm{mg} / \mathrm{kg} \text { i.p. } \times 5 \\
\text { daily }\end{array}$ & $\begin{array}{l}\text { Acute on day } 7 \\
10 \& 14\end{array}$ & $\begin{array}{l}\text { 1-6 } \mu \mathrm{g} / \mathrm{kg} \text { s.c. reduced heat hyperalgesia (day } 7 \text { ), cold allodynia } \\
\text { (day 10-14)and mechanical allodynia (day 10) }\end{array}$ & (11) \\
\hline E139 & $\begin{array}{l}\text { Inhibits voltage- } \\
\text { gated } \mathrm{Na}^{+} \text {channels }\end{array}$ & $\begin{array}{l}\text { Female BALB/c } \\
\text { mice }\end{array}$ & $\begin{array}{l}2 \mathrm{mg} / \mathrm{kg} \text { i.p. } \times 5 \\
\text { daily }\end{array}$ & Acute on day 7 & $10-40 \mathrm{mg} / \mathrm{kg}$ i.p. reduced thermal hyperalgesia & (7) \\
\hline Ralfinamide & $\begin{array}{l}\text { Inhibits voltage- } \\
\text { gated } \mathrm{Na}^{+} \text {channels }\end{array}$ & $\begin{array}{l}\text { Male C57BL/6 } \\
\text { and ICR mice }\end{array}$ & $\begin{array}{l}2 \mathrm{mg} / \mathrm{kg} \text { i.p. } \times 5 \\
\text { daily }\end{array}$ & $\begin{array}{l}\text { Once allodynia } \\
\text { established }\end{array}$ & $\begin{array}{l}5-20 \mathrm{mg} / \mathrm{kg} \text { intragastric dose-dependently alleviated mechanical } \\
\text { allodynia for up to } 300 \mathrm{~min} \text { (highest dose) }\end{array}$ & $(12)$ \\
\hline Lamotrigine & $\begin{array}{l}\text { Inhibits voltage- } \\
\text { gated } \mathrm{Na}^{+} \text {channels }\end{array}$ & Male Wistar rats & $\begin{array}{l}2 \mathrm{mg} / \mathrm{kg} \text { i.p. } \times 4 \\
\text { alternate days }\end{array}$ & $\begin{array}{l}\text { From day } 26, \\
\text { daily for } 5 \text { days }\end{array}$ & $\begin{array}{l}40 \mathrm{mg} / \mathrm{kg} \text { oral gavage significantly reduced thermal and } \\
\text { mechanical hyperalgesia }\end{array}$ & (13) \\
\hline HC-030031 & TRPA1 antagonist & $\begin{array}{l}\text { Male C57BL/6 } \\
\text { mice }\end{array}$ & $6 \mathrm{mg} / \mathrm{kg}$ i.p. $\times 1$ & Acute on day 8 & 300 mg/kg intragastric reduced mechanical allodynia & (14) \\
\hline HC-067047 & TRPV4 antagonist & $\begin{array}{l}\text { Male C57BL/6 } \\
\text { mice }\end{array}$ & $6 \mathrm{mg} / \mathrm{kg}$ i.p. $\times 1$ & Acute on day 8 & $10 \mathrm{mg} / \mathrm{kg}$ i.p. reduced mechanical allodynia & (14) \\
\hline AMD3100 & CXCR4 antagonist & $\begin{array}{l}\text { Male Sprague } \\
\text { Dawley rats }\end{array}$ & $\begin{array}{l}2 \mathrm{mg} / \mathrm{kg} \text { i.p. } \times 4 \\
\text { alternate days }\end{array}$ & $\begin{array}{l}\text { Acute on day } \\
20\end{array}$ & $10 \mathrm{mg} / \mathrm{kg}$ i.p. did not suppress mechanical or cold allodynia & (15) \\
\hline FSLLRY-amide & PAR2 antagonist & Male ICR mice & $\begin{array}{l}1 \mathrm{mg} / \mathrm{kg} \text { i.p. } \times 4 \\
\text { alternate days }\end{array}$ & $\begin{array}{l}\text { Once allodynia } \\
\text { established }\end{array}$ & $\begin{array}{l}0.1-10 \mathrm{nmol} \text { intrathecal reversed mechanical allodynia and heat } \\
\text { hyperalgesia }\end{array}$ & (16) \\
\hline $\begin{array}{l}\text { FeTMPyP \& } \\
\text { MnTE-2-PyP }\end{array}$ & $\begin{array}{l}\text { Peroxynitrite } \\
\text { decomposition }\end{array}$ & $\begin{array}{l}\text { Male Sprague } \\
\text { Dawley rats }\end{array}$ & $\begin{array}{l}2 \mathrm{mg} / \mathrm{kg} \text { i.p. } \times 4 \\
\text { alternate days }\end{array}$ & $\begin{array}{l}\text { Acute on day } \\
16\end{array}$ & $\begin{array}{l}10 \mathrm{mg} / \mathrm{kg} \text { i.p. and } 1 \mathrm{nmol} / \mathrm{kg} \text { intrathecal reduced mechanical } \\
\text { allodynia and hyperalgesia }\end{array}$ & (17) \\
\hline $\begin{array}{l}\text { Ibudilast } \\
\text { (AV411) }\end{array}$ & $\begin{array}{l}\text { Suppresses glial } \\
\text { activation }\end{array}$ & $\begin{array}{l}\text { Male Sprague } \\
\text { Dawley rats }\end{array}$ & $\begin{array}{l}1 \mathrm{mg} / \mathrm{kg} \text { i.p. } \times 4 \\
\text { alternate days }\end{array}$ & $\begin{array}{l}\text { Twice daily on } \\
\text { days 19-25 }\end{array}$ & $\begin{array}{l}7.5 \mathrm{mg} / \mathrm{kg} \text { i.p. resulted in sustained attenuation of mechanical } \\
\text { allodynia }\end{array}$ & (18) \\
\hline IL-1RA & $\begin{array}{l}\text { IL-1 receptor } \\
\text { antagonist }\end{array}$ & $\begin{array}{l}\text { Male Sprague } \\
\text { Dawley rats }\end{array}$ & $\begin{array}{l}1 \mathrm{mg} / \mathrm{kg} \text { i.p. } \times 4 \\
\text { alternate days }\end{array}$ & $\begin{array}{l}\text { Acute on day } \\
18\end{array}$ & $\begin{array}{l}150 \mu g \text { intrathecal transiently ( } 3 \text { hours) reverses mechanical } \\
\text { allodynia }\end{array}$ & (19) \\
\hline $\begin{array}{l}\text { Acetyl-L- } \\
\text { carnitine }\end{array}$ & $\begin{array}{l}\text { Neuroregenerative } \\
\text { properties }\end{array}$ & $\begin{array}{l}\text { Male Sprague } \\
\text { Dawley rats }\end{array}$ & $\begin{array}{l}2 \mathrm{mg} / \mathrm{kg} \text { i.p. } \times 4 \\
\text { alternate days }\end{array}$ & $\begin{array}{l}\text { Daily on days } \\
17-26\end{array}$ & $\begin{array}{l}100 \mathrm{mg} / \mathrm{kg} \text { oral gavage significantly inhibited mechanical } \\
\text { allodynia, the effect lasted until } 4 \text { days post final treatment }\end{array}$ & (20) \\
\hline Olesoxime & $\begin{array}{l}\text { Neuroregenerative } \\
\text { (mitochondria) }\end{array}$ & $\begin{array}{l}\text { Male Sprague } \\
\text { Dawley rats }\end{array}$ & $\begin{array}{l}2 \mathrm{mg} / \mathrm{kg} \text { i.p. } \times 4 \\
\text { alternate days }\end{array}$ & $\begin{array}{l}\text { Daily on days } \\
27-31\end{array}$ & $\begin{array}{l}10-100 \mathrm{mg} / \mathrm{kg} \text { oral gavage significantly reduced mechanical } \\
\text { hyperalgesia and allodynia }\end{array}$ & (21) \\
\hline
\end{tabular}

\section{Table 5.4: Preclinical results for the treatment of paclitaxel-induced neuropathic pain}

References: (1) Flatters and Bennett (2004), (2) Rahn et al. (2008), (3) Pascual et al. (2010), (4) Mori et al. (2014), (5) Deng et al. (2015), (6) Pascual et al. (2005), (7) Thangamani et al. (2013), (8) Deng et al. (2016), (9) Matsumoto et al. (2006), (10) Xiao et al. (2007), (11) Nieto et al. (2008), (12) Liang et al. (2018), (13) Chogtu et al. (2011), (14) Materazzi et al. (2012), (15) Deng et al. (2012), (16) Chen et al. (2011), (17) Doyle et al. (2012), (18) Ledeboer et al. (2006), (19) Ledeboer et al. (2007), (20) 芯

Flatters et al. (2006), (21) Xiao et al. (2009) 


\subsection{Aims and hypotheses}

SalA and the novel analogues 16-Ethynyl SalA and 16-Bromo SalA have been shown to have antinociceptive effects in acute models of pain in mice. The aim of the present chapter was to assess the effects of the KOPr agonists in a model of paclitaxel-induced neuropathic pain. In addition, these carbon-16 analogues were compared to $\beta$-THP SalB, a carbon-2 analogue of SalA. $\beta$-THP SalB has antinociceptive effects in acute models of pain (Paton et al., 2017) and, therefore, was further assessed in the paclitaxel-induced neuropathic pain model. It was hypothesised that the KOPr agonists would have antinociceptive effects via both acute administration and, due to the fact that the novel compounds have less $\beta$-arrestin-associated side effects, would show reduced tolerance in the chronic administration model.

The specific aims of the study were to:

1. Establish the paclitaxel-induced neuropathic pain model using mechanical and cold allodynia as measures of neuropathic pain.

2. Assess the antinociceptive potency and efficacy of the novel KOPr agonists, 16-Ethynyl SalA, 16-Bromo SalA and $\beta$-THP SalB, in the acute dose-response model with comparisons between the sexes.

3. Further establish a chronic KOPr agonist treatment model for animals with paclitaxel-induced neuropathic pain.

4. In the chronic administration model, evaluate the potency and tolerance effects of selected KOPr agonists that showed the most promise in the dose-response procedure.

5. Assess the effects of the KOPr agonists on myelin degeneration using transmission electron microscopy. 


\subsection{Results}

\subsubsection{Establishing a model of paclitaxel-induced neuropathic pain in mice}

The dosage regimen for inducing the paclitaxel-induced neuropathic pain model was based on methodology from Deng et al. (2015), with $4 \times 4 \mathrm{mg} / \mathrm{kg}$ injections on alternate days. This methodology was selected as the study showed robust paclitaxel-induced effects in both female and male C57BL/6J mice. The animals were closely monitored over the 15 days for the paclitaxel effects to be established consistently. The animals were weighed every alternate day to assess the effect of paclitaxel on the general health of the mice. A three-way repeated measures mixed ANOVA with a Greenhouse-Geisser correction was run to understand the effects of treatment, sex and time on the percentage weight change. The three-way interaction of treatment, sex and time was not significant $\left[F_{(3.952,458.4)}=29.016\right.$, $p=0.133]$ and none of the two-way interactions were significant $(p>0.05 ;$ Figure 5.2$)$. This indicates that paclitaxel treatment does not affect the body weight measurements of the mice in either sex.

To further monitor the well-being of the mice, the paclitaxel-treated animals from the first experimental replicate were also observed for spontaneous pain behaviours using the grimace scale on days $0,4,7,11$ and 15 ( $n=12$ male paclitaxel-treated animals). Only a single mouse showed any spontaneous grimace scale behaviours, observed on both day 4 and 7 (Figure 5.3). No other pain behaviours were observed in any of the animals. The grimace scale behaviours were not used as a measure of pain beyond the first experimental replicate.

The effects of paclitaxel on mechanical and cold allodynia were carried out over 7 experimental replicates. The complete results were combined to understand the effect of paclitaxel treatment on mechanical and cold withdrawal thresholds, and if there was a different effect of paclitaxel on the sexes. Initially, all of the baseline measurements from day 0 were compared to test for any inherent sex differences. Unpaired t-test analysis found there was no difference in the mechanical threshold between the sexes $\left[\mathrm{t}_{(118)}=0.3782, p=0.7059\right.$ ] (Figure 5.4A); however, females had a significantly longer reaction time to the cold acetone stimulus compared to males on day $0\left[\mathrm{t}_{(118)}=3.79, p=0.0002\right]$ (Figure 5.4B).

\subsubsection{Mechanical withdrawal thresholds}

A three-way repeated measures mixed ANOVA was run to understand the effects of treatment, sex and time on the mechanical withdrawal thresholds on days $2-15$, as the day 0 measurements were baseline pre-treatment values as analysed above. A reflect and square root transformation was carried out to correct the negatively-skewed data and improve the homogeneity of variances as assessed by the Levene's test for equality of variances. Mauchly's test of sphericity indicated that the assumption 
of sphericity had been violated, so a Greenhouse-Geisser correction was applied. The three-way interaction of treatment, sex and time was not statistically significant $\left[\mathrm{F}_{(6.183,717.2)}=2.053, p=0.055\right]$, meaning that the treatment did not affect the sexes differently over time. There was a statistically significant two-way interaction between treatment and time $\left[F_{(6.183,717.2)}=66.771, p<0.0005\right]$ and between treatment and $\operatorname{sex}\left[F_{(1,116)}=9.744, p=0.002\right]$. Statistical significance of a simple main effect was accepted at a Bonferroni-adjusted alpha level of 0.006 due to multiple families of comparisons. There were statistically significant simple main effects of treatment at days 4-15 $(p<0.006)$, specifically the day 15 values which are measured prior to the dose-response procedure were significantly different $\left[F_{(1,116)}=808.585, p<0.0005\right]$ (Figure 5.5B). The simple main effects of sex were not significant at any day $\left(p>0.006\right.$; day $15\left[\mathrm{~F}_{(1,116)}=1.132, p=0.290\right]$ Figure 5.5B). Overall, this shows that the paclitaxel treatment group were significantly different to the vehicle treatment group, but that paclitaxel affected each sex the same on each day.

\subsubsection{Cold stimulus reaction times}

A three-way repeated measures mixed ANOVA was run to understand the effects of treatment, sex and time on the reaction times to the cold acetone stimulus on days 2-15. A logarithmic transformation was carried out to correct the positively-skewed data and improve the homogeneity of variances. The assumption of sphericity was violated so a Greenhouse-Geisser correction was applied. The three-way interaction of treatment, sex and time was statistically significant $\left[F_{(6.618,767.7)}=\right.$ 3.284, $p=0.002$ ] (Figure 5.5C). Statistical significance of a simple main effect was accepted at a Bonferroni-adjusted alpha level of 0.006 . There was not a significant simple two-way interaction of treatment and sex at any time point $(p>0.006)$, however, there was a significant main effect of both treatment and sex on days $2-15$ ( $p<0.006$; Figure $5.5 \mathrm{C}$ ). Specifically, on day 15 prior to the commencement of the acute dose-response procedure, there was no two-way interaction of treatment and sex $\left[F_{(1,116)}=3.381, p=0.069\right]$, however, there was a main effect of treatment $\left[F_{(1,116)}=\right.$ $662.034, p<0.0005]$ and $\operatorname{sex}\left[\mathrm{F}_{(1,116)}=24.907, p<0.0005\right]$ (Figure 5.5D). Overall, this means that there is an effect of paclitaxel treatment and an inherent difference between the sexes reaction to the cold stimulus, however, the effect of paclitaxel on the sexes is equal, so that the same sex differences are maintained over the time period.

\subsubsection{Outcome of establishing the paclitaxel-induced neuropathic pain model}

After treating the mice with an accumulative dose of $16 \mathrm{mg} / \mathrm{kg}$ of paclitaxel, the animals showed no sign of weight loss and minimal spontaneous behavioural signs of pain. It was found that female mice inherently had a longer reaction time to the cold stimulus and this sex difference was maintained with paclitaxel treatment. By day 15, mice administered paclitaxel had significantly lower mechanical withdrawal thresholds and had increased reaction times to the cold acetone stimulus. 


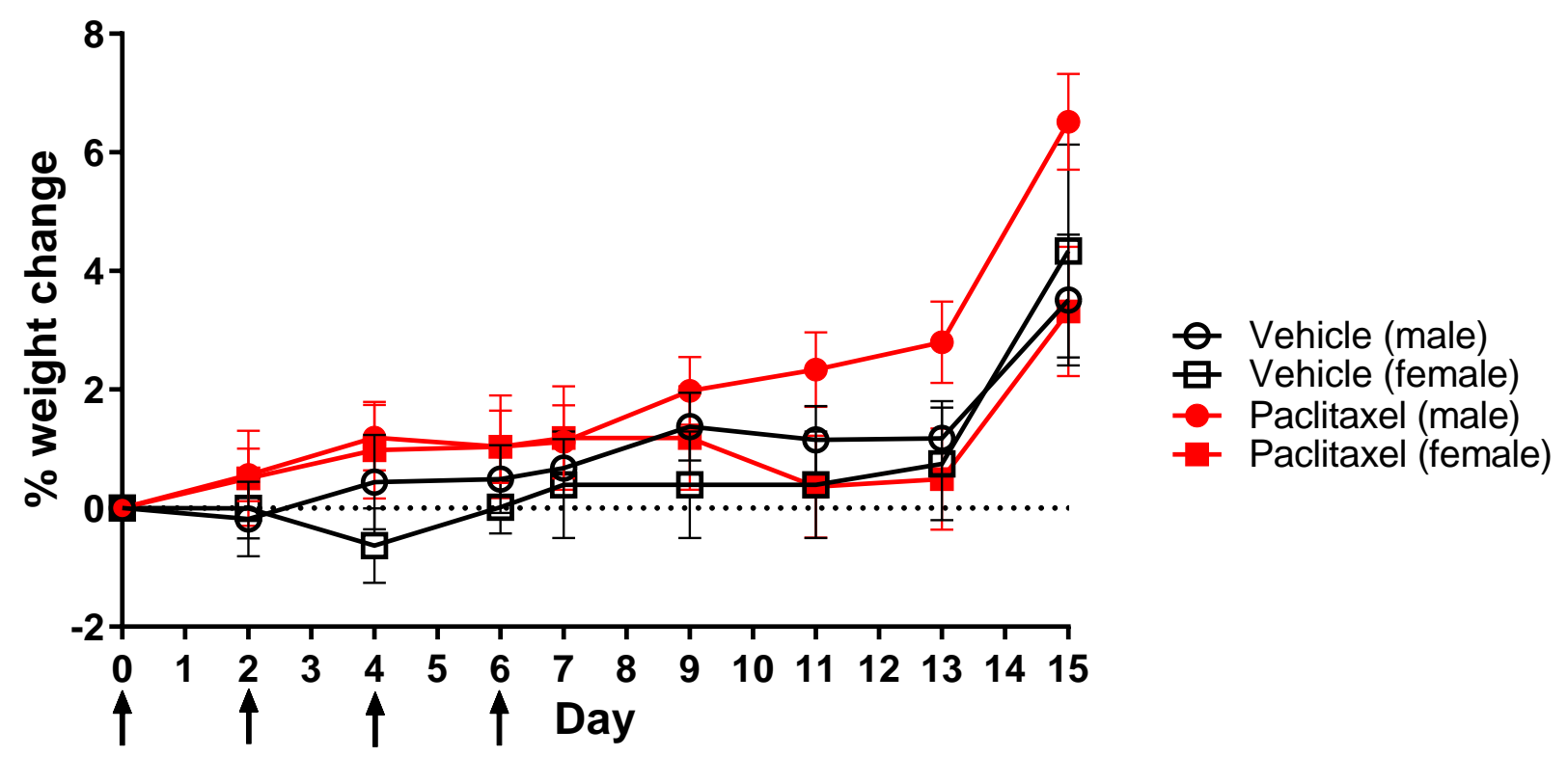

Figure 5.2: Paclitaxel treatment did not cause a weight change in mice

The weight of the mice undergoing the acute paclitaxel experiment was closely monitored. No significant difference was found between the vehicle and paclitaxel-treated mice. Arrows indicate paclitaxel ( $4 \mathrm{mg} / \mathrm{kg}$ i.p.) or vehicle treatment. Weight was always measured prior to the administration of treatment. Values presented as mean \pm SEM, vehicle male $n=18$, vehicle female $n=16$, paclitaxel male $n=50$, paclitaxel female $n=36$. 


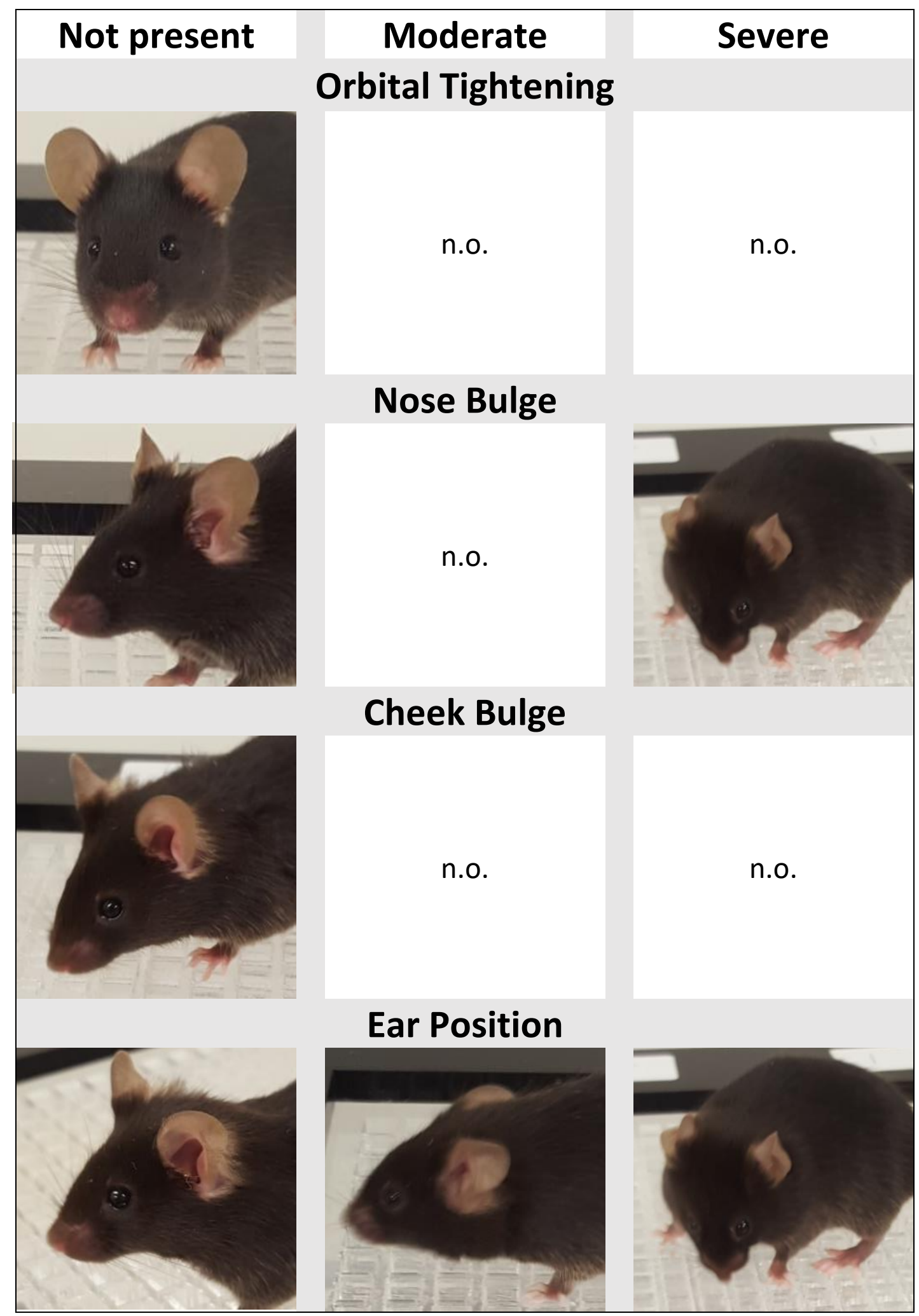

Figure 5.3: Representative images of the observed changes in the mouse grimace scale following administration of paclitaxel

Images were taken of spontaneous grimace scale behaviour during the first replicate of the acute paclitaxel experiment on days $0,4,7,11$ and $15(n=12$ paclitaxel-treated animals). A single mouse showed nose bulge and ear position changes, observed on day 4 and 7 . The other behaviours were not observed (n.o.) in any mouse on any day. 

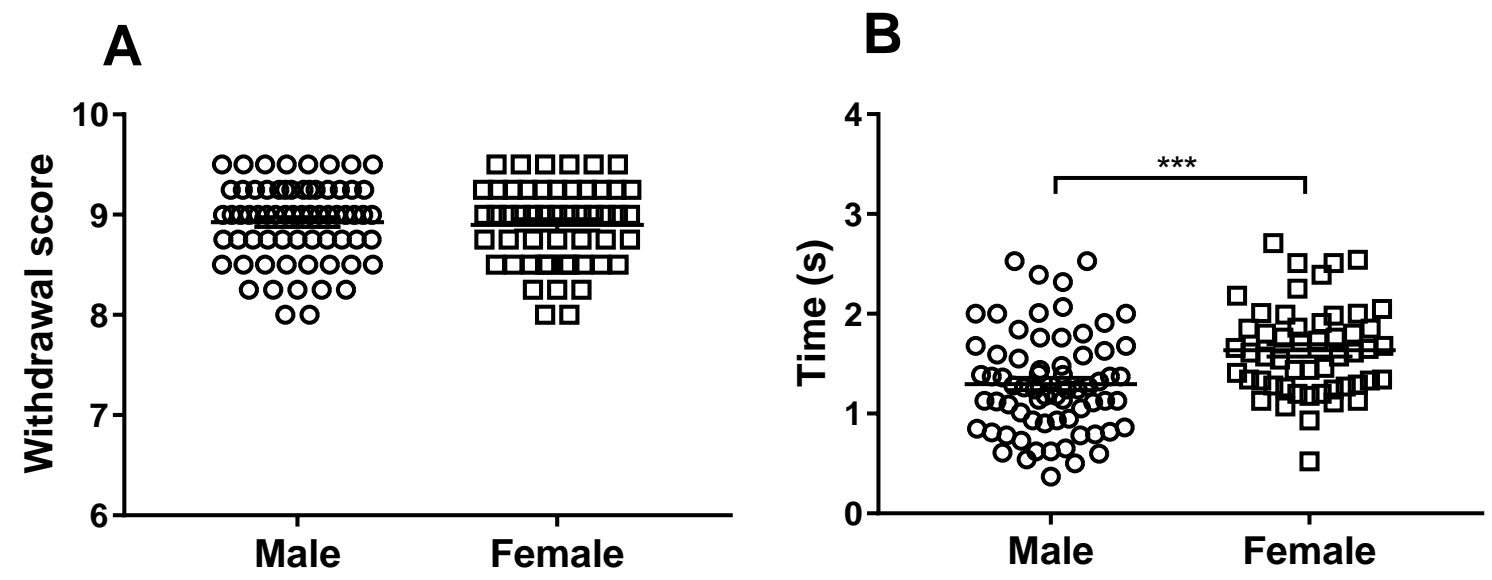

Figure 5.4: Female mice have longer baseline reaction times to a cold acetone stimulus

(A) The baseline (day 0) mechanical thresholds were not different between the sexes. (B) The baseline reaction times to a cold acetone stimulus were significantly higher in females compared to males. Unpaired t-test. Values presented as individual data points, with a line indicating the mean \pm SEM. Male $n=68$, female $n=52$. 

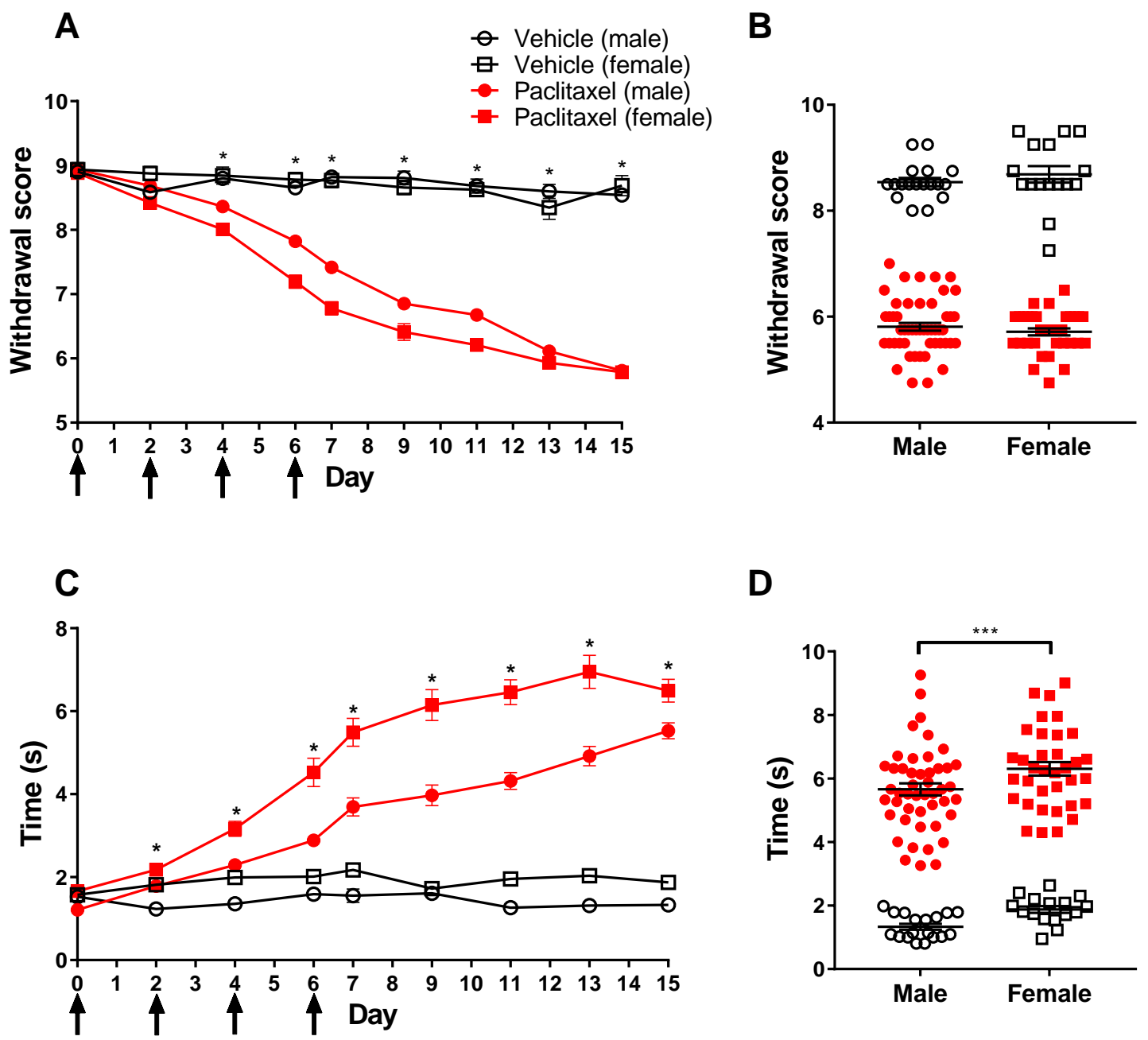

D

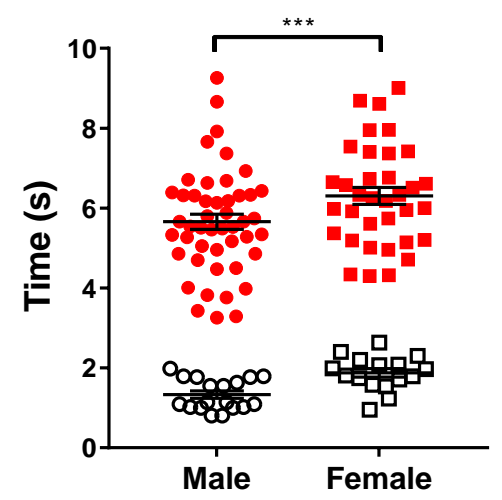

Figure 5.5: Paclitaxel treatment induces mechanical and cold allodynia in male and female mice

(A) Paclitaxel treatment induced mechanical allodynia on days 4-15, shown as a reduction in withdrawal score and measured using von Frey filaments. (B) The day 15 results show an effect of treatment but not sex. (C) Paclitaxel treatment had a significant effect of treatment on days 2-15, seen as an increase in reaction time to a cold acetone stimulus. (D) On day 15 there was an effect of treatment and sex, but no interaction of both factors. Arrows indicate days that treatment was administered. Three-way mixed ANOVA. ${ }^{*} p<0.006$ shows the significant effect of treatment at given time point. Values presented as SEM \pm mean, with individual data points shown in (B) and (D), vehicletreated males $n=18$, vehicle-treated females $n=16$, paclitaxel-treated males $n=50$, paclitaxel-treated females $n=36$. 


\subsubsection{Cumulative dose-response effects of kappa opioid receptor agonists}

Following the initiation of paclitaxel-induced mechanical and cold allodynia, the dose-response effects of the novel KOPr agonists were measured on day 15. Cumulative doses of the KOPr agonists, morphine or vehicle controls were administered subcutaneously and both the mechanical and cold allodynia measurements taken at $\mathbf{3 0}$ min time points post-injection. Non-linear regression analysis was used to determine the potency $\left(E_{50}\right.$ and $\left.I D_{50}\right)$ by comparing the values to morphine. There were no significant differences in the baseline or day 15 measures of mechanical or cold allodynia effects when the mice were grouped dependent on the subsequent dose-response treatment, showing the mice were assigned to treatments in an unbiased manner (see Appendix D.1 and D.2).

\subsubsection{Mechanical allodynia}

The treatment curves were analysed and separated for sex that showed a different curve fit each data set, demonstrating a significant effect of treatment $\left[\mathrm{F}_{(20,410)}=12.72, p<0.0001\right]$ (Figure 5.6). Equivalent treatments with vehicle did not have any antinociceptive effect over time (see Appendix D.3). The ED $D_{50}$ values were compared and showed a significant interaction of treatment and $\operatorname{sex}\left[\mathrm{F}_{(4,370)}=8.099, p<\right.$ 0.0001] (Table 5.5). Treatment with U50,488 showed males had a significantly more potent $E_{50}$ value compared to the females $(p=0.0136)$, whilst the opposite was found with SalA treatment, with SalA treatment in females significantly more potent than in males $(p=0.0040)$. For morphine, 16 -Ethynyl SalA and 16-Bromo SalA there was no significant effect between the sexes. When the male treatment groups were compared to morphine, only 16-Ethynyl SalA was significantly more potent $(p=0.0152)$. When comparing to the female morphine treatment group, the females treated with SalA $(p=0.0098)$ and 16-Ethynyl SalA ( $p=0.0242)$ were significantly more potent.

\subsubsection{Cold allodynia}

Analysis of the treatment curves showed a different curve fit each data set, indicating a significant effect of treatment $\left[F_{(20,410)}=19.41, p<0.0001\right]$ (Figure 5.6 and Figure 5.7). Equivalent treatments with vehicle did not have any antinociceptive effect on the cold stimulus over time (see Appendix D.3). When the data was separated for sex, two-way ANOVA found that there was no interaction of treatment $x$ sex $\left[\mathrm{F}_{(4,370)}=0.2473, p=0.9112\right]$ (Table 5.6). Since there was no interaction, the sexes were combined to understand the main effects of each of the treatments. With the data combined, there was a significant effect of treatment on potency $\left[\mathrm{F}_{(5,420)}=6.797, p<0.0001\right]$ (Table 5.7). SalA ( $p$ $=0.0034)$, 16-Ethynyl SalA $(p<0.0001)$ and $\beta$-THP SalB (males only, $p=0.0002$ ) had significantly more potent antinociceptive effects compared to morphine. 
A

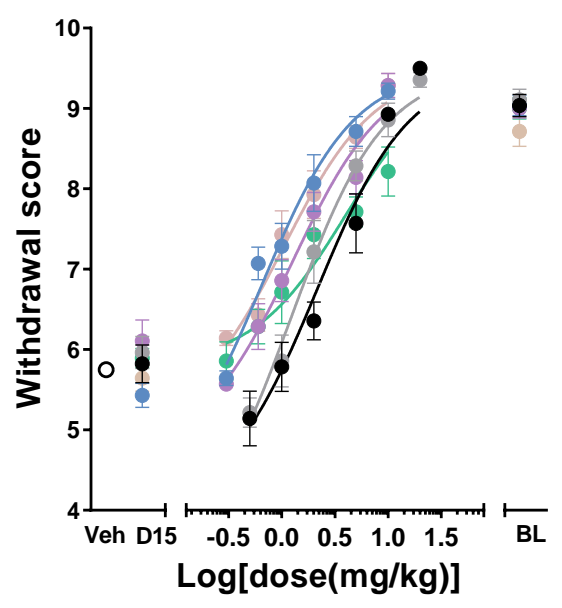

C

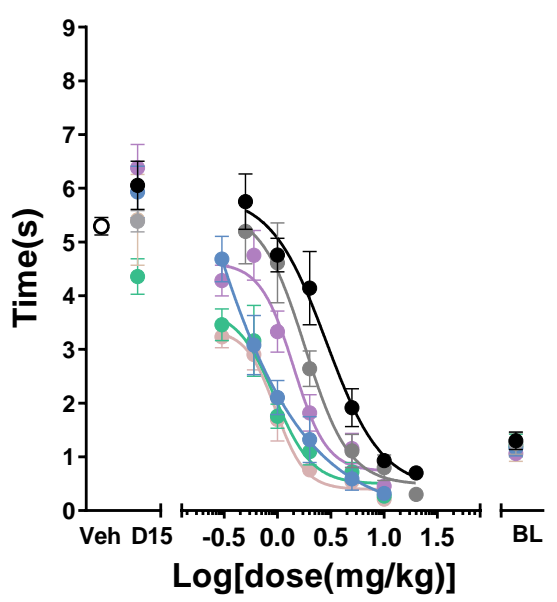

B

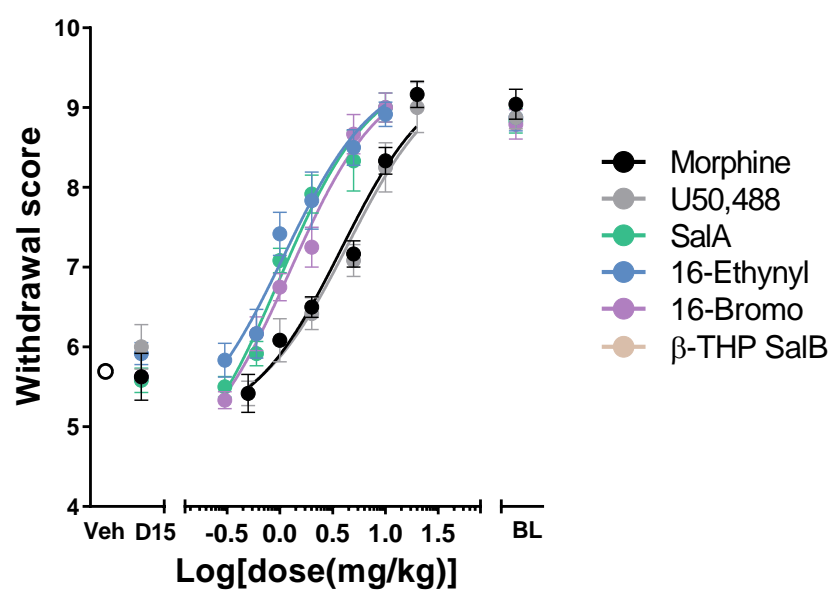

D

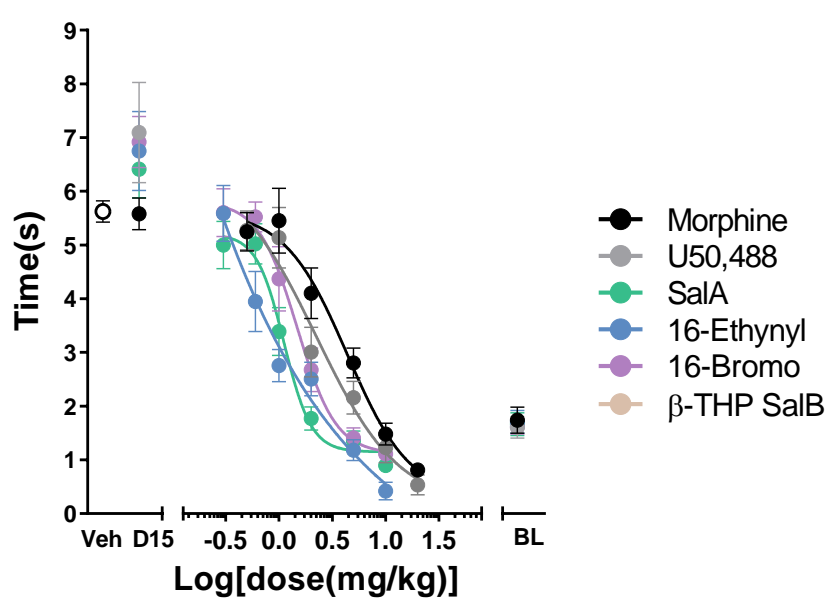

Figure 5.6: Antinociceptive dose-response effects of novel KOPr agonists in mice separated by sex with paclitaxel-induced neuropathic pain

Antinociceptive dose-response effects of morphine and KOPr agonists comparing male and female mice with established paclitaxel-induced neuropathic pain. Mechanical allodynia was measured in (A) males and (B) females. Cold allodynia was measured in (C) males and (D) females. Veh refers to paclitaxel-treated animals treated with vehicle as opposed to the KOPr agonists. D15 refers to the values on day 15 post-paclitaxel prior to the dose-response experiment. BL refers to pre-paclitaxel baseline values. $n=7$ all treatments in males (except vehicle $n=8$ ), and $n=6$ for all treatments in females. Values presented as mean \pm SEM. 

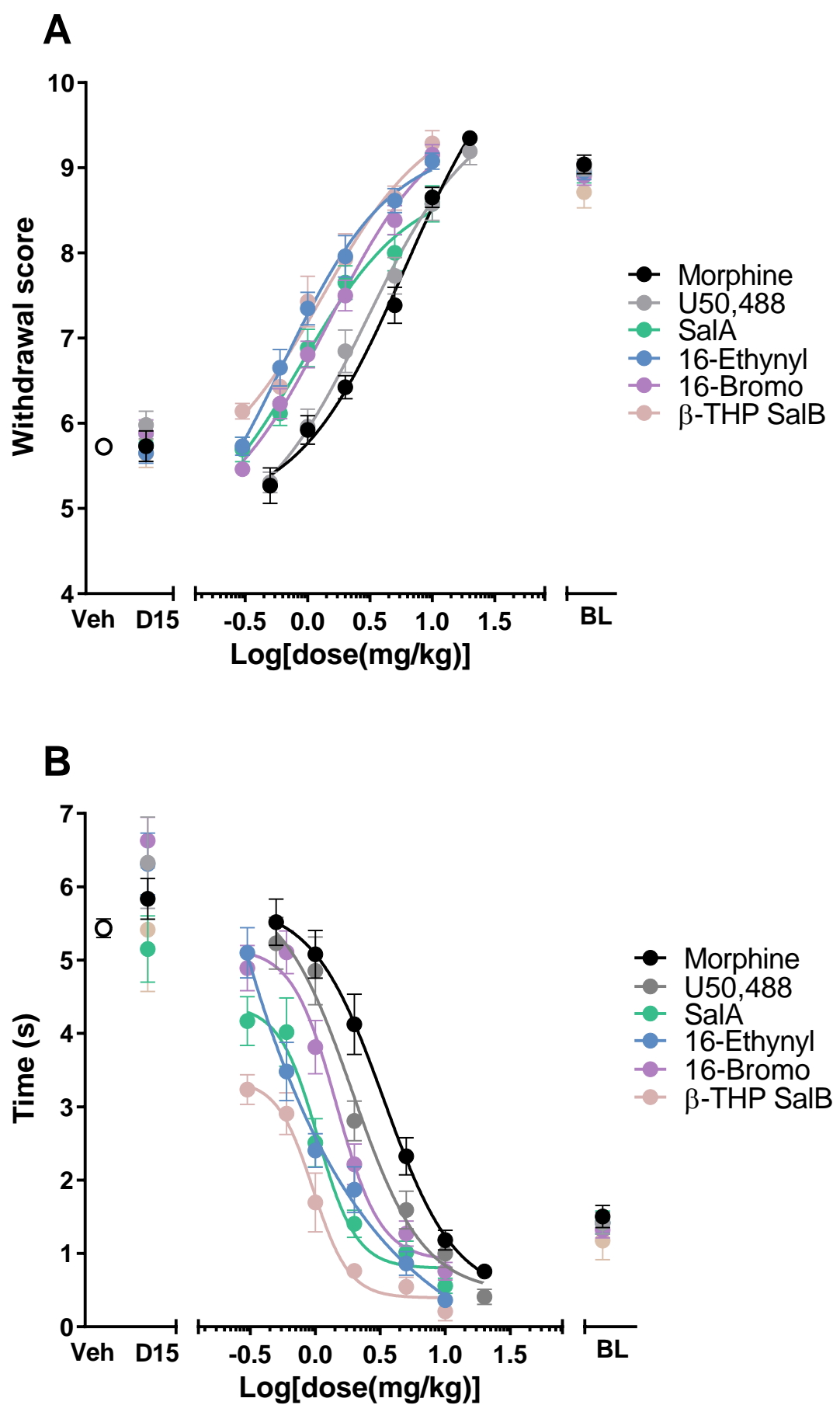

Figure 5.7: Antinociceptive dose-response effects of novel KOPr agonists in combined male and female mice with established paclitaxel-induced neuropathic pain

Antinociceptive dose-response effects of morphine and KOPr agonists in combined male and female mice with established paclitaxel-induced neuropathic pain with (A) mechanical and (B) cold allodynia. Veh refers to paclitaxel-treated animals treated with vehicle as opposed to the KOPr agonists. D15 refers to the values on day 15 post-paclitaxel prior to the dose-response experiment. BL refers to prepaclitaxel baseline values. $n=7$ for $\beta$-THP SalB (males only), all other opioid receptor agonist treatments $n=13$ ( 7 males, 6 females) and $n=14$ for vehicle treatment ( 8 males, 6 females). Values presented as mean \pm SEM. 


\begin{tabular}{|c|c|c|c|c|c|c|c|c|c|c|c|c|}
\hline \multirow[t]{2}{*}{$\begin{array}{l}\text { Opioid receptor } \\
\text { agonist }\end{array}$} & \multicolumn{2}{|c|}{$\begin{array}{l}\text { ED }_{50} \text { value } \\
(\mathrm{mg} / \mathrm{kg})\end{array}$} & \multicolumn{2}{|c|}{$\operatorname{logED}_{50} \pm \mathrm{SEM}$} & \multicolumn{6}{|c|}{ Two-way ANOVA comparisons } & \multicolumn{2}{|c|}{$R^{2}$ value } \\
\hline & Male & Female & Male & Female & \multicolumn{2}{|c|}{ Male vs Female } & \multicolumn{2}{|c|}{$\begin{array}{l}\text { Male vs. Male } \\
\text { treated with morphine }\end{array}$} & \multicolumn{2}{|c|}{$\begin{array}{l}\text { Female vs. Female } \\
\text { treated with morphine }\end{array}$} & Male & Female \\
\hline Morphine & 2.16 & 3.83 & $0.334 \pm 0.106$ & $0.583 \pm 0.070$ & $>0.9999$ & n.s. & - & & - & & 0.828 & 0.893 \\
\hline U50,488 & 1.23 & 4.38 & $0.0898 \pm 0.111$ & $0.641 \pm 0.093$ & 0.0136 & $*$ & $>0.9999$ & n.s. & $>0.9999$ & n.s. & 0.853 & 0.808 \\
\hline SalA & 3.94 & 0.992 & $0.596 \pm 0.122$ & $-0.0035 \pm 0.1011$ & 0.0040 & $* *$ & $>0.9999$ & n.s. & 0.0098 & $* *$ & 0.546 & 0.842 \\
\hline 16-Ethynyl SalA & 0.645 & 1.08 & $-0.191 \pm 0.124$ & $0.0346 \pm 0.126$ & $>0.9999$ & n.s. & 0.0152 & $*$ & 0.0242 & $*$ & 0.800 & 0.760 \\
\hline 16-Bromo SalA & 1.27 & 1.25 & $0.104 \pm 0.098$ & $0.0954 \pm 0.0842$ & $>0.9999$ & n.s. & $>0.9999$ & n.s. & 0.0925 & n.s. & 0.819 & 0.881 \\
\hline$\beta$-THP SalB & 1.04 & & $0.0166 \pm 0.110$ & & & & $>0.9999$ & n.s. & & & 0.806 & \\
\hline
\end{tabular}

Table 5.5: Dose-response effects of the KOPr agonists in female and male mice with established paclitaxel-induced mechanical allodynia

The potency $\left(E D_{50}\right)$ of the antinociceptive effects of the opioid receptor agonists were measured in mice of both sexes. U50,488 had more potent effects in males compared to females, whereas SalA was more potent in females. When the KOPr treatments for each sex were compared to morphine, 16-Ethynyl SalA was significantly more potent in males and females. SalA was more potent than morphine in females only. Non-linear regression analysis. Two-way ANOVA with Bonferroni post-tests. $\mathrm{n}=13$ (7 males, 6 females). n.s. $=$ not significant, ${ }^{*} p<0.05,{ }^{* *} p<0.01$. 


\begin{tabular}{|c|c|c|c|c|c|c|}
\hline \multirow[t]{2}{*}{$\begin{array}{l}\text { Opioid receptor } \\
\text { agonist }\end{array}$} & \multicolumn{2}{|c|}{$\begin{array}{c}I_{50} \text { value } \\
(\mathrm{mg} / \mathrm{kg})\end{array}$} & \multicolumn{2}{|c|}{$\log I D_{50} \pm S E M$} & \multicolumn{2}{|c|}{$R^{2}$ value } \\
\hline & Male & Female & Male & Female & Male & Female \\
\hline Morphine & 2.06 & 3.64 & $0.314 \pm 0.117$ & $0.561 \pm 0.101$ & 0.782 & 0.800 \\
\hline U50,488 & 1.06 & 2.08 & $0.0267 \pm 0.168$ & $0.319 \pm 0.120$ & 0.735 & 0.793 \\
\hline SalA & 0.597 & 1.03 & $-0.224 \pm 0.191$ & $0.0132 \pm 0.118$ & 0.652 & 0.794 \\
\hline 16-Ethynyl SalA & 0.312 & 0.712 & $-0.506 \pm 0.204$ & $-0.148 \pm 0.137$ & 0.734 & 0.780 \\
\hline 16-Bromo SalA & 1.20 & 1.44 & $0.0801 \pm 0.129$ & $0.157 \pm 0.107$ & 0.743 & 0.802 \\
\hline
\end{tabular}

Table 5.6: Dose-response effects of the KOPr agonists in female and male mice with established paclitaxel-induced cold allodynia

Non-linear regression analysis was used to calculate the potency $\left(I_{50}\right)$ of the antinociceptive effects of the opioid receptor agonists measured in mice of both sexes. Two-way ANOVA showed there was no significant interaction of treatment and sex. $n=13$ ( 7 males, 6 females). 


\begin{tabular}{|c|c|c|c|c|c|}
\hline $\begin{array}{l}\text { Opioid receptor } \\
\text { agonist }\end{array}$ & $\begin{array}{c}I_{50} \text { value } \\
(\mathrm{mg} / \mathrm{kg})\end{array}$ & $\log \mathrm{ID}_{50} \pm \mathrm{SEM}$ & \multicolumn{2}{|c|}{$\begin{array}{l}p \text { value for } I D_{50} \\
\text { compared to } \\
\text { morphine }\end{array}$} & $R^{2}$ value \\
\hline Morphine & 2.71 & $0.433 \pm 0.078$ & - & & 0.781 \\
\hline U50,488 & 1.46 & $0.165 \pm 0.103$ & 0.3899 & n.s. & 0.749 \\
\hline SalA & 0.822 & $-0.0853 \pm 0.128$ & 0.0034 & $* *$ & 0.774 \\
\hline 16-Ethynyl SalA & 0.477 & $-0.321 \pm 0.123$ & $<0.0001$ & $* * * *$ & 0.725 \\
\hline 16-Bromo SalA & 1.31 & $0.119 \pm 0.091$ & 0.1928 & n.s. & 0.635 \\
\hline $\begin{array}{c}\beta \text {-THP SalB } \\
\text { (males only) }\end{array}$ & 0.481 & $-0.318 \pm 0.157$ & 0.0002 & $* * *$ & 0.774 \\
\hline
\end{tabular}

Table 5.7: Antinociceptive dose-response effects of KOPr agonists in combined male and female mice with established paclitaxel-induced cold allodynia

Non-linear regression analysis was used to calculate the potency $\left(I D_{50}\right)$ of the antinociceptive effects to cold stimulus on day 15 following the first injection of paclitaxel in mice, with data for both sexes combined. SalA, 16-Ethynyl SalA and $\beta$-THP SalB (males only) had more potent antinociceptive effects than morphine. The efficacy of the treatments were not significantly different. One-way ANOVA with Bonferroni post-tests. $\beta$-THP SalB $n=7$ males only, all other agonists $n=13$ ( 7 males, 6 females). n.s. $=$ not significant, $* * p<0.01, * * * * p<0.0001$. 


\subsubsection{Antagonism of the kappa opioid receptor}

The novel SalA analogues 16-Ethynyl SalA and 16-Bromo SalA were antagonised the KOPr by pretreating with nor-BNI. Pre-treatment with nor-BNI was administered 24 hours before the doseresponse experiment to selectively antagonise the KOPr (Munro et al., 2012). The day 15 values, prior the dose-response procedure, were not significantly different between treatment groups, indicating that antagonising the KOPr on day 14 had no effect on the established neuropathic pain (see Appendix D.4). The non-linear regression analysis of the mechanical allodynia results showed that a different curve fit each treatment group $\left[\mathrm{F}_{(9,216)}=78.47, p<0.0001\right]$ (Figure 5.8A). One-way ANOVA analysis showed a significant effect of treatment at the final KOPr agonist dose of $10 \mathrm{mg} / \mathrm{kg}\left[\mathrm{F}_{(3,34)}=129.9, p<\right.$ 0.0001] (Figure 5.8B). Bonferroni post-tests showed a significant difference with pre-treatment of norBNI for 16-Ethynyl SalA $(p<0.0001)$ and 16-Bromo SalA $(p<0.0001)$. One-way ANOVA analysis of the cold allodynia data also showed a significant effect of treatment $\left[\mathrm{F}_{(3,34)}=96.54, p<0.0001\right]$ (Figure 5.8D) and there was a significant effect of pre-treatment with nor-BNI for 16-Ethynyl SalA $(p<0.0001)$ and 16-Bromo SalA $(p<0.0001)$. The results show that the antinociceptive actions of the novel SalA analogues are mediated via the KOPr.

\subsubsection{Outcome of cumulative dose-response treatment of paclitaxel-induced neuropathic pain}

Overall, the antinociceptive effects of the KOPr agonists for the treatment of mechanical allodynia showed a significant interaction of sex and treatment. U50,488 was more potent in males compared to females but had no improvement over morphine for either sex. SalA was more potent as a treatment in females and was more potent than morphine in females. 16-Ethynyl SalA affected the sexes the same and was more potent than morphine for both males and females. For the treatment of cold allodynia, the combined sexes data showed SalA and 16-Ethynyl SalA were more potent than morphine. In both mechanical and cold allodynia, 16-Bromo SalA was equally potent to morphine. Finally, antagonist studies showed that the antinociceptive effects of 16-Ethynyl SalA and 16-Bromo SalA were mediated by the KOPr. 
A

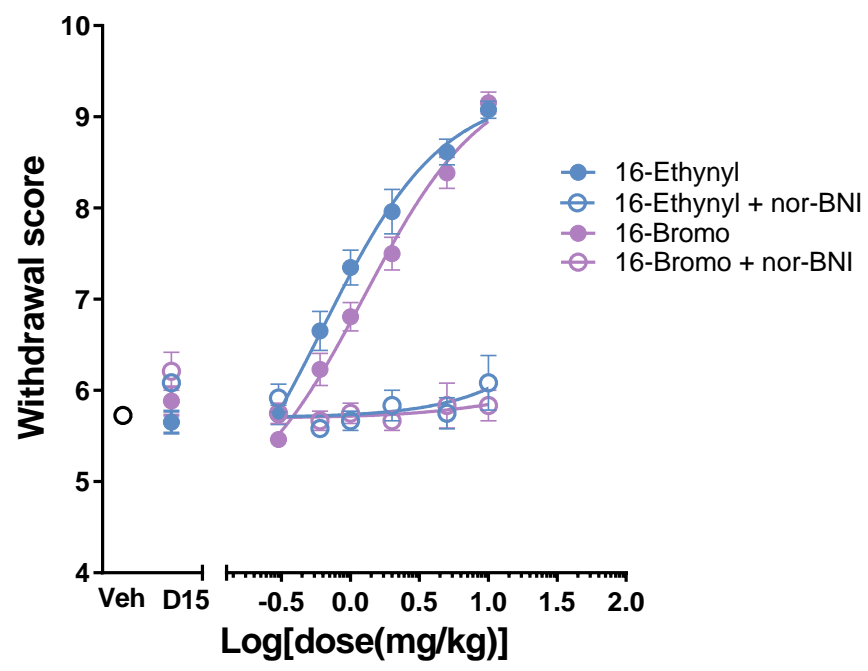

C

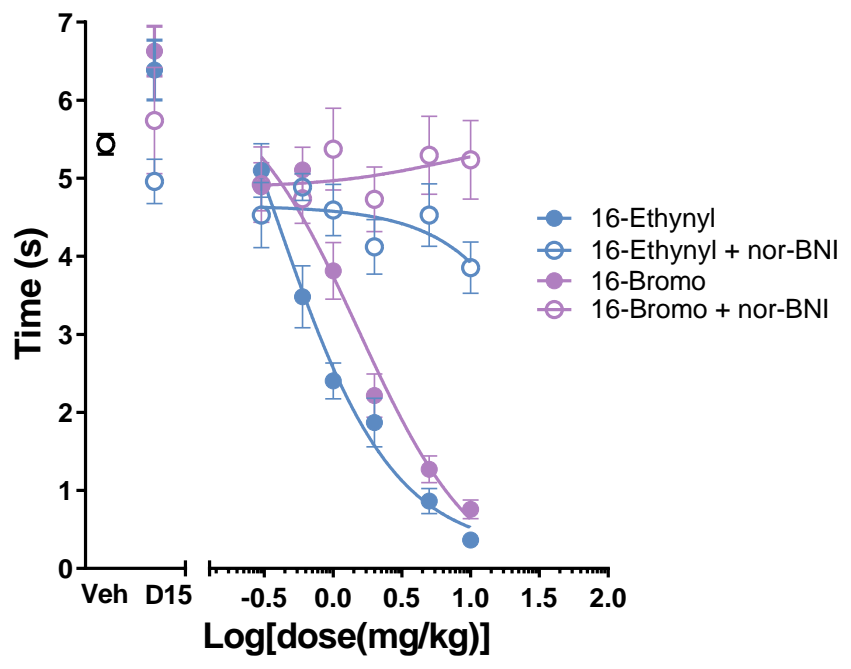

B

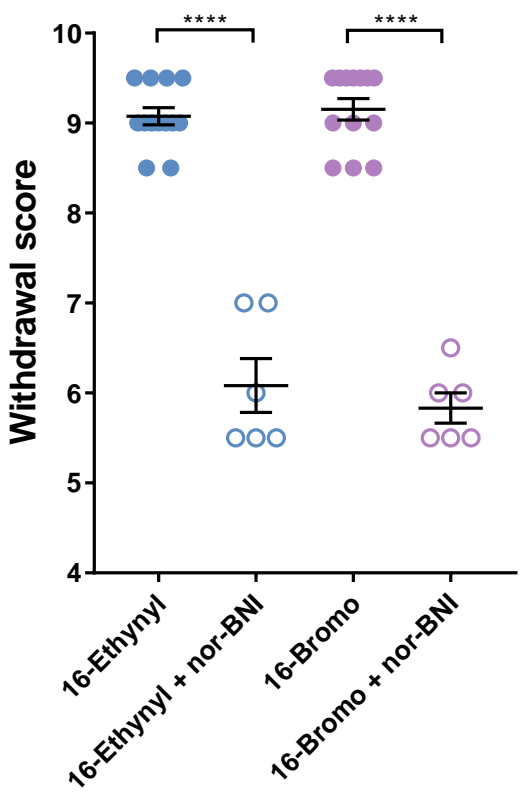

D

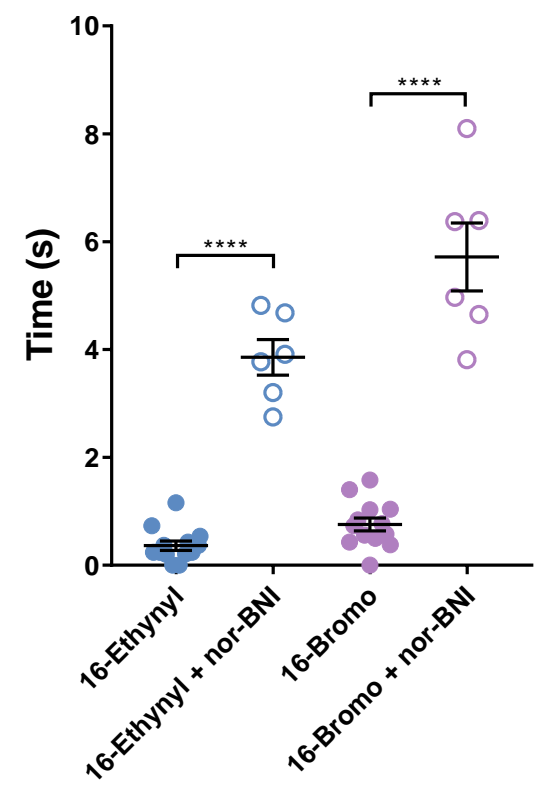

Figure 5.8: KOPr antagonism reduces the antinociceptive effect of the novel KOPr agonists

The selective KOPr antagonist nor-BNI was administered 24 hours prior to the commencement of the dose-response procedure for treatment with the novel KOPr agonists 16-Ethynyl SalA and 16-Bromo SalA. (A) Dose-response curve of antinociceptive effects against mechanical allodynia. (B) Nor-BNI reduced the antinociceptive effects of the KOPr agonists $(10 \mathrm{mg} / \mathrm{kg})$ to the mechanical stimulus. (C) Dose-response curve of antinociceptive effects against cold allodynia. (D) Nor-BNI reduced the antinociceptive effects of the KOPr agonists $(10 \mathrm{mg} / \mathrm{kg})$ to the thermal stimulus. One-way ANOVA with Bonferroni post-tests. Values presented as mean \pm SEM, with individual data points presented (B) and (D). $n=13$ for KOPr agonist treatment without nor-BNI, $n=6$ for groups with nor-BNI pre-treatment. $* * * * p<0.0001$. 


\subsubsection{Early initiation of chronic daily treatments on day 7}

Due to the efficacy of the novel KOPr agonists 16-Ethynyl SalA and 16-Bromo SalA at treating paclitaxel-induced mechanical and cold allodynia in the acute dose-response model, the novel agonists were further tested in a chronic model of paclitaxel. Chronic opioid treatment is more representative of a possible clinical situation and was also used to test the opioid tolerance effects over time. Initially, it was decided to begin treatment on day 7 , to potentially prevent the further development of neuropathic pain soon after the initial onset of symptoms. The mice were matched to treatment groups based on the day 6 allodynia values (see Appendix E.1). The treatment with either morphine, the traditional KOPr agonist U50,488, or the novel agonists 16-Ethynyl SalA and 16-Bromo SalA began on day 7 , with daily administrations up to day 24 . On the odd-numbered days and the final day 24 , the treatment was given 30 min prior to the behavioural measurements. On the evennumbered days, the behaviours were measured prior to the administration of the treatment to test whether the treatment was reducing overall the neuropathic pain condition or only temporally limiting the symptoms (for experimental design see Figure 2.3A).

\subsubsection{Treatment of mechanical allodynia}

The experimental replicate was carried out with a sample size of 6 , with 3 males and 3 females. Threeway repeated measures mixed ANOVA showed there was no three-way interaction of sex, treatment and time $\left[\mathrm{F}_{(29.286,134.7)}=1.459, p=0.078\right]$ and no effect of sex and treatment $\left[\mathrm{F}_{(5,23)}=0.654, p=0.662\right]$, therefore, the sexes were combined for further analysis. Two-way repeated measures ANOVA was used to understand the effect of treatment and time on the mechanical withdrawal thresholds for the days where treatment occurred before the behaviour. There was a significant interaction of treatment $x$ time $\left[F_{(45,261)}=4.332, p<0.0001\right]$ (Figure 5.9A). Bonferroni multiple comparisons showed a significant difference between the vehicle/vehicle negative control and the vehicle/paclitaxel positive control on all days from 9-24 $(p<0.0001)$. Compared to the vehicle/paclitaxel control group, morphine treatment significantly increased the withdrawal thresholds on days 9-24 and U50,488 increased the thresholds on days 17-24 $(p<0.05)$. The novel compounds increased the level of mechanical allodynia at the earlier time points of day 11-13 for 16-Ethynyl SalA and day 11 for 16 -Bromo SalA $(p<0.05)$. At day $23,16-$ Ethynyl SalA reduced the level of mechanical allodynia $(p<0.05)$. To understand the main effect of treatment, the AUC was calculated for days 7-24. One-way ANOVA showed a significant effect of treatment $\left[\mathrm{F}_{(5,29)}=57.57, p<0.0001\right]$ (Figure 5.9B). Compared to the vehicle/paclitaxel treatment group, only morphine had a significant improvement in mechanical withdrawal threshold $(p<0.0001)$, and the morphine treatment group was not significantly different to the vehicle/vehicle control $(p>$ $0.9999)$. U50,488 ( $p=0.4257), 16$-Ethynyl SalA $(p>0.9999)$ and 16-Bromo SalA $(p>0.9999)$ were not significantly different to the vehicle/paclitaxel control. 


\subsubsection{Treatment of cold allodynia}

For the cold allodynia measurements, there was no three-way interaction of sex, treatment and time $\left[\mathrm{F}_{(21.486,98.836)}=1.098, p=0.362\right]$ and no effect of sex and treatment $\left[\mathrm{F}_{(5,23)}=0.878, p=0.511\right]$, therefore, the sexes were combined for further analysis. To test the effects of the opioid treatments on the reaction times to the acetone stimulus, a two-way ANOVA showed an interaction of treatment $x$ time $\left[\mathrm{F}_{(45,261)}=4.35, p<0.0001\right]$ (Figure $5.9 \mathrm{C}$ ). Bonferroni post-tests showed a significant effect between the vehicle/vehicle negative control and the vehicle/paclitaxel treatment on day 11 and 15-24 ( $p<$ 0.05). The opioid treatments produced a reduction in the cold stimulus reaction times, for morphine on days 17 and 23-24; U50,488 on days 17-19 and 23-24; 16-Ethynyl SalA on days 17-19 and 23-24; and 16-Bromo SalA on days 17 and $24(p<0.05)$. On day 13, 16-Ethynyl SalA increased the level of cold allodynia compared to the vehicle/paclitaxel control $(p=0.0040)$. The AUC analysis showed a significant effect of treatment $\left[F_{(5,29)}=11.47, p<0.0001\right]$ (Figure 5.9D). The Bonferroni post-tests showed a significant difference between vehicle/paclitaxel treatment and U50,488/paclitaxel treatment $(p=0.0017)$, in fact, $\mathrm{U} 50,488 /$ paclitaxel treatment was not different to the vehicle/vehicle negative control $(p=0.5496)$. Morphine $(p>0.9999), 16-$ Ethynyl SalA $(p>0.9999)$ and $16-$ Bromo SalA $(p=0.7334)$ were not significantly different to the positive control. 

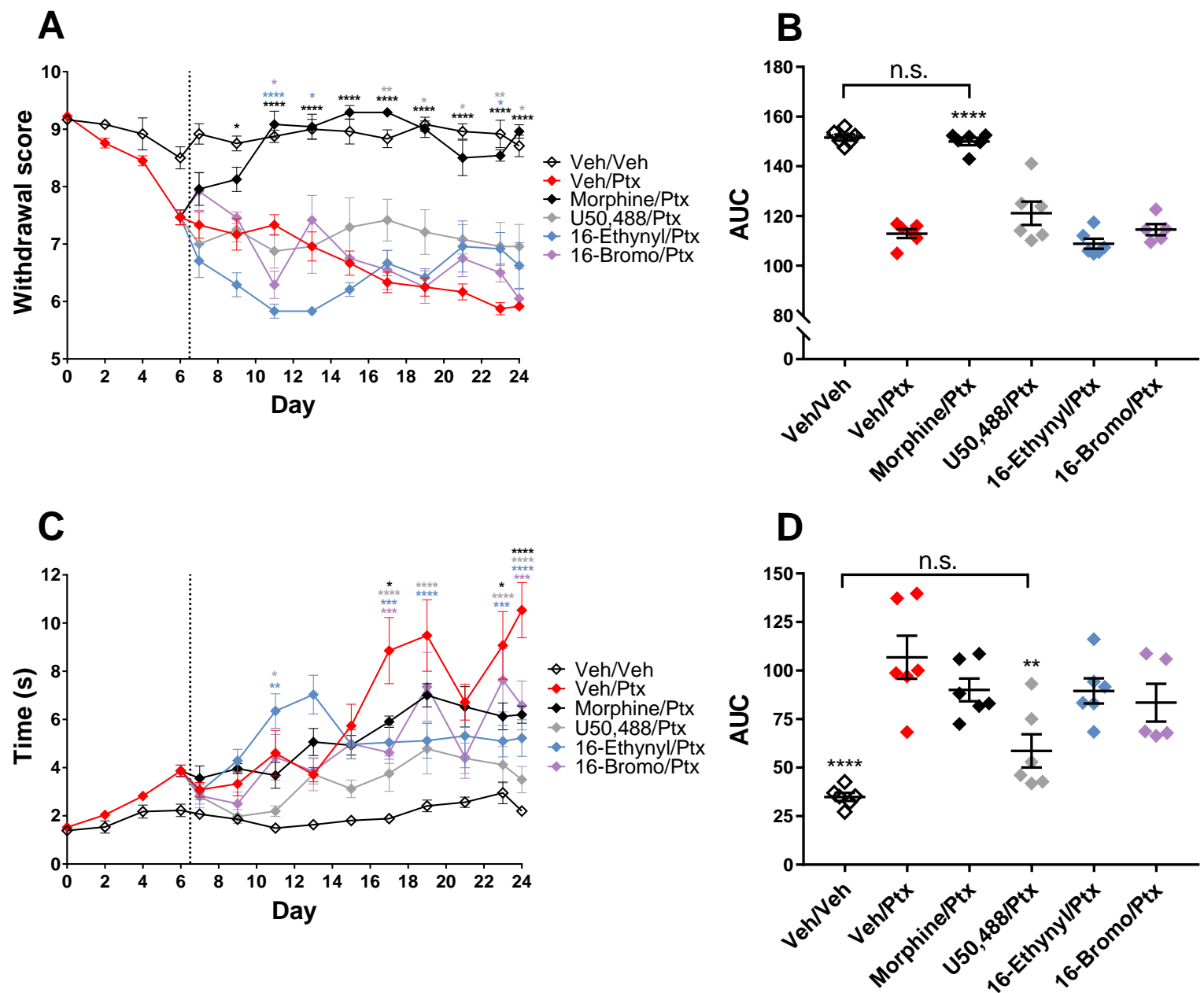

Figure 5.9: Antinociceptive effects of early initiation of chronic daily treatments in mice with paclitaxel-induced neuropathic pain

(A) Time course showing the effects of chronic opioid treatment on paclitaxel (ptx)-induced mechanical allodynia. (B) Area under the curve (AUC) analysis showing the main effect of treatment. Morphine (4.3 mg/kg i.p. daily) reduced mechanical thresholds back to vehicle levels. (C) Time course showing the effects of chronic opioid treatment on paclitaxel-induced cold allodynia. (D) AUC analysis showed the traditional KOPr agonist U50,488 (3.2 mg/kg i.p. daily) reduced cold reaction times to the same as vehicle control levels. Overall, 16 -Ethynyl SalA ( $1.3 \mathrm{mg} / \mathrm{kg}$ i.p. daily) and 16-Bromo SalA (1.9 $\mathrm{mg} / \mathrm{kg}$ i.p. daily) did not reduce mechanical or cold allodynia compared to the vehicle/paclitaxel control treatment group. Two-way ANOVA with Bonferroni post-tests. n.s. $=$ not significant, ${ }^{*} p<0.05$, ${ }^{* *} p<0.01,{ }^{* * *} p<0.005,{ }^{* * * *} p<0.0001$ compared to the vehicle/paclitaxel control treatment group. Values presented as mean \pm SEM, with individual data points presented (B) and (D), $n=6$ for all treatment groups, except 16-Bromo SalA treatment group $n=5$. 


\subsubsection{Progression of neuropathic pain in the early initiation of treatment model}

Two-way repeated measures ANOVA was used to understand the effect of the opioid treatments on the progression of the neuropathic pain on the even-numbered days where behaviour was measured prior to the administration of treatment. There was a significant two-way interaction of treatment $x$ time on mechanical withdrawal thresholds $\left[F_{(40,232)}=3.002, p<0.0001\right]$ (Figure 5.10A). Bonferroni post-tests showed the vehicle/vehicle and vehicle/paclitaxel control groups were significantly different at all days from 8-24 $(p<0.05)$. Compared to the vehicle/paclitaxel treatment group, only 16-Ethynyl SalA was statistically significant, showing increased mechanical allodynia on days 10-14 (p $<0.05$ ). The AUC analysis showed a significant effect of treatment $\left[F_{(5,29)}=30.48, p<0.0001\right]$ (Figure 5.10B). None of the opioid treatments were significantly different compared to the vehicle/paclitaxel treatment group $(p>0.05)$.

For the cold allodynia measurements, there was a two-way interaction of treatment $\mathrm{x}$ time on the days where behaviour was measured before the administration of treatment $\left[F_{(40,232)}=3.489, p<\right.$ 0.0001 ] (Figure 5.10C). The vehicle control groups were significantly different on days $12-24(p<0.01)$. The traditional agonist U50,488 significantly reduced the effects of paclitaxel on days 12 and 22-24. Morphine, 16-Ethynyl SalA and 16-Bromo SalA treatment all reduced the level of cold allodynia compared to the vehicle/paclitaxel treatment group on days $22-24(p<0.0001)$. The AUC analysis showed a significant effect of treatment $\left[\mathrm{F}_{(5,29)}=3.049, p<0.0001\right]$ (Figure 5.10D). Compared to the vehicle/paclitaxel treatment group, U50,488 $(p=0.0113)$ and 16 -Bromo SalA $(p=0.0446)$ significantly reduced the cold allodynia levels. Morphine $(p>0.9999)$ and 16-Ethynyl SalA $(p>0.9999)$ were not significantly different to the positive control.

\subsubsection{Outcome of early initiation of the chronic treatment model}

Overall, the effects of the early initiation of treatment on day 7 showed that morphine was potent at treating mechanical allodynia, whereas U50,488 was potent at treating cold allodynia. In terms of the progression of the neuropathic pain state, none of the treatments altered the mechanical allodynia, whereas U50,488 and 16-Bromo SalA reduced the level of cold allodynia. 

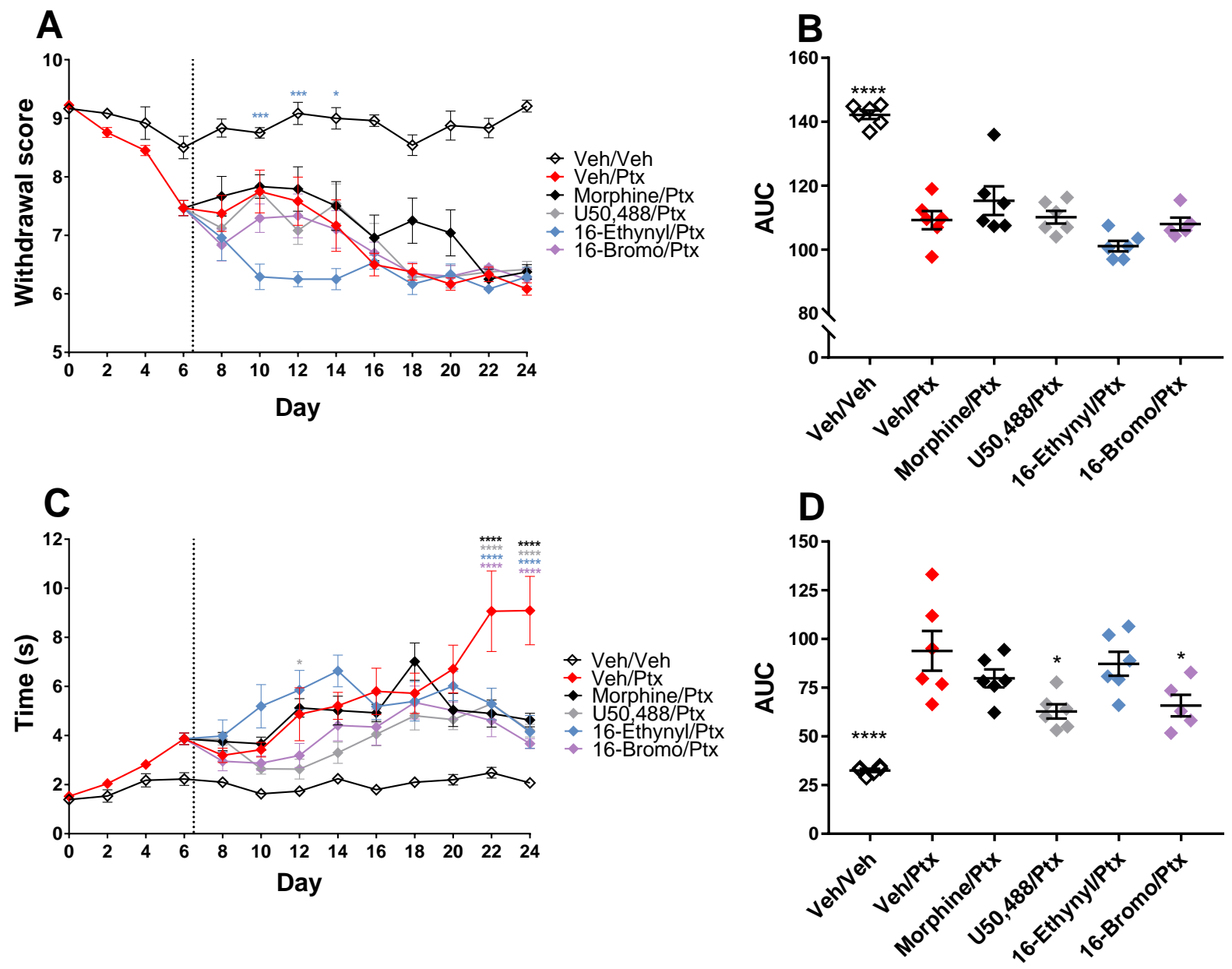

Figure 5.10: Progression of paclitaxel-induced neuropathic pain in mice following the early initiation of daily treatments

(A) Time course showing the level of paclitaxel (ptx)-induced mechanical allodynia. (B) Area under the curve (AUC) analysis showing the main effect of treatment. None of the opioid treatments reduced the paclitaxel-induced mechanical allodynia. (C) Time course showing the level of paclitaxel-induced cold allodynia. (D) AUC analysis showed the KOPr agonists U50,488 (3.2 mg/kg i.p. daily) and 16-Bromo SalA ( $1.9 \mathrm{mg} / \mathrm{kg}$ i.p. daily) reduced cold reaction times compared to the vehicle/paclitaxel treatment group. Neither morphine $(4.3 \mathrm{mg} / \mathrm{kg}$ i.p. daily) or 16 -Ethynyl SalA $(1.3 \mathrm{mg} / \mathrm{kg}$ i.p. daily) reduced the AUC for mechanical or cold allodynia. Two-way ANOVA with Bonferroni post-tests. ${ }^{*} p<0.05,{ }^{* *} p<$ $0.01, * * * p<0.005, * * * * p 0.0001$ compared to the vehicle/paclitaxel control treatment group. Values presented as mean $\pm S E M$, with individual data points presented (B) and (D), $n=6$ for all treatment groups, except 16-Bromo SalA treatment group $n=5$. 


\subsubsection{Initiation of chronic daily treatments on day 16}

The effects of the KOPr agonists at treating and preventing paclitaxel-induced neuropathic pain when administered on day 7 were not very potent. Therefore, the antinociceptive effects were further tested in mice with well-established paclitaxel-induced neuropathic pain. The treatments began on day 16 when the mice had consistent mechanical and cold allodynia. The longer duration for the neuropathic pain effects to be established allowed for more effective matching of the animals into treatment groups with an approximately equal paclitaxel-induced effects (see Appendix E.2), as with the day 7 initiation of treatment, it could not be predicted how the neuropathic pain symptoms were going to continue to progress in each mouse. The dosage of the opioid receptor agonist treatments was also increased to approximately the $\mathrm{ED}_{80}$ value from the acute dose-response experiment.

\subsubsection{Treatment of mechanical allodynia}

To understand the effects of sex, treatment and time on the paclitaxel-induced mechanical allodynia, a three-way repeated measures mixed ANOVA was used. A square root transformation was carried out to correct the positively-skewed data and improve the homogeneity of variances as assessed by the Levene's test for equality of variances. Mauchly's test of sphericity indicated that the assumption of sphericity had been violated, so a Greenhouse-Geisser correction was applied. The three-way interaction of treatment, sex and time was not statistically significant $\left[\mathrm{F}_{(40.528,486.33)}=1.034, p=0.418\right]$ (Figure 5.11A). There was a two-way interaction of treatment and time $\left[\mathrm{F}_{(40.528,486.33)}=9.637, p<\right.$ $0.0005]$, as well as treatment and $\operatorname{sex}\left[\mathrm{F}_{(5,60)}=3.966, p=0.004\right]$, however, there was not an effect of sex and time $\left[\mathrm{F}_{(8.106,486.33)}=1.645, p=0.109\right]$. This means that the treatment had an effect and that the treatment affects the sexes differently, but the effect on the sexes was the same over time. The main effect of treatment were compared at each time point. U50,488 and 16-Ethynyl SalA reduced the paclitaxel-induced mechanical allodynia effects on all days 16-38 $(p<0.0001)$. Morphine treatment had antinociceptive effects on days 16-30, and 16-Bromo SalA on days 16-36 ( $p<0.05$; Figure 5.11A). The effects of sex and treatment were investigated by first comparing the sexes within each treatment group. The U50,488 treatment showed a significant effect, with less pain shown in the males compared to females $(p<0.0005)$, similarly, 16-Ethynyl SalA treated the males more effectively $(p=$ 0.003; Figure 5.11B). The main treatment effects within the males group showed that morphine, U50,488, 16-Ethynyl SalA and 16-Bromo SalA all reduce the paclitaxel-induced mechanical allodynia levels $(p<0.0005$; Figure 5.11D). Furthermore, U50,488 treatment reduced the mechanical withdrawal thresholds back to equivalent to the vehicle/vehicle treatment group $(p>0.9999)$. The treatments in the females showed that morphine, U50,488, 16-Ethynyl SalA and 16-Bromo SalA all reduce the paclitaxel-induced mechanical allodynia $(p<0.0005)$, however, all of the treatment groups were significantly different to the vehicle/vehicle group (Figure 5.11F). 

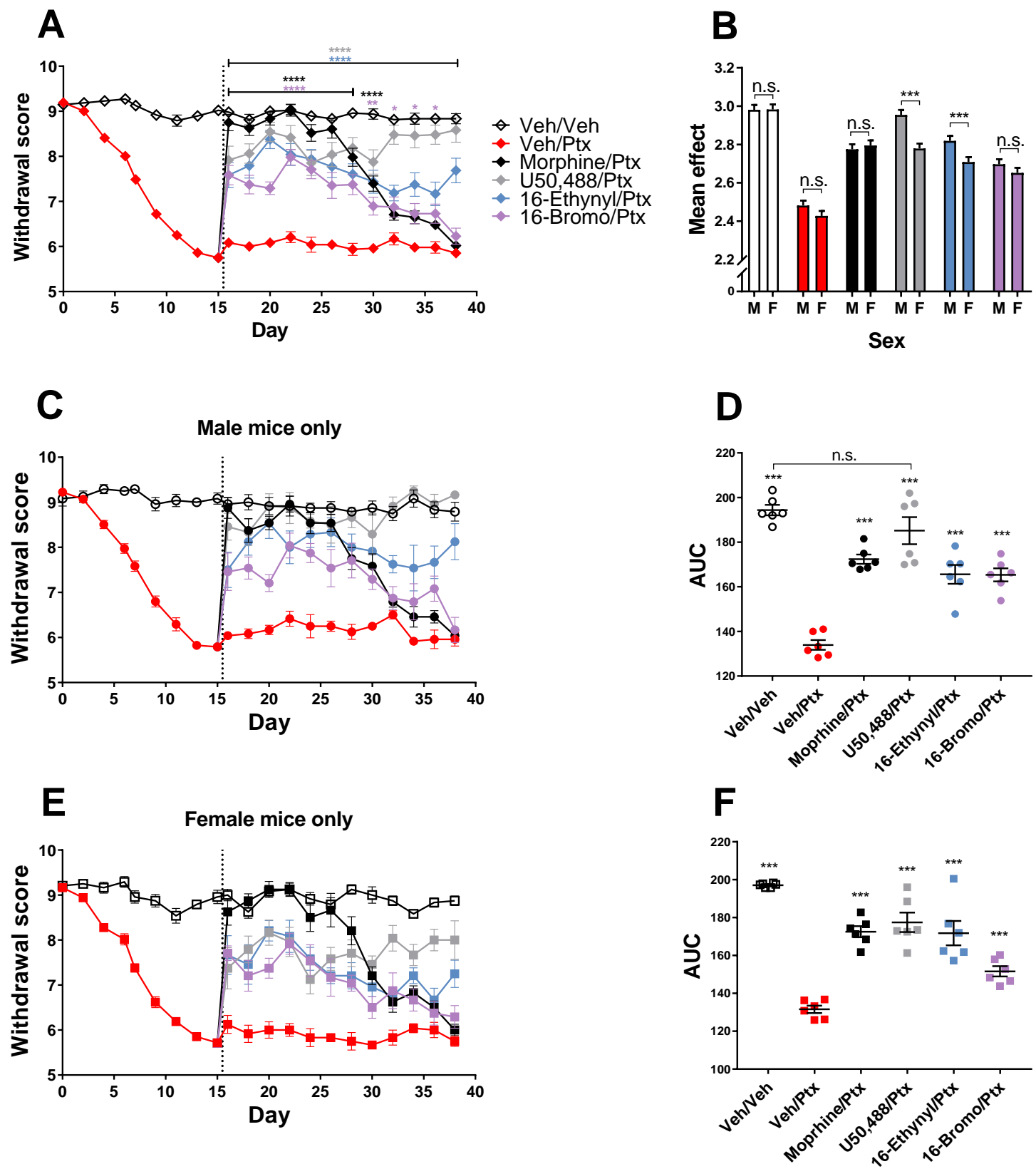

Figure 5.11: Antinociceptive effects of opioid receptor agonists on paclitaxel-induced mechanical allodynia following initiation of chronic daily treatment on day 16

(A) Time course of the treatment effects on paclitaxel-induced mechanical allodynia. (B) Main interaction effects of treatment and sex showed that U50,488 (10 mg/kg i.p. daily) and 16-Ethynyl SalA $(3 \mathrm{mg} / \mathrm{kg}$ i.p. daily) were more effective at treating males. (C) Time course of the treatment effects in males. (D) Comparison of the treatments within the male animals showed morphine $(10 \mathrm{mg} / \mathrm{kg}$ i.p. daily), U50,488, 16-Ethynyl SalA and 16-Bromo SalA (4 mg/kg i.p. daily) all reduced the paclitaxelinduced effects, with U50,488 reducing the mechanical thresholds to vehicle/vehicle levels. (E) Time course of the treatment effects in males. (F) Comparison of the treatments within the male animals showed all opioid treatments reduced the paclitaxel-induced effects. Three-way mixed ANOVA with Bonferroni post-tests. n.s. $=$ not significant, ${ }^{*} p<0.05,{ }^{* *} p<0.01,{ }^{* * *} p<0.005,{ }^{* * *} p<0.0001$ compared to the vehicle/paclitaxel control treatment group. Values presented as mean \pm SEM, with individual data points presented (D) and (F), $n=12$ for all treatment groups ( 6 males, 6 females). 


\subsubsection{Treatment of cold allodynia}

A three-way repeated measured mixed ANOVA was also used to understand the effects of treatment, sex and time on the reaction times to the cold acetone stimulus. A logarithmic transformation was carried out to correct the positively-skewed data and improve the homogeneity of variances as assessed by the Levene's test for equality of variances. Mauchly's test of sphericity indicated that the assumption of sphericity had not been violated. There was a significant three-way interaction of treatment, sex and time $\left[F_{(55,660)}=1.481, p=0.016\right]$ (Figure 5.12A). The interaction of treatment and sex were compared at every time point and the statistical significance of an interaction effect was accepted at a Bonferroni-adjusted alpha level of 0.004. There was not a significant simple two-way interaction of treatment and sex at any time point $(p>0.004)$, however, there was a significant main effect of treatment on every day $(p<0.0005)$. Therefore, the effects of treatment were further assessed. Morphine, U50,488 and 16-Ethynyl SalA treatment reduced the paclitaxel-induced effects on days 16-38 ( $p<0.05$; Figure 5.12A). 16-Bromo SalA had significant effects at days 16-24, 28, 32 and 36-38 $(p<0.05)$. Since there was no effect of treatment and sex, only the main effects of treatment were assessed and show that U50,488, 16-Ethynyl SalA and 16-Bromo SalA all reduce the paclitaxelinduced cold allodynia levels ( $p<0.001$; Figure 5.12B). Furthermore, U50,488 treatment reduced the cold reaction times back to the same level as the vehicle/vehicle treatment group $(p>0.9999)$. 

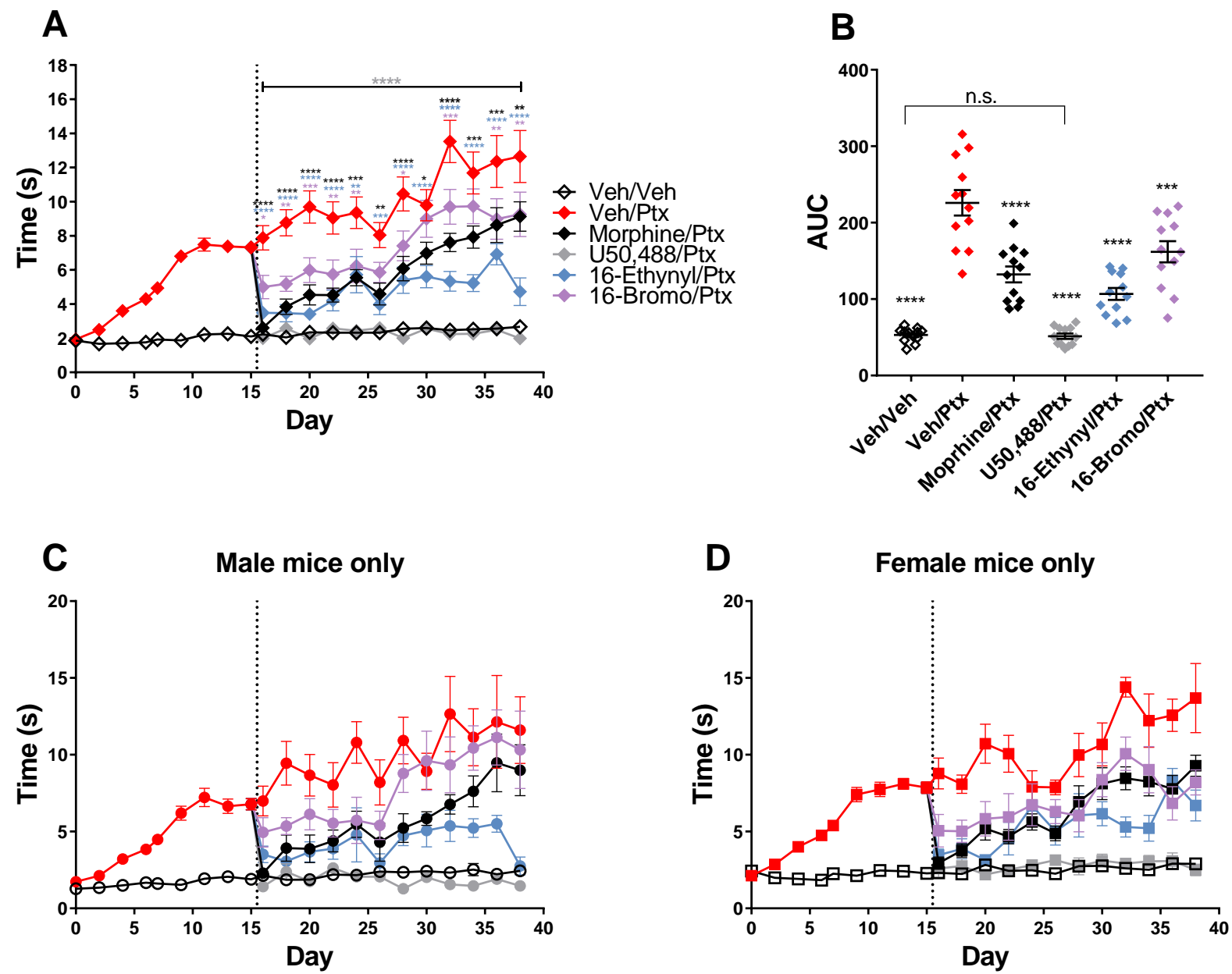

Figure 5.12: Antinociceptive effects of opioid receptor agonists on paclitaxel-induced cold allodynia following initiation of chronic daily treatment on day 16

(A) Time course showing the effect of treatment on paclitaxel-induced cold allodynia. (B) Main effects of treatment show that morphine $(10 \mathrm{mg} / \mathrm{kg}$ i.p. daily), U50,488 (10 mg/kg i.p. daily), 16-Ethynyl SalA ( $3 \mathrm{mg} / \mathrm{kg}$ i.p. daily) and 16-Bromo SalA ( $4 \mathrm{mg} / \mathrm{kg}$ i.p. daily) all reduced the paclitaxel-induced effects, with U50,488 reducing the mechanical thresholds to vehicle/vehicle levels. (C) Effect of treatment over time in male animals. (D) Effect of treatment over time in female animals. Three-way mixed ANOVA with Bonferroni post-tests. n.s. = not significant, ${ }^{*} p<0.05,{ }^{* *} p<0.01,{ }^{* * *} p<0.005,{ }^{* * * *} p<$ 0.0001 compared to the vehicle/paclitaxel control treatment group. Values presented as mean $\pm S E M$, with individual data points presented (B), $n=12$ for all treatment groups ( 6 males, 6 females). 


\subsubsection{Reduction of neuropathic pain}

On the odd-numbered days, the behavioural tests were measured prior to the administration of treatment, to test whether the treatments were resolving the paclitaxel-induced neuropathic pain. A three-way repeated measures mixed ANOVA was used to understand the effects of treatment, sex and time on the mechanical withdrawal thresholds. Mauchly's test of sphericity indicated that the assumption of sphericity had been violated, so a Greenhouse-Geisser correction was applied. There was no three-way interaction $\left[\mathrm{F}_{(37.747,452.97)}=1.385, p=0.068\right]$ or two-way interaction of treatment and time $\left[\mathrm{F}_{(37.747,452.97)}=1.390, p=0.066\right]$ (Figure 5.13A). However, there was a two-way interaction of treatment and $\operatorname{sex}\left[\mathrm{F}_{(5,60)}=4.992, p=0.001\right]$. Comparison of the sexes within each treatment showed that there was a significantly less mechanical allodynia in the males in the vehicle/paclitaxel group ( $p$ $=0.037)$, as well as for the treatments morphine $(p<0.0005)$, U50,488 $(p<0.0005), 16$-Ethynyl SalA $(p=0.020)$ and 16-Bromo SalA $(p<0.0005$; Figure 5.13B). Within the male animals, there was a significant improvement between the vehicle/paclitaxel group and morphine treatment $(p=0.002)$ as well as 16 -Bromo SalA treatment ( $p=0.001$; Figure 5.13D). Among the females, only the positive and negative controls were significantly different (Figure 5.13F).

A three-way repeated measures ANOVA was carried out to understand the effects of treatment, sex and time on the reaction times to a cold acetone stimulus prior to the administration of the opioid receptor agonist treatments. A logarithmic transformation was carried out to correct the positivelyskewed data and improve the homogeneity of variances as assessed by the Levene's test for equality of variances. Mauchly's test of sphericity indicated that the assumption of sphericity had been violated, so a Greenhouse-Geisser correction was applied. There was no three-way interaction $\left[\mathrm{F}_{(37.131,445.48)}=1.222, p=0.179\right]$, and no statistically significant two-way interaction between treatment and time $\left[\mathrm{F}_{(37.131,445.48)}=1.382, p=0.071\right]$ or treatment and $\operatorname{sex}\left[\mathrm{F}_{(5,60)}=0.779, p=0.569\right]$. However, there was a main effect of treatment $\left[\mathrm{F}_{(5,60)}=44.348, p<0.0005\right]$. Compared to the vehicle/paclitaxel treatment group, only the $U 50,488$ treatment reduced the level of cold allodynia ( $p=0.001$; Figure $5.14 B)$. 
A

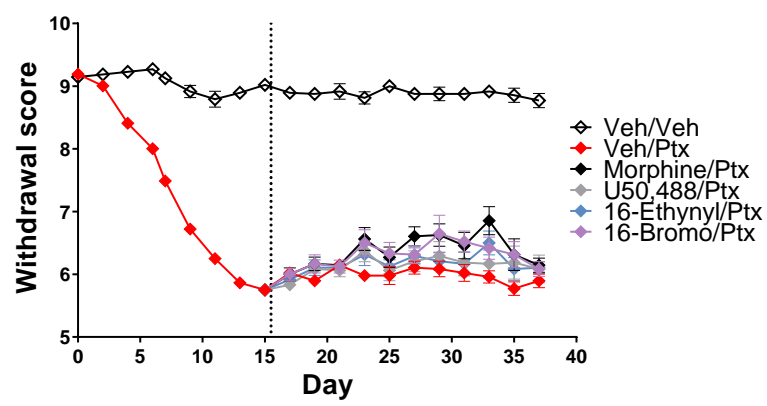

C

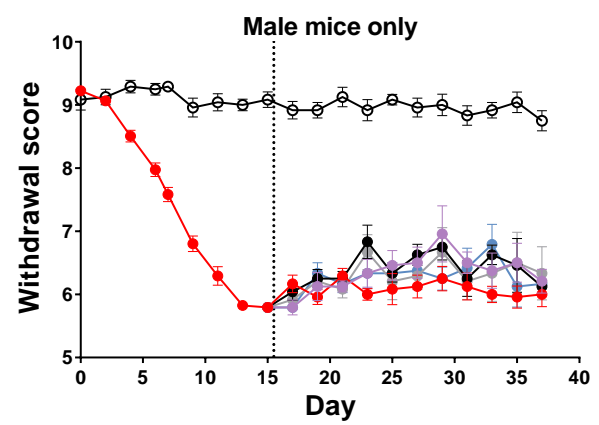

\section{E}

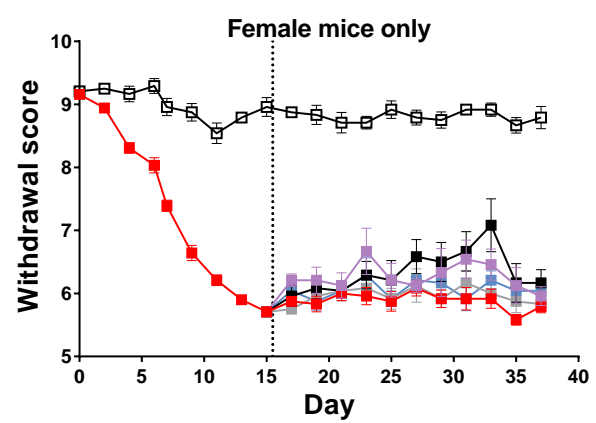

B

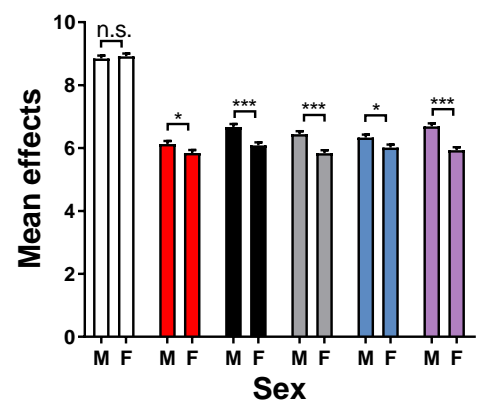

D

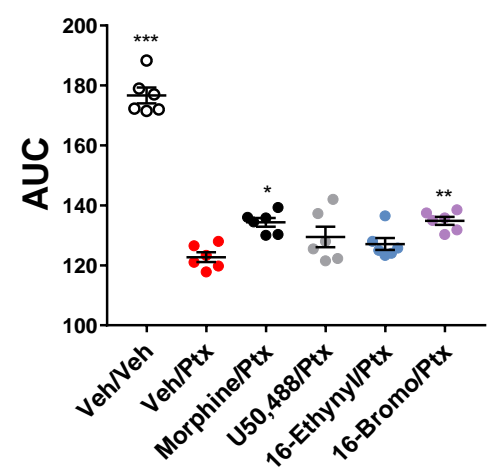

$\mathbf{F}$

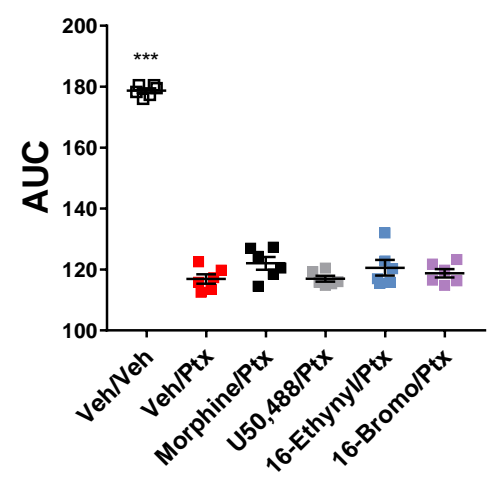

Figure 5.13: Mechanical allodynia with measurement before treatment

(A) Time course of the antinociceptive effects of the opioid receptor agonists on paclitaxel-induced cold allodynia 24 hours after administration of treatment. (B) Main effects of each treatment compared by sex. Vehicle, morphine (10 mg/kg i.p. daily), U50,488 (10 mg/kg i.p. daily), 16-Ethynyl SalA ( $3 \mathrm{mg} / \mathrm{kg}$ i.p. daily) and 16-Bromo SalA ( $4 \mathrm{mg} / \mathrm{kg}$ i.p. daily) treatment in mice with established paclitaxel-induced cold allodynia, showed less allodynia in male mice. (C) Time course of antinociceptive effects in male mice. (D) The main effect of treatment in male mice. Morphine and 16Bromo SalA treatment groups had significantly less allodynia compared to the vehicle/paclitaxel treatment group. (E) Time course of antinociceptive effects in female mice. (F) The main effect of treatment in female mice, with only the vehicle control groups showing a significant difference. Threeway mixed ANOVA with Bonferroni post-tests. n.s. = not significant, ${ }^{*} p<0.05,{ }^{* *} p<0.01,{ }^{* * *} p<0.005$ compared to the vehicle/paclitaxel control treatment group. Values presented as mean $\pm \mathrm{SEM}$, with individual data points presented (D) and (F), $n=12$ for all treatment groups ( 6 males, 6 females). 

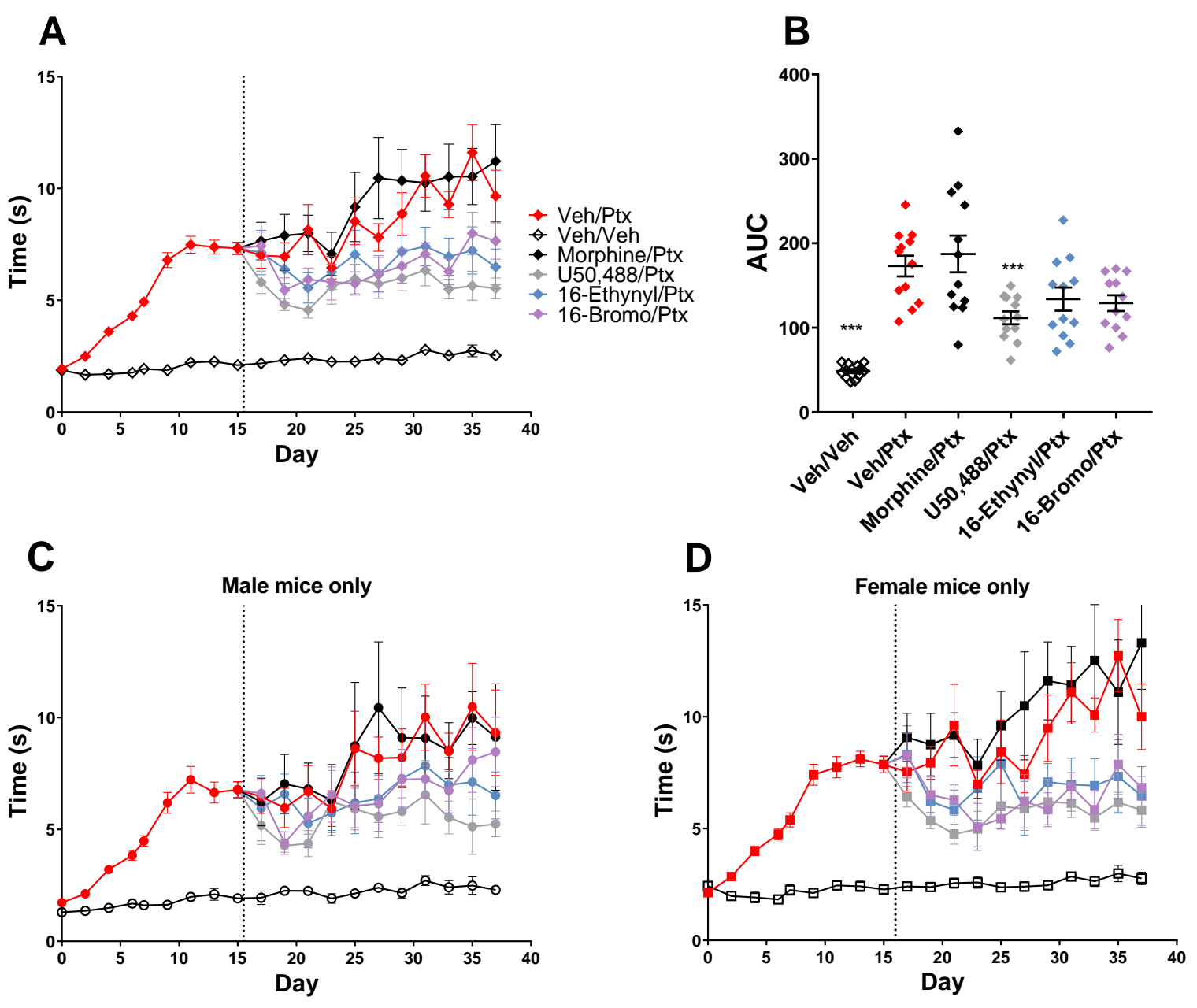

Figure 5.14: Cold allodynia with measurement before daily treatment

(A) Time course of the antinociceptive effects 24 hours following the administration of the opioid receptor treatment on cold allodynia. (B) The main effects of treatment showed U50,488 $(10 \mathrm{mg} / \mathrm{kg}$ i.p.) significantly reduced the paclitaxel-induced cold allodynia. (C) Time course of antinociceptive effects in male mice. (D) Time course of antinociceptive effects in female mice. Three-way mixed ANOVA with Bonferroni post-tests. ${ }^{*} p<0.05,{ }^{* *} p<0.01,{ }^{* * *} p<0.005$ compared to the vehicle/paclitaxel control treatment group. Values presented as mean $\pm \mathrm{SEM}$, with individual data points presented (B), $n=12$ for all treatment groups ( 6 males, 6 females). 


\subsubsection{Transmission electron microscopy}

Paclitaxel treatment leads to degeneration of myelin and vacuolated mitochondria in the axons of the sciatic nerve. Following the chronic administration experiment, the sciatic nerves of male mice treated with vehicle/vehicle, vehicle/paclitaxel or U50,488/paclitaxel were collected and imaged using TEM. To measure the integrity of the myelin sheath, g-ratios were calculated for each animal by dividing the diameter of the axon by the diameter of the outer myelin sheath. Normal distribution was assumed due to the small sample size, with one-way ANOVA showing a significant effect of treatment $\left[F_{(2,6)}=\right.$ 1.203, $p=0.0056$ ] (Figure 5.15A). Bonferroni post-tests showed the paclitaxel treatment significantly reduced the g-ratio compared to the vehicle control $(p=0.0200)$. The $U 50,488$ treatment in mice with established paclitaxel-induced neuropathic pain did not improve the g-ratio compared to the vehicle/paclitaxel treatment group ( $p>0.9999$ ) and was significantly lower than the vehicle/vehicle control $(p=0.0077)$.

The percentage of atypical mitochondria in the myelinated axons were compared between the treatment groups showing a significant effect of treatment $\left[F_{(2,6)}=2.079, p=0.0012\right]$ (Figure 5.15B). Paclitaxel treatment induced a higher percentage of swollen and vacuolated mitochondria. Both the vehicle $(p=0.0032)$ and $\mathrm{U} 50,488(p=0.0054)$ treatment groups in paclitaxel-intoxicated mice had an increased proportion of atypical mitochondria compared to the vehicle/vehicle treatment group. There was no difference between the vehicle/paclitaxel group and the U50,488 treatment group ( $p$ > 0.9999). 

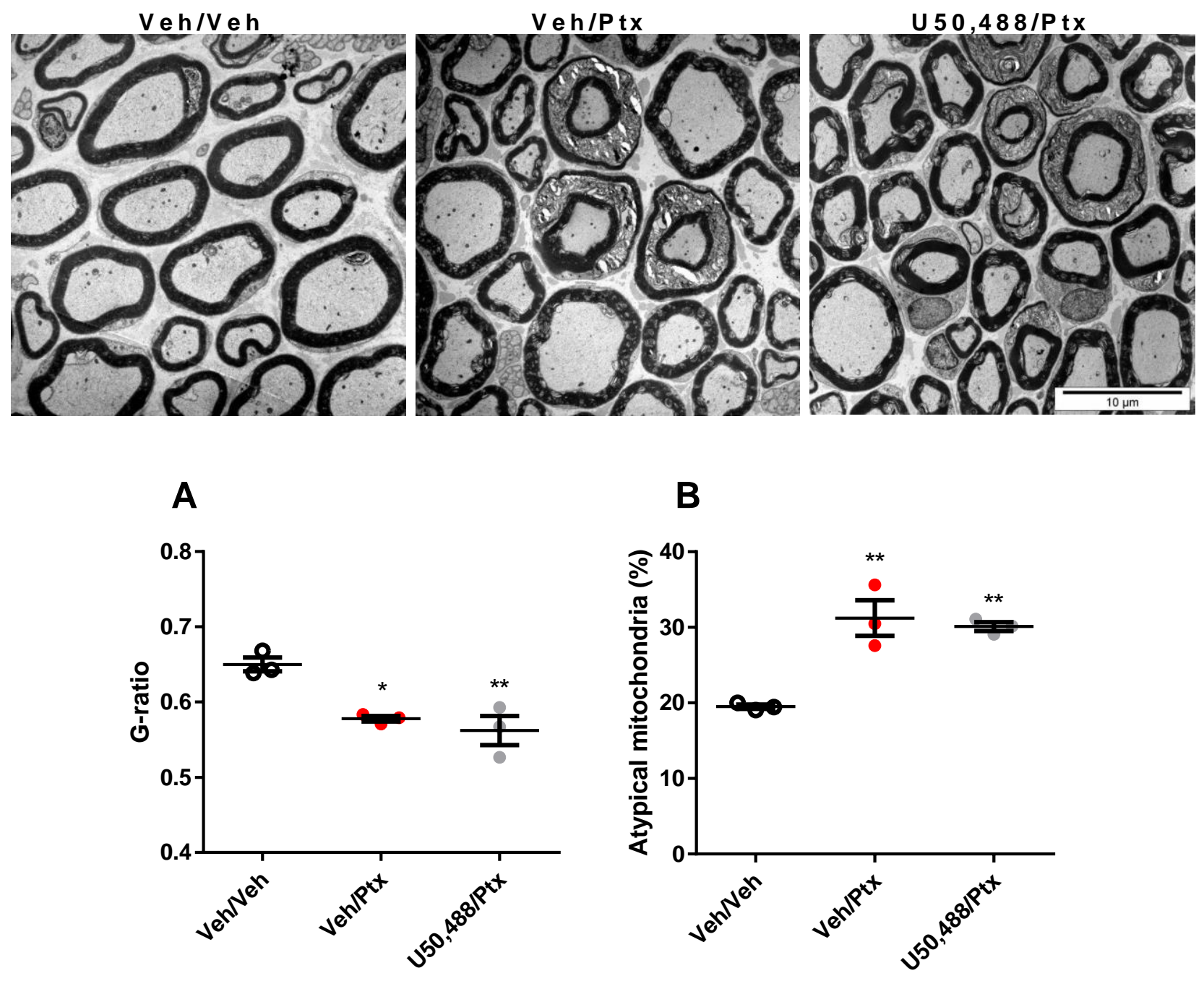

\section{Figure 5.15: Ultrastructure of the sciatic nerve}

Representative transmission electron microscopy images of the vehicle/vehicle, vehicle/paclitaxel and U50,488/paclitaxel treatment groups. (A) G-ratio measurements in the sciatic nerves are calculated by the ratio of the axon diameter to myelin diameter. Paclitaxel treatment significantly reduced the g-ratio compared to vehicle treatment. Chronic U50,488 treatments did not reverse the effects of paclitaxel. (B) Quantitative analysis of the percentage of atypical mitochondria. Paclitaxel treatment significantly increased the percentage of atypical mitochondria, however, U50,488 treatments did not reduce the effect of paclitaxel. One-way ANOVA with Bonferroni post-tests. ${ }^{*} p<0.05,{ }^{* *} p<0.01$ compared to the vehicle/vehicle control group. Values presented as individual data points, with a line indicating the mean \pm SEM. $n=3$ male mice per treatment group. 100 myelinated nerves $/$ mouse assessed for G-ratio analysis. All mitochondria found in 100 myelinated nerves assessed/mouse (at least 300 mitochondria/animal). 


\subsubsection{Outcome of the initiation of chronic treatment on day 16 model}

Overall, in the initiation of chronic treatment on day 16 model, sex differences in the treatments were only present in the mechanical allodynia data. U50,488 and 16-Ethynyl SalA were more potent in males as opposed to females. When comparing the individual sex treatment groups, all opioid treatments reduced the effects in both females and males, however, U50,488 was particularly potent in males, by reducing the thresholds back to vehicle/vehicle level. Similarly, in the combined sex data for the treatment of cold allodynia, all opioid treatments reduced the level of pain, however, U50,488 reduced the pain back to vehicle/vehicle levels. In the progression of neuropathic pain studies, morphine and 16-Bromo SalA modestly reduced the mechanical allodynia in male mice and U50,488 reduced cold allodynia. Due to the potent effects of U50,488, the ultrastructure of the sciatic nerve was assessed in a sample of male mice. The results showed that U50,488 treatments did not reverse the demyelination and percentage of atypical mitochondria induced by paclitaxel treatment.

A summary of the sex differences across all of the paclitaxel-induced neuropathic pain procedures are presented in Table 5.8 on the following page. 


\begin{tabular}{|c|c|c|c|c|c|c|c|}
\hline Experimental procedure & Veh/Veh & Veh/Ptx & Morphine/Ptx & U50,488/Ptx & 16-Ethynyl/Ptx & 16-Bromo/Ptx & SalA/Ptx \\
\hline \multicolumn{8}{|l|}{ Baseline levels (day 0) } \\
\hline -mechanical allodynia & $\sigma^{x}=$ o & - & - & - & - & - & - \\
\hline -cold allodynia & $\sigma^{\pi}<$ Q & - & - & - & - & - & - \\
\hline \multicolumn{8}{|l|}{ Day 15 levels } \\
\hline -mechanical allodynia & $\sigma^{x}=$ o & $\sigma^{x}=$ o & - & - & - & - & - \\
\hline -cold allodynia & $\sigma<q$ & $\sigma^{n}<q$ & - & - & - & - & - \\
\hline \multicolumn{8}{|l|}{ Dose-response effects } \\
\hline -mechanical allodynia & - & - & $\sigma^{\prime}=$ Q & $\sigma^{\top}<$ Q & $\sigma^{\#}=q^{\#}$ & $\sigma^{\prime}=q$ & $\sigma^{\top}>Q^{\#}$ \\
\hline -cold allodynia & - & - & $\sigma^{n}=q$ & $\sigma^{n}=q$ & $\sigma^{x}=q$ & $\sigma^{x}=q$ & $\sigma^{n}=q$ \\
\hline \multicolumn{8}{|l|}{ Chronic admin on day 16} \\
\hline -mechanical allodynia & $\sigma^{n}=q$ & $\sigma^{n}=q$ & $\sigma^{*}=$ o* & $\sigma^{*}<$ \&* & $\sigma^{*}<$ o* & $\sigma^{*}=$ Q* & - \\
\hline -cold allodynia & $\sigma^{\prime}=$ Q & $\sigma^{x}=q$ & $\sigma^{\pi}=$ Q & $\sigma^{\prime}=$ Q & $\sigma^{\prime}=$ Q & $\sigma^{x}=$ Q & - \\
\hline \multicolumn{8}{|l|}{ Chronic ptx development } \\
\hline -mechanical allodynia & $\sigma^{\prime}=q$ & $\sigma^{\prime}<$ Q & $\sigma^{*}<$ Q & n.e. & n.e. & $\sigma^{*}<$ \& & - \\
\hline -cold allodynia & $\sigma^{x}=$ Q & $\sigma^{x}=q$ & n.e. & $\sigma^{x}=q$ & n.e. & n.e. & - \\
\hline
\end{tabular}

Table 5.8: Summary of the sex differences in the paclitaxel-induced neuropathic pain experimental results

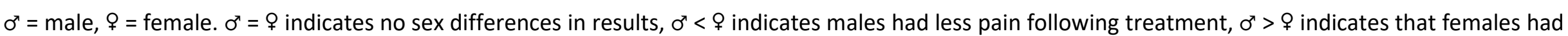
less pain following treatment. "indicates that treatment was significantly more potent than morphine treatment in the corresponding sex. ${ }^{* i n d i c a t e s}$ that treatment had significantly less allodynia compared to the vehicle/paclitaxel group in the corresponding sex. n.e. $=$ no effect of treatment on either sex. 


\subsection{Discussion}

Pain management is a major problem in cancer patients. Pain can be caused by the active tumour growth and by chemotherapy treatments, with $68 \%$ of patients reporting CINP symptoms within the first month of treatment (Seretny et al., 2014). This is often the reason for limiting or stopping potentially life-saving chemotherapy treatment (Holmes et al., 1991; Rowinsky et al., 1993). Currently there is no recommended therapy for prevention of CINP (Hershman et al., 2014). For patients with established CINP, there is one recommended analgesic treatment, however, duloxetine has limited effects at treating paclitaxel-induced neuropathic pain (Smith et al., 2013). Currently, opioid therapies, such as morphine, are used as a third-line treatment for CINP as many side effects, such as respiratory depression and gastrointestinal transit, as well as addiction and tolerance are associated with longterm use of MOPr agonists (Chu et al., 2006; Compton \& Volkow, 2006). Therefore, this study investigated the effects of novel KOPr agonists, which are known to be non-rewarding (Vonvoigtlander et al., 1983), and are not associated with respiratory depression (Freye et al., 1983) or gastrointestinal transit (Porreca et al., 1984).

\subsubsection{Establishment of the paclitaxel-induced neuropathic pain model in mice}

The paclitaxel-induced neuropathic pain model had not been previously carried out in the Kivell laboratory. The dosage regimen for inducing the paclitaxel-induced neuropathic pain model was selected as $4 \times 4 \mathrm{mg} / \mathrm{kg}$ injections on alternate days based on methodology in Deng et al. (2015). In this study, paclitaxel-induced pain effects were established in both female and male C57BL/6J mice. A previous study in C57BL/6 mice had used $1 \times 6 \mathrm{mg} / \mathrm{kg}$ injection (Materazzi et al., 2012), however, this had only been tested for mechanical allodynia in male mice. The majority of studies administer the paclitaxel treatment over multiple days, typically on 4 alternate days, to induce robust neuropathic pain effects ( 15 of the 21 studies in Table 5.4 were administered on 4 alternate days; 3 additional studies were administered on 5 consecutive days).

Since it was unknown if the animals would show any signs of adverse effects, the animals in the first experimental replicate were closely monitored. The effect of paclitaxel on the general health of the mice appeared to be minimal. The paclitaxel-treated animals of either sex did not show any changes in weight compared to the vehicle treated animals (Figure 5.1). The 12 male mice in the first experimental replicate were assessed for spontaneous pain behaviours using the grimace scale. Only one mouse showed signs of pain behaviour (Figure 5.2), which was observed on both day 4 and 7 , but not observed after this on day 11 or 15 . This finding is similar to previous studies which have shown mice and rats had no obvious signs of spontaneous pain and had normal weight gain (Smith et al., 
2004; Hwang et al., 2012). This was important as we wanted to induce the stimulation-evoked neuropathic pain without causing any further complications or other symptoms in the mice.

The baseline allodynic effects were measured on day 0 prior to the first injection of paclitaxel and assessed for sex differences in the response to mechanical and cold stimulation. There was no significant difference between the males and females in response to the mechanical stimulus (Figure 5.4A), however, it was found that females respond for a significantly longer time following stimulation of the paw with a cold stimulus (Figure 5.4B). In terms of the induction of neuropathic pain, there was a significant effect of treatment from days 4-15 for mechanical allodynia (Figure 5.5A). There was no effect of sex at any of the time points. However, for the cold allodynia measurements, there was a significant main effects of treatment and sex on the days 2-15 (Figure 5.5C). The results show that paclitaxel induces cold allodynia equally in both sexes, however, since the females began with a longer reaction time, this longer response is maintained and on day 15 females responded for a longer time than males (Figure 5.5D). This is interesting as previous studies have shown no differences between the sexes. In a study using NMRI mice, the females had increased paw licking using the acetone model of cold allodynia on days 7-11, however, measurements on days 13 and 15 were not significantly different to males (Naji-Esfahani et al., 2016). The same study found no sex differences in the development of mechanical allodynia measured using von Frey filaments. Similarly, mechanical allodynia had no differences between the sexes in C57BL/6 mice (Smith et al., 2004) and Sprague Dawley rats (Hwang et al., 2012).

\subsubsection{Dose-response effects of opioid receptor agonists}

We believe this is the first instance of KOPr agonists being used to treat paclitaxel-induced neuropathic pain in rodents, and therefore the dose-response effects were initially assessed to ascertain the efficacy and potency of each treatment. The KOPr agonists were compared to morphine to relate to a clinically available treatment. In the mechanical threshold testing, U50,488 was significantly more potent in males compared to females (Table 5.5). In comparison, SalA treatment was more potent in females. The other treatments did not show any differences between the sexes. No sex differences in morphine treatment have previously been shown in Sprague Dawley rats with paclitaxel-induced neuropathic pain, where an acute morphine treatment at both $2 \mathrm{mg} / \mathrm{kg}$ and $5 \mathrm{mg} / \mathrm{kg}$ i.p. had the same effect against mechanical allodynia in males and females (Hwang et al., 2012). Interestingly, in rhesus macaques, SalA has been shown to have potential sex differences in the pharmacokinetic effects, with females showing a slower elimination from plasma $(37.9 \pm 5.6 \mathrm{~min}$ in males vs. $80.0 \pm 13.1 \mathrm{~min}$ in females) and a larger area under the concentration-time curve $(572 \pm 100 \mathrm{ng} \mathrm{min} / \mathrm{ml}$ in males vs. 1087 $\pm 46 \mathrm{ng} \mathrm{min} / \mathrm{ml}$ in females) following i.v. injection of SalA (Schmidt et al., 2005a). Previous studies have 
shown U50,488 exhibits higher antinociceptive potency in males when measured with the tail withdrawal assay (reviewed in Rasakham \& Liu-Chen, 2011).

The potency of each treatment was then further compared within each sex. When compared to the males treated with morphine, only the males treated with 16-Ethynyl SalA showed more potent effects (Table 5.5). Among the females, SalA and 16-Ethynyl SalA treatments were more potent than morphine (Table 5.5). For the cold allodynia testing, there was found to be no significant interaction between treatment and sex, and therefore the sexes were combined to find the effect of treatment. SalA, 16-Ethynyl SalA and $\beta$-THP SalB (males only) were significantly more potent compared to morphine (Table 5.7). Therefore, 16-Ethynyl SalA was more potent than morphine in all aspects of the dose-response results. Similar results were presented in Chapter 4, where 16-Ethynyl SalA was more potent and efficacious than the traditional agonist U50,488 in the warm water tail withdrawal assay (Figure 4.1 and Table 4.3). The dose-response effects of 16-Ethynyl SalA and 16-Bromo SalA on mechanical and cold allodynia were reversed by administering a pre-treatment of the KOPr antagonist nor-BNI (Figure 5.8).

Whilst the effects of KOPr agonists have not been previously tested in paclitaxel-induced neuropathic pain, multiple MOPr agonists have been assessed (see Table 5.4). In male Sprague Dawley rats, the dose-dependent effects against paclitaxel-induced mechanical pain showed that fentanyl was the most potent, followed by oxycodone and morphine (Mori et al., 2014). A further study in male Sprague Dawley rats found that $2.5 \mathrm{mg} / \mathrm{kg}$ i.p. morphine reduced both mechanical allodynia and thermal hyperalgesia, which was reversed by pre-treating with naloxone (Pascual et al., 2010). Methadone was also found to be effective at $2.5 \mathrm{mg} / \mathrm{kg}$ i.p. in thermal hyperalgesia and at $5 \mathrm{mg} / \mathrm{kg}$ i.p. in mechanical allodynia (Pascual et al., 2010). Another study measuring the effects of morphine in paclitaxelintoxicated Sprague Dawley rats found that acute treatment with $8 \mathrm{mg} / \mathrm{kg}$ i.p. reduced the mechanical allodynia by $50 \%$ (Flatters \& Bennett, 2004), compared to the current study which found an $\mathrm{ED}_{50}$ of $2.16 \mathrm{mg} / \mathrm{kg}$ s.c. in male mice. However, strain differences can have a large effect on the paclitaxelinduced neuropathic model, and therefore, it is not accurate to compare between Sprague Dawley

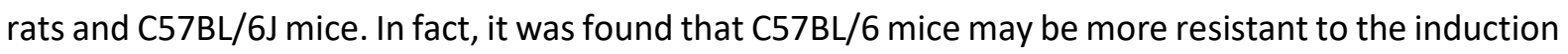
of mechanical allodynia by paclitaxel compared to other strains of mice (Smith et al., 2004). After extensive searching, no studies have been found that assess the effects of MOPr agonists in a mouse model of paclitaxel-induced neuropathic pain. 


\subsubsection{Chronic administration of the opioid receptor agonists}

Once the dose-response effects had been studied, the paclitaxel-induced neuropathic pain model was used to assess the effects of chronic administration of the opioid receptor agonist treatments. In the initial study, the opioid receptor agonists were administered beginning on day 7 , with the expectation that treatment could be initiated at the point where mechanical and cold allodynia becomes pronounced from the vehicle treatment, to mimic a typical clinical situation, and that the opioid treatments would limit the further progression of the neuropathy. A lower dose, based on the approximate $\mathrm{ED}_{60}$ value was selected to limit the sedative effects of the opioid receptor agonists. One experimental replicate was carried out with a sample size of 6 ( 3 males and 3 females), however, upon analysis of the results, a different treatment schedule was selected as the primary focus of this study. This left the results of this early initiation of treatment with a sample size too small to statistically study the sex differences, and therefore the sexes were combined to give the main effects of treatment over time.

In the treatment of mechanical allodynia, morphine reduced the effects induced by paclitaxel, and the AUC analysis showed no difference between morphine therapy in paclitaxel-treated mice than in vehicle/vehicle-treated mice (Figure 5.9A and B). Treatment with U50,488 increased the withdrawal thresholds on days 17-24, however, the novel KOPr agonists 16-Ethynyl SalA and 16-Bromo SalA showed an increased level of mechanical allodynia on days 11 and 13 compared to the vehicle/paclitaxel treatment (Figure 5.9A). 16-Ethynyl SalA treatment significantly reduced mechanical allodynia on day 23. In the cold allodynia experiments, the U50,488 treatment reduced the reaction times back to the same level as the vehicle/vehicle treatment (Figure 5.9C and D). Again, 16-Ethynyl SalA increased the level of pain on day 11, however, the treatment reduced pain on days 17-19 and 23-24. Morphine treatment reduced cold stimulation pain on days 17 and 23-24 and 16-Bromo SalA treatment reduced pain on days 17 and 24 (Figure 5.9C). Overall, the data shows that morphine was effective at treating mechanical allodynia over 18 days of treatment, whereas U50,488 was effective at treating cold allodynia. It is unclear as to why particularly the 16-Ethynyl SalA treatment group showed an increased level of pain compared to the vehicle/paclitaxel treatment, however, it could be that since the paclitaxel-induced effects were not well-established before the initiation of treatment, the animals could not be assigned to treatment groups with matched levels of neuropathic pain. In fact, animals from the same cage were given the same treatment, and it is possible that the animals that happened to receive the 16-Ethynyl SalA therapy were more sensitive to the neuropathic effects of paclitaxel. In the further chronic administration procedures, animals were carefully matched into treatment groups with equivalent average levels of allodynia. 
On alternate days during the chronic administration experiment, the behavioural tests were carried out prior to the opioid receptor agonist treatment to assess the progression of the paclitaxel-induced neuropathic pain. In the time course of the mechanical allodynia results, once more 16-Ethynyl SalA was shown to increase the level of pain on days 10-14 (Figure 5.10A). Comparing the overall treatment effects, none of the treatments altered level of mechanical allodynia (Figure 5.10B). In comparison, all of the opioid receptor agonists treatment groups showed a reduction in the cold allodynia on days 22 and 24 (Figure 5.10C). The overall treatment effects showed that U50,488 and 16-Bromo SalA treatment was associated with less mechanical allodynia (Figure 5.10D). However, it is unclear as to whether this means that the treatments had long-lasting effects, showing antinociceptive potential 24 hours following administration; if the treatments limited the progression or further development of the paclitaxel-induced neuropathy; or if the mice assigned to these treatment groups by chance were less sensitive to the effects of paclitaxel. It is unlikely that the antinociceptive effects were still present 24 hours following administration as the KOPr agonists are not known to be this long-lasting. In the next experimental procedure, the opioid receptor agonists were administered on day 16 , once the paclitaxel-induced effects were well-established. In this instance, the mice were matched to each treatment group by comparing the mechanical allodynia scores on day 15 (see Appendix E.2) and the doses were increased to approximately the $\mathrm{ED}_{80}$ value. For the treatment of mechanical allodynia, there was no three-way effect of treatment, sex and time, so the overall treatment effects were assessed over time for the combined sexes. U50,488 and 16-Ethynyl SalA significantly improved the mechanical allodynia at all days 16-38 (Figure 5.11A). Morphine treatment reduced the paclitaxel effects up until day 30, when it appears the animals became tolerant to the antinociceptive effects. Similarly, 16-Bromo SalA reduced mechanical allodynia until day 36. The mean effects of the treatments compared by sex showed that U50,488 and 16-Ethynyl SalA were more effective at treating the male mice (Figure 5.11B). When comparing within the sexes, all of the opioid receptor agonist treatments of each sex showed improvement in treating mechanical allodynia compared to the vehicle/paclitaxel control (Figure 5.11D and F). However, only males treated with U50,488 reduced mechanical threshold score back to vehicle level (Figure 5.11D). This is similar to the dose-response results, which showed that U50,488 was more potent in male mice (Table 5.5).

The opioid receptor agonists were also effective against the cold allodynia symptoms in mice with established paclitaxel-induced neuropathic pain. In this analysis there were no significant effects of sex, so the sexes were combined. The morphine, U50,488 and 16-Ethynyl SalA treatment groups had significant effects at all of the days 16-38 (Figure 5.12A). 16-Bromo SalA significantly reduced the paclitaxel-induced cold allodynia effects at days 16-24, 28, 32 and 36-38. When comparing the main effects of the treatments, all of the opioid receptor agonists reduced the level of allodynia compared 
to the vehicle/paclitaxel control, however, only $\mathrm{U} 50,488$ was not significantly different to the vehicle/vehicle control group (Figure 5.12B). Therefore, both in the mechanical allodynia scores in males and the cold allodynia reaction times in both sexes, U50,488 significantly reversed the effects of paclitaxel over 23 days, with no apparent tolerance effects. In the warm water $\left(55^{\circ}\right)$ tail withdrawal assay in C57BL/6 mice, U50,488 has been shown to cause tolerance effects, however, this was with an escalating dose scheme up to $75 \mathrm{mg} / \mathrm{kg}$ i.p. over 4 days (McLaughlin et al., 2004), whereas this was not the case in the current study that used $10 \mathrm{mg} / \mathrm{kg}$ i.p. treatment daily. Interestingly, in a partial spinal nerve ligation model of neuropathic pain in male C57BL/6 mice, phosphorylated $\mathrm{KOPr}$ immunoreactivity was increased in the L4-5 dorsal horn regions of the spinal cord, specifically in both GABAergic neurons and GFAP-positive astrocytes (Xu et al., 2004). KOPr knock-out mice also showed increased mechanical allodynia and thermal heat hyperalgesia. However, because of this endogenous KOPr activation in the mice with neuropathic pain, treatment with $U 50,488$ showed increased tolerance compared to sham, and this tolerance effect was absent in prodynorphin or GRK3 knockout mice (Xu et al., 2004). However, the endogenous KOPr effect may be mediated via the NMDA receptor, as sciatic nerve ligation leads to increased dynorphin, which at increased concentrations also activates NMDA receptors (Vanderah et al., 1996). KOPr antagonism with nor-BNI in mice and rats leads to increased levels of mechanical and thermal allodynia, however, co-administration of nor-BNI and ketamine lead to the abolishment of the neuropathic pain symptoms (Obara et al., 2003). In comparing to the current study, because U50,488 did not show the tolerance effects associated with endogenous KOPr activation, it could be that the endogenous KOPr system is not activated to the same extent in the paclitaxel-induced neuropathic pain model, however, this effect in paclitaxelinduced neuropathic pain has not been studied. 


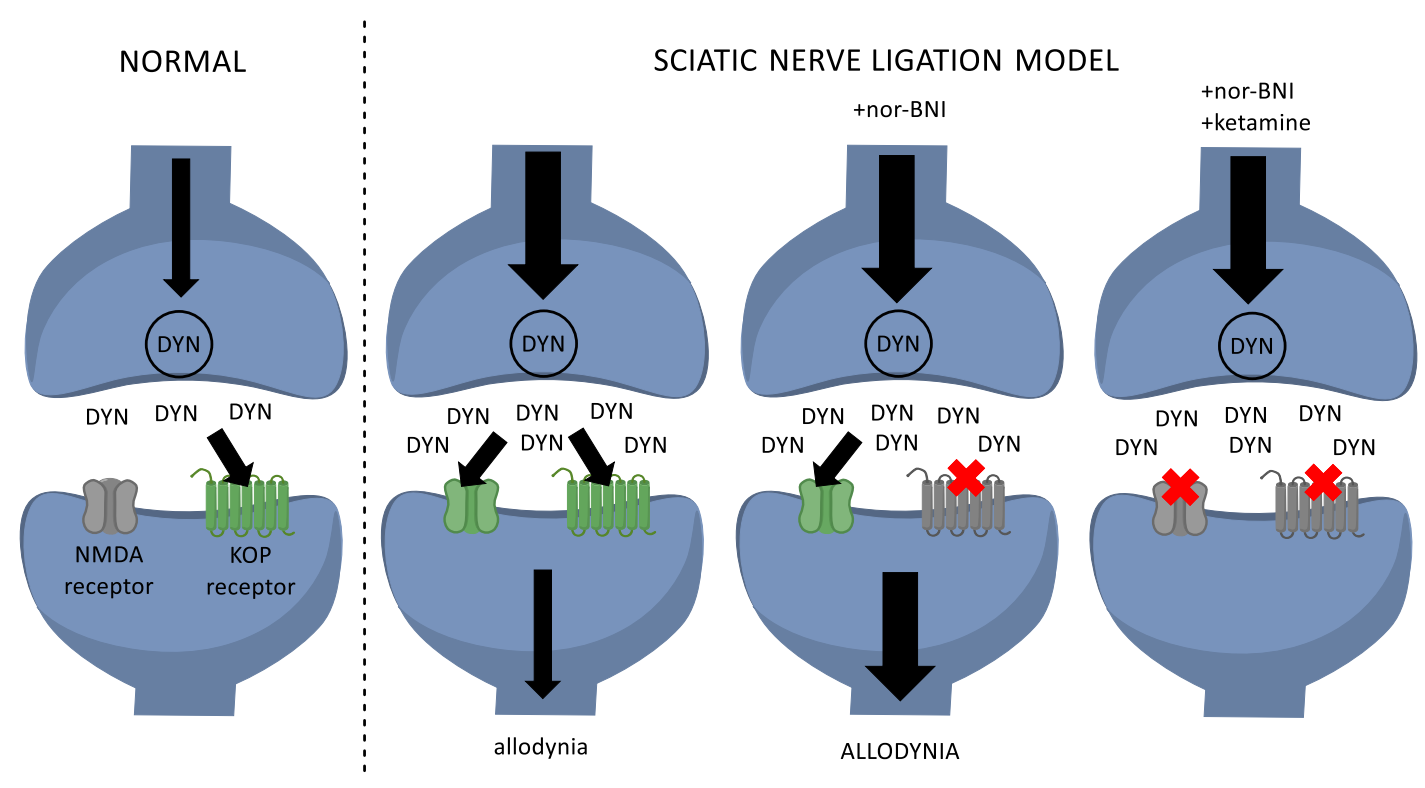

Figure 5.16: Proposed mechanism for the effects of dynorphin and the KOPr and NMDA receptor in a model of neuropathic pain

During normal physiological dynorphin signalling, the majority of the effects are mediated via the kappa opioid (KOP) receptor. However, in the sciatic nerve ligation model in mice and rats, dynorphin signalling increases, which activates the N-methyl-D-aspartate (NMDA) receptor leading to the development of allodynia. When the KOPr antagonist nor-binaltorphimine (nor-BNI) is administered, the level of mechanical and thermal allodynia is increased. With coadministration of nor-BNI and the NMDA receptor antagonist ketamine, the allodynic effects are removed. Figure adapted from Obara et al. (2003).

SalA has been previously shown to reduce neuropathic pain in a sciatic nerve ligature model in male Wistar rats (Coffeen et al., 2018). In this study, SalA was injected directly into the insular cortex, and reduced the mechanical and thermal allodynia measured immediately and $30 \mathrm{~min}$ after injection. Pretreatment with nor-BNI and the CB1 receptor antagonist AM251 reversed the effects of SalA (Coffeen et al., 2018). Furthermore, an extract of $S$. divinorum, which contained both SalA and SalB as well as other substances found in the leaves of the plant, reduced mechanical and thermal sciatic nerve ligature neuropathic pain when administered at 100-200 mg/kg i.p. (Simon-Arceo et al., 2017). However, no previous studies were found for the treatment of CINP with SalA. However, one study found that U50,488 treated cancer-induced bone pain (Edwards et al., 2018), suggesting it could be used as a target for both tumour-mediated and chemotherapy-mediated pain. 
In the chronic administration model, the animals were also tested every second day to measure the continued development of the paclitaxel-induced neuropathic effects. For the mechanical testing, there was a significant interaction between treatment and sex, with all the paclitaxel-treated animals showing increased mechanical allodynia in the female mice (Figure 5.13B). This included the vehicle/paclitaxel control, perhaps showing that in this experiment, females are more sensitive to paclitaxel than males. This is contrary to the day 15 values previously assessed that showed no sex differences (Figure 5.5), however, the chronic administration model was carried out over a longer period of time, which may be required to observe these sex differences. In the $30 \mathrm{~min}$ post-treatment measurements, the same vehicle/paclitaxel mice did not show sex differences in the withdrawal thresholds (Figure 5.11), further complicating the analysis. However, it has been shown that male and female mice have differing stress-induced antinociception mechanisms (Mogil et al., 1997), and since these days where the treatment is administered first are likely to be more stressful on the animals, this effect could play a role. When compared within the sexes, $\mathrm{U} 50,488$ and 16 -Bromo SalA had less mechanical allodynia in the male animals compared to the vehicle/paclitaxel control (Figure 5.13D). There was no difference between the females, except between the positive and negative controls (Figure 5.13F). In the cold allodynia testing, the combined sex data show $U 50,488$ significantly reduced the cold allodynia pain (Figure 5.14B). Therefore, since U50,488 does not have a long antinociceptive duration of action (Millan et al., 1987), it could be hypothesised that there is another mechanism of action occurring that is potentially reducing the paclitaxel-induced neuropathy.

CINP is a condition that predominantly affects the nerves of the PNS and therefore the ultrastructure of the sciatic nerve was studied to assess the damage to the myelin sheath and the mitochondria. The results showed that there was a decrease in the g-ratio following treatment with paclitaxel (Figure 5.15A). Treatment with U50,488 did not alter the paclitaxel-induced myelin damage. Similarly, paclitaxel treatment induced a higher percentage of atypical mitochondria compared to the vehicle/vehicle treatment, and U50,488 treatment did not alter this effect (Figure 5.15B). This paclitaxel-induced increase in g-ratio and high atypical mitochondrial occurrence in the sciatic nerve has also shown by Chen et al. (2017b), where co-treatment with minoxidil prevented these paclitaxelinduced effects. Similarly, lithium treatment prevented the development of demyelination in the sciatic nerve of C57BL/6 female mice (Mo et al., 2012). However, these two studies administered the treatment one hour before the paclitaxel injections, therefore acting as prevention therapy, as opposed to the current study that started treatment once paclitaxel-induced neuropathic pain was established on day 16. After searching the literature, no studies were found where treatment of established neuropathic pain lead to alterations in the myelin damage in the PNS, and it may only be possible to prevent this damage, not repair it. 


\subsubsection{Limitations and future directions}

One of the major limitations of this study is that fact that the opioid receptor agonists have sedative properties at high doses. In the previous chapter, it was shown that 16-Ethynyl SalA and 16-Bromo SalA at $1-2 \mathrm{mg} / \mathrm{kg}$ and morphine at $10 \mathrm{mg} / \mathrm{kg}$ had motor coordination deficits in the rotarod performance assay (Figure 4.10). Since the treatments affect motor coordination, the ability of the animal to physically withdraw the paw from the mechanical or cold stimulus could be affected, appearing to show antinociceptive effects. However, in the chronic administration model for treatment of cold allodynia, there was no instance where the animals did not react to the stimulation at all (Figure 5.12), indicating that the treatments did allow for the coordination required to selectively withdraw the paw applied with acetone. It was initially attempted to use lower doses of approximately the $\mathrm{ED}_{60}$ value in the chronic administration model, however, these doses were less effective and were subsequently increased for further experiments. The rotarod performance assay could be carried out on paclitaxel-treated animals that have received the opioid receptor agonist treatment to test the effect in this model. Rotarod performance has been tested in C57BL/6J mice that have been treated with paclitaxel, and these animals produced no motor deficits (Deng et al., 2015). Another study in female C57BL/6 mice found a decrease in the rotarod performance 2 weeks post-paclitaxel, but this motor deficit effect was recovered by week 3-4 (Mo et al., 2012). Therefore, it could be tested if the compounds cause any motor deficits beyond that caused by paclitaxel. However, since the higher doses are needed to produce antinociceptive effects, there is not much that could be done to reduce the motor incoordination effects except to design new improved KOPr agonists that have limited sedative effects.

To improve on the behavioural testing methodologies, an electronic von Frey instrument could be used to get a more precise measurement of the paw withdrawal threshold. The current methodology uses a series of manual filaments and a simplified up-down system used to measure the withdrawal threshold. The specific up-down system used here is an improvement on the traditional methodology described in Chaplan et al. (1994) which requires between 4-9 applications of the von Frey filaments per measurement. In the simplified up-down methodology, there are a standard 5 applications for every measurement (Bonin et al., 2014), which removes the variation in the amount of stimulation each animal receives, which is especially important when measurements are repeatedly recorded such as in the dose-response experiment. However, use of an electronic von Frey instrument would eliminate the need for multiple applications and the results are measured in force, rather than a score calculated by estimating the threshold based on which filaments causes the paw to withdraw. 
The acetone model of cold allodynia could also be further improved. The problems with this model are the potential to cause mechanical stimulation from the syringe when the acetone bubble is applied to the paw. The idea is to isolate the cold allodynia effects as any mechanical stimulation would interfere with the interpretation of the results. In addition, the acetone could have direct effects on the skin or olfactory cues during application. Furthermore, the technique of application, the volume of acetone applied and room temperature could all affect the rate at which the acetone evaporates, potentially extending the length of the stimulus and therefore the animal's reaction time. As a further model, hyperalgesic reactions to a heat stimulus could also be measured using the Hargreaves test.

The strain of rodent used in experiments can have a large effect on the outcome of the results. In the current study, C57BL/6J mice were selected as this strain had been used in previous studies in males and females showing mechanical and cold allodynia (Ward et al., 2011; Materazzi et al., 2012; Ward et al., 2014; Deng et al., 2015; Chen et al., 2017b; Liang et al., 2018). However, in a comparison of 10 inbred mouse strains, C57BL/6 mice were relatively lower responders to mechanical stimulus, however, there was no difference in the cold reaction measurements (Smith et al., 2004). However, these mice were given a $4 \mathrm{mg} / \mathrm{kg}$ cumulative dose of paclitaxel, compared to $16 \mathrm{mg} / \mathrm{kg}$ in the current study which represents a more typical paclitaxel regime relative to other preclinical studies (Table 5.4). The dose used in this study gives a robust effect on mechanical and cold allodynia, which was repeated over many experimental replicates. Although if the preclinical results in Table 5.4 are compared to the clinical trial data in Table 5.2, there are many examples, including gabapentin, amitriptyline, lamotrigine and ketamine, which were shown to be effective in a rodent model but ineffective compared to placebo in a clinical trial. This could indicate that better models need to be used to increase the translation of the preclinical results into effective therapeutics.

Interestingly, MOPr/KOPr heterodimers in the spinal cord are found in female and male Sprague Dawley rats at levels from highest to lowest expression in the following order: proestrous, then diestrous females, followed by males, suggesting that oestrogen levels lead to the formation of these heterodimers (Chakrabarti et al., 2010). Dimerisation of the receptors could be important for the antinociceptive effects of the KOPr agonists. For female mice the oestrous cycle is only 4-5 days in length, and it was decided for these experiments not to assess the stage of cycle or attempt to synchronise the cycles. However, in future experiments, this information may lead to new insights into the female pain levels and reduce variability in the results.

The study compared the effects of the KOPr agonists to morphine, as the aim of the research is to find improved opioid receptor agonists that do not have the addictive effects traditionally produced by MOPr agonists. Currently, MOPr agonists are used as a third-line treatment for CINP due to the 
addictive effects, and other side effects including respiratory depression (Finnerup et al., 2015; Grace et al., 2016). Therefore, this study aimed to test the efficacy of the KOPr agonists, with the plan that they could replace MOPr agonists as an improved third-line treatment. However, considering how effective the KOPr agonists were in the paclitaxel-induced neuropathic pain model, these compounds could be developed as a first-line treatment. Especially, for U50,488, as this compound did not show any reduction in the effects over time, indicating that there were not any tolerance effects over the 23 day treatment period. Since U50,488 is known to produce side effects, this structure would be a promising chemical scaffold for the development of improved biased KOPr agonists for the treatment of CINP. Another interesting compound is nalfurafine, which is the clinically available KOPr agonist used in Japan to treatment haemodialysis-induced pruritus (Kumagai et al., 2010), since this compound has already been assessed for side effects and deemed suitable for use in the clinic (Ueno et al., 2013; Nakao et al., 2016). For comparison, the compounds could be compared to a clinically available first-line treatment such as duloxetine or gabapentin.

It would be interesting to understand the effects of the KOPr on CINP, considering the effect of the endogenous dynorphin peptide in nerve ligation models of neuropathic pain (Obara et al., 2003). In terms of measuring the behavioural effects, KOPr knock-out mice could be administered with paclitaxel and test whether there any changes in the induction of mechanical and cold allodynia. If there is a similar effect to the nerve ligation model, then with the KOPr knocked out, there would be an increase in the level of allodynia. In the acute dose-response experiment, nor-BNI was administered to paclitaxel-treated animals on day 14,24 hours prior to the dose-response procedure. There was not any difference between the day 15 pre-treatment values of the nor-BNI treated animals compared to the animals that did not receive nor-BNI (see Appendix D.4). If there are no endogenous effects of the KOPr system in the development of paclitaxel-induced neuropathic pain, the knock-out mice could be used to show that the effects of the compounds tested in the chronic administration are mediated via the KOPr. The KOPr mediated effects of the novel compounds were tested in the dose-response model (Figure 5.8), however, the chronic effects of the compounds need to be assessed. Once confirmed that the effects are mediated via the KOPr, the effects of the receptor expression, phosphorylation and internalisation could be assessed in the lumbar spinal cord. Typically, tolerance effects would show an increase in the phosphorylation and internalisation of the receptor, however, since U50,488 did not show tolerance over the time period measured, this compound could be having a different effect.

Further studies should be performed to understand the exact mechanism occurring. Treatment with U50,488 significantly altered the cold allodynia progression in the days where behaviour was measured prior to the administration of treatment (Figure 5.14). It could be hypothesised that there 
is another mechanism of action, potentially reducing the paclitaxel-induced neuropathy. In the current study, the peripheral sciatic nerve was examined using TEM as CINP is primarily neurotoxic in the PNS. The results showed that the measure was sensitive enough to see differences in the positive and negative control, however, U50,488 treatment did not alter the effect of the paclitaxel (Figure 5.15). It is known that astrocyte activation in the CNS is involved in the neuropathogenesis of paclitaxelinduced neuropathic pain (Zhang et al., 2012) and microglia have been shown to be involved in some studies but not in others (Peters, 2007; Zhang et al., 2012). In addition, CD68-positive macrophage infiltration occurs in the peripheral sites of the DRG and sciatic nerve (Peters, 2007; Tasnim et al., 2016). From the sciatic nerve, lumbar spinal cord and DRG tissue collected at the end of the chronic administration model, antibodies for GFAP, OX42 and CD68 could be used to identify and understand the effects of the opioid treatments on the activation and presence of these cells. Interestingly, it was recently shown that KOPr activation promotes oligodendrocyte differentiation and neuron remyelination and repair to damaged neurons both in vivo and in vitro (Du et al., 2016; Mei et al., 2016). One paper found that paclitaxel administration leads to myelin degeneration in the dorsal column of the spinal cord in C57BL/6 mice, however, the level of degeneration was not quantified, with only representative images presented in the paper (Tasnim et al., 2016). Paclitaxel has limited access to the CNS as it does not readily cross the blood-brain barrier (Fellner et al., 2002) and therefore less damage likely occurs in the spinal cord. However, it could be interesting to study the myelin degeneration in the lumbar spinal cord where the sensory nerves from the hind legs innervate the spinal cord. Myelin could be investigated using toluidine blue staining or further TEM sections which would also give information on the level of damage to the mitochondria. 


\subsection{Summary}

In the dose-response experiment for the treatment of mechanical allodynia, $\mathrm{U} 50,488$ was more potent in male mice, whereas, SalA was more potent in females. Comparing within the sexes, males treated with 16-Ethynyl SalA had more potent effects than males treated with morphine; and females treated with SalA or 16-Ethynyl SalA had more potent effects than females treated with morphine. For the treatment of cold allodynia, the sexes were not significantly different. SalA and 16-Ethynyl SalA treatments were significantly more potent than morphine treatment. Pre-treatment with nor-BNI reversed the effects of the novel compounds 16-Ethynyl SalA and 16-Bromo SalA.

In the chronic administration model with treatments beginning on day $16, \mathrm{U} 50,488$ and 16-Ethynyl SalA were more potent in males compared to females for the treatment of mechanical allodynia. When compared within the individual sexes, all opioid treatments significantly reduced the allodynic effects of paclitaxel, with U50,488 treated males showing the same mechanical threshold scores as vehicle/vehicle treatment. For the cold reaction times, there were no sex differences. For the overall treatment, all opioid receptor agonists reduced the paclitaxel-induced cold allodynia, however, only U50,488 reduced the reaction times back to vehicle level. The ultrastructure of the sciatic nerve showed paclitaxel treatment reduced the g-ratio of axon/myelin diameter and increased the percentage of atypical mitochondria. Treatment with U50,488 did not alter these paclitaxel-induced effects.

In conclusion, the KOPr agonist U50,488 was the most promising treatment for paclitaxel-induced neuropathic pain as mechanical allodynia in males and cold allodynia in both sexes were reduced to baseline levels. U50,488 is known to have KOPr-mediated side effects and is not a clinically viable therapy, however, the novel KOPr agonists also showed promising results, as both compounds significantly reduced mechanical and cold allodynia. This study is the first evidence that KOPr agonists could be useful at treating paclitaxel-induced neuropathic pain and could be used to design further drugs for the treatment of this difficult to manage condition. 


\section{Chapter 6: General discussion}

\subsection{Significance of the study}

Many people are currently suffering from chronic pain without effective management, with high social and economic consequences. Current analgesic therapies include MOPr agonists which are highly addictive, with opioid overdoses now the leading cause of accidental death in the USA, causing 42,249 deaths in 2016 (Jones et al., 2018). Therefore, there is a need to develop effective non-addictive analgesic medications. The aim of this study was to screen and evaluate the antinociceptive effect of several novel compounds in preclinical animal behavioural models of nociceptive, inflammatory and neuropathic pain. To aid in the development of safe non-opioid analgesic therapeutics for chronic inflammatory pain, a bioactive lipid class compound was assessed. The NAE compound DHEA has previously been shown to have immune-modulatory effects in vitro, however, the in vivo effects had not been assessed. The parent compound DHA and other NAE class lipids have antinociceptive effects in inflammatory pain, so it was hypothesised that DHEA would reduce formaldehyde-induced inflammatory pain. In a second strategy to replace addictive MOPr agonists, KOPr agonists with limited abuse potential were investigated for antinociceptive effects in nociceptive and inflammatory pain. The parent compound SalA has been shown to have an antinociceptive effect in vivo, however, the short duration of action and aversive side effects have limited any clinical development. It was hypothesised that altering the functional group at the carbon-16 position would increase the duration of action and potency at the KOPr and alter the bias factor towards fewer side effects. Finally, selected KOPr agonists were assessed in a paclitaxel-induced neuropathic pain model. There is currently only one recommended treatment for CIPN, however, duloxetine is weaker at treating paclitaxel-induced neuropathic pain compared to the neuropathic pain induced by other chemotherapies (Smith et al., 2013). KOPr activation has previously been shown to have antinociceptive effects in nerve damage models of neuropathic pain (Sounvoravong et al., 2004), however, no previous studies have investigated the effects of KOPr in CINP. Therefore, this study is important in understanding the antinociceptive effects of compounds with limited abuse potential and could aid in the development of pain medications to replace the current addictive MOPr agonists that are causing an increasing number of deaths worldwide. 


\subsection{Key findings}

\subsubsection{N-docosahexaenoyl ethanolamine reduces inflammatory pain independently of the cannabinoid system and without side effects}

DHEA is structurally related to the endocannabinoid anandamide and previous studies found DHEA had a weak binding affinity to the cannabinoid receptors in vitro (Sheskin et al., 1997; Brown et al., 2010). Therefore, it was hypothesised that any antinociceptive effects in the intraplantar formaldehyde model would be mediated via the cannabinoid receptors. The results show that DHEA is effective at reducing formaldehyde-induced nociceptive and inflammatory pain, however, from the results presented in this study, it appears that the effects are independent of the cannabinoid system. Similarly, DHEA did not show side effects in the behavioural models that are associated with cannabinoid receptor activation. Meijerink et al. (2015) found the CB1 and CB2 receptors and PPARs were not involved in the anti-inflammatory effects of DHEA in vitro, but instead, DHEA reduced levels of eicosanoids produced by COX-2. With the addition of this information, it is possible that the effects presented here for DHEA could be due to COX-2 inhibition, which is the analgesic mechanism of action of NSAIDs. This could explain why DHEA shows different results in the intraplantar formaldehyde test compared to anandamide which is predominately mediated by the CB1 receptor (Calignano et al., 1998). Furthermore, there is evidence that DHEA can cross the blood-brain barrier (Sonti et al., 2017), however, the lack of any effect at the current concentration against the spinal reflex of the warm water tail withdrawal assay, could indicate that the antinociceptive effects are not occurring at the spinal cord level.

\subsubsection{The novel analogues have a longer duration of action compared to Salvinorin A}

KOPr agonists are a promising alternative to addictive MOPr agonists for the treatment of pain. The naturally-occurring KOPr agonist SalA has previously been shown to have antinociceptive properties, however, a short duration of action and aversive side effects have limited the potential for clinical development. Therefore, this section of the study aimed to study two analogues in a range of nociceptive and inflammatory pain models and compare the results to SalA. 16-Ethynyl SalA and 16Bromo SalA both had a longer antinociceptive duration of action than SalA in the warm water tail withdrawal assay and the hot plate test. The novel compounds may have improved pharmacokinetic profiles compared to SalA. It has been previously shown that carbon-2 alterations of the SalA structure, such as the analogue EOM SalB, are more metabolically stable (Ewald et al., 2017), while less is known about the effects of carbon-16 alterations, it has been predicted that modifications at 
this position may hinder the action of cytochrome P450 enzymes (Wilson et al., 1990). Since the short duration of action of SalA is one factor that has limited the clinical development of the KOPr agonist, the longer duration for the novel analogues is a promising development.

\subsubsection{6-Ethynyl SalA has potent antinociceptive effects in the intraplantar formaldehyde model of inflammatory pain}

In the intraplantar formaldehyde test, SalA and 16-Ethynyl SalA significantly reduced nociceptive pain and inflammatory pain when administered by both i.p. and local injection. SalA and 16-Ethynyl SalA and 16-Bromo SalA reduced paw oedema when administered via i.p. injection. Flow cytometry analysis of the footpad tissue revealed a significant reduction in neutrophil cell counts following administration of SalA, which was further confirmed in H\&E stained sections. In previous studies, SalA had already been shown to have potent effects in the intraplantar formaldehyde model (Aviello et al., 2011; Guida et al., 2012) and it appears that 16-Ethynyl SalA has equivalent effects in this model, showing that the structural change at the carbon-16 position has not altered the effectiveness of the KOPr agonist at reducing inflammatory pain.

\subsubsection{6-Ethynyl SalA has reduced side effects compared to parent compound Salvinorin A}

In the present thesis, 16-Ethynyl SalA did not have an effect on body temperature, whilst previous work has shown that SalA reduced body temperature between 20-40 min following administration (Kumar, 2014). 16-Ethynyl SalA did show sedative effects for the same duration as SalA, however, the sedative effect was reduced compared to morphine. In Sprague Dawley rats, it has been shown that 16-Ethynyl SalA does not cause sedation in spontaneous locomotor tests at the same dose that attenuated drug-seeking behaviour (Riley et al., 2014). Previous studies from our laboratory have shown that 16-Ethynyl SalA has reduced side effects compared to the parent compound SalA and the traditional KOPr agonist U50,488. 16-Ethynyl SalA did not cause anxiety in the elevated plus maze or light-dark test or have depressive-like effects in the forced swim test. In CPA, 16-Ethynyl SalA did not show aversive effects and did not show memory impairments in the novel recognition task. These results suggest that 16-Ethynyl SalA may have potential to be developed as an analgesic with reduced side effects and may be a biased agonist, however, this can only be established once the complete signalling pathways have been investigated. 


\subsubsection{KOPr agonists have potential as a novel therapeutic for the treatment of paclitaxel-induced neuropathic pain}

CINP is a common side effect of treating cancer and can limit the dose and length of chemotherapy treatments. There are very limited options for treatment of CINP, with only one recommended therapeutic. The aim of this study was to test the potential of KOPr agonists as a potential treatment compared with morphine. Overall, U50,488 and 16-Ethynyl SalA appear to be potential therapeutics for this condition. 16-Ethynyl SalA was more potent than morphine in the acute dose-response. 16Ethynyl SalA also reduced allodynia when administered chronically, however, U50,488 was more effective, reducing the pain back to baseline vehicle levels over the 23 days of treatment and did not appear to have a tolerance effect. U50,488 is not suitable for clinical development due to a number of prohibitive side effects, however, 16-Ethynyl SalA or biased structural analogues of U50,488 may be potential pharmacotherapies for this difficult to treat condition.

\subsubsection{U50,488 and 16-Ethynyl SalA are more effective in male mice with established paclitaxel-induced neuropathic pain}

Under current NIH guidelines, increased emphasis has been put on comparing the effects of pharmacotherapies in both sexes, hopefully improving the translation of animal studies into the clinic (Clayton \& Collins, 2014). Interestingly, the current study found sex differences in different KOPr agonist treatments for established paclitaxel-induced neuropathic pain. In the dose-response effects of the opioid receptor agonists, it was found that for treating mechanical allodynia, U50,488 was more effective in male mice, whereas SalA was more effective in female mice. Furthermore, in the chronic administration experiment, U50,488 and 16-Ethynyl SalA were more effective in male mice. This indicates that there may be fundamental differences in the sexes that means that the females are less sensitive to the KOPr agonists, and may indicate that women need a higher dose to get the equivalent analgesic benefit if developed further into clinical trials. 


\subsection{Limitations and future directions}

The selection of the KOPr agonists tested in this study was partially based on the fact that there were reduced side effects in Sprague Dawley rats studies carried out in the Kivell laboratory. However, the current study used mice to assess the nociceptive effects. Ideally, the side effects would be repeated in mice at the same doses required to produce antinociceptive effects. Importantly, the CPA paradigm should be used to assess the aversive effects of the KOPr agonists in mice, as this is an important side effect of KOPr agonists. The effect of KOPr agonists on stress, mood and dysphoria, may limit the viability of developing these drugs into the clinic, especially when the alternative is to use MOPr agonists which have euphoric effects. There is strong evidence that the negative side effects of KOPr agonists are mediated by the $\beta$-arrestin pathway (Bruchas \& Chavkin, 2010) and previous studies have shown that p38 MAPK is involved in the aversive properties of KOPr agonists (Bruchas et al., 2007; Ehrich et al., 2015). However, a study using the SalA analogue RB-64 by the Roth laboratory group has shown that aversion is mediated via the G-protein pathway (White et al., 2015). U69,593, SalA and RB-64 showed aversion in CPA in both wildtype and $\beta$-arrestin-2 $\mathrm{K} / \mathrm{O}$ mice, however, it is unclear whether p38 MAPK is still involved in aversion in a $\beta$-arrestin-independent manner (White et al., 2015). Further elucidation of the KOPr signalling pathways is required. Currently, the G-protein bias calculations are typically carried out based on in vitro assays, sometimes only assessing one protein on each side of the signalling pathway, or using multiple cell lines. An improvement is to create a "web of bias" using multiple signalling molecules to generate a better picture of the pathway activation. However, this is further complicated by translation into in vivo studies, and the fact that signalling bias in different regions of the brain or different neurons may have different outcomes.

Another promising avenue for creating analgesics with reduced side effects is multi-targeting both the KOPr and MOPr. In this way, the antinociceptive effects of MOPr and KOPr activation could be combined, however, the euphoric effect of MOPr activation could be dampened down by the KOPr component of the treatment. Previous studies have looked at this in terms of co-administration of two agonists (Negus et al., 2008; Rech et al., 2012) or development of dual agonists that simultaneously activate both receptors (Provencher et al., 2013; Turnaturi et al., 2016; Bera et al., 2018). Administration of $\mathrm{U} 50,488$ has been shown to attenuate morphine-induced place preference and sedation (Bolanos et al., 1996) and antagonise DAMGO-induced respiratory depression (Dosaka-Akita et al., 1993). Similarly, administration of the KOPr agonist spiradoline suppressed fentanyl preference and fentanyl reversed spiradoline aversion in rats (Rech et al., 2010). Furthermore, co-administration of spiradoline and fentanyl had additive antinociceptive effects in nociceptive and visceral pain models in rats (Rech et al., 2012). The compounds from this study have the potential to be co-administered 
with a MOPr agonist, and the antinociceptive, rewarding and aversive properties of the combined treatment tested.

However, another issue with KOPr activation is the hallucinogenic effects. Since SalA is not a traditional hallucinogen, it is unclear whether rodent models used to test serotonergic or glutamatergic hallucinogens would be useful at measuring the unique experience associated with SalA. It is likely that using non-human primates would be a better model, as the homology of the relevant brain structures may be important. An animal model needs to be established and validated using SalA before the analogues are tested to ascertain whether they have improvements. One paper did study the effects in zebrafish and found that SalA induced changes in swimming behaviour that could be interpreted as hallucinatory behaviour (Braida et al., 2007).

In the pursuit of developing SalA and analogues into potential therapeutics, there are chemical limitations that may reduce the likelihood of success. SalA and all of the analogues created thus far are not soluble in saline and must be dissolved in a solvent and emulsifier to stop the compounds precipitating out of solution. In terms of clinical development, it is optimal if drugs are orally bioavailable as this allows patients to treat themselves, does not have the discomfort and infection risks associated with i.v. administration and increases patient compliance with a treatment regimen. There is doubt over the oral availability of SalA, although the novel analogue PR-38 has been shown to be orally active (Sałaga et al., 2014). There are also limitations in the total synthesis of SalA, as currently analogues are synthesised from SalA that is extracted from the plant. This could be a problem in terms of generating enough product at the commercial scale. Furthermore, the legality of Salvia divinorum could hinder manufacture. The development of KOPr agonists as analgesic medications could be very important, however, a new chemical scaffold may need to be investigated for optimal clinical outcomes.

The translation of animal research into successful human clinical trials has not always yielded favourable outcomes, with many prospective compounds failing at Phase I. Often the results obtained using animal models cannot be reproduced by other laboratory groups, commonly due to poor experimental design or insufficient reporting of methodology and housing conditions. Publishing bodies are increasingly requiring more robust reporting on animal procedures, as laid out in the ARRIVE (Animal Research: Reporting of In Vivo Experiments) guidelines for publishing (Kilkenny et al., 2010). Mice are the most commonly used animal model, with the advantages of having a relatively similar genome to humans that can be manipulated to be a useful genetic or molecular tool to model many human conditions (reviewed in Vandamme, 2014). The inbred rodent strains are useful models, however, due to the limited genetic variability, results are not always consistent between different 
breeds of animals (for example, mouse vs. rat) and variability exists between different strains of animals (for example C57BL/6 vs BALB/c mouse strains). It is important that new pain models are rigorously tested and validated in specific animal strains before further experimentation is carried out.

In the past, it has been standard practice to carry out experiments in male animals only to decrease variability caused by the different sexes. However, the USA National Institutes of Health are now requiring more extensive studies using both sexes (Clayton \& Collins, 2014), as the importance of gender differences are becoming more apparent. This is apparent from the results of this thesis, showing that the KOPr agonists have different effectiveness in different sexes. Furthermore, the gender of the experimenter can also have an impact on the results, with a recent study showing male experimenters performing assays of antinociception in animal models can lead to stress-induced analgesia, significantly affecting the results of the study (Sorge et al., 2014). To mitigate this effect in the current thesis, if a male observer was present, the mice were acclimatised to this person for at least $60 \mathrm{~min}$ prior to the commencement of the procedure. In addition, if the animals observe cage mates in pain this leads to sensitisation to pain themselves (Langford et al., 2006). These differences emphasise the importance of careful experimental design when working with animal models, as it is important to isolate and observe only the behaviours in the animals caused by the administration of the drug rather than any other external factors. 


\subsection{Final conclusions}

MOPr agonists are highly addictive, as evidenced by the current opioid crisis in the USA and increasing rates of opioid overdoses worldwide. This thesis identified two classes of compounds as potential therapeutics that do not have addictive effects. This was the first study to understand the antinociceptive in vivo effects of DHEA, which was found to have applications in treating inflammatory pain. Whereas the novel KOPr agonist 16-Ethynyl SalA was found be potent at treating both inflammatory and neuropathic pain with fewer side effects than the parent compound SalA. Interestingly, the traditional KOPr agonist was found to be particularly effective at treating paclitaxelinduced neuropathic pain, while this compound is not suitable for clinical development, these results warrant further investigation of structural analogues of U50,488 for this type of pain. Furthermore, it was found that U50,488 and 16-Ethynyl SalA were more effective at treating male mice with paclitaxelinduced allodynia, perhaps indicating treatment doses need to be tailored to the different sexes. This was the first study to identify KOPr agonists as a treatment for chemotherapy-induced neuropathic pain and may aid in the development of new therapeutics for treating this condition which currently has very limited treatment options. 


\section{Appendices}

\section{Appendix A: Solutions}

10X Phosphate buffered saline (PBS) pH 7.4

$\begin{array}{lll} & \text { Concentration } & \mathrm{g} / \mathrm{L} \\ \mathrm{NaCl} & 1.4 \mathrm{M} & 80.0 \\ \mathrm{KCl} & 26.8 \mathrm{mM} & 2.0 \\ \mathrm{Na}_{2} \mathrm{HPO}_{4} & 81.0 \mathrm{mM} & 26.8 \\ \mathrm{KH}_{2} \mathrm{PO}_{4} & 14.7 \mathrm{mM} & 2.4\end{array}$

Make up to total volume with distilled water, adjust to $\mathrm{pH}$ 7.4.

\section{Eosin Y solution}

Eosin $Y \quad 1 \%(w / v)$

Glacial acetic acid $\quad 0.5 \%$

Ethanol $\quad 70 \%$

Make up to total volume with water. Dissolve Eosin $Y$ in water, add ethanol and glacial acetic acid. Stir before use.

\section{Fluorescence-activated cell sorter (FACS) buffer}

BSA $\quad 0.1 \%$

Sodium azide $\quad 0.01 \%$

Make up to total volume with $1 \mathrm{X}$ PBS.

\section{4\% Formaldehyde in PBS pH 7.4}

Paraformaldehyde $\quad 40 \mathrm{~g}$

Preheat $900 \mathrm{~mL}$ of $1 \mathrm{X}$ PBS to $50{ }^{\circ} \mathrm{C}$ and add the paraformaldehyde powder (never allow to boil). Add drops of $\mathrm{NaOH}$ until the solution becomes clear. Make up to $1 \mathrm{~L}$ with PBS. Cool and adjust to $\mathrm{pH} 7.4$ with $\mathrm{HCl}$.

Piperazine-1,4-bis(2-ethanesulfonic acid) (PIPES) buffer pH 7.4

\section{Concentration}

PIPES $\quad 0.1 \mathrm{M}$

$\mathrm{NaOH} \quad 1 \mathrm{~N}$

Glutaraldehyde $\quad 2.5 \%$

Make up to $1 \mathrm{~L}$ with PIPES, $\mathrm{NaOH}$ and distilled water, adjust to $\mathrm{pH}$ 7.4. Add glutaraldehyde to solution immediately prior to use. 


\section{Appendix B: Analysis of routes of administration of vehicle control in the intraplantar formaldehyde test}

Comparison of the vehicle controls for the i.p. and i.pl. administration in the intraplantar formaldehyde model showed that there was significantly more pain following the i.pl. administration of the vehicle in both phase I and II.
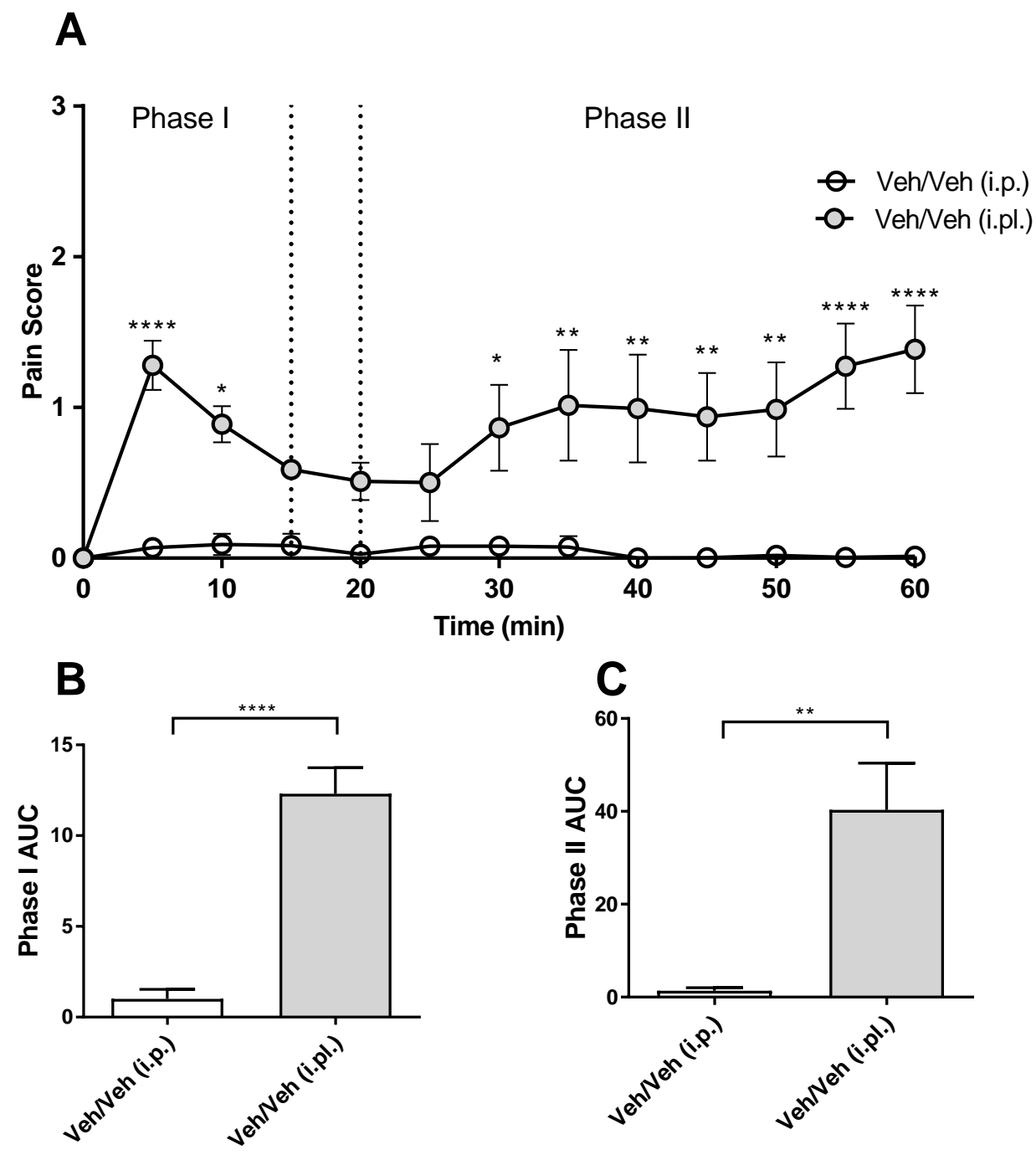

Figure B.1: The vehicle control administered via intraplantar injection produces an antinociceptive effect

(A) Time course of pain behaviour following i.pl. injection of the PBS vehicle. The treatment vehicle (2:1:7 of DMSO:Tween-80:saline) was administered either via i.p. or i.pl. injection, with the i.pl. injection route showing a significant level of pain. Two-way ANOVA with Bonferroni post-tests. (B-C) Area under the curve (AUC) was calculated for phase I nociceptive pain (0-15 $\mathrm{min}, \mathrm{B})$ and phase II inflammatory pain (20-60 min, C). The i.pl. administration of the vehicle showed a significant level of pain compared to the i.p. administration in both phases. Two-tailed unpaired Student's t-test. ${ }^{*} p<$ $0.05, * * p<0.01, * * * p<0.001, * * * * p<0.0001$. Values presented as mean $\pm \mathrm{SEM}, \mathrm{n}=7$. 


\section{Appendix C: Warm water tail withdrawal dose-response experiment}

\section{C.1 Vehicle control treatment group}

As a control for the cumulative dose-response tail withdrawal test, animals received the equivalent number of vehicle injections. The vehicle control showed no antinociceptive effect and no increase in effect over time indicating that the results of the KOPr agonists were not due to any sedative effects of the vehicle or stress-induced analgesia.

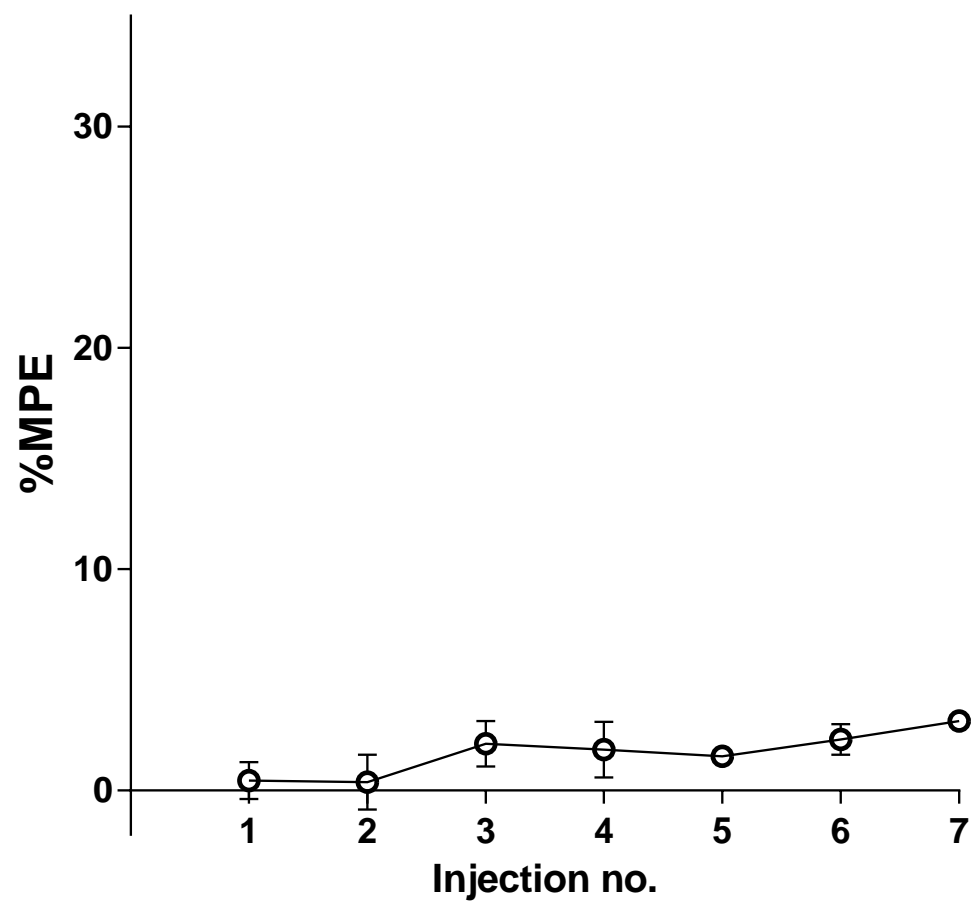

Figure C.1: Vehicle control group in the warm water dose-response experiment

The mice were administered vehicle s.c. injections in the same number as the KOPr agonist treatment groups. One-way repeated measures ANOVA showed no significant effect between the successive of injections $\left[F_{(2.373,11.86)}=1.018, p=0.4032\right]$. Values presented as mean $\pm S E M, n=6$. 


\section{C.2 Comparison of morphine and U50,488 in the warm water tail withdrawal dose-response experiment}

The effects of the KOPr agonists were compared to the traditional KOPr agonist U50,488. However, when U50,488 was compared to the MOPr agonist morphine, the potencies were not significantly different but the efficacy of morphine was significantly higher than $U 50,488$, indicating a fundamental difference between the effects of MOPr agonists and KOPr agonists in this model of pain.

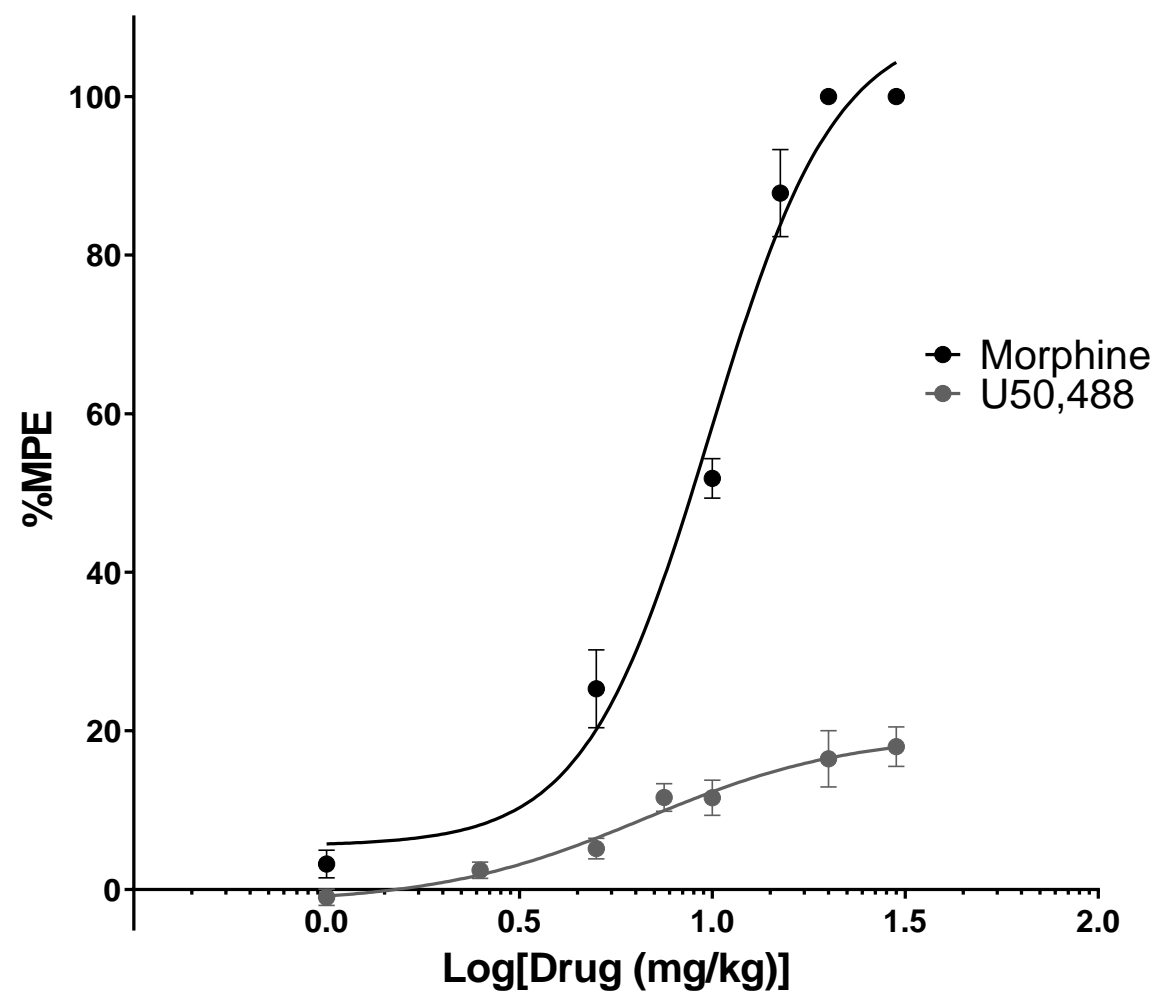

Figure C.2: Morphine is more efficacious than U50,488 in the warm water tail withdrawal assay

Cumulative dose-response effects of morphine and U50,488. The maximal possible effect (\%MPE) at each dose was calculated as a percentage based on the pre-treatment baseline latencies for each individual mouse. The data sets are significantly different, in that the same curve does not fit both data sets $\left[F_{(4,70)}=226.4, p<0.0001\right]$. The potency $\left(E D_{50}\right)$ values were not significantly different (Unpaired two-tailed t-test, $p=0.2827$ ) with $9.86 \mathrm{mg} / \mathrm{kg}$ for morphine and $6.28 \mathrm{mg} / \mathrm{kg}$ for U50,488. However, the $E_{\max }$ values of $100 \%$ for morphine and $19.7 \pm 4.6 \%$ for U50,488 were significantly different (Unpaired two-tailed t-test, $p<0.0001$ ). Values presented as mean $\pm S E M, n=6$. The U50,488 dataset is also presented in Paton et al. (2017) and Figure 4.1. 


\section{Appendix D: Acute dose-response controls in the paclitaxel-induced neuropathic pain model}

\section{D.1 Baseline withdrawal thresholds}

The baseline (day 0 ) values were compared between the assigned treatment groups assigned for the dose-response experiment. It was found that there were no inherent differences between the baseline values of the paclitaxel-treated animals.

A

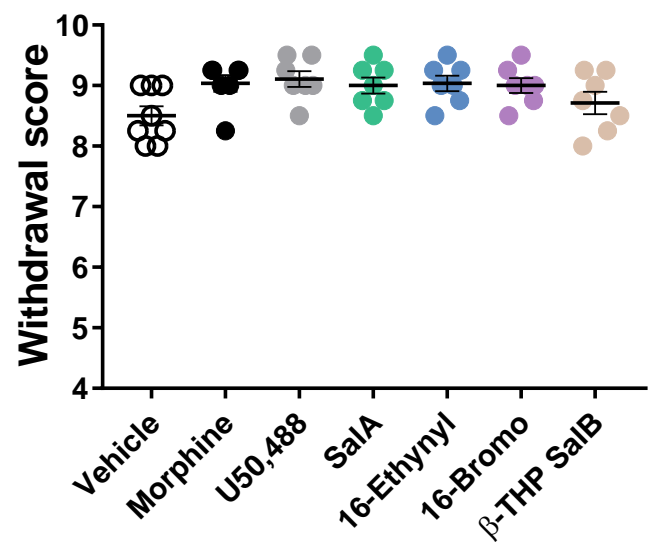

C

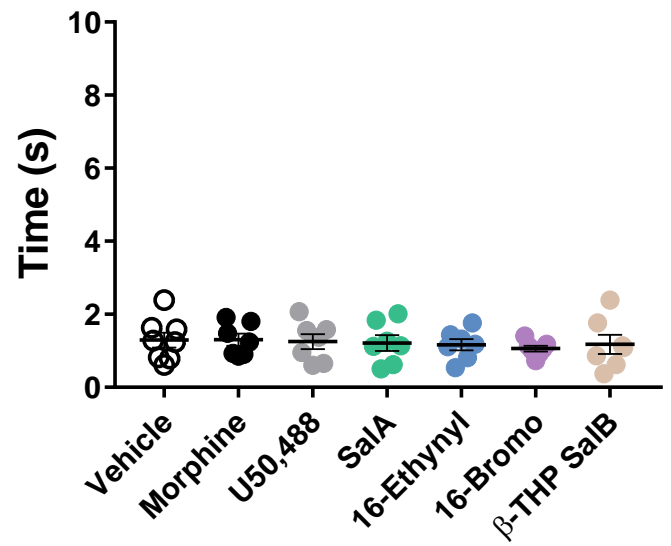

B
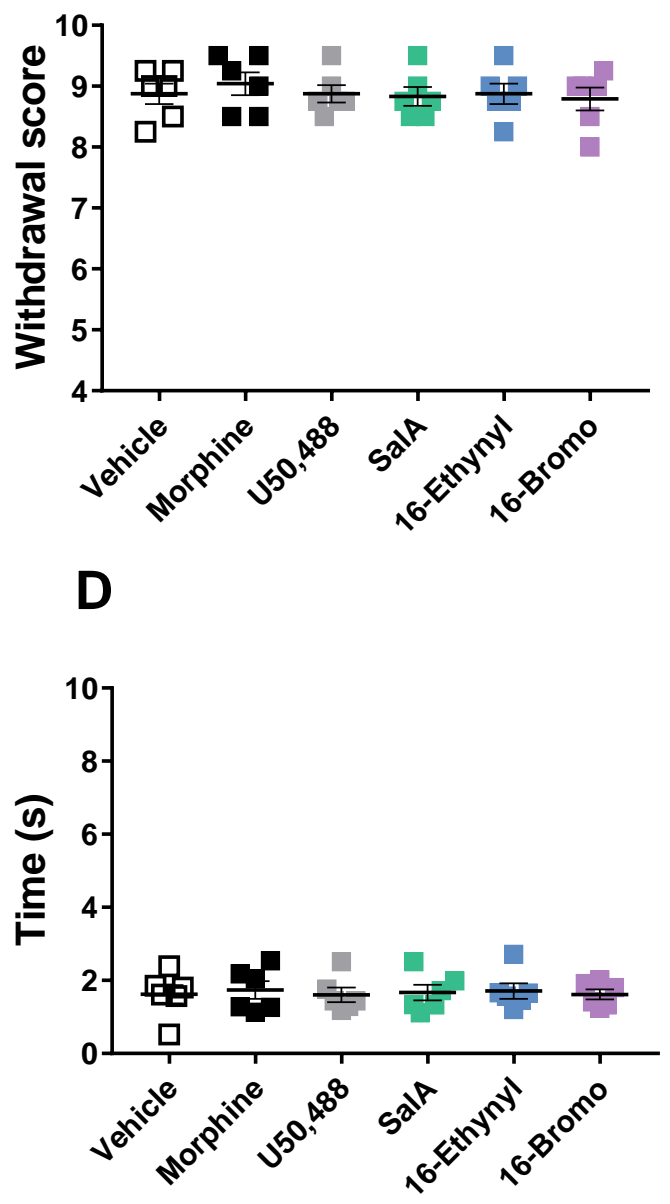

Figure D.1: The day 0 pre-paclitaxel mechanical and cold threshold values were not different between upcoming assigned dose-response treatment groups

The baseline values on day 0 , before the first injection of paclitaxel, were not significantly different when separated by the day 15 treatment group. Mechanical allodynia in (A) males and (B) females. Cold allodynia in (C) males and (D) females. $n=7$ for male groups, except $n=8$ for vehicle treatment, $\mathrm{n}=6$ for all female treatment groups. Values presented as individual data points, with a line indicating the mean \pm SEM. 


\section{D.2 Day 15 pre-treatment withdrawal thresholds}

The day 15 values were compared depending on the assigned treatment in the dose-response procedure. There was no difference between the treatment groups for mechanical allodynia and cold allodynia for either sex.

A

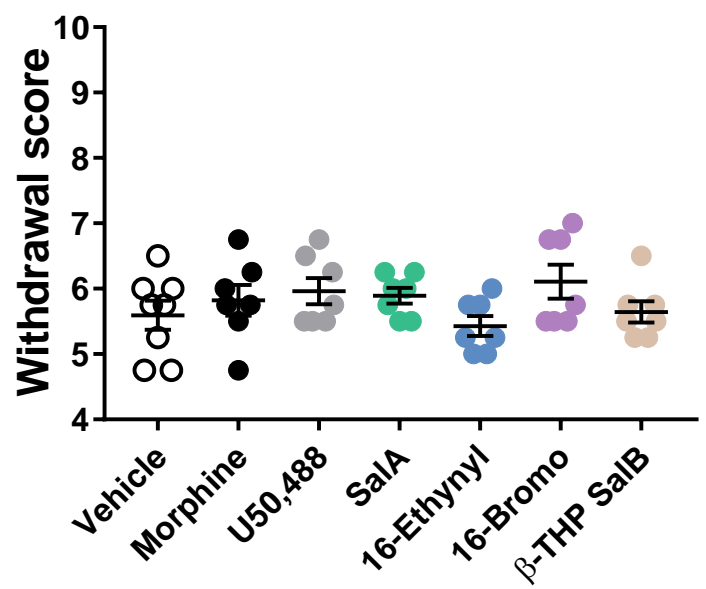

C

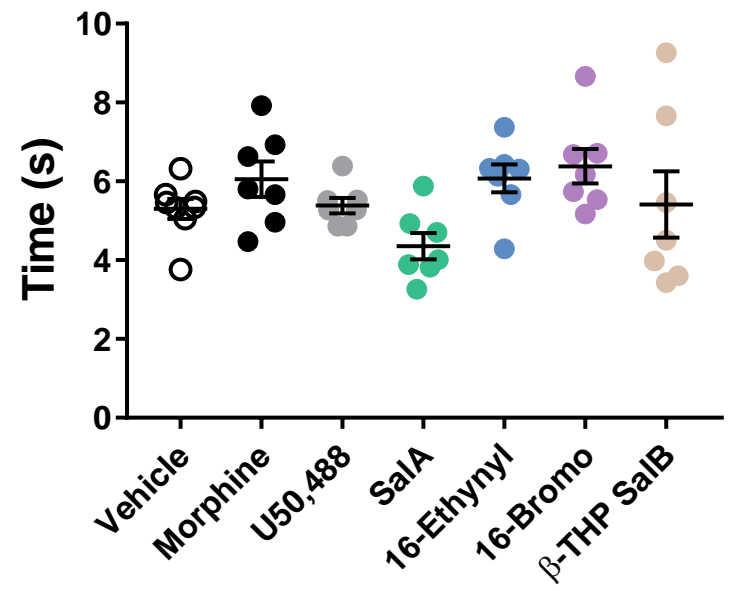

B

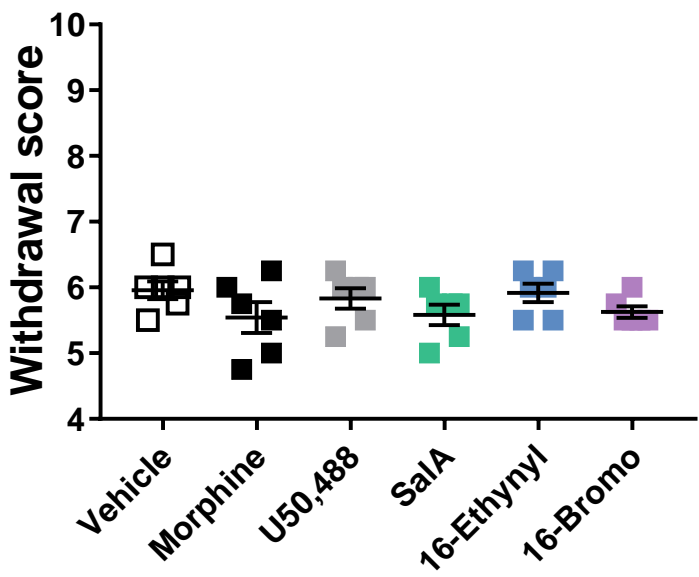

D

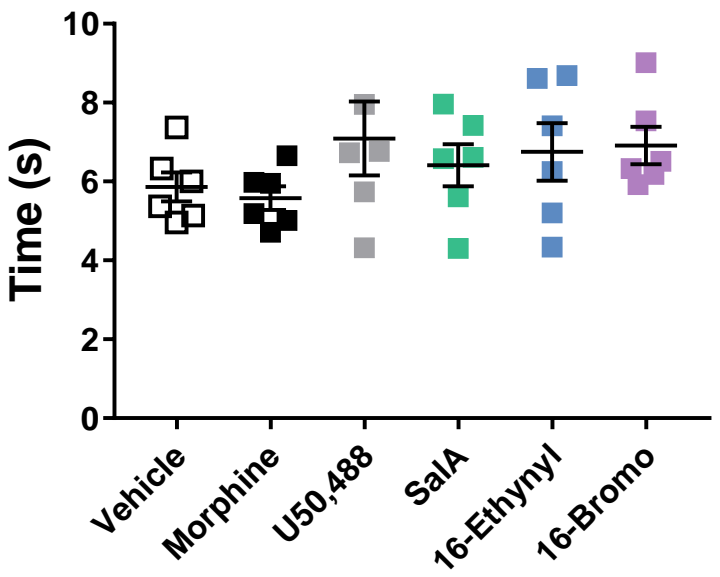

Figure D.2: The day 15 post-paclitaxel mechanical and cold allodynia values were not different between assigned treatment groups

The baseline values on day 15 post-paclitaxel were not significantly different when separated by the dose-response treatment group. Mechanical allodynia in (A) males and (B) females. Cold allodynia in (C) males and (D) females. $n=7$ for male groups, except $n=8$ for vehicle treatment, $n=6$ for all female treatment groups. Values presented as individual data points, with a line indicating the mean \pm SEM. 


\section{D.3 Vehicle treatment in the dose-response procedure}

For comparison in the dose-response procedure, an equivalent number of vehicle treatments were given to paclitaxel-treated mice. The results showed that the vehicle had no antinociceptive effect and successive injections did not have any effect over time.

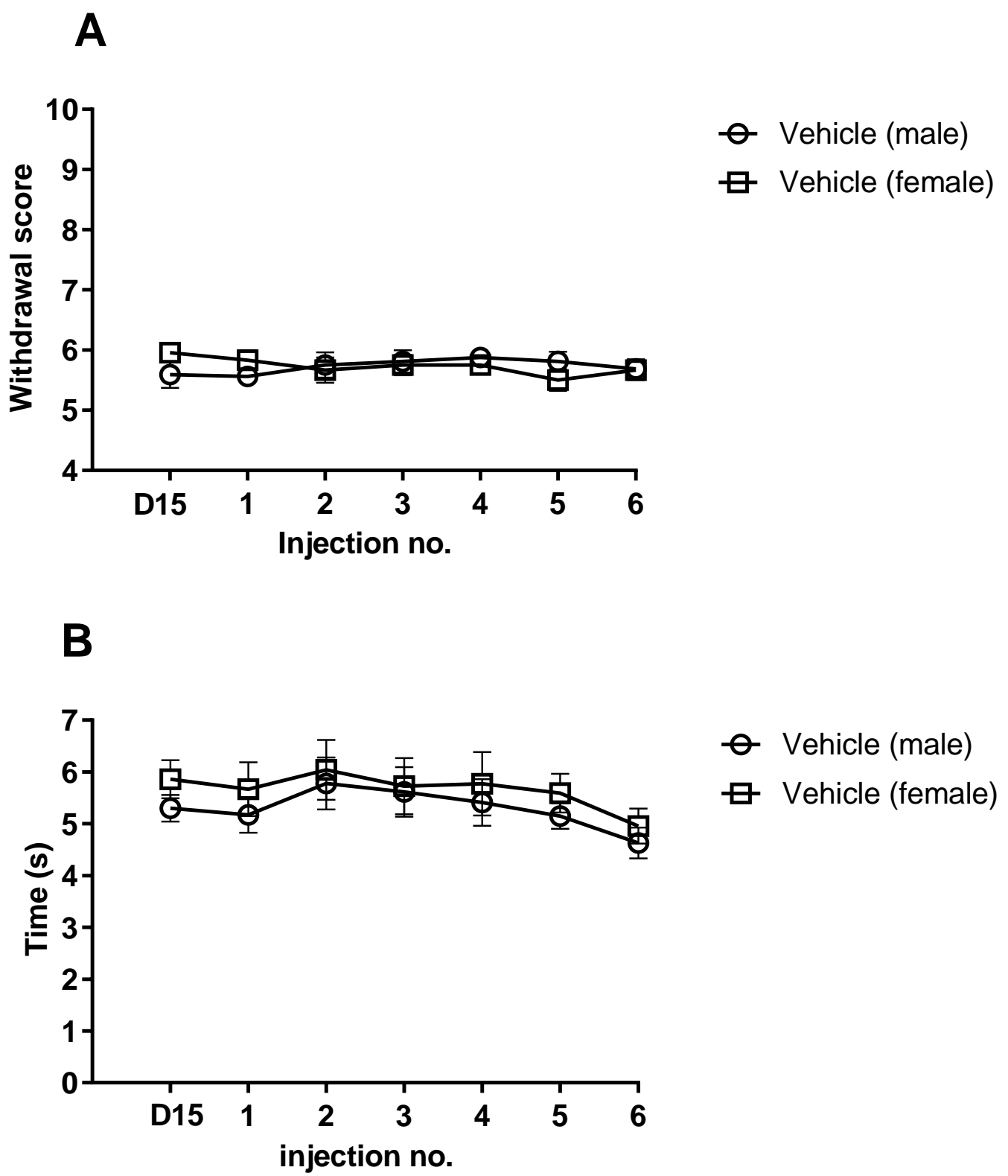

Figure D.3: Vehicle treatment did not have an antinociceptive effect in mice with established paclitaxel-induced neuropathic pain

Vehicle controls were administered to mice with paclitaxel-induced neuropathic pain on day 15 , with the same number of injections as the opioid receptor agonists. There was no significant effect of vehicle over time for $(A)$ mechanical allodynia $\left[F_{(6,72)}=1.189, p=0.3219\right]$ and $(B)$ for cold allodynia $\left[F_{(6,72)}=0.1399, p=0.9904\right]$. One-way repeated measures ANOVA. D15 refers to the values on day 15 prior to vehicle treatment. $n=8$ for males, $n=6$ for females. Values presented as mean \pm SEM. 


\section{D.4 Day 15 pre-treatment withdrawal thresholds for antagonist study}

The selectively antagonise the KOPrs, the antagonist nor-BNI was administered 24 hours (on day 14) prior to the beginning of the dose-response procedure. Comparing between the assigned treatment groups on day 15 before the start of the dose-response procedure, there were no significant differences between any of the groups. This indicates that the nor-BNI treatment did not alter the level of neuropathic pain induced by paclitaxel.
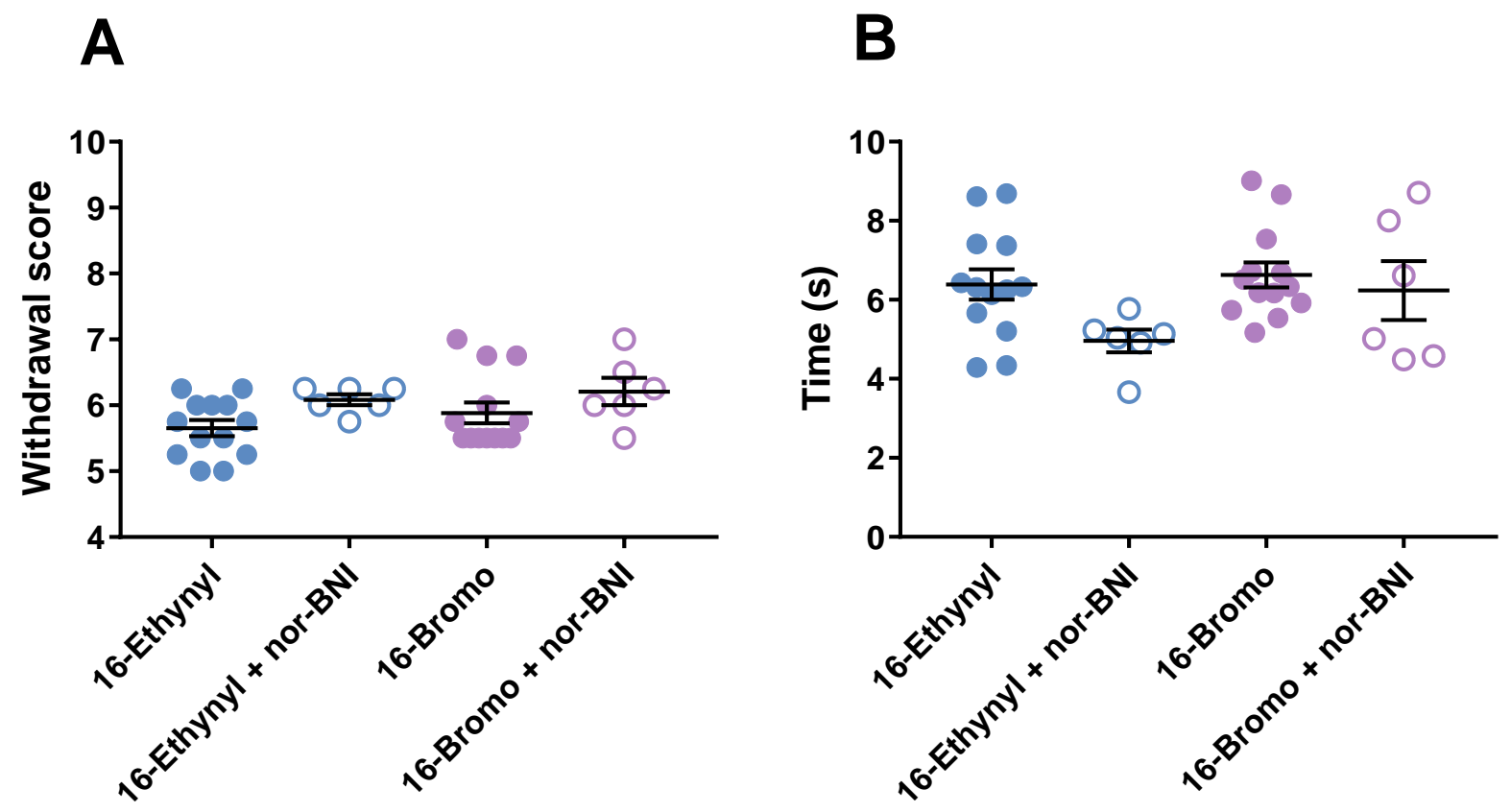

Figure D.4: KOPr antagonism with nor-BNI did not affect the day 15 pre-treatment values

Nor-BNI treatment was administered 24 hours prior to the dose-response procedure (on day 14). There was no significant difference between the day 15 values prior to the commencement of the dose-response antinociceptive effects against (A) mechanical allodynia $\left[\mathrm{F}_{(3,34)}=2.306, p=0.0942\right]$ and (B) cold allodynia $\left[\mathrm{F}_{(3,34)}=2.369, p=0.0879\right] . \mathrm{n}=13$ for KOPr agonist treatment without nor-BNI, $\mathrm{n}=6$ for groups with nor-BNI pre-treatment. Values presented as mean \pm SEM. 


\section{Appendix E: Controls for the chronic administration procedure in the paclitaxel-induced neuropathic pain model}

\section{E.1 Early initiation of treatment on day 7 baseline values}

The baseline values on day 6 were compared between the assigned treatment groups in the paclitaxeltreated animals before the initiation of treatment on day 7 . The results showed no significant difference between the groups.

A

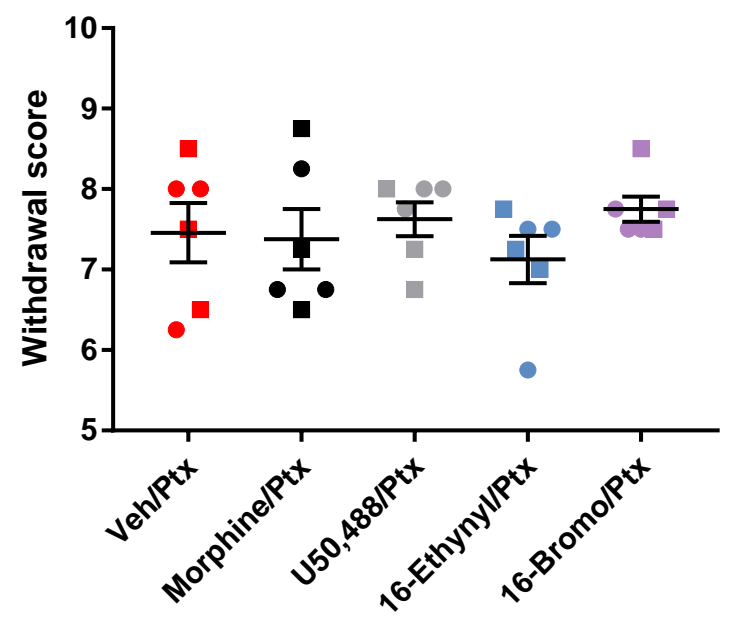

B

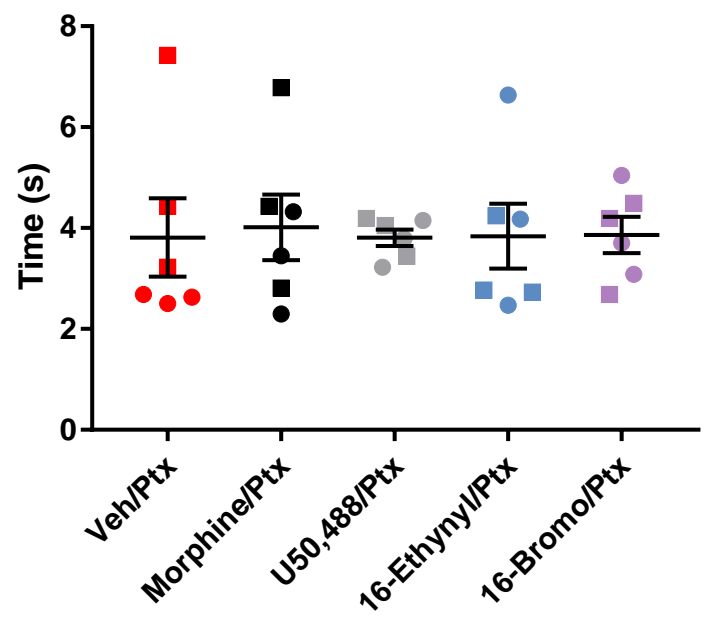

Figure E.1: Day 6 values for early initiation of chronic opioid receptor agonist treatment procedure

The allodynic values on day 6 were not significantly different when separated by the assigned treatment group, in measuring (A) mechanical allodynia and (B) cold allodynia. Values presented as individual data points, with a line indicating the mean \pm SEM. $n=6$. 


\section{E.2 Day 15 values for mechanical allodynia}

The baseline values on day 15 were compared between the assigned treatment groups in the paclitaxel-treated animals before the initiation of the daily treatment on day 16 . The results showed no significant difference between the groups of either sex.

A

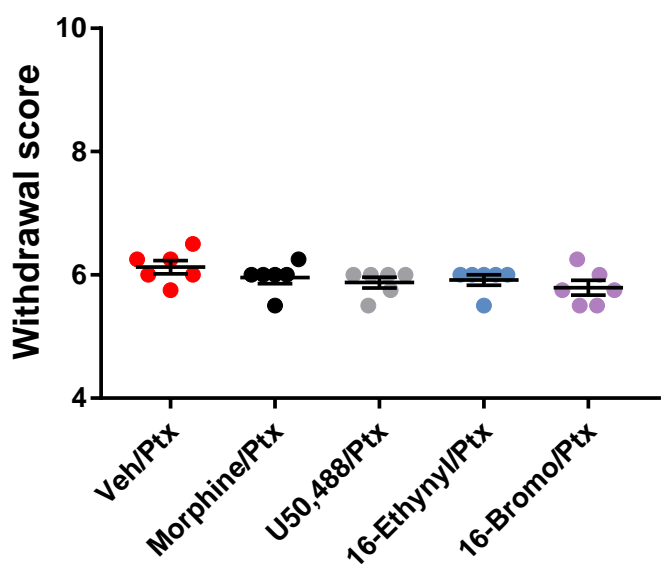

B

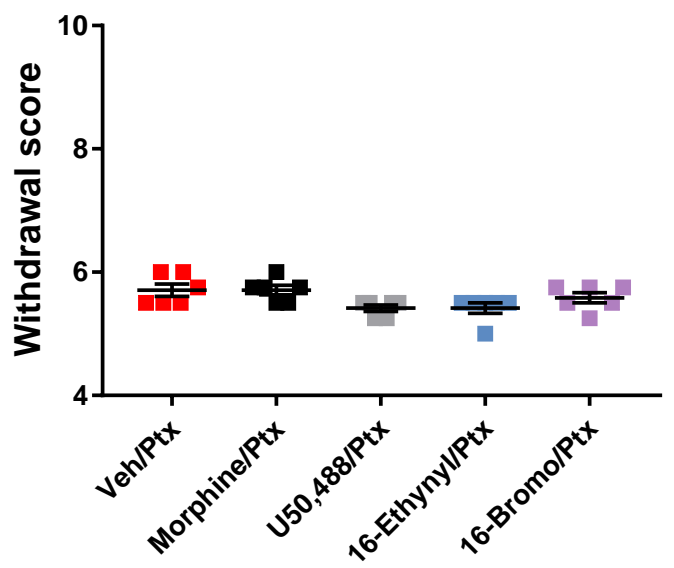

Figure E.2: Mechanical withdrawal thresholds on day 15 prior to assignment of treatment groups

The allodynic values on day 15 were not significantly different when separated by the assigned treatment group in (A) males and (B) females. Values presented as individual data points, with a line indicating the mean \pm SEM. $n=6$. 


\section{E.3 Day 15 values for cold allodynia}

The baseline values on day 15 were compared between the assigned treatment groups in the paclitaxel-treated animals before the initiation of treatment on day 16.

A

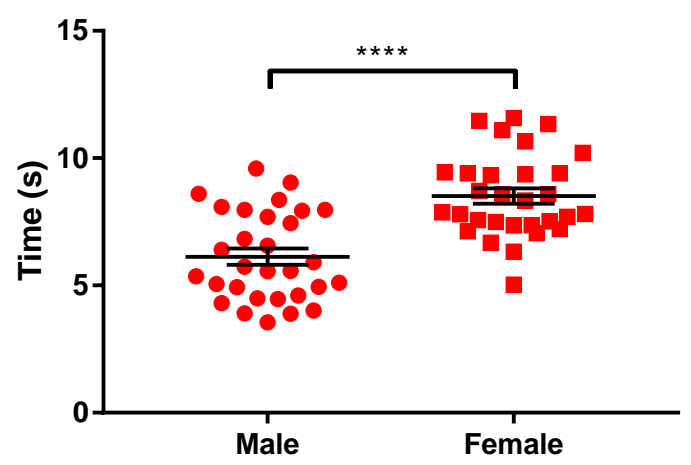

B

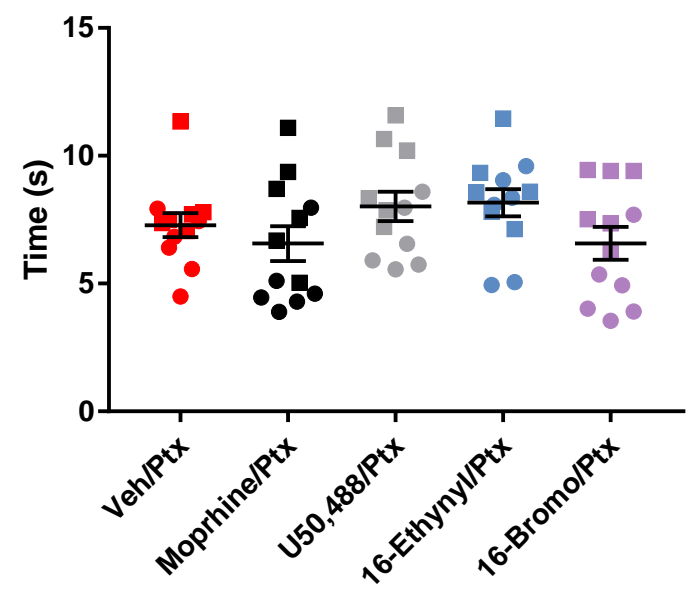

Figure E.3: Cold reaction times on day 15 prior to assignment of treatment groups

(A) There was a difference between the males and females in the cold reaction times on day 15 prior to the beginning of treatments. (B) The allodynic values on day 15 were not significantly different when separated by the assigned treatment group in the combined sexes. Values presented as individual data points, with a line indicating the mean \pm SEM. $n=12$ ( 6 males, 6 females). 


\section{Appendix F: List of publications}

The following papers were published during the course of this PhD study:

Kivell, B. M., Paton, K. F., Kumar, N., Morani, A. S., Culverhouse, A., Shepherd, A., Welsh, S. A., Biggerstaff, A., Crowley, R. S., \& Prisinzano, T. E. (2018). Kappa Opioid Receptor Agonist Mesyl Sal B Attenuates Behavioral Sensitization to Cocaine with Fewer Aversive Side-Effects than Salvinorin A in Rodents. Molecules, 23(10), 2602. doi:10.3390/molecules23102602

Paton, K. F., Kumar, N., Crowley, R. S., Harper, J. L., Prisinzano, T. E., \& Kivell, B. M. (2017). The analgesic and anti-inflammatory effects of Salvinorin A analogue beta-tetrahydropyran Salvinorin B in mice. European Journal of Pain, 21(6), 1039-1050. doi:10.1002/ejp.1002

Sherwood, A. M., Crowley, R. S., Paton, K. F., Biggerstaff, A., Neuenswander, B., Day, V. W., Kivell, B. M., \& Prisinzano, T. E. (2017). Addressing structural flexibility at the A-ring on Salvinorin A: Discovery of a potent Kappa-opioid agonist with enhanced metabolic stability. Journal of Medicinal Chemistry, 60(9), 3866-3878. doi:10.1021/acs.jmedchem.7b00148

Crowley, R. S., Riley, A. P., Sherwood, A. M., Groer, C. E., Shivaperumal, N., Biscaia, M., Paton, K. F., Schneider, S., Provasi, D., Kivell, B. M., Filizola, M., \& Prisinzano, T. E. (2016). Synthetic Studies of Neoclerodane Diterpenes from Salvia divinorum: Identification of a potent and centrally acting mu opioid analgesic with reduced abuse liability. Journal of Medicinal Chemistry, 59(24), 11027-11038. doi:10.1021/acs.jmedchem.6b01235 


\section{References}

Abramo, F., Campora, L., Albanese, F., della Valle, M. F., Cristino, L., Petrosino, S., Di Marzo, V., \& Miragliotta, V. (2014). Increased levels of palmitoylethanolamide and other bioactive lipid mediators and enhanced local mast cell proliferation in canine atopic dermatitis. BMC Veterinary Research, 10(1), 21. doi:10.1186/1746-6148-10-21

Adams, J., Unett, D., Anthony, T., Gatlin, J., \& Gaidarov, I. (2018). APD371: a potent, highly selective, full agonist of the human CB 2 receptor with sustained analgesic effects in rodents. The Journal of Pain, 19(3), S2. doi:10.1016/j.jpain.2017.12.014

Addington, J., \& Freimer, M. (2016). Chemotherapy-induced peripheral neuropathy: an update on the current understanding. F1000Research, 5, F1000 Faculty Rev-1466. doi:10.12688/f1000research.8053.1

Addy, P. H. (2012). Acute and post-acute behavioral and psychological effects of salvinorin A in humans. Psychopharmacology, 220(1), 195-204. doi:10.1007/s00213-011-2470-6

Addy, P. H., Garcia-Romeu, A., Metzger, M., \& Wade, J. (2015). The subjective experience of acute, experimentally-induced Salvia divinorum inebriation. Journal of psychopharmacology (Oxford, England), 29(4), 426-435. doi:10.1177/0269881115570081

Adler, M. W., Bradley, E., Martinez, R., \& Geller, E. B. (1991). Production of hypothermia in the guinea pig by a kappa-agonist opioid alone and in combination with chlorpromazine. Pharmacology Biochemistry and Behavior, 4O(1), 129-132. doi:10.1016/0091-3057(91)90333-W

Adriaensen, H., Gybels, J., Handwerker, H. O., \& Van Hees, J. (1983). Response properties of thin myelinated (A-delta) fibers in human skin nerves. Journal of Neurophysiology, 49(1), 111-122. doi:10.1152/jn.1983.49.1.111

Ahern, G. P. (2003). Activation of TRPV1 by the satiety factor oleoylethanolamide. Journal of Biological Chemistry, 278(33), 30429-30434. doi:10.1074/jbc.M305051200

Akiyama, T., Carstens, M. I., Piecha, D., Steppan, S., \& Carstens, E. (2015). Nalfurafine suppresses pruritogen- and touch-evoked scratching behavior in models of acute and chronic itch in mice. Acta Dermato-Venereologica, 95(2), 147-150. doi:10.2340/00015555-1879

Al Hilli, F., \& Wright, E. A. (1983). The effects of changes in the environmental temperature on the growth of tail bones in the mouse. British Journal of Experimental Pathology, 64(1), 34-42.

Albert-Vartanian, A., Boyd, M. R., Hall, A. L., Morgado, S. J., Nguyen, E., Nguyen, V. P., Patel, S. P., Russo, L. J., Shao, A. J., \& Raffa, R. B. (2016). Will peripherally restricted kappa-opioid receptor agonists (pKORAs) relieve pain with less opioid adverse effects and abuse potential? Journal of Clinical Pharmacy and Therapeutics, 41(4), 371-382. doi:10.1111/jcpt.12404

Alessandri-Haber, N., Dina, O. A., Joseph, E. K., Reichling, D. B., \& Levine, J. D. (2008). Interaction of transient receptor potential vanilloid 4, integrin, and SRC tyrosine kinase in mechanical hyperalgesia. Journal of Neuroscience, 28(5), 1046-1057. doi:10.1523/JNEUROSCl.449707.2008

Alessandri-Haber, N., Joseph, E., Dina, O. A., Liedtke, W., \& Levine, J. D. (2005). TRPV4 mediates painrelated behavior induced by mild hypertonic stimuli in the presence of inflammatory mediator. Pain, 118(1-2), 70-79. doi:10.1016/j.pain.2005.07.016

Allen, D. T., \& Kiernan, J. A. (1994). Permeation of proteins from the blood into peripheral-nerves and ganglia. Neuroscience, 59(3), 755-764. doi:10.1016/0306-4522(94)90192-9

Alloui, A., Zimmermann, K., Mamet, J., Duprat, F., Noel, J., Chemin, J., Guy, N., Blondeau, N., Voilley, N., Rubat-Coudert, C., Borsotto, M., Romey, G., Heurteaux, C., Reeh, P., Eschalier, A., \& Lazdunski, M. (2006). TREK-1, a K+ channel involved in polymodal pain perception. EMBO Journal, 25(11), 2368-2376. doi:10.1038/sj.emboj.7601116

Aloe, L., Leon, A., \& Levi-Montalcini, R. (1993). A proposed autacoid mechanism controlling mastocyte behaviour. Agents and Actions, 39 Spec No, C145-147. 
Alvarez, A. M. (2015). Analgesic effects of pharmacological and cannabinoids treatments for Trigeminal Neuralgia in patients with MS. Journal of Neurology and Neuroscience, 06(04). doi:10.21767/2171-6625.100041

Ameri, A. (1999). The effects of cannabinoids on the brain. Progress in Neurobiology, 58(4), 315-348. doi:10.1016/S0301-0082(98)00087-2

Amir, R., \& Devor, M. (2003). Electrical excitability of the soma of sensory neurons is required for spike invasion of the soma, but not for through-conduction. Biophysical Journal, 84(4), 2181-2191. doi:10.1016/S0006-3495(03)75024-3

Amminger, G. P., Schafer, M. R., Papageorgiou, K., Klier, C. M., Cotton, S. M., Harrigan, S. M., Mackinnon, A., McGorry, P. D., \& Berger, G. E. (2010). Long-chain omega-3 fatty acids for indicated prevention of psychotic disorders: a randomized, placebo-controlled trial. Archives of General Psychiatry, 67(2), 146-154. doi:10.1001/archgenpsychiatry.2009.192

Andersson, D. A., Gentry, C., Moss, S., \& Bevan, S. (2008). Transient receptor potential A1 is a sensory receptor for multiple products of oxidative stress. Journal of Neuroscience, 28(10), 2485-2494. doi:10.1523/JNEUROSCI.5369-07.2008

Ansonoff, M. A., Zhang, J., Czyzyk, T., Rothman, R. B., Stewart, J., Xu, H., Zjwiony, J., Siebert, D. J., Yang, F., Roth, B. L., \& Pintar, J. E. (2006). Antinociceptive and hypothermic effects of Salvinorin A are abolished in a novel strain of kappa-opioid receptor-1 knockout mice. Journal of Pharmacology and Experimental Therapeutics, 318(2), 641-648. doi:10.1124/jpet.106.101998

Apkarian, A. V., Bushnell, M. C., Treede, R. D., \& Zubieta, J. K. (2005). Human brain mechanisms of pain perception and regulation in health and disease. European Journal of Pain (London, England), 9(4), 463-484. doi:10.1016/j.ejpain.2004.11.001

Appleby, S. B., Ristimaki, A., Neilson, K., Narko, K., \& Hla, T. (1994). Structure of the human cyclooxygenase-2 gene. Biochemical Journal, 302 (Pt 3)(3), 723-727. doi:10.1042/bj3020723

Arendt-Nielsen, L., Olesen, A. E., Staahl, C., Menzaghi, F., Kell, S., Wong, G. Y., \& Drewes, A. M. (2009). Analgesic efficacy of peripheral kappa-opioid receptor agonist CR665 compared to oxycodone in a multi-modal, multi-tissue experimental human pain model: selective effect on visceral pain. Anesthesiology, 111(3), 616-624. doi:10.1097/ALN.0b013e3181af6356

Arif-Rahu, M., \& Grap, M. J. (2010). Facial expression and pain in the critically ill non-communicative patient: state of science review. Intensive and Critical Care Nursing, 26(6), 343-352. doi:10.1016/j.iccn.2010.08.007

Arita, M., Yoshida, M., Hong, S., Tjonahen, E., Glickman, J. N., Petasis, N. A., Blumberg, R. S., \& Serhan, C. N. (2005). Resolvin E1, an endogenous lipid mediator derived from omega-3 eicosapentaenoic acid, protects against 2,4,6-trinitrobenzene sulfonic acid-induced colitis. Proceedings of the National Academy of Sciences of the United States of America, 102(21), 7671-7676. doi:10.1073/pnas.0409271102

Armati, P. J., \& Mathey, E. K. (2013). An update on Schwann cell biology-immunomodulation, neural regulation and other surprises. Journal of the Neurological Sciences, 333(1-2), 68-72. doi:10.1016/j.jns.2013.01.018

Auh, Q. S., \& Ro, J. Y. (2012). Effects of peripheral kappa opioid receptor activation on inflammatory mechanical hyperalgesia in male and female rats. Neuroscience Letters, 524(2), 111-115. doi:10.1016/j.neulet.2012.07.018

Aviello, G., Borrelli, F., Guida, F., Romano, B., Lewellyn, K., De Chiaro, M., Luongo, L., Zjawiony, J. K., Maione, S., Izzo, A. A., \& Capasso, R. (2011). Ultrapotent effects of salvinorin A, a hallucinogenic compound from Salvia divinorum, on LPS-stimulated murine macrophages and its anti-inflammatory action in vivo. Journal of Molecular Medicine (Berlin, Germany), 89(9), 891-902. doi:10.1007/s00109-011-0752-4

Aydin, C., Grace, C. E., \& Gordon, C. J. (2011). Effect of physical restraint on the limits of thermoregulation in telemetered rats. Experimental Physiology, 96(11), 1218-1227. doi:10.1113/expphysiol.2011.060301 
Aziz, M. T., Good, B. L., \& Lowe, D. K. (2014). Serotonin-norepinephrine reuptake inhibitors for the management of chemotherapy-induced peripheral neuropathy. Annals of Pharmacotherapy, 48(5), 626-632. doi:10.1177/1060028014525033

Baggott, M. J., Erowid, E., Erowid, F., Galloway, G. P., \& Mendelson, J. (2010). Use patterns and selfreported effects of Salvia divinorum: an internet-based survey. Drug and Alcohol Dependence, 111(3), 250-256. doi:10.1016/j.drugalcdep.2010.05.003

Bagnol, D., Mansour, A., Akil, H., \& Watson, S. J. (1997). Cellular localization and distribution of the cloned mu and kappa opioid receptors in rat gastrointestinal tract. Neuroscience, 81(2), 579591. doi:10.1016/S0306-4522(97)00227-3

Baker, A. K., \& Meert, T. F. (2002). Functional effects of systemically administered agonists and antagonists of mu, delta, and kappa opioid receptor subtypes on body temperature in mice. Journal of Pharmacology and Experimental Therapeutics, 302(3), 1253-1264. doi:10.1124/jpet.102.037655

Baker, L. E., Panos, J. J., Killinger, B. A., Peet, M. M., Bell, L. M., Haliw, L. A., \& Walker, S. L. (2009). Comparison of the discriminative stimulus effects of salvinorin $A$ and its derivatives to U69,593 and U50,488 in rats. Psychopharmacology, 203(2), 203-211. doi:10.1007/s00213-008-1458-3

Baliki, M. N., \& Apkarian, A. V. (2015). Nociception, pain, negative moods, and behavior selection. Neuron, 87(3), 474-491. doi:10.1016/j.neuron.2015.06.005

Bals-Kubik, R., Ableitner, A., Herz, A., \& Shippenberg, T. S. (1993). Neuroanatomical sites mediating the motivational effects of opioids as mapped by the conditioned place preference paradigm in rats. Journal of Pharmacology and Experimental Therapeutics, 264(1), 489-495.

Balvers, M. G., Verhoeckx, K. C., Plastina, P., Wortelboer, H. M., Meijerink, J., \& Witkamp, R. F. (2010). Docosahexaenoic acid and eicosapentaenoic acid are converted by 3T3-L1 adipocytes to Nacyl ethanolamines with anti-inflammatory properties. Biochimica et Biophysica Acta (BBA) Bioenergetics, 1801(10), 1107-1114. doi:10.1016/j.bbalip.2010.06.006

Balvers, M. G., Wortelboer, H. M., Witkamp, R. F., \& Verhoeckx, K. C. (2013). Liquid chromatographytandem mass spectrometry analysis of free and esterified fatty acid $\mathrm{N}$-acyl ethanolamines in plasma and blood cells. Analytical Biochemistry, 434(2), 275-283. doi:10.1016/j.ab.2012.11.008

Bang, H., Kim, Y., \& Kim, D. (2000). TREK-2, a new member of the mechanosensitive tandem-pore K+ channel family. Journal of Biological Chemistry, 275(23), 17412-17419. doi:10.1074/jbc.M000445200

Barberger-Gateau, P., Raffaitin, C., Letenneur, L., Berr, C., Tzourio, C., Dartigues, J. F., \& Alperovitch, A. (2007). Dietary patterns and risk of dementia: the Three-City cohort study. Neurology, 69(20), 1921-1930. doi:10.1212/01.wnl.0000278116.37320.52

Barbosa, D. S., Cecchini, R., El Kadri, M. Z., Rodriguez, M. A., Burini, R. C., \& Dichi, I. (2003). Decreased oxidative stress in patients with ulcerative colitis supplemented with fish oil omega-3 fatty acids. Nutrition, 19(10), 837-842. doi:10.1016/\$0899-9007(03)00162-X

Barriere, D. A., Rieusset, J., Chanteranne, D., Busserolles, J., Chauvin, M. A., Chapuis, L., Salles, J., Dubray, C., \& Morio, B. (2012). Paclitaxel therapy potentiates cold hyperalgesia in streptozotocin-induced diabetic rats through enhanced mitochondrial reactive oxygen species production and TRPA1 sensitization. Pain, 153(3), 553-561. doi:10.1016/j.pain.2011.11.019

Bartley, E. J., \& Fillingim, R. B. (2013). Sex differences in pain: a brief review of clinical and experimental findings. British Journal of Anaesthesia, 111(1), 52-58. doi:10.1093/bja/aet127

Bartok, R. E., \& Craft, R. M. (1997). Sex differences in opioid antinociception. Journal of Pharmacology and Experimental Therapeutics, 282(2), 769-778.

Barton, D. L., Wos, E. J., Qin, R., Mattar, B. I., Green, N. B., Lanier, K. S., Bearden, J. D., 3rd, Kugler, J. W., Hoff, K. L., Reddy, P. S., Rowland, K. M., Jr., Riepl, M., Christensen, B., \& Loprinzi, C. L. (2011). A double-blind, placebo-controlled trial of a topical treatment for chemotherapyinduced peripheral neuropathy: NCCTG trial N06CA. Supportive Care in Cancer, 19(6), 833841. doi:10.1007/s00520-010-0911-0 
Barzegar-Fallah, A., Alimoradi, H., Mehrzadi, S., Barzegar-Fallah, N., Zendedel, A., Abbasi, A., \& Dehpour, A. R. (2014). The neuroprotective effect of tropisetron on vincristine-induced neurotoxicity. Neurotoxicology, 41, 1-8. doi:10.1016/j.neuro.2013.12.002

Bastuji, H., Frot, M., Perchet, C., Magnin, M., \& Garcia-Larrea, L. (2016). Pain networks from the inside: Spatiotemporal analysis of brain responses leading from nociception to conscious perception. Human Brain Mapping, 37(12), 4301-4315. doi:10.1002/hbm.23310

Bautista, D. M., Siemens, J., Glazer, J. M., Tsuruda, P. R., Basbaum, A. I., Stucky, C. L., Jordt, S. E., \& Julius, D. (2007). The menthol receptor TRPM 8 is the principal detector of environmental cold. Nature, 448(7150), 204-208. doi:10.1038/nature05910

Baykan, B., Ertas, M., Karli, N., Uluduz, D., Uygunoglu, U., Ekizoglu, E., Kocasoy Orhan, E., Saip, S., Zarifoglu, M., \& Siva, A. (2015). Migraine incidence in 5 years: a population-based prospective longitudinal study in Turkey. Journal of Headache and Pain, 16(1), 103. doi:10.1186/s10194015-0589-2

Beguin, C., Duncan, K. K., Munro, T. A., Ho, D. M., Xu, W., Liu-Chen, L. Y., Carlezon, W. A., Jr., \& Cohen, B. M. (2009). Modification of the furan ring of salvinorin A: identification of a selective partial agonist at the kappa opioid receptor. Bioorganic and Medicinal Chemistry, 17(3), 1370-1380. doi:10.1016/j.bmc.2008.12.012

Beguin, C., Potter, D. N., Dinieri, J. A., Munro, T. A., Richards, M. R., Paine, T. A., Berry, L., Zhao, Z., Roth, B. L., Xu, W., Liu-Chen, L. Y., Carlezon, W. A., Jr., \& Cohen, B. M. (2008). Nmethylacetamide analog of salvinorin $\mathrm{A}$ : a highly potent and selective kappa-opioid receptor agonist with oral efficacy. Journal of Pharmacology and Experimental Therapeutics, 324(1), 188-195. doi:10.1124/jpet.107.129023

Beijers, A., Mols, F., Dercksen, W., Driessen, C., \& Vreugdenhil, G. (2014). Chemotherapy-induced peripheral neuropathy and impact on quality of life 6 months after treatment with chemotherapy. Journal of Community and Supportive Oncology, 12(11), 401-406. doi:10.12788/jcso.0086

Belcheva, M. M., Clark, A. L., Haas, P. D., Serna, J. S., Hahn, J. W., Kiss, A., \& Coscia, C. J. (2005). Mu and kappa opioid receptors activate ERK/MAPK via different protein kinase $C$ isoforms and secondary messengers in astrocytes. Journal of Biological Chemistry, 280(30), 27662-27669. doi:10.1074/jbc.M502593200

Belluzzi, A., Brignola, C., Campieri, M., Pera, A., Boschi, S., \& Miglioli, M. (1996). Effect of an entericcoated fish-oil preparation on relapses in Crohn's disease. New England Journal of Medicine, 334(24), 1557-1560. doi:10.1056/NEJM199606133342401

Bennett, D. L., \& Woods, C. G. (2014). Painful and painless channelopathies. Lancet Neurology, 13(6), 587-599. doi:10.1016/S1474-4422(14)70024-9

Bennett, G. J., \& Xie, Y. K. (1988). A peripheral mononeuropathy in rat that produces disorders of pain sensation like those seen in man. Pain, 33(1), 87-107. doi:10.1016/0304-3959(88)90209-6

Bensch, K. G., \& Malawista, S. E. (1968). Microtubule crystals: a new biophysical phenomenon induced by Vinca alkaloids. Nature, 218(5147), 1176-1177. doi:10.1038/2181176a0

Bera, I., Marathe, M. V., Payghan, P. V., \& Ghoshal, N. (2018). Identification of novel hits as highly prospective dual agonists for mu and kappa opioid receptors: an integrated in silico approach. Journal of Biomolecular Structure and Dynamics, 36(2), 279-301. doi:10.1080/07391102.2016.1275810

Berge, O. G., Garcia-Cabrera, I., \& Hole, K. (1988). Response latencies in the tail-flick test depend on tail skin temperature. Neuroscience Letters, 86(3), 284-288. doi:10.1016/03043940(88)90497-1

Berger, A., Crozier, G., Bisogno, T., Cavaliere, P., Innis, S., \& Di Marzo, V. (2001). Anandamide and diet: inclusion of dietary arachidonate and docosahexaenoate leads to increased brain levels of the corresponding $\mathrm{N}$-acylethanolamines in piglets. Proceedings of the National Academy of Sciences of the United States of America, 98(11), 6402-6406. doi:10.1073/pnas.101119098 
Berke, D. S., Reidy, D. E., Miller, J. D., \& Zeichner, A. (2017). Take it like a man: Gender-threatened men's experience of gender role discrepancy, emotion activation, and pain tolerance. Psychology of Men \& Masculinity, 18(1), 62. doi:10.1037/men0000036

Berterame, S., Erthal, J., Thomas, J., Fellner, S., Vosse, B., Clare, P., Hao, W., Johnson, D. T., Mohar, A., Pavadia, J., Samak, A. K., Sipp, W., Sumyai, V., Suryawati, S., Toufiq, J., Yans, R., \& Mattick, R. P. (2016). Use of and barriers to access to opioid analgesics: a worldwide, regional, and national study. Lancet, 387(10028), 1644-1656. doi:10.1016/S0140-6736(16)00161-6

Bhalla, S., Zhang, Z., Patterson, N., \& Gulati, A. (2010). Effect of endothelin-A receptor antagonist on $\mathrm{mu}$, delta and kappa opioid receptor-mediated antinociception in mice. European Journal of Pharmacology, 635(1-3), 62-71. doi:10.1016/j.ejphar.2010.03.003

Bileviciute-Ljungar, I., Saxne, T., \& Spetea, M. (2006). Anti-inflammatory effects of contralateral administration of the kappa-opioid agonist $\mathrm{U}-50,488 \mathrm{H}$ in rats with unilaterally induced adjuvant arthritis. Rheumatology, 45(3), 295-302. doi:10.1093/rheumatology/kei156

Bileviciute-Ljungar, I., \& Spetea, M. (2004). Contralateral, ipsilateral and bilateral treatments with the kappa-opioid receptor agonist U-50,488H in mononeuropathic rats. European Journal of Pharmacology, 494(2-3), 139-146. doi:10.1016/j.ejphar.2004.04.043

Binder, W., Machelska, H., Mousa, S., Schmitt, T., Riviere, P. J., Junien, J. L., Stein, C., \& Schafer, M. (2001). Analgesic and antiinflammatory effects of two novel kappa-opioid peptides. Anesthesiology, 94(6), 1034-1044.

Bisogno, T., Delton-Vandenbroucke, I., Milone, A., Lagarde, M., \& Di Marzo, V. (1999). Biosynthesis and inactivation of $\mathrm{N}$-arachidonoylethanolamine (anandamide) and $\mathrm{N}$ docosahexaenoylethanolamine in bovine retina. Archives of Biochemistry and Biophysics, 370(2), 300-307. doi:10.1006/abbi.1999.1410

Blasbalg, T. L., Hibbeln, J. R., Ramsden, C. E., Majchrzak, S. F., \& Rawlings, R. R. (2011). Changes in consumption of omega-3 and omega- 6 fatty acids in the United States during the 20th century. American Journal of Clinical Nutrition, 93(5), 950-962. doi:10.3945/ajcn.110.006643

Bober, B. G., Gutierrez, E., Plaxe, S., Groisman, A., \& Shah, S. B. (2015). Combinatorial influences of paclitaxel and strain on axonal transport. Experimental Neurology, 271, 358-367. doi:10.1016/j.expneurol.2015.06.023

Boehmerle, W., Splittgerber, U., Lazarus, M. B., McKenzie, K. M., Johnston, D. G., Austin, D. J., \& Ehrlich, B. E. (2006). Paclitaxel induces calcium oscillations via an inositol 1,4,5-trisphosphate receptor and neuronal calcium sensor 1-dependent mechanism. Proceedings of the National Academy of Sciences of the United States of America, 103(48), 18356-18361. doi:10.1073/pnas.0607240103

Boehmerle, W., Zhang, K., Sivula, M., Heidrich, F. M., Lee, Y., Jordt, S. E., \& Ehrlich, B. E. (2007). Chronic exposure to paclitaxel diminishes phosphoinositide signaling by calpain-mediated neuronal calcium sensor-1 degradation. Proceedings of the National Academy of Sciences of the United States of America, 104(26), 11103-11108. doi:10.1073/pnas.0701546104

Bohn, L. M., Gainetdinov, R. R., Lin, F. T., Lefkowitz, R. J., \& Caron, M. G. (2000). Mu-opioid receptor desensitization by beta-arrestin-2 determines morphine tolerance but not dependence. Nature, 408(6813), 720-723. doi:10.1038/35047086

Bolanos, C. A., Garmsen, G. M., Clair, M. A., \& McDougall, S. A. (1996). Effects of the kappa-opioid receptor agonist $\mathrm{U}-50,488$ on morphine-induced place preference conditioning in the developing rat. European Journal of Pharmacology, 317(1), 1-8.

Bonin, R. P., Bories, C., \& De Koninck, Y. (2014). A simplified up-down method (SUDO) for measuring mechanical nociception in rodents using von Frey filaments. Molecular Pain, 10(1), 26. doi:10.1186/1744-8069-10-26

Borrelli, F., \& Izzo, A. A. (2009). Role of acylethanolamides in the gastrointestinal tract with special reference to food intake and energy balance. Best Practice \& Research: Clinical Endocrinology \& Metabolism, 23(1), 33-49. doi:10.1016/j.beem.2008.10.003 
Bosch-Bouju, C., \& Layé, S. (2016). Dietary omega-6/omega-3 and endocannabinoids: Implications for brain health and diseases Cannabinoids in Health and Disease: InTechOpen.

Bourne, S., Machado, A. G., \& Nagel, S. J. (2014). Basic anatomy and physiology of pain pathways. Neurosurgery Clinics of North America, 25(4), 629-638. doi:10.1016/j.nec.2014.06.001

Boutaud, O., Aronoff, D. M., Richardson, J. H., Marnett, L. J., \& Oates, J. A. (2002). Determinants of the cellular specificity of acetaminophen as an inhibitor of prostaglandin $H(2)$ synthases. Proceedings of the National Academy of Sciences of the United States of America, 99(10), 7130-7135. doi:10.1073/pnas.102588199

Boychuk, D. G., Goddard, G., Mauro, G., \& Orellana, M. F. (2015). The effectiveness of cannabinoids in the management of chronic nonmalignant neuropathic pain: a systematic review. J Oral Facial Pain Headache, 29(1), 7-14. doi:10.11607/ofph.1274

Braida, D., Capurro, V., Zani, A., Rubino, T., Vigano, D., Parolaro, D., \& Sala, M. (2009). Potential anxiolytic- and antidepressant-like effects of salvinorin A, the main active ingredient of Salvia divinorum, in rodents. British Journal of Pharmacology, 157(5), 844-853. doi:10.1111/j.14765381.2009.00230.x

Braida, D., Donzelli, A., Martucci, R., Capurro, V., \& Sala, M. (2011). Learning and memory impairment induced by salvinorin $A$, the principal ingredient of Salvia divinorum, in wistar rats. International Journal of Toxicology, 30(6), 650-661. doi:10.1177/1091581811418538

Braida, D., Limonta, V., Pegorini, S., Zani, A., Guerini-Rocco, C., Gori, E., \& Sala, M. (2007). Hallucinatory and rewarding effect of salvinorin A in zebrafish: kappa-opioid and CB1-cannabinoid receptor involvement. Psychopharmacology, 190(4), 441-448. doi:10.1007/s00213-006-0639-1

Branco, J. C., Bannwarth, B., Failde, I., Abello Carbonell, J., Blotman, F., Spaeth, M., Saraiva, F., Nacci, F., Thomas, E., Caubere, J. P., Le Lay, K., Taieb, C., \& Matucci-Cerinic, M. (2010). Prevalence of fibromyalgia: a survey in five European countries. Seminars in Arthritis and Rheumatism, 39(6), 448-453. doi:10.1016/j.semarthrit.2008.12.003

Brandolini, L., Benedetti, E., Ruffini, P. A., Russo, R., Cristiano, L., Antonosante, A., d'Angelo, M., Castelli, V., Giordano, A., Allegretti, M., \& Cimini, A. (2017). CXCR1/2 pathways in paclitaxelinduced neuropathic pain. Oncotarget, 8(14), 23188-23201. doi:10.18632/oncotarget.15533

Brauchi, S., Orta, G., Salazar, M., Rosenmann, E., \& Latorre, R. (2006). A hot-sensing cold receptor: Cterminal domain determines thermosensation in transient receptor potential channels. Journal of Neuroscience, 26(18), 4835-4840. doi:10.1523/JNEUROSCI.5080-05.2006

Breivik, H., Collett, B., Ventafridda, V., Cohen, R., \& Gallacher, D. (2006). Survey of chronic pain in Europe: prevalence, impact on daily life, and treatment. European Journal of Pain (London, England), 10(4), 287-333. doi:10.1016/j.ejpain.2005.06.009

Brenna, J. T. (2002). Efficiency of conversion of alpha-linolenic acid to long chain n-3 fatty acids in man. Current Opinion in Clinical Nutrition and Metabolic Care, 5(2), 127-132.

Brenna, J. T., Salem, N., Jr., Sinclair, A. J., Cunnane, S. C., International Society for the Study of Fatty, A., \& Lipids, I. (2009). alpha-Linolenic acid supplementation and conversion to n-3 long-chain polyunsaturated fatty acids in humans. Prostaglandins, Leukotrienes, and Essential Fatty Acids, 80(2-3), 85-91. doi:10.1016/j.plefa.2009.01.004

Brierley, S. M., Hughes, P. A., Page, A. J., Kwan, K. Y., Martin, C. M., O'Donnell, T. A., Cooper, N. J., Harrington, A. M., Adam, B., Liebregts, T., Holtmann, G., Corey, D. P., Rychkov, G. Y., \& Blackshaw, L. A. (2009). The ion channel TRPA1 is required for normal mechanosensation and is modulated by algesic stimuli. Gastroenterology, 137(6), 2084-2095 e2083. doi:10.1053/j.gastro.2009.07.048

Brown, I., Cascio, M. G., Wahle, K. W., Smoum, R., Mechoulam, R., Ross, R. A., Pertwee, R. G., \& Heys, S. D. (2010). Cannabinoid receptor-dependent and -independent anti-proliferative effects of omega-3 ethanolamides in androgen receptor-positive and -negative prostate cancer cell lines. Carcinogenesis, 31(9), 1584-1591. doi:10.1093/carcin/bgq151

Bruchas, M. R., \& Chavkin, C. (2010). Kinase cascades and ligand-directed signaling at the kappa opioid receptor. Psychopharmacology, 210(2), 137-147. doi:10.1007/s00213-010-1806-y 
Bruchas, M. R., Land, B. B., Aita, M., Xu, M., Barot, S. K., Li, S., \& Chavkin, C. (2007). Stress-induced p38 mitogen-activated protein kinase activation mediates kappa-opioid-dependent dysphoria. Journal of Neuroscience, 27(43), 11614-11623. doi:10.1523/JNEUROSCI.3769-07.2007

Bruchas, M. R., Macey, T. A., Lowe, J. D., \& Chavkin, C. (2006). Kappa opioid receptor activation of p38 MAPK is GRK3- and arrestin-dependent in neurons and astrocytes. Journal of Biological Chemistry, 281(26), 18081-18089. doi:10.1074/jbc.M513640200

Bruchas, M. R., Schindler, A. G., Shankar, H., Messinger, D. I., Miyatake, M., Land, B. B., Lemos, J. C., Hagan, C. E., Neumaier, J. F., Quintana, A., Palmiter, R. D., \& Chavkin, C. (2011). Selective p38alpha MAPK deletion in serotonergic neurons produces stress resilience in models of depression and addiction. Neuron, 71(3), 498-511. doi:10.1016/j.neuron.2011.06.011

Bruijnzeel, A. W., Qi, X., Guzhva, L. V., Wall, S., Deng, J. V., Gold, M. S., Febo, M., \& Setlow, B. (2016). Behavioral characterization of the effects of cannabis smoke and anandamide in rats. PloS One, 11(4), e0153327. doi:10.1371/journal.pone.0153327

Burdge, G. (2004). Alpha-linolenic acid metabolism in men and women: nutritional and biological implications. Current Opinion in Clinical Nutrition and Metabolic Care, 7(2), 137-144. doi:10.1097/00075197-200403000-00006

Burgos, E., Gomez-Nicola, D., Pascual, D., Martin, M. I., Nieto-Sampedro, M., \& Goicoechea, C. (2012). Cannabinoid agonist WIN 55,212-2 prevents the development of paclitaxel-induced peripheral neuropathy in rats. Possible involvement of spinal glial cells. European Journal of Pharmacology, 682(1-3), 62-72. doi:10.1016/j.ejphar.2012.02.008

Burr, G. O., \& Burr, M. M. (1930). On the nature and role of the fatty acids essential in nutrition. Journal of Biological Chemistry, 86(2), 0587-0621.

Bushnell, M. C., Case, L. K., Ceko, M., Cotton, V. A., Gracely, J. L., Low, L. A., Pitcher, M. H., \& Villemure, C. (2015). Effect of environment on the long-term consequences of chronic pain. Pain, 156 Suppl 1(0 1), S42-49. doi:10.1097/01.j.pain.0000460347.77341.bd

Bushnell, M. C., Ceko, M., \& Low, L. A. (2013). Cognitive and emotional control of pain and its disruption in chronic pain. Nature Reviews: Neuroscience, 14(7), 502-511. doi:10.1038/nrn3516

Busserolles, J., Tsantoulas, C., Eschalier, A., \& Lopez Garcia, J. A. (2016). Potassium channels in neuropathic pain: advances, challenges, and emerging ideas. Pain, 157 Suppl 1, S7-14. doi:10.1097/j.pain.0000000000000368

Butelman, E. R., Mandau, M., Tidgewell, K., Prisinzano, T. E., Yuferov, V., \& Kreek, M. J. (2007). Effects of salvinorin A, a kappa-opioid hallucinogen, on a neuroendocrine biomarker assay in nonhuman primates with high kappa-receptor homology to humans. Journal of Pharmacology and Experimental Therapeutics, 320(1), 300-306. doi:10.1124/jpet.106.112417

Butelman, E. R., Prisinzano, T. E., Deng, H., Rus, S., \& Kreek, M. J. (2009). Unconditioned behavioral effects of the powerful kappa-opioid hallucinogen salvinorin $A$ in nonhuman primates: fast onset and entry into cerebrospinal fluid. Journal of Pharmacology and Experimental Therapeutics, 328(2), 588-597. doi:10.1124/jpet.108.145342

Cabral, G. A., \& Griffin-Thomas, L. (2009). Emerging role of the cannabinoid receptor CB2 in immune regulation: therapeutic prospects for neuroinflammation. Expert Reviews in Molecular Medicine, 11, e3. doi:10.1017/S1462399409000957

Cahill, C. M., Taylor, A. M., Cook, C., Ong, E., Moron, J. A., \& Evans, C. J. (2014). Does the kappa opioid receptor system contribute to pain aversion? Frontiers in Pharmacology, 5(253), 253. doi:10.3389/fphar.2014.00253

Calcagnetti, D. J., Helmstetter, F. J., \& Fanselow, M. S. (1988). Analgesia produced by centrally administered DAGO, DPDPE and $\mathrm{U} 50488 \mathrm{H}$ in the formalin test. European Journal of Pharmacology, 153(1), 117-122. doi:10.1016/0014-2999(88)90595-X

Calder, P. C. (2008). Polyunsaturated fatty acids, inflammatory processes and inflammatory bowel diseases. Molecular Nutrition \& Food Research, 52(8), 885-897. doi:10.1002/mnfr.200700289 
Calder, P. C. (2011). Fatty acids and inflammation: the cutting edge between food and pharma. European Journal of Pharmacology, 668 Suppl 1, S50-58. doi:10.1016/j.ejphar.2011.05.085

Calder, P. C. (2015). Marine omega-3 fatty acids and inflammatory processes: Effects, mechanisms and clinical relevance. Biochimica et Biophysica Acta (BBA) - Bioenergetics, 1851(4), 469-484. doi:10.1016/j.bbalip.2014.08.010

Calignano, A., La Rana, G., Giuffrida, A., \& Piomelli, D. (1998). Control of pain initiation by endogenous cannabinoids. Nature, 394(6690), 277-281. doi:10.1038/28393

Calignano, A., La Rana, G., \& Piomelli, D. (2001). Antinociceptive activity of the endogenous fatty acid amide, palmitylethanolamide. European Journal of Pharmacology, 419(2-3), 191-198. doi:10.1016/S0014-2999(01)00988-8

Campana, G., \& Rimondini, R. (2015). Mechanical nociception measurement in mice and rats with automated Von Frey equipment. Methods in Molecular Biology, 1230, 229-231. doi:10.1007/978-1-4939-1708-2_18

Campbell, G., Darke, S., Bruno, R., \& Degenhardt, L. (2015). The prevalence and correlates of chronic pain and suicidality in a nationally representative sample. Australian and New Zealand Journal of Psychiatry, 49(9), 803-811. doi:10.1177/0004867415569795

Carlezon, W. A., Jr., Beguin, C., DiNieri, J. A., Baumann, M. H., Richards, M. R., Todtenkopf, M. S., Rothman, R. B., Ma, Z., Lee, D. Y., \& Cohen, B. M. (2006). Depressive-like effects of the kappaopioid receptor agonist salvinorin $A$ on behavior and neurochemistry in rats. Journal of Pharmacology and Experimental Therapeutics, 316(1), 440-447. doi:10.1124/jpet.105.092304

Caterina, M. J., Rosen, T. A., Tominaga, M., Brake, A. J., \& Julius, D. (1999). A capsaicin-receptor homologue with a high threshold for noxious heat. Nature, 398(6726), 436-441. doi:10.1038/18906

Caterina, M. J., Schumacher, M. A., Tominaga, M., Rosen, T. A., Levine, J. D., \& Julius, D. (1997). The capsaicin receptor: a heat-activated ion channel in the pain pathway. Nature, 389(6653), 816824. doi:10.1038/39807

Cavaletti, G., Cavalletti, E., Oggioni, N., Sottani, C., Minoia, C., D'Incalci, M., Zucchetti, M., Marmiroli, P., \& Tredici, G. (2000). Distribution of paclitaxel within the nervous system of the rat after repeated intravenous administration. Neurotoxicology, 21(3), 389-393. doi:10.1046/j.15298027.2001.01008-5.x

Ceccarelli, I., Scaramuzzino, A., Massafra, C., \& Aloisi, A. M. (2003). The behavioral and neuronal effects induced by repetitive nociceptive stimulation are affected by gonadal hormones in male rats. Pain, 104(1-2), 35-47. doi:10.1016/S0304-3959(02)00460-8

Cederholm, T. (2017). Fish consumption and omega-3 fatty acid supplementation for prevention or treatment of cognitive decline, dementia or Alzheimer's disease in older adults - any news? Current Opinion in Clinical Nutrition and Metabolic Care, 20(2), 104-109. doi:10.1097/MCO.0000000000000350

Celerier, E., Rivat, C., Jun, Y., Laulin, J. P., Larcher, A., Reynier, P., \& Simonnet, G. (2000). Long-lasting hyperalgesia induced by fentanyl in rats: preventive effect of ketamine. Anesthesiology, 92(2), 465-472.

Cerrato, S., Brazis, P., Della Valle, M. F., Miolo, A., Petrosino, S., Di Marzo, V., \& Puigdemont, A. (2012). Effects of palmitoylethanolamide on the cutaneous allergic inflammatory response in Ascaris hypersensitive Beagle dogs. Veterinary Journal, 191(3), 377-382. doi:10.1016/j.tvjl.2011.04.002

Cervero, F. (2009). Visceral versus somatic pain: similarities and differences. Digestive Diseases, 27 Suppl 1(Suppl. 1), 3-10. doi:10.1159/000268115

Chakrabarti, S., Liu, N. J., \& Gintzler, A. R. (2010). Formation of mu-/kappa-opioid receptor heterodimer is sex-dependent and mediates female-specific opioid analgesia. Proceedings of the National Academy of Sciences of the United States of America, 107(46), 20115-20119. doi:10.1073/pnas.1009923107 
Chan, S. S., Luben, R., Olsen, A., Tjonneland, A., Kaaks, R., Lindgren, S., Grip, O., Bergmann, M. M., Boeing, H., Hallmans, G., Karling, P., Overvad, K., Veno, S. K., van Schaik, F., Bueno-deMesquita, B., Oldenburg, B., Khaw, K. T., Riboli, E., \& Hart, A. R. (2014). Association between high dietary intake of the n-3 polyunsaturated fatty acid docosahexaenoic acid and reduced risk of Crohn's disease. Alimentary Pharmacology and Therapeutics, 39(8), 834-842. doi:10.1111/apt.12670

Chang, J., Versloot, J., Fashler, S. R., McCrystal, K. N., \& Craig, K. D. (2015). Pain assessment in children: validity of facial expression items in observational pain scales. Clinical Journal of Pain, 31(3), 189-197. doi:10.1097/AJP.0000000000000103

Chang, W., Berta, T., Kim, Y. H., Lee, S., Lee, S. Y., \& Ji, R. R. (2018). Expression and role of voltagegated sodium channels in human dorsal root ganglion neurons with special focus on Nav1.7, species differences, and regulation by paclitaxel. Neuroscience Bulletin, 34(1), 4-12. doi:10.1007/s12264-017-0132-3

Chapkin, R. S., Akoh, C. C., \& Miller, C. C. (1991). Influence of dietary n-3 fatty acids on macrophage glycerophospholipid molecular species and peptidoleukotriene synthesis. Journal of Lipid Research, 32(7), 1205-1213.

Chaplan, S. R., Bach, F. W., Pogrel, J. W., Chung, J. M., \& Yaksh, T. L. (1994). Quantitative assessment of tactile allodynia in the rat paw. Journal of Neuroscience Methods, 53(1), 55-63. doi:10.1016/0165-0270(94)90144-9

Chapman, C. R., Casey, K. L., Dubner, R., Foley, K. M., Gracely, R. H., \& Reading, A. E. (1985). Pain measurement: an overview. Pain, 22(1), 1-31. doi:10.1016/0304-3959(85)90145-9

Chavkin, C., James, I. F., \& Goldstein, A. (1982). Dynorphin is a specific endogenous ligand of the kappaopioid receptor. Science, 215(4531), 413-415. doi:10.1126/science.6120570

Chavkin, C., Sud, S., Jin, W., Stewart, J., Zjawiony, J. K., Siebert, D. J., Toth, B. A., Hufeisen, S. J., \& Roth, B. L. (2004). Salvinorin A, an active component of the hallucinogenic sage salvia divinorum is a highly efficacious kappa-opioid receptor agonist: structural and functional considerations. Journal of Pharmacology and Experimental Therapeutics, 308(3), 1197-1203. doi:10.1124/jpet.103.059394

Che, T., Majumdar, S., Zaidi, S. A., Ondachi, P., McCorvy, J. D., Wang, S., Mosier, P. D., Uprety, R., Vardy, E., Krumm, B. E., Han, G. W., Lee, M. Y., Pardon, E., Steyaert, J., Huang, X. P., Strachan, R. T., Tribo, A. R., Pasternak, G. W., Carroll, F. I., Stevens, R. C., Cherezov, V., Katritch, V., Wacker, D., \& Roth, B. L. (2018). Structure of the nanobody-stabilized active state of the kappa opioid receptor. Cell, 172(1-2), 55-67 e15. doi:10.1016/j.cell.2017.12.011

Chen, X., McClatchy, D. B., Geller, E. B., Tallarida, R. J., \& Adler, M. W. (2005). The dynamic relationship between mu and kappa opioid receptors in body temperature regulation. Life Sciences, 78(4), 329-333. doi:10.1016/j.Ifs.2005.04.084

Chen, Y.-F., Chen, L.-H., Yeh, Y.-M., Wu, P.-Y., Chen, Y.-F., Chang, L.-Y., Chang, J.-Y., \& Shen, M.-R. (2017a). Minoxidil is a potential neuroprotective drug for paclitaxel-induced peripheral neuropathy. Scientific Reports, 7, 45366. doi:10.1038/srep45366

Chen, Y., Mestek, A., Liu, J., \& Yu, L. (1993). Molecular cloning of a rat k-opioid receptor reveals sequence similarities to the $\mu$ - and $\delta$-opioid receptors. Biochemical Journal, 295(3), 625-628. doi:10.1042/bj2950625

Chen, Y., Yang, C., \& Wang, Z. J. (2011). Proteinase-activated receptor 2 sensitizes transient receptor potential vanilloid 1 , transient receptor potential vanilloid 4 , and transient receptor potential ankyrin 1 in paclitaxel-induced neuropathic pain. Neuroscience, 193, 440-451. doi:10.1016/j.neuroscience.2011.06.085

Chen, Y. C., Auer-Grumbach, M., Matsukawa, S., Zitzelsberger, M., Themistocleous, A. C., Strom, T. M., Samara, C., Moore, A. W., Cho, L. T., Young, G. T., Weiss, C., Schabhuttl, M., Stucka, R., Schmid, A. B., Parman, Y., Graul-Neumann, L., Heinritz, W., Passarge, E., Watson, R. M., Hertz, J. M., Moog, U., Baumgartner, M., Valente, E. M., Pereira, D., Restrepo, C. M., Katona, I., Dusl, M., Stendel, C., Wieland, T., Stafford, F., Reimann, F., von Au, K., Finke, C., Willems, P. J., Nahorski, 
M. S., Shaikh, S. S., Carvalho, O. P., Nicholas, A. K., Karbani, G., McAleer, M. A., Cilio, M. R., McHugh, J. C., Murphy, S. M., Irvine, A. D., Jensen, U. B., Windhager, R., Weis, J., Bergmann, C., Rautenstrauss, B., Baets, J., De Jonghe, P., Reilly, M. M., Kropatsch, R., Kurth, I., Chrast, R., Michiue, T., Bennett, D. L., Woods, C. G., \& Senderek, J. (2015). Transcriptional regulator PRDM12 is essential for human pain perception. Nature Genetics, 47(7), 803-808. doi:10.1038/ng.3308

Chen, Y. F., Chen, L. H., Yeh, Y. M., Wu, P. Y., Chen, Y. F., Chang, L. Y., Chang, J. Y., \& Shen, M. R. (2017b). Minoxidil is a potential neuroprotective drug for paclitaxel-induced peripheral neuropathy. Scientific Reports, 7, 45366. doi:10.1038/srep45366

Cheng, C., Guo, G. F., Martinez, J. A., Singh, V., \& Zochodne, D. W. (2010). Dynamic plasticity of axons within a cutaneous milieu. Journal of Neuroscience, 30(44), 14735-14744. doi:10.1523/JNEUROSCI.2919-10.2010

Chiu, Y. T., Chen, C., Yu, D., Schulz, S., \& Liu-Chen, L. Y. (2017). Agonist-dependent and -independent kappa opioid receptor phosphorylation: Distinct phosphorylation patterns and different cellular outcomes. Molecular Pharmacology, 92(5), 588-600. doi:10.1124/mol.117.108555

Chogtu, B., Bairy, K. L., Smitha, D., Dhar, S., \& Himabindu, P. (2011). Comparison of the efficacy of carbamazepine, gabapentin and lamotrigine for neuropathic pain in rats. Indian Journal of Pharmacology, 43(5), 596-598. doi:10.4103/0253-7613.84980

Choi, Y., Yoon, Y. W., Na, H. S., Kim, S. H., \& Chung, J. M. (1994). Behavioral signs of ongoing pain and cold allodynia in a rat model of neuropathic pain. Pain, 59(3), 369-376. doi:10.1016/03043959(94)90023-X

Chou, J. Z., Chait, B. T., Wang, R., \& Kreek, M. J. (1996). Differential biotransformation of dynorphin A (1-17) and dynorphin A (1-13) peptides in human blood, ex vivo. Peptides, 17(6), 983-990. doi:10.1016/0196-9781(96)00154-4

Chouchkov, C. (2012). Cutaneous receptors (Vol. 54): Springer Science \& Business Media.

Christensen, M. D., Everhart, A. W., Pickelman, J. T., \& Hulsebosch, C. E. (1996). Mechanical and thermal allodynia in chronic central pain following spinal cord injury. Pain, 68(1), 97-107. doi:10.1016/S0304-3959(96)03224-1

Chu, L. F., Clark, D. J., \& Angst, M. S. (2006). Opioid tolerance and hyperalgesia in chronic pain patients after one month of oral morphine therapy: a preliminary prospective study. Journal of Pain, 7(1), 43-48. doi:10.1016/j.jpain.2005.08.001

Cicero, T. J., Ellis, M. S., Surratt, H. L., \& Kurtz, S. P. (2014). The changing face of heroin use in the United States: a retrospective analysis of the past 50 years. JAMA Psychiatry, 71(7), 821-826. doi:10.1001/jamapsychiatry.2014.366

Clapper, J. R., Moreno-Sanz, G., Russo, R., Guijarro, A., Vacondio, F., Duranti, A., Tontini, A., Sanchini, S., Sciolino, N. R., Spradley, J. M., Hohmann, A. G., Calignano, A., Mor, M., Tarzia, G., \& Piomelli, D. (2010). Anandamide suppresses pain initiation through a peripheral endocannabinoid mechanism. Nature Neuroscience, 13(10), 1265-1270. doi:10.1038/nn.2632

Clarke, T. C., Black, L. I., Stussman, B. J., Barnes, P. M., \& Nahin, R. L. (2015). Trends in the use of complementary health approaches among adults: United States, 2002-2012. National health statistics reports(79), 1-16. doi:10.1097/HNP.0000000000000088

Clayton, J. A., \& Collins, F. S. (2014). Policy: NIH to balance sex in cell and animal studies. Nature, 509(7500), 282-283. doi:10.1038/509282a

Coburn, A. F., \& Moore, L. V. (1943). Nutrition as a conditioning factor in the rheumatic state. American Journal of Diseases of Children, 65(5), 744-756. doi:10.1001/archpedi.1943.02010170066008

Coffeen, U., Canseco-Alba, A., Simon-Arceo, K., Almanza, A., Mercado, F., Leon-Olea, M., \& Pellicer, F. (2018). Salvinorin A reduces neuropathic nociception in the insular cortex of the rat. European Journal of Pain (London, England), 22(2), 311-318. doi:10.1002/ejp.1120

Coghill, R. C., McHaffie, J. G., \& Yen, Y. F. (2003). Neural correlates of interindividual differences in the subjective experience of pain. Proceedings of the National Academy of Sciences of the United States of America, 100(14), 8538-8542. doi:10.1073/pnas.1430684100 
Cohen, S. P., \& Mao, J. (2014). Neuropathic pain: mechanisms and their clinical implications. British Medical Journal, 348(f7656), 1-12. doi:10.1136/bmj.f7656

Colburn, R. W., Lubin, M. L., Stone, D. J., Jr., Wang, Y., Lawrence, D., D'Andrea, M. R., Brandt, M. R., Liu, Y., Flores, C. M., \& Qin, N. (2007). Attenuated cold sensitivity in TRPM8 null mice. Neuron, 54(3), 379-386. doi:10.1016/j.neuron.2007.04.017

Cole, R. L., Konradi, C., Douglass, J., \& Hyman, S. E. (1995). Neuronal adaptation to amphetamine and dopamine: molecular mechanisms of prodynorphin gene regulation in rat striatum. Neuron, 14(4), 813-823. doi:10.1016/0896-6273(95)90225-2

Colleoni, M., \& Sacerdote, P. (2010). Murine models of human neuropathic pain. Biochimica et Biophysica Acta (BBA) - Bioenergetics, 1802(10), 924-933. doi:10.1016/j.bbadis.2009.10.012

Collier, D. S., \& Pain, J. A. (1985). Non-steroidal anti-inflammatory drugs and peptic ulcer perforation. Gut, 26(4), 359-363. doi:10.1136/gut.26.4.359

Compton, D. R., Johnson, M. R., Melvin, L. S., \& Martin, B. R. (1992). Pharmacological profile of a series of bicyclic cannabinoid analogs - Classification as cannabimimetic agents. Journal of Pharmacology and Experimental Therapeutics, 260(1), 201-209.

Compton, W. M., \& Volkow, N. D. (2006). Major increases in opioid analgesic abuse in the United States: concerns and strategies. Drug and Alcohol Dependence, 81(2), 103-107. doi:10.1016/j.drugalcdep.2005.05.009

Costa, B., Comelli, F., Bettoni, I., Colleoni, M., \& Giagnoni, G. (2008). The endogenous fatty acid amide, palmitoylethanolamide, has anti-allodynic and anti-hyperalgesic effects in a murine model of neuropathic pain: involvement of CB(1), TRPV1 and PPARgamma receptors and neurotrophic factors. Pain, 139(3), 541-550. doi:10.1016/j.pain.2008.06.003

Costa, R., Bicca, M. A., Manjavachi, M. N., Segat, G. C., Dias, F. C., Fernandes, E. S., \& Calixto, J. B. (2018). Kinin receptors sensitize TRPV4 channel and induce mechanical hyperalgesia: Relevance to paclitaxel-induced peripheral neuropathy in mice. Molecular Neurobiology, 55(3), 2150-2161. doi:10.1007/s12035-017-0475-9

Coste, B., Mathur, J., Schmidt, M., Earley, T. J., Ranade, S., Petrus, M. J., Dubin, A. E., \& Patapoutian, A. (2010). Piezo1 and Piezo2 are essential components of distinct mechanically activated cation channels. Science, 330(6000), 55-60. doi:10.1126/science.1193270

Courad, J. P., Besse, D., Delchambre, C., Hanoun, N., Hamon, M., Eschalier, A., Caussade, F., \& Cloarec, A. (2001). Acetaminophen distribution in the rat central nervous system. Life Sciences, 69(12), 1455-1464. doi:10.1016/S0024-3205(01)01228-0

Cox, J. J., Reimann, F., Nicholas, A. K., Thornton, G., Roberts, E., Springell, K., Karbani, G., Jafri, H., Mannan, J., Raashid, Y., Al-Gazali, L., Hamamy, H., Valente, E. M., Gorman, S., Williams, R., McHale, D. P., Wood, J. N., Gribble, F. M., \& Woods, C. G. (2006). An SCN9A channelopathy causes congenital inability to experience pain. Nature, 444(7121), 894-898. doi:10.1038/nature05413

Craft, R. M. (2007). Modulation of pain by estrogens. Pain, 132 Suppl 1, S3-12. doi:10.1016/j.pain.2007.09.028

Crawley, J. N., Corwin, R. L., Robinson, J. K., Felder, C. C., Devane, W. A., \& Axelrod, J. (1993). Anandamide, an endogenous ligand of the cannabinoid receptor, induces hypomotility and hypothermia in vivo in rodents. Pharmacology Biochemistry and Behavior, 46(4), 967-972. doi:10.1016/0091-3057(93)90230-Q

Cremon, C., Stanghellini, V., Barbaro, M. R., Cogliandro, R. F., Bellacosa, L., Santos, J., Vicario, M., Pigrau, M., Alonso Cotoner, C., Lobo, B., Azpiroz, F., Bruley des Varannes, S., Neunlist, M., DeFilippis, D., luvone, T., Petrosino, S., Di Marzo, V., \& Barbara, G. (2017). Randomised clinical trial: the analgesic properties of dietary supplementation with palmitoylethanolamide and polydatin in irritable bowel syndrome. Alimentary Pharmacology and Therapeutics, 45(7), 909-922. doi:10.1111/apt.13958

Cristina-Silva, C., Martins, V., Gargaglioni, L. H., \& Bicego, K. C. (2017). Mu and kappa opioid receptors of the periaqueductal gray stimulate and inhibit thermogenesis, respectively, during 
psychological stress in rats. Pflügers Archiv. European Journal of Physiology, 469(9), 11511161. doi:10.1007/s00424-017-1966-2

Crowley, R. S., Riley, A. P., Sherwood, A. M., Groer, C. E., Shivaperumal, N., Biscaia, M., Paton, K., Schneider, S., Provasi, D., Kivell, B. M., Filizola, M., \& Prisinzano, T. E. (2016). Synthetic studies of neoclerodane diterpenes from Salvia divinorum: Identification of a potent and centrally acting mu opioid analgesic with reduced abuse liability. Journal of Medicinal Chemistry, 59(24), 11027-11038. doi:10.1021/acs.jmedchem.6b01235

Cryer, B., Barnett, M. A., Wagner, J., \& Wilcox, C. M. (2016). Overuse and misperceptions of nonsteroidal anti-inflammatory drugs in the United States. American Journal of the Medical Sciences, 352(5), 472-480. doi:10.1016/j.amjms.2016.08.028

Culverhouse, A. D. (2015). Exploring the aversive and anxiogenic effects of novel kappa opioid receptor agonists in rats. Master's degree in Science, Victoria University of Wellington.

D'Agostino, G., La Rana, G., Russo, R., Sasso, O., lacono, A., Esposito, E., Mattace Raso, G., Cuzzocrea, S., Loverme, J., Piomelli, D., Meli, R., \& Calignano, A. (2009). Central administration of palmitoylethanolamide reduces hyperalgesia in mice via inhibition of NF-kappaB nuclear signalling in dorsal root ganglia. European Journal of Pharmacology, 613(1-3), 54-59. doi:10.1016/j.ejphar.2009.04.022

D'amour, F. E., \& Smith, D. L. (1941). A method for determining loss of pain sensation. Journal of Pharmacology and Experimental Therapeutics, 72(1), 74-79.

Dahan, A., Sarton, E., Teppema, L., Olievier, C., Nieuwenhuijs, D., Matthes, H. W., \& Kieffer, B. L. (2001). Anesthetic potency and influence of morphine and sevoflurane on respiration in mu-opioid receptor knockout mice. Anesthesiology, 94(5), 824-832.

Dai, Y. (2016). TRPs and pain. Seminars in Immunopathology, 38(3), 277-291. doi:10.1007/s00281-0150526-0

Dar, M. S. (2000). Cerebellar CB1 receptor mediation of $\triangle 9$-THC-induced motor incoordination and its potentiation by ethanol and modulation by the cerebellar adenosinergic $A 1$ receptor in the mouse. Brain Research, 864(2), 186-194. doi:10.1016/s0006-8993(00)02103-x

Dasarathy, S., Dasarathy, J., Khiyami, A., Yerian, L., Hawkins, C., Sargent, R., \& McCullough, A. J. (2015). Double-blind randomized placebo-controlled clinical trial of omega 3 fatty acids for the treatment of diabetic patients with nonalcoholic steatohepatitis. Journal of Clinical Gastroenterology, 49(2), 137-144. doi:10.1097/MCG.0000000000000099

Day, R., Lazure, C., Basak, A., Boudreault, A., Limperis, P., Dong, W., \& Lindberg, I. (1998). Prodynorphin processing by proprotein convertase 2 . Cleavage at single basic residues and enhanced processing in the presence of carboxypeptidase activity. Journal of Biological Chemistry, 273(2), 829-836. doi:10.1074/jbc.273.2.829

De Ruddere, L., \& Craig, K. D. (2016). Understanding stigma and chronic pain: a-state-of-the-art review. Pain, 157(8), 1607-1610. doi:10.1097/j.pain.0000000000000512

Deng, L., Ding, W., \& Granstein, R. D. (2003). Thalidomide inhibits tumor necrosis factor-alpha production and antigen presentation by Langerhans cells. Journal of Investigative Dermatology, 121(5), 1060-1065. doi:10.1046/j.1523-1747.2003.12565.x

Deng, L., Guindon, J., Cornett, B. L., Makriyannis, A., Mackie, K., \& Hohmann, A. G. (2015). Chronic cannabinoid receptor 2 activation reverses paclitaxel neuropathy without tolerance or cannabinoid receptor 1-dependent withdrawal. Biological Psychiatry, 77(5), 475-487. doi:10.1016/j.biopsych.2014.04.009

Deng, L., Guindon, J., Vemuri, V. K., Thakur, G. A., White, F. A., Makriyannis, A., \& Hohmann, A. G. (2012). The maintenance of cisplatin- and paclitaxel-induced mechanical and cold allodynia is suppressed by cannabinoid $\mathrm{CB}(2)$ receptor activation and independent of CXCR4 signaling in models of chemotherapy-induced peripheral neuropathy. Molecular Pain, 8, 71. doi:10.1186/1744-8069-8-71

Deng, L., Lee, W. H., Xu, Z., Makriyannis, A., \& Hohmann, A. G. (2016). Prophylactic treatment with the tricyclic antidepressant desipramine prevents development of paclitaxel-induced neuropathic 
pain through activation of endogenous analgesic systems. Pharmacological Research, 114, 7589. doi:10.1016/j.phrs.2016.10.007

Dennis, E. A., \& Norris, P. C. (2015). Eicosanoid storm in infection and inflammation. Nature Reviews: Immunology, 15(8), 511-523. doi:10.1038/nri3859

DePaoli, A. M., Hurley, K. M., Yasada, K., Reisine, T., \& Bell, G. (1994). Distribution of kappa opioid receptor mRNA in adult mouse brain: an in situ hybridization histochemistry study. Molecular and Cellular Neuroscience, 5(4), 327-335. doi:10.1006/mcne.1994.1039

DeSanty, K. P., \& Dar, M. S. (2001). Cannabinoid-induced motor incoordination through the cerebellar CB1 receptor in mice. Pharmacology Biochemistry and Behavior, 69(1-2), 251-259. doi:10.1016/s0091-3057(01)00539-1

Deuis, J. R., \& Vetter, I. (2016). The thermal probe test: A novel behavioral assay to quantify thermal paw withdrawal thresholds in mice. Temperature (Austin), 3(2), 199-207. doi:10.1080/23328940.2016.1157668

Devane, W. A., Hanus, L., Breuer, A., Pertwee, R. G., Stevenson, L. A., Griffin, G., Gibson, D., Mandelbaum, A., Etinger, A., \& Mechoulam, R. (1992). Isolation and structure of a brain constituent that binds to the cannabinoid receptor. Science, 258(5090), 1946-1949. doi:10.1126/science.1470919

Devor, M. (1999). Unexplained peculiarities of the dorsal root ganglion. Pain, Suppl 6, S27-35. doi:10.1016/S0304-3959(99)00135-9

Dhaka, A., Viswanath, V., \& Patapoutian, A. (2006). TRP ion channels and temperature sensation. Annual Review of Neuroscience, 29, 135-161. doi:10.1146/annurev.neuro.29.051605.112958

Dhar, S., Seth, J., \& Parikh, D. (2014). Systemic side-effects of topical corticosteroids. Indian Journal of Dermatology, 59(5), 460-464. doi:10.4103/0019-5154.139874

Di Chiara, G., \& Imperato, A. (1988). Opposite effects of mu and kappa opiate agonists on dopamine release in the nucleus accumbens and in the dorsal caudate of freely moving rats. Journal of Pharmacology and Experimental Therapeutics, 244(3), 1067-1080.

Di Marzo, V., Bisogno, T., \& De Petrocellis, L. (2007). Endocannabinoids and related compounds: walking back and forth between plant natural products and animal physiology. Chemistry \& Biology, 14(7), 741-756. doi:10.1016/j.chembiol.2007.05.014

Dichi, I., Frenhane, P., Dichi, J. B., Correa, C. R., Angeleli, A. Y., Bicudo, M. H., Rodrigues, M. A., Victoria, C. R., \& Burini, R. C. (2000). Comparison of omega-3 fatty acids and sulfasalazine in ulcerative colitis. Nutrition, 16(2), 87-90. doi:10.1016/S0899-9007(99)00231-2

DiMattio, K. M., Ehlert, F. J., \& Liu-Chen, L. Y. (2015). Intrinsic relative activities of kappa opioid agonists in activating Galpha proteins and internalizing receptor: Differences between human and mouse receptors. European Journal of Pharmacology, 761, 235-244. doi:10.1016/j.ejphar.2015.05.054

Dogra, S., \& Yadav, P. N. (2015). Biased agonism at kappa opioid receptors: Implication in pain and mood disorders. European Journal of Pharmacology, 763(Pt B), 184-190. doi:10.1016/j.ejphar.2015.07.018

Dosaka-Akita, K., Tortella, F. C., Holaday, J. W., \& Long, J. B. (1993). The kappa opioid agonist U$50,488 \mathrm{H}$ antagonizes respiratory effects of mu opioid receptor agonists in conscious rats. Journal of Pharmacology and Experimental Therapeutics, 264(2), 631-637.

Dougherty, P. M., Cata, J. P., Cordella, J. V., Burton, A., \& Weng, H. R. (2004). Taxol-induced sensory disturbance is characterized by preferential impairment of myelinated fiber function in cancer patients. Pain, 109(1-2), 132-142. doi:10.1016/j.pain.2004.01.021

Dowell, D., Arias, E., Kochanek, K., Anderson, R., Guy, G. P., Jr., Losby, J. L., \& Baldwin, G. (2017). Contribution of opioid-involved poisoning to the change in life expectancy in the United States, 2000-2015. JAMA, 318(11), 1065-1067. doi:10.1001/jama.2017.9308

Doyle, T., Chen, Z., Muscoli, C., Bryant, L., Esposito, E., Cuzzocrea, S., Dagostino, C., Ryerse, J., Rausaria, S., Kamadulski, A., Neumann, W. L., \& Salvemini, D. (2012). Targeting the overproduction of 
peroxynitrite for the prevention and reversal of paclitaxel-induced neuropathic pain. Journal of Neuroscience, 32(18), 6149-6160. doi:10.1523/JNEUROSCI.6343-11.2012

Du, C., Duan, Y., Wei, W., Cai, Y., Chai, H., Lv, J., Du, X., Zhu, J., \& Xie, X. (2016). Kappa opioid receptor activation alleviates experimental autoimmune encephalomyelitis and promotes oligodendrocyte-mediated remyelination. Nature communications, 7, 11120. doi:10.1038/ncomms11120

Dubin, A. E., \& Patapoutian, A. (2010). Nociceptors: the sensors of the pain pathway. Journal of Clinical Investigation, 120(11), 3760-3772. doi:10.1172/JCl42843

Dubuisson, D., \& Dennis, S. G. (1977). The formalin test: a quantitative study of the analgesic effects of morphine, meperidine, and brain stem stimulation in rats and cats. Pain, 4(2), 161-174. doi:10.1016/0304-3959(77)90130-0

Duenas, M., Salazar, A., Ojeda, B., Fernandez-Palacin, F., Mico, J. A., Torres, L. M., \& Failde, I. (2015). A nationwide study of chronic pain prevalence in the general spanish population: identifying clinical subgroups through cluster analysis. Pain Medicine, 16(4), 811-822. doi:10.1111/pme.12640

Duggett, N. A., Griffiths, L. A., McKenna, O. E., de Santis, V., Yongsanguanchai, N., Mokori, E. B., \& Flatters, S. J. (2016). Oxidative stress in the development, maintenance and resolution of paclitaxel-induced painful neuropathy. Neuroscience, 333, 13-26. doi:10.1016/j.neuroscience.2016.06.050

Dullemeijer, C., Durga, J., Brouwer, I. A., van de Rest, O., Kok, F. J., Brummer, R. J., van Boxtel, M. P., \& Verhoef, P. (2007). n-3 fatty acid proportions in plasma and cognitive performance in older adults. American Journal of Clinical Nutrition, 86(5), 1479-1485. doi:10.1093/ajcn/86.5.1479

Durand, J. P., Deplanque, G., Montheil, V., Gornet, J. M., Scotte, F., Mir, O., Cessot, A., Coriat, R., Raymond, E., Mitry, E., Herait, P., Yataghene, Y., \& Goldwasser, F. (2012). Efficacy of venlafaxine for the prevention and relief of oxaliplatin-induced acute neurotoxicity: results of EFFOX, a randomized, double-blind, placebo-controlled phase III trial. Annals of Oncology, 23(1), 200-205. doi:10.1093/annonc/mdr045

Dykstra, L. A., Gmerek, D. E., Winger, G., \& Woods, J. H. (1987). Kappa opioids in rhesus monkeys. I. Diuresis, sedation, analgesia and discriminative stimulus effects. Journal of Pharmacology and Experimental Therapeutics, 242(2), 413-420.

Ebner, S. R., Roitman, M. F., Potter, D. N., Rachlin, A. B., \& Chartoff, E. H. (2010). Depressive-like effects of the kappa opioid receptor agonist salvinorin $A$ are associated with decreased phasic dopamine release in the nucleus accumbens. Psychopharmacology, 210(2), 241-252. doi:10.1007/s00213-010-1836-5

Echeverry, S., Shi, X. Q., Yang, M., Huang, H., Wu, Y., Lorenzo, L. E., Perez-Sanchez, J., Bonin, R. P., De Koninck, Y., \& Zhang, J. (2017). Spinal microglia are required for long-term maintenance of neuropathic pain. Pain, 158(9), 1792-1801. doi:10.1097/j.pain.0000000000000982

Eddy, N. B., \& Leimbach, D. (1953). Synthetic analgesics. II. Dithienylbutenyl-and dithienylbutylamines. Journal of Pharmacology and Experimental Therapeutics, 107(3), 385-393.

Edwards, K. A., Havelin, J. J., McIntosh, M. I., Ciccone, H. A., Pangilinan, K., Imbert, I., Largent-Milnes, T. M., King, T., Vanderah, T. W., \& Streicher, J. M. (2018). A kappa opioid receptor agonist blocks bone cancer pain without altering bone loss, tumor size, or cancer cell proliferation in a mouse model of cancer-induced bone pain. Journal of Pain. doi:10.1016/j.jpain.2018.01.002

Ehrich, J. M., Messinger, D. I., Knakal, C. R., Kuhar, J. R., Schattauer, S. S., Bruchas, M. R., Zweifel, L. S., Kieffer, B. L., Phillips, P. E., \& Chavkin, C. (2015). Kappa opioid receptor-induced aversion requires p38 MAPK activation in VTA dopamine neurons. Journal of Neuroscience, 35(37), 12917-12931. doi:10.1523/JNEUROSCI.2444-15.2015

Emken, E. A., Adlof, R. O., Rakoff, H., Rohwedder, W. K., \& Gulley, R. M. (1990). Metabolism in vivo of deuterium-labelled linolenic and linoleic acids in humans. Biochemical Society Transactions, 18(5), 766-769. doi:10.1042/bst0180766 
Emmerson, P. J., Liu, M. R., Woods, J. H., \& Medzihradsky, F. (1994). Binding-affinity and selectivity of opioids at mu-receptor, delta-receptor and kappa-receptor in monkey brain membranes. Journal of Pharmacology and Experimental Therapeutics, 271(3), 1630-1637.

Endoh, T., Matsuura, H., Tajima, A., Izumimoto, N., Tajima, C., Suzuki, T., Saitoh, A., Suzuki, T., Narita, M., Tseng, L., \& Nagase, H. (1999). Potent antinociceptive effects of TRK-820, a novel kappaopioid receptor agonist. Life Sciences, 65(16), 1685-1694. doi:10.1016/S0024-3205(99)004178

Endoh, T., Matsuura, H., Tanaka, C., \& Nagase, H. (1992). Nor-binaltorphimine: a potent and selective kappa-opioid receptor antagonist with long-lasting activity in vivo. Archives Internationales de Pharmacodynamie et Therapie, 316, 30-42.

Endoh, T., Tajima, A., Suzuki, T., Kamei, J., Narita, M., Tseng, L., \& Nagase, H. (2000). Characterization of the antinociceptive effects of TRK-820 in the rat. European Journal of Pharmacology, 387(2), 133-140. doi:10.1016/S0014-2999(99)00815-8

Engstrom Ruud, L., Wilhelms, D. B., Eskilsson, A., Vasilache, A. M., Elander, L., Engblom, D., \& Blomqvist, A. (2013). Acetaminophen reduces lipopolysaccharide-induced fever by inhibiting cyclooxygenase-2. Neuropharmacology, 71, 124-129. doi:10.1016/j.neuropharm.2013.03.012

Ewald, A. W. M. (2015). Investigating the anti-cocaine effects of novel kappa opioid receptor agonists: Behavioural and cellular actions of Salvinorin A analogues. Doctor of Philosophy, Victoria University of Wellington.

Ewald, A. W. M., Bosch, P. J., Culverhouse, A., Crowley, R. S., Neuenswander, B., Prisinzano, T. E., \& Kivell, B. M. (2017). The C-2 derivatives of salvinorin A, ethoxymethyl ether Sal B and betatetrahydropyran Sal B, have anti-cocaine properties with minimal side effects. Psychopharmacology, 234(16), 2499-2514. doi:10.1007/s00213-017-4637-2

Ewertz, M., Qvortrup, C., \& Eckhoff, L. (2015). Chemotherapy-induced peripheral neuropathy in patients treated with taxanes and platinum derivatives. Acta Oncologica, 54(5), 587-591. doi:10.3109/0284186X.2014.995775

Fallon, M. T., Wilcock, A., Kelly, C. A., Paul, J., Lewsley, L. A., Norrie, J., \& Laird, B. J. A. (2018). Oral ketamine vs placebo in patients with cancer-related neuropathic pain: A randomized clinical trial. JAMA Oncol. doi:10.1001/jamaoncol.2018.0131

Fantegrossi, W. E., Kugle, K. M., Valdes, L. J., 3rd, Koreeda, M., \& Woods, J. H. (2005). Kappa-opioid receptor-mediated effects of the plant-derived hallucinogen, salvinorin $A$, on inverted screen performance in the mouse. Behavioural Pharmacology, 16(8), 627-633.

Fayaz, A., Croft, P., Langford, R. M., Donaldson, L. J., \& Jones, G. T. (2016). Prevalence of chronic pain in the UK: a systematic review and meta-analysis of population studies. BMJ Open, 6(6), e010364. doi:10.1136/bmjopen-2015-010364

Fehrenbacher, J. C., Vasko, M. R., \& Duarte, D. B. (2001). Models of inflammation: Carrageenan- or complete Freund's adjuvant (CFA)-induced edema and hypersensitivity in the rat. Current Protocols in Pharmacology: John Wiley \& Sons, Inc.

Felder, C. C., Briley, E. M., Axelrod, J., Simpson, J. T., Mackie, K., \& Devane, W. A. (1993). Anandamide, an endogenous cannabimimetic eicosanoid, binds to the cloned human cannabinoid receptor and stimulates receptor-mediated signal transduction. Proceedings of the National Academy of Sciences of the United States of America, 90(16), 7656-7660. doi:10.1073/pnas.90.16.7656

Felder, C. C., Nielsen, A., Briley, E. M., Palkovits, M., Priller, J., Axelrod, J., Nguyen, D. N., Richardson, J. M., Riggin, R. M., Koppel, G. A., Paul, S. M., \& Becker, G. W. (1996). Isolation and measurement of the endogenous cannabinoid receptor agonist, anandamide, in brain and peripheral tissues of human and rat. FEBS Letters, 393(2-3), 231-235. doi:10.1016/00145793(96)00891-5

Fellner, S., Bauer, B., Miller, D. S., Schaffrik, M., Fankhanel, M., Spruss, T., Bernhardt, G., Graeff, C., Farber, L., Gschaidmeier, H., Buschauer, A., \& Fricker, G. (2002). Transport of paclitaxel (Taxol) across the blood-brain barrier in vitro and in vivo. Journal of Clinical Investigation, 110(9), 1309-1318. doi:10.1172/JCI15451 
Ferreira, S. H. (1972). Prostaglandins, aspirin-like drugs and analgesia. Nature: New Biology, 240(102), 200-203. doi:10.1038/10.1038/newbio240200a0

Ferreira, S. H., Moncada, S., \& Vane, J. R. (1997). Prostaglandins and the mechanism of analgesia produced by aspirin-like drugs. 1973. British Journal of Pharmacology, 120(4 Suppl), 401-412; discussion 399-400. doi:10.1111/j.1476-5381.1997.tb06823.x

Fichna, J., Dicay, M., Lewellyn, K., Janecka, A., Zjawiony, J. K., MacNaughton, W. K., \& Storr, M. A. (2012). Salvinorin A has antiinflammatory and antinociceptive effects in experimental models of colitis in mice mediated by KOR and CB1 receptors. Inflammatory Bowel Diseases, 18(6), 1137-1145. doi:10.1002/ibd.21873

Fields, H. L., Malick, A., \& Burstein, R. (1995). Dorsal horn projection targets of ON and OFF cells in the rostral ventromedial medulla. Journal of Neurophysiology, 74(4), 1742-1759. doi:10.1152/jn.1995.74.4.1742

Fields, H. L., Vanegas, H., Hentall, I. D., \& Zorman, G. (1983). Evidence that disinhibition of brain stem neurones contributes to morphine analgesia. Nature, 306(5944), 684-686. doi:10.1038/306684a0

Fillingim, R. B., King, C. D., Ribeiro-Dasilva, M. C., Rahim-Williams, B., \& Riley, J. L., 3rd. (2009). Sex, gender, and pain: a review of recent clinical and experimental findings. Journal of Pain, 10(5), 447-485. doi:10.1016/j.jpain.2008.12.001

Finan, P. H., Goodin, B. R., \& Smith, M. T. (2013). The association of sleep and pain: an update and a path forward. Journal of Pain, 14(12), 1539-1552. doi:10.1016/j.jpain.2013.08.007

Finnerup, N. B., Attal, N., Haroutounian, S., McNicol, E., Baron, R., Dworkin, R. H., Gilron, I., Haanpaa, M., Hansson, P., Jensen, T. S., Kamerman, P. R., Lund, K., Moore, A., Raja, S. N., Rice, A. S., Rowbotham, M., Sena, E., Siddall, P., Smith, B. H., \& Wallace, M. (2015). Pharmacotherapy for neuropathic pain in adults: a systematic review and meta-analysis. Lancet Neurology, 14(2), 162-173. doi:10.1016/S1474-4422(14)70251-0

Fitzcharles, M. A., Baerwald, C., Ablin, J., \& Hauser, W. (2016). Efficacy, tolerability and safety of cannabinoids in chronic pain associated with rheumatic diseases (fibromyalgia syndrome, back pain, osteoarthritis, rheumatoid arthritis): A systematic review of randomized controlled trials. Schmerz, 30(1), 47-61. doi:10.1007/s00482-015-0084-3

Flatters, S. J., \& Bennett, G. J. (2004). Ethosuximide reverses paclitaxel- and vincristine-induced painful peripheral neuropathy. Pain, 109(1-2), 150-161. doi:10.1016/j.pain.2004.01.029

Flatters, S. J., \& Bennett, G. J. (2006). Studies of peripheral sensory nerves in paclitaxel-induced painful peripheral neuropathy: evidence for mitochondrial dysfunction. Pain, 122(3), 245-257. doi:10.1016/j.pain.2006.01.037

Flatters, S. J., Dougherty, P. M., \& Colvin, L. A. (2017). Clinical and preclinical perspectives on Chemotherapy-Induced Peripheral Neuropathy (CIPN): a narrative review. British Journal of Anaesthesia, 119(4), 737-749. doi:10.1093/bja/aex229

Flatters, S. J., Xiao, W. H., \& Bennett, G. J. (2006). Acetyl-L-carnitine prevents and reduces paclitaxelinduced painful peripheral neuropathy. Neuroscience Letters, 397(3), 219-223. doi:10.1016/j.neulet.2005.12.013

Flower, R. J., \& Vane, J. R. (1972). Inhibition of prostaglandin synthetase in brain explains the antipyretic activity of paracetamol (4-acetamidophenol). Nature, 240(5381), 410-411. doi:10.1038/240410a0

Forman, A. (1990). Peripheral neuropathy in cancer patients: clinical types, etiology, and presentation. Part 2. Oncology (Williston Park, N.Y.), 4(2), 85-89.

Fortin, P. R., Lew, R. A., Liang, M. H., Wright, E. A., Beckett, L. A., Chalmers, T. C., \& Sperling, R. I. (1995). Validation of a meta-analysis: the effects of fish oil in rheumatoid arthritis. Journal of Clinical Epidemiology, 48(11), 1379-1390. doi:10.1016/0895-4356(95)00028-3

France, C. P., Medzihradsky, F., \& Woods, J. H. (1994). Comparison of kappa opioids in rhesus monkeys: behavioral effects and receptor binding affinities. Journal of Pharmacology and Experimental Therapeutics, 268(1), 47-58. 
Freeman, K. B., Naylor, J. E., Prisinzano, T. E., \& Woolverton, W. L. (2014). Assessment of the kappa opioid agonist, salvinorin $A$, as a punisher of drug self-administration in monkeys. Psychopharmacology, 231(14), 2751-2758. doi:10.1007/s00213-014-3436-2

Freye, E., Hartung, E., \& Schenk, G. K. (1983). Bremazocine - an opiate that induces sedation and analgesia without respiratory depression. Anesthesia and Analgesia, 62(5), 483-488.

Fride, E., \& Mechoulam, R. (1993). Pharmacological activity of the cannabinoid receptor agonist, anandamide, a brain constituent. European Journal of Pharmacology, 231(2), 313-314. doi:10.1016/0014-2999(93)90468-W

Gallantine, E. L., \& Meert, T. F. (2008). Antinociceptive and adverse effects of mu- and kappa-opioid receptor agonists: a comparison of morphine and U50488-H. Basic \& Clinical Pharmacology \& Toxicology, 103(5), 419-427. doi:10.1111/j.1742-7843.2008.00306.x

Gaoni, Y., \& Mechoulam, R. (1964). Isolation, structure, and partial synthesis of an active constituent of hashish. Journal of the American Chemical Society, 86(8), 1646-1647. doi:10.1021/ja01062a046

Gaskin, D. J., \& Richard, P. (2012). The economic costs of pain in the United States. Journal of Pain, 13(8), 715-724. doi:10.1016/j.jpain.2012.03.009

Gasser, H. S. (1941). The classification of nerve fibers. The Ohio Journal of Science, 41(3), 145-159.

Gasser, H. S., \& Erlanger, J. (1927). The role played by the sizes of the consituent fibers of a nerve trunk in determining the form of its action potential wave. American Journal of Physiology - Legacy Content, 80(3), 522. doi:10.1152/ajplegacy.1927.80.3.522

Gaumond, I., Arsenault, P., \& Marchand, S. (2002). The role of sex hormones on formalin-induced nociceptive responses. Brain Research, 958(1), 139-145. doi:10.1016/S0006-8993(02)036612

Gavhane, Y. N., \& Yadav, A. V. (2012). Loss of orally administered drugs in GI tract. Saudi Pharmaceutical Journal, 20(4), 331-344. doi:10.1016/j.jsps.2012.03.005

GBD Mortality and Causes of Death Collaborators. (2016). Global, regional, and national life expectancy, all-cause mortality, and cause-specific mortality for 249 causes of death, 19802015: a systematic analysis for the Global Burden of Disease Study 2015. Lancet, 388(10053), 1459-1544. doi:10.1016/S0140-6736(16)31012-1

Gee, N. S., Brown, J. P., Dissanayake, V. U., Offord, J., Thurlow, R., \& Woodruff, G. N. (1996). The novel anticonvulsant drug, gabapentin (Neurontin), binds to the alpha2delta subunit of a calcium channel. Journal of Biological Chemistry, 271(10), 5768-5776. doi:10.1074/jbc.271.10.5768

Geffeney, S. L., \& Goodman, M. B. (2012). How we feel: ion channel partnerships that detect mechanical inputs and give rise to touch and pain perception. Neuron, 74(4), 609-619. doi:10.1016/j.neuron.2012.04.023

Gehrke, B. J., Chefer, V. I., \& Shippenberg, T. S. (2008). Effects of acute and repeated administration of salvinorin A on dopamine function in the rat dorsal striatum. Psychopharmacology, 197(3), 509-517. doi:10.1007/s00213-007-1067-6

Gemes, G., Koopmeiners, A., Rigaud, M., Lirk, P., Sapunar, D., Bangaru, M. L., Vilceanu, D., Garrison, S. R., Ljubkovic, M., Mueller, S. J., Stucky, C. L., \& Hogan, Q. H. (2013). Failure of action potential propagation in sensory neurons: mechanisms and loss of afferent filtering in C-type units after painful nerve injury. Journal of Physiology, 591(4), 1111-1131. doi:10.1113/jphysiol.2012.242750

Gentry, C., Stoakley, N., Andersson, D. A., \& Bevan, S. (2010). The roles of iPLA2, TRPM8 and TRPA1 in chemically induced cold hypersensitivity. Molecular Pain, 6, 4. doi:10.1186/1744-8069-6-4

Gewandter, J. S., Mohile, S. G., Heckler, C. E., Ryan, J. L., Kirshner, J. J., Flynn, P. J., Hopkins, J. O., \& Morrow, G. R. (2014). A phase III randomized, placebo-controlled study of topical amitriptyline and ketamine for chemotherapy-induced peripheral neuropathy (CIPN): a University of Rochester CCOP study of 462 cancer survivors. Supportive Care in Cancer, 22(7), 1807-1814. doi:10.1007/s00520-014-2158-7 
Giffin, N. (2016). Epidemiology of cluster headache in a DGH Headache Clinic. Journal of Neurology Neurosurgery and Psychiatry, 87(12), e1-e1. doi:10.1136/jnnp-2016-315106.69

Glass, M., Dragunow, M., \& Faull, R. L. (1997). Cannabinoid receptors in the human brain: a detailed anatomical and quantitative autoradiographic study in the fetal, neonatal and adult human brain. Neuroscience, 77(2), 299-318. doi:10.1016/S0306-4522(96)00428-9

Glick, S. D., Maisonneuve, I. M., Raucci, J., \& Archer, S. (1995). Kappa opioid inhibition of morphine and cocaine self-administration in rats. Brain Research, 681(1-2), 147-152. doi:10.1016/00068993(95)00306-B

Goldstein, A., Tachibana, S., Lowney, L. I., Hunkapiller, M., \& Hood, L. (1979). Dynorphin-(1-13), an extraordinarily potent opioid peptide. Proceedings of the National Academy of Sciences of the United States of America, 76(12), 6666-6670. doi:10.1073/pnas.76.12.6666

Gordon, C. J., Aydin, C., Repasky, E. A., Kokolus, K. M., Dheyongera, G., \& Johnstone, A. F. (2014). Behaviorally mediated, warm adaptation: a physiological strategy when mice behaviorally thermoregulate. Journal of Thermal Biology, 44, 41-46. doi:10.1016/j.jtherbio.2014.06.006

Gornstein, E. L., \& Schwarz, T. L. (2014). The paradox of paclitaxel neurotoxicity: Mechanisms and unanswered questions. Neuropharmacology, 76 Pt A, 175-183. doi:10.1016/j.neuropharm.2013.08.016

Gornstein, E. L., \& Schwarz, T. L. (2017). Neurotoxic mechanisms of paclitaxel are local to the distal axon and independent of transport defects. Experimental Neurology, 288, 153-166. doi:10.1016/j.expneurol.2016.11.015

Grace, P. M., Strand, K. A., Galer, E. L., Urban, D. J., Wang, X., Baratta, M. V., Fabisiak, T. J., Anderson, N. D., Cheng, K., Greene, L. I., Berkelhammer, D., Zhang, Y., Ellis, A. L., Yin, H. H., Campeau, S., Rice, K. C., Roth, B. L., Maier, S. F., \& Watkins, L. R. (2016). Morphine paradoxically prolongs neuropathic pain in rats by amplifying spinal NLRP3 inflammasome activation. Proceedings of the National Academy of Sciences of the United States of America, 113(24), E3441-3450. doi:10.1073/pnas.1602070113

Grenald, S. A., Young, M. A., Wang, Y., Ossipov, M. H., Ibrahim, M. M., Largent-Milnes, T. M., \& Vanderah, T. W. (2017). Synergistic attenuation of chronic pain using mu opioid and cannabinoid receptor 2 agonists. Neuropharmacology, 116(Supplement C), 59-70. doi:10.1016/j.neuropharm.2016.12.008

Grosso, G., Pajak, A., Marventano, S., Castellano, S., Galvano, F., Bucolo, C., Drago, F., \& Caraci, F. (2014). Role of omega-3 fatty acids in the treatment of depressive disorders: a comprehensive meta-analysis of randomized clinical trials. Plos One, 9(5), e96905. doi:10.1371/journal.pone.0096905

Grudt, T. J., \& Williams, J. T. (1993). Kappa-opioid receptors also increase potassium conductance. Proceedings of the National Academy of Sciences of the United States of America, 90(23), 11429-11432. doi:10.1073/pnas.90.23.11429

Guerrero, A. T. G., Verri, W. A., Cunha, T. M., Silva, T. A., Schivo, I. R. S., Dal-Secco, D., Canetti, C., Rocha, F. A. C., Parada, C. A., Cunha, F. Q., \& Ferreira, S. H. (2008). Involvement of LTB4 in zymosan-induced joint nociception in mice: participation of neutrophils and PGE2. Journal of Leukocyte Biology, 83(1), 122-130. doi:10.1189/jlb.0207123

Guida, F., Luongo, L., Aviello, G., Palazzo, E., De Chiaro, M., Gatta, L., Boccella, S., Marabese, I., Zjawiony, J. K., Capasso, R., Izzo, A. A., de Novellis, V., \& Maione, S. (2012). Salvinorin A reduces mechanical allodynia and spinal neuronal hyperexcitability induced by peripheral formalin injection. Mol Pain, 8(1), 60. doi:10.1186/1744-8069-8-60

Guindon, J., De Lean, A., \& Beaulieu, P. (2006). Local interactions between anandamide, an endocannabinoid, and ibuprofen, a nonsteroidal anti-inflammatory drug, in acute and inflammatory pain. Pain, 121(1-2), 85-93. doi:10.1016/j.pain.2005.12.007

Gupta, A., Gomes, I., Bobeck, E. N., Fakira, A. K., Massaro, N. P., Sharma, I., Cave, A., Hamm, H. E., Parello, J., \& Devi, L. A. (2016). Collybolide is a novel biased agonist of kappa-opioid receptors 
with potent antipruritic activity. Proceedings of the National Academy of Sciences of the United States of America, 113(21), 6041-6046. doi:10.1073/pnas.1521825113

Hammack, J. E., Michalak, J. C., Loprinzi, C. L., Sloan, J. A., Novotny, P. J., Soori, G. S., Tirona, M. T., Rowland, K. M., Jr., Stella, P. J., \& Johnson, J. A. (2002). Phase III evaluation of nortriptyline for alleviation of symptoms of cis-platinum-induced peripheral neuropathy. Pain, 98(1-2), 195203. doi:10.1016/S0304-3959(02)00047-7

Han, J. S., \& Xie, C. W. (1982). Dynorphin: potent analgesic effect in spinal cord of the rat. Life Sciences, 31(16-17), 1781-1784.

Hansen, H. S. (1986). The essential nature of linoleic-acid in mammals. Trends in Biochemical Sciences, 11(6), 263-265. doi:10.1016/0968-0004(86)90191-X

Hansen, H. S., \& Artmann, A. (2008). Endocannabinoids and nutrition. Journal of Neuroendocrinology, 20 Suppl 1, 94-99. doi:10.1111/j.1365-2826.2008.01687.x

Hansen, N., Uceyler, N., Palm, F., Zelenka, M., Biko, L., Lesch, K. P., Gerlach, M., \& Sommer, C. (2011). Serotonin transporter deficiency protects mice from mechanical allodynia and heat hyperalgesia in vincristine neuropathy. Neuroscience Letters, 495(2), 93-97. doi:10.1016/j.neulet.2011.03.035

Hara, T., Chiba, T., Abe, K., Makabe, A., Ikeno, S., Kawakami, K., Utsunomiya, I., Hama, T., \& Taguchi, K. (2013). Effect of paclitaxel on transient receptor potential vanilloid 1 in rat dorsal root ganglion. Pain, 154(6), 882-889. doi:10.1016/j.pain.2013.02.023

Harding, W. W., Tidgewell, K., Byrd, N., Cobb, H., Dersch, C. M., Butelman, E. R., Rothman, R. B., \& Prisinzano, T. E. (2005). Neoclerodane diterpenes as a novel scaffold for mu opioid receptor ligands. Journal of Medicinal Chemistry, 48(15), 4765-4771. doi:10.1021/jm048963m

Hargreaves, K., Dubner, R., Brown, F., Flores, C., \& Joris, J. (1988). A new and sensitive method for measuring thermal nociception in cutaneous hyperalgesia. Pain, 32(1), 77-88. doi:10.1016/0304-3959(88)90026-7

Heinricher, M. M., Barbaro, N. M., \& Fields, H. L. (1989). Putative nociceptive modulating neurons in the rostral ventromedial medulla of the rat - Firing of on-cells and off-cells is related to nociceptive responsiveness. Somatosensory and Motor Research, 6(4), 427-439. doi:10.3109/08990228909144685

Henderson, J. V., Harrison, C. M., Britt, H. C., Bayram, C. F., \& Miller, G. C. (2013). Prevalence, causes, severity, impact, and management of chronic pain in Australian general practice patients. Pain Medicine, 14(9), 1346-1361. doi:10.1111/pme.12195

Hershman, D. L., Lacchetti, C., Dworkin, R. H., Lavoie Smith, E. M., Bleeker, J., Cavaletti, G., Chauhan, C., Gavin, P., Lavino, A., Lustberg, M. B., Paice, J., Schneider, B., Smith, M. L., Smith, T., Terstriep, S., Wagner-Johnston, N., Bak, K., Loprinzi, C. L., \& American Society of Clinical Oncology. (2014). Prevention and management of chemotherapy-induced peripheral neuropathy in survivors of adult cancers: American Society of Clinical Oncology clinical practice guideline. Journal of Clinical Oncology, 32(18), 1941-1967. doi:10.1200/JCO.2013.54.0914

Ho, W. S., Barrett, D. A., \& Randall, M. D. (2008). 'Entourage' effects of N-palmitoylethanolamide and $\mathrm{N}$-oleoylethanolamide on vasorelaxation to anandamide occur through TRPV1 receptors. British Journal of Pharmacology, 155(6), 837-846. doi:10.1038/bjp.2008.324

Hoareau, L., Buyse, M., Festy, F., Ravanan, P., Gonthier, M. P., Matias, I., Petrosino, S., Tallet, F., d'Hellencourt, C. L., Cesari, M., Di Marzo, V., \& Roche, R. (2009). Anti-inflammatory effect of palmitoylethanolamide on human adipocytes. Obesity (Silver Spring), 17(3), 431-438. doi:10.1038/oby.2008.591

Hoeijmakers, J. G., Faber, C. G., Merkies, I. S., \& Waxman, S. G. (2015). Painful peripheral neuropathy and sodium channel mutations. Neuroscience Letters, 596, 51-59. doi:10.1016/j.neulet.2014.12.056

Hojo, M., Sudo, Y., Ando, Y., Minami, K., Takada, M., Matsubara, T., Kanaide, M., Taniyama, K., Sumikawa, K., \& Uezono, Y. (2008). mu-Opioid receptor forms a functional heterodimer with 
cannabinoid CB1 receptor: electrophysiological and FRET assay analysis. Journal of Pharmacological Sciences, 108(3), 308-319. doi:10.1254/jphs.08244FP

Hoke, A. (2012). Animal models of peripheral neuropathies. Neurotherapeutics, 9(2), 262-269. doi:10.1007/s13311-012-0116-y

Hole, K., \& Tjolsen, A. (1993). The tail-flick and formalin tests in rodents: changes in skin temperature as a confounding factor. Pain, 53(3), 247-254. doi:10.1016/0304-3959(93)90220-J

Holmes, F. A., Walters, R. S., Theriault, R. L., Forman, A. D., Newton, L. K., Raber, M. N., Buzdar, A. U., Frye, D. K., \& Hortobagyi, G. N. (1991). Phase II trial of taxol, an active drug in the treatment of metastatic breast cancer. Journal of the National Cancer Institute, 83(24), 1797-1805. doi:10.1093/jnci/83.24.1797

Hooker, J. M., Munro, T. A., Beguin, C., Alexoff, D., Shea, C., Xu, Y., \& Cohen, B. M. (2009). Salvinorin A and derivatives: protection from metabolism does not prolong short-term, whole-brain residence. Neuropharmacology, 57(4), 386-391. doi:10.1016/j.neuropharm.2009.06.044

Hoover, V., Marlowe, D. B., Patapis, N. S., Festinger, D. S., \& Forman, R. F. (2008). Internet access to Salvia divinorum: implications for policy, prevention, and treatment. Journal of Substance Abuse Treatment, 35(1), 22-27. doi:10.1016/j.jsat.2007.07.011

Hu, H. J., Bhave, G., \& Gereau, R. W. t. (2002). Prostaglandin and protein kinase A-dependent modulation of vanilloid receptor function by metabotropic glutamate receptor 5: potential mechanism for thermal hyperalgesia. Journal of Neuroscience, 22(17), 7444-7452. doi:10.1523/JNEUROSCI.22-17-07444.2002

Huma, Z., Ireland, K., \& Maxwell, D. J. (2015). The spino-bulbar-cerebellar pathway: Activation of neurons projecting to the lateral reticular nucleus in the rat in response to noxious mechanical stimuli. Neuroscience Letters, 591, 197-201. doi:10.1016/j.neulet.2015.02.047

Hunskaar, S., Berge, O. G., \& Hole, K. (1986). A modified hot-plate test sensitive to mild analgesics. Behavioural Brain Research, 21(2), 101-108. doi:10.1016/0166-4328(86)90088-4

Hunskaar, S., Fasmer, O. B., \& Hole, K. (1985). Formalin test in mice, a useful technique for evaluating mild analgesics. Journal of Neuroscience Methods, 14(1), 69-76. doi:10.1016/01650270(85)90116-5

Hunskaar, S., \& Hole, K. (1987). The formalin test in mice: dissociation between inflammatory and noninflammatory pain. Pain, 30(1), 103-114. doi:10.1016/0304-3959(87)90088-1

Hutton, F., Kivell, B., \& Boyle, O. (2016). "Quite a profoundly strange experience": An analysis of the experiences of Salvia divinorum users. Journal of Psychoactive Drugs, 48(3), 206-213. doi:10.1080/02791072.2016.1179376

Hwang, B. Y., Kim, E. S., Kim, C. H., Kwon, J. Y., \& Kim, H. K. (2012). Gender differences in paclitaxelinduced neuropathic pain behavior and analgesic response in rats. Korean Journal of Anesthesiology, 62(1), 66-72. doi:10.4097/kjae.2012.62.1.66

ladarola, M. J., Brady, L. S., Draisci, G., \& Dubner, R. (1988). Enhancement of dynorphin gene expression in spinal cord following experimental inflammation: stimulus specificity, behavioral parameters and opioid receptor binding. Pain, 35(3), 313-326. doi:10.1016/03043959(88)90141-8

IASP Task Force on Taxonomy. (1994). PART III pain terms, a current list with definitions and notes on usage: Classification of chronic pain-descriptions of chronic pain syndromes and definitions of pain terms. Seattle: IASP Press.

Imai, S., Koyanagi, M., Azimi, Z., Nakazato, Y., Matsumoto, M., Ogihara, T., Yonezawa, A., Omura, T., Nakagawa, S., Wakatsuki, S., Araki, T., Kaneko, S., Nakagawa, T., \& Matsubara, K. (2017). Taxanes and platinum derivatives impair Schwann cells via distinct mechanisms. Scientific Reports, 7(1), 5947. doi:10.1038/s41598-017-05784-1

Inan, S., \& Cowan, A. (2004). Kappa opioid agonists suppress chloroquine-induced scratching in mice. European Journal of Pharmacology, 502(3), 233-237. doi:10.1016/j.ejphar.2004.09.010

Irwin, S., Houde, R. W., Bennett, D. R., Hendershot, L. C., \& Seevers, M. H. (1951). The effects of morphine, methadone and meperidine on some reflex responses of spinal animals to 
nociceptive stimulation. Journal of Pharmacology and Experimental Therapeutics, 101(2), 132143.

Ismayilova, N., \& Shoaib, M. (2010). Alteration of intravenous nicotine self-administration by opioid receptor agonist and antagonists in rats. Psychopharmacology, 210(2), 211-220. doi:10.1007/s00213-010-1845-4

Itoi, K., Horiba, N., Tozawa, F., Sakai, Y., Sakai, K., Abe, K., Demura, H., \& Suda, T. (1996). Major role of $3^{\prime}, 5^{\prime}$-cyclic adenosine monophosphate-dependent protein kinase A pathway in corticotropinreleasing factor gene expression in the rat hypothalamus in vivo. Endocrinology, 137(6), 23892396. doi:10.1210/endo.137.6.8641191

Jaggar, S. I., Hasnie, F. S., Sellaturay, S., \& Rice, A. S. (1998). The anti-hyperalgesic actions of the cannabinoid anandamide and the putative CB2 receptor agonist palmitoylethanolamide in visceral and somatic inflammatory pain. Pain, 76(1-2), 189-199. doi:10.1016/S03043959(98)00041-4

Jaggi, A. S., Jain, V., \& Singh, N. (2011). Animal models of neuropathic pain. Fundamental and Clinical Pharmacology, 25(1), 1-28. doi:10.1111/j.1472-8206.2009.00801.x

Janes, K., Esposito, E., Doyle, T., Cuzzocrea, S., Tosh, D. K., Jacobson, K. A., \& Salvemini, D. (2014). A3 adenosine receptor agonist prevents the development of paclitaxel-induced neuropathic pain by modulating spinal glial-restricted redox-dependent signaling pathways. Pain, 155(12), 2560-2567. doi:10.1016/j.pain.2014.09.016

Jardin, I., Lopez, J. J., Diez, R., Sanchez-Collado, J., Cantonero, C., Albarran, L., Woodard, G. E., Redondo, P. C., Salido, G. M., Smani, T., \& Rosado, J. A. (2017). TRPs in pain sensation. Frontiers in Physiology, 8, 392. doi:10.3389/fphys.2017.00392

Ji, R. R., Xu, Z. Z., \& Gao, Y. J. (2014). Emerging targets in neuroinflammation-driven chronic pain. Nature Reviews: Drug Discovery, 13(7), 533-548. doi:10.1038/nrd4334

Jia, M., Wu, C., Gao, F., Xiang, H., Sun, N., Peng, P., Li, J., Yuan, X., Li, H., Meng, X., Tian, B., Shi, J., \& Li, M. (2017). Activation of NLRP3 inflammasome in peripheral nerve contributes to paclitaxelinduced neuropathic pain. Molecular Pain, 13, 1744806917719804. doi:10.1177/1744806917719804

Jin, H. W., Flatters, S. J., Xiao, W. H., Mulhern, H. L., \& Bennett, G. J. (2008). Prevention of paclitaxelevoked painful peripheral neuropathy by acetyl-L-carnitine: effects on axonal mitochondria, sensory nerve fiber terminal arbors, and cutaneous Langerhans cells. Experimental Neurology, 210(1), 229-237. doi:10.1016/j.expneurol.2007.11.001

Johansen, J. P., Fields, H. L., \& Manning, B. H. (2001). The affective component of pain in rodents: direct evidence for a contribution of the anterior cingulate cortex. Proceedings of the National Academy of Sciences of the United States of America, 98(14), 8077-8082. doi:10.1073/pnas.141218998

John, S., Luben, R., Shrestha, S. S., Welch, A., Khaw, K. T., \& Hart, A. R. (2010). Dietary n-3 polyunsaturated fatty acids and the aetiology of ulcerative colitis: a UK prospective cohort study. European Journal of Gastroenterology and Hepatology, 22(5), 602-606. doi:10.1097/MEG.0b013e3283352d05

John, T. F., French, L. G., \& Erlichman, J. S. (2006). The antinociceptive effect of salvinorin A in mice. European Journal of Pharmacology, 545(2-3), 129-133. doi:10.1016/j.ejphar.2006.06.077

Jones, C. M., Einstein, E. B., \& Compton, W. M. (2018). Changes in synthetic opioid involvement in drug overdose deaths in the United States, 2010-2016. JAMA, 319(17), 1819-1821. doi:10.1001/jama.2018.2844

Jordt, S. E., Bautista, D. M., Chuang, H. H., McKemy, D. D., Zygmunt, P. M., Hogestatt, E. D., Meng, I. D., \& Julius, D. (2004). Mustard oils and cannabinoids excite sensory nerve fibres through the TRP channel ANKTM1. Nature, 427(6971), 260-265. doi:10.1038/nature02282

Kajihara, Y., Murakami, M., Imagawa, T., Otsuguro, K., Ito, S., \& Ohta, T. (2010). Histamine potentiates acid-induced responses mediating transient receptor potential V1 in mouse primary sensory neurons. Neuroscience, 166(1), 292-304. doi:10.1016/j.neuroscience.2009.12.001 
Kalliomaki, J., Annas, P., Huizar, K., Clarke, C., Zettergren, A., Karlsten, R., \& Segerdahl, M. (2013). Evaluation of the analgesic efficacy and psychoactive effects of AZD1940, a novel peripherally acting cannabinoid agonist, in human capsaicin-induced pain and hyperalgesia. Clinical and Experimental Pharmacology and Physiology, 40(3), 212-218. doi:10.1111/1440-1681.12051

Kalynovska, N., Adamek, P., \& Palecek, J. (2017). TRPV1 receptors contribute to mediate paclitaxelinduced c-Fos expression in spinal cord dorsal horn neurons. Physiological Research, 66(3), 549-552.

Kandel, E. R., Schwartz, J. H., Jessell, T. M., Siegelbaum, S. A., \& Hudspeth, A. J. (2000). Principles of neural science (Vol. 4): McGraw-hill New York.

Karli, N., Akis, N., Zarifoglu, M., Akgoz, S., Irgil, E., Ayvacioglu, U., Calisir, N., Haran, N., \& Akdogan, O. (2006). Headache prevalence in adolescents aged 12 to 17: a student-based epidemiological study in Bursa. Headache, 46(4), 649-655. doi:10.1111/j.1526-4610.2006.00362.x

Kash, T., \& Li, C. (2018). Kappa opioid receptor modulation on GABAergic inputs onto ventral periaqueductal gray dopamine neurons. bioRxiv. doi:10.1101/389536

Katz, J., \& Rosenbloom, B. N. (2015). The golden anniversary of Melzack and Wall's gate control theory of pain: Celebrating 50 years of pain research and management. Pain Research and Management, 20(6), 285-286. doi:10.1155/2015/865487

Kautio, A. L., Haanpaa, M., Saarto, T., \& Kalso, E. (2008). Amitriptyline in the treatment of chemotherapy-induced neuropathic symptoms. Journal of Pain and Symptom Management, 35(1), 31-39. doi:10.1016/j.jpainsymman.2007.02.043

Kawakami, K., Chiba, T., Katagiri, N., Saduka, M., Abe, K., Utsunomiya, I., Hama, T., \& Taguchi, K. (2012). Paclitaxel increases high voltage-dependent calcium channel current in dorsal root ganglion neurons of the rat. Journal of Pharmacological Sciences, 120(3), 187-195.

Kennedy, J., Roll, J. M., Schraudner, T., Murphy, S., \& McPherson, S. (2014). Prevalence of persistent pain in the U.S. adult population: new data from the 2010 national health interview survey. Journal of Pain, 15(10), 979-984. doi:10.1016/j.jpain.2014.05.009

Keogh, E., \& Eccleston, C. (2006). Sex differences in adolescent chronic pain and pain-related coping. Pain, 123(3), 275-284. doi:10.1016/j.pain.2006.03.004

Keppel Hesselink, J. M., Kopsky, D. J., \& Witkamp, R. F. (2014). Palmitoylethanolamide (PEA)'Promiscuous' anti-inflammatory and analgesic molecule at the interface between nutrition and pharma. PharmaNutrition, 2(1), 19-25. doi:10.1016/j.phanu.2013.11.127

Khanna, C., Rosenberg, M., \& Vail, D. M. (2015). A review of paclitaxel and novel formulations including those suitable for use in dogs. Journal of Veterinary Internal Medicine, 29(4), 1006-1012. doi:10.1111/jvim.12596

Kharebava, G., Rashid, M. A., Lee, J. W., Sarkar, S., Kevala, K., \& Kim, H. Y. (2015). Ndocosahexaenoylethanolamine regulates Hedgehog signaling and promotes growth of cortical axons. Biology Open, 4(12), 1660-1670. doi:10.1242/bio.013425

Kidd, J. F., Pilkington, M. F., Schell, M. J., Fogarty, K. E., Skepper, J. N., Taylor, C. W., \& Thorn, P. (2002). Paclitaxel affects cytosolic calcium signals by opening the mitochondrial permeability transition pore. Journal of Biological Chemistry, 277(8), 6504-6510. doi:10.1074/jbc.M106802200

Kilkenny, C., Browne, W., Cuthill, I. C., Emerson, M., \& Altman, D. G. (2010). Animal research: Reporting in vivo experiments: The ARRIVE guidelines. British Journal of Pharmacology, 160(7), 15771579. doi:10.1111/j.1476-5381.2010.00872.x

Killinger, B. A., Peet, M. M., \& Baker, L. E. (2010). Salvinorin A fails to substitute for the discriminative stimulus effects of LSD or ketamine in Sprague-Dawley rats. Pharmacology Biochemistry and Behavior, 96(3), 260-265. doi:10.1016/j.pbb.2010.05.014

Kim, H. Y., Moon, H. S., Cao, D., Lee, J., Kevala, K., Jun, S. B., Lovinger, D. M., Akbar, M., \& Huang, B. X. (2011a). N-Docosahexaenoylethanolamide promotes development of hippocampal neurons. Biochemical Journal, 435(2), 327-336. doi:10.1042/BJ20102118 
Kim, H. Y., Spector, A. A., \& Xiong, Z. M. (2011b). A synaptogenic amide Ndocosahexaenoylethanolamide promotes hippocampal development. Prostaglandins and Other Lipid Mediators, 96(1-4), 114-120. doi:10.1016/j.prostaglandins.2011.07.002

Kim, J. H., Dougherty, P. M., \& Abdi, S. (2015). Basic science and clinical management of painful and non-painful chemotherapy-related neuropathy. Gynecologic Oncology, 136(3), 453-459. doi:10.1016/j.ygyno.2015.01.524

Kim, M. J., Shin, H. J., Won, K. A., Yang, K. Y., Ju, J. S., Park, Y. Y., Park, J. S., Bae, Y. C., \& Ahn, D. K. (2012). Progesterone produces antinociceptive and neuroprotective effects in rats with microinjected lysophosphatidic acid in the trigeminal nerve root. Mol Pain, 8, 16. doi:10.1186/1744-8069-8-16

Kim, S. H., \& Chung, J. M. (1992). An experimental model for peripheral neuropathy produced by segmental spinal nerve ligation in the rat. Pain, 50(3), 355-363. doi:10.1016/03043959(92)90041-9

Kishioka, S., Kiguchi, N., Kobayashi, Y., Yamamoto, C., Saika, F., Wakida, N., Ko, M. C., \& Woods, J. H. (2013). Pharmacokinetic evidence for the long-lasting effect of nor-binaltorphimine, a potent kappa opioid receptor antagonist, in mice. Neuroscience Letters, 552, 98-102. doi:10.1016/j.neulet.2013.07.040

Kivell, B., \& Prisinzano, T. E. (2010). Kappa opioids and the modulation of pain. Psychopharmacology, 210(2), 109-119. doi:10.1007/s00213-010-1819-6

Kivell, B., Uzelac, Z., Sundaramurthy, S., Rajamanickam, J., Ewald, A., Chefer, V., Jaligam, V., Bolan, E., Simonson, B., Annamalai, B., Mannangatti, P., Prisinzano, T. E., Gomes, I., Devi, L. A., Jayanthi, L. D., Sitte, H. H., Ramamoorthy, S., \& Shippenberg, T. S. (2014). Salvinorin A regulates dopamine transporter function via a kappa opioid receptor and ERK1/2-dependent mechanism. Neuropharmacology, 86, 228-240. doi:10.1016/j.neuropharm.2014.07.016

Kivell, B. M., Paton, K. F., Kumar, N., Morani, A. S., Culverhouse, A., Shepherd, A., Welsh, S. A., Biggerstaff, A., Crowley, R. S., \& Prisinzano, T. E. (2018). Kappa Opioid Receptor Agonist Mesyl Sal B Attenuates Behavioral Sensitization to Cocaine with Fewer Aversive Side-Effects than Salvinorin A in Rodents. Molecules, 23(10), 2602. doi:10.3390/molecules23102602

Knowlton, W. M., Daniels, R. L., Palkar, R., McCoy, D. D., \& McKemy, D. D. (2011). Pharmacological blockade of TRPM8 ion channels alters cold and cold pain responses in mice. PloS One, 6(9), e25894. doi:10.1371/journal.pone.0025894

Knowlton, W. M., Palkar, R., Lippoldt, E. K., McCoy, D. D., Baluch, F., Chen, J., \& McKemy, D. D. (2013). A sensory-labeled line for cold: TRPM8-expressing sensory neurons define the cellular basis for cold, cold pain, and cooling-mediated analgesia. Journal of Neuroscience, 33(7), 28372848. doi:10.1523/JNEUROSCI.1943-12.2013

Kolaczkowska, E., \& Kubes, P. (2013). Neutrophil recruitment and function in health and inflammation. Nature Reviews Immunology, 13(3), 159-175. doi:10.1038/nri3399

Koster, R., Anderson, M., \& Debeer, E. J. (1959). Acetic acid for analgesic screening. Federation Proceedings, 18(1), 412-412.

Koyama, T., McHaffie, J. G., Laurienti, P. J., \& Coghill, R. C. (2005). The subjective experience of pain: where expectations become reality. Proceedings of the National Academy of Sciences of the United States of America, 102(36), 12950-12955. doi:10.1073/pnas.0408576102

Kreibich, A. S., \& Blendy, J. A. (2004). cAMP response element-binding protein is required for stress but not cocaine-induced reinstatement. The Journal of Neuroscience, 24(30), 6686. doi:10.1523/JNEUROSCI.1706-04.2004

Kris-Etherton, P. M., Grieger, J. A., \& Etherton, T. D. (2009). Dietary reference intakes for DHA and EPA. Prostaglandins, Leukotrienes, and Essential Fatty Acids, 81(2-3), 99-104. doi:10.1016/j.plefa.2009.05.011

Kris-Etherton, P. M., Harris, W. S., Appel, L. J., \& American Heart Association. Nutrition, C. (2002). Fish consumption, fish oil, omega-3 fatty acids, and cardiovascular disease. Circulation, 106(21), 2747-2757. doi:10.1161/01.cir.0000038493.65177.94 
Kroenke, K., Outcalt, S., Krebs, E., Bair, M. J., Wu, J., Chumbler, N., \& Yu, Z. (2013). Association between anxiety, health-related quality of life and functional impairment in primary care patients with chronic pain. General Hospital Psychiatry, 35(4), 359-365. doi:10.1016/j.genhosppsych.2013.03.020

Kuda, O. (2017). Bioactive metabolites of docosahexaenoic acid. Biochimie, 136, 12-20. doi:10.1016/j.biochi.2017.01.002

Kudryavtseva, N. N., Gerrits, M. A., Avgustinovich, D. F., Tenditnik, M. V., \& Van Ree, J. M. (2004). Modulation of anxiety-related behaviors by mu- and kappa-opioid receptor agonists depends on the social status of mice. Peptides, 25(8), 1355-1363. doi:10.1016/j.peptides.2004.05.005

Kuehl, F. A., Jacob, T. A., Ganley, O. H., Ormond, R. E., \& Meisinger, M. A. P. (1957). The identification of $\mathrm{N}$-(2-Hydroxyethyl)-Palmitamide as a naturally occurring anti-inflammatory agent. Journal of the American Chemical Society, 79(20), 5577-5578. doi:10.1021/ja01577a066

Kuehn, B. M. (2007). Prescription drug abuse rises globally. JAMA, 297(12), 1306. doi:10.1001/jama.297.12.1306

Kumagai, H., Ebata, T., Takamori, K., Muramatsu, T., Nakamoto, H., \& Suzuki, H. (2010). Effect of a novel kappa-receptor agonist, nalfurafine hydrochloride, on severe itch in 337 haemodialysis patients: a Phase III, randomized, double-blind, placebo-controlled study. Nephrology, Dialysis and Transplantation, 25(4), 1251-1257. doi:10.1093/ndt/gfp588

Kumar, N. (2014). Kappa opioid receptor agonists: New targets in the treatment of pain. Master's degree in Science, Victoria University of Wellington.

Kuner, R. (2010). Central mechanisms of pathological pain. Nature Medicine, 16(11), 1258-1266. doi:10.1038/nm.2231

Kuo, C. F., Grainge, M. J., Zhang, W., \& Doherty, M. (2015). Global epidemiology of gout: prevalence, incidence and risk factors. Nature Reviews Rheumatology, 11(11), 649-662. doi:10.1038/nrrheum.2015.91

Kutrzeba, L. M., Karamyan, V. T., Speth, R. C., Williamson, J. S., \& Zjawiony, J. K. (2009). In vitro studies on metabolism of salvinorin A. Pharmaceutical Biology, 47(11), 1078-1084. doi:10.3109/13880200903002222

Kuzmin, A. V., Semenova, S., Gerrits, M. A., Zvartau, E. E., \& Van Ree, J. M. (1997). Kappa-opioid receptor agonist $\mathrm{U} 50,488 \mathrm{H}$ modulates cocaine and morphine self-administration in drugnaive rats and mice. European Journal of Pharmacology, 321(3), 265-271. doi:10.1016/S00142999(96)00961-2

Kwan, K. Y., Allchorne, A. J., Vollrath, M. A., Christensen, A. P., Zhang, D. S., Woolf, C. J., \& Corey, D. P. (2006). TRPA1 contributes to cold, mechanical, and chemical nociception but is not essential for hair-cell transduction. Neuron, 50(2), 277-289. doi:10.1016/j.neuron.2006.03.042

La Regina, A., Petrillo, P., Sbacchi, M., \& Tavani, A. (1988). Interaction of U-69,593 with $\mu-$, $\partial$ - and kopioid binding sites and its analgesic and intestinal effects in rats. Life Sciences, 42(3), 293301. doi:10.1016/0024-3205(88)90638-8

LaBuda, C. J., \& Fuchs, P. N. (2000). A behavioral test paradigm to measure the aversive quality of inflammatory and neuropathic pain in rats. Experimental Neurology, 163(2), 490-494. doi:10.1006/exnr.2000.7395

Lahti, R. A., Mickelson, M. M., McCall, J. M., \& Von Voigtlander, P. F. (1985). [3H]U-69593 a highly selective ligand for the opioid kappa receptor. European Journal of Pharmacology, 109(2), 281-284. doi:10.1016/0014-2999(85)90431-5

Lai, J., Luo, M. C., Chen, Q., Ma, S., Gardell, L. R., Ossipov, M. H., \& Porreca, F. (2006). Dynorphin A activates bradykinin receptors to maintain neuropathic pain. Nature Neuroscience, 9(12), 1534-1540. doi:10.1038/nn1804

Lalanne, L., Ayranci, G., Kieffer, B. L., \& Lutz, P. E. (2014). The kappa opioid receptor: from addiction to depression, and back. Front Psychiatry, 5, 170. doi:10.3389/fpsyt.2014.00170

Lamb, K., Tidgewell, K., Simpson, D. S., Bohn, L. M., \& Prisinzano, T. E. (2012). Antinociceptive effects of herkinorin, a MOP receptor agonist derived from salvinorin $A$ in the formalin test in rats: 
new concepts in mu opioid receptor pharmacology: from a symposium on new concepts in mu-opioid pharmacology. Drug and Alcohol Dependence, 121(3), 181-188. doi:10.1016/j.drugalcdep.2011.10.026

Lamont, L. A., Tranquilli, W. J., \& Grimm, K. A. (2000). Physiology of pain. Veterinary Clinics of North America: Small Animal Practice, 30(4), 703-728, v. doi:10.1016/S0195-5616(08)70003-2

Langford, D. J., Bailey, A. L., Chanda, M. L., Clarke, S. E., Drummond, T. E., Echols, S., Glick, S., Ingrao, J., Klassen-Ross, T., Lacroix-Fralish, M. L., Matsumiya, L., Sorge, R. E., Sotocinal, S. G., Tabaka, J. M., Wong, D., van den Maagdenberg, A. M., Ferrari, M. D., Craig, K. D., \& Mogil, J. S. (2010). Coding of facial expressions of pain in the laboratory mouse. Nature Methods, 7(6), 447-449. doi:10.1038/nmeth.1455

Langford, D. J., Crager, S. E., Shehzad, Z., Smith, S. B., Sotocinal, S. G., Levenstadt, J. S., Chanda, M. L., Levitin, D. J., \& Mogil, J. S. (2006). Social modulation of pain as evidence for empathy in mice. Science, 312(5782), 1967-1970. doi:10.1126/science.1128322

LaPointe, N. E., Morfini, G., Brady, S. T., Feinstein, S. C., Wilson, L., \& Jordan, M. A. (2013). Effects of eribulin, vincristine, paclitaxel and ixabepilone on fast axonal transport and kinesin-1 driven microtubule gliding: implications for chemotherapy-induced peripheral neuropathy. Neurotoxicology, 37, 231-239. doi:10.1016/j.neuro.2013.05.008

Latremoliere, A., \& Woolf, C. J. (2009). Central sensitization: a generator of pain hypersensitivity by central neural plasticity. Journal of Pain, 10(9), 895-926. doi:10.1016/j.jpain.2009.06.012

Laughlin, T. M., Vanderah, T. W., Lashbrook, J., Nichols, M. L., Ossipov, M., Porreca, F., \& Wilcox, G. L. (1997). Spinally administered dynorphin A produces long-lasting allodynia: involvement of NMDA but not opioid receptors. Pain, 72(1-2), 253-260. doi:10.1016/S0304-3959(97)00046-8

Law, P. Y., Reggio, P. H., \& Loh, H. H. (2013). Opioid receptors: toward separation of analgesic from undesirable effects. Trends in Biochemical Sciences, 38(6), 275-282. doi:10.1016/j.tibs.2013.03.003

Le Merrer, J., Becker, J. A., Befort, K., \& Kieffer, B. L. (2009). Reward processing by the opioid system in the brain. Physiological Reviews, 89(4), 1379-1412. doi:10.1152/physrev.00005.2009

Ledeboer, A., Jekich, B. M., Sloane, E. M., Mahoney, J. H., Langer, S. J., Milligan, E. D., Martin, D., Maier, S. F., Johnson, K. W., Leinwand, L. A., Chavez, R. A., \& Watkins, L. R. (2007). Intrathecal interleukin-10 gene therapy attenuates paclitaxel-induced mechanical allodynia and proinflammatory cytokine expression in dorsal root ganglia in rats. Brain, Behavior, and Immunity, 21(5), 686-698. doi:10.1016/j.bbi.2006.10.012

Ledeboer, A., Liu, T., Shumilla, J. A., Mahoney, J. H., Vijay, S., Gross, M. I., Vargas, J. A., Sultzbaugh, L., Claypool, M. D., Sanftner, L. M., Watkins, L. R., \& Johnson, K. W. (2006). The glial modulatory drug AV411 attenuates mechanical allodynia in rat models of neuropathic pain. Neuron Glia Biology, 2(4), 279-291. doi:10.1017/S1740925X0700035X

Leipold, E., Hanson-Kahn, A., Frick, M., Gong, P., Bernstein, J. A., Voigt, M., Katona, I., Oliver Goral, R., Altmuller, J., Nurnberg, P., Weis, J., Hubner, C. A., Heinemann, S. H., \& Kurth, I. (2015). Coldaggravated pain in humans caused by a hyperactive NaV1.9 channel mutant. Nature communications, 6, 10049. doi:10.1038/ncomms10049

Lennertz, R. C., Kossyreva, E. A., Smith, A. K., \& Stucky, C. L. (2012). TRPA1 mediates mechanical sensitization in nociceptors during inflammation. PloS One, 7(8), e43597. doi:10.1371/journal.pone.0043597

Leong, A. G., Herst, P. M., \& Harper, J. L. (2012). Indigenous New Zealand honeys exhibit multiple antiinflammatory activities. Innate Immunity, 18(3), 459-466. doi:10.1177/1753425911422263

Lev-Tzion, R., Griffiths, A. M., Leder, O., \& Turner, D. (2014). Omega 3 fatty acids (fish oil) for maintenance of remission in Crohn's disease. Cochrane Database of Systematic Reviews(2), CD006320. doi:10.1002/14651858.CD006320.pub4

Levy, R. A., \& Proudfit, H. K. (1979). Analgesia produced by microinjection of baclofen and morphine at brain stem sites. European Journal of Pharmacology, 57(1), 43-55. doi:10.1016/00142999(79)90102-x 
Li, C., Pleil, K. E., Stamatakis, A. M., Busan, S., Vong, L., Lowell, B. B., Stuber, G. D., \& Kash, T. L. (2012). Presynaptic inhibition of gamma-aminobutyric acid release in the bed nucleus of the stria terminalis by kappa opioid receptor signaling. Biological Psychiatry, 71(8), 725-732. doi:10.1016/j.biopsych.2011.11.015

$\mathrm{Li}, \mathrm{J} . \mathrm{X}$. (2013). The application of conditioning paradigms in the measurement of pain. European Journal of Pharmacology, 716(1-3), 158-168. doi:10.1016/j.ejphar.2013.03.002

Li, S., Zhu, J., Chen, C., Chen, Y. W., Deriel, J. K., Ashby, B., \& Liu-Chen, L. Y. (1993). Molecular cloning and expression of a rat kappa opioid receptor. Biochemical Journal, 295 (Pt 3)(3), 629-633. doi:10.1042/bj2950629

Li, Y., North, R. Y., Rhines, L. D., Tatsui, C. E., Rao, G., Edwards, D. D., Cassidy, R. M., Harrison, D. S., Johansson, C. A., Zhang, H., \& Dougherty, P. M. (2018). DRG voltage-gated sodium channel 1.7 is upregulated in paclitaxel-induced neuropathy in rats and in humans with neuropathic pain. Journal of Neuroscience, 38(5), 1124-1136. doi:10.1523/JNEUROSCI.0899-17.2017

Li, Y., Zhang, H., Zhang, H., Kosturakis, A. K., Jawad, A. B., \& Dougherty, P. M. (2014). Toll-like receptor 4 signaling contributes to Paclitaxel-induced peripheral neuropathy. Journal of Pain, 15(7), 712-725. doi:10.1016/j.jpain.2014.04.001

Liang, X., Yu, G., \& Su, R. (2018). Effects of ralfinamide in models of nerve injury and chemotherapyinduced neuropathic pain. European Journal of Pharmacology, 823, 27-34. doi:10.1016/j.ejphar.2018.01.041

Lichtman, A. H., Smith, F. L., \& Martin, B. R. (1993). Evidence that the antinociceptive tail-flick response is produced independently from changes in either tail-skin temperature or core temperature. Pain, 55(3), 283-295. doi:10.1016/0304-3959(93)90003-8

Lieberman, A., \& Landon, D. (1976). The peripheral nerve. Sensory Ganglia, Chapman and Hall, London, 188-278.

Lima-Garcia, J. F., Dutra, R. C., da Silva, K., Motta, E. M., Campos, M. M., \& Calixto, J. B. (2011). The precursor of resolvin $\mathrm{D}$ series and aspirin-triggered resolvin $\mathrm{D} 1$ display anti-hyperalgesic properties in adjuvant-induced arthritis in rats. British Journal of Pharmacology, 164(2), 278293. doi:10.1111/j.1476-5381.2011.01345.x

Lin, L., Yang, H., \& Jones, P. J. (2012). Quantitative analysis of multiple fatty acid ethanolamides using ultra-performance liquid chromatography-tandem mass spectrometry. Prostaglandins, Leukotrienes, and Essential Fatty Acids, 87(6), 189-195. doi:10.1016/j.plefa.2012.09.001

Lindholm, S., Werme, M., Brene, S., \& Franck, J. (2001). The selective kappa-opioid receptor agonist $\mathrm{U} 50,488 \mathrm{H}$ attenuates voluntary ethanol intake in the rat. Behavioural Brain Research, 120(2), 137-146. doi:10.1016/S0166-4328(00)00368-5

Linley, J. E., Rose, K., Ooi, L., \& Gamper, N. (2010). Understanding inflammatory pain: ion channels contributing to acute and chronic nociception. Pflugers Archiv : European Journal of Physiology, 459(5), 657-669. doi:10.1007/s00424-010-0784-6

Liu-Chen, L. Y. (2004). Agonist-induced regulation and trafficking of kappa opioid receptors. Life Sciences, 75(5), 511-536. doi:10.1016/j.lfs.2003.10.041

Liu, C. C., Lu, N., Cui, Y., Yang, T., Zhao, Z. Q., Xin, W. J., \& Liu, X. G. (2010). Prevention of paclitaxelinduced allodynia by minocycline: Effect on loss of peripheral nerve fibers and infiltration of macrophages in rats. Mol Pain, 6, 76. doi:10.1186/1744-8069-6-76

Lo Verme, J., Fu, J., Astarita, G., La Rana, G., Russo, R., Calignano, A., \& Piomelli, D. (2005). The nuclear receptor peroxisome proliferator-activated receptor-alpha mediates the anti-inflammatory actions of palmitoylethanolamide. Molecular Pharmacology, 67(1), 15-19. doi:10.1124/mol.104.006353

Lokesh, B. R., Hsieh, H. L., \& Kinsella, J. E. (1986). Peritoneal macrophages from mice fed dietary (n-3) polyunsaturated fatty acids secrete low levels of prostaglandins. Journal of Nutrition, 116(12), 2547-2552. doi:10.1093/jn/116.12.2547

Lolignier, S., Bonnet, C., Gaudioso, C., Noel, J., Ruel, J., Amsalem, M., Ferrier, J., Rodat-Despoix, L., Bouvier, V., Aissouni, Y., Prival, L., Chapuy, E., Padilla, F., Eschalier, A., Delmas, P., \& 
Busserolles, J. (2015). The Nav1.9 channel is a key determinant of cold pain sensation and cold allodynia. Cell Reports, 11(7), 1067-1078. doi:10.1016/j.celrep.2015.04.027

Lolignier, S., Gkika, D., Andersson, D., Leipold, E., Vetter, I., Viana, F., Noel, J., \& Busserolles, J. (2016). New Insight in Cold Pain: Role of Ion Channels, Modulation, and Clinical Perspectives. Journal of Neuroscience, 36(45), 11435-11439. doi:10.1523/JNEUROSCI.2327-16.2016

Long, D. A., \& Martin, A. J. (1956). Factor in arachis oil depressing sensitivity to tuberculin in B.C.G.infected guineapigs. Lancet, 270(6921), 464-466. doi:10.1016/S0140-6736(56)90529-3

Lorente-Cebrian, S., Costa, A. G., Navas-Carretero, S., Zabala, M., Laiglesia, L. M., Martinez, J. A., \& Moreno-Aliaga, M. J. (2015). An update on the role of omega-3 fatty acids on inflammatory and degenerative diseases. Journal of Physiology and Biochemistry, 71(2), 341-349. doi:10.1007/s13105-015-0395-y

Lourdudoss, C., Wolk, A., Nise, L., Alfredsson, L., \& Vollenhoven, R. V. (2017). Are dietary vitamin D, omega-3 fatty acids and folate associated with treatment results in patients with early rheumatoid arthritis? Data from a Swedish population-based prospective study. BMJ Open, 7(6), e016154. doi:10.1136/bmjopen-2017-016154

Lu, H. P., Minervini, V., \& France, C. P. (2017). Interactions between mu and kappa opioid receptor agonists: Antinociceptive and adverse effects in rats. FASEB Journal, 31(1 supplement), 985917. doi:10.1096/fasebj.31.1_supplement.985.17

Luis-Delgado, O. E., Barrot, M., Rodeau, J. L., Schott, G., Benbouzid, M., Poisbeau, P., Freund-Mercier, M. J., \& Lasbennes, F. (2006). Calibrated forceps: a sensitive and reliable tool for pain and analgesia studies. Journal of Pain, 7(1), 32-39. doi:10.1016/j.jpain.2005.07.011

Lukas, G., Brindle, S. D., \& Greengard, P. (1971). The route of absorption of intraperitoneally administered compounds. Journal of Pharmacology and Experimental Therapeutics, 178(3), 562-564.

Lukoshkova, E. V. (1976). Conduction velocity and excitability of A and C fibers of cat mesenteric nerves. Neurophysiology, 7(3), 211-217. doi:10.1007/bf01063306

Lumpkin, E. A., Marshall, K. L., \& Nelson, A. M. (2010). The cell biology of touch. Journal of Cell Biology, 191(2), 237-248. doi:10.1083/jcb.201006074

Luo, M. C., Chen, Q., Ossipov, M. H., Rankin, D. R., Porreca, F., \& Lai, J. (2008). Spinal dynorphin and bradykinin receptors maintain inflammatory hyperalgesia. Journal of Pain, 9(12), 1096-1105. doi:10.1016/j.jpain.2008.06.005

Lynch, M. E., Cesar-Rittenberg, P., \& Hohmann, A. G. (2014). A double-blind, placebo-controlled, crossover pilot trial with extension using an oral mucosal cannabinoid extract for treatment of chemotherapy-induced neuropathic pain. Journal of Pain and Symptom Management, 47(1), 166-173. doi:10.1016/j.jpainsymman.2013.02.018

Ma, W., Zhang, Y., Bantel, C., \& Eisenach, J. C. (2005). Medium and large injured dorsal root ganglion cells increase TRPV-1, accompanied by increased alpha2C-adrenoceptor co-expression and functional inhibition by clonidine. Pain, 113(3), 386-394. doi:10.1016/j.pain.2004.11.018

Macpherson, L. J., Geierstanger, B. H., Viswanath, V., Bandell, M., Eid, S. R., Hwang, S., \& Patapoutian, A. (2005). The pungency of garlic: activation of TRPA1 and TRPV1 in response to allicin. Current Biology, 15(10), 929-934. doi:10.1016/j.cub.2005.04.018

Mague, S. D., Pliakas, A. M., Todtenkopf, M. S., Tomasiewicz, H. C., Zhang, Y., Stevens, W. C., Jr., Jones, R. M., Portoghese, P. S., \& Carlezon, W. A., Jr. (2003). Antidepressant-like effects of kappaopioid receptor antagonists in the forced swim test in rats. Journal of Pharmacology and Experimental Therapeutics, 305(1), 323-330. doi:10.1124/jpet.102.046433

Malan, T. P., Ossipov, M. H., Gardell, L. R., Ibrahim, M., Bian, D., Lai, J., \& Porreca, F. (2000). Extraterritorial neuropathic pain correlates with multisegmental elevation of spinal dynorphin in nerve-injured rats. Pain, 86(1-2), 185-194. doi:10.1016/S0304-3959(00)00243-8

Maldonado, R., Saiardi, A., Valverde, O., Samad, T. A., Roques, B. P., \& Borrelli, E. (1997). Absence of opiate rewarding effects in mice lacking dopamine D2 receptors. Nature, 388(6642), 586-589. doi:10.1038/41567 
Manchikanti, L., Singh, V., Falco, F. J., Benyamin, R. M., \& Hirsch, J. A. (2014). Epidemiology of low back pain in adults. Neuromodulation, 17 Suppl 2, 3-10. doi:10.1111/ner.12018

Mangaiarkkarasi, A., Rameshkannan, S., \& Ali, R. M. (2015). Effect of gabapentin and pregabalin in rat model of Taxol induced neuropathic pain. Journal of Clinical and Diagnostic Research, 9(5), FF11-14. doi:10.7860/JCDR/2015/13373.5955

Mansson, E., Bare, L., \& Yang, D. (1994). Isolation of a human kappa opioid receptor cDNA from placenta. Biochemical and Biophysical Research Communications, 202(3), 1431-1437. doi:10.1006/bbrc.1994.2091

Manzano, G. M., Giuliano, L. M., \& Nobrega, J. A. (2008). A brief historical note on the classification of nerve fibers. Arquivos de Neuro-Psiquiatria, 66(1), 117-119. doi:10.1590/50004282X2008000100033

Maqueda, A. E., Valle, M., Addy, P. H., Antonijoan, R. M., Puntes, M., Coimbra, J., Ballester, M. R., Garrido, M., Gonzalez, M., Claramunt, J., Barker, S., Johnson, M. W., Griffiths, R. R., \& Riba, J. (2015). Salvinorin-A induces intense dissociative effects, blocking external sensory perception and modulating interoception and sense of body ownership in humans. International Journal of Neuropsychopharmacology, 18(12), pyv065. doi:10.1093/ijnp/pyv065

Marchand, F., Perretti, M., \& McMahon, S. B. (2005). Role of the immune system in chronic pain. Nature Reviews: Neuroscience, 6(7), 521-532. doi:10.1038/nrn1700

Marchand, S. (2008). The physiology of pain mechanisms: from the periphery to the brain. Rheumatic Diseases Clinics of North America, 34(2), 285-309. doi:10.1016/j.rdc.2008.04.003

Margolis, E. B., Lock, H., Chefer, V. I., Shippenberg, T. S., Hjelmstad, G. O., \& Fields, H. L. (2006). Kappa opioids selectively control dopaminergic neurons projecting to the prefrontal cortex. Proceedings of the National Academy of Sciences of the United States of America, 103(8), 2938-2942. doi:10.1073/pnas.0511159103

Martin, M., Matifas, A., Maldonado, R., \& Kieffer, B. L. (2003). Acute antinociceptive responses in single and combinatorial opioid receptor knockout mice: distinct mu, delta and kappa tones. European Journal of Neuroscience, 17(4), 701-708. doi:10.1046/j.1460-9568.2003.02482.x

Martin, V. T. (2009). Ovarian hormones and pain response: a review of clinical and basic science studies. Gender Medicine, 6 Suppl 2, 168-192. doi:10.1016/j.genm.2009.03.006

Materazzi, S., Fusi, C., Benemei, S., Pedretti, P., Patacchini, R., Nilius, B., Prenen, J., Creminon, C., Geppetti, P., \& Nassini, R. (2012). TRPA1 and TRPV4 mediate paclitaxel-induced peripheral neuropathy in mice via a glutathione-sensitive mechanism. Pflügers Archiv. European Journal of Physiology, 463(4), 561-569. doi:10.1007/s00424-011-1071-x

Matsuda, L. A., Lolait, S. J., Brownstein, M. J., Young, A. C., \& Bonner, T. I. (1990). Structure of a cannabinoid receptor and functional expression of the cloned cDNA. Nature, 346(6284), 561564. doi:10.1038/346561a0

Matsumoto, M., Inoue, M., Hald, A., Xie, W., \& Ueda, H. (2006). Inhibition of paclitaxel-induced A-fiber hypersensitization by gabapentin. Journal of Pharmacology and Experimental Therapeutics, 318(2), 735-740. doi:10.1124/jpet.106.103614

Mauderli, A. P., Acosta-Rua, A., \& Vierck, C. J. (2000). An operant assay of thermal pain in conscious, unrestrained rats. Journal of Neuroscience Methods, 97(1), 19-29. doi:10.1016/S01650270(00)00160-6

McCurdy, C. R., Sufka, K. J., Smith, G. H., Warnick, J. E., \& Nieto, M. J. (2006). Antinociceptive profile of salvinorin A, a structurally unique kappa opioid receptor agonist. Pharmacology Biochemistry and Behavior, 83(1), 109-113. doi:10.1016/j.pbb.2005.12.011

McGaraughty, S., Chu, K. L., Perner, R. J., Didomenico, S., Kort, M. E., \& Kym, P. R. (2010). TRPA1 modulation of spontaneous and mechanically evoked firing of spinal neurons in uninjured, osteoarthritic, and inflamed rats. Molecular Pain, 6, 14. doi:10.1186/1744-8069-6-14

McLaughlin, J. P., Myers, L. C., Zarek, P. E., Caron, M. G., Lefkowitz, R. J., Czyzyk, T. A., Pintar, J. E., \& Chavkin, C. (2004). Prolonged kappa opioid receptor phosphorylation mediated by G-protein 
receptor kinase underlies sustained analgesic tolerance. Journal of Biological Chemistry, 279(3), 1810-1818. doi:10.1074/jbc.M305796200

McLaughlin, J. P., Xu, M., Mackie, K., \& Chavkin, C. (2003). Phosphorylation of a carboxyl-terminal serine within the kappa-opioid receptor produces desensitization and internalization. Journal of Biological Chemistry, 278(36), 34631-34640. doi:10.1074/jbc.M304022200

McNamara, C. R., Mandel-Brehm, J., Bautista, D. M., Siemens, J., Deranian, K. L., Zhao, M., Hayward, N. J., Chong, J. A., Julius, D., Moran, M. M., \& Fanger, C. M. (2007). TRPA1 mediates formalininduced pain. Proceedings of the National Academy of Sciences of the United States of America, 104(33), 13525-13530. doi:10.1073/pnas.0705924104

Mei, F., Mayoral, S. R., Nobuta, H., Wang, F., Desponts, C., Lorrain, D. S., Xiao, L., Green, A. J., Rowitch, D., Whistler, J., \& Chan, J. R. (2016). Identification of the kappa-opioid receptor as a therapeutic target for oligodendrocyte remyelination. Journal of Neuroscience, 36(30), 79257935. doi:10.1523/JNEUROSCI.1493-16.2016

Meijerink, J., Balvers, M., \& Witkamp, R. (2013). N-Acyl amines of docosahexaenoic acid and other n3 polyunsatured fatty acids - from fishy endocannabinoids to potential leads. British Journal of Pharmacology, 169(4), 772-783. doi:10.1111/bph.12030

Meijerink, J., Plastina, P., Vincken, J. P., Poland, M., Attya, M., Balvers, M., Gruppen, H., Gabriele, B., \& Witkamp, R. F. (2011). The ethanolamide metabolite of DHA, docosahexaenoylethanolamine, shows immunomodulating effects in mouse peritoneal and RAW264.7 macrophages: evidence for a new link between fish oil and inflammation. British Journal of Nutrition, 105(12), 1798-1807. doi:10.1017/S0007114510005635

Meijerink, J., Poland, M., Balvers, M. G., Plastina, P., Lute, C., Dwarkasing, J., van Norren, K., \& Witkamp, R. F. (2015). Inhibition of COX-2-mediated eicosanoid production plays a major role in the anti-inflammatory effects of the endocannabinoid $\mathrm{N}$-docosahexaenoylethanolamine (DHEA) in macrophages. British Journal of Pharmacology, 172(1), 24-37. doi:10.1111/bph.12747

Mello, N. K., \& Negus, S. S. (2000). Interactions between kappa opioid agonists and cocaine - Preclinical studies. New Medications for Drug Abuse, 909(1), 104-132. doi:10.1111/j.17496632.2000.tb06678.x

Melzack, R., \& Wall, P. D. (1965). Pain mechanisms: a new theory. Science, 150(3699), 971-979. doi:10.1126/science.150.3699.971

Memon, T., Chase, K., Leavitt, L. S., Olivera, B. M., \& Teichert, R. W. (2017). TRPA1 expression levels and excitability brake by KV channels influence cold sensitivity of TRPA1-expressing neurons. Neuroscience, 353(Supplement C), 76-86. doi:10.1016/j.neuroscience.2017.04.001

Mendell, L. M. (2014). Constructing and deconstructing the gate theory of pain. Pain, 155(2), 210-216. doi:10.1016/j.pain.2013.12.010

Mendelson, J. E., Coyle, J. R., Lopez, J. C., Baggott, M. J., Flower, K., Everhart, E. T., Munro, T. A., Galloway, G. P., \& Cohen, B. M. (2011). Lack of effect of sublingual salvinorin A, a naturally occurring kappa opioid, in humans: a placebo-controlled trial. Psychopharmacology, 214(4), 933-939. doi:10.1007/s00213-010-2103-5

Meng, F., Xie, G. X., Thompson, R. C., Mansour, A., Goldstein, A., Watson, S. J., \& Akil, H. (1993). Cloning and pharmacological characterization of a rat kappa opioid receptor. Proceedings of the National Academy of Sciences of the United States of America, 90(21), 9954-9958. doi:10.1073/pnas.90.21.9954

Meng, H., Johnston, B., Englesakis, M., Moulin, D. E., \& Bhatia, A. (2017). Selective cannabinoids for chronic neuropathic pain: A systematic review and meta-analysis. Anesthesia \& Analgesia, 125(5), 1638-1652. doi:10.1213/ANE.0000000000002110

Mense, S. (2008). Muscle pain: mechanisms and clinical significance. Deutsches Ärzteblatt International, 105(12), 214-219. doi:10.3238/artzebl.2008.0214

Michalski, J. P., \& Kothary, R. (2015). Oligodendrocytes in a nutshell. Frontiers in Cellular Neuroscience, 9, 340. doi:10.3389/fncel.2015.00340 
Michel, M. C., \& Charlton, S. J. (2018). Biased agonism in drug discovery - is it too soon to choose a path? Molecular Pharmacology. doi:10.1124/mol.117.110890

Millan, M. J., Czlonkowski, A., Pilcher, C. W., Almeida, O. F., Millan, M. H., Colpaert, F. C., \& Herz, A. (1987). A model of chronic pain in the rat: functional correlates of alterations in the activity of opioid systems. Journal of Neuroscience, 7(1), 77-87. doi:10.1523/JNEUROSCI.07-0100077.1987

Minami, M., Toya, T., Katao, Y., Maekawa, K., Nakamura, S., Onogi, T., Kaneko, S., \& Satoh, M. (1993). Cloning and expression of a cDNA for the ratk-opioid receptor. FEBS Letters, 329(3), 291-295. doi:10.1016/0014-5793(93)80240-u

Ministry of Health. (2016). Annual update of key results 2015/16: New Zealand Health Survey. Retrieved from Wellington: Ministry of Health

Mo, M., Erdelyi, I., Szigeti-Buck, K., Benbow, J. H., \& Ehrlich, B. E. (2012). Prevention of paclitaxelinduced peripheral neuropathy by lithium pretreatment. The FASEB Journal, 26(11), 46964709. doi:10.1096/fj.12-214643

Mogil, J. S. (2009). Animal models of pain: progress and challenges. Nature Reviews: Neuroscience, 10(4), 283-294. doi:10.1038/nrn2606

Mogil, J. S. (2012). Sex differences in pain and pain inhibition: multiple explanations of a controversial phenomenon. Nature Reviews: Neuroscience, 13(12), 859-866. doi:10.1038/nrn3360

Mogil, J. S., Richards, S. P., O'Toole, L. A., Helms, M. L., Mitchell, S. R., Kest, B., \& Belknap, J. K. (1997). Identification of a sex-specific quantitative trait locus mediating nonopioid stress-induced analgesia in female mice. Journal of Neuroscience, 17(20), 7995-8002. doi:10.1523/JNEUROSCI.17-20-07995.1997

Mols, F., Beijers, T., Vreugdenhil, G., \& van de Poll-Franse, L. (2014). Chemotherapy-induced peripheral neuropathy and its association with quality of life: a systematic review. Supportive Care in Cancer, 22(8), 2261-2269. doi:10.1007/s00520-014-2255-7

Momin, A., \& McNaughton, P. A. (2009). Regulation of firing frequency in nociceptive neurons by proinflammatory mediators. Experimental Brain Research, 196(1), 45-52. doi:10.1007/s00221009-1744-2

Morani, A. S., Ewald, A., Prevatt-Smith, K. M., Prisinzano, T. E., \& Kivell, B. M. (2013). The 2-methoxy methyl analogue of salvinorin $A$ attenuates cocaine-induced drug seeking and sucrose reinforcements in rats. European Journal of Pharmacology, 720(1-3), 69-76. doi:10.1016/j.ejphar.2013.10.050

Morani, A. S., Kivell, B., Prisinzano, T. E., \& Schenk, S. (2009). Effect of kappa-opioid receptor agonists U69593, U50488H, spiradoline and salvinorin A on cocaine-induced drug-seeking in rats. Pharmacology Biochemistry and Behavior, 94(2), 244-249. doi:10.1016/j.pbb.2009.09.002

Morani, A. S., Schenk, S., Prisinzano, T. E., \& Kivell, B. M. (2012). A single injection of a novel kappa opioid receptor agonist salvinorin $A$ attenuates the expression of cocaine-induced behavioral sensitization in rats. Behavioural Pharmacology, 23(2), 162-170. doi:10.1097/FBP.0b013e3283512c1e

Moreira, F. X., Carvalho, F., de Lourdes Bastos, M., \& de Pinho, P. G. (2014). Analytical investigation of legal high products containing Salvia divinorum traded in smartshops and internet. Forensic Science International, 242(Supplement C), 255-260. doi:10.1016/j.forsciint.2014.07.009

Mori, T., Kanbara, T., Harumiya, M., Iwase, Y., Masumoto, A., Komiya, S., Nakamura, A., Shibasaki, M., Kanemasa, T., Sakaguchi, G., \& Suzuki, T. (2014). Establishment of opioid-induced rewarding effects under oxaliplatin- and Paclitaxel-induced neuropathy in rats. Journal of Pharmacological Sciences, 126(1), 47-55. doi:10.1254/jphs.14134FP

Mucha, R. F., \& Herz, A. (1985). Motivational properties of kappa and mu opioid receptor agonists studied with place and taste preference conditioning. Psychopharmacology, 86(3), 274-280. doi:10.1007/BF00432213 
Muhuri, P. K., Gfroerer, J. C., \& Davies, C. (2013). Associations of nonmedical pain reliever use and initiation of heroin use in the United States. Center for Behavioral Health Statistics and Quality Data Review.

Munro, T. A., Berry, L. M., Van't Veer, A., Beguin, C., Carroll, F. I., Zhao, Z., Carlezon, W. A., Jr., \& Cohen, B. M. (2012). Long-acting kappa opioid antagonists nor-BNI, GNTI and JDTic: pharmacokinetics in mice and lipophilicity. BMC Pharmacology, 12, 5. doi:10.1186/1471-2210-12-5

Munro, T. A., Duncan, K. K., Xu, W., Wang, Y., Liu-Chen, L. Y., Carlezon, W. A., Jr., Cohen, B. M., \& Beguin, C. (2008). Standard protecting groups create potent and selective kappa opioids: salvinorin B alkoxymethyl ethers. Bioorganic and Medicinal Chemistry, 16(3), 1279-1286. doi:10.1016/j.bmc.2007.10.067

Munro, T. A., \& Rizzacasa, M. A. (2003). Salvinorins D-F, new neoclerodane diterpenoids from Salvia divinorum, and an improved method for the isolation of Salvinorin A. Journal of Natural Products, 66(5), 703-705. doi:10.1021/np0205699

Nag, S., \& Mokha, S. S. (2009). Testosterone is essential for alpha(2)-adrenoceptor-induced antinociception in the trigeminal region of the male rat. Neuroscience Letters, 467(1), 48-52. doi:10.1016/j.neulet.2009.10.016

Nahin, R. L. (2015). Estimates of pain prevalence and severity in adults: United States, 2012. Journal of Pain, 16(8), 769-780. doi:10.1016/j.jpain.2015.05.002

Naji-Esfahani, H., Vaseghi, G., Safaeian, L., Pilehvarian, A. A., Abed, A., \& Rafieian-Kopaei, M. (2016). Gender differences in a mouse model of chemotherapy-induced neuropathic pain. Laboratory Animals, 50(1), 15-20. doi:10.1177/0023677215575863

Nakamoto, K., Nishinaka, T., Mankura, M., Fujita-Hamabe, W., \& Tokuyama, S. (2010). Antinociceptive effects of docosahexaenoic acid against various pain stimuli in mice. Biological \& Pharmaceutical Bulletin, 33(6), 1070-1072. doi:10.1248/bpb.33.1070

Nakao, K., Hirakata, M., Miyamoto, Y., Kainoh, M., Wakasa, Y., \& Yanagita, T. (2016). Nalfurafine hydrochloride, a selective kappa opioid receptor agonist, has no reinforcing effect on intravenous self-administration in rhesus monkeys. Journal of Pharmacological Sciences, 130(1), 8-14. doi:10.1016/j.jphs.2015.11.008

Nave, K. A., \& Werner, H. B. (2014). Myelination of the nervous system: mechanisms and functions. Annual Review of Cell and Developmental Biology, 30(1), 503-533. doi:10.1146/annurevcellbio-100913-013101

Negus, S. S., Mello, N. K., Portoghese, P. S., \& Lin, C. E. (1997). Effects of kappa opioids on cocaine selfadministration by rhesus monkeys. Journal of Pharmacology and Experimental Therapeutics, 282(1), 44-55.

Negus, S. S., Schrode, K., \& Stevenson, G. W. (2008). Mu/kappa opioid interactions in rhesus monkeys: implications for analgesia and abuse liability. Experimental and Clinical Psychopharmacology, 16(5), 386-399. doi:10.1037/a0013088

Ness, T. J., \& Gebhart, G. F. (1988). Colorectal distension as a noxious visceral stimulus: physiologic and pharmacologic characterization of pseudaffective reflexes in the rat. Brain Research, 450(1-2), 153-169. doi:10.1016/0006-8993(88)91555-7

Neuringer, M., Connor, W. E., Lin, D. S., Barstad, L., \& Luck, S. (1986). Biochemical and functional effects of prenatal and postnatal omega 3 fatty acid deficiency on retina and brain in rhesus monkeys. Proceedings of the National Academy of Sciences of the United States of America, 83(11), 4021-4025. doi:10.1073/pnas.83.11.4021

Neuringer, M., Connor, W. E., Van Petten, C., \& Barstad, L. (1984). Dietary omega-3 fatty acid deficiency and visual loss in infant rhesus monkeys. Journal of Clinical Investigation, 73(1), 272-276. doi:10.1172/JCl111202

Nicol, A. L., Sieberg, C. B., Clauw, D. J., Hassett, A. L., Moser, S. E., \& Brummett, C. M. (2016). The Association Between a History of Lifetime Traumatic Events and Pain Severity, Physical Function, and Affective Distress in Patients With Chronic Pain. Journal of Pain, 17(12), 13341348. doi:10.1016/j.jpain.2016.09.003 
Nieto, F. R., Entrena, J. M., Cendan, C. M., Pozo, E. D., Vela, J. M., \& Baeyens, J. M. (2008). Tetrodotoxin inhibits the development and expression of neuropathic pain induced by paclitaxel in mice. Pain, 137(3), 520-531. doi:10.1016/j.pain.2007.10.012

Nishi, M., Takeshima, H., Fukuda, K., Kato, S., \& Mori, K. (1993). cDNA cloning and pharmacological characterization of an opioid receptor with high affinities for kappa-subtype-selective ligands. FEBS Letters, 330(1), 77-80. doi:10.1016/0014-5793(93)80923-i

Nishi, M., Takeshima, H., Mori, M., Nakagawara, K., \& Takeuchi, T. (1994). Structure and chromosomal mapping of genes for the mouse kappa-opioid receptor and an opioid receptor homologue (MOR-C). Biochemical and Biophysical Research Communications, 205(2), 1353-1357. doi:10.1006/bbrc.1994.2814

Nobre, M. E., Correia, A. O., Borges Mde, B., Sampaio, T. M., Chakraborty, S. A., Goncalves Dde, O., Brito, G. A., Leal, L. K., Felipe, C. F., Lucetti, D. L., Arida, R. M., \& Viana, G. S. (2013). Eicosapentaenoic acid and docosahexaenoic acid exert anti-inflammatory and antinociceptive effects in rodents at low doses. Nutrition Research, 33(5), 422-433. doi:10.1016/j.nutres.2013.02.011

Noel, J., Zimmermann, K., Busserolles, J., Deval, E., Alloui, A., Diochot, S., Guy, N., Borsotto, M., Reeh, P., Eschalier, A., \& Lazdunski, M. (2009). The mechano-activated K+ channels TRAAK and TREK1 control both warm and cold perception. EMBO Journal, 28(9), 1308-1318. doi:10.1038/emboj.2009.57

Noli, C., Della Valle, M. F., Miolo, A., Medori, C., Schievano, C., \& Skinalia Clinical Research Group. (2015). Efficacy of ultra-micronized palmitoylethanolamide in canine atopic dermatitis: an open-label multi-centre study. Veterinary Dermatology, 26(6), 432-440, e101. doi:10.1111/vde.12250

Nozadze, I., Tsiklauri, N., Gurtskaia, G., \& Tsagareli, M. G. (2016). Role of thermo TRPA1 and TRPV1 channels in heat, cold, and mechanical nociception of rats. Behavioural Pharmacology, 27(1), 29-36. doi:10.1097/FBP.0000000000000176

Obara, I., Mika, J., Schafer, M. K., \& Przewlocka, B. (2003). Antagonists of the kappa-opioid receptor enhance allodynia in rats and mice after sciatic nerve ligation. British Journal of Pharmacology, 140(3), 538-546. doi:10.1038/sj.bjp.0705427

Ohta, T., Ikemi, Y., Murakami, M., Imagawa, T., Otsuguro, K., \& Ito, S. (2006). Potentiation of transient receptor potential V1 functions by the activation of metabotropic 5-HT receptors in rat primary sensory neurons. Journal of Physiology, 576(Pt 3), 809-822. doi:10.1113/jphysiol.2006.112250

Okie, S. (2010). A flood of opioids, a rising tide of deaths. New England Journal of Medicine, 363(21), 1981-1985. doi:10.1056/NEJMp1011512

Old, E. A., Clark, A. K., \& Malcangio, M. (2015). The role of glia in the spinal cord in neuropathic and inflammatory pain. In H.-G. Schaible (Ed.), Pain Control (pp. 145-170). Berlin, Heidelberg: Springer Berlin Heidelberg.

Olesen, A. E., Kristensen, K., Staahl, C., Kell, S., Wong, G. Y., Arendt-Nielsen, L., \& Drewes, A. M. (2013). A population pharmacokinetic and pharmacodynamic study of a peripheral kappa-opioid receptor agonist CR665 and oxycodone. Clinical Pharmacokinetics, 52(2), 125-137. doi:10.1007/s40262-012-0023-8

Ossipov, M. H., Morimura, K., \& Porreca, F. (2014). Descending pain modulation and chronification of pain. Curr Opin Support Palliat Care, 8(2), 143-151. doi:10.1097/SPC.0000000000000055

Ostadhadi, S., Haj-Mirzaian, A., Nikoui, V., Kordjazy, N., \& Dehpour, A. R. (2016). Involvement of opioid system in antidepressant-like effect of the cannabinoid CB1 receptor inverse agonist AM-251 after physical stress in mice. Clinical and Experimental Pharmacology and Physiology, 43(2), 203-212. doi:10.1111/1440-1681.12518

Outcalt, S. D., Kroenke, K., Krebs, E. E., Chumbler, N. R., Wu, J., Yu, Z., \& Bair, M. J. (2015). Chronic pain and comorbid mental health conditions: independent associations of posttraumatic stress 
disorder and depression with pain, disability, and quality of life. Journal of Behavioral Medicine, 38(3), 535-543. doi:10.1007/s10865-015-9628-3

Pan, C. H., Chang, C. C., Su, C. T., \& Tsai, P. S. (2016). Trends in irritable bowel syndrome incidence among Taiwanese adults during 2003-2013: A population-based study of sex and age differences. PloS One, 11(11), e0166922. doi:10.1371/journal.pone.0166922

Parikh, D., Hamid, A., Friedman, T. C., Nguyen, K., Tseng, A., Marquez, P., \& Lutfy, K. (2011). Stressinduced analgesia and endogenous opioid peptides: the importance of stress duration. European Journal of Pharmacology, 650(2-3), 563-567. doi:10.1016/j.ejphar.2010.10.050

Pascual, D., Goicoechea, C., Burgos, E., \& Martin, M. I. (2010). Antinociceptive effect of three common analgesic drugs on peripheral neuropathy induced by paclitaxel in rats. Pharmacology Biochemistry and Behavior, 95(3), 331-337. doi:10.1016/j.pbb.2010.02.009

Pascual, D., Goicoechea, C., Suardiaz, M., \& Martin, M. I. (2005). A cannabinoid agonist, WIN 55,2122, reduces neuropathic nociception induced by paclitaxel in rats. Pain, 118(1-2), 23-34. doi:10.1016/j.pain.2005.07.008

Paton, K. F., Kumar, N., Crowley, R. S., Harper, J. L., Prisinzano, T. E., \& Kivell, B. M. (2017). The analgesic and anti-inflammatory effects of Salvinorin A analogue beta-tetrahydropyran Salvinorin B in mice. European Journal of Pain (London, England), 21(6), 1039-1050. doi:10.1002/ejp.1002

Patrono, C. (1994). Aspirin as an antiplatelet drug. New England Journal of Medicine, 330(18), 12871294. doi:10.1056/NEJM199405053301808

Peart, J. N., Gross, E. R., Reichelt, M. E., Hsu, A., Headrick, J. P., \& Gross, G. J. (2008). Activation of kappa-opioid receptors at reperfusion affords cardioprotection in both rat and mouse hearts. Basic Research in Cardiology, 103(5), 454-463. doi:10.1007/s00395-008-0726-z

Pease-Raissi, S. E., Pazyra-Murphy, M. F., Li, Y., Wachter, F., Fukuda, Y., Fenstermacher, S. J., Barclay, L. A., Bird, G. H., Walensky, L. D., \& Segal, R. A. (2017). Paclitaxel reduces axonal Bclw to initiate IP3R1-dependent axon degeneration. Neuron, 96(2), 373-386 e376. doi:10.1016/j.neuron.2017.09.034

Peckys, D., \& Landwehrmeyer, G. B. (1999). Expression of mu, kappa, and delta opioid receptor messenger RNA in the human CNS: a 33P in situ hybridization study. Neuroscience, 88(4), 1093-1135. doi:10.1016/S0306-4522(98)00251-6

Pedersen, L. H., \& Blackburn-Munro, G. (2006). Pharmacological characterisation of place escape/avoidance behaviour in the rat chronic constriction injury model of neuropathic pain. Psychopharmacology, 185(2), 208-217. doi:10.1007/s00213-005-0281-3

Peet, M. M., \& Baker, L. E. (2011). Salvinorin B derivatives, EOM-Sal B and MOM-Sal B, produce stimulus generalization in male Sprague-Dawley rats trained to discriminate salvinorin $A$. Behavioural Pharmacology, 22(5-6), 450-457. doi:10.1097/FBP.0b013e328349fc1b

Pelissier, T., Paeile, C., Soto-Moyano, R., Saavedra, H., \& Hernández, A. (1990). Analgesia produced by intrathecal administration of the $\mathrm{k}$ opioid agonist, $\mathrm{U}-50,488 \mathrm{H}$, on formalin-evoked cutaneous pain in the rat. European Journal of Pharmacology, 190(3), 287-293. doi:10.1016/00142999(90)94192-Z

Peng, J., Sarkar, S., \& Chang, S. L. (2012). Opioid receptor expression in human brain and peripheral tissues using absolute quantitative real-time RT-PCR. Drug and Alcohol Dependence, 124(3), 223-228. doi:10.1016/j.drugalcdep.2012.01.013

Pereira, V., Busserolles, J., Christin, M., Devilliers, M., Poupon, L., Legha, W., Alloui, A., Aissouni, Y., Bourinet, E., Lesage, F., Eschalier, A., Lazdunski, M., \& Noel, J. (2014). Role of the TREK2 potassium channel in cold and warm thermosensation and in pain perception. Pain, 155(12), 2534-2544. doi:10.1016/j.pain.2014.09.013

Pertwee, R. G. (2007). GPR55: a new member of the cannabinoid receptor clan? British Journal of Pharmacology, 152(7), 984-986. doi:10.1038/sj.bjp.0707464

Pertwee, R. G. (2012). Targeting the endocannabinoid system with cannabinoid receptor agonists: pharmacological strategies and therapeutic possibilities. Philosophical Transactions of the 
Royal Society of London. Series B: Biological Sciences, 367(1607), 3353-3363. doi:10.1098/rstb.2011.0381

Peskar, B. M. (2001). Role of cyclooxygenase isoforms in gastric mucosal defence. Journal of Physiology, Paris, 95(1-6), 3-9. doi:10.1016/S0928-4257(01)00003-1

Peters, C. M. (2007). Intravenous paclitaxel administration in the rat induces a peripheral sensory neuropathy characterized by macrophage infiltration and injury to sensory neurons and their supporting cells. Experimental Neurology, 203. doi:10.1016/j.expneurol.2006.07.022

Peterson, L. D., Jeffery, N. M., Thies, F., Sanderson, P., Newsholme, E. A., \& Calder, P. C. (1998). Eicosapentaenoic and docosahexaenoic acids alter rat spleen leukocyte fatty acid composition and prostaglandin E2 production but have different effects on lymphocyte functions and cellmediated immunity. Lipids, 33(2), 171-180. doi:10.1007/s11745-998-0193-y

Petty, R. E., Hunt, D. W., Mathers, D. M., McCormick, A. Q., Barker, H., Southwood, T. R., \& Corson, L. (1994). Experimental arthritis and uveitis in rats associated with Mycobacterium butyricum. Journal of Rheumatology, 21(8), 1491-1496.

Phinney, S. D., Clarke, S. D., Odin, R. S., Moldawer, L. L., Blackburn, G. L., \& Bistrian, B. R. (1993). Thermogenesis secondary to transdermal water loss causes growth retardation in essential fatty acid-deficient rats. Metabolism, 42(8), 1022-1026. doi:10.1016/0026-0495(93)90017-I

Pinho-Ribeiro, F. A., Verri, W. A., Jr., \& Chiu, I. M. (2017). Nociceptor sensory neuron-immune interactions in pain and inflammation. Trends in Immunology, 38(1), 5-19. doi:10.1016/j.it.2016.10.001

Pliakas, A. M., Carlson, R. R., Neve, R. L., Konradi, C., Nestler, E. J., \& Carlezon, W. A., Jr. (2001). Altered responsiveness to cocaine and increased immobility in the forced swim test associated with elevated CAMP response element-binding protein expression in nucleus accumbens. Journal of Neuroscience, 21(18), 7397-7403. doi:10.1523/JNEUROSCI.21-18-07397.2001

Plourde, M., \& Cunnane, S. C. (2007). Extremely limited synthesis of long chain polyunsaturates in adults: implications for their dietary essentiality and use as supplements. Applied Physiology, Nutrition, and Metabolism, 32(4), 619-634. doi:10.1139/H07-034

Pogozheva, I. D., Przydzial, M. J., \& Mosberg, H. I. (2005). Homology modeling of opioid receptor-ligand complexes using experimental constraints. The AAPS Journal, 7(2), E434-448. doi:10.1208/aapsj070243

Porreca, F., Mosberg, H. I., Hurst, R., Hruby, V. J., \& Burks, T. F. (1984). Roles of mu, delta and kappa opioid receptors in spinal and supraspinal mediation of gastrointestinal transit effects and hotplate analgesia in the mouse. Journal of Pharmacology and Experimental Therapeutics, 230(2), 341-348.

Prevatt-Smith, K. M., Lovell, K. M., Simpson, D. S., Day, V. W., Douglas, J. T., Bosch, P., Dersch, C. M., Rothman, R. B., Kivell, B., \& Prisinzano, T. E. (2011). Potential Drug Abuse Therapeutics Derived from the Hallucinogenic Natural Product Salvinorin A. MedChemComm, 2(12), 1217-1222. doi:10.1039/C1MD00192B

Price, M. P., Mcllwrath, S. L., Xie, J., Cheng, C., Qiao, J., Tarr, D. E., Sluka, K. A., Brennan, T. J., Lewin, G. R., \& Welsh, M. J. (2001). The DRASIC cation channel contributes to the detection of cutaneous touch and acid stimuli in mice. Neuron, 32(6), 1071-1083. doi:10.1016/S0896-6273(01)005475

Prior, R., Van Helleputte, L., Benoy, V., \& Van Den Bosch, L. (2017). Defective axonal transport: A common pathological mechanism in inherited and acquired peripheral neuropathies. Neurobiology of Disease, 105, 300-320. doi:10.1016/j.nbd.2017.02.009

Prisinzano, T. E. (2005). Psychopharmacology of the hallucinogenic sage Salvia divinorum. Life Sciences, 78(5), 527-531. doi:10.1016/j.lfs.2005.09.008

Privette, T. H., \& Terrian, D. M. (1995). Kappa opioid agonists produce anxiolytic-like behavior on the elevated plus-maze. Psychopharmacology, 118(4), 444-450. doi:10.1007/BF02245945 
Proudfoot, C. J., Garry, E. M., Cottrell, D. F., Rosie, R., Anderson, H., Robertson, D. C., FleetwoodWalker, S. M., \& Mitchell, R. (2006). Analgesia mediated by the TRPM8 cold receptor in chronic neuropathic pain. Current Biology, 16(16), 1591-1605. doi:10.1016/j.cub.2006.07.061

Provencher, B. A., Sromek, A. W., Li, W., Russell, S., Chartoff, E., Knapp, B. I., Bidlack, J. M., \& Neumeyer, J. L. (2013). Synthesis and pharmacological evaluation of aminothiazolomorphinans at the mu and kappa opioid receptors. Journal of Medicinal Chemistry, 56(21), 8872-8878. doi:10.1021/jm401290y

Puehler, W., Rittner, H. L., Mousa, S. A., Brack, A., Krause, H., Stein, C., \& Schafer, M. (2006). Interleukin-1 beta contributes to the upregulation of kappa opioid receptor mrna in dorsal root ganglia in response to peripheral inflammation. Neuroscience, 141(2), 989-998. doi:10.1016/j.neuroscience.2006.03.078

Qureshi, A. A., Hosoi, J., Xu, S., Takashima, A., Granstein, R. D., \& Lerner, E. A. (1996). Langerhans cells express inducible nitric oxide synthase and produce nitric oxide. Journal of Investigative Dermatology, 107(6), 815-821. doi:10.1111/1523-1747.ep12330572

Racine, M., Tousignant-Laflamme, Y., Kloda, L. A., Dion, D., Dupuis, G., \& Choiniere, M. (2012). A systematic literature review of 10 years of research on sex/gender and experimental pain perception - part 1: are there really differences between women and men? Pain, 153(3), 602618. doi:10.1016/j.pain.2011.11.025

Radhakrishnan, R., Moore, S. A., \& Sluka, K. A. (2003). Unilateral carrageenan injection into muscle or joint induces chronic bilateral hyperalgesia in rats. Pain, 104(3), 567-577. doi:10.1016/S03043959(03)00114-3

Rahn, E. J., Zvonok, A. M., Thakur, G. A., Khanolkar, A. D., Makriyannis, A., \& Hohmann, A. G. (2008). Selective activation of cannabinoid CB2 receptors suppresses neuropathic nociception induced by treatment with the chemotherapeutic agent paclitaxel in rats. Journal of Pharmacology and Experimental Therapeutics, 327(2), 584-591. doi:10.1124/jpet.108.141994

Ramirez-Maestre, C., \& Esteve, R. (2014). The role of sex/gender in the experience of pain: resilience, fear, and acceptance as central variables in the adjustment of men and women with chronic pain. Journal of Pain, 15(6), 608-618 e601. doi:10.1016/j.jpain.2014.02.006

Randall, L. O., \& Selitto, J. J. (1957). A method for measurement of analgesic activity on inflamed tissue. Archives Internationales de Pharmacodynamie et Therapie, 111(4), 409-419.

Ranganathan, M., Schnakenberg, A., Skosnik, P. D., Cohen, B. M., Pittman, B., Sewell, R. A., \& D'Souza, D. C. (2012). Dose-related behavioral, subjective, endocrine, and psychophysiological effects of the kappa opioid agonist Salvinorin A in humans. Biological Psychiatry, 72(10), 871-879. doi:10.1016/j.biopsych.2012.06.012

Rankovic, Z., Brust, T. F., \& Bohn, L. M. (2016). Biased agonism: An emerging paradigm in GPCR drug discovery. Bioorganic and Medicinal Chemistry Letters, 26(2), 241-250. doi:10.1016/j.bmcl.2015.12.024

Rao, R. D., Flynn, P. J., Sloan, J. A., Wong, G. Y., Novotny, P., Johnson, D. B., Gross, H. M., Renno, S. I., Nashawaty, M., \& Loprinzi, C. L. (2008). Efficacy of lamotrigine in the management of chemotherapy-induced peripheral neuropathy: a phase 3 randomized, double-blind, placebocontrolled trial, N01C3. Cancer, 112(12), 2802-2808. doi:10.1002/cncr.23482

Rao, R. D., Michalak, J. C., Sloan, J. A., Loprinzi, C. L., Soori, G. S., Nikcevich, D. A., Warner, D. O., Novotny, P., Kutteh, L. A., Wong, G. Y., \& North Central Cancer Treatment, G. (2007). Efficacy of gabapentin in the management of chemotherapy-induced peripheral neuropathy: a phase 3 randomized, double-blind, placebo-controlled, crossover trial (NOOC3). Cancer, 110(9), 2110-2118. doi:10.1002/cncr.23008

Rasakham, K., \& Liu-Chen, L. Y. (2011). Sex differences in kappa opioid pharmacology. Life Sciences, 88(1-2), 2-16. doi:10.1016/j.lfs.2010.10.007

Rech, R. H., Briggs, S. L., \& Mokler, D. J. (2010). Fentanyl and spiradoline interactions in a placeconditioning black-white shuttle-box. Pharmaceuticals, 4(1), 101-116. doi:10.3390/ph401101 
Rech, R. H., Mokler, D. J., \& Briggs, S. L. (2012). Effects of combined opioids on pain and mood in mammals. Pain Research and Treatment, 2012, 145965. doi:10.1155/2012/145965

Reed, D. E., \& Vanner, S. J. (2017). Emerging studies of human visceral nociceptors. American Journal of Physiology: Gastrointestinal and Liver Physiology, 312(3), G201-G207. doi:10.1152/ajpgi.00391.2016

Ren, M. F., Lu, C. H., \& Han, J. S. (1985). Dynorphin-A-(1-13) antagonizes morphine analgesia in the brain and potentiates morphine analgesia in the spinal cord. Peptides, 6(6), 1015-1020. doi:10.1016/0196-9781(85)90423-1

Reynolds, D. V. (1969). Surgery in the rat during electrical analgesia induced by focal brain stimulation. Science, 164(3878), 444-445. doi:10.1126/science.164.3878.444

Riley, A. P., Groer, C. E., Young, D., Ewald, A. W., Kivell, B. M., \& Prisinzano, T. E. (2014). Synthesis and kappa-opioid receptor activity of furan-substituted salvinorin $A$ analogues. Journal of Medicinal Chemistry, 57(24), 10464-10475. doi:10.1021/jm501521d

Rittner, H. I., \& Stein, C. (2005). Involvement of cytokines, chemokines and adhesion molecules in opioid analgesia. European Journal of Pain, 9(2), 109-112. doi:10.1016/j.ejpain.2004.05.009

Rivière, P. J. M. (2004). Peripheral kappa-opioid agonists for visceral pain. British Journal of Pharmacology, 141(8), 1331-1334. doi:10.1038/sj.bjp.0705763

Rizzuto, R., De Stefani, D., Raffaello, A., \& Mammucari, C. (2012). Mitochondria as sensors and regulators of calcium signalling. Nature Reviews Molecular Cell Biology, 13(9), 566-578. doi:10.1038/nrm3412

Roberts, M. H., \& Rees, H. (1986). The antinociceptive effects of stimulating the pretectal nucleus of the rat. Pain, 25(1), 83-93. doi:10.1016/0304-3959(86)90011-4

Roeckel, L. A., Le Coz, G. M., Gaveriaux-Ruff, C., \& Simonin, F. (2016). Opioid-induced hyperalgesia: Cellular and molecular mechanisms. Neuroscience, 338, 160-182. doi:10.1016/j.neuroscience.2016.06.029

Romero, T. R., \& Duarte, I. D. (2012). N-palmitoyl-ethanolamine (PEA) induces peripheral antinociceptive effect by ATP-sensitive K+-channel activation. Journal of Pharmacological Sciences, 118(2), 156-160. doi:10.1254/jphs.11150FP

Rosland, J. H., Tjolsen, A., Maehle, B., \& Hole, K. (1990). The formalin test in mice: effect of formalin concentration. Pain, 42(2), 235-242. doi:10.1016/0304-3959(90)91167-H

Ross, R. A. (2003). Anandamide and vanilloid TRPV1 receptors. British Journal of Pharmacology, 140(5), 790-801. doi:10.1038/sj.bjp.0705467

Rossi, A., Pace, S., Tedesco, F., Pagano, E., Guerra, G., Troisi, F., Werner, M., Roviezzo, F., Zjawiony, J. K., Werz, O., Izzo, A. A., \& Capasso, R. (2016). The hallucinogenic diterpene salvinorin A inhibits leukotriene synthesis in experimental models of inflammation. Pharmacological Research, 106, 64-71. doi:10.1016/j.phrs.2016.01.032

Roth, B. L., Baner, K., Westkaemper, R., Siebert, D., Rice, K. C., Steinberg, S., Ernsberger, P., \& Rothman, R. B. (2002). Salvinorin A: a potent naturally occurring nonnitrogenous kappa opioid selective agonist. Proceedings of the National Academy of Sciences of the United States of America, 99(18), 11934-11939. doi:10.1073/pnas.182234399

Rothman, S. (1941). Physiology of itching. Physiological Reviews, 21(2), 357-381.

Rowinsky, E. K., Chaudhry, V., Forastiere, A. A., Sartorius, S. E., Ettinger, D. S., Grochow, L. B., Lubejko, B. G., Cornblath, D. R., \& Donehower, R. C. (1993). Phase I and pharmacologic study of paclitaxel and cisplatin with granulocyte colony-stimulating factor: neuromuscular toxicity is dose-limiting. Journal of Clinical Oncology, 11(10), 2010-2020. doi:10.1200/JCO.1993.11.10.2010

Ruda, M. A., ladarola, M. J., Cohen, L. V., \& Young, W. S., 3rd. (1988). In situ hybridization histochemistry and immunocytochemistry reveal an increase in spinal dynorphin biosynthesis in a rat model of peripheral inflammation and hyperalgesia. Proceedings of the National Academy of Sciences of the United States of America, 85(2), 622-626. 
Rudd, R. A., Seth, P., David, F., \& Scholl, L. (2016). Increases in drug and opioid-involved overdose deaths - United States, 2010-2015. MMWR: Morbidity and Mortality Weekly Report, 65(5051), 1445-1452. doi:10.15585/mmwr.mm655051e1

Rusin, K. I., Giovannucci, D. R., Stuenkel, E. L., \& Moises, H. C. (1997). Kappa-opioid receptor activation modulates $\mathrm{Ca} 2+$ currents and secretion in isolated neuroendocrine nerve terminals. Journal of Neuroscience, 17(17), 6565-6574. doi:10.1523/JNEUROSCI.17-17-06565.1997

Russell, W. M. S., \& Burch, R. L. (1959). The Principles of Humane Experimental Technique. London: Methuen.

Saddi, G., \& Abbott, F. V. (2000). The formalin test in the mouse: a parametric analysis of scoring properties. Pain, 89(1), 53-63. doi:10.1016/S0304-3959(00)00348-1

Safrany-Fark, A., Petrovszki, Z., Kekesi, G., Keresztes, C., Benedek, G., \& Horvath, G. (2015). Telemetry monitoring for non-invasive assessment of changes in core temperature after spinal drug administration in freely moving rats. Journal of Pharmacological and Toxicological Methods, 72, 19-25. doi:10.1016/j.vascn.2015.01.002

Sałaga, M., Polepally, P. R., Sobczak, M., Grzywacz, D., Kamysz, W., Sibaev, A., Storr, M., Do Rego, J. C., Zjawiony, J. K., \& Fichna, J. (2014). Novel orally available Salvinorin A analog PR-38 inhibits gastrointestinal motility and reduces abdominal pain in mouse models mimicking irritable bowel syndrome. Journal of Pharmacology and Experimental Therapeutics, 350(1), 69. doi:10.1124/jpet.114.214239

Samieri, C., Feart, C., Letenneur, L., Dartigues, J. F., Peres, K., Auriacombe, S., Peuchant, E., Delcourt, C., \& Barberger-Gateau, P. (2008). Low plasma eicosapentaenoic acid and depressive symptomatology are independent predictors of dementia risk. American Journal of Clinical Nutrition, 88(3), 714-721. doi:10.1093/ajen/88.3.714

Samimi, M., Jamilian, M., Asemi, Z., \& Esmaillzadeh, A. (2015). Effects of omega-3 fatty acid supplementation on insulin metabolism and lipid profiles in gestational diabetes: Randomized, double-blind, placebo-controlled trial. Clinical Nutrition, 34(3), 388-393. doi:10.1016/j.clnu.2014.06.005

Samineni, V. K., Premkumar, L. S., \& Faingold, C. L. (2017). Neuropathic pain-induced enhancement of spontaneous and pain-evoked neuronal activity in the periaqueductal gray that is attenuated by gabapentin. Pain, 158(7), 1241-1253. doi:10.1097/j.pain.0000000000000905

Sapunar, D., Kostic, S., Banozic, A., \& Puljak, L. (2012). Dorsal root ganglion - a potential new therapeutic target for neuropathic pain. Journal of Pain Research, 5, 31-38. doi:10.2147/JPR.S26603

Schaible, H. G., Richter, F., Ebersberger, A., Boettger, M. K., Vanegas, H., Natura, G., Vazquez, E., \& Segond von Banchet, G. (2009). Joint pain. Experimental Brain Research, 196(1), 153-162. doi:10.1007/s00221-009-1782-9

Schattauer, S. S., Kuhar, J. R., Song, A., \& Chavkin, C. (2017). Nalfurafine is a G-protein biased agonist having significantly greater bias at the human than rodent form of the kappa opioid receptor. Cellular Signalling, 32, 59-65. doi:10.1016/j.cellsig.2017.01.016

Schenk, S., Partridge, B., \& Shippenberg, T. S. (1999). U69593, a kappa-opioid agonist, decreases cocaine self-administration and decreases cocaine-produced drug-seeking. Psychopharmacology, 144(4), 339-346. doi:10.1007/s002130051016

Schenk, S., Partridge, B., \& Shippenberg, T. S. (2001). Effects of the kappa-opioid receptor agonist, U69593, on the development of sensitization and on the maintenance of cocaine selfadministration. Neuropsychopharmacology, 24(4), 441-450. doi:10.1016/S0893$133 \times(00) 00190-1$

Schiff, P. B., Fant, J., \& Horwitz, S. B. (1979). Promotion of microtubule assembly in vitro by taxol. Nature, 277(5698), 665-667. doi:10.1038/277665a0

Schmidt, M. D., Schmidt, M. S., Butelman, E. R., Harding, W. W., Tidgewell, K., Murry, D. J., Kreek, M. J., \& Prisinzano, T. E. (2005a). Pharmacokinetics of the plant-derived kappa-opioid 
hallucinogen salvinorin $A$ in nonhuman primates. Synapse, 58(3), 208-210. doi:10.1002/syn.20191

Schmidt, M. S., Prisinzano, T. E., Tidgewell, K., Harding, W., Butelman, E. R., Kreek, M. J., \& Murry, D. J. (2005b). Determination of Salvinorin $A$ in body fluids by high performance liquid chromatography-atmospheric pressure chemical ionization. Journal of Chromatography. B: Analytical Technologies in the Biomedical and Life Sciences, 818(2), 221-225. doi:10.1016/j.jchromb.2004.12.041

Schulz, R., Wehmeyer, A., \& Schulz, K. (2002). Visualizing preference of G protein-coupled receptor kinase 3 for the process of kappa-opioid receptor sequestration. Molecular Pharmacology, 61(6), 1444-1452. doi:10.1124/mol.61.6.1444

Seely, K. A., Brents, L. K., Franks, L. N., Rajasekaran, M., Zimmerman, S. M., Fantegrossi, W. E., \& Prather, P. L. (2012). AM-251 and rimonabant act as direct antagonists at mu-opioid receptors: implications for opioid/cannabinoid interaction studies. Neuropharmacology, 63(5), 905-915. doi:10.1016/j.neuropharm.2012.06.046

Seki, T., Awamura, S., Kimura, C., Ide, S., Sakano, K., Minami, M., Nagase, H., \& Satoh, M. (1999). Pharmacological properties of TRK-820 on cloned mu-, delta- and kappa-opioid receptors and nociceptin receptor. European Journal of Pharmacology, 376(1-2), 159-167. doi:10.1016/S0014-2999(99)00369-6

Seltzman, H. H., Shiner, C., Hirt, E. E., Gilliam, A. F., Thomas, B. F., Maitra, R., Snyder, R., Black, S. L., Patel, P. R., Mulpuri, Y., \& Spigelman, I. (2016). Peripherally selective cannabinoid 1 receptor (CB1R) agonists for the treatment of neuropathic pain. Journal of Medicinal Chemistry, 59(16), 7525-7543. doi:10.1021/acs.jmedchem.6b00516

Seretny, M., Currie, G. L., Sena, E. S., Ramnarine, S., Grant, R., MacLeod, M. R., Colvin, L. A., \& Fallon, M. (2014). Incidence, prevalence, and predictors of chemotherapy-induced peripheral neuropathy: A systematic review and meta-analysis. Pain, 155(12), 2461-2470. doi:10.1016/j.pain.2014.09.020

Serhan, C. N., Chiang, N., \& Van Dyke, T. E. (2008). Resolving inflammation: dual anti-inflammatory and pro-resolution lipid mediators. Nature Reviews: Immunology, 8(5), 349-361. doi:10.1038/nri2294

Serhan, C. N., Clish, C. B., Brannon, J., Colgan, S. P., Chiang, N., \& Gronert, K. (2000). Novel functional sets of lipid-derived mediators with antiinflammatory actions generated from omega-3 fatty acids via cyclooxygenase 2-nonsteroidal antiinflammatory drugs and transcellular processing. Journal of Experimental Medicine, 192(8), 1197-1204. doi:10.1084/jem.192.8.1197

Serhan, C. N., Hong, S., Gronert, K., Colgan, S. P., Devchand, P. R., Mirick, G., \& Moussignac, R. L. (2002). Resolvins: a family of bioactive products of omega-3 fatty acid transformation circuits initiated by aspirin treatment that counter proinflammation signals. Journal of Experimental Medicine, 196(8), 1025-1037. doi:10.1084/jem.20020760

Shaw, J. S., Carroll, J. A., Alcock, P., \& Main, B. G. (1989). ICI 204448: a k-opioid agonist with limited access to the CNS. British Journal of Pharmacology, 96(4), 986-992. doi:10.1111/j.14765381.1989.tb11911.x

Sherrington, C. (1906). Yale University Mrs. Hepsa Ely Silliman memorial lectures. The integrative action of the nervous system. New Haven, CT, US: Yale University Press. doi:10.1037/13798000

Sherwood, A. M., Crowley, R. S., Paton, K. F., Biggerstaff, A., Neuenswander, B., Day, V. W., Kivell, B. M., \& Prisinzano, T. E. (2017a). Addressing Structural Flexibility at the A-Ring on Salvinorin A: Discovery of a Potent Kappa-Opioid Agonist with Enhanced Metabolic Stability. Journal of Medicinal Chemistry, 60(9), 3866-3878. doi:10.1021/acs.jmedchem.7b00148

Sherwood, A. M., Williamson, S. E., Crowley, R. S., Abbott, L. M., Day, V. W., \& Prisinzano, T. E. (2017b). Modular approach to pseudo-neoclerodanes as designer kappa-opioid ligands. Organic Letters, 19(19), 5414-5417. doi:10.1021/acs.orglett.7b02684 
Sheskin, T., Hanus, L., Slager, J., Vogel, Z., \& Mechoulam, R. (1997). Structural requirements for binding of anandamide-type compounds to the brain cannabinoid receptor. Journal of Medicinal Chemistry, 40(5), 659-667. doi:10.1021/jm960752x

Shibata, M., Ohkubo, T., Takahashi, H., \& Inoki, R. (1989). Modified formalin test: characteristic biphasic pain response. Pain, 38(3), 347-352. doi:10.1016/0304-3959(89)90222-4

Shimizu, S. (2004). Routes of administration The laboratory mouse (pp. 527-541): Elsevier.

Shinto, L., Quinn, J., Montine, T., Dodge, H. H., Woodward, W., Baldauf-Wagner, S., Waichunas, D., Bumgarner, L., Bourdette, D., Silbert, L., \& Kaye, J. (2014). A randomized placebo-controlled pilot trial of omega-3 fatty acids and alpha lipoic acid in Alzheimer's disease. Journal of Alzheimer's Disease, 38(1), 111-120. doi:10.3233/JAD-130722

Shivaperumal, N. (2017). Investigating the analgesic properties of Kurkinorin, a novel mu-opioid receptor analogue of Salvinorin A. Master's degree in Biomedical Science, Vicotoria University of Wellington.

Shook, J. E., Watkins, W. D., \& Camporesi, E. M. (1990). Differential roles of opioid receptors in respiration, respiratory disease, and opiate-induced respiratory depression. American Review of Respiratory Disease, 142(4), 895-909. doi:10.1164/ajrccm/142.4.895

Siau, C., \& Bennett, G. J. (2006). Dysregulation of cellular calcium homeostasis in chemotherapyevoked painful peripheral neuropathy. Anesthesia \& Analgesia, 102(5), 1485-1490. doi:10.1213/01.ane.0000204318.35194.ed

Siau, C., Xiao, W., \& Bennett, G. J. (2006). Paclitaxel- and vincristine-evoked painful peripheral neuropathies: loss of epidermal innervation and activation of Langerhans cells. Experimental Neurology, 201(2), 507-514. doi:10.1016/j.expneurol.2006.05.007

Siebert, D. J. (1994). Salvia divinorum and salvinorin A: new pharmacologic findings. Journal of Ethnopharmacology, 43(1), 53-56. doi:10.1016/0378-8741(94)90116-3

Siemens, J., Zhou, S., Piskorowski, R., Nikai, T., Lumpkin, E. A., Basbaum, A. I., King, D., \& Julius, D. (2006). Spider toxins activate the capsaicin receptor to produce inflammatory pain. Nature, 444(7116), 208-212. doi:10.1038/nature05285

Sierra, S., Gomes, I., \& Devi, L. A. (2017). Class A GPCRs: Cannabinoid and opioid receptor heteromers. In K. Herrick-Davis, G. Milligan, \& G. Di Giovanni (Eds.), G-Protein-Coupled Receptor Dimers (pp. 173-206). Cham: Springer International Publishing.

Sikand, P., \& Premkumar, L. S. (2007). Potentiation of glutamatergic synaptic transmission by protein kinase C-mediated sensitization of TRPV1 at the first sensory synapse. Journal of Physiology, 581(Pt 2), 631-647. doi:10.1113/jphysiol.2006.118620

Sikandar, S., \& Dickenson, A. H. (2012). Visceral pain: the ins and outs, the ups and downs. Current Opinion in Supportive and Palliative Care, 6(1), 17-26. doi:10.1097/SPC.0b013e32834f6ec9

Simon-Arceo, K., Gonzalez-Trujano, M. E., Coffeen, U., Fernandez-Mas, R., Mercado, F., Almanza, A., Contreras, B., Jaimes, O., \& Pellicer, F. (2017). Neuropathic and inflammatory antinociceptive effects and electrocortical changes produced by Salvia divinorum in rats. Journal of Ethnopharmacology, 206, 115-124. doi:10.1016/j.jep.2017.05.016

Simone, D. A., Baumann, T. K., \& LaMotte, R. H. (1989). Dose-dependent pain and mechanical hyperalgesia in humans after intradermal injection of capsaicin. Pain, 38(1), 99-107. doi:10.1016/0304-3959(89)90079-1

Simonin, F., Gaveriaux-Ruff, C., Befort, K., Matthes, H., Lannes, B., Micheletti, G., Mattei, M. G., Charron, G., Bloch, B., \& Kieffer, B. (1995). kappa-Opioid receptor in humans: cDNA and genomic cloning, chromosomal assignment, functional expression, pharmacology, and expression pattern in the central nervous system. Proceedings of the National Academy of Sciences of the United States of America, 92(15), 7006-7010. doi:10.1073/pnas.92.15.7006

Simonin, F., Valverde, O., Smadja, C., Slowe, S., Kitchen, I., Dierich, A., Le Meur, M., Roques, B. P., Maldonado, R., \& Kieffer, B. L. (1998). Disruption of the kappa-opioid receptor gene in mice enhances sensitivity to chemical visceral pain, impairs pharmacological actions of the selective 
kappa-agonist U-50,488H and attenuates morphine withdrawal. EMBO Journal, 17(4), 886897. doi:10.1093/emboj/17.4.886

Simonson, B., Morani, A. S., Ewald, A. W., Walker, L., Kumar, N., Simpson, D., Miller, J. H., Prisinzano, T. E., \& Kivell, B. M. (2015). Pharmacology and anti-addiction effects of the novel kappa opioid receptor agonist Mesyl Sal B, a potent and long-acting analogue of salvinorin A. British Journal of Pharmacology, 172(2), 515-531. doi:10.1111/bph.12692

Sinclair, A. J. (1975). Incorporation of Radioactive Polyunsaturated Fatty-Acids into Liver and Brain of Developing Rat. Lipids, 10(3), 175-184. doi:10.1007/Bf02534156

Sinn, N., Milte, C. M., Street, S. J., Buckley, J. D., Coates, A. M., Petkov, J., \& Howe, P. R. (2012). Effects of $n-3$ fatty acids, EPA v. DHA, on depressive symptoms, quality of life, memory and executive function in older adults with mild cognitive impairment: a 6-month randomised controlled trial. British Journal of Nutrition, 107(11), 1682-1693. doi:10.1017/S0007114511004788

Siscovick, D. S., Barringer, T. A., Fretts, A. M., Wu, J. H., Lichtenstein, A. H., Costello, R. B., Kris-Etherton, P. M., Jacobson, T. A., Engler, M. B., Alger, H. M., Appel, L. J., Mozaffarian, D., American Heart Association Nutrition Committee of the Council on Lifestyle and Cardiometabolic Health, Council on Epidemiology and Prevention, Council on Cardiovascular Disease in the Young, Council on Cardiovascular and Stroke Nursing, \& Council on Clinical Cardiology. (2017). Omega-3 polyunsaturated fatty acid (fish oil) supplementation and the prevention of clinical cardiovascular disease: A science advisory from the American Heart Association. Circulation, 135(15), e867-e884. doi:10.1161/CIR.0000000000000482

Sisignano, M., Baron, R., Scholich, K., \& Geisslinger, G. (2014). Mechanism-based treatment for chemotherapy-induced peripheral neuropathic pain. Nature Reviews: Neurology, 10(12), 694707. doi:10.1038/nrneurol.2014.211

Skaper, S. D. (2017). Mast cells and glia as targets for the anandamide congener palmitoylethanolamide: an anti-inflammatory and neuroprotective lipid signaling molecule. In M. Melis (Ed.), Endocannabinoids and Lipid Mediators in Brain Functions (pp. 347-369). Cham: Springer International Publishing.

Skaper, S. D., Facci, L., \& Giusti, P. (2013). Glia and mast cells as targets for palmitoylethanolamide, an anti-inflammatory and neuroprotective lipid mediator. Molecular Neurobiology, 48(2), 340352. doi:10.1007/s12035-013-8487-6

Skoubis, P. D., Matthes, H. W., Walwyn, W. M., Kieffer, B. L., \& Maidment, N. T. (2001). Naloxone fails to produce conditioned place aversion in mu-opioid receptor knock-out mice. Neuroscience, 106(4), 757-763. doi:10.1016/S0306-4522(01)00333-5

Smith, E. M., Pang, H., Cirrincione, C., Fleishman, S., Paskett, E. D., Ahles, T., Bressler, L. R., Fadul, C. E., Knox, C., Le-Lindqwister, N., Gilman, P. B., Shapiro, C. L., \& Alliance for Clinical Trials in Oncology. (2013). Effect of duloxetine on pain, function, and quality of life among patients with chemotherapy-induced painful peripheral neuropathy: a randomized clinical trial. JAMA, 309(13), 1359-1367. doi:10.1001/jama.2013.2813

Smith, P. B., Compton, D. R., Welch, S. P., Razdan, R. K., Mechoulam, R., \& Martin, B. R. (1994). The pharmacological activity of anandamide, a putative endogenous cannabinoid, in mice. Journal of Pharmacology and Experimental Therapeutics, 270(1), 219-227.

Smith, S. B., Crager, S. E., \& Mogil, J. S. (2004). Paclitaxel-induced neuropathic hypersensitivity in mice: responses in 10 inbred mouse strains. Life Sciences, 74(21), 2593-2604. doi:10.1016/j.lfs.2004.01.002

Smith, W. L., Meade, E. A., \& Dewitt, D. L. (1997). Interaction of PGH synthase isozymes-1 and -2 with nonsteroidal anti-inflammatory drugs. Advances in Experimental Medicine and Biology, 400A, 189-196.

Smith, Y. R., Stohler, C. S., Nichols, T. E., Bueller, J. A., Koeppe, R. A., \& Zubieta, J. K. (2006). Pronociceptive and antinociceptive effects of estradiol through endogenous opioid neurotransmission in women. Journal of Neuroscience, 26(21), 5777-5785. doi:10.1523/JNEUROSCI.5223-05.2006 
Sobanski, P., Krajnik, M., Shaqura, M., Bloch-Boguslawska, E., Schafer, M., \& Mousa, S. A. (2014). The presence of mu-, delta-, and kappa-opioid receptors in human heart tissue. Heart and Vessels, 29(6), 855-863. doi:10.1007/s00380-013-0456-5

Soeberdt, M., Molenveld, P., Storcken, R. P., Bouzanne des Mazery, R., Sterk, G. J., Autar, R., Bolster, M. G., Wagner, C., Aerts, S. N., van Holst, F. R., Wegert, A., Tangherlini, G., Frehland, B., Schepmann, D., Metze, D., Lotts, T., Knie, U., Lin, K. Y., Huang, T. Y., Lai, C. C., Stander, S., Wunsch, B., \& Abels, C. (2017). Design and synthesis of enantiomerically pure decahydroquinoxalines as potent and selective kappa-opioid receptor agonists with antiinflammatory activity in vivo. Journal of Medicinal Chemistry, 60(6), 2526-2551. doi:10.1021/acs.jmedchem.6b01868

Sonti, S., Duclos, R. I., Jr., Tolia, M., \& Gatley, S. J. (2017). N-Docosahexaenoylethanolamine (synaptamide): Carbon-14 radiolabeling and metabolic studies. Chemistry and Physics of Lipids. doi:10.1016/j.chemphyslip.2017.11.002

Sorge, R. E., Martin, L. J., Isbester, K. A., Sotocinal, S. G., Rosen, S., Tuttle, A. H., Wieskopf, J. S., Acland, E. L., Dokova, A., Kadoura, B., Leger, P., Mapplebeck, J. C., McPhail, M., Delaney, A., Wigerblad, G., Schumann, A. P., Quinn, T., Frasnelli, J., Svensson, C. I., Sternberg, W. F., \& Mogil, J. S. (2014). Olfactory exposure to males, including men, causes stress and related analgesia in rodents. Nature Methods, 11(6), 629-632. doi:10.1038/nmeth.2935

Sotocinal, S. G., Sorge, R. E., Zaloum, A., Tuttle, A. H., Martin, L. J., Wieskopf, J. S., Mapplebeck, J. C., Wei, P., Zhan, S., Zhang, S., McDougall, J. J., King, O. D., \& Mogil, J. S. (2011). The Rat Grimace Scale: a partially automated method for quantifying pain in the laboratory rat via facial expressions. Molecular Pain, 7(1), 55. doi:10.1186/1744-8069-7-55

Sounvoravong, S., Takahashi, M., Nakashima, M. N., \& Nakashima, K. (2004). Disability of development of tolerance to morphine and $\mathrm{U}-50,488 \mathrm{H}$, a selective kappa-opioid receptor agonist, in neuropathic pain model mice. Journal of Pharmacological Sciences, 94(3), 305-312. doi:10.1254/jphs.94.305

Spencer, R. L., Hruby, V. J., \& Burks, T. F. (1988). Body temperature response profiles for selective mu, delta and kappa opioid agonists in restrained and unrestrained rats. Journal of Pharmacology and Experimental Therapeutics, 246(1), 92-101.

Spetea, M., Eans, S. O., Ganno, M. L., Lantero, A., Mairegger, M., Toll, L., Schmidhammer, H., \& McLaughlin, J. P. (2017). Selective kappa receptor partial agonist HS666 produces potent antinociception without inducing aversion after i.c.v. administration in mice. British Journal of Pharmacology, 174(15), 2444-2456. doi:10.1111/bph.13854

Sprouse-Blum, A. S., Smith, G., Sugai, D., \& Parsa, F. D. (2010). Understanding endorphins and their importance in pain management. Hawaii Medical Journal, 69(3), 70-71.

St Sauver, J. L., Warner, D. O., Yawn, B. P., Jacobson, D. J., McGree, M. E., Pankratz, J. J., Melton, L. J., 3rd, Roger, V. L., Ebbert, J. O., \& Rocca, W. A. (2013). Why patients visit their doctors: assessing the most prevalent conditions in a defined American population. Mayo Clinic Proceedings, 88(1), 56-67. doi:10.1016/j.mayocp.2012.08.020

Stein, A. T., Ufret-Vincenty, C. A., Hua, L., Santana, L. F., \& Gordon, S. E. (2006). Phosphoinositide 3kinase binds to TRPV1 and mediates NGF-stimulated TRPV1 trafficking to the plasma membrane. Journal of General Physiology, 128(5), 509-522. doi:10.1085/jgp.200609576

Stevens, A. J., \& Higgins, M. D. (2017). A systematic review of the analgesic efficacy of cannabinoid medications in the management of acute pain. Acta Anaesthesiologica Scandinavica, 61(3), 268-280. doi:10.1111/aas.12851

Sufka, K. J. (1994). Conditioned place preference paradigm: a novel approach for analgesic drug assessment against chronic pain. Pain, 58(3), 355-366. doi:10.1016/0304-3959(94)90130-9

Sugiura, T., Kondo, S., Sukagawa, A., Tonegawa, T., Nakane, S., Yamashita, A., Ishima, Y., \& Waku, K. (1996). Transacylase-mediated and phosphodiesterase-mediated synthesis of $\mathrm{N}$ arachidonoylethanolamine, an endogenous cannabinoid-receptor ligand, in rat brain 
microsomes. Comparison with synthesis from free arachidonic acid and ethanolamine. European Journal of Biochemistry, 240(1), 53-62. doi:10.1111/j.1432-1033.1996.0053h.x

Sugiura, T., Tominaga, M., Katsuya, H., \& Mizumura, K. (2002). Bradykinin lowers the threshold temperature for heat activation of vanilloid receptor 1. Journal of Neurophysiology, 88(1), 544-548. doi:10.1152/jn.2002.88.1.544

Suzuki, M., Mizuno, A., Kodaira, K., \& Imai, M. (2003). Impaired pressure sensation in mice lacking TRPV4. Journal of Biological Chemistry, 278(25), 22664-22668. doi:10.1074/jbc.M302561200

Suzuki, T., Shiozaki, Y., Masukawa, Y., Misawa, M., \& Nagase, H. (1992). The role of mu- and kappaopioid receptors in cocaine-induced conditioned place preference. Japanese Journal of Pharmacology, 58(4), 435-442. doi:10.1254/jjp.58.435

Svingos, A. L., Chavkin, C., Colago, E. E., \& Pickel, V. M. (2001). Major coexpression of kappa-opioid receptors and the dopamine transporter in nucleus accumbens axonal profiles. Synapse, 42(3), 185-192. doi:10.1002/syn.10005

Swain, N., \& Johnson, M. (2014). Chronic pain in New Zealand: a community sample. New Zealand Medical Journal, 127(1388), 21-30.

Szmuszkovicz, J., \& Von Voigtlander, P. F. (1982). Benzeneacetamide amines: structurally novel nonm mu opioids. Journal of Medicinal Chemistry, 25(10), 1125-1126. doi:10.1021/jm00352a005

Szolcsanyi, J., Sebok, B., \& Bartho, L. (1985). Capsaicin, sensation and flare reaction: the concept of bidirectional axon reflex. Substance $P$ metabolism and biological actions, 234.

Takaishi, K., Eisele, J. H., Jr., \& Carstens, E. (1996). Behavioral and electrophysiological assessment of hyperalgesia and changes in dorsal horn responses following partial sciatic nerve ligation in rats. Pain, 66(2-3), 297-306. doi:10.1016/0304-3959(96)03023-0

Talavera, K., Gees, M., Karashima, Y., Meseguer, V. M., Vanoirbeek, J. A., Damann, N., Everaerts, W., Benoit, M., Janssens, A., Vennekens, R., Viana, F., Nemery, B., Nilius, B., \& Voets, T. (2009). Nicotine activates the chemosensory cation channel TRPA1. Nature Neuroscience, 12(10), 1293-1299. doi:10.1038/nn.2379

Tang, A. H., \& Collins, R. J. (1985). Behavioral effects of a novel kappa opioid analgesic, U-50488, in rats and rhesus monkeys. Psychopharmacology, 85(3), 309-314. doi:10.1007/BF00428193

Tasnim, A., Rammelkamp, Z., Slusher, A. B., Wozniak, K., Slusher, B. S., \& Farah, M. H. (2016). Paclitaxel causes degeneration of both central and peripheral axon branches of dorsal root ganglia in mice. BMC Neuroscience, 17(1), 47. doi:10.1186/s12868-016-0285-4

Taussig, R., Iniguez-Lluhi, J. A., \& Gilman, A. G. (1993). Inhibition of adenylyl cyclase by Gi alpha. Science, 261(5118), 218-221. doi:10.1126/science.8327893

Taylor, V. A., Chang, L., Rainville, P., \& Roy, M. (2017). Learned expectations and uncertainty facilitate pain during classical conditioning. Pain, 158(8), 1528-1537. doi:10.1097/j.pain.0000000000000948

Teksin, Z. S., Lee, I. J., Nemieboka, N. N., Othman, A. A., Upreti, V. V., Hassan, H. E., Syed, S. S., Prisinzano, T. E., \& Eddington, N. D. (2009). Evaluation of the transport, in vitro metabolism and pharmacokinetics of Salvinorin A, a potent hallucinogen. European Journal of Pharmaceutics and Biopharmaceutics, 72(2), 471-477. doi:10.1016/j.ejpb.2009.01.002

Thangamani, D., Edafiogho, I. O., \& Masocha, W. (2013). The anticonvulsant enaminone E139 attenuates paclitaxel-induced neuropathic pain in rodents. The Scientific World Journal, 2013(Article ID 240508). doi:10.1155/2013/240508

Thibault, K., Van Steenwinckel, J., Brisorgueil, M. J., Fischer, J., Hamon, M., Calvino, B., \& Conrath, M. (2008). Serotonin 5-HT2A receptor involvement and Fos expression at the spinal level in vincristine-induced neuropathy in the rat. Pain, 140(2), 305-322. doi:10.1016/j.pain.2008.09.006

Tidgewell, K., Harding, W. W., Schmidt, M., Holden, K. G., Murry, D. J., \& Prisinzano, T. E. (2004). A facile method for the preparation of deuterium labeled salvinorin A: synthesis of [2,2,2-2H3]salvinorin A. Bioorganic and Medicinal Chemistry Letters, 14(20), 5099-5102. doi:10.1016/j.bmcl.2004.07.081 
Titeler, M., Lyon, R. A., \& Glennon, R. A. (1988). Radioligand binding evidence implicates the brain 5HT2 receptor as a site of action for LSD and phenylisopropylamine hallucinogens. Psychopharmacology, 94(2), 213-216. doi:10.1007/BF00176847

Tjolsen, A., Berge, O. G., Hunskaar, S., Rosland, J. H., \& Hole, K. (1992). The formalin test: an evaluation of the method. Pain, 51(1), 5-17. doi:10.1016/0304-3959(92)90003-T

Tjolsen, A., Lund, A., Berge, O. G., \& Hole, K. (1989). An improved method for tail-flick testing with adjustment for tail-skin temperature. Journal of Neuroscience Methods, 26(3), 259-265. doi:10.1016/0165-0270(89)90124-6

Tjolsen, A., Lund, A., \& Hole, K. (1991). Antinociceptive effect of paracetamol in rats is partly dependent on spinal serotonergic systems. European Journal of Pharmacology, 193(2), 193201. doi:10.1016/0014-2999(91)90036-P

Toblin, R. L., Mack, K. A., Perveen, G., \& Paulozzi, L. J. (2011). A population-based survey of chronic pain and its treatment with prescription drugs. Pain, 152(6), 1249-1255. doi:10.1016/j.pain.2010.12.036

Tominaga, M., Caterina, M. J., Malmberg, A. B., Rosen, T. A., Gilbert, H., Skinner, K., Raumann, B. E., Basbaum, A. I., \& Julius, D. (1998). The cloned capsaicin receptor integrates multiple painproducing stimuli. Neuron, 21(3), 531-543. doi:10.1016/S0896-6273(00)80564-4

Tominaga, M., Wada, M., \& Masu, M. (2001). Potentiation of capsaicin receptor activity by metabotropic ATP receptors as a possible mechanism for ATP-evoked pain and hyperalgesia. Proceedings of the National Academy of Sciences of the United States of America, 98(12), 6951-6956. doi:10.1073/pnas.111025298

Tong, G., Sun, Z., Wei, X., Gu, C., Kaye, A. D., Wang, Y., Li, J., Zhang, Q., Guo, H., Yu, S., Yi, D., \& Pei, J. (2011). U50,488H postconditioning reduces apoptosis after myocardial ischemia and reperfusion. Life Sciences, 88(1-2), 31-38. doi:10.1016/j.Ifs.2010.10.018

Tsou, K., \& Jang, C. S. (1964). Studies on the site of analgesic action of morphine by intracerebral microinjection. Scientia Sinica, 13, 1099-1109.

Tsujino, H., Kondo, E., Fukuoka, T., Dai, Y., Tokunaga, A., Miki, K., Yonenobu, K., Ochi, T., \& Noguchi, K. (2000). Activating transcription factor 3 (ATF3) induction by axotomy in sensory and motoneurons: A novel neuronal marker of nerve injury. Molecular and Cellular Neuroscience, 15(2), 170-182. doi:10.1006/mcne.1999.0814

Tsuno, N., Yukimasa, A., Yoshida, O., Suzuki, S., Nakai, H., Ogawa, T., Fujiu, M., Takaya, K., Nozu, A., Yamaguchi, H., Matsuda, H., Funaki, S., Yamanada, N., Tanimura, M., Nagamatsu, D., Asaki, T., Horita, N., Yamamoto, M., Hinata, M., Soga, M., Imai, M., Morioka, Y., Kanemasa, T., Sakaguchi, G., \& Iso, Y. (2017). Pharmacological evaluation of novel (6-aminopyridin-3-yl)(4(pyridin-2-yl)piperazin-1-yl) methanone derivatives as TRPV4 antagonists for the treatment of pain. Bioorganic and Medicinal Chemistry, 25(7), 2177-2190. doi:10.1016/j.bmc.2017.02.047

Turnaturi, R., Arico, G., Ronsisvalle, G., Parenti, C., \& Pasquinucci, L. (2016). Multitarget opioid ligands in pain relief: New players in an old game. European Journal of Medicinal Chemistry, 108, 211 228. doi:10.1016/j.ejmech.2015.11.028

Turner, P. V., Brabb, T., Pekow, C., \& Vasbinder, M. A. (2011). Administration of substances to laboratory animals: routes of administration and factors to consider. Journal of the American Association for Laboratory Animal Science : JAALAS, 50(5), 600-613.

Ueno, Y., Mori, A., \& Yanagita, T. (2013). One year long-term study on abuse liability of nalfurafine in hemodialysis patients. International Journal of Clinical Pharmacology and Therapeutics, 51(11), 823-831. doi:10.5414/CP201852

United Nations Office of Drug and Crime. (2017). World Drug Report. United Nations publication, Sales No. E.17.XI.6.

Vadivelu, N., Mitra, S., \& Hines, R. L. (2011). Peripheral opioid receptor agonists for analgesia: a comprehensive review. J Opioid Manag, $7(1), \quad$ 55-68. doi:10.1097/01.SA.0000415552.38044.37 
Valdes, L. J., 3rd. (1994). Salvia divinorum and the unique diterpene hallucinogen, Salvinorin (divinorin) A. Journal of Psychoactive Drugs, 26(3), 277-283. doi:10.1080/02791072.1994.10472441

Valdes, L. J., Diaz, J. L., \& Paul, A. G. (1983). Ethnopharmacology of ska Maria Pastora (Salvia divinorum, Epling and Jativa-M.). Journal of Ethnopharmacology, 7(3), 287-312. doi:10.1016/03788741(83)90004-1

Vallejo, R., Barkin, R. L., \& Wang, V. C. (2011). Pharmacology of opioids in the treatment of chronic pain syndromes. Pain Physician, 14(4), E343-360.

van den Bent, M. J., van Raaij-van den Aarssen, V. J., Verweij, J., Doorn, P. A., \& Sillevis Smitt, P. A. (1997). Progression of paclitaxel-induced neuropathy following discontinuation of treatment. Muscle \& Nerve, 20(6), 750-752. doi:10.1002/(SICI)1097-4598(199706)20:6<750::AIDMUS15>3.0.CO;2-Y

van Gelder, B. M., Tijhuis, M., Kalmijn, S., \& Kromhout, D. (2007). Fish consumption, n-3 fatty acids, and subsequent 5-y cognitive decline in elderly men: the Zutphen Elderly Study. American Journal of Clinical Nutrition, 85(4), 1142-1147. doi:10.1093/ajcn/85.4.1142

Vandamme, T. F. (2014). Use of rodents as models of human diseases. Journal of Pharmacy \& Bioallied Sciences, 6(1), 2-9. doi:10.4103/0975-7406.124301

Vanderah, T. W., Largent-Milnes, T., Lai, J., Porreca, F., Houghten, R. A., Menzaghi, F., Wisniewski, K., Stalewski, J., Sueiras-Diaz, J., Galyean, R., Schteingart, C., Junien, J. L., Trojnar, J., \& Riviere, P. J. (2008). Novel D-amino acid tetrapeptides produce potent antinociception by selectively acting at peripheral kappa-opioid receptors. European Journal of Pharmacology, 583(1), 6272. doi:10.1016/j.ejphar.2008.01.011

Vanderah, T. W., Laughlin, T., Lashbrook, J. M., Nichols, M. L., Wilcox, G. L., Ossipov, M. H., Malan, T. P., Jr., \& Porreca, F. (1996). Single intrathecal injections of dynorphin A or des-Tyr-dynorphins produce long-lasting allodynia in rats: blockade by MK-801 but not naloxone. Pain, 68(2-3), 275-281. doi:10.1016/S0304-3959(96)03225-3

Vanderah, T. W., Schteingart, C. D., Trojnar, J., Junien, J. L., Lai, J., \& Riviere, P. J. (2004). FE200041 (DPhe-D-Phe-D-Nle-D-Arg-NH2): A peripheral efficacious kappa opioid agonist with unprecedented selectivity. Journal of Pharmacology and Experimental Therapeutics, 310(1), 326-333. doi:10.1124/jpet.104.065391

Vane, J. R., \& Botting, R. M. (1998). Anti-inflammatory drugs and their mechanism of action. Inflammation Research, 47(2), 78-87.

Varadi, A., Marrone, G. F., Eans, S. O., Ganno, M. L., Subrath, J. J., Le Rouzic, V., Hunkele, A., Pasternak, G. W., McLaughlin, J. P., \& Majumdar, S. (2015). Synthesis and characterization of a dual kappa-delta opioid receptor agonist analgesic blocking cocaine reward behavior. ACS Chemical Neuroscience, 6(11), 1813-1824. doi:10.1021/acschemneuro.5b00153

Vardeh, D., \& Naranjo, J. F. (2017). Peripheral and Central Sensitization. In R. J. Yong, M. Nguyen, E. Nelson, \& R. D. Urman (Eds.), Pain Medicine: An Essential Review (pp. 15-17). Cham: Springer International Publishing.

Vartiainen, P., Heiskanen, T., Sintonen, H., Roine, R. P., \& Kalso, E. (2016). Health-related quality of life and burden of disease in chronic pain measured with the 15D instrument. Pain, 157(10), 22692276. doi:10.1097/j.pain.0000000000000641

Varvel, S. A., Bridgen, D. T., Tao, Q., Thomas, B. F., Martin, B. R., \& Lichtman, A. H. (2005). Delta9tetrahydrocannbinol accounts for the antinociceptive, hypothermic, and cataleptic effects of marijuana in mice. Journal of Pharmacology and Experimental Therapeutics, 314(1), 329-337. doi:10.1124/jpet.104.080739

Veigas, J. M., Williams, P. J., Halade, G., Rahman, M. M., Yoneda, T., \& Fernandes, G. (2011). Fish oil concentrate delays sensitivity to thermal nociception in mice. Pharmacological Research, 63(5), 377-382. doi:10.1016/j.phrs.2011.02.004

Victor, T. W., Hu, X., Campbell, J. C., Buse, D. C., \& Lipton, R. B. (2010). Migraine prevalence by age and sex in the United States: a life-span study. Cephalalgia, 30(9), 1065-1072. doi:10.1177/0333102409355601 
Vinals, X., Moreno, E., Lanfumey, L., Cordomi, A., Pastor, A., de La Torre, R., Gasperini, P., Navarro, G., Howell, L. A., Pardo, L., Lluis, C., Canela, E. I., McCormick, P. J., Maldonado, R., \& Robledo, P. (2015). Cognitive impairment induced by delta9-tetrahydrocannabinol occurs through heteromers between cannabinoid CB1 and serotonin 5-HT2A receptors. PLoS Biology, 13(7), e1002194. doi:10.1371/journal.pbio.1002194

Virtanen, J. K., Mursu, J., Voutilainen, S., Uusitupa, M., \& Tuomainen, T. P. (2014). Serum omega-3 polyunsaturated fatty acids and risk of incident type 2 diabetes in men: the Kuopio Ischemic Heart Disease Risk Factor study. Diabetes Care, 37(1), 189-196. doi:10.2337/dc13-1504

Viscusi, E. R., Webster, L., Kuss, M., Daniels, S., Bolognese, J. A., Zuckerman, S., Soergel, D. G., Subach, R. A., Cook, E., \& Skobieranda, F. (2016). A randomized, phase 2 study investigating TRV130, a biased ligand of the mu-opioid receptor, for the intravenous treatment of acute pain. Pain, 157(1), 264-272. doi:10.1097/j.pain.0000000000000363

Vitkova, J., Loucka, M., Bocek, J., \& Vaculin, S. (2015). The effect of acclimatization and ambient temperature on heat withdrawal threshold in rats. European Journal of Pain (London, England), 19(1), 21-27. doi:10.1002/ejp.515

Von Frey, M. (1896). Untersuchungen über die sinnesfunctionen der menschlichen haut: 1. abhandlung: Druckempfindung und schmerz (Vol. 23): S. Hirzel.

Von Voigtlander, P. F., \& Lewis, R. A. (1982). U-50,488, a selective kappa opioid agonist: comparison to other reputed kappa agonists. Progress in Neuro-Psychopharmacology and Biological Psychiatry, 6(4-6), 467-470. doi:10.1016/S0278-5846(82)80130-9

Vonvoigtlander, P. F., Lahti, R. A., \& Ludens, J. H. (1983). U-50,488: a selective and structurally novel non-Mu (kappa) opioid agonist. Journal of Pharmacology and Experimental Therapeutics, 224(1), 7-12.

Vowles, K. E., McEntee, M. L., Julnes, P. S., Frohe, T., Ney, J. P., \& van der Goes, D. N. (2015). Rates of opioid misuse, abuse, and addiction in chronic pain: a systematic review and data synthesis. Pain, 156(4), 569-576. doi:10.1097/01.j.pain.0000460357.01998.f1

Vunck, S. A., Snider, S. E., van den Oord, E. J. C. G., \& Beardsley, P. M. (2011). The kappa opioid receptor agonist, U50,488, exacerbates conditioned fear in mice. FASEB Journal, 25(1 Supplement), 617.620.

Walentiny, D. M., Vann, R. E., Warner, J. A., King, L. S., Seltzman, H. H., Navarro, H. A., Twine, C. E., Jr., Thomas, B. F., Gilliam, A. F., Gilmour, B. P., Carroll, F. I., \& Wiley, J. L. (2010). Kappa opioid mediation of cannabinoid effects of the potent hallucinogen, salvinorin $A$, in rodents. Psychopharmacology, 210(2), 275-284. doi:10.1007/s00213-010-1827-6

Walker, K. M., Urban, L., Medhurst, S. J., Patel, S., Panesar, M., Fox, A. J., \& McIntyre, P. (2003). The VR1 antagonist capsazepine reverses mechanical hyperalgesia in models of inflammatory and neuropathic pain. Journal of Pharmacology and Experimental Therapeutics, 304(1), 56-62. doi:10.1124/jpet.102.042010

Wang, Y. H., Sun, J. F., Tao, Y. M., Chi, Z. Q., \& Liu, J. G. (2010). The role of kappa-opioid receptor activation in mediating antinociception and addiction. Acta Pharmacologica Sinica, 31(9), 1065-1070. doi:10.1038/aps.2010.138

Wang, Y. J., Hang, A., Lu, Y. C., Long, Y., Zan, G. Y., Li, X. P., Wang, Q., Zhao, Z. X., He, L., Chi, Z. Q., \& Liu, J. G. (2016). kappa Opioid receptor activation in different brain regions differentially modulates anxiety-related behaviors in mice. Neuropharmacology, 110(Pt A), 92-101. doi:10.1016/j.neuropharm.2016.04.022

Wang, Z., Gardell, L. R., Ossipov, M. H., Vanderah, T. W., Brennan, M. B., Hochgeschwender, U., Hruby, V. J., Malan, T. P., Jr., Lai, J., \& Porreca, F. (2001). Pronociceptive actions of dynorphin maintain chronic neuropathic pain. Journal of Neuroscience, 21(5), 1779-1786. doi:10.1523/JNEUROSCI.21-05-01779.2001

Wani, M. C., Taylor, H. L., Wall, M. E., Coggon, P., \& McPhail, A. T. (1971). Plant antitumor agents. VI. The isolation and structure of taxol, a novel antileukemic and antitumor agent from Taxus 
brevifolia. Journal of the American Chemical Society, 93(9), 2325-2327. doi:10.1021/ja00738a045

Ward, S. J., McAllister, S. D., Kawamura, R., Murase, R., Neelakantan, H., \& Walker, E. A. (2014). Cannabidiol inhibits paclitaxel-induced neuropathic pain through 5-HT(1A) receptors without diminishing nervous system function or chemotherapy efficacy. British Journal of Pharmacology, 171(3), 636-645. doi:10.1111/bph.12439

Ward, S. J., Ramirez, M. D., Neelakantan, H., \& Walker, E. A. (2011). Cannabidiol prevents the development of cold and mechanical allodynia in paclitaxel-treated female C57BI6 mice. Anesthesia \& Analgesia, 113(4), 947-950. doi:10.1213/ANE.0b013e3182283486

Watanabe, H., Vriens, J., Prenen, J., Droogmans, G., Voets, T., \& Nilius, B. (2003). Anandamide and arachidonic acid use epoxyeicosatrienoic acids to activate TRPV4 channels. Nature, 424(6947), 434-438. doi:10.1038/nature01807

Weaver, B. A. (2014). How Taxol/paclitaxel kills cancer cells. Molecular Biology of the Cell, 25(18), 2677-2681. doi:10.1091/mbc.E14-04-0916

Welsh, S. A. (2017). The effect of novel kappa opioid peptide receptor agonists on learning and memory in rats. Master's degree in Biomedical Science, Victoria University of Wellington.

Wemmie, J. A., Taugher, R. J., \& Kreple, C. J. (2013). Acid-sensing ion channels in pain and disease. Nature Reviews: Neuroscience, 14(7), 461-471. doi:10.1038/nrn3529

Wetzel, C., Hu, J., Riethmacher, D., Benckendorff, A., Harder, L., Eilers, A., Moshourab, R., Kozlenkov, A., Labuz, D., Caspani, O., Erdmann, B., Machelska, H., Heppenstall, P. A., \& Lewin, G. R. (2007). A stomatin-domain protein essential for touch sensation in the mouse. Nature, 445(7124), 206-209. doi:10.1038/nature05394

White, K. L., Robinson, J. E., Zhu, H., DiBerto, J. F., Polepally, P. R., Zjawiony, J. K., Nichols, D. E., Malanga, C. J., \& Roth, B. L. (2015). The G protein-biased kappa-opioid receptor agonist RB-64 is analgesic with a unique spectrum of activities in vivo. Journal of Pharmacology and Experimental Therapeutics, 352(1), 98-109. doi:10.1124/jpet.114.216820

Wilson, J. L., Nayanar, V., \& Walker, J. S. (1996). The site of anti-arthritic action of the kappa-opioid, $\mathrm{U}-50,488 \mathrm{H}$, in adjuvant arthritis: importance of local administration. British Journal of Pharmacology, 118(7), 1754-1760. doi:10.1111/j.1476-5381.1996.tb15601.x

Wilson, W. C., Simon, J., \& Garst, J. E. (1990). The effects of selected bulky substituents on the pulmonary toxicity of 3-furyl ketones in mice. Journal of Animal Science, 68(4), 1072-1076.

Wise, L. E., Cannavacciulo, R., Cravatt, B. F., Martin, B. F., \& Lichtman, A. H. (2008). Evaluation of fatty acid amides in the carrageenan-induced paw edema model. Neuropharmacology, 54(1), 181188. doi:10.1016/j.neuropharm.2007.06.003

Wittert, G., Hope, P., \& Pyle, D. (1996). Tissue distribution of opioid receptor gene expression in the rat. Biochemical and Biophysical Research Communications, 218(3), 877-881. doi:10.1006/bbrc.1996.0156

Wood, J. T., Williams, J. S., Pandarinathan, L., Janero, D. R., Lammi-Keefe, C. J., \& Makriyannis, A. (2010). Dietary docosahexaenoic acid supplementation alters select physiological endocannabinoid-system metabolites in brain and plasma. Journal of Lipid Research, 51(6), 1416-1423. doi:10.1194/jlr.M002436

Woolf, C. J. (2010). What is this thing called pain? Journal of Clinical Investigation, 120(11), 3742-3744. doi:10.1172/JCl45178

Woolf, C. J. (2011). Central sensitization: implications for the diagnosis and treatment of pain. Pain, 152(3 Suppl), S2-15. doi:10.1016/j.pain.2010.09.030

Woolfe, G., \& Macdonald, A. D. (1944). The evaluation of the analgesic action of Pethidine hydrochloride (Demerol). Journal of Pharmacology and Experimental Therapeutics, 80(3), 300307.

Wozniak, K. M., Vornov, J. J., Wu, Y., Nomoto, K., Littlefield, B. A., DesJardins, C., Yu, Y., Lai, G., Reyderman, L., Wong, N., \& Slusher, B. S. (2016). Sustained accumulation of microtubulebinding chemotherapy drugs in the peripheral nervous system: Correlations with time course 
and neurotoxic severity. Cancer Research, 76(11), 3332-3339. doi:10.1158/0008-5472.CAN15-2525

Wu, H., Wacker, D., Mileni, M., Katritch, V., Han, G. W., Vardy, E., Liu, W., Thompson, A. A., Huang, X. P., Carroll, F. I., Mascarella, S. W., Westkaemper, R. B., Mosier, P. D., Roth, B. L., Cherezov, V., \& Stevens, R. C. (2012a). Structure of the human kappa-opioid receptor in complex with JDTic. Nature, 485(7398), 327-332. doi:10.1038/nature10939

Wu, J. H., Micha, R., Imamura, F., Pan, A., Biggs, M. L., Ajaz, O., Djousse, L., Hu, F. B., \& Mozaffarian, D. (2012b). Omega-3 fatty acids and incident type 2 diabetes: a systematic review and metaanalysis. British Journal of Nutrition, 107 Suppl 2(S2), S214-227. doi:10.1017/S0007114512001602

Wu, S., Ding, Y., Wu, F., Li, R., Hou, J., \& Mao, P. (2015). Omega-3 fatty acids intake and risks of dementia and Alzheimer's disease: a meta-analysis. Neuroscience \& Biobehavioral Reviews, 48, 1-9. doi:10.1016/j.neubiorev.2014.11.008

Xiao, W., Boroujerdi, A., Bennett, G. J., \& Luo, Z. D. (2007). Chemotherapy-evoked painful peripheral neuropathy: analgesic effects of gabapentin and effects on expression of the alpha-2-delta type-1 calcium channel subunit. Neuroscience, 144(2), 714-720. doi:10.1016/j.neuroscience.2006.09.044

Xiao, W. H., Zheng, F. Y., Bennett, G. J., Bordet, T., \& Pruss, R. M. (2009). Olesoxime (cholest-4-en-3one, oxime): analgesic and neuroprotective effects in a rat model of painful peripheral neuropathy produced by the chemotherapeutic agent, paclitaxel. Pain, 147(1-3), 202-209. doi:10.1016/j.pain.2009.09.006

Xiao, W. H., Zheng, H., Zheng, F. Y., Nuydens, R., Meert, T. F., \& Bennett, G. J. (2011). Mitochondrial abnormality in sensory, but not motor, axons in paclitaxel-evoked painful peripheral neuropathy in the rat. Neuroscience, 199, 461-469. doi:10.1016/j.neuroscience.2011.10.010

Xie, G. X., Meng, F., Mansour, A., Thompson, R. C., Hoversten, M. T., Goldstein, A., Watson, S. J., \& Akil, H. (1994). Primary structure and functional expression of a guinea pig kappa opioid (dynorphin) receptor. Proceedings of the National Academy of Sciences of the United States of America, 91(9), 3779-3783. doi:10.1073/pnas.91.9.3779

Xin, L., Geller, E. B., \& Adler, M. W. (1997). Body temperature and analgesic effects of selective mu and kappa opioid receptor agonists microdialyzed into rat brain. Journal of Pharmacology and Experimental Therapeutics, 281(1), 499-507.

Xu, H., Blair, N. T., \& Clapham, D. E. (2005). Camphor activates and strongly desensitizes the transient receptor potential vanilloid subtype 1 channel in a vanilloid-independent mechanism. Journal of Neuroscience, 25(39), 8924-8937. doi:10.1523/JNEUROSCI.2574-05.2005

Xu, H., Delling, M., Jun, J. C., \& Clapham, D. E. (2006). Oregano, thyme and clove-derived flavors and skin sensitizers activate specific TRP channels. Nature Neuroscience, 9(5), 628-635. doi:10.1038/nn1692

Xu, M., Petraschka, M., McLaughlin, J. P., Westenbroek, R. E., Caron, M. G., Lefkowitz, R. J., Czyzyk, T. A., Pintar, J. E., Terman, G. W., \& Chavkin, C. (2004). Neuropathic pain activates the endogenous kappa opioid system in mouse spinal cord and induces opioid receptor tolerance. Journal of Neuroscience, 24(19), 4576-4584. doi:10.1523/JNEUROSCI.5552-03.2004

Yamamizu, K., Hamada, Y., \& Narita, M. (2015). kappa Opioid receptor ligands regulate angiogenesis in development and in tumours. British Journal of Pharmacology, 172(2), 268-276. doi:10.1111/bph.12573

Yamamoto, T., Ohno, M., \& Ueki, S. (1988). A selective kappa-opioid agonist, U-50,488H, blocks the development of tolerance to morphine analgesia in rats. European Journal of Pharmacology, 156(1), 173-176. doi:10.1016/0014-2999(88)90162-8

Yan, F., Bikbulatov, R. V., Mocanu, V., Dicheva, N., Parker, C. E., Wetsel, W. C., Mosier, P. D., Westkaemper, R. B., Allen, J. A., Zjawiony, J. K., \& Roth, B. L. (2009). Structure-based design, synthesis, and biochemical and pharmacological characterization of novel salvinorin $A$ 
analogues as active state probes of the kappa-opioid receptor. Biochemistry, 48(29), 68986908. doi:10.1021/bi900605n

Yasuda, K., Raynor, K., Kong, H., Breder, C. D., Takeda, J., Reisine, T., \& Bell, G. I. (1993). Cloning and functional comparison of kappa and delta opioid receptors from mouse brain. Proceedings of the National Academy of Sciences of the United States of America, 90(14), 6736-6740. doi:10.1073/pnas.90.14.6736

Yezierski, R. P., \& Park, S. H. (1993). The mechanosensitivity of spinal sensory neurons following intraspinal injections of quisqualic acid in the rat. Neuroscience Letters, 157(1), 115-119. doi:10.1016/0304-3940(93)90656-6

Yilmaz, E., \& Gold, M. S. (2015). Sensory neuron subpopulation-specific dysregulation of intracellular calcium in a rat model of chemotherapy-induced peripheral neuropathy. Neuroscience, 300 , 210-218. doi:10.1016/j.neuroscience.2015.05.019

Yilmaz, E., Watkins, S. C., \& Gold, M. S. (2017). Paclitaxel-induced increase in mitochondrial volume mediates dysregulation of intracellular $\mathrm{Ca}(2+)$ in putative nociceptive glabrous skin neurons from the rat. Cell Calcium, 62, 16-28. doi:10.1016/j.ceca.2017.01.005

Yogev, S., Cooper, R., Fetter, R., Horowitz, M., \& Shen, K. (2016). Microtubule Organization Determines Axonal Transport Dynamics. Neuron, 92(2), 449-460. doi:10.1016/j.neuron.2016.09.036

Young, D. R. (2015). Pre-clinical anti-addictive and side-effect profiles of novel kappa-opioid agonists. Master's degree in Biomedical Science, Victoria University of Wellington.

Zador, F., Lenart, N., Csibrany, B., Santha, M., Molnar, M., Tuka, B., Samavati, R., Klivenyi, P., Vecsei, L., Marton, A., Vizler, C., Nagy, G. M., Borsodi, A., Benyhe, S., \& Paldy, E. (2015). Low dosage of rimonabant leads to anxiolytic-like behavior via inhibiting expression levels and G-protein activity of kappa opioid receptors in a cannabinoid receptor independent manner. Neuropharmacology, 89, 298-307. doi:10.1016/j.neuropharm.2014.10.008

Zasadil, L. M., Andersen, K. A., Yeum, D., Rocque, G. B., Wilke, L. G., Tevaarwerk, A. J., Raines, R. T., Burkard, M. E., \& Weaver, B. A. (2014). Cytotoxicity of paclitaxel in breast cancer is due to chromosome missegregation on multipolar spindles. Science Translational Medicine, 6(229), 229ra243. doi:10.1126/scitrans/med.3007965

Zeynalov, E., Nemoto, M., Hurn, P. D., Koehler, R. C., \& Bhardwaj, A. (2006). Neuroprotective effect of selective kappa opioid receptor agonist is gender specific and linked to reduced neuronal nitric oxide. Journal of Cerebral Blood Flow and Metabolism, 26(3), 414-420. doi:10.1038/sj.jcbfm.9600196

Zhang, H., Boyette-Davis, J. A., Kosturakis, A. K., Li, Y., Yoon, S. Y., Walters, E. T., \& Dougherty, P. M. (2013). Induction of monocyte chemoattractant protein-1 (MCP-1) and its receptor CCR2 in primary sensory neurons contributes to paclitaxel-induced peripheral neuropathy. Journal of Pain, 14(10), 1031-1044. doi:10.1016/j.jpain.2013.03.012

Zhang, H., \& Dougherty, P. M. (2014). Enhanced excitability of primary sensory neurons and altered gene expression of neuronal ion channels in dorsal root ganglion in paclitaxel-induced peripheral neuropathy. Anesthesiology, 120(6), 1463-1475. doi:10.1097/ALN.0000000000000176

Zhang, H., Li, Y., de Carvalho-Barbosa, M., Kavelaars, A., Heijnen, C. J., Albrecht, P. J., \& Dougherty, P. M. (2016). Dorsal root ganglion infiltration by macrophages contributes to paclitaxel chemotherapy-induced peripheral neuropathy. Journal of Pain, 17(7), 775-786. doi:10.1016/j.jpain.2016.02.011

Zhang, H., Yoon, S. Y., Zhang, H., \& Dougherty, P. M. (2012). Evidence that spinal astrocytes but not microglia contribute to the pathogenesis of Paclitaxel-induced painful neuropathy. Journal of Pain, 13(3), 293-303. doi:10.1016/j.jpain.2011.12.002

Zhang, L. S., Wang, J., Chen, J. C., Tao, Y. M., Wang, Y. H., Xu, X. J., Chen, J., Xu, Y. G., Xi, T., Hu, X. W., Wang, Y. J., \& Liu, J. G. (2015). Novel kappa-opioid receptor agonist MB-1C-OH produces potent analgesia with less depression and sedation. Acta Pharmacologica Sinica, 36(5), 565571. doi:10.1038/aps.2014.145 
Zhang, N., Inan, S., Cowan, A., Sun, R., Wang, J. M., Rogers, T. J., Caterina, M., \& Oppenheim, J. J. (2005a). A proinflammatory chemokine, CCL3, sensitizes the heat- and capsaicin-gated ion channel TRPV1. Proceedings of the National Academy of Sciences of the United States of America, 102(12), 4536-4541. doi:10.1073/pnas.0406030102

Zhang, X., Li, L., \& McNaughton, P. A. (2008). Proinflammatory mediators modulate the heat-activated ion channel TRPV1 via the scaffolding protein AKAP79/150. Neuron, 59(3), 450-461. doi:10.1016/j.neuron.2008.05.015

Zhang, Y., Butelman, E. R., Schlussman, S. D., Ho, A., \& Kreek, M. J. (2005b). Effects of the plant-derived hallucinogen salvinorin $A$ on basal dopamine levels in the caudate putamen and in a conditioned place aversion assay in mice: agonist actions at kappa opioid receptors. Psychopharmacology, 179(3), 551-558. doi:10.1007/s00213-004-2087-0

Zheng, H., Xiao, W. H., \& Bennett, G. J. (2011). Functional deficits in peripheral nerve mitochondria in rats with paclitaxel- and oxaliplatin-evoked painful peripheral neuropathy. Experimental Neurology, 232(2), 154-161. doi:10.1016/j.expneurol.2011.08.016

Zheng, J. (2013). Molecular mechanism of TRP channels. Comprehensive Physiology, 3(1), 221-242. doi:10.1002/cphy.c120001

Zhou, Y., Crowley, R. S., Ben, K., Prisinzano, T. E., \& Kreek, M. J. (2017). Synergistic blockade of alcohol escalation drinking in mice by a combination of novel kappa opioid receptor agonist Mesyl Salvinorin B and naltrexone. Brain Research, 1662, 75-86. doi:10.1016/j.brainres.2017.02.027

Zhu, J., Chen, C., Xue, J. C., Kunapuli, S., DeRiel, J. K., \& Liu-Chen, L. Y. (1995). Cloning of a human kappa opioid receptor from the brain. Life Sciences, 56(9), PL201-207. doi:10.1016/00243205(94)00507-0

Zollner, C., Shaqura, M. A., Bopaiah, C. P., Mousa, S., Stein, C., \& Schafer, M. (2003). Painful inflammation-induced increase in mu-opioid receptor binding and G-protein coupling in primary afferent neurons. Molecular Pharmacology, 64(2), 202-210. doi:10.1124/mol.64.2.202 